\title{
Mapping of Corrosion Sites in Advanced Gas-cooled Reactor Fuel Cladding in Long Term Pond Storage
}

\author{
Clark, Ronald N.
}

How to cite:

Clark, Ronald N. (2018) Mapping of Corrosion Sites in Advanced Gas-cooled Reactor Fuel Cladding in Long Term Pond Storage. Doctoral thesis, Swansea University.

http://cronfa.swan.ac.uk/Record/cronfa40783

Use policy:

This item is brought to you by Swansea University. Any person downloading material is agreeing to abide by the terms of the repository licence: copies of full text items may be used or reproduced in any format or medium, without prior permission for personal research or study, educational or non-commercial purposes only. The copyright for any work remains with the original author unless otherwise specified. The full-text must not be sold in any format or medium without the formal permission of the copyright holder. Permission for multiple reproductions should be obtained from the original author.

Authors are personally responsible for adhering to copyright and publisher restrictions when uploading content to the repository.

Please link to the metadata record in the Swansea University repository, Cronfa (link given in the citation reference above.)

http://www.swansea.ac.uk/library/researchsupport/ris-support/ 


\section{MAPPING OF CORROSION SITES IN \\ ADVANCED GAS-COOLED REACTOR FUEL \\ CLADDING FOR LONG TERM POND \\ STORAGE}

Ronald Nuuchin Clark, MPhys.

789741

Academic Supervisor: Prof. Geraint Williams, Swansea University

Industrial Supervisor: Dr. Steve Walters, National Nuclear Laboratory

Industrial Sponsor: Nuclear Decommissioning Authority Discipline: Materials Engineering

Submitted to Swansea University in fulfilment of the requirements for the Degree of Doctor of Philosophy

Materials Engineering

College of Engineering

Swansea University 


\begin{abstract}
This thesis aims to investigate sites where corrosion initiates on an austenitic stainless steel used to encapsulate advanced gas-cooled nuclear reactor fuel when in long term pond storage and subsequently provide insight into what happens when corrosion propagates. The combination of both a critical temperature and radiation dose within the reactor core may lead to very small quantities of this stainless steel becoming sensitised. The first chapter is a discussion on the literature and past study on the subject. Within the second chapter, analysis of the stainless steel in an as-received format is undertaken, and is relevant, given that only a very small quantity of cladding may be rendered sensitised. This chapter includes an investigation into $\mathrm{Cl}^{-}$concentration and temperature dependence. The third chapter focuses on identifying the initiation points of localised intergranular corrosion (IGC) in freely corroding and perturbed environments. An in-situ time-lapse microscopy and scanning vibrating electrode technique investigation are used in conjunction with post corrosion microscopy to study areas affected by IGC. The fourth chapter describes a higher resolution approach to investigating the initiation point of IGC. A scanning Kelvin probe force microscope is used to map the surfaces for areas in which corrosion may occur, while an atomic force microscope was used to provide high resolution images of tens of grains undergoing in-situ IGC. The aim of the final chapter is to provide an insight into reducing corrosion rates using an $\mathrm{NaOH}$ pre-treatment. $\mathrm{NaOH}$ is used in two different concentrations to study the effect on the free corrosion and breakdown potentials $\left(E_{\text {corr }}\right.$ and $E_{b}$ ) on as-received and sensitised 20/25/Nb. This study will provide a scientific backbone that the industry may use to target areas of further research, helping provide ongoing safe storage of spent nuclear fuel in the UK.
\end{abstract}




\section{Declaration}

This work has not previously been accepted in substance for any degree and is not being concurrently submitted in candidature for any degree.

Signed: (Candidate) Date:

\section{Statement 1}

This thesis is the result of my own investigations, except where otherwise stated. Where correction services have been used, the extent and nature of the correction is clearly marked in a footnote(s). Other sources are acknowledged by footnotes giving explicit references. A bibliography is appended.

Signed:

(Candidate) Date:

\section{Statement 2}

I hereby give consent for my thesis, if accepted, to be available for photocopying and for inter-library loan, and for the title and summary to be made available to outside organisations.

Signed: (Candidate) Date: 


\section{Acknowledgments}

I would to take this opportunity to extend my gratitude to the Nuclear Decommissioning Authority (NDA) for their funding through their PhD bursary scheme. They have provided not only an interesting project in which to research, but also through their annual seminar, provided a stage in which to advertise the work in this thesis to others. The NDA provide the time for a focus group meeting once a year, typically held at a nuclear licensed site, and also time for a one-to-one meeting with an appointed industrial supervisor. I would like to acknowledge the National Nuclear Laboratory for providing an industrial supervisor who helped coordinate the project (meetings, technical Q\&A, sourcing materials) on behalf of the sponsor. A thank you to Springfields Fuels Limited (Westinghouse Electric UK) for providing SS samples. Also a thank you to May Chan of Manchester University for providing both her time and equipment for thermal processing of specimens in the early stages of this project.

Furthermore I would like to thank Swansea University's two schemes SPECIFIC and MACH1 for their use of facilities and equipment. I offer a thank you to Dr. Justin Searle who helped provide many hours of training and help in setting up the time-lapse atomic force microscopy equipment. He has helped image corrosion in-situ on the scale of tens of grains.

In particular I would like to gratefully acknowledge my academic supervisor Prof. Geraint Williams who was a source for ideas throughout the duration of the project. He provided me with a constant stream of suggestions and helped correlate ideas in which to pursue the task of researching the beast that is corrosion. I would also like to thank my industrial supervisor, Dr. Steve Walters, for providing an industrial focus all the way through the project. He kept the project exciting, giving interesting avenues in which the project could be taken.

I would like to mention my secondary school Physics teacher Mr. Will Davies for inspiring me to pursue a career in the sciences. I never would have taken the opportunity to become a scientist were it not for him saying 'you can do it'.

Finally I would like to dedicate this thesis to my family, but most importantly my parents Christopher and Bunsin Clark for supporting me throughout my life, they have always been there when I have needed them. 


\section{Table of Contents}

0.1 Glossary . . . . . . . . . . . . . . . . . . xvii

1 Literature Review $\quad 1$

1.1 The Advanced Gas-cooled Reactor . . . . . . . . . . . . . . 2

1.1 .1 History . . . . . . . . . . . . . . . . . 2

1.1 .2 Fuel Cladding . . . . . . . . . . . . . . . . . . . 3

1.1.3 AGR Fuel Element Manufacture . . . . . . . . . . . . 5

1.1.4 Spent AGR Fuel Storage . . . . . . . . . . . . . . . . . 7

1.1.5 Carbon Deposition ... . . . . . . . . . . . . . 11

1.1.6 AGR Stainless Steels in Sodium Hydroxide . . . . . . . . . 13

1.2 Fundamentals of Corrosion . . . . . . . . . . . . . . . . 20

1.2.1 Thermodynamics . . . . . . . . . . . . . . 20

1.2.2 Nernst Equation and Pourbaix . . . . . . . . . . . . . 23

1.2.3 Electrochemical Kinetics and Mixed Potential Theory . . . 30

1.3 Localised Corrosion . . . . . . . . . . . . . . . . . . . . 35

1.3.1 Pitting Corrosion . . . . . . . . . . . . . 35

1.3.2 Intergranular Corrosion . . . . . . . . . . . . . . 40

2 Experimental Techniques and Procedures $\quad 55$

2.1 Specimen Preparation . . . . . . . . . . . . . . . . 55

2.2 Heat Treatment . . . . . . . . . . . . . . . . . 56

2.2.1 DL-EPR Experimentation . . . . . . . . . . . . 56

2.3 DC Electrochemistry Experiments . . . . . . . . . . . . . 60

2.3.1 Reference Electrodes . . . . . . . . . . . . . . 62

2.4 SVET . . . . . . . . . . . . . . . . . . 63

2.5 Scanning Probe Microscopy . . . . . . . . . . . . . . . . . . 69

2.6 Overview of Experiments Undertaken in this Thesis . . . . . . . . 70

3 Corrosion of 20/25/Nb Stainless Steel Studied using DC Electrochemistry and SVET Methods $\quad \mathbf{7 5}$

3.1 Introduction . . . . . . . . . . . . . . . . . . 76

3.2 Experimental . . . . . . . . . . . . . . . . . . . 77

3.3 Unsensitised Stainless Steel . . . . . . . . . . . . . . . . . . . 82

3.3.1 Chloride Dependence . . . . . . . . . . . . . . . . 82

3.3.2 Temperature Dependence . . . . . . . . . . . . . 87

3.3.3 SVET Crevice Corrosion . . . . . . . . . . . . . . . . . 92

3.3.4 Green and Blue Cladding . . . . . . . . . . . . . . . . 95

3.4 Thermally Sensitised Stainless Steel . . . . . . . . . . . . . . . . 99

3.4.1 Degree of Sensitisation . . . . . . . . . . . . . . . 99 
3.4.2 Grain Size Calculation . . . . . . . . . . . . . . . 103

3.4.3 Analysis of Scale . . . . . . . . . . . . . . . . . 107

3.4.4 SVET Analysis of Specimens Containing Scale . . . . . . . 116

3.4.5 Investigation of Carbon Coated SS . . . . . . . . . . 125

3.4.6 Effects of Crevice Formers on Sensitised Tube . . . . . . . 127

3.5 Conclusions . . . . . . . . . . . . . . . . . . 133

4 Where does IGC Initiate? A Combined SVET and Time-Lapse Microscopy Approach 137

4.1 Introduction . . . . . . . . . . . . . . . . . . . . . . . . . 138

4.2 Experimental . . . . . . . . . . . . . . . . . . . . . 139

4.3 Results and Discussion . . . . . . . . . . . . . . . . . . . . 142

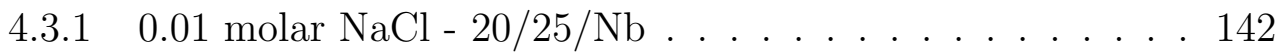

4.3.2 3 molar $\mathrm{NaCl}-20 / 25 / \mathrm{Nb}$. . . . . . . . . . . . . . 148

4.3.3 Sensitised 310 Stainless Steel in 0.2 molar $\mathrm{NaCl}$. . . . . . 154

4.3.4 SVET Analysis - 1000 ppm Chloride - Sensitised 20/25/Nb

Stainless Steel . . . . . . . . . . . . . . . 157

4.4 Conclusions . . . . . . . . . . . . . . . . . . . . . 173

5 A Combined in-situ Investigation into Intergranular Corrosion $\begin{array}{lr}\text { on Stainless Steel } & 176\end{array}$

5.1 Introduction . . . . . . . . . . . . . . . . . . . 177

5.2 Experimental . . . . . . . . . . . . . . . . . . . . 179

5.2.1 Specimen and Solution Preparation . . . . . . . . . . 179

5.2.2 In-situ Time-Lapse Microscopy . . . . . . . . . . . . . . . 180

5.2 .3 Atomic Force Microscopy . . . . . . . . . . . . . . . . . . . 180

5.2.4 Scanning Kelvin Probe Force Microscopy . . . . . . . . . . 181

5.2.5 AFM Post Processing . . . . . . . . . . . . . . . . . . . . . 182

5.3 Results and Discussion . . . . . . . . . . . . . . . . . . . . . 182

5.3 .1 In-situ TLM . . . . . . . . . . . . . . . . . . . . . . . . 182

5.3.2 In-situ Electrochemical Atomic Force Microscopy . . . . . 191

5.4 Conclusions . . . . . . . . . . . . . . . . . . 195

6 Inhibition of IGC: The Caustic Dosing Approach 197

6.1 Introduction . . . . . . . . . . . . . . . . . . . . 198

6.1.1 Experimental Procedure . . . . . . . . . . . . . . . 199

6.2 Study 1: The Effect of $\mathrm{NaOH}$ on Unsensitised SS . . . . . . . . . 201

6.2.1 Experimental Procedure . . . . . . . . . . . . . . 201

6.2 .2 Results and Discussion . . . . . . . . . . . . . . . 201

6.3 Study 2: The Effect of $\mathrm{NaOH}$ on Sensitised SS . . . . . . . . . . 207

6.3 .1 Introduction . . . . . . . . . . . . . . 207

6.3.2 Experimental Procedure . . . . . . . . . . . . . . 207

6.3.3 Results and Discussion . . . . . . . . . . . . . . . . . 208

6.4 Study 3: An In-situ Experiment Using $\mathrm{NaOH}$ as a Pretreatment for Corrosion Inhibition ． . . . . . . . . . . . . . . 219

6.4 Introduction . . . . . . . . . . . . . . . . . . 219

6.4.2 Experimental Procedure . . . . . . . . . . . . . . . . 219

6.4.3 Results and Discussion . . . . . . . . . . . . . . . . . 220

6.5 Conclusions . . . . . . . . . . . . . . . . . . . . . . . 239 
7 Conclusions and Discussion of Future Work

8 Bibliography

248 


\section{List of Figures}

1.1 Fuel element at EDF Barnwood . . . . . . . . . . . . . . . . 3

1.2 AGR fuel production at Springfields Ltd. [29] . . . . . . . . . 6

1.3 Cross section of the AGR fuel element showing fuel pins [30] . . 8

1.4 Fuel cladding conditions as a function of distance for stringer located at Hinkley Point B, B4286 [33] . . . . . . . . . . . . . 9

1.5 The current Sellafield spent fuel route, 2011 Sellafield Plan [38] . . 10

1.6 Corrosion rate as a function of Cr content within a general SS [44]. An exponential decrease in corrosion rate leads to negligible corrosion once the minimum $\approx 12.5 \% \mathrm{Cr}$ is present. . . . . . . . . . 14

1.7 Pourbaix diagram for $\mathrm{Fe}[16] \ldots \ldots$. . . . . . . . . . . 15

$1.8 \mathrm{NaOH}$ isocorrosion diagram, solid lines represent a corrosion rate of $0.1 \mathrm{~mm} \cdot \mathrm{yr}-1$. S30400, S31600 and N08904 are ANSI types 304, 316, 904 respectively. Without chloride [44] . . . . . . . . . 15

1.9 Major alloying elements of a general SS (pure Fe, Ni, Cr and Mo) in $150 \mathrm{~g} \mathrm{dm}^{-3}\left(3.75 \mathrm{~mol} \mathrm{dm}^{-3}\right) \mathrm{NaOH}$ at $90^{\circ} \mathrm{C}[49] \ldots . . . . .16$

1.10 Pourbaix diagram of elemental $\mathrm{Nb}$ with a dissolved metal ion concentration of $10^{-5} \mathrm{~mol}[52]$. Note that this diagram is for elemental $\mathrm{Nb}$, not $\mathrm{NbC}$.

1.11 Peak current densities as a function of $\mathrm{NaOH}$ concentration and temperature, obtained from Robin [53] . . . . . . . . . 20

1.12 Free energy curve taken from Trethewey and Chamberlain [10] . . 22

1.13 Schematic of a wet corrosion cell. (i) Illustrates the case of two different metals immersed in the same electrolyte, but connected together. For example Fe and Zn. (ii) Illustrates the case where a single metal is immersed, with anodes and cathodes separated spatially, but on the same metal. A represents the anode (i.e. Zn), $\mathrm{C}$ represents the cathode (i.e. Fe), the current flux is the flux of electrons through the metal (ii) or metal contact in case (i), the diffusion of metal cations is shown by the ionic flux within the electrolyte. This schematic was recreated from Akid [56]. . . . . .

1.14 Pourbaix diagram of Fe with soluble species concentration $10^{-5}$ mol $d m^{-3}$ and common stable oxides present at $298 \mathrm{~K}[16]$. . . .

1.15 Pourbaix diagram of $\mathrm{Cr}$ with soluble species concentration $10^{-5}$ mol $d m^{-3}$ and common stable oxides present at $298 \mathrm{~K}[16] \ldots$

1.16 Pourbaix diagram of $\mathrm{Ni}$ with soluble species concentration $10^{-5}$ mol $d m^{-3}$ and common stable oxides present at $298 \mathrm{~K}[16]$. . . .

1.17 Pourbaix diagram for austenitic stainless steel (Fe, Cr, Ni) with soluble Fe, Cr, Ni species concentration $10^{-5} \mathrm{~mol} \mathrm{dm}^{-3}$ and common stable oxides present at $573 \mathrm{~K}\left(300{ }^{\circ} \mathrm{C}\right)[16] \ldots . . . . .$. 
1.18 The exponential relationship between current density and polarisation.

1.19 A Tafel plot showing the relationship between polarisation and current is linear if the polarisation is plotted against the logarithm of current density. The diagonal lines represent the individual anodic metal oxidation reaction and the cathodic metal reduction reaction occurring at the electrode. . . . . . . . . . . . .

1.20 An Evans diagram for a system undergoing the oxygen reduction reaction. This graph shows all four reactions occurring on the electrodes, which have been added for ease of the viewer. . . . . .

1.21 Separation between anode and cathode which can occur in pitting corrosion. . . . . . . . . . . . . . . .

1.22 Tomograms of 304 stainless steel pin after (a) 1 minute, (b) 6 minutes. The pin was exposed to $1 \mathrm{Mol} d m^{-3} \mathrm{NaCl}$ and polarised galvanostatically to $500 \mu \mathrm{A}$. Metal is white [71]. . . . . . . . 38

1.23 Inverse Kirkendall Mechanism [101] . . . . . . . . . . . . . . . . . 43

1.24 Concentration of $\mathrm{Cr}$ as a function of temperature for a range of different stainless steels. From [104] . . . . . . . . . . . 43

1.25 AGR fuel stringer (Hinkley point B) . . . . . . . . . . . . . 44

1.26 Line profiles across grain boundary from the lower elements for inservice fuel stringer B4286. The irradiation temperature is given

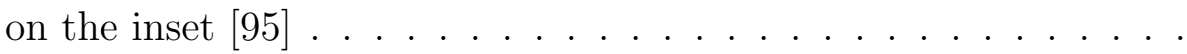

1.27 Line profiles across grain boundary for the same in-service fuel stringer B4286 for the upper elements. Note the $\mathrm{x}$ axis is of a different scale to the previous image $[95] \ldots \ldots . . . . .$.

1.28 Line profiles across grain boundary from an in-service, low burn-up fuel stringer (D1433) at different locations along the stringer [95] .

1.29 Compositional profile across grain boundary. Before (left), and after (right) post irradiation anneal $[95] \ldots . . . . . . .$.

1.30 Modelled and predicted compositional profiles across the grain boundary of different positions of an in-service stringer. The units on the y-axis are in atomic percent (at.\%). 13.2 at.\% corresponds to 12 wt.\% [107] [108] . . . . . . . . . . . . . . .

1.31 Time-temperature-precipitation curves for 20/25/Nb stainless steel [113] 53

2.1 KSCN concentration effects on the microstructure of 304 SS during

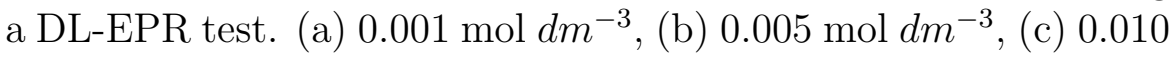
mol $d m^{-3}$, (d) $0.050 \mathrm{~mol} d m^{-3}$. [118] . . . . . . . . . . . . 58

2.2 The relationship between the log current and potential for the DLEPR test [117]. . . . . . . . . . . . . . . . . . . . . . . 59

2.3 Vickers hardness indent used for depth analysis . . . . . . . . . 59

2.4 Example potentiodynamic curve (shown with a starting and final potential). . . . . . . . . . . . . 63

2.5 The saturated calomel electrode $[58] \ldots \ldots$

2.6 Diagram of the SVET probe and speaker set-up obtained from Williams $[87] \ldots \ldots \ldots \ldots \ldots \ldots$ 
2.7 SVET tube cell used for calibration. Two Pt electrodes are seperated by a narrow vertical tube. This image was obtained from Williams [87] . . . . . . . . . . . . . . . .

2.8 Typical SVET calibration plot of SVET detection signal against user selected current density. There exists a linear relationship over a range of current density values . . . . . . . . . . .

3.1 Tight fitting o-rings were used to introduce crevice corrosion. 4 o-rings were used within a maximum tube length of $1.5 \mathrm{~cm} . . .$.

3.2 Comparison of typical potentiodynamic curves for unsensitised SS in a range of chloride ion concentrations. . . . . . . . . . . . 84

3.3 Graph showing the relationship between $\left[\mathrm{Cl}^{-}\right]$and potential. OCP values are from separate OCP experiments and are shown in table 3.186

$3.4\left[\mathrm{Cl}^{-}\right]$dependence at $60{ }^{\circ} \mathrm{C}$ at $\mathrm{pH} 5.8$ for 316L and $904 \mathrm{~L}$ stainless steels $[130] \ldots \ldots \ldots$. . . . . . . . . . . . . . . . . . . . . 88

3.5 Temperature dependence of $E_{b}\left(-7.3 m V K^{-1}\right)$ and $E_{\text {corr }}\left(0.65 \mathrm{mVK}^{-1}\right)$ observed in fixed, $1 \mathrm{~mol} \mathrm{dm}^{-3} \mathrm{NaCl}$ electrolyte at a nominal $\mathrm{pH}$ of 7 . . . . . . . . . . . . . . . . . . . . . 89

3.6 Temperature dependence of $316 \mathrm{~L}$ and $904 \mathrm{~L}$ stainless steels in 0.6 mol $d m^{-3} \mathrm{NaCl}[130] \ldots \ldots$. . . . . . . . . . . . . . . . . . . . . . 90

3.7 Comparison of typical potentiodynamic curves for unsensitised SS at a fixed chloride ion concentrations $\left(1 \mathrm{~mol} d m^{-3} \mathrm{NaCl}\right)$ and varying electrolyte temperature. . . . . . . . . . . . .

3.8 SVET scans obtained after $0,3,6,9$ hours in $1 \mathrm{~mol} d m^{-3} \mathrm{NaCl}$ for unsensitised SS, polarised to $375 \mathrm{mV}$ (Vs. SCE). . . . . . . . 93

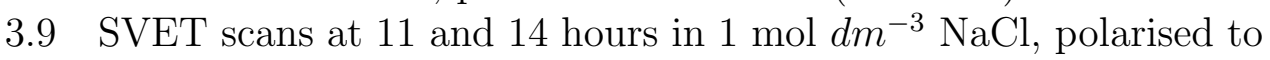
$375 \mathrm{mV}$. A photograph of the surface following 15 hours immersion is also included . . . . . . . . . . . . . . . .

3.10 'Green' oxide unsensitised SS compared to the unsensitised control

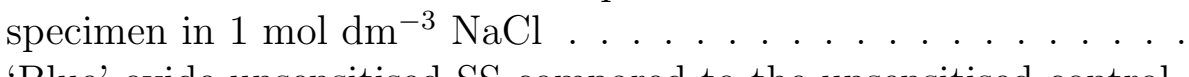

3.11 'Blue' oxide unsensitised SS compared to the unsensitised control specimen in $1 \mathrm{~mol} \mathrm{dm}^{-3} \mathrm{NaCl}$. . . . . . . . . . . . . . . . . 98

3.12 Surface of the sensitised $20 / 25 / \mathrm{Nb}$ specimen following the DLEPR test . . . . . . . . . . . . . . . . . 101

3.13 Micrograph of roughened surface following DL-EPR test, 10x . . . 101

3.14 Micrograph of roughened surface following DL-EPR test, 50x . . . 101

3.15 Offset mounted SS specimen schematic of the specimen cross section, where the dark box represents the oxide layer. The orange colouring represents highly sensitised grain boundaries relative to the grey area (less sensitised material). The green box is an area suitable for TLM or SVET investigation of a highly sensitised area adjacent to the oxide. . . . . . . . . . . . . . . . 102

3.16 Offset mounted SS specimen in resin with the oxide layer clearly shown, the polished metal surface along with the interface between the two (the more sensitised material) . . . . . . . . . . 102 
3.17 Grain size measurements for the as received microstructure - ASTM E112 data plotted [128]. Solid lines represent the average result, dotted lines indicate minimum and maximum sizes corresponding to one standard deviation. . . . . . . . . . . . . . 103

3.18 Grain size measurements for the sensitised microstructure. The solid lines represent the average result, dotted lines indicate minimum and maximum sizes corresponding to one standard deviation. 104

3.19 Tube before furnace treatment . . . . . . . . . . . . . . 107

3.20 20-25-Nb SS after solution anneal and ageing treatment . . . . . . 107

3.21 Thermally sensitised SS cross sections imaged using SEM. The micrographs show the thickness of the scale at different locations on the sample (a-d).

3.22 XRD analysis of the thermally sensitised SS showing the presence of haematite.

3.23 Waterfall plot of XRD data for the thermally sensitised SS, covered by the haematite scale. . . . . . . . . . . . . . . . . . . . 111

3.24 Waterfall plot of XRD data for the thermally sensitised SS, 20-30 degrees.

3.25 SEM image of thermally sensitised SS cross section showing areas of interest, corresponding to the EDS map in figure 3.21.

3.26 Cross section of the SEM micrograph in figure 3.25 analysed using EDS. The false colour map shows an oxide rich layer adjacent to the metal and the presence of niobium precipitates.

3.27 Annotated AFM topographical image of the thermally sensitised SS. The lower half of the image shows the scale (white), whereas the upper part of the image shows the metal surface and carbides which decorate the grain boundaries. The grain boundaries at these locations could also contain residual haematite.

3.28 SVET surface current density maps. The SVET lock-in amplifier was set to $2 \mathrm{mV}$ sensitivity. The specimen was immersed in 0.5 mol $d^{-3} \mathrm{NaCl}$ over a 24 hour period. (i) 0 hours, (ii) 6 hours, (iii) 12 hours, (iv) 18 hours, (v) 24 hours, (vi) post corrosion image. The scale bar units are in $\mathrm{Am}^{-2}$.

3.29 SVET surface current density maps. The SVET lock-in amplifier was set to $200 \mu \mathrm{V}$ sensitivity. The specimen was immersed in 0.5 mol $d^{-3} \mathrm{NaCl}$ over a 87 hour period. (i) 0 hours, (ii) 36 hours, (iii) 72 hours, (iv) 87 hours, (v) post corrosion image. The scale bar units are in $\mathrm{Am}^{-2}$.

3.30 Images showing the surface before (a) and after (b) immersion at open circuit potentials in $1 \mathrm{~mol} \mathrm{dm}^{-3} \mathrm{NaCl}$. (c) A montage of the highly sensitised region after corrosion by optical microscopy. (d-h) SVET surface current density maps, with a 3 hour interval, the scale bar is shown in (i). The total immersion time was 24 hours. 121

3.31 Optical micrographs of IGC in the region in figure 3.30(c) recorded at different magnifications. . . . . . . . . . . . . . . . .

3.32 Cathodic branch scan of thermally sensitised SS with and without scale removed. The sample was polarised from $-650 \mathrm{mV}$ (vs. SCE) to $-150 \mathrm{mV}$ (vs. SCE). . . . . . . . . . . . . . . . . 12 
3.33 ZRA experiment sensitised SS. The uncoated sensitised tube was the working electrode, the counter electrode and reference was connected to the carbon coated sensitised SS tube. The SS electrodes were immersed in $10 \mathrm{ppm}\left[\mathrm{Cl}^{-}\right]$. The lengths in the legend refer to the length of working electrode immersed within the electrolyte. The length of the non-carbon coated, sensitised counter electrode was $4 \mathrm{~cm}$ and kept constant. . . . . . . . . . . . .

3.34 Post corrosion photographs of sensitised tubes. These specimens were uncoated. (Top) With crevice formers (Bottom) without

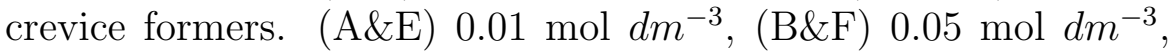
(C\&G) $0.5 \mathrm{~mol} d m^{-3},(\mathrm{D} \& \mathrm{H}) 5 \mathrm{~mol} d m^{-3}$. . . . . . . . . . . . 129

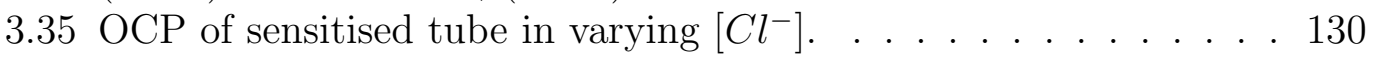

3.36 OCP of sensitised tube in varying $\left[\mathrm{Cl}^{-}\right]$. A crevice environment has been induced on the surface of the tubes. . . . . . . . . . . . . 130

3.37 OCP of sensitised tubes. Crevice formers were used, and specimens immersed in $5 \mathrm{~mol} \mathrm{dm}^{-3} \mathrm{NaCl}$. The green curve shows the specimen with additional $\mathrm{H}_{2} \mathrm{O}_{2}$ added.

3.38 Evans diagram showing the effect of $\mathrm{H}_{2} \mathrm{O}_{2}$ addition on current density [136]. The two black squares show the increase in current density following a shift in concentration from $10 \mathrm{ppb}$ to $100 \mathrm{ppb}$

3.39 Macrographs of surface after immersion in $5 \mathrm{~mol} \mathrm{dm}^{-3} \mathrm{NaCl}$ with crevice formers . . . . . . . . . . . . . . . . . 132

3.40 Macrographs of surface after immersion in $5 \mathrm{~mol} \mathrm{dm}^{-3} \mathrm{NaCl}$ crevice formers and $20 \mathrm{mM} \mathrm{H}_{2} \mathrm{O}_{2}$ added . . . . . . . . . . . . . . . . . 132

4.1 Annotated micrograph of the exposed area showing the location of the zero line for measurement of distance of anode to scale. The

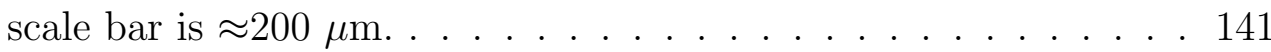

4.2 TLM stills from $0.01 \mathrm{~mol} \mathrm{dm}^{-3} \mathrm{NaCl}$ time lapse polarisation study. The study was published as an extended abstract at NACE Corrosion 2016 by Clark [114] . . . . . . . . . . . . . . . . . . . . . . 142

4.3 Crops of figure 4.2. Annotations show the $\mathrm{NbC}$ inclusion which is not affected by the IGC . . . . . . . . . . . . . . . . . . . . 143

4.4 Schematic of how NbC inclusions are affected by the corrosion of the surrounding alloy. The red particulates are NbC inclusions. At (i) no corrosion has yet occurred, at (ii) a pit initiates, (iii) the pit propagates, but does not affect the inclusion, (iv) the corrosion causes other $\mathrm{NbC}$ to fall by gravity to the bottom of the pit. An alternative schematic for stage (ii) is provided in figure 4.5 . . . . 145

4.5 An alternative schematic for the case provided in figure 4.4(ii) . . 146

4.6 Post corrosion SEM micrographs of (unsensitised) SS in $0.01 \mathrm{~mol}$ $d m^{-3} \mathrm{NaCl}$ with pits witnessed at locations around $\mathrm{NbC}$. The example supports case (b), where $\mathrm{NbC}$ fall by gravity into pits, whilst the surrounding matrix is dissolved . . . . . . . . . . . . . 147 
4.7 (i-x) Crops of SS in $3 \mathrm{~mol} d m^{-3} \mathrm{NaCl}$ under freely corroding conditions. The crops have been selected to show the locations in which anodes initiated, from the TLM images at 20x. These have been marked in red boxes for ease of viewing. (xi) graticule scale bar, with the same crop factor applied. Each division represents $10 \mu \mathrm{m} \ldots \ldots \ldots \ldots \ldots$. . . . . . . . . . . 150

4.8 Post corrosion micrographs of the specimen surface. (Left) optical microscopy image showing crevice sites which were more dominant after metastable IGC. (centre) SEM of crevice at site a, (right) SEM of crevice at site b. . . . . . . . . . . . . . . . . . . 151

4.9 The exposed surface before corrosion had occurred. . . . . . . . 152

4.10 The exposed surface following corrosion. . . . . . . . . . . . . 152

4.11 SEM micrograph of the surface $(5 \mathrm{KeV})$, annotated to show the location of anodes from the post corrosion optical image. . . . . . 152

4.12 SEM micrograph of the surface (20 KeV), annotated showing the location of anodes from the post corrosion optical image. . . . . . 152

4.13 Distances in microns and percent of anodes from haematite scale. $2 / 3$ of anodes initiated within $50 \%$ of the scale. . . . . . . 153

4.14 Thermally sensitised $310 \mathrm{SS}$ polarised to past $E_{b}(900 \mathrm{mV}$ vs $\mathrm{Ag} / \mathrm{AgCl}$ ) (image iii). Images (iv - vi) show rapid IGC propagation caused by polarisation past $E_{b} \ldots \ldots$. . . . . . . . 154

4.15 Crops of metastable events. Each of these sites passivated. . . . . 156

4.16 Cropped images of stable IGC initiation on the sensitised 310 SS in $0.2 \mathrm{~mol} \mathrm{dm}^{-3}$. Image (ii) has been annotated to show the initiation location. (iv) shows the propagation of IGC to areas more heavily depleted of Cr. . . . . . . . . . . . . . . . . 156

4.17 SVET surface current density maps (ii-vii). Image (i) is a montage of the surface pre corrosion, with image (ii) showing a montage of the surface post corrosion. The units given in the scale bar are in $\mathrm{Am}^{-2}$. At the bottom of each surface current density map is the time from the start of the experiment and potential used. The different sites and cross section used in figure 4.18 are labelled in (iv).

4.18 Evolution of SVET derived current density $\left(J_{Z}\right)$ for the anode at site $\mathrm{C}$ (left) and $\mathrm{D}$ (right). The cross section was made through the centre of the anode. z59 refers to image 4.17ii, before corrosion was detected, and is plotted in light orange, z60 shows the maximum anodic current density recorded for site $\mathrm{D}$ at $87 \mathrm{Am}^{-2}$. The final SVET scan taken, z65 is plotted in dark orange with a maximum of $20 \mathrm{Am}^{-2}$. . . . . . . . . . . . . . . . . 160

4.19 Macroscope image of the surface post corrosion. Each division is equivalent to $1 \mathrm{~mm}$, the entire bar is $4 \mathrm{~mm}$. . . . . . . . . 161

4.20 SEM map of the surface post immersion . . . . . . . . . . . . . 161

4.21 Site D $(1400 \mathrm{x})$. Nb-rich inclusions are present within the pit itself, shown as white in the BSE micrograph. . . . . . . . . . . . 164

4.22 SEM of intergranular pitting corrosion at site $\mathrm{F}$ (left) and $\mathrm{E}$ (right). Nb-rich inclusions are shown as white. The large $\approx 50 \mu \mathrm{m}$ particulates are a result of contaminants and not $\mathrm{NbC}$. 
4.23 SEM and respective EDS measurement for site A (150x)

4.24 SEM and respective EDS measurement at (i) pit site A. Note in these images the $\mathrm{Nb}$ and $\mathrm{Cl}$ signals are both shown in pink. (ii) away from the pit site. In this image the Nb signal is shown in red. 165

4.25 SEM and respective EDS measurement for site B (170x). There is distinct $\mathrm{Nb}$ enrichment at the lower location of the image close to the pit. $\mathrm{O}$ and $\mathrm{Cl}$ signals are also associated with the lower part of the image. . . . . . . . . . . . . . . . . 166

4.26 EDS map for site B (170x) with the SEM image overlaid. Yellow represents $\mathrm{Nb}$. The scale bar is $200 \mu \mathrm{m} . \quad$. . . . . . . . . . . . 167

4.27 As above, site B, at 400x magnification. The scale bar is $100 \mu \mathrm{m} .167$

4.28 As above, site B, at 800x magnification. The scale bar is $50 \mu \mathrm{m} \quad{ }_{1} 167$

4.29 SEM and respective EDS measurement for site D (figure 4.21) at a lower magnification, 200x. The map confirms the increased concentration of $\mathrm{Nb}$ rich particulates closer to the pit. . . . . . . 168

4.30 SEM and respective EDS measurement for site C (400x). The scale bar is $100 \mu \mathrm{m} \ldots \ldots \ldots$. . . . . . . . . . . . . 169

4.31 EDS measurement for site F (450x) (figure 4.22). The image is overlaid above the SEM image for easier viewing. . . . . . . . . . 169

4.32 Site G lacy cover from figure 4.37. The SEM and EDS maps have been overlaid at 1500x. The scale bar is $20 \mu \mathrm{m}$. . . . . . . . . . 169

4.33 Site G (i) pit cavity, (ii) lacy cover. . . . . . . . . . . . . . 171

4.34 Higher magnification image of the pit site (i) in area G. . . . . . . 171

4.35 An image from location (i) in images 4.33 4.34, inside the pit cavity at site G. Note the number of Nb-rich inclusions present, and the scale bar, showing many inclusions within a small area. . . . . . . 171

4.36 Site G lacy cover (figure 4.33(ii)) (500x) . . . . . . . . . . . . . 172

4.37 Site $\mathrm{G}$ lacy cover (1500x). The red arrow shows the high density of Nb-rich inclusions present in the pit, and the blue arrow shows the locations where IGC has caused the removal of $\mathrm{Cr}$ carbides through undercutting of the adjacent metal. . . . . . . . . . . . . 172

4.38 Site G lacy cover $(2500 \mathrm{x})$. The arrow shows the abundance of Nb-rich inclusions present within the pit (under the lacy cover). . 172

5.1 Carbides decorate the grain boundaries of thermally sensitised 20/25/Nb stainless steel. The image was obtained using a HSAFM.

5.2 Carbides present along grain boundaries of thermally sensitised 20/25/Nb stainless steel (a). (b) shows the surface following polarisation $\left(500 \mathrm{mV}\right.$ vs. Pt) in $5 \mathrm{mgkg}^{-1}\left[\mathrm{Cl}^{-}\right]$. . . . . . . . . . . 178

5.3 (a) 50x optical montage (22 composited images) of the surface before immersion, (b) SEM micrograph of surface before immersion,

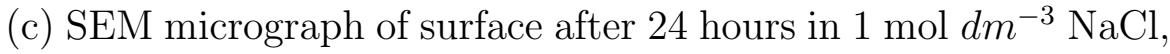

(d) 20x optical micrograph post corrosion . . . . . . . . . . . . . . 184

5.4 SEM images of NbC inclusions and corresponding EDS mapping a) backscattered electron images, b) carbon EDS map, c) niobium EDS map . . . . . . . . . . . . . . . . . . . . 185 
5.5 Optical TLM images of the sensitised SS 20-25-Nb immersed in $1 \mathrm{~mol} d m^{-3} \mathrm{NaCl}$ showing the progression of corrosion product depositing on the exposed surface. a) 0, b) 6, c) 18, d) 24 hours . 186

5.6 Open circuit potential as a function of time (vs. SCE) for the TLM specimen immersed in $1 \mathrm{~mol} \mathrm{dm}^{-3} \mathrm{NaCl}$. Arrows at c.0, 6, 18, 24 hours have been added to assist the reader. The peak at $\mathrm{c}$. $65,000 \mathrm{~s}$ (18 hour) corresponds to the trapped gas movement, or gas evolution seen in figure 5.5 . . . . . . . . . . . . 187

5.7 Cropped optical TLM images of the sensitised SS alloy in $1 \mathrm{~mol}$ $d m^{-3} \mathrm{NaCl}$ showing the development of IGC over time under the influence of potential control. (a-d) Show the progression of several anodes within a period of $270 \mathrm{~s}$ where the current density increased exponentially from $\approx-150 \mathrm{mV} \ldots \ldots . . . \ldots$

5.8 Potentiodynamic curve for the above TLM specimen immersed in $1 \mathrm{~mol} \mathrm{dm}^{-3} \mathrm{NaCl}$ undergoing polarisation. Current density is shown as a function of applied potential (vs. SCE). The experiment started at $-350 \mathrm{mV}$ vs. SCE. We have annotated the graph to show the specimen breakdown potential at c. $-150 \mathrm{mV}$ vs. SCE. The graph appears to plateau at $86 \mathrm{mV}$ vs. SCE. . . . . . . . . 190

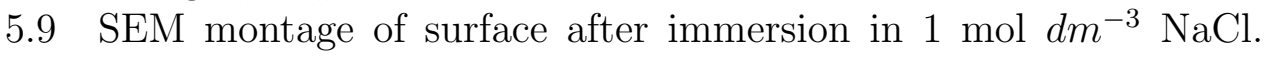
The specimen has been subjected to polarisation. The perforated cover at the scale is visible in this image, as is the large amount of NbC present within the excavation. . . . . . . . . .

5.10 In-situ optical TLM images of the development of IGC and increase

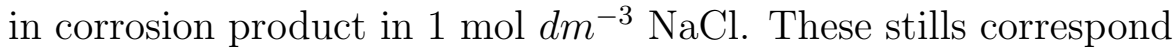
to those in figure 5.11; (a) 30 minutes, (b) 60 minutes, (c) 120 minutes, (d) 240 minutes. . . . . . . . . . . . . . . .

In-situ EC-AFM images of the sensitised SS 20-25-Nb immersed

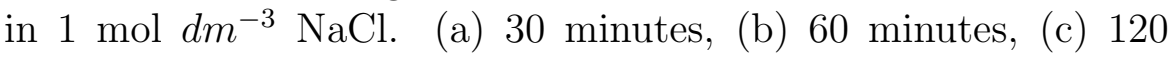
minutes, (d) 240 minutes. The images depict the development of a grain boundary swelling effect, pit covers, intergrannular pitting and consequential corrosion product covering the exposed surface. The arrow in image (a) shows what is a possible NbC inclusion. The swelling effect may be an artefact of the relative height scaling used . . . . . . . . . . . . . . . . . . 192

5.12 Centre frame AFM image at 310 minutes (a) 2D map (b) 3D map 195

5.13 AFM topography and KPM maps. (a) Sensitised SS Metal:scale interface, (b) Corresponding SKP-FM map, (c) Cr carbide present on sensitised SS, (d) Corresponding SKP-FM map, (e) Unsensitised, etched SS NbC, (f) Corresponding SKP-FM map . . . . . . 196

6.1 AGR Fuel cladding, 20/25/Nb stainless steel fuel cladding compo-

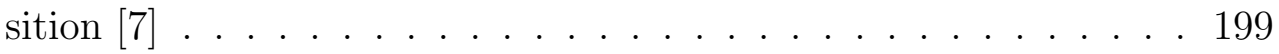

6.2 Unsensitised SS in $1 \mathrm{~mol} d m^{-3} \mathrm{NaCl}$, varying additions of $\mathrm{HCl}$ and

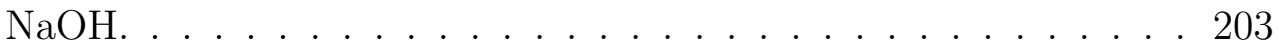

6.3 Potentiodynamic experiment on unsensitised specimen in $1 \mathrm{~mol}$

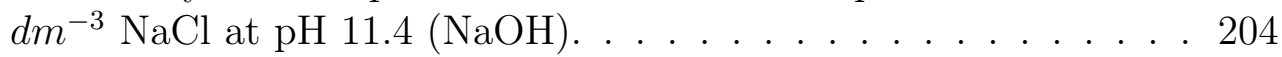


6.4 Potentiodynamic experiment on unsensitised specimen in $1 \mathrm{~mol}$ $d m^{-3} \mathrm{NaCl}$ at $\mathrm{pH} 12.5(\mathrm{NaOH})$

6.5 Potentiodynamic experiment on unsensitised specimen in $1 \mathrm{~mol}$

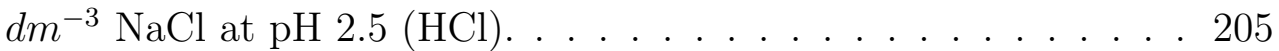

6.6 Potentiodynamic experiment on unsensitised specimen in $1 \mathrm{~mol}$

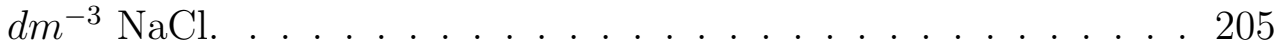

6.7 Breakdown and free corrosion potentials as a function of electrolyte $\mathrm{pH}$ for unsensitised SS. . . . . . . . . . . . . . . 206

6.8 Sensitised SS with and without $\mathrm{NaOH}$ inhibitor $\left(2.5 \mathrm{mM} \mathrm{dm}^{-3}\right)$, as a function of $\left[\mathrm{Cl}^{-}\right](0,1$ and $10 \mathrm{ppm}) \ldots \ldots . \ldots 209$

6.9 Sensitised SS with and without $\mathrm{NaOH}$ inhibitor $\left(2.5 \mathrm{mM} \mathrm{dm} \mathrm{m}^{-3}\right)$, as a function of $\left[\mathrm{Cl}^{-}\right](10,100,1000,10000 \mathrm{ppm}) \ldots 209$

6.10 Polarisation resistance experiments as a function of $\left[\mathrm{Cl}^{-}\right]$in dis-

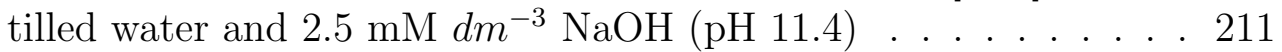

6.11 Change in potential (Eb-Ecorr) for sensitised SS specimens immersed in neutral $\mathrm{pH}$ and $\mathrm{NaOH}$ as a function of $\left[\mathrm{Cl}^{-}\right]$. . . . . . 212

$6.12 \mathrm{ppm}\left[\mathrm{Cl}^{-}\right]$potentiodynamic experiments without $\mathrm{NaOH}$. . . . . 214

$6.13 \mathrm{ppm}\left[\mathrm{Cl}^{-}\right]$potentiodynamic experiments with $\mathrm{pH} 11.4 \mathrm{NaOH}$. . . 214

$6.140 \mathrm{ppm}\left[\mathrm{Cl}^{-}\right]$potentiodynamic experiments in neutral $\mathrm{pH}$ and 11.4 $(\mathrm{NaOH}) \ldots \ldots \ldots \ldots \ldots . \ldots \ldots$

$6.151 \mathrm{ppm}\left[\mathrm{Cl}^{-}\right]$potentiodynamic experiments in neutral $\mathrm{pH}$ and 11.4 $(\mathrm{NaOH}) \ldots \ldots \ldots \ldots \ldots \ldots \ldots$

$6.1610 \mathrm{ppm}\left[\mathrm{Cl}^{-}\right]$potentiodynamic experiments in neutral $\mathrm{pH}$ and 11.4

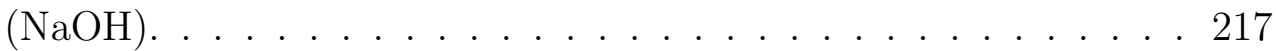

$6.17100 \mathrm{ppm}\left[\mathrm{Cl}^{-}\right]$potentiodynamic experiments in neutral $\mathrm{pH}$ and $11.4(\mathrm{NaOH}) \ldots \ldots \ldots \ldots \ldots \ldots . \ldots \ldots$

$6.181000 \mathrm{ppm}\left[\mathrm{Cl}^{-}\right]$potentiodynamic experiments in neutral $\mathrm{pH}$ and $11.4(\mathrm{NaOH})$

$6.1910,000 \mathrm{ppm}\left[\mathrm{Cl}^{-}\right]$potentiodynamic experiments in neutral $\mathrm{pH}$ and $11.4(\mathrm{NaOH})$.

6.20 Specimen used for the $\mathrm{pH} 13 \mathrm{NaOH}$ experiment following 1 day of immersion. The inclusion dissolution occurred at 28,800 s. OCP data at $2.5 \mathrm{mmol} d \mathrm{~m}^{-3}$ for 4 days shown in inset . . . . . . . . .

6.21 Images of inclusions in $0.1 \mathrm{~mol} d^{-3} \mathrm{NaOH}$ (20x crop). Colour change witnessed after $\approx 28 \mathrm{Ks}$. . . . . . . . . . . . . . . . 222

6.22 Inclusions captured using optical (50x crop) and SEM techniques

6.23 EDS mapping of the set of inclusions. (From left to right) secondary electron image, nickel, iron, niobium, carbon, chromium

6.24 Annotated AFM image to show location of background and inclusion cross sections. This inclusion was present in the in-situ TLM experiment. . . . . . . . . . . . . . . 225

6.25 Cross section of background and inclusion. . . . . . . . . . . . 225

6.26 Suggested cross sectional area for the inclusion above the surface (shown in green). . . . . . . . . . . . . . . . . 226

6.27 Annotated AFM image to show location of background and inclusion cross sections. This inclusion was present in the in-situ TLM experiment. . . . . . . . . . . . . . . 227

6.28 Cross section of background and inclusion. . . . . . . . . . . . 227 
6.29 NbC inclusion; AFM topography (left), SKPFM Volta potential map (right) . . . . . . . . . . . . . . . . . . . 228

6.30 LPR dependency as a function of $\left[\mathrm{Cl}^{-}\right] \ldots \ldots$. . . . . . . . . 230

6.31 Images of inclusions after 4 days immersion in $\mathrm{pH} 13 \mathrm{NaOH}$. (A, B) 3D topography of image in (C). Image (D) shows an inclusion which has large excavation around edges. The lower half of the image shows an artefact, relating to the AFM instrumentation . . 231

6.32 Cross section of inclusion site following 4 days immersion in $0.1 \mathrm{moldm}^{-3}$

$\mathrm{NaOH}$ and corresponding AFM image . . . . . . . . . . . 233

6.33 Cross section of inclusion site following 4 days immersion in 0.1 moldm$^{-3}$ $\mathrm{NaOH}$ and corresponding AFM image. . . . . . . . . . . . 234

6.34 SEM micrographs of the stainless steel following 4 days immersion in 0.1 moldm $^{-3} \mathrm{NaOH}$ ( $\left.\mathrm{pH} 13\right)$. The arrows show the fracturing effect which appears to have modified the inclusions. . . . . . . 235

6.354 day immersion in $\mathrm{NaOH}$ SEM-EDS showing a single NbC inclusion.236

6.364 day immersion in $\mathrm{NaOH}$ SEM-EDS showing a group of $\mathrm{NbC}$ inclusions. The fractured effect in the SEM image is either visual, or the EDS detector cannot resolve these features. . . . . . . . 236

6.374 day immersion in $\mathrm{NaOH}$ SEM-EDS. The fracture witnessed with a second set of $\mathrm{NbC}$ inclusions. The EDS maps show no change in $\mathrm{Nb}$ enrichment at the crack sites. . . . . . . . . . . . . . 236

$6.38 \mathrm{NbC}$ site following $\mathrm{pH} 13 \mathrm{NaOH}$ treatment for 4 weeks . . . . . . 238

$6.39 \mathrm{NbC}$ site following $\mathrm{pH} 13 \mathrm{NaOH}$ treatment for 4 weeks . . . . . . 238

6.40 Point based acquisition of NbC inclusion following pH $13 \mathrm{NaOH}$ treatment . . . . . . . . . . . . . . 239 


\section{List of Tables}

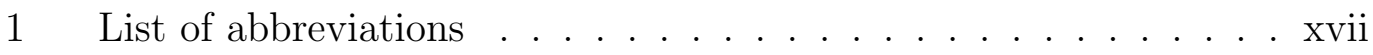

1.1 UK AGR build and expected decommissioning dates [3] . . . . . 2

1.2 Current and past UK nuclear reactors. AGRs are composed of two reactor units per site. The UK has one PWR located at the

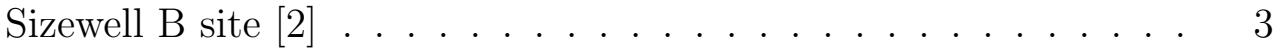

1.3 AGR cladding composition $[28] \ldots \ldots \ldots \ldots$

1.4 Spent AGR fuel cladding performance [37]. . . . . . . . . . . . . 11

1.5 Corrosion rates of $\mathrm{Nb}$ in basic media, recreated from [52] . . . . . 18

2.1 Overview of experiments undertaken within this thesis. U and $\mathrm{S}$ refer to unsensitised and thermally sensitised material condition .

2.2 Conversion between sodium chloride in units of molarity and as parts per million chloride . . . . . . . . . . . . . . 74

$3.1 E_{C o r r}, E_{b}$ and $\Delta E_{A v}$. for unsensitised specimens in varying concentrations of $\mathrm{NaCl}$. The final column shows $E_{\text {corr }}$ from separate OCP experiments. All potentials are with respect to SCE. . . . .

3.2 Table showing free corrosion and breakdown potentials for unsensitised specimens at a fixed $\left[\mathrm{Cl}^{-}\right]$, with varying $\mathrm{T}$. All potentials are with respect to $\mathrm{SCE}$; and error values quoted represent one standard deviation from the mean. . . . . . . . . . . 87

3.3 Degree of Sensitisation - the DL-EPR test (*Rp - repassivation) . 100

3.4 Grain size measurement ASTM E112 for as received and sensitised SS specimens for line and circle intercept procedures. The numbers shown are ASTM grain size numbers ' $G$ ' and is unitless. 'S.D.' represents one standard deviation. . . . . . . . . . . . 105

3.5 Average grain sizes and respective minimum and maximums (one standard deviation) derived from grain size measurement using the circular intercept procedure. These were taken from the circular intersect results. The values are derived from ASTM E112 standard datasheet. . . . . . . . . . . . . . . . . . 106

4.1 Composition of 310 stainless steel [137] . . . . . . . . . . . . 139

6.1 Average potentials of unsensitised SS, and respective uncertainty (one standard deviation). . . . . . . . . . . . . . . 202

6.2 Sodium Hydroxide LPR results (neutral $\mathrm{pH}$ included for compar-

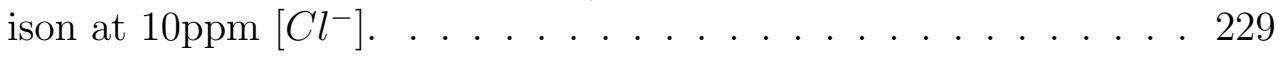




\subsection{Glossary}

Table 1: List of abbreviations

\begin{tabular}{|l|l|}
\hline Acronym & Description \\
\hline AGR & Advanced Gas Cooled Reactor \\
GDF & Geological Disposal Facility \\
THORP & Thermal Oxide Reprocessing Plant \\
SEM & Scanning Electron Microscope \\
TEM & Transmission Electron Microscope \\
EDS/EDX & Energy Dispersive X-ray Spectroscopy \\
SVET & Scanning Vibrating Electrode Technique \\
TLM & Time-Lapse Microscopy \\
SKP & Scanning Kelvin Probe \\
AFM & Atomic Force Microscopy \\
SKPFM & Scanning Kelvin Probe Force Microscopy \\
IGC & Intergranular Corrosion \\
DOS & Degree of Sensitisation \\
IKE & Inverse Kirkendall Effect \\
RIS & Radiation Induced Segregation \\
SCE & Saturated Calomel Electrode \\
ZRA & Zero Resistance Ammeter \\
OCP & Open Circuit Potential \\
\hline
\end{tabular}


Chapter 1

\section{Literature Review}




\subsection{The Advanced Gas-cooled Reactor}

\subsubsection{History}

The advanced gas-cooled reactor (AGR) is a nuclear reactor which uses carbon dioxide gas $\left(\mathrm{CO}_{2}\right)$ as a coolant. There are 14 AGRs in operation in Britain, located at 6 different sites (table 1.1). A stainless steel fuel cladding alloy was produced especially for the programme following testing and development at the Windscale AGR test reactor (Sellafield, Cumbria) during the 1960s and through to the 1980s. AGR designs differ from PWR and BWR (pressurised/boiling water reactor) designs in that the AGR uses a gas rather than water as a coolant. The AGR is the UK's second generation gas-cooled reactor design using carbon dioxide; building on previous experience from the Magnox (MAGnesium NoOXide) gas reactor design. The AGR operates at increased gas temperatures than Magnox reactors; allowing greater thermal efficiencies, thereby reducing the cost of electricity. At such temperatures initially proposed for the AGR design, the magnesium alloy fuel cladding would have simply melted. Therefore thin gauge stainless steel (SS) provided fuel encapsulation. Poorer neutron absorption qualities meant that uranium metal was no longer feasible, and instead lightly enriched uranium dioxide was used [1] [2]. Table 1.2 shows current UK AGR power stations and expected closure dates.

\begin{tabular}{|l|l|l|l|}
\hline Location & $\begin{array}{l}\text { Construction } \\
\text { started }\end{array}$ & First Power & $\begin{array}{l}\text { Expected Decom- } \\
\text { missioning date } \\
(2016)\end{array}$ \\
\hline Dungeness B & 1965 & 1985 & 2028 \\
Hartlepool & 1968 & 1989 & 2024 \\
Heysham 1 & 1970 & 1989 & 2024 \\
Heysham 2 & 1980 & 1989 & 2030 \\
Hinkley Point B & 1967 & 1976 & 2023 \\
Hunterston B & 1967 & 1976 & 2023 \\
Torness & 1980 & 1988 & 2030 \\
\hline
\end{tabular}

Table 1.1: UK AGR build and expected decommissioning dates [3] 


\begin{tabular}{|c|c|c|c|}
\hline Reactor Type & Fuel Source & $\begin{array}{l}\text { Capacity } \quad \text { (net } \\
\left.M W_{e}\right)\end{array}$ & First Power \\
\hline Magnox & Uranium metal & 490 & 1971 \\
\hline AGR & $\begin{array}{l}\text { Enriched Ura- } \\
\text { nium dioxide }\end{array}$ & $2 \times(545$ to 625$)$ & 1976 \\
\hline PWR & $\begin{array}{ll}\text { Enriched } & \text { Ura- } \\
\text { nium oxide } & \end{array}$ & 1188 & 1995 \\
\hline
\end{tabular}

Table 1.2: Current and past UK nuclear reactors. AGRs are composed of two reactor units per site. The UK has one PWR located at the Sizewell B site [2]

\subsubsection{Fuel Cladding}

The role of the fuel cladding is to provide encapsulation of fuel. In an AGR the cladding takes the form of a tube, which holds lightly enriched uranium dioxide fuel in the form of cylindrical pellets. Thirty-six of these tubes form what is known as fuel element, an example is shown in figure 1.1. The fuel element sleeve itself is constructed of graphite which acts as a moderator. The moderator is used to decrease neutron energies from fast to thermal energies $(\sim 1 / 40 \mathrm{eV})$ so that they are effective for the fission process [4] [5] [6]. Depending on the AGR, up to 8 of these fuel elements are stacked atop one another to form what is known as a 'fuel stringer' [7].

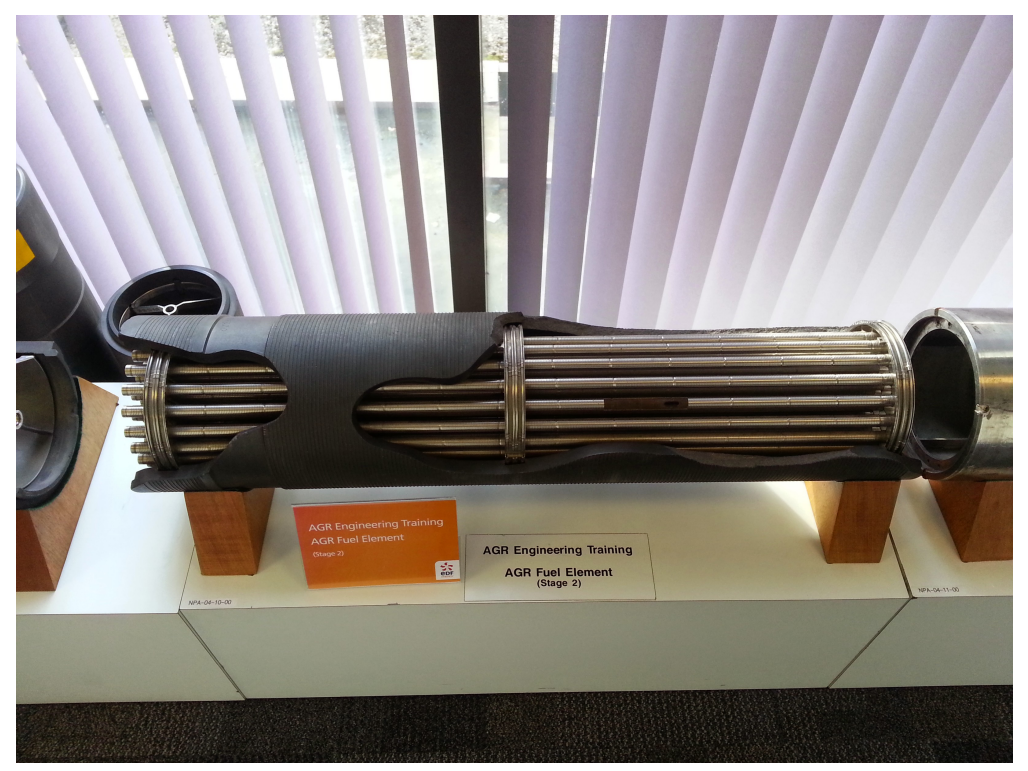

Figure 1.1: Fuel element at EDF Barnwood

Stainless steels can be classified into 4 types: Ferritic (body-centred cubic, 
BCC), Austenitic (face-centred cubic, FCC), and Martensitic (BCC or bodycentred tetragonal, BCT) [8]. Alloys containing ferritic and martensitic crystal structures are called duplex stainless steels. Austenitic 300 series stainless steels have a chromium-nickel $(\mathrm{Cr}-\mathrm{Ni})$ base. The most common $\mathrm{SS}$ grade is today is austenitic. Type 304 has a composition of $18 \mathrm{wt} \% \mathrm{Cr} 8 \mathrm{wt} \% \mathrm{Ni}$, and has satisfactory corrosion resistance for most standard applications (cutlery, kitchen sinks, pans, indoor architectural applications).

The fuel cladding used within an AGR is a bespoke grade, belonging to the austenitic family of stainless steels. It is commonly referred to as $20 / 25 / \mathrm{Nb}$, named after the main constituents of the alloy, chromium (Cr), nickel (Ni) and a stabiliser, niobium $(\mathrm{Nb})$. The alloying composition is shown in table 1.3. Corrosion rates for conventional austenitic SS (316L, 904L) are low (less than 0.005 milli-inches per year, 5000 hours salt spray, seawater), with resistance to corrosion being dependant on the alloying content of $\mathrm{Cr}$, Mo and $\mathrm{Ni}$ [9]. It is generally believed that in order for a stainless steel to remain 'stainless', the alloy needs to contain Cr levels of at least 13.5\% [10] [11] [12] [13]. This ensures resistance to general corrosion, such as that which may occur in iron. As such, it is desirable to alloy high amounts of $\mathrm{Cr}$ in a stainless steel for better corrosion resistance. There is however a finite limit the amount of $\mathrm{Cr}$ within the alloy; as sigma phase and chromium nitrides begin to precipitate from solution, leading to reduction in ductility, toughness and even corrosion resistance [14]. Cr is the passivating element in the stainless steel, forming an invisible, self-healing, non-porous, nanometer-scale, protective film rich in $\mathrm{Cr}$ [15]. The thin-oxide films rebuild in most environments; greatly lowering corrosion rates for SS. In most normal cases the SS are said to be passive, as the rate at which metal is consumed is smaller than the rate at which the passivating film rebuilds [16]. Films have been reported to be 1.3 and $1.5 \mathrm{~nm}$ for type $304 \mathrm{~L}$ and $316 \mathrm{~L}$ respectively using depth profiling $\mathrm{x}-$ ray photoelectron spectroscopy [17]. The same author showed these passive films contain $\mathrm{Cr}$ (iii), Fe (ii) and $\mathrm{Fe}(\mathrm{iii})$ type oxides, with an $\mathrm{Cr}$ (iii) hydroxide forming 
the outer film. Thus it is expected that the film thickness on this austenitic SS will be within the same range. Newman [18] considers, under most instances, the $\mathrm{Cr}_{2} \mathrm{O}_{3}$ film to be less than $2 \mathrm{~nm}$. Should the passive protective chromium-iron oxide film rupture (depassivation) Ni has the positive effect of lowering corrosion rates [19]. Carbon content in the $20 / 25 / \mathrm{Nb}$ alloy is $0.04-0.08$ wt. $\%$, which is a result of the addition of $\mathrm{Nb}$. $\mathrm{Nb}$ has a high affinity for $\mathrm{C}$, forming inclusions of niobium carbide (NbC), lowering $\mathrm{C}$ content within the matrix [10] [20]. This is known as stabilisation, aiding prevention of sensitisation which may occur at weld locations [21] [22]. The $\mathrm{C}$ content in this alloy is the same as standard type 304 and 316 SS. Nowadays, modern metallurgical manufacturing techniques allow carbon content to be reduced even further. The argon oxygen decarburisation (AOD) or vacuum oxygen decarurisation (VOD) processes may be used to produce low carbon content 'L' types (0.03 wt. \% C) [23] [24] [25] [26] [27].

Table 1: Composition of $20 / 25 / \mathrm{Nb}$ stainless steel for Mk.4 fuel.

\begin{tabular}{|l|l|}
\hline Element & Analysis Wt\% \\
\hline Nickel & $24-26$ \\
\hline Chromium & $19-21$ \\
\hline Manganese & $0.55-0.85$ \\
\hline Silicon & $0.45-0.75$ \\
\hline Sulphur & $<0.02$ \\
\hline Phosphorous & $<0.02$ \\
\hline Cobalt & $<0.015$ \\
\hline Boron & $<0.001$ \\
\hline Niobium * & $>0.08$ but $>8 \times(\mathrm{C}+\mathrm{N})$ \\
\hline Titanium & $<0.05$ \\
\hline Carbon * & $0.040-0.080$ \\
\hline Nitrogen * & $0.040-0.080$ \\
\hline Iron & balance \\
\hline
\end{tabular}

* Previous versions of fuel cladding alloy have observed the following constraints (all in wt\%): Mk2 $-\mathrm{Nb}>0.7$ but $>10 \times \mathrm{C}, \mathrm{C}=0.01$ to $0.05, \mathrm{~N}$ not specified but typically 0.005 to 0.010 . $\mathrm{Mk3}-\mathrm{Nb}>0.8$ but $>10 \times(\mathrm{C}+\mathrm{N}), \mathrm{C}=0.025$ to 0.065 and $(\mathrm{C}+\mathrm{N})>0.075$.

Table 1.3: AGR cladding composition [28]

\subsubsection{AGR Fuel Element Manufacture}

Stainless steel AGR fuel elements (tubes, sleeve, fuel enrichment) are produced by Westinghouse Fuels Limited. The SS fuel cladding tubes are composed of two 
main parts; a $900 \mathrm{~mm}$ tube and an end cap, both made from the 20/25/Nb SS alloy. Each of these tube structures has helical ribs machined onto the outer surface for increased thermal conductivity of pin to coolant, and to ensure turbulent coolant flow. Helical ribs run the length of the tube; fabricated from a tube of larger diameter using a lathe.

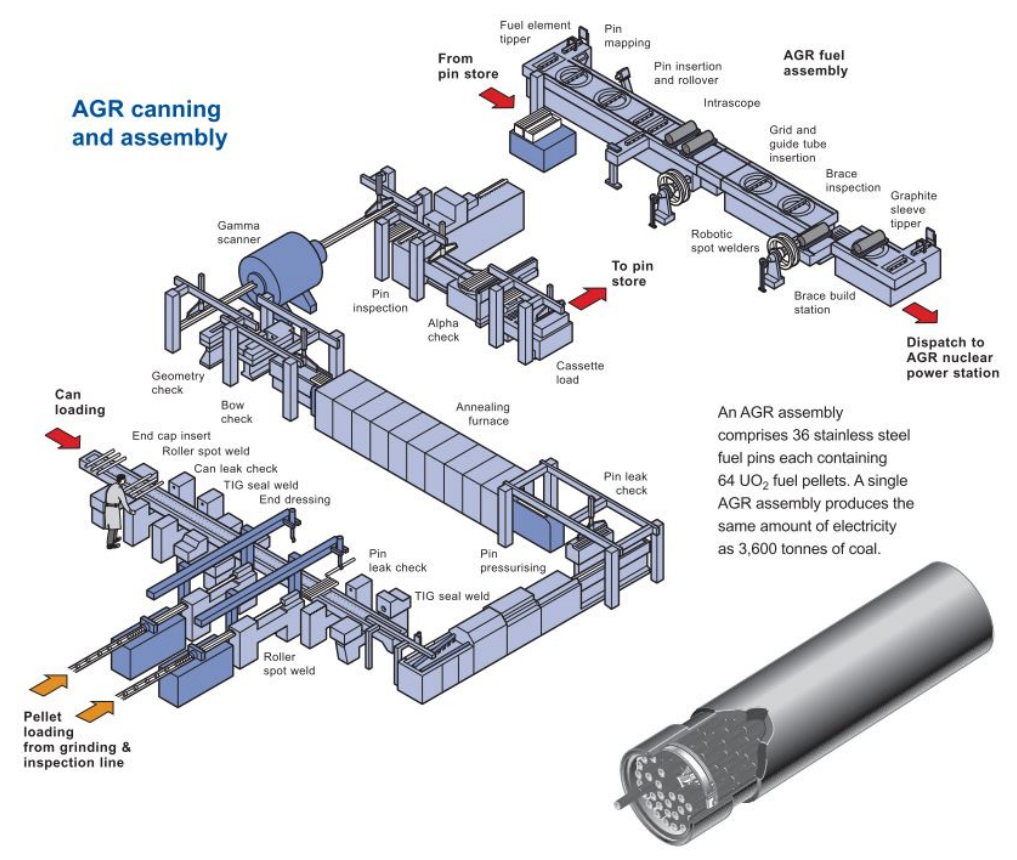

Figure 1.2: AGR fuel production at Springfields Ltd. [29]

Following lathe machining, an end cap is inserted onto one end of the tube. The end cap is formed from a flat bar of the material. The SS flat bar is cold worked in order to introduce dislocations which strengthen the material, and is subsequently annealed at $1050{ }^{\circ} \mathrm{C}$. The tube also receives cold work prior to an anneal at $950{ }^{\circ} \mathrm{C}$. End caps resemble a sewing thimble of slightly smaller diameter which allows it to sit flush inside the can. This is then twice welded to one end of the fuel can; a roller spot weld first fuses the inside side wall to the cap by electric current, whilst a tungsten inert gas (TIG) weld satisfies the joining of the two rims together. The can end is then checked for leaks, dressed, and then insulated with a sintox aluminium-oxide disc.

The tubes are subsequently loaded with toroid-shaped Uranium dioxide $\left(\mathrm{UO}_{2}\right)$ pellets. $\mathrm{UO}_{2}$ within the AGR is slightly enriched, to levels of $2.2-2.7 \%$ [2]. The 
hollow centre is added to allow for any expansion and contraction which can occur under irradiation after the fuel assembly has been completed. In addition, pellets are fixed in place by an anti-stacking groove (ASG) in order to limit movement of pellets and expansion along the length of the can. Every fifth pellet has a circumferential notch ground into it. The ASGs are added at a later stage. Once each pin has been loaded with 64 pellets the tube is filled with helium to promote heat transfer. A second sintox disc and end cap is inserted and, as before, double welded; encapsulating the pellets. Tubes, now with end caps, are moved to a hydraulic bath where the outside pressure is 1200 bar under water. ASGs are formed where the tube wall deforms at notches under pressure. The tubes undergo a leak test before travelling to a furnace where they are annealed at $930^{\circ} \mathrm{C}$ in hydrogen to relieve work stresses. Finally the tubes are checked to ensure they meet the correct geometry (i.e. not bowed), before final packaging into a graphite sleeve. Each element consists of 36 of these tubes, held in position by metal braces, a grid plate and a tie bar. The grid plate is manufactured from a single piece of the same cladding alloy, machined by computer control. The brace is constructed from SS stabilised with titanium (Ti), instead of Niobium.

These braces are located at the top and middle and bottom of the element. In order to fix pins to the grid plate the ends are swagged into place. This decreases the ability for the pin to rotate within the grid plate. Further information on other components used within the assembly is provided in figure 1.3.

\subsubsection{Spent AGR Fuel Storage}

During the course of operation AGRs are refuelled and fuel is sometimes reshuffled, in order to maintain a nominal energy throughput. Fuel assemblies stay in the reactor for years at a time, this time is known as fuel burn-up. Burn-up times may be longer or shorter depending on the neutron flux required and the plant owner's policy. AGR fuel burn-up has been increasing over time. Initial fuels had a stringer mean irradiation of $5 \mathrm{GWd} / t_{e}$ rising to $22 \mathrm{GWd} / t_{e}$ in 1996 , with 


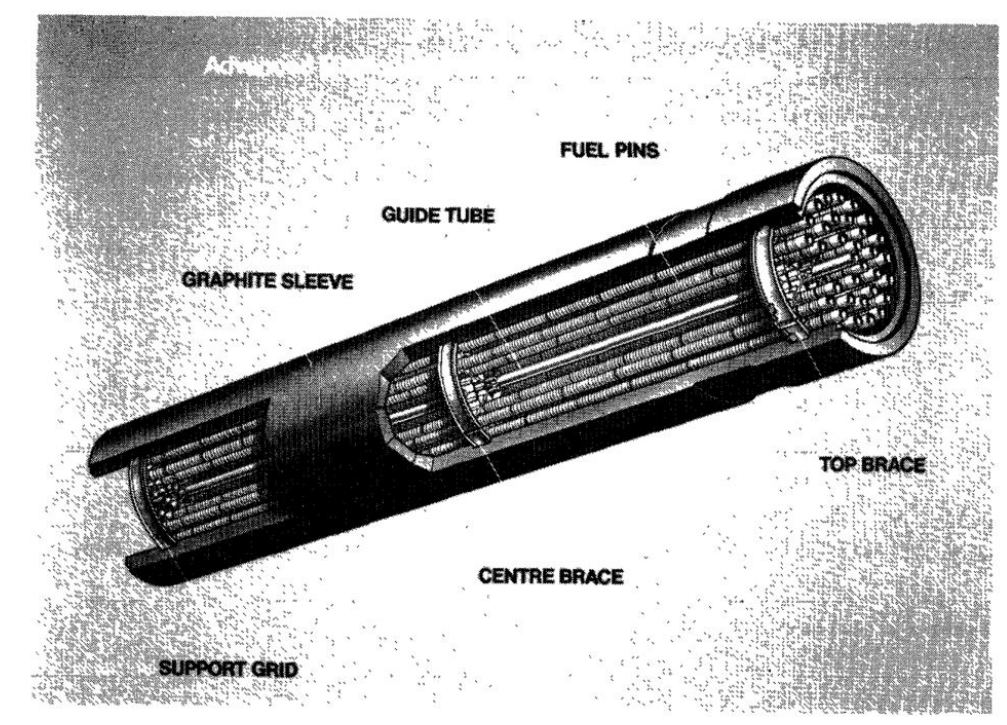

Figure 1.3: Cross section of the AGR fuel element showing fuel pins [30]

increases planned to $30 \mathrm{GWd} / t_{e}$ [31] [32]. Though longer durations may be more economic for the plant owner, integrity of the fuel cladding may be impacted as it will be irradiated for longer periods of time.

Taylor [33] investigated sensitisation of stainless steel AGR fuel cladding from the Hinkley Point B and Hunterston AGR stations. A modified version of the Strauss test [34] was used to compare specimens that had been in reactor for a number of years. In this experiment polished specimens are immersed in $37.5 \mathrm{~g}$ $\mathrm{CuSO}_{4} \cdot 5 \mathrm{H}_{2} \mathrm{O}, 37.5 \mathrm{~g} \mathrm{H}_{2} \mathrm{SO}_{4}, 328 \mathrm{ml}$ of $\mathrm{H}_{2} \mathrm{O}$. Cu powder was added to cover the specimens, then the mixture was boiled for a period of 72 hours. The Strauss test is a measure of the depth of attack from each surface of the specimen. The investigation found that only material irradiated at a temperature range of 350$520{ }^{\circ} \mathrm{C}$ became sensitised. Peak sensitisation, where the depth of attack from the polished face was greatest, was found for specimens in the range $380-440{ }^{\circ} \mathrm{C}$. This corresponds to elements located at the coolest part of the gas flow at the bottom of the reactor. The fuel irradiation temperature and burn up for Hinkley Point B fuel stringer are given in figure 1.4. Results from the Strauss tests show attack depth was proportional to increased burn up, at the temperatures ranging between $380-440^{\circ} \mathrm{C}$. The attack depth was minimal for specimens at $460-600^{\circ} \mathrm{C}$.

The AGR spent fuel assembly initially cools in a gas flow allowing short- 


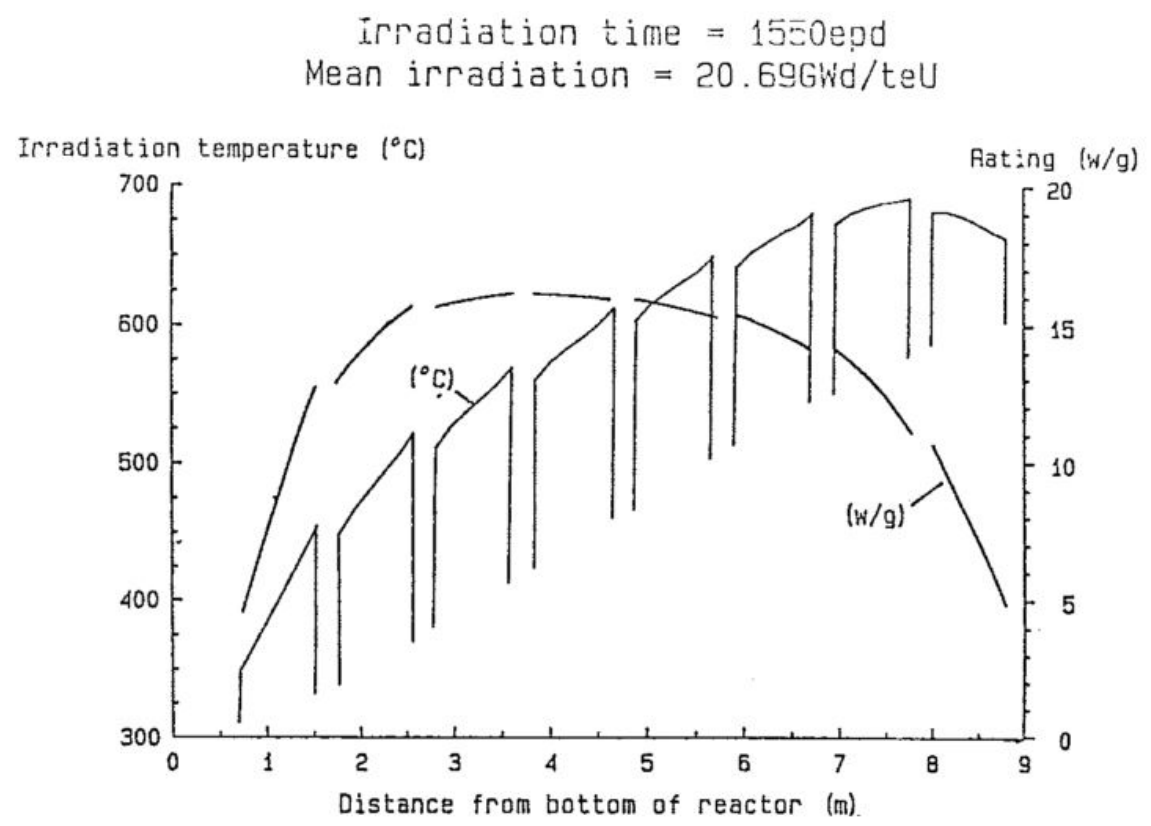

Figure 1.4: Fuel cladding conditions as a function of distance for stringer located at Hinkley Point B, B4286 [33]

lived fission products to decay to low enough levels for stringer dismantling. The individual fuel elements from the stringers are then cooled in roofed, borated ponds for several months giving operators greater control over pond chemistry and maintain low $\left[\mathrm{Cl}^{-}\right][31]$ [35]. Boric acid provides criticality control, and the $\mathrm{pH}$ of ponds is neutral, through the addition of sodium hydroxide $(\mathrm{NaOH})$.

Once fission products have decayed to safe levels, spent fuel elements are transported from the nuclear power station to Sellafield. The thermal oxide reprocessing plant (THORP) provides interim storage for a period of less than ten years in storage ponds before spent fuel can be safely dismantled and subsequently, reprocessed by chemical separation [36] . Upon delivery, spent AGR fuel is immersed in ponds containing $\mathrm{NaOH}$ with a $\mathrm{pH}$ target range of $\mathrm{pH} 11.35$ to $\mathrm{pH}$ 11.45. Sellafield has shown $\mathrm{NaOH}$ is an effective corrosion inhibitor for spent AGR fuel cladding over a period of at least 20 years [31] [37].

The AGR spent fuel storage route from power station to disposal is shown in figure 1.5. The fuel handling plant provides storage for AGR fuel at the target range of 11.35 to 11.45 as described above. THORP receipt and storage (TRS) is a roofed, enclosed pond and water chemistry tightly controlled; demineralised 
water at neutral $\mathrm{pH}$ with chloride ion concentration $\left(\left[C l^{-}\right]\right)<0.5 \mathrm{ppm}$. TRS does not employ a $\mathrm{NaOH}$ treatment stage due to the different types of fuel in the pond (as of 2016).

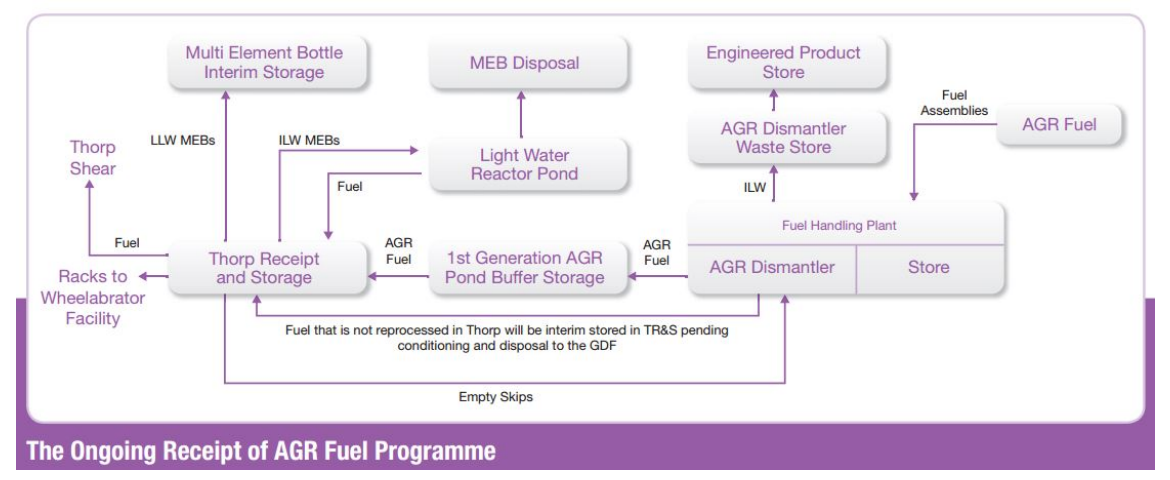

Figure 1.5: The current Sellafield spent fuel route, 2011 Sellafield Plan [38]

Early post storage examination (PIE) performed on irradiated SS showed corrosion rates were $<0.2 \mu \mathrm{m} y r^{-1}$ which, extrapolated for a 80 year duration in-pond represents a 5\% thickness loss [37]. These experiments were conducted on the brace material through weight loss experiments. The brace material is stabilised by $\mathrm{Ti}$, instead of $\mathrm{Nb}$, therefore giving a 'upper limit' for corrosion rate. Original immersion tests of the fuel pins (SS tubes) in 1-100 ppm $\left[\mathrm{Cl}^{-}\right]$failed within a range of $80-350$ days, but with $\mathrm{NaOH}$ inhibitor (10 ppm $\left[\mathrm{Cl}^{-}\right], 200 \mathrm{ppm}$ $\left.\left[\mathrm{OH}^{-}\right]\right)$pins were stable for over a year. Sellafield predict if $\left[\mathrm{Cl}^{-}\right]$is maintained below $0.5 \mathrm{ppm}$, with pond temperatures of $30^{\circ} \mathrm{C}$ and pond $\mathrm{pH}$ at 11.7 then IGC can be inhibited.

As previously discussed earlier in this section, fuel elements 1 and 2 are most sensitive to becoming sensitised, and thus most susceptible to IGC [33]. Post irradiation examination (PIE) conducted by Kyffin and Hiller [37] showed that $50 \%$ of fuel pins from these elements failed when in stored in $>1 \mathrm{ppm}\left[\mathrm{Cl}^{-}\right]$ when irradiated $>15 \mathrm{GWd} / t_{e}$. On the other hand, $\mathrm{NaOH}$ dosed ponds showed no fuel cladding failures. Cross sections of $\mathrm{NaOH}$ treated specimens showed IGC attack up to $40 \mu \mathrm{m}$ depth, with only $3 \%$ showing deeper IGC cracks. More recent inhibitor studies on more up-to-date high burn-up fuel cladding have been conducted by Sellafield $\left(<38 \mathrm{GWd} / t_{e}\right)$. Specimens showed the SS were sensitised, 
but no more severely depleted of Cr than lower burn-up fuel cladding from the original tests. In $2 \mathrm{ppm}\left[\mathrm{Cl}^{-}\right]\left(50{ }^{\circ} \mathrm{C}\right)$ immersion tests $\mathrm{IGC}$ was inhibited using pH 11.0 NaOH. The full results of the study are shown in table 1.4. Fuel cladding stored at pH $9 \mathrm{NaOH}$ did inhibit corrosion, but showed a lesser inhibitory effect.

To conclude, fuel cladding has been safely stored in in ponds using $\mathrm{NaOH}$ (pH 11.4) and has not failed since 1989. ${ }^{1}$

\begin{tabular}{|l|l|l|}
\hline$\left[C l^{-}\right], \mathrm{ppm}$ & $\mathrm{NaOH}, \mathrm{pH}$ & Condition \\
\hline 0.4 & No & Failure, 450 days \\
0.4 & Yes, 9 & No failure $>1000$ days \\
1.0 & No & Failure, 200 days \\
1.0 & Yes, 9 & No failure $>1250$ days \\
2.0 & Yes, 11.0 & IGC arrested $\left(50^{\circ} \mathrm{C}\right)$ \\
2.5 & No & Failure, 100 days \\
2.5 & Yes, 9 & No failure $>400$ days \\
$\begin{array}{l}\text { Typical AGR pond } \\
\text { chemistry (c. 0.2-1.0) }\end{array}$ & Yes, 11.4 & $>26$ years \\
\hline
\end{tabular}

Table 1.4: Spent AGR fuel cladding performance [37].

\subsubsection{Carbon Deposition}

Carbon dioxide $\left(\mathrm{CO}_{2}\right.$ the AGR coolant gas) decomposes due to radiation forming oxygen radicals which may either recombine with carbon monoxide $(\mathrm{CO})$ to form $\mathrm{CO}_{2}$, or react with the moderator (graphite) to form $(\mathrm{CO})$.

$$
\mathrm{CO}_{2} \rightarrow \mathrm{CO}+\mathrm{O}(\text { radiolytic })
$$

$$
\mathrm{O}+\mathrm{C} \rightarrow \mathrm{CO}
$$

Should the oxygen combine with the graphite (the moderator), then the mass of solid graphite will decrease. This may limit the life of the reactor. As a consequence, the coolant chemistry can be tailored to minimise the oxidation of the moderator by using methane gas.

\footnotetext{
${ }^{1}$ Taken at time that reference was published; 2015.
} 


$$
\mathrm{CH}_{4}+3 \mathrm{CO}_{2} \rightarrow 2 \mathrm{H}_{2} \mathrm{O}+4 \mathrm{CO}
$$

The addition of methane can have an effect on spent AGR fuel cladding however, as post-service inspections have revealed the presence of carbon deposits on the surfaces of the AGR fuel cladding stainless steel. The mechanism is as follows:

$$
\begin{gathered}
\mathrm{CH}_{4} \rightarrow \mathrm{CH}_{3}^{\bullet}+\mathrm{H}^{\bullet} \\
\mathrm{CH}_{3}^{\bullet} \rightarrow \mathrm{CH}_{2}^{\bullet \bullet}+H^{\bullet} \\
2 \mathrm{CH}_{2}^{\bullet \bullet} \rightarrow \mathrm{C}_{2} \mathrm{H}_{4}
\end{gathered}
$$

Radiation causes the methane to be converted to the unsaturated hydrocarbon ethene, which is deposited on the SS. This occurs at Ni sites, as Ni is less prone to oxidation compared to other constituents within the alloy. Ni sites act as a catalyst, and causes carbon to be deposited on the site due to decomposition of the $C O$ according to the Boudouard reaction:

$$
2 \mathrm{CO} \rightarrow \mathrm{C}+\mathrm{CO}_{2}
$$

This is a redox reaction and describes the chemical equilibrium between graphite $\mathrm{C}, \mathrm{CO}$ and $\mathrm{CO}_{2}$. At temperatures below $700{ }^{\circ} \mathrm{C}$ the thermodynamic equilibrium favours the formation of $\mathrm{CO}_{2}$ compared to $\mathrm{CO}$ formation [39]. This continues by extrinsic Ni catalysis [40]. Such deposition could even affect the performance of the AGR fuel in reactor pond storage conditions. This affects the heat transfer properties of the AGR fuel. Carbon, in the form of graphite, is a natural cathode [41] and as a result may cause galvanic corrosion in combination with the sensitised SS. The impact of this carbon deposition is threefold, result- 
ing in: lower plant output, reduced graphite moderator and the possibility of producing a Galvanic cell in cooling storage ponds. Currently this may be minimised by carbonyl sulphide (COS) addition in reactor to poison the Ni catalysis reaction.

\subsubsection{AGR Stainless Steels in Sodium Hydroxide}

Sodium hydroxide ( $\mathrm{NaOH}$, caustic soda), a strong base (i.e. highly dissociated with water) is an important chemical worldwide. It is created in large quantities by the electrolysis industry, and is used by the nuclear industry in the UK to delay the onset of localised corrosion. Caustic soda was identified as a corrosion inhibitor for AGR fuel cladding in lab-scale experiments in the early 1980s, and has been utilised in select cooling ponds since 1985 [31]. Spent AGR fuel has been safely maintained by this process within the UK for over 30 years [42]. Caustic dosing of pond water leads to a positive impact on the corrosion properties of stainless steel advanced gas-cooled reactor (AGR) fuel cladding alloy, 20/25/Nb [43]. After a number of years in reactor, SNF is removed, and the fuel replaced. SNF is safely managed and immersed in spent fuel cooling ponds; and after an interim period in borated station ponds, SNF is transported to larger ponds at Sellafield site, some of which are caustic dosed. Stainless steels (SS) are highly resistant to generalised corrosion. Corrosion resistance as a general rule, increases with chromium content. However, neutron irradiation, in-reactor, can lead to the activation of the radiation induced segregation (RIS) mechanism as explained in earlier chapters. This causes the SS to become less corrosion resistant. Figure 1.6 shows the general relationship between $\mathrm{Cr}$ content and corrosion rate. Should the $\mathrm{Cr}$ content drop below $\approx 12 \%$ wt., corrosion rates increase exponentially; therefore it is important to safely manage RIS affected SS.

The combination of both RIS (thus sensitisation) and the presence of $\mathrm{Cl}^{-}$may elevate corrosion rates. A recent study using the same SS (20/25/Nb) showed that grain boundary triple points and $\mathrm{Nb}$ rich precipitates are initiation sites 


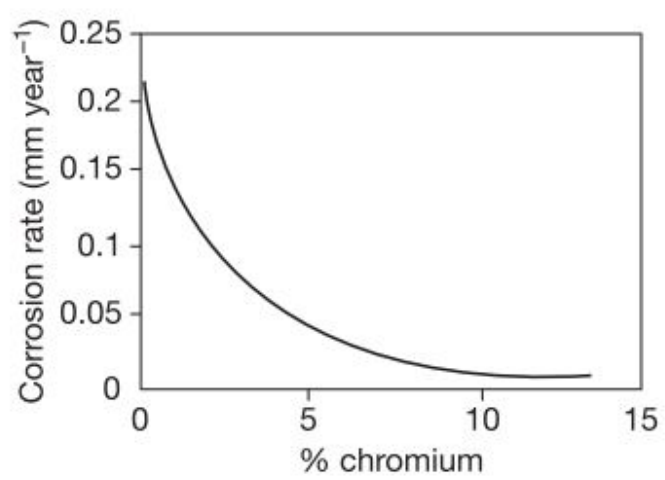

Figure 1.6: Corrosion rate as a function of Cr content within a general SS [44]. An exponential decrease in corrosion rate leads to negligible corrosion once the minimum $\approx 12.5 \% \mathrm{Cr}$ is present.

for localised corrosion, in the sensitised and stabilised microstructures respectively [45]. This is in agreement with previous research conducted in this thesis. Chapter 5 showed that grain boundary intersections and triple points are initiation sites through the time-lapse microscopy method. SVET in combination with SEM-EDS showed that Nb-rich inclusions were concentrated at pit sites, suggesting that IGC has a tendency to initiate at locations with a high number densities of $\mathrm{Nb}$ precipitates (section 4.3.4). This current investigation aims to investigate a process using $\mathrm{NaOH}$ to suppress the effect of Nb-rich inclusions as initiation sites, to investigate if this could be used to reduce corrosion rates further.

Corrosion rates for stainless steels can be reduced by increasing the $\mathrm{pH}$ of the electrolyte [46] [47]. The Pourbaix diagram for iron, the metal actively undergoing dissolution, suggests that immersion within basic environments leads to passivation (shown in figure 1.7).

Excellent resistance to weak bases, for example ammonium hydroxide, is found with 18-8 SS. NaOH, a strong base, can be introduced to an electrolyte to levels of 50 wt.\% for 18-8 SS [47]. An isocorrosion diagram for austenitic stainless steel type 304, 316 and 904 in $\mathrm{NaOH}$ is provided in figure 1.8, the solid lines represent a corrosion rate of $0.1 \mathrm{~mm} \cdot y^{-1}$. Although bases can be used as a corrosion inhibitor, they can also induce corrosion in some metals should an incorrect concentration of base be used. Hydroxides can react to the metal to form soluble 


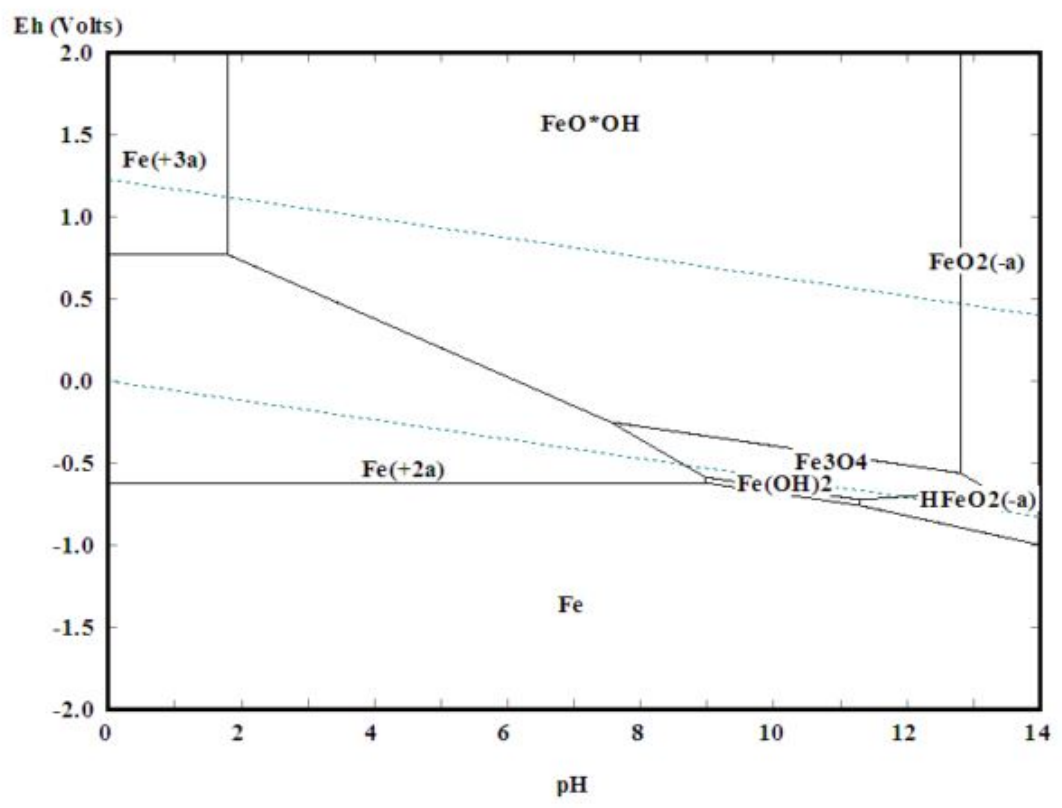

Figure 1.7: Pourbaix diagram for Fe [16]

oxyanions. An example is in aluminium forming aluminium hydroxide from hydroxides, and then reacting further with more hydroxides to form aluminate [48].

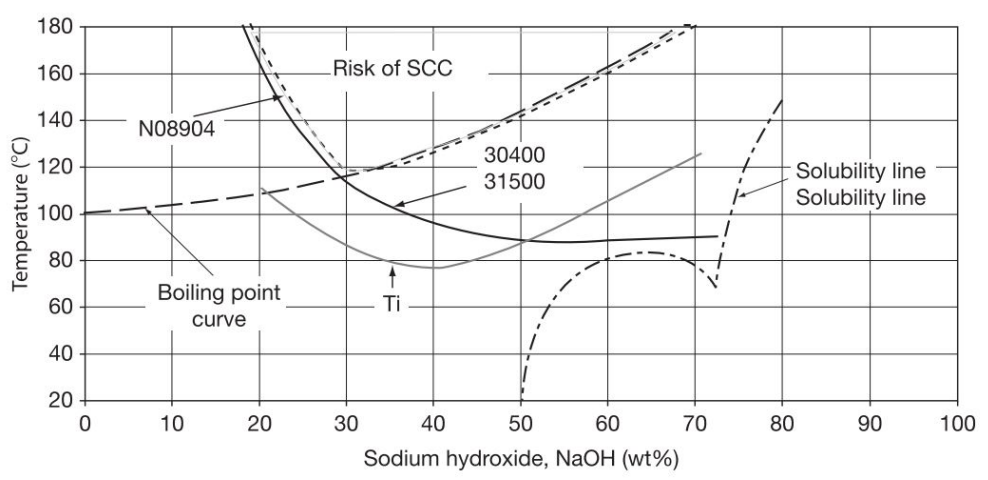

Figure 1.8: $\mathrm{NaOH}$ isocorrosion diagram, solid lines represent a corrosion rate of $0.1 \mathrm{~mm} \cdot \mathrm{yr}-1$. S30400, S31600 and N08904 are ANSI types 304, 316, 904 respectively. Without chloride [44]

The main constituents of an austenitic SS are iron, nickel and chromium (from largest amount to smallest). In addition some SS have molybdenum which is used to increase resistance when in the active region of the polarisation curve. The impact of sodium hydroxide on these key components of the SS is shown in figure 1.9. These alloying elements were researched by Bhattacharya and Singh [49]. 
Fe has the most cathodic open circuit potential when compared to the other elements in the SS. Very close to its open circuit potential the Fe undergoes active dissolution, followed by passivation due to the formation of magnetite $\left(\mathrm{Fe}_{3} \mathrm{O}_{4}\right)$, a stable phase at the first anodic peak. The second peak, within the transpassive region, is attributed to the oxidation of $\mathrm{Fe}_{3} \mathrm{O}_{4}$ to $\mathrm{Fe}_{2} \mathrm{O}_{3}$ (ferric oxide), again lowering current density. $\mathrm{Ni}$ has a breakdown potential that is more noble than Fe, which displays a single peak. This peak represents the formation of nickel oxide, causing a decrease in current density such that along the passive region current density is one order of magnitude lower compared to that of $\mathrm{Fe}$. $\mathrm{Cr}$ undergoes passivation without the same active-passive peak behaviour as with Fe and Ni. Molybdenum has an OCP between that of $\mathrm{Fe}$ and $\mathrm{Ni}$, and undergoes an exponential increase in current density almost immediately without any passivation.

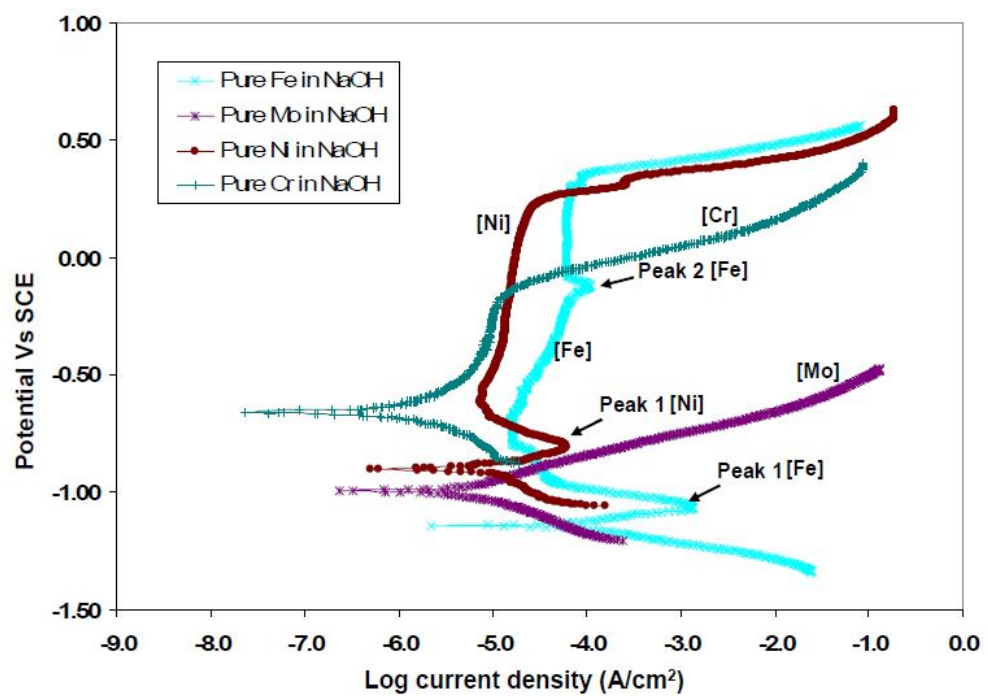

Figure 1.9: Major alloying elements of a general SS (pure $\mathrm{Fe}, \mathrm{Ni}, \mathrm{Cr}$ and $\mathrm{Mo}$ ) in $150 \mathrm{~g} d m^{-3}\left(3.75 \mathrm{~mol} d m^{-3}\right) \mathrm{NaOH}$ at $90^{\circ} \mathrm{C}[49]$

It is well known that intermetallic inclusions play an important role in the initiation of corrosion in SS, and there are several publications to support this. In-situ AFM studies have shown that MnS inclusions, present in 304 SS are a site for corrosion initiation [50]. Ryan et al. [13] used SEM and SIMS to investigate $316 \mathrm{~F}$, a high sulphur grade SS which increased the probability of 
MnS inclusions. The group estimated, from SIMS and elemental analysis that the alloy adjacent to the inclusions could have as little as $10 \%$ at. Cr, compared to $\approx 18 \%$ at. Cr within the bulk. Below the limit for successful passivation, with the authors suggesting $\approx 13 \% \mathrm{Cr}$ needed to make the SS 'stainless'. This depleted region is within the order of 200-400 nm, making it difficult to study. The group suggest that this region of $\mathrm{Cr}$ depletion adjacent to the inclusion allows for 'rapid, high-rate metal dissolution events'. Sulphur forms a pit cover and occludes mass transport, allowing an aggressive pit chemistry to form within, the process becomes autocatyltic and pitting ensues. The process of pitting is described in earlier chapters. Phuah showed that localised corrosion occurred around $\mathrm{NbC}$ inclusions in 20/25/Nb SS, post corrosion [51]. Therefore if NbC inclusions could be removed in some way, corrosion initiation would be suppressed, leading to a lower corrosion rate.

Lyon describes elemental niobium and its corrosion of in [52]. The element has a BCC structure, with the sixth highest melting point of all elements, and exceptional corrosion resistance following very stable passive oxide film formation. $\mathrm{Nb}$ is passive at almost all $\mathrm{pH}$ and potentials, as shown in figure 1.10, due to the very stable passive film ( $\mathrm{Nb}$ is more active than $\mathrm{Zn}$ ). Lyon states the metals must be passive in most environments due to the extreme difficulty in finding literature data on the electrochemical dissolution of $\mathrm{Nb}$, other than in extreme conditions.

Lyon discusses the performance in such conditions first by strong acids, finding that in 20,40,80\% sulphuric, and 20,38\% hydrochloric acids at temperatures between $75^{\circ} \mathrm{C}$ and $95^{\circ} \mathrm{C} \mathrm{Nb}$ is passive. In stark contrast to the passivity of $\mathrm{Nb}$ in acids is its noteworthy dissolution in strong alkali. Nb was found to freely corrode in concentrations of $\mathrm{NaOH}$ above $10 \%\left(>25^{\circ} \mathrm{C}\right)$. Corrosion of $\mathrm{Nb}$ occurs through the dissolution of the passive film, rather than through the pitting process. An overview of corrosion rates in basic media are tabulated in table 1.5.

Robin investigated the behaviour of metallic Nb in concentrated $\mathrm{NaOH}$ in 2004 [53]. The results showed that commercially pure $\mathrm{Nb}$ underwent corrosion at the free 


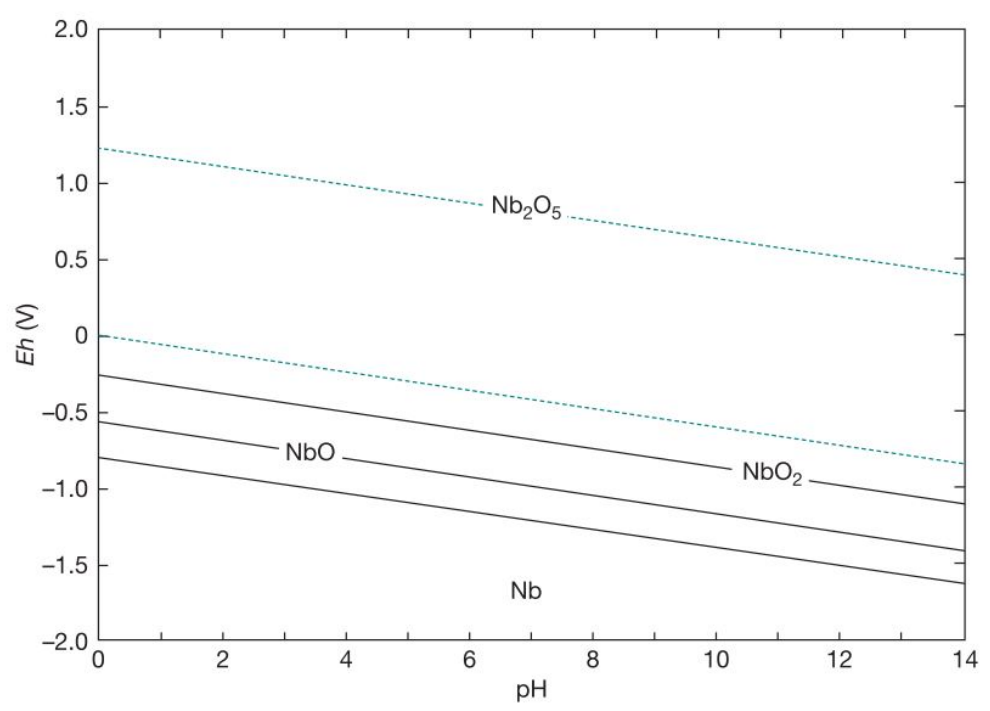

Figure 1.10: Pourbaix diagram of elemental $\mathrm{Nb}$ with a dissolved metal ion concentration of $10^{-5} \mathrm{~mol}$ [52]. Note that this diagram is for elemental $\mathrm{Nb}$, not $\mathrm{NbC}$.

\begin{tabular}{|l|l|l|}
\hline Solution & Temperature $^{\circ} \mathrm{C}$ & Corrosion rate $\left(\mu m \cdot\right.$ year $\left.^{-1}\right)$ \\
\hline $\mathrm{NaOH} \mathrm{1 \%}$ & 98 & $>750$ \\
$\mathrm{NaOH} 5 \%$ & 98 & $>1200$ \\
$\mathrm{NaOH} \mathrm{10 \%}$ & 98 & $>2000$ \\
$\mathrm{KOH} \mathrm{1 \%}$ & 98 & 600 \\
$\mathrm{KOH} \mathrm{5 \%}$ & 98 & $>2500$ \\
$\mathrm{Na}_{2} \mathrm{CO}_{3} 10 \%$ & 98 & $>1500$ \\
$\mathrm{~K}_{2} \mathrm{CO}_{3} 10 \%$ & 98 & $>1500$ \\
$\mathrm{Na}_{2} \mathrm{PO}_{4} 25 \%$ & 98 & $>1300$ \\
$\mathrm{Na}_{2} \mathrm{~S} 10 \%^{2}$ & 98 & 90 \\
\hline
\end{tabular}

Table 1.5: Corrosion rates of $\mathrm{Nb}$ in basic media, recreated from [52].

corrosion potential in 10, 15 and 30 wt.\% $\mathrm{NaOH}$ at 25,50 and $75^{\circ} \mathrm{C}$. Results from electrochemical measurement as a function of $\mathrm{NaOH}$ concentration and temperature are shown in figure 1.11. The graph shows that, at $10 \mathrm{wt} \%$ and above, temperature has a large impact when $\mathrm{NaOH}$ concentration is fixed. Increasing the concentration, and fixing the temperature has less of an effect, presumably because the threshold concentration has already been reached, and by modifying the kinetics (raising the temperature) rates of reaction increase. Both Lyons and Robin display strong evidence that although $\mathrm{Nb}$ metal is stable across most potentials and $\mathrm{pH}$, it's passive oxide film begins to dissolve in a range of concentrated basic mediums. The greatest corrosion rates occur in potassium hydroxide 
$(>5 \% \mathrm{KOH})$ and concentrated $\mathrm{NaOH}(>10 \%)$. If using $\mathrm{NaOH}$, once a threshold concentration of $\mathrm{NaOH}$ is reached (Robin used concentrations above $10 \mathrm{wt} \%$ $\mathrm{NaOH}$ ) changing the kinetics (temperature control) leads to an increased peak current density response. 


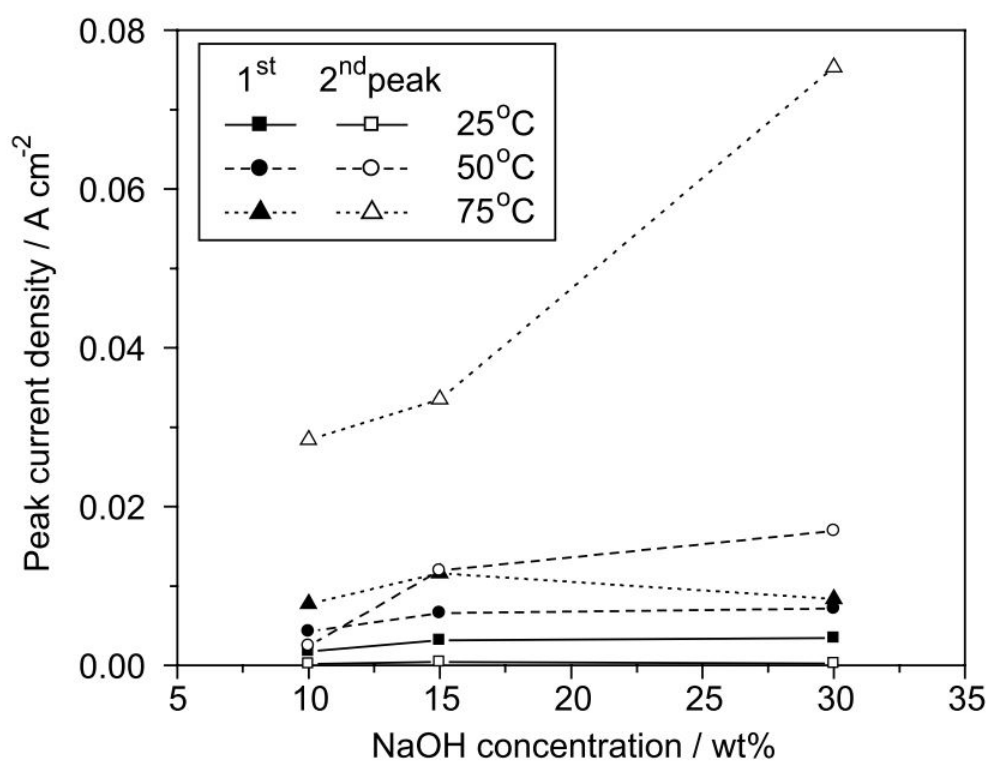

Figure 1.11: Peak current densities as a function of $\mathrm{NaOH}$ concentration and temperature, obtained from Robin [53]

These findings suggest that $\mathrm{NbC}$, as inclusions, may also be affected in a similar fashion. As it was not possible to experiment using concentrated strong $(\mathrm{NaOH})$ basic electrolytes at high temperature, a room temperature environment was used instead. Lower concentrations were also used to study the effect to of $\mathrm{NaOH}$ on $\mathrm{NbC}$ inclusions, as $\mathrm{Nb}$ metal does not correspond directly to its compound as $\mathrm{NbC}$.

\subsection{Fundamentals of Corrosion}

\subsubsection{Thermodynamics}

All metals, given time, will corrode eventually. Metals, when processed and removed from their ores, are at a higher energy state than their corrosion products. The fundamental underlying materials science declares that all materials will always have a preference to reside in its lowest energy state, as per the second law of thermodynamics:

"All spontaneous changes occur with a release of free energy from a system to its surroundings at constant temperature and pressure." 
Thus favourable states are those with a negative change in Gibbs free energy, (equation 1.7).

$$
\Delta G=G_{2}-G_{1}
$$

The process of corrosion is seen as an unfortunate consequence of fundamental thermodynamics, returning the metal to it's lowest energy state. This is best be shown in figure 1.12. The metal, once extracted and worked upon, is in an unstable high-energy state and therefore is thermodynamically unstable with respect to it's oxide, a lower energy state. Corrosion is therefore unavoidable as $\Delta E$ is negative for metals returning to their oxides. The free energy barrier, $\Delta G^{*}$ (energy of activation), exists between the reactants and products and is what keeps metals from spontaneously corroding into their oxides (a non-reversible process). This barrier can be overcome if energy is supplied to the system. More corrosion resistant metals can have larger values of $\Delta G^{*}$, which slow the kinetics such that corrosion only occurs at a slow rate. The opposite is true for less corrosion resistant metals.

Part of the reason why some metals corrode more readily than others can be answered from the thermodynamics above. Iron (Fe) for example suffers more from corrosion compared to gold $(\mathrm{Au})$ for instance, as Fe is more thermodynamically unstable. Kinetics (rates of reaction) are also important, as the free energy is a function of the reaction co-ordinate. Thermodynamics are favourable for $\mathrm{Fe}$ to undergo spontaneous corrosion, but in practice the surface is quickly covered by a thin layer of metal oxide, which for some metals and their alloys can effectively prevent further reactions from taking place. This is known as passivation; and in such a scenario kinetics limits the corrosion, even though the reactions are thermodynamically favourable. For corrosion to occur the rate of dissolution must be greater than the rate that the passive film rebuilds, if this is not the case, the metal is 'passive'.

By adding water to the metal $\Delta G^{*}$ is lowered; especially if it contains ionic 


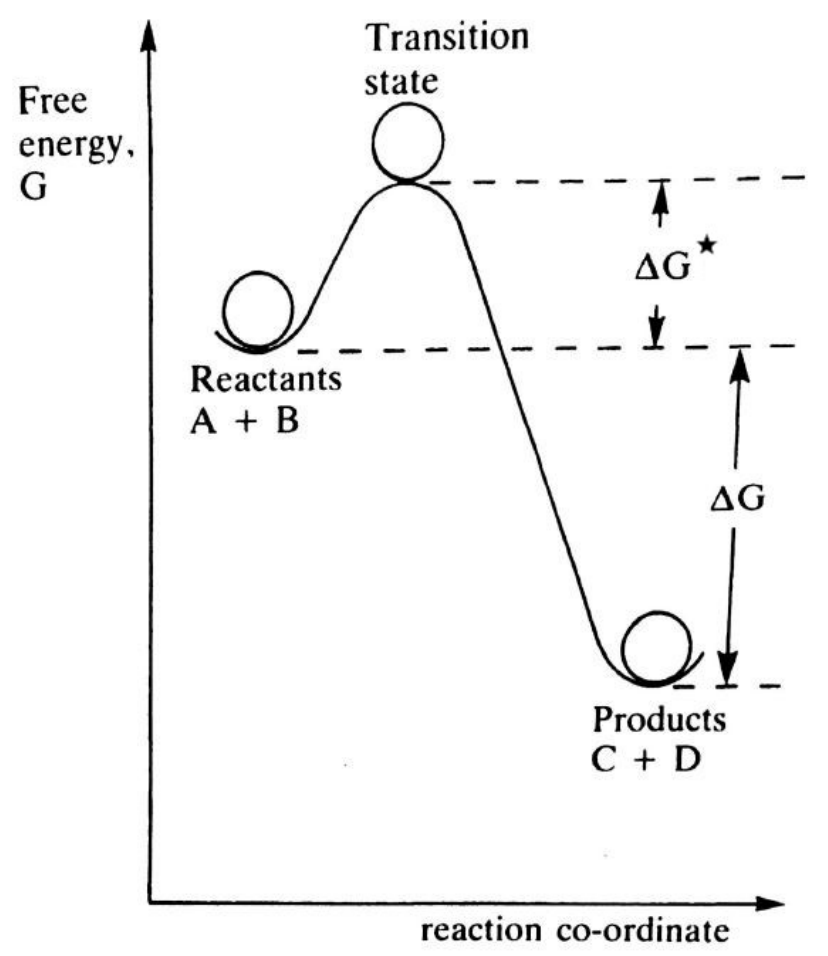

Figure 1.12: Free energy curve taken from Trethewey and Chamberlain [10]

salts. Aqueous species capable of carrying a charge, such as the transfer of metal cations by salts in solution is called an electrolyte. Corrosion is a redox process and comprises two half reactions; reduction and oxidation. This is an electrochemical process in which electrons are donated in the oxidation half reaction, and in order to balance the reaction, are consumed in the reduction half reaction. Consider a system of two metals connected together, immersed within an aqueous electrolyte such as salt containing water, such as is provided in figure 1.13. In order for corrosion to occur, electrons flow from one metal to the other in order to satisfy both parts of the redox reaction. Both oxidation and reduction reactions occur at the same time, and thus at the same rate, i.e. no net increase of electrons or net consumption of electrons [54]. The process of corrosion can occur on a single metal, with anodes and cathodes separated spatially [16]. From the above system, the site where electron transfer initiates is called the anode; and the oxidation half reaction that occurs here is called the anodic reaction. The site where electrons are consumed, or reduced, is called the cathode, and this process 
is called the cathodic reaction. This system containing an anode, cathode and a medium in which ionic species can undergo mass transport (electrolyte) is called a wet corrosion cell. The general anodic reaction of metal dissolution (oxidation) is as follows in equation where $M$ represents the metal, and $n$, the number of electrons transferred 1.8. Equation 1.9) shows the processes occurring at the cathode at very low $\mathrm{pH}<<7$. This is known as the hydrogen evolution reaction, and occurs in acidic conditions. In such a scenario, the high excess of protons from the acid (hydrogen ions) react with electrons from the metal, reducing hydrogen cations into hydrogen gas. For all neutral and basic conditions oxygen reduction takes place (equation 1.10). As the concentration of protons is much lower when acidic media is not used, the hydrogen reduction reaction is unlikely, unless oxygen diffusion is limited (anaerobic conditions). Equation 1.10 shows how electrons generated at the anode, react with adsorbed water and oxygen on the surface of the metal to form hydroxyl anions. From this stage the process continues by combining equations 1.9 and 1.10. Equations 1.8, 1.9 and 1.10 are well understood [55] [10]

$$
\begin{gathered}
M \leftrightarrow M_{n}^{+}+n e^{-} \\
2 H^{+}+2 e^{-} \leftrightarrow H_{2} \\
2 \mathrm{H}_{2} \mathrm{O}+\mathrm{O}_{2}+4 e^{-} \leftrightarrow 4 O H^{-}
\end{gathered}
$$

\subsubsection{Nernst Equation and Pourbaix}

Walther Nernst derived an equation to describe the half reaction, relating reactant to product ratio (equation $1.11[54]$ ). $E^{\text {rev }}$ is the reversible potential at equilibrium for a half reaction. $\mathrm{R}$, T represent the molar gas constant $\left(8.314 \mathrm{JK}^{-1} \mathrm{~mol}^{-1}\right)$ and temperature in Kelvin $(298 \mathrm{~K})$ respectively. The variables within the braces 


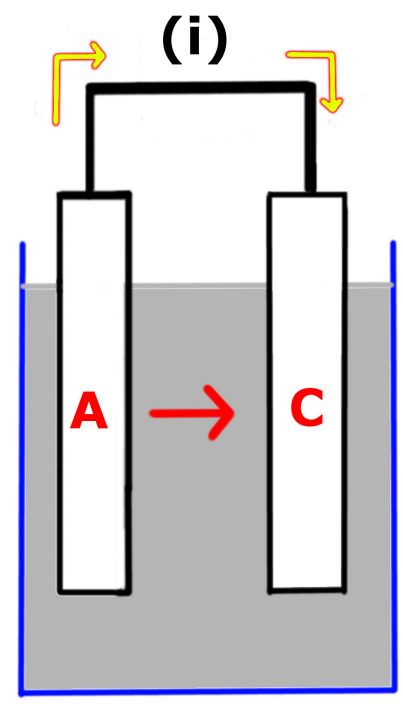

\section{Current Flux $\longrightarrow$ \\ Ionic Flux $\longrightarrow$}

\section{(ii)}

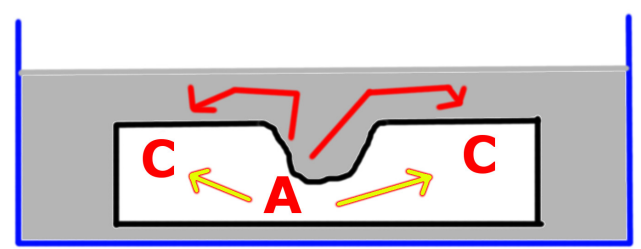

Figure 1.13: Schematic of a wet corrosion cell. (i) Illustrates the case of two different metals immersed in the same electrolyte, but connected together. For example Fe and Zn. (ii) Illustrates the case where a single metal is immersed, with anodes and cathodes separated spatially, but on the same metal. A represents the anode (i.e. Zn), C represents the cathode (i.e. Fe), the current flux is the flux of electrons through the metal (ii) or metal contact in case (i), the diffusion of metal cations is shown by the ionic flux within the electrolyte. This schematic was recreated from Akid [56]. 
represent the concentration of oxidised:reduced species. n, represents the number of moles of electrons transferred within the reaction, and F is Faraday's constant. The superscript j, k constitutes the stoichiometric coefficents in half reaction experiments. The Nernst equation links the likelihood for a specimen to undergo the anodic or cathodic half reactions to thermodynamics. Therefore it can be used as an estimate for the expected outcome of a reaction at thermodynamic equilibrium (i.e. not kinetic) . For example, net cathodic reactions will proceed when the potential is less anodic than $E^{r e v}$. Likewise, the anodic reactions will occur when potentials are more negative than $E^{\text {rev }}[54]$.

$$
E^{r e v}=E^{0}+\frac{R T}{n F} \ln \left[\frac{\Pi\left(a_{o x}\right)^{j}}{\Pi\left(a_{\text {red }}\right)^{k}}\right]
$$

The Nernst equation can be simplified by taking the ratio of products to reactants within the braces. Fundamental thermodynamic theory (Henry's law) allows the assumption that activity and concentration can be equated (equation 1.12). The activity coefficient can be taken as being equal to one. This holds true when using dilute concentrations. Using Raoult's law that states when the mole fraction is close to unity as in equation 1.13. Collating the Nernst equation (1.11), Henry's law (1.12) and Raoult's laws (1.13) together, equation 1.14 can be obtained.

$$
\begin{gathered}
a_{A}=\gamma c_{A} \approx c_{A}=[A] \\
\text { Activty }=\text { Molefraction } \\
E^{\text {rev }}=E^{0}+\frac{R T}{n F} \ln \frac{[\text { Products }]}{[\text { Reactants }]}
\end{gathered}
$$

Marcel Pourbaix applied the Nernst equaion to his studies to construct diagrams of $\mathrm{E}$ as a function of $\mathrm{pH}$ [56]. These are known as Pourbaix diagrams and 
show areas where the metal is immune, passive, or corroding when immersed in an electrolyte. Pourbaix defined a metal undergoing active corrosion as one where the concentration of its ions is $\geq 10^{-6} \mathrm{~mol} \mathrm{dm}^{-3}$. Metals classed as immune have a species concentration below this figure, while those that are protected by an insoluble layer are said to be passivated. Pourbaix diagrams allow a corrosion engineer to evaluate the state of the metal if $\mathrm{pH}$ and $\mathrm{E}$ are known. All potentials are with respect to a reference electrode, in most cases (such as those shown in figures 1.17, 1.14 and 1.15) this is a standard hydrogen electrode (SHE). Note that the diagrams here have been taken from [16], and are based on the species concentration of $10^{-5}$ mold $m^{-3}$, rather than $10^{-6}$ moldm ${ }^{-3}$ which is more common [57]. The solid lines are calculated by using electrode potentials and the Nernst equation using equilibrium constants for different reactions. The upper dashed line is the region of oxidation and the lower, the region in which reduction occurs. For the Fe system in water this is most likely the oxygen reduction reaction, rather than the hydrogen evolution reaction, which would occur in an acid. The potential of a saturated calomel electrode (SCE, such as is used in this thesis) is $+242 \mathrm{mV}$ with respect to the SHE. More information on reference electrodes is given in section 2.3.1.

Take as an example the Fe-water system at room temperature (figure 1.14), where the metal is immune at all $\mathrm{pH}$ at $\sim-1000 \mathrm{mV}$ (SHE). If E increases postitively past $\sim-600 \mathrm{mV}$ (SHE) the metal undergoes corrosion, and is in the active state as $\mathrm{Fe}^{2+}$ are produced. However if the electrolyte is basic ( $\mathrm{pH} 9$ to $\mathrm{pH} 12$ ) at $\sim-600 \mathrm{mV}$ (SHE), the material is said to be in the passive regime, whereby iron hydroxides can form. Further information on Pourbaix diagrams can be found in [58]. The Cr-water system is shown in figure 1.15. The horizontal line at -1000 $\mathrm{mV}$ (SHE) means that the reaction of $\mathrm{Cr}$ forming $\mathrm{Cr}^{2+}$ is not dependant on $\mathrm{pH}$. Above this line the oxidised product is stable, and below the solid $\mathrm{Cr}$ is stable with respect to the oxidised product. Taking the previous example at $\sim-600 \mathrm{mV}$ (SHE), $\mathrm{Cr}^{2+}$ is stable until the $\mathrm{pH}$ increases to 5 . For this fixed potential, but 
for more basic $\mathrm{pH}$, a passive $\mathrm{Cr}$ oxide is formed $\left(\mathrm{Cr}_{2} \mathrm{O}_{3}\right)$ this is an example of a linear negative relationship with $\mathrm{pH}$. The Pourbaix diagram for $\mathrm{Ni}$ is displayed in figure 1.16. The formation of $\mathrm{Ni}$ ions $\left(N i^{2+}\right)$ from $\mathrm{Ni}$ occurs at $\sim-400 \mathrm{mV}(\mathrm{SHE})$, at $\mathrm{pH} 0$ to $\sim \mathrm{pH} 9$. At more basic $\mathrm{pH}$, the nickel oxide $(\mathrm{NiO})$ formation can occur, which is insoluble in water. 


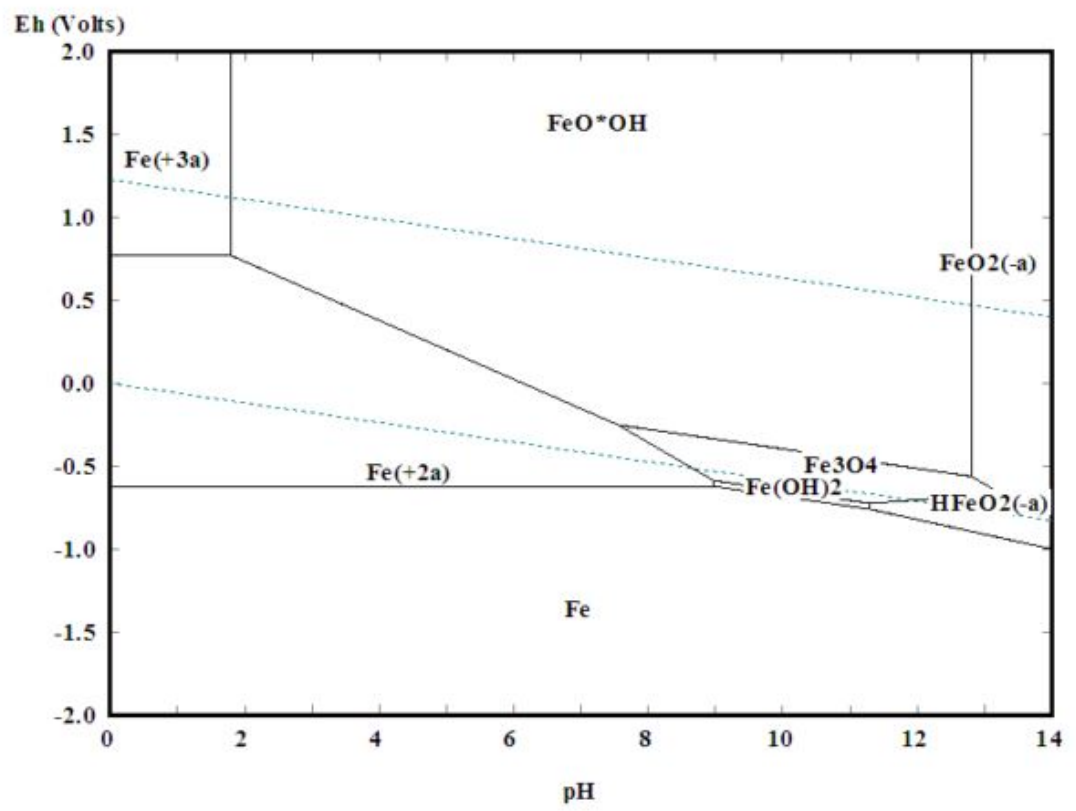

Figure 1.14: Pourbaix diagram of Fe with soluble species concentration $10^{-5} \mathrm{~mol}$ $d m^{-3}$ and common stable oxides present at $298 \mathrm{~K}[16]$

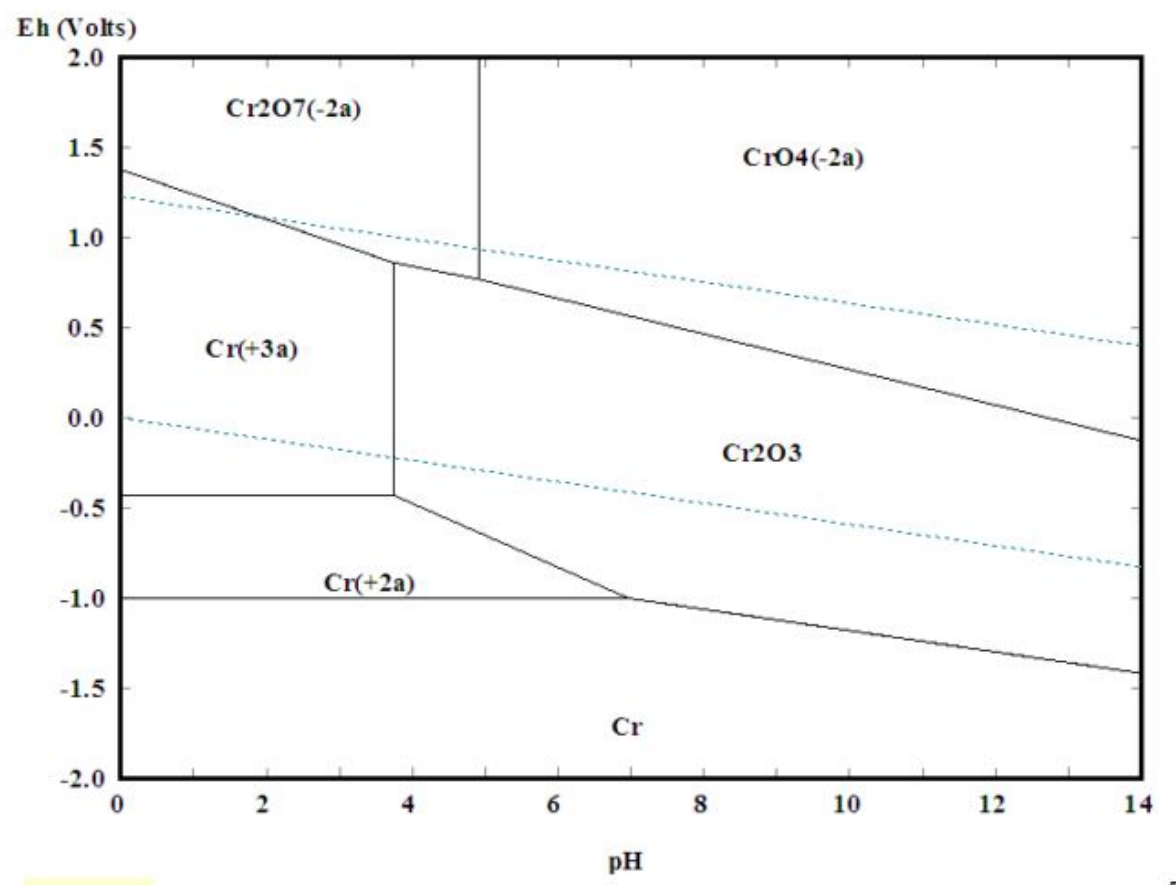

Figure 1.15: Pourbaix diagram of Cr with soluble species concentration $10^{-5} \mathrm{~mol}$ $d m^{-3}$ and common stable oxides present at $298 \mathrm{~K}$ [16] 


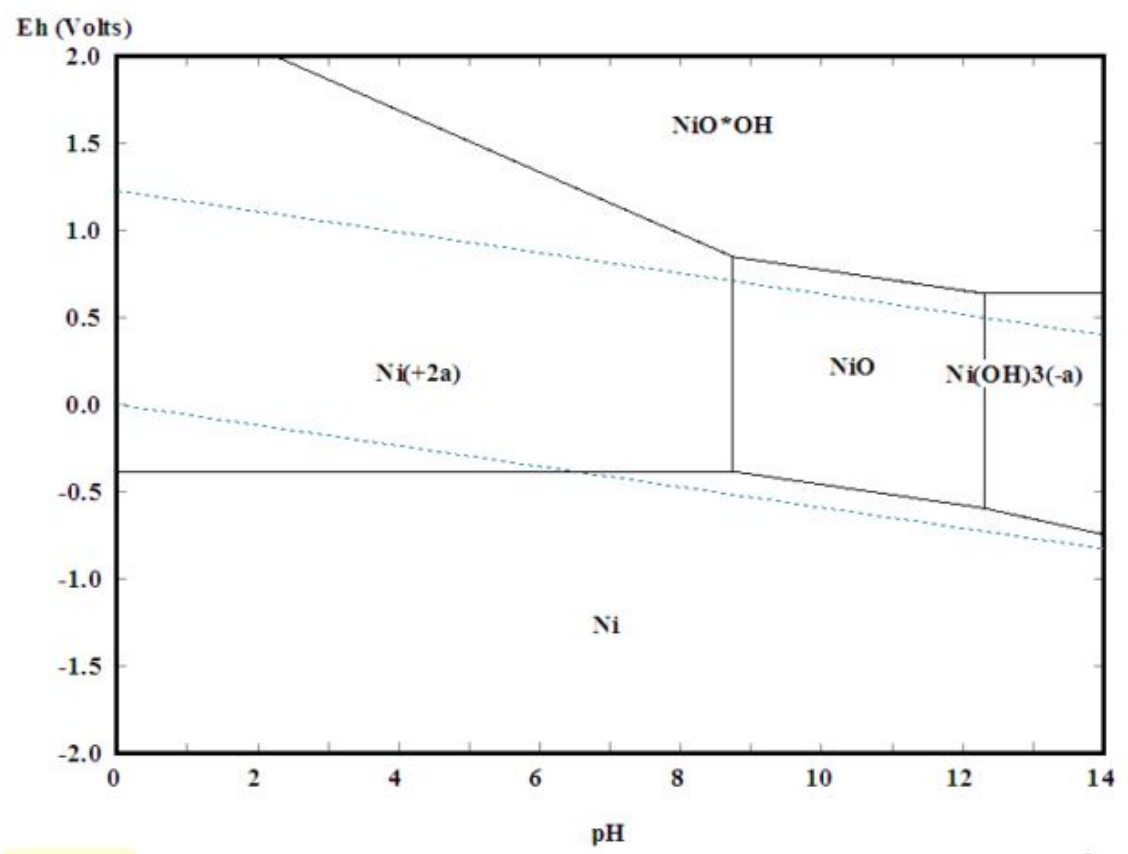

Figure 1.16: Pourbaix diagram of Ni with soluble species concentration $10^{-5} \mathrm{~mol}$ $d m^{-3}$ and common stable oxides present at $298 \mathrm{~K}[16]$

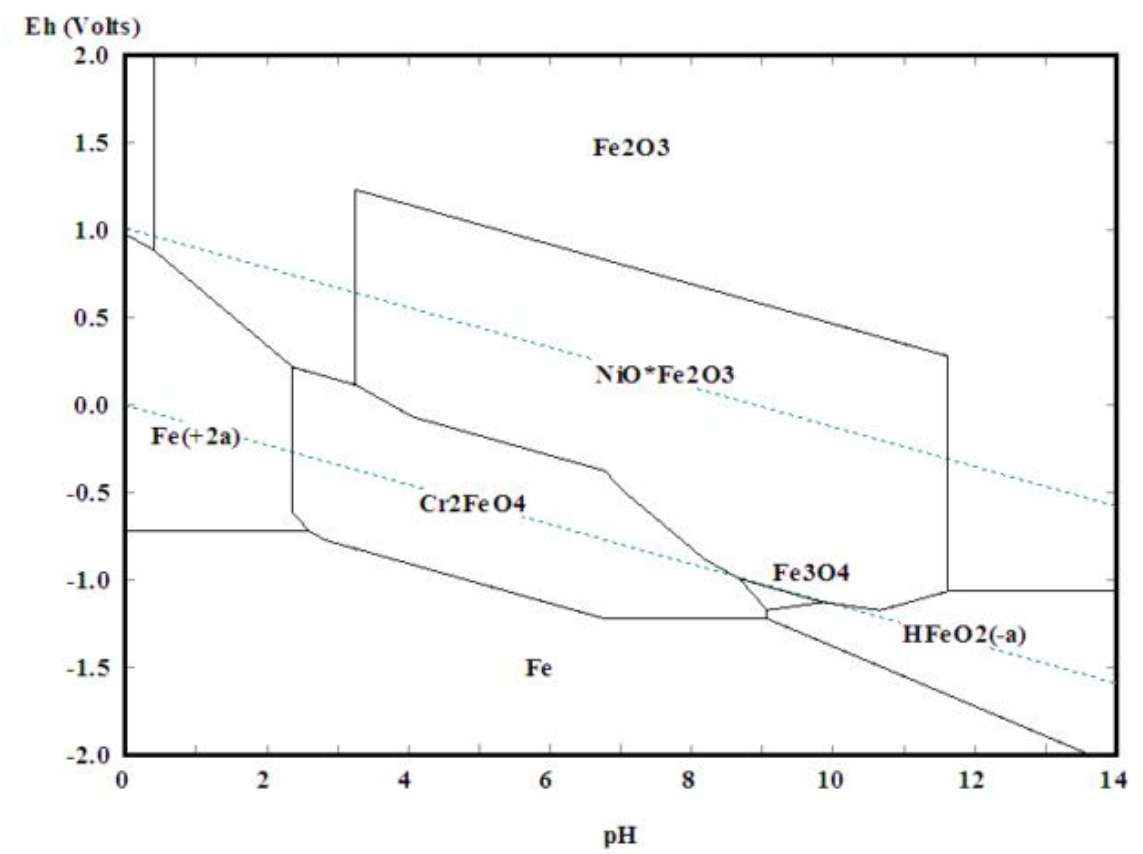

Figure 1.17: Pourbaix diagram for austenitic stainless steel (Fe, Cr, Ni) with soluble Fe, Cr, Ni species concentration $10^{-5} \mathrm{~mol} d m^{-3}$ and common stable oxides present at $573 \mathrm{~K}\left(300{ }^{\circ} \mathrm{C}\right)[16]$ 


\subsubsection{Electrochemical Kinetics and Mixed Potential The- ory}

The Nernst equation uses thermodynamics to describe the likelihood for a metal to undergo either of the two half-reactions, oxidation or reduction. It does not however give any information on rates of reaction; and therefore provides no timescale for said corrosion to occur. As the half reactions involve electron transfer either by oxidation (generation of electrons) or reduction (electron consumption) there is a change in current, which can be measured. This net electron transfer gives information on the rates of reaction, when normalised, to a current density (dividing by unit area) [16]. From the above section (1.2.2) it is shown that if, $E^{r e v} \neq E^{o}$, either a net anodic or cathodic reaction will occur. The exchange current density, $i_{o}$, is the where there is no net anodic or cathodic current. In such a case the two reactions can still occur at the same rate, and corrosion is still ongoing.

$$
\begin{aligned}
& i_{a}=i_{c}=i_{0} \\
& i_{a}-i_{c}=0
\end{aligned}
$$

In a non-equilibrium state, the metal is said to be be polarised with respect to its equilibrium potential, by some quantity known as an over-potential, $\eta$. This is simply the change in potential from the potential predicted by the Nernst equation, (equation 1.17). Where $\mathrm{E}$ is the potential of the metal undergoing corrosion, and $E_{e} q$ is the potential predicted by the Nernst equation.

$$
\eta=E-E_{e q}
$$

$i_{a}, i_{c}$ can both be linked to the Gibbs activation potential, which can also be related to $\eta$. The anodic relationship is shown in equation 1.21 and the re- 
lationship for the cathodic reaction is shown in equation 1.22. Equation 1.21 shows the individual anodic reaction occurring at the electrode (equation 1.19), the dissolution of metal into metal cations. Equation 1.21 shows the individual cathodic half reaction occurring at the electrode (equation 1.20), i.e. the consumption of metal cations and electrons to return to metal. Both equations 1.21 and 1.22 can also be used to describe the corresponding cathodic corrosion reactions given in equation 1.9 and 1.10. $\alpha$ is related to the electrode used and is available in tables of data, known as the Tafel constant. Now the overpotential (a function of E, as described above in eq 1.17) can be plotted as an exponential function of the two branches of current density, i, as shown in figure 1.18. This shows the link between $E^{r e v}$ and $i_{o}$, whereby the rates of reaction for each of the half-reactions generally increase with increasing potential difference away from $E^{r e v}$, hence the exponential relationships. However these exponential reaction rates become limited by the transfer of charge at the interface at large potentials, as given in equation 1.18. The location of $i_{0}$ is where the anodic and cathodic reactions occur at the same rate, which occurs at $E^{\text {rev }}$. If this is plotted as a log-linear graph, it is known as a Tafel plot as shown in figure 1.19.

$$
\begin{gathered}
E-E^{r e v}=\operatorname{blog}\left(\frac{i}{i_{o}}\right) \\
M \longrightarrow M^{Z+}+z e^{-} \\
M^{Z+}+z e^{-} \longrightarrow M \\
i_{a}=i_{0} \exp \left[\frac{(1-\alpha) N F}{R T}\left(E-E_{e q}\right)\right] \\
i_{c}=i_{0} \exp \left[\frac{(-\alpha) N F}{R T}\left(E-E_{e q}\right)\right]
\end{gathered}
$$




$$
\log i=\log i_{o}+\frac{E-E^{r e v}}{b}
$$

These reactions describe the anodic and cathodic reactions which occur at one of the electrodes, as described earlier. Typical E versus $\log |i|$ relationships for all four reactions, metal oxidation, metal reduction, hydroxide oxidation and oxygen reduction are plotted as in figure 1.20. The gradient of the lines is equal to $\frac{1}{b}$ as given by equation 1.23 if plotted as $\log |i|$ versus E. Otherwise, the gradient is equal to b. The metal reduction and hydroxide oxidation lines are usually removed from the Evans diagram, but have been included for ease of understanding for the reader. The corrosion rate can be determined from the Evans diagram as it is a function of $i_{c o r r}$, which is shown on the $\mathrm{x}$ axis. The metallic system undergoing corrosion has a mixed potential $E_{c o r r}$ which is also the point in which the two curves intersect along the y axis. This is different from the values obtained by the Nernst equation. 


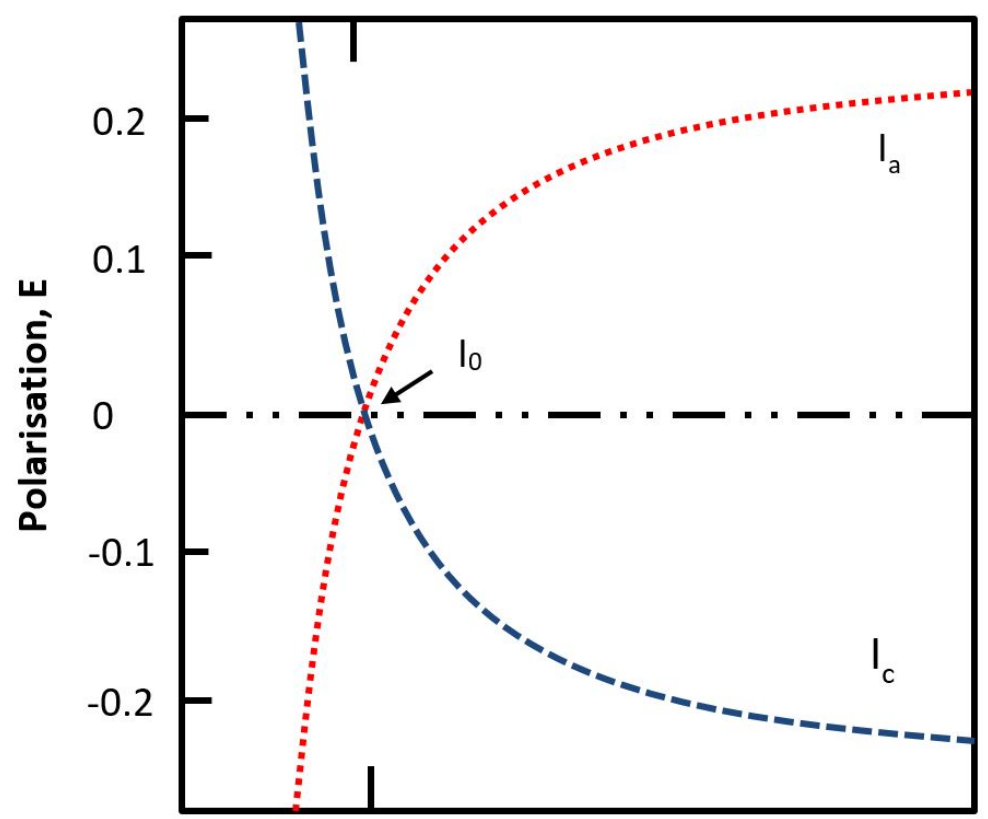

Current Density, i

Figure 1.18: The exponential relationship between current density and polarisation.

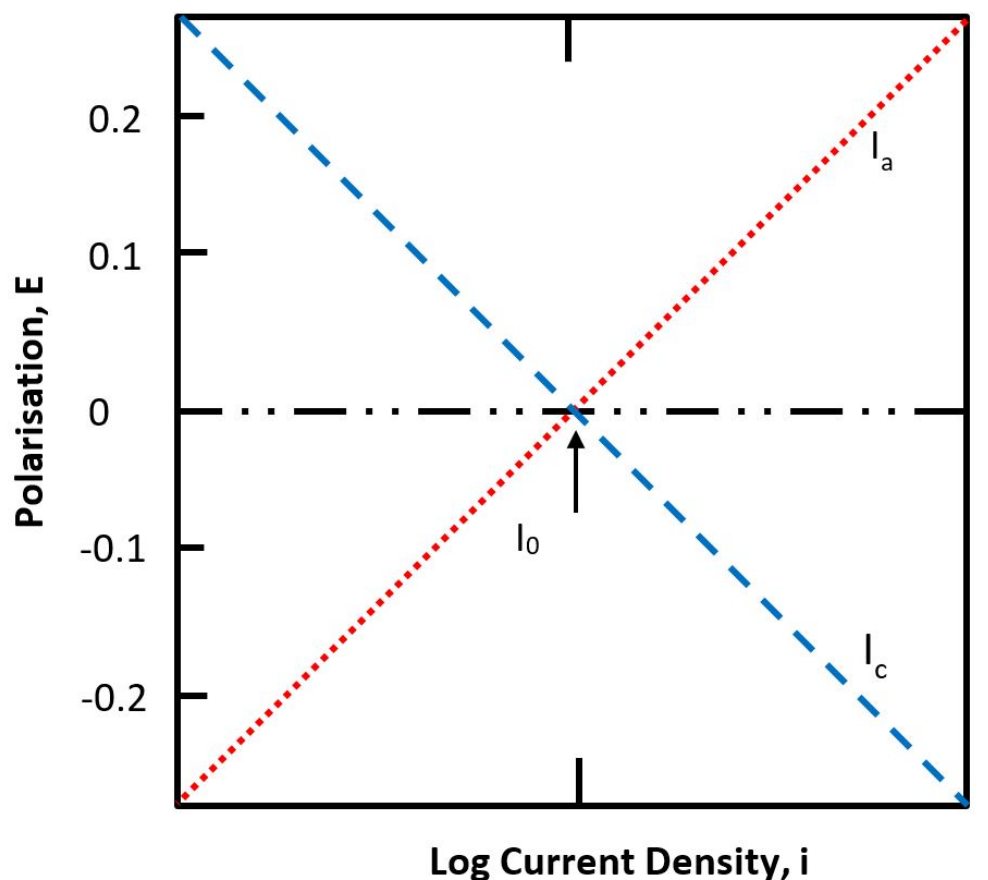

Figure 1.19: A Tafel plot showing the relationship between polarisation and current is linear if the polarisation is plotted against the logarithm of current density. The diagonal lines represent the individual anodic metal oxidation reaction and the cathodic metal reduction reaction occurring at the electrode. 


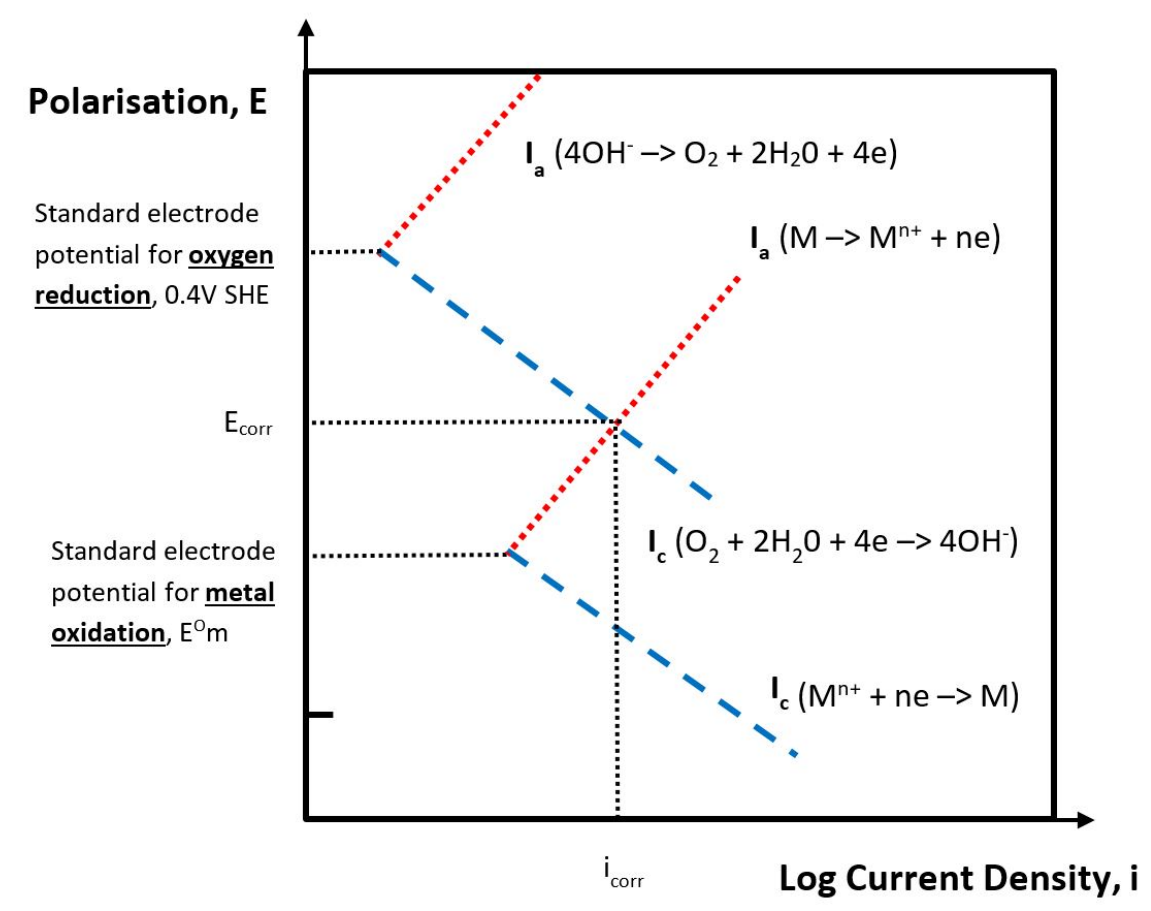

Figure 1.20: An Evans diagram for a system undergoing the oxygen reduction reaction. This graph shows all four reactions occurring on the electrodes, which have been added for ease of the viewer. 


\subsection{Localised Corrosion}

\subsubsection{Pitting Corrosion}

Stainless steels are able to passivate by the formation of a natural protective oxide layer which forms on the surface prior to immersion. This layer, while protective, is susceptible to highly localised attack in solutions containing halide ions, and particularly chloride ions $\left(\mathrm{Cl}^{-}\right)$[59] [60] [61] [62]. Pitting corrosion is a form of localised corrosion; where select, discrete areas of the surface undergo metal dissolution. Galvele [63] developed a model for pitting corrosion, introduced in 1976, which described the pit initiation conditions, the hydrolysis reaction within the pit, and the critical proton concentration required for pitting. In the pitting corrosion process, the two half reactions governing corrosion are spatially separated [64]. A schematic shows the separation in figure 1.21.

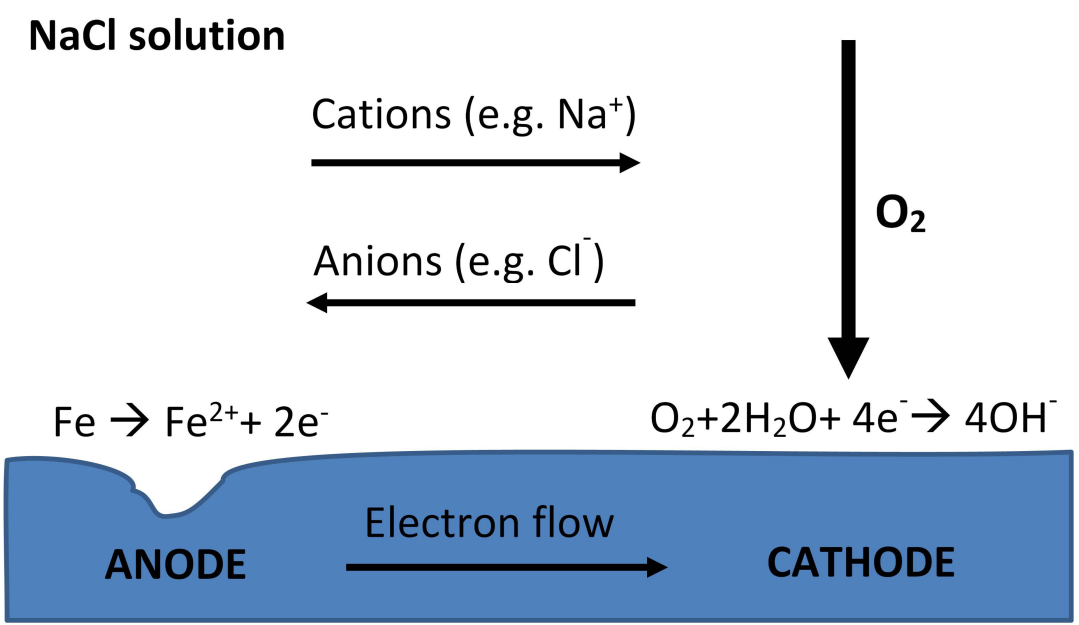

Figure 1.21: Separation between anode and cathode which can occur in pitting corrosion.

The pit cover and dimensions restrict oxygen transport. Thus as the anodic half reaction is concentrated at the pit site, the cathodic half reaction occurs at the surface where the oxygen transport is less limited, favouring the oxygen reduction reaction. The processes occurring at each site are shown in figure 1.21. As oxygen reduction causes a local change in $\mathrm{pH}$, it inhibits pit formation at sites 
close to the active pit site. Pitting corrosion, when compared to general corrosion, can propagate more rapidly [65]. Cavity-like, hemispherical pits and holes are characteristic of this type of corrosion; which may burrow through the entire gauge of the metal, even if the remainder of the metal remains largely corrosionfree. This type of corrosion is difficult to detect visually during the propagation phase, as the pit dimensions are often deep, but the diameter of the pit may be small and are often covered by a lace-like structure. Schwenk noted the difficulty in detecting these cavities, hence used a fine pin to detect covered pits on stainless steels [66]. Once the initiation phase has completed, corrosion pits on stainless steels either repassivate, or propagate, thereby becoming autocatalytic. In order for stable pit growth to be maintained, generally the pit environment is many times more concentrated than that of the bulk electrolyte. Ryan et al. states that there is a critical concentration for pit stability, that is for it to continue propagation, has to be $\geq 70 \%$ of saturated metal chloride for 300 series austenitic stainless steels [67]. Laycock et al. also note this requirement [68]. In these environments the solution may become supersaturated, thereby precipitating to form a film within the pit, most often at the bottom. For a SS it is usually $\mathrm{FeCl}_{2}$. This salt film formation limits transport of ions to and from the site of metal dissolution. Furthermore, pits often tend to form by burrowing under the surface of the stainless steel, such that a perforated 'lacy' cover forms over the pit [69]. In addition to the salt film, these covers are essential to pit stability and help to limit diffusion of the vital pit chemistry away from the pit. Pit covers also make the pits more difficult to spot, as the holes within the lace-like structure are much smaller than the cavity of the pit itself. The lacy cover is essential to ongoing pit growth, at least in the early stages. Rosenfield and Danilov [70] studied the effect removing the pit cover during pit growth. The group found that the majority of pit covers that were punctured underwent repassivation. The application of synchrotron x-ray tomography is a promising technique that has allowed the in-situ imaging of pit nucleation and growth as a function of time. 
This has been applied by Ghahari [71] [72] on 304 stainless steel as shown in figure 1.22. From the figure it can easily be seen that the pits nucleate and form hemispherical shaped cavities along the surface of the metal. The prominent pit develops the characteristic lacy cover, which hinders diffusion and keeps the pit cavity concentrated with metal cations and the aggressive anions.

Both the processes and models of pitting of metals are well described in literature [60] [65] [66] [73]. More information on pit covers, and modelling of pit covers are given in [68] [69]. Pitting corrosion can be broadly described in two stages: initiation and propagation. As noted prior, propagation requires a number of factors to be maintained, such as cathode area, pit geometry, mass transport, pit composition and any required driving force (polarisation) [65]. Initiation on the other hand is strongly related to both metastable events and stable events. Pit initiation of course is required for pit propagation to occur and therefore, unstable (metastable) and stable pits must follow the same initiation processes [74]. Propagation therefore is the most crucial stage in order to maintain pit development. The process of pitting corrosion on stainless steels can be broken down into more stages, as follows:

1. Loss of passivity. Breakdown generally occurs either through adsorption of ions, penetration by ions or thining/breaking of the passive oxide film [75] [63]. Austenitic stainless steels are mainly composed of $\mathrm{Fe}$ and Ni, which are particularly susceptible to the $\mathrm{Cl}^{-}$anion; compared to others such as $\mathrm{Br}^{-}$or $I^{-}$. The $\mathrm{Cl}^{-}$anion may penetrate the oxide film due to its small diameter, allowing it to diffuse through the film [76]. From this point the anions can adsorb onto the bare metal surface.

2. Nucleation of metastable pits occurs where the passive film starts to undergo breakdown [77] [78] [79] [80]. The reasoning for the nucleation of the metastable pits is debated. One school of thought [81] is that the surface of the stainless steel constantly undergoes a series of random current density fluctuations that are a function of time and space. Should these small 


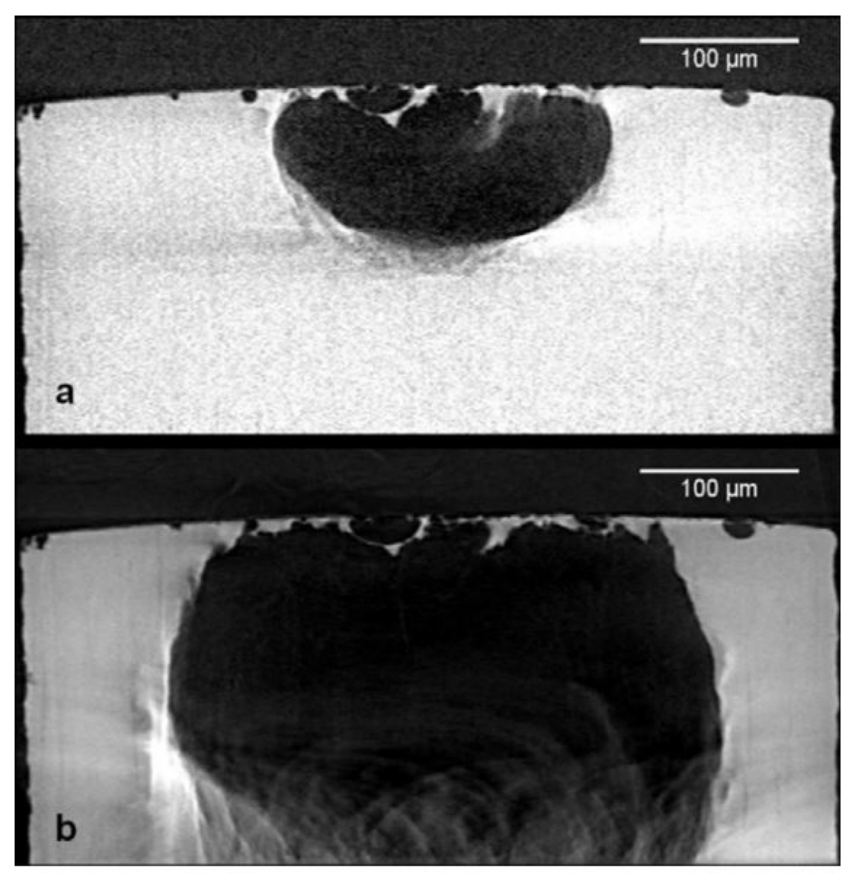

Figure 1.22: Tomograms of 304 stainless steel pin after (a) 1 minute, (b) 6 minutes. The pin was exposed to $1 \mathrm{Mol} d m^{-3} \mathrm{NaCl}$ and polarised galvanostatically to $500 \mu \mathrm{A}$. Metal is white [71].

changes in current density be amplified (application of external polarisation for example) these small fluctuations nucleate, leading to metastable pitting. This was studied by Zhu and Williams using an scanning electrochemical microscope (SECM) with 304L SS microelectrodes, whereby the onset of metastable pitting was shown as fluctuations within the order of picoamps [81].

3. Metastable pitting events may grow in terms of current and become successful pit initiation sites, as studied by Issacs [82]. Acidification occurs within the pits due to water hydrolysis; this is the reaction between metal cations and water, forming metal hydroxide and protons. Pit covers begin to form, which allow the pit to maintain aggressive chemistry for the pitting process.

4. Most of these pits may passivate, whereby the process simply ceases. Rupture of pit covers also leads to repassivation in the majority of cases as the aggressive chemistry becomes diluted [70]. 
5. Stable pitting occurs in localised areas which do not passivate. This leads to an increase in metal ion dissolution, and hence current density. In such a case the charge imbalance (due to the excess of metal cations) anions present in the electrolyte, such as chloride, diffuse toward the pit to maintain charge balance, leading to further corrosion. This leads to a subsequent increase in metal cations, causing further acidification (water hydrolysis) and attraction of aggressive anions. The metal is said to be in an active state as the process is auto catalytic.

Pitting corrosion may initiate at different locations on stainless steels, and can depend on the environment. Different phases within the metal are often cited as locations where corrosion may initiate. This may be due to a difference in composition, shape, and/or size of the phase [83] [84]. This is of course inclusive of defects or inclusions such as chromium carbides, manganese-sulphide, and niobium carbides [13] [61]. This also includes surface effects on the metal, such as scratches, where the protective oxide film may be ruptured. Corrosion performance is very dependent on surface finish, with rough surfaces being more susceptible to corrosion [85]. Holness and Harrison [86] suggest the increased surface area on rough surfaces provides more locations in which $C l^{-}$anions can accumulate. Fine polished surfaces in contrast are similar to clean-cut surfaces and therefore lead to a lower susceptibility for anions to penetrate and destroy the film. Another possibility is that rough finishes from the grinding process create deep enough grooves which would form sites for crevice and pitting corrosion. Corrosion may also initiate at areas where local oxygen concentrations are low, as stainless steels require oxygen in order to form the passive $\mathrm{Cr}$ oxide film. This is known as differential aeration corrosion. This process occurs in pits, (Evans) droplets and crevices, whereby diffusion of oxygen is limited in different parts of the cell. Differences in pH may cause corrosion. Iron is in a region of immunity at $\mathrm{pH} 12$; though is susceptible to attack in acidic conditions $\mathrm{pH}<7$ (Figure 1.14). In a situation where the stainless steel is in contact with an electrolyte, pits and 
crevice sites may be highly acidic $(\mathrm{pH}<0)$ [13]. The acidic pit environment is formed from the reaction between metal cations and water, forming metal hydroxides and protons (hydrolysis of water). This causes accelerated corrosion as per the proposed Fontana-Greene mechanism for crevice corrosion [87].

Breakdown potential at pit and crevice sites can be hundreds of millivolts lower than that of the metal surface; as a consequence of IR drop (which is related to pit and crevice geometry) [88]. These environments are more harsh than that of the bulk electrolyte, which help the continued aggressive growth of the excavation [13] [71]. It has been shown that stainless steel immersed 0.5 mol $d m^{-3} \mathrm{NaCl}$ electrolyte at neutral $\mathrm{pH}$ can have a local pit $\mathrm{pH}$ of less than 1.0 and a $\left[\mathrm{Cl}^{-}\right]$over 12 times that of the bulk solution $\left(6.2 \mathrm{~mol} \mathrm{dm}^{-3}\right)$ [57]. Electrons generated at the anode flow through the bulk metal to the site of the cathode which is likely to be the larger free surface more suitable for the oxygen reduction reaction, where oxygen availability is plentiful. It is the increase in concentration of positive metal cations (oxidation) within the pit/crevice that causes the migration of $\left[\mathrm{OH}^{-}\right]$and $\left[\mathrm{Cl}^{-}\right]$anions toward the anode. This is a result of charge imbalance. Similarly, metal cations undergo mass transport towards the reduction reaction site. It is this flux of products from the two half reactions which creates a deposition on the surface of the metal. For $\mathrm{Fe}^{2+}$ the cations first become oxidised to $\mathrm{Fe}^{3+}$ (from dissolved $\mathrm{O}_{2}$ ). This is then hydrolysed at the high $\mathrm{pH}$ outside the pit.

\subsubsection{Intergranular Corrosion}

Intergranular corrosion (IGC) is known to affect austenitic stainless steels. [10] [89], alloys of aluminium [90], highly corrosion resistant Ni-based alloy 600 [91], and multiple other metallic systems [92]. Here the process of IGC within the austenitic stainless steel system is discussed, firstly through weld decay, and then through a radiation mechanism.

Weld decay is a process in which IGC occurs at weld locations. Here, weld 
decay is given a brief description, and a more thorough understanding can be obtained in [93]. The area around the fusion zone (the 'weld') is known as the heat affected zone (HAZ). Local areas within the HAZ can undergo a process known as sensitisation whereby chromium carbide intermetallics form at grain boundaries, resulting in a reduction of chromium $(\mathrm{Cr})$ adjacent to the grain boundary. These metal carbides are formed once a critical temperature is passed, allowing the diffusion of $\mathrm{Cr}$ to carbon, and precipitate at the boundary of the grain [10]. Trethewey and Chamberlain [10] note that precipitation occurs (type 304) within the temperature range of $600-850{ }^{\circ} \mathrm{C}$, reducing to $300-320{ }^{\circ} \mathrm{C}$ if carbides have already pre nucleated prior to welding. The process of sensitisation creates highly localised Galvanic cells, whereby the more noble matrix couples with Cr-depleted zones. Once the amount of Cr drops below the important threshold, 12 wt.\% [94], the depleted zones do not produce the passive oxide film required for protection. This leads to corrosion along grain boundaries, and can result in the loss of entire grains of metal.

The mechanism in which stainless steel AGR fuel cladding becomes susceptible to IGC is not through a heat-induced mechanism, but instead occurs because of the radiation present within the core of a nuclear reactor. This is an important difference. Radiation induced segregation (RIS) is the mechanism in which sensitisation occurs in service, rather than through heat only. This is caused by protracted exposure to a combination of the high neutron flux and elevated temperature within the reactor core [95]. This leads to the redistribution of alloying elements at a microstructural level. Defect sites located within the matrix, caused by neutron radiation, interact with grain boundaries, but also interact with each other [96]. This can include recombination and back diffusion. Thus, sensitisation can be modelled, though the more complex nature of RIS means it is more difficult to 'quantitatively predict' grain boundary depletion than the thermal mechanism. In fact, models have been created and used for grain boundary RIS in the past by Perks and Murphy [97], Allen and Was in 1998 [98], Norris 
et al. [95] and Simonen and Bruemmer [96]. English, Murphy and Perks conducted a review in 1990 [99]. The high neutron flux causes vacancies which, through a process of diffusion, move towards grain boundaries, leading to a flux of atoms away from the boundaries. The composition of these zones are dependant on the vacancy gradient. This is known as the inverse Kirkendall model (IKE) [100] [101] [102]. A diagram of IKE is depicted in figure 1.23. As a result of vacancy formation and their diffusion, counter diffusion of atoms must occur to areas where the vacancies were present. The major elements diffuse away from the grain boundaries, but the composition profile is dependent on the elements' diffusivity [102]. Nickel (Ni) becomes enriched at grain boundaries because it is the slowest diffuser, along with silicon, which is lightly enriched. Cr in austenitic stainless steels has the fastest diffusivity, which leads to its depletion at the grain boundary [96] [101] [103].

As explained within section 1.1.4, the coolest part of the AGR stringer (elements 1 and 2) that are primarily effected by the RIS mechanism, due to a dependence on irradiation temperature [104]. At too low a temperature the concentration of defects increase and, rather than diffuse to sinks, annihilate. At higher temperatures equilibrium concentrations of the elements are reached because thermal diffusion dominates. Kyffin notes that temperatures in the 350-520 - $\mathrm{C}$ range (irradiation temperature) represent the window in which sensitisation affects the AGR 20/25/Nb stainless steel [37]. The peak sensitisation occurs at $380-440^{\circ} \mathrm{C}$ [33]. Allen and Was show (figure 1.24) the temperature window in which Cr becomes depleted for a stainless steel grade (20 Cr, 24 Ni, Fe balance) which has similar levels of major elements to that of the AGR clad.

Norris et al. [95] showed through microscopy methods the concentrations of major alloying elements present at grain boundaries for the AGR stainless steel. Analytical field emission gun scanning transmission electron microscopy (FEGSTEM) was used and showed the segregation of the major alloying elements at grain boundaries for different locations on the stringer. The fuel elements are 


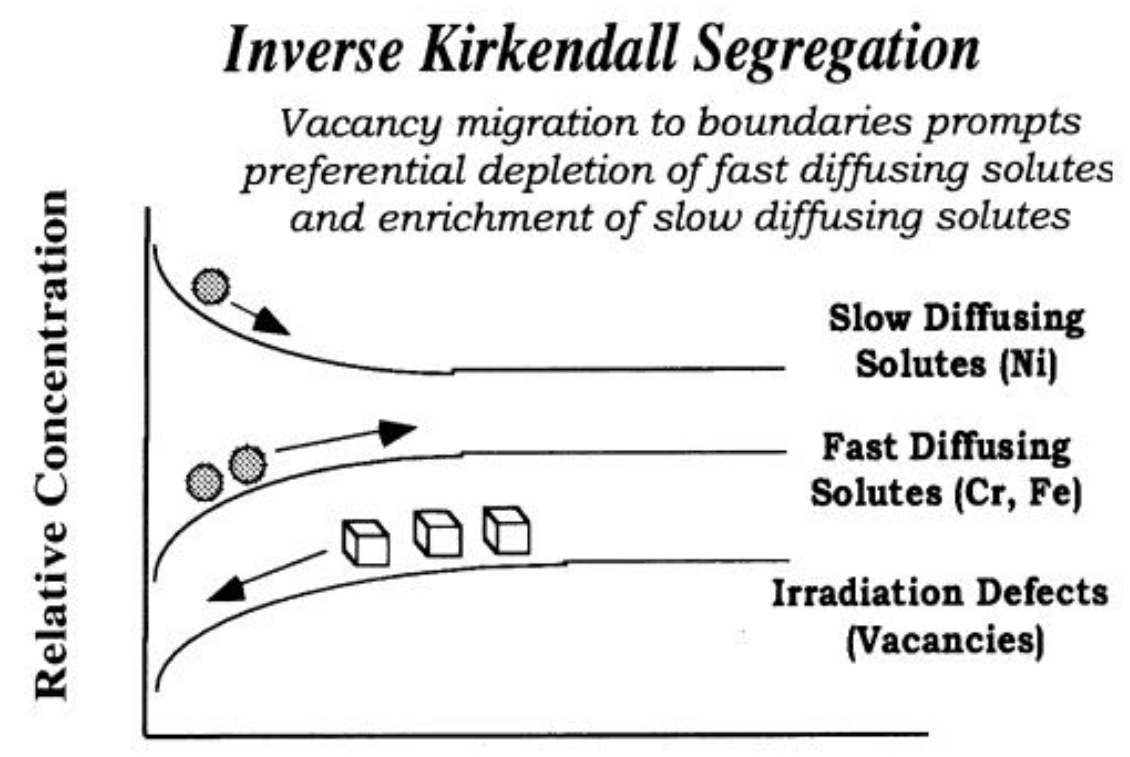

Distance from Grain Boundary

Figure 1.23: Inverse Kirkendall Mechanism [101]

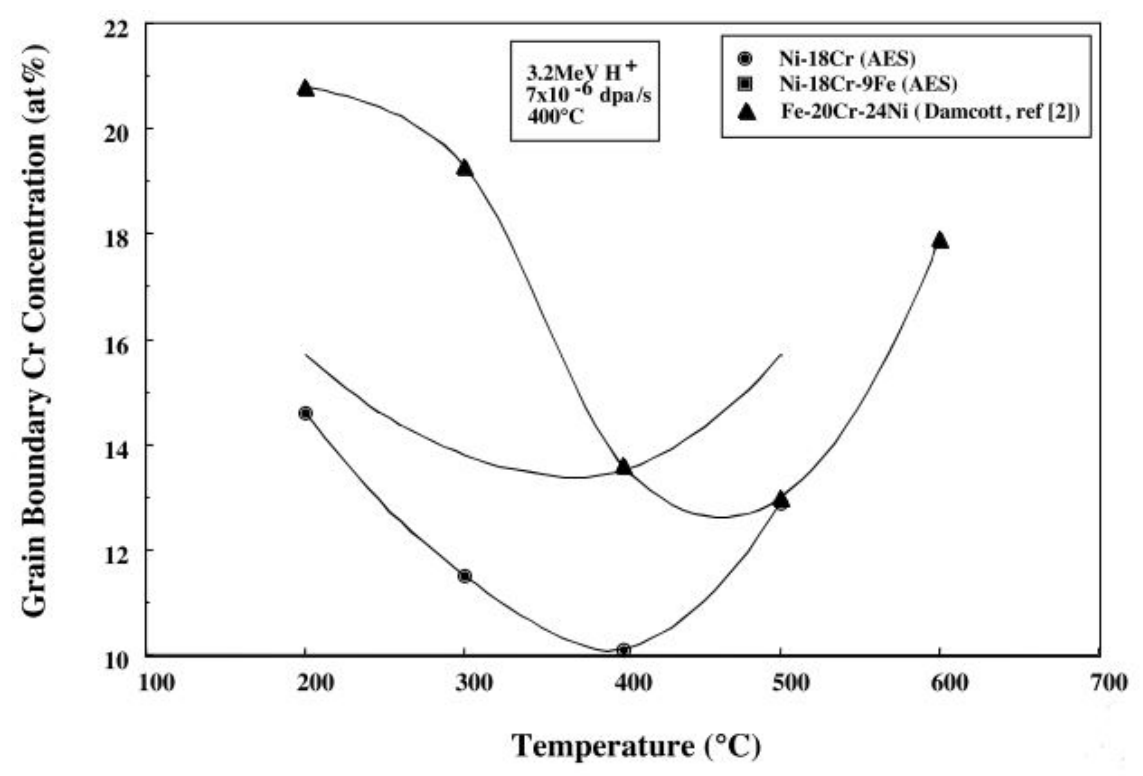

Figure 1.24: Concentration of $\mathrm{Cr}$ as a function of temperature for a range of different stainless steels. From [104] 


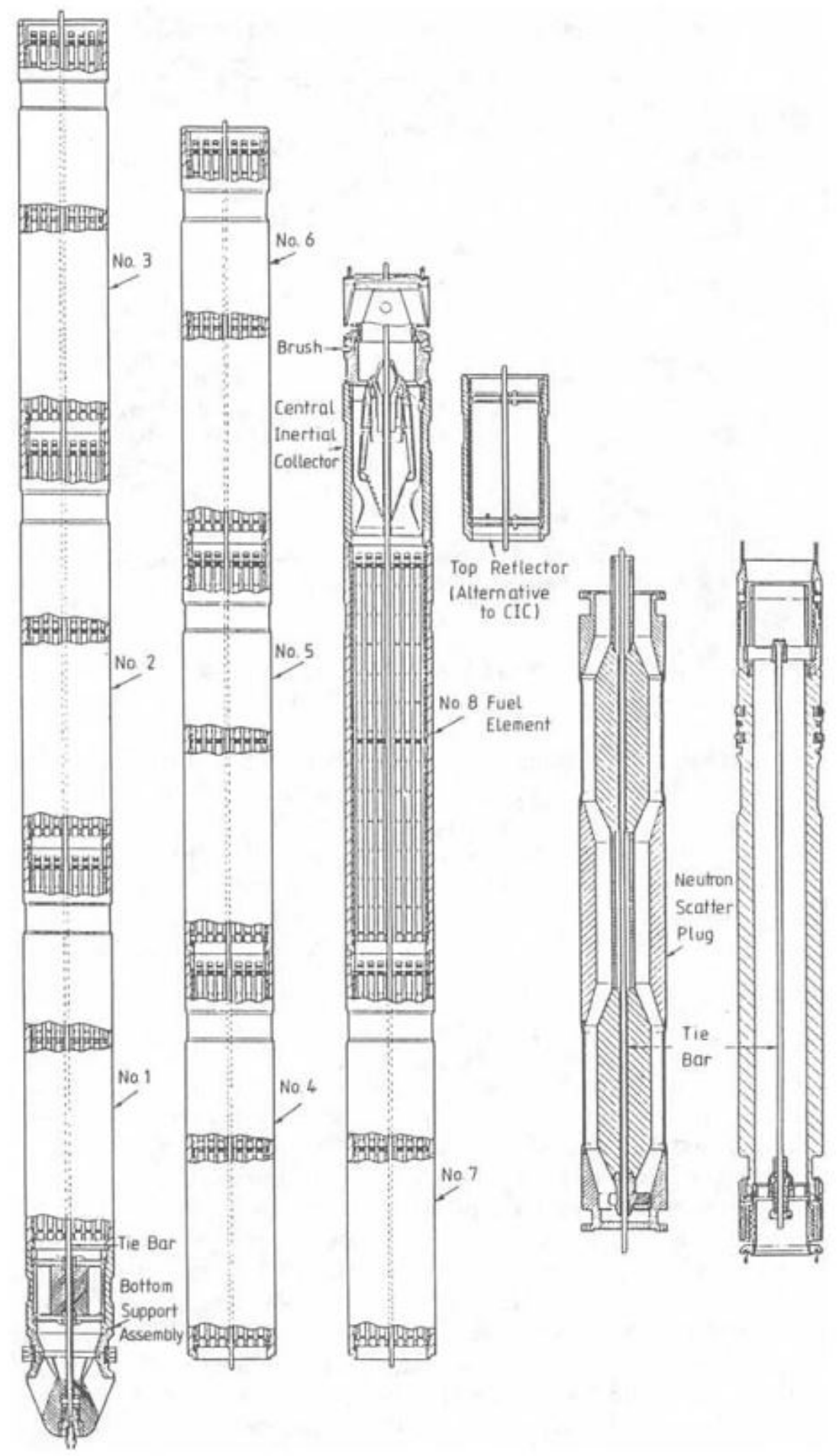

Figure 1.25: AGR fuel stringer (Hinkley point B) 
stacked vertically, with element 1 located at the bottom, through to 7 or 8 depending on the AGR station. Figure 1.25 shows a schematic of an AGR fuel stringer (the bottom of the stringer is shown on the left). Due to the thermal gradient within the core, each of the elements are subjected to different irradiation temperatures. The graphs for irradiated AGR stainless steel element concentration as a function of distance from the grain boundary are given in figures 1.26, 1.27. The figures reproduced from Norris et al. [95] show that Cr and Fe become depleted at areas adjacent to the grain boundary across all irradiation temperatures, and that grain boundaries experience Ni and Si enrichment. There is a dependence on temperature, as not all locations adjacent to grain boundaries are rendered sensitised, with the peak occurring at $420^{\circ} \mathrm{C}$, where $\mathrm{Cr}$ drops below 12 wt.\%. The effect of RIS diminishes as a function of temperature. The depletion zones for $\mathrm{Cr}$ and Fe increase in width, but do lead to a decrease in severity, as the amount of $\mathrm{Cr}$ depletion decreases either side of $420^{\circ} \mathrm{C}$. The enrichment profile for $\mathrm{Ni}$ is also narrower at lower temperatures. This is likely to be caused by the domination of the thermal diffusion mechanism at increased temperatures. Strauss tests revealed that the greatest depth of attack occurred with at $420^{\circ} \mathrm{C}$, with a dose of 3.5 dpa. In the Strauss corrosion tests, specimens outside the $350-520^{\circ} \mathrm{C}$ range showed less corrosion compared to those that were within the window. The depletion profile for $\mathrm{Cr}$ was also the widest under such conditions. The group also investigated low burn-up cladding and found that RIS was detected early on during service (figure 1.28). A 2 hour anneal at $600^{\circ} \mathrm{C}$ leads to reduced segregation of alloying elements, thus increasing the $\mathrm{Cr}$ levels above the critical threshold (figure 1.29). The annealed specimen showed corrosion at depths of up to $50 \mu \mathrm{m}$. As a comparison, the Strauss test led to the disintegration of the irradiated, non-annealed specimen. These temperature and dose variations are thus somewhat reduced by a post service anneal.

As explained earlier, IKE can be modelled to give compositional profiles across a grain boundary. The groundwork for the model was laid out by Perks et 

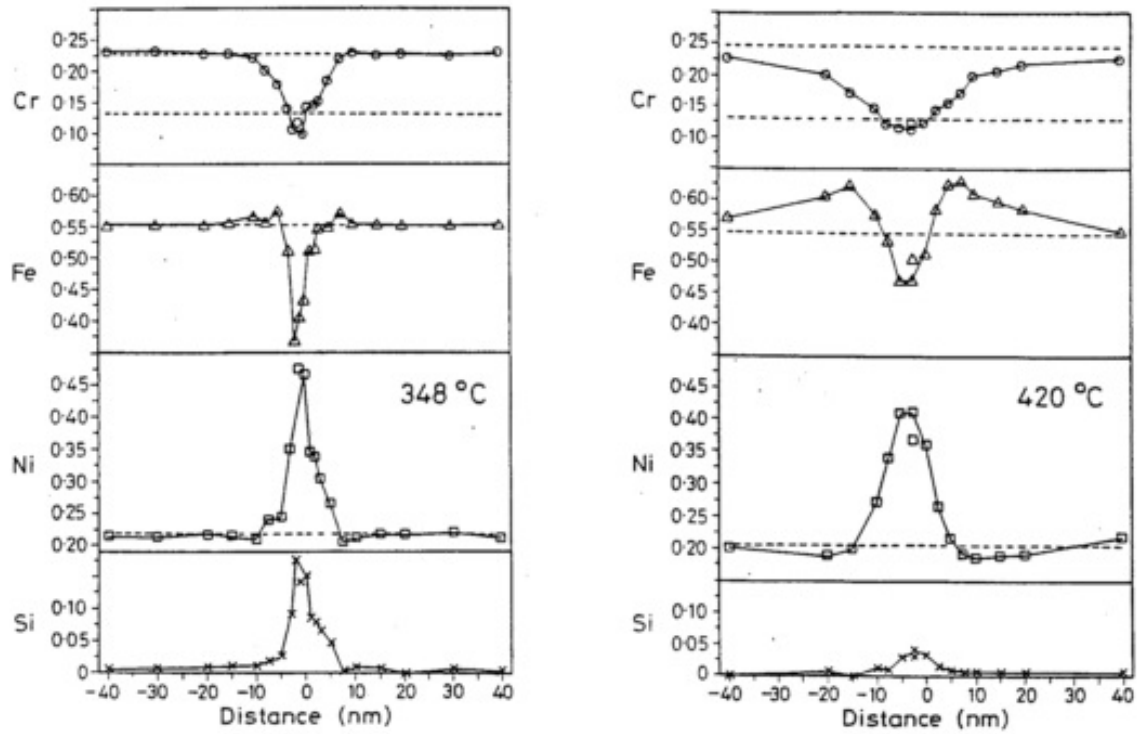

Figure 1.26: Line profiles across grain boundary from the lower elements for inservice fuel stringer B4286. The irradiation temperature is given on the inset [95]
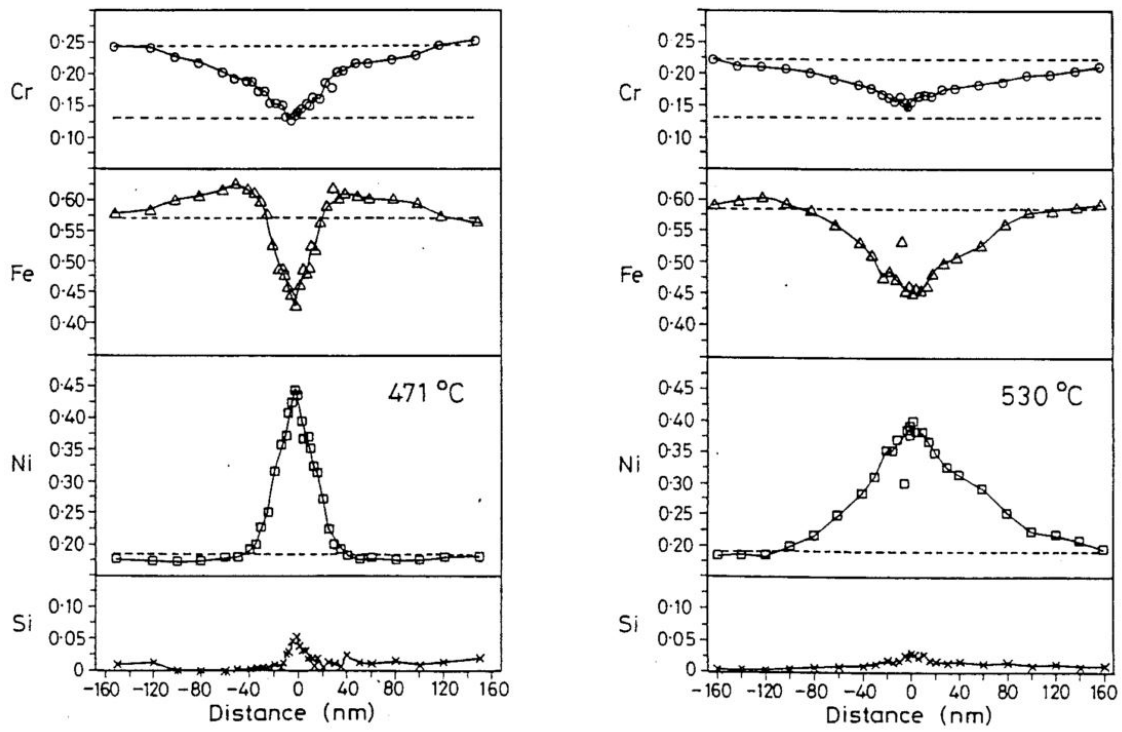

Figure 1.27: Line profiles across grain boundary for the same in-service fuel stringer B4286 for the upper elements. Note the $\mathrm{x}$ axis is of a different scale to the previous image [95] 

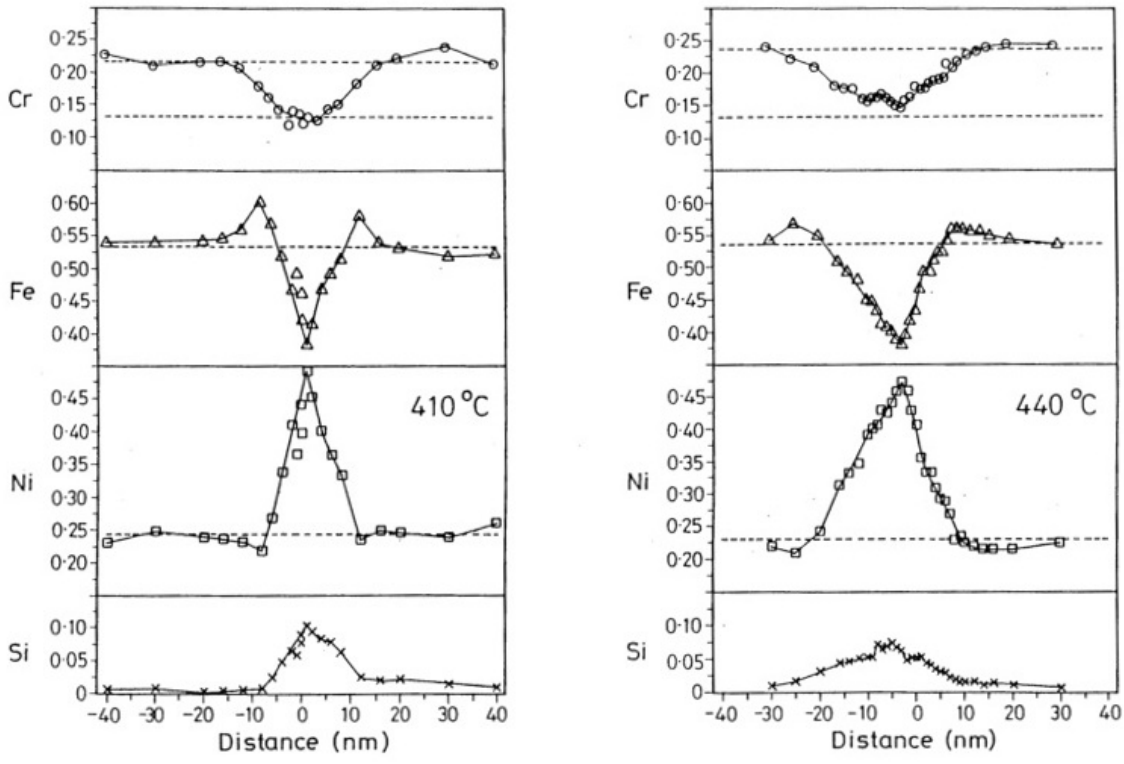

Figure 1.28: Line profiles across grain boundary from an in-service, low burn-up fuel stringer (D1433) at different locations along the stringer [95]
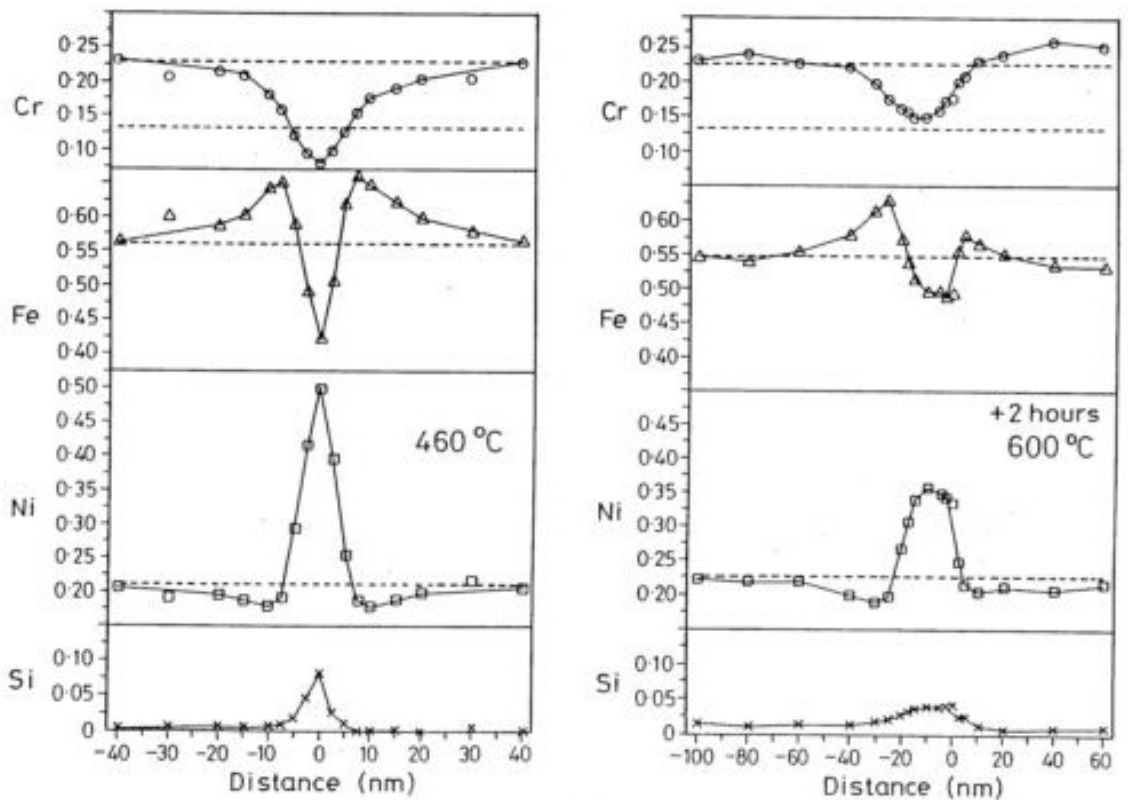

Figure 1.29: Compositional profile across grain boundary. Before (left), and after (right) post irradiation anneal [95] 
al. [105] [106] [97]. The results of the AGR RIS model developed by Norris et al. [107] are shown in figure 1.30. The model shows a good agreement with the measured values at low temperatures, but overpredicted the widths of the depleted and enhanced zones (Cr, Ni respectively) at higher temperatures (455, $481^{\circ} \mathrm{C}$ ). The models however provide useful information on the parameters for the RIS mechanism. It allows the researcher to reasonably predict the Cr content if the irradiation temperature and dose are known.

\section{Mechanisms Causing IGC}

Radiation-induced Segregation There are a number of ways to sensitise AGR 20/25/Nb stainless steel. In order to induce sensitisation by the RIS mechanism, a source of radiation is needed. These sources can be either neutron or proton based. In-service material is affected by neutron radiation. It is possible that the material may also be irradiated at neutron beam sources also such as ISIS in the UK. For authentic RIS-affected material, the 20/25/Nb stainless steel must be present in-reactor for a number of years until it has the appropriate radiation dose for experiment. Ion beam irradiation (protons) is a promising alternative to induce RIS within $20 / 25 / \mathrm{Nb}$ stainless steel. Though there are technical and practical implications which are yet to be resolved which are mainly considerations based on temperature and beam energy given that ion beam irradiation introduces significant energy density within the target material, necessitating careful temperature control. Both of the above methods induce sensitisation through IKE, leading to RIS, but both methods have their disadvantages. Reactor-irradiated material must be in-reactor for a significant amount of time (several years) before discharge and accessibility for examination. Proton irradiated specimens will also be radioactive, but generally to a much smaller extent. A study by the Dalton Cumbria Facility [109] is decribed below. After an irradiation period using 20/25/Nb stainless steel specimen (59 hours, $3 \mathrm{MeV}$ proton beam, $9.8 \mu \mathrm{A}$ average current) the dose was $1.7 \mu \mathrm{Sv}^{-1}$. 

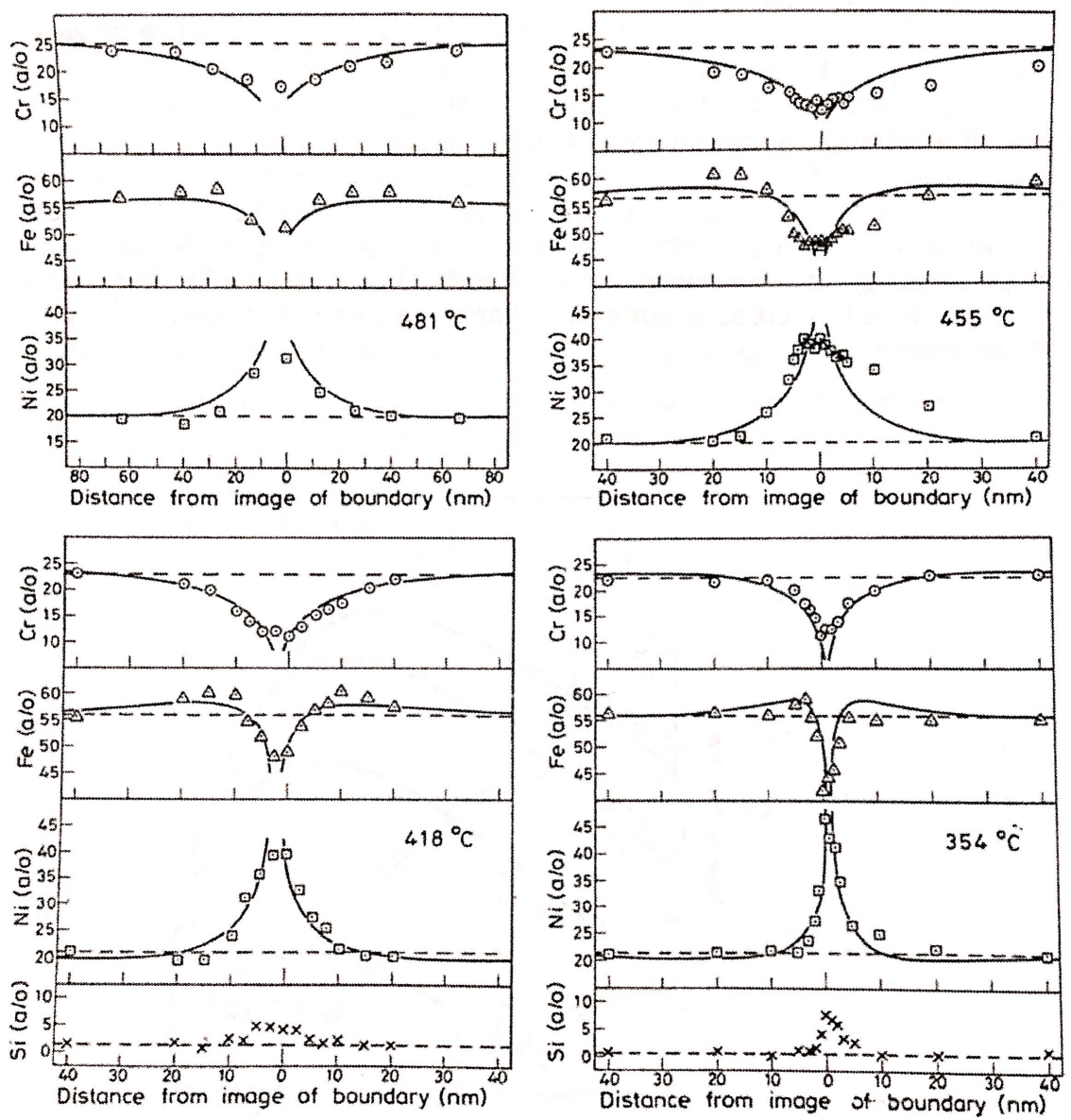

Figure 1.30: Modelled and predicted compositional profiles across the grain boundary of different positions of an in-service stringer. The units on the yaxis are in atomic percent (at.\%). 13.2 at.\% corresponds to 12 wt.\% [107] [108] 
${ }^{55}$ Co was present after 30 minutes, the half life of this fission product was 18 hours. Following 27 days after the irradiation, the dose decreased to $0.2 \mu \mathrm{Sv} h^{-1}$, however transmutation leads to the formation of ${ }^{57} \mathrm{Co}$ which has a half life of 272 days. The time taken to produce proton irradiated stainless steel is difficult, as an increase in current leads to an increase in the dose of radiation. As such, both radiation-induced mechanisms have special requirements for the safety of workers (facilities, radiation shielding, personal protective equipment). These materials will be radioactive after irradiation and, in order to conduct experimentation may need to be reduced in size to lower the dose rate to workers, which limits the number and type of experiment that can be conducted.

Thermally-induced Sensitisation An alternative method is to simulate sensitisation using thermal treatment, and has been used in the past to study 20/25/Nb stainless steels [110]. 20/25/Nb is a stabilised alloy, meaning that the matrix has a low carbon concentration as much of it will be chemically bound as $\mathrm{Nb}$ carbide. As such a two stage process is needed to produce sensitisation. This type of sensitisation mechanism is more akin to the HAZ on welded stainless steels, rather than the RIS mechanism. In order to form Cr carbides, pre-existing $\mathrm{Nb}$ carbides located at both grain boundaries and the matrix are dissolved using a high temperature anneal. The aim of the anneal to increase the amount of carbon within the matrix, which would otherwise have been constrained as NbC. Moss and Sykes [110] required 1050 and $1150{ }^{\circ} \mathrm{C}$ for 30 minutes to dissolve the intermetallics. Argon gas was used to avoid oxidation and decarburisation effects.

Upon the second thermal treatment (hereby referred to as 'ageing') Cr carbides (MC, $M_{6} C, M_{23} C_{6}$ ) precipitate at grain boundary, causing Cr-depleted zones along the GB. The Cr carbides predominantly form at the grain boundary because the boundary itself acts as a faster pathway for $\mathrm{Cr}$ diffusion, when compared to the bulk. As Cr is depleted by the nucleation and growth of Cr-rich precipitates, Cr must be replenished. This occurs by through the collector plate mechanism, via grain boundary diffusion from the matrix, causing a reduction of 
Cr along grain boundaries [111] [110]. Three temperatures (550, 600 and $\left.650{ }^{\circ} \mathrm{C}\right)$ were used to heat treat specimens up to periods of 500 hours. Moss et al. used the Strauss test to assess the degree of sensitisation. In conclusion, the optimum sensitisation was achieved with a $1150{ }^{\circ} \mathrm{C}$ solution anneal and 50 hour ageing at $600{ }^{\circ} \mathrm{C} .1150{ }^{\circ} \mathrm{C}$ was preferred for the solution anneal, as compared to the lower $1050{ }^{\circ} \mathrm{C}$ anneal showed greater IGC.

Ecob et al. [112] studied the 20/25/Nb microstructure in respect to creep properties, post thermal treatment. It is not only mixed metal carbides that can nucleate, as nickel niobium silicide $\left(N_{18} N b_{6} S i_{7}\right)$ can precipitate (also known as G-phase). Sigma phase, a brittle intermetallic consisting of Fe and Cr, can also be precipitate during treatment [113]. The precipitation of these phases is not dependant solely on temperature, but also dependant on kinetics. Powell et al. [113] studied specimens for periods of up to 15,000 hours and performed transmission and scanning electron microscopy (TEM and STEM), along with other techniques. Powell states that for a temperature of $600{ }^{\circ} \mathrm{C}, M_{23} C_{6}$ is the only precipitate phase present at the grain boundary over 300 hours. This is first identified after 200 hours. The matrix should contain $\mathrm{Nb}(\mathrm{CN})$, which is identified after 200 hours. If studies by Powell et Al. are followed, more stable G-phase can be expected to precipitate after 500 hours, $M_{23} C_{6}$ after 200 hours, and sigma phase after 1000 hours at grain boundary regions. Within the Matrix $\mathrm{Nb}(\mathrm{CN})$ should nucleate after 200 hours, then $\mathrm{G}$ and sigma phase after 10,000 hours. Powell notes other phase transformations that occur with temperature. According to Powell, at $500{ }^{\circ} \mathrm{C}$ the kinetics are very slow at this temperature, and only G-phase and $M_{23} C_{6}$ precipitate after 5000 hours. Higher temperatures of 575 and $600{ }^{\circ} \mathrm{C}$ showed similarities with $650{ }^{\circ} \mathrm{C}$ specimens, although the amount of $M_{23} C_{6}$ was greater at the lower temperatures. From the study Powell et al. was able to produce a time-temperature-precipitation graph, as shown in figure 1.31. This graph shows that $M_{23} C_{6}$ does precipitate at grain boundaries, in agreement with Moss and Sykes [110]. As the study was not based around corrosion, but 
more regarding what phases precipitate at what timescale in regard to creep properties, it cannot be said if this temperature and time lead to most highly sensitised microstructure as Moss and Sykes state.

Though both thermal sensitised and neutron-irradiated methods will produce Cr-depleted GBs, the sensitisation mechanism does produce differences. NbC inclusions remain within the irradiated (reactor and proton specimens), as the process is dependent on the vacancies formed by irradiation. When specimens are heat treated (thermal sensitisation), NbC inclusions are dissolved during the initial thermal sensitisation treatment (solution anneal). Though, the solution anneal is unlikely to ever completely dissolve all $\mathrm{NbC}$ inclusions, so a finite quantity will still remain. Hence, there is a lower probability for Cr-carbide formation in irradiated specimens, due principally to the presence of the $\mathrm{Nb}$ stabiliser. $\mathrm{NbC}$ constrains carbon levels, thus free carbon within the matrix is reduced, limiting the availability for $\mathrm{Cr}$ for Cr-carbide formation. Another difference is the width of the Cr-depleted zone, where thermal sensitised specimens exhibit wider zones $(>30 \mathrm{~nm})$ in comparison to narrow $(5-15 \mathrm{~nm})$ regions produced in reactor specimens [95].

\section{Intergranular Pitting Corrosion}

The term intergranular pitting corrosion has been used to describe the process in which the specimens used in this work appear to corrode. This may be attributed to the material not being highly sensitised, resulting in IGC propagation not being continuous, instead appearing more pit-like. The process in which the intergranular pitting mechanism is postulated to take place is detailed below:

\section{Metastable corrosion occurs}

2. Pit initiation at a susceptible site. The studies in Chapter 6 have shown that grain boundary intersections (including triple point grain boundaries) are suitable locations for corrosion to initiate in this thermally sensitised stainless steel. The grain boundary intersections are postulated to poten- 


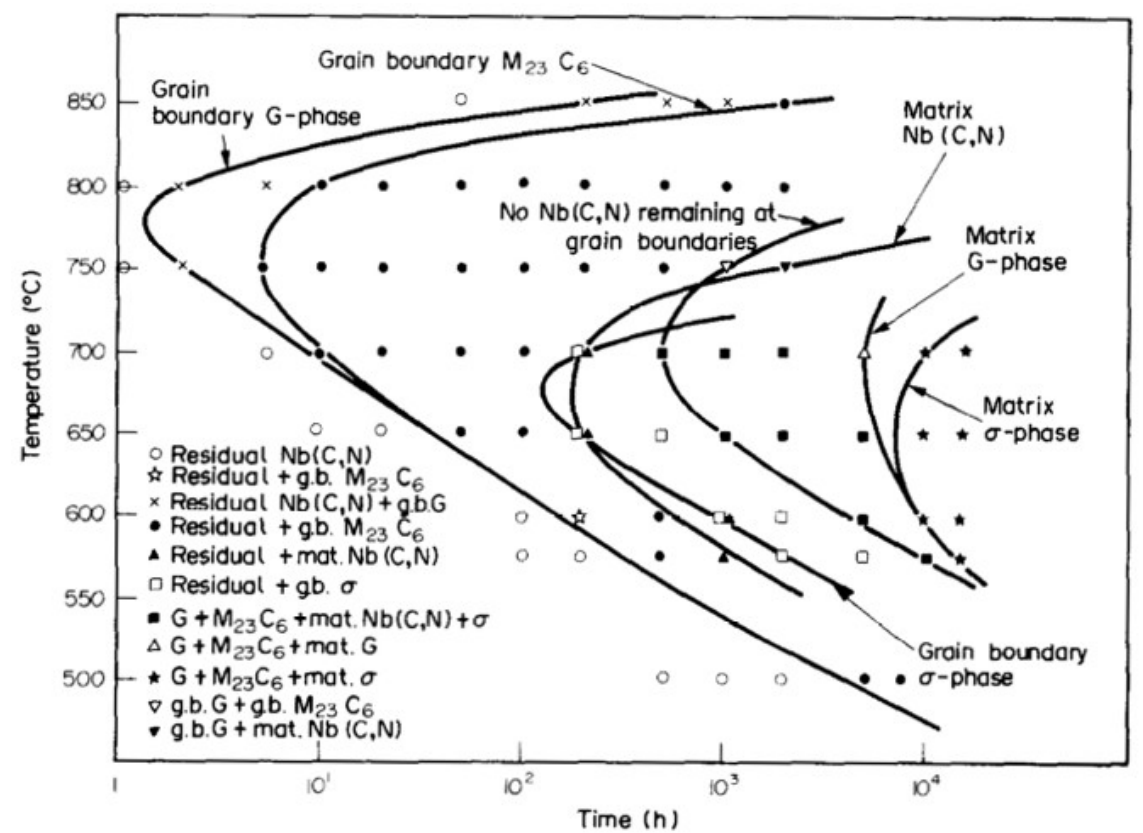

Figure 1.31: Time-temperature-precipitation curves for 20/25/Nb stainless steel [113]

tially be more heavily chromium depleted (the Cr depletion is due to the formation of $\mathrm{Cr}$ carbides, reducing local $\mathrm{Cr}$ ). These findings are in agreement with the findings by Chan [45] and a study by Clark [114]. The study in Chapter 7 shows that the $\mathrm{Nb}$ rich inclusions are more noble than that of the matrix, and an in-situ electrochemical AFM experiment showed that pits initiated adjacent to the grain boundary, close to the location of an inclusion, thought to be NbC. It is speculated that the NbC may therefore act as cathodic activators, allowing corrosion to occur around these inclusions

3. Autocatalytic propagation of the pit. Inside the pit the oxidation reaction creates an excess of metal cations, leading to:

(a) Charge imbalance, leading to diffusion of anions such as chloride into the pit, creating supersaturated salt films on the lower surface and walls of the pit itself

(b) Hydrolysis of water. This is the reaction between the metal cations and water, forming metal hydroxide and protons, thus leading to the 
acidification of the pit [63]

4. IGC of Cr-depleted grain boundaries within the pit, excavating the metal sub-surface. Above the surface, Cr-depleted regions suffer from IGC and lacy covers form to conserve the critical pit chemistry, as observed by Burnett et al. [115]

Burnett et al. [115] observed the how the two corrosion mechanisms (pitting corrosion and IGC) act both in synergy and in competition in a sensitised $316 \mathrm{H}$ stainless steel. The sensitised stainless steel $\left(650{ }^{\circ} \mathrm{C}, 24\right.$ hours, Ar atmosphere) was subjected to potentiodynamic polarisation in $0.1 \mathrm{~mol} \mathrm{dm}^{-3} \mathrm{NaCl}$, and imaged using in-situ X-ray computed tomography (CT) with $3.4 \mu \mathrm{m}$ voxels. The authors studied the pits using multiple techniques to move from the millimetre to sub nanometre length scales. Following pit initiation the material was studied using at higher resolution X-ray CT $(0.8 \mu \mathrm{m}$ voxels $)$ and then destructively examined using serial FIB sectioning to obtain even greater resolution. The authors also performed electron backscatter diffraction to attain information on high angle grain boundaries and transmission electron diffraction EDS to attain nanometre resolution with elemental composition information ahead of the IGC path. The authors did not show what caused corrosion to initiate in the very early stages. What the research did show is that once the pits had formed the IGC mechanism was favoured over further propagation of the pit itself. The authors argue that is likely due to the combination of both aggressive pit chemistry and that the sensitised grain boundaries formed 'rapid diffusion pathways'. 


\section{Chapter 2}

\section{Experimental Techniques and Procedures}

\subsection{Specimen Preparation}

Un-irradiated stainless steel cladding was obtained from Westinghouse Fuels Ltd, via the National Nuclear Laboratory as either tube or flat strip. The strip, of $20 \times 3.5 \mathrm{~cm}$ (thickness $0.80 \mathrm{~mm}$ ) dimensions, was cut to smaller lengths, and then finally to $20 \mathrm{~mm}$ squares by means of a hand guillotine. The resulting squares were subsequently ground and polished manually using a Buehler Ecomet 300 machine. Grinding was performed using coarse P240 through to fine P2500 (P240, P400, P600, P800, P1200, P2500, Buehler CarbiMet 2 silicon carbide pads). Kemet $6 \mu \mathrm{m}$, and subsequently $1 \mu \mathrm{m}$ water-based diamond suspension was used in conjunction with Buehler MetaDi lubricant for polishing. Following polishing, specimens were given a final rinse with ethanol to remove particulates. Stainless steel tubes were cut using a linear precision saw (Buehler Isomet 5000) using low feed rate $\left(10 \mathrm{~mm}^{-s}\right)$ and high blade speed $(4000 \mathrm{rpm})$. In situations where the specimens were examined using microscopy techniques, Struers durocit or Epofix resin was used to mount specimens. When experiments required an electrical connection, a wire was spot welded to the specimen prior to being set 
in resin.

To reveal the microstructure an electrolytic etch was used. The specimen and Pt gauze counter electrode were connected to a DC power supply, and then immersed in a solution of $10 \mathrm{wt} \%$ Oxalic acid. A DC potential of $6 \mathrm{~V}$ was used for a period of 10 seconds in order to expose grain boundaries [116]. The sample was rinsed using distilled water and finally cleaned with ethanol.

\subsection{Heat Treatment}

Specimens underwent two stage thermal processing in order to sensitise the SS, depleting Cr from the grain boundaries (described in section 1.3.2). The need for a two stage treatment was considered on the basis of both temperature and duration. The merits of different furnace temperatures and durations were discussed previously in section 1.3.2. The first stage consisted of a high temperature solution anneal at $1150{ }^{\circ} \mathrm{C}$ for 30 minutes in an argon atmosphere. This was followed by a water quench. The high temperature anneal results in incomplete niobium carbide (NbC) dissolution (not all NbC may be dissolved), thereby increasing free carbon content within the matrix. The water quench, at room temperature, ensured minimal back diffusion of niobium with carbon to reform NbC. Following the solution anneal, the carbon content is greater than the base specification, rendering the SS less stabilised. The second heat treatment was undertaken at a lower temperature of $600{ }^{\circ} \mathrm{C}$ in air for a longer period of 2 weeks (c.336 hours). This second treatment allowed chromium carbides (Cr-carbides) to form at grain boundaries, thereby depleting $\mathrm{Cr}$, rendering the stainless steel sensitised.

\subsubsection{DL-EPR Experimentation}

Following thermal treatment the surface of the SS is covered in a dark oxide layer. In later chapters this is shown to be a type of iron oxide, characterised as Haematite by X-ray diffraction (see section 3.4.3). In order to quantify the degree 
of sensitisation, a double-loop electropotentiokinetic reactivation (DL-EPR) test was undertaken. The international standard, ISO 12732:2006 [117] was followed to conduct the experiment. Sulphuric acid $\left(0.5 \mathrm{~mol} \mathrm{dm}^{-3}\right)$ and potassium thio-

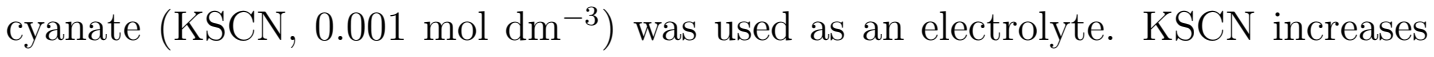
activity occurring on the surface when polarised, and thus increases the level of corrosion during both forward and reverse scans, compared to the sole acid [118]. The effect of KSCN concentration is shown in figure 2.1.

A potentiostat was used to polarise the SS from the free corrosion potential (active in the test environment) through to the passive region. This is illustrated by the anodic trace marked as ' 1 ' in figure 2.2 . The SS passes a region in which grain boundaries are activated, and then it is polarised in the cathodic direction as per the cathodic trace '2' in figure 2.2. $Q_{p}$ and $Q_{r}$ in figure 2.2 denote the charge of the anodic and repasssivation loops respectively which are obtained by integration. The relative change in maximum current from the peak in trace 1 (anodic max current, $I_{a}$ ) and trace 2 (reactivation max current, $I_{r}$ ) forms a ratio. Both the ratio of current and charge can be used to quantify the degree of sensitisation (DOS). The experiment works on the basis that the grain boundaries have a different breakdown potential to the matrix, where chromium levels are greater. For sensitised material, the grain boundaries are reactivated during the cathodic scan, due to grain boundary dissolution, so instead of repassivation the current rises. The magnitude of the reactivation peak is dependant on the level of chromium depletion. According to the standard, at the end of the experiment, the specimens should be imaged using a microscope. The grain size and corroded grain boundary areas can then be estimated. The variation in DOS as a function of depth was studied by using a Vickers hardness indent. In order to remove the indent as an initiation site, it was positioned away from the area exposed to the electrolyte. As the indent from the Vickers hardness machine left an inverted square pyramid shape, polish depth could be calculated by mathematical calculation of the dimensions of the indent. 

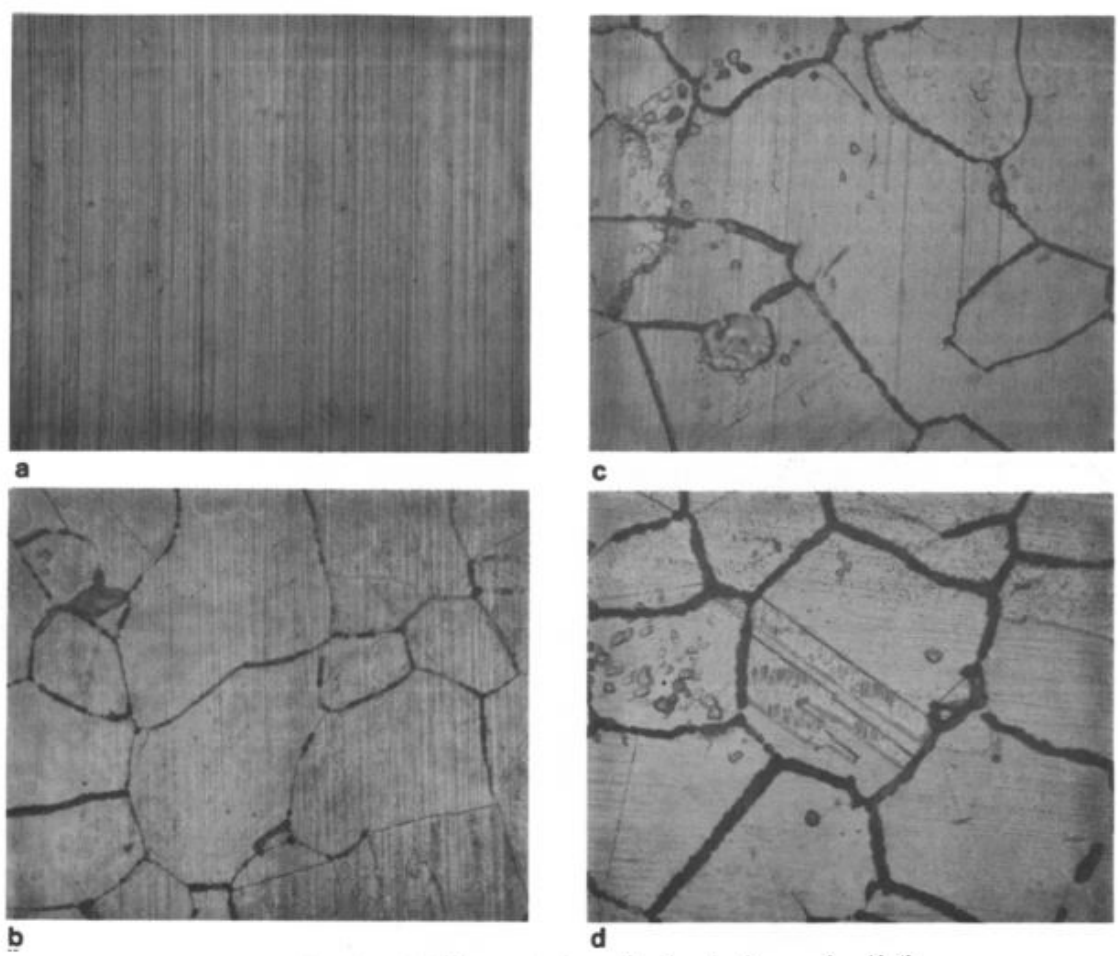

Figure 2.1: KSCN concentration effects on the microstructure of 304 SS during

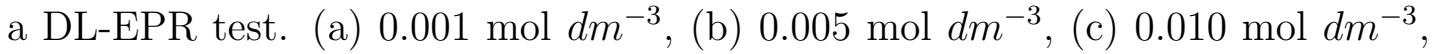
(d) $0.050 \mathrm{~mol} \mathrm{dm^{-3 }}$. [118]

Figure 2.3 shows an example of one of the indents used and its relevant dimensions. Once the indent depth was calculated, the DL-EPR test could be conducted. It was determined that the quantifiers for DOS used would be the ratio of $Q_{r} / Q_{p}$ and $I_{r} / I_{p}$. If the ratios remained similar the DOS could be considered constant. If this was not the case then DOS could be said to vary as a function of depth. After the DL-EPR experiment was conducted, a fresh surface was created by polishing the specimen to ensure there were no pits or IGC present which would impact further experimentation. The indent dimensions were re-measured and then recorded. An assumption was made that polishing was always normal to the pad so that the quantity of material (and indent) removed was uniform across the surface. 


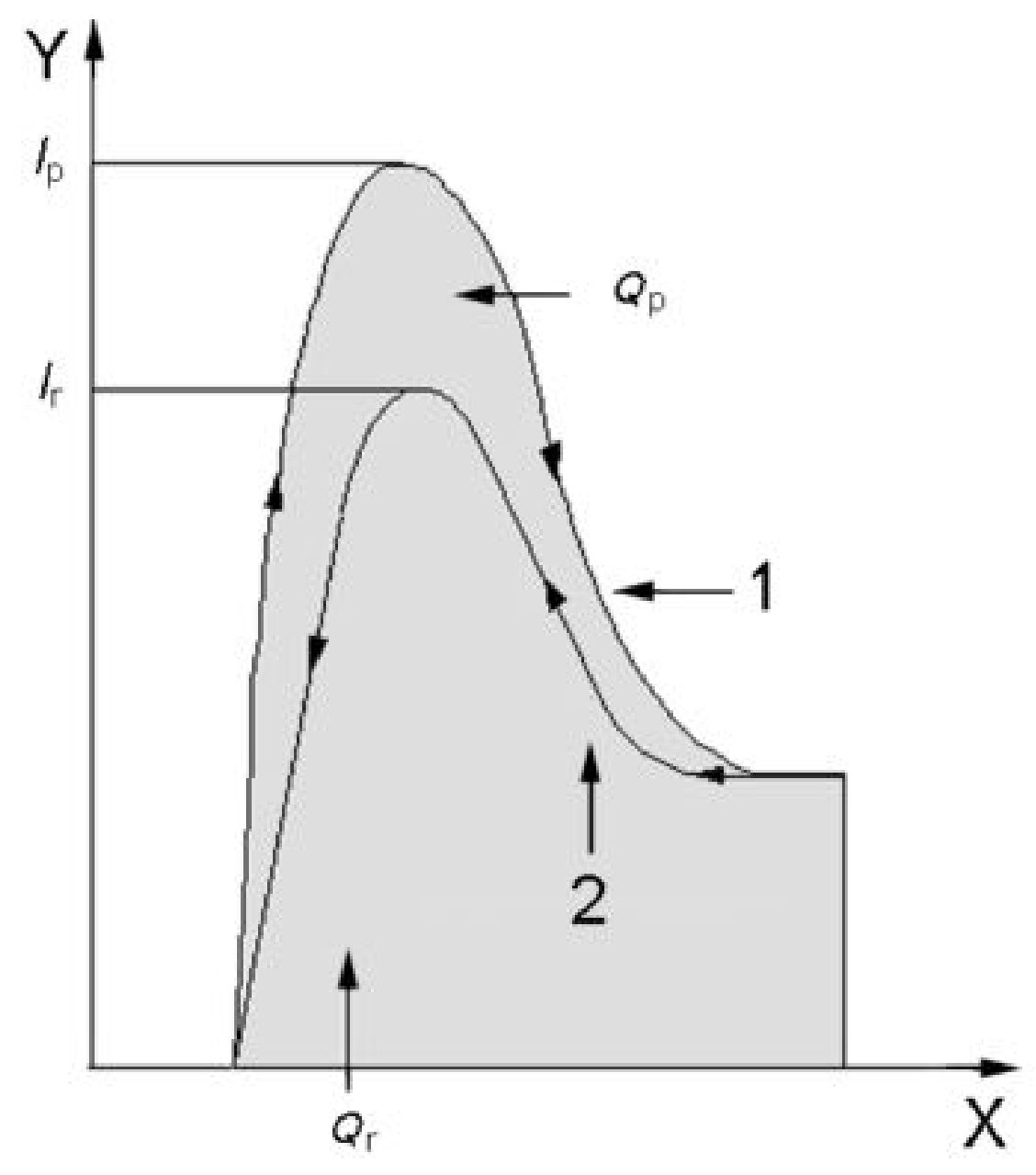

Figure 2.2: The relationship between the log current and potential for the DLEPR test [117].

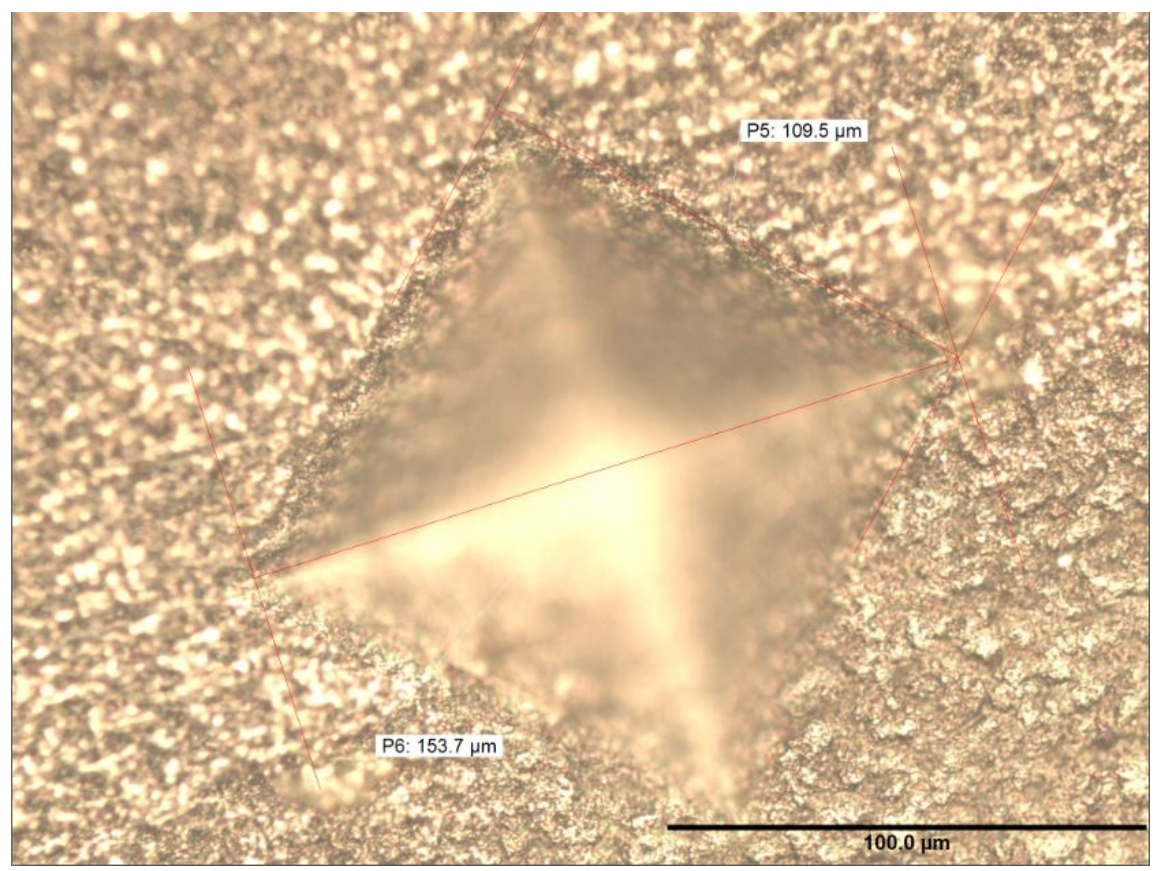

Figure 2.3: Vickers hardness indent used for depth analysis 


\subsection{Electrochemistry Experiments}

Potentiostat Set-up A Solartron SI1280 potentiostat was used for standard Direct Current (DC) electrochemistry experiments. Polarised time-lapse microscopy (TLM) and polarised SVET experiments used a battery operated potentiostat (Palmsens 3). The instruments had both potentiostat and Galvanostat functionality. The following experiment types were used: open circuit potential $(\mathrm{OCP})$, linear polarisation resistance (LPR), zero resistance ammetry (ZRA), and potentiostatic/potentiodynamic experimentation.

Generally, most experiments involved the use of a three electrode cell set-up, in which a working electrode (WE), counter electrode (CE), and reference electrode (RE) were immersed in a common electrolyte. An exception to this was the open circuit experiment, where only WE and RE were used. The potential of the WE, comprising the SS of interest, was varied with respect to a saturated calomel electrode (SCE), which was used as the RE in all experiments unless stated. Platinum $(\mathrm{Pt})$ gauze was used as a $\mathrm{CE}$, and is an ideal material for this purpose as it is a noble metal, and is hence stable in the majority of experimental solutions used and at the applied potentials employed. When applied potentials give a net anodic current to the $\mathrm{WE}$, the $\mathrm{CE}$ is the site of the cathodic reaction. Likewise, when there is a net cathodic current on the WE, the CE is the site of the anodic reaction. However if the free corrosion potential was measured as a function of time, in the absence of external perturbation (i.e. polarisation), the oxidation and reduction reactions occur on the working electrode.

OCP OCP measurements were conducted using a two electrode cell comprising the WE and RE. The potentiostat measures the potential difference between the two electrodes as a function of time. This is a mixed potential as both reactions are occurring at the same time on the single working electrode. OCP experiments can give information on the reactions occurring on the surface. The technique can been coupled with in-situ experiments (TLM, SVET). The tech- 
nique is complimentary, and provides electrochemical information, alongside the spatially-resolved maps of current density (SVET) and, in the case of TLM, insitu images of the microstructure, with respect to time.

Potentiostatic Experiments The potentiostatic method imposed a single, constant potential to the WE, measured with respect to the RE. As the cell is potential controlled $\mathrm{CE}$ and $\mathrm{RE}$ are used. The experiment measures current density as a function of time, which can help in the study of metastable events where corrosion initiation does not occur under freely corroding conditions.

Zero resistance ammeter Zero resistance ammeter (ZRA) experiments allowed current to be measured between two electrodes as a function of time. The instrument coupled two different electrodes and imposed a $0 \mathrm{~V}$ potential difference between them. Thus the technique could be used for monitoring Galvanic coupling between two different metals, for example Fe and Zn. The technique can also be used to couple two similar specimens i.e. two different grades of stainless steel, with one having a greater alloying composition. ZRA can also be used to investigate the suitability for one metal to provide cathodic protection to another. If by changing the metal expected to be the cathode (i.e. Fe) to metal expected to be the anode (i.e. Zn) area ratio (i.e. a cathode two times larger than the anode), an increase in measured current is observed then the expected cathode (Fe) is likely to be protected when coupled with $\mathrm{Zn}$.

Potentiodynamic Experiments Potentiodynamic experiments were conducted using a three electrode cell. An OCP experiment was run beforehand to both establish the starting potential $E_{1}$, and also allow the potential between the electrode, electrolyte, and counter-electrode to equilibrate. This was typically a ten to thirty minute OCP experiment; and was a compromise between sample throughput and to find a semi-steady state free corrosion potential $\left(E_{c o r r}\right)$. Once the free corrosion potential was recorded; a suitable starting potential was chosen 
which was negative than that of $E_{\text {corr }}$. This potential was then swept from $E_{1}$ to the final potential $E_{2}$ using a constant sweep rate. $E_{2}$ was chosen to be sufficiently positive such that the breakdown potential $E_{b}$ was reached. Once the potential was equal to $E_{2}$, the measurement ceases. An example potentiodynamic curve is shown in figure 2.4, showing key locations on the curve. The diagram shows the breakdown potential $\left(E_{b}\right)$, and pitting potential $E_{p i t}$, the locations on the curve where stable pitting occurs, thus the current density increases exponentially as shown on the graph. In addition to $E_{1}$ and $E_{2}$ further potentials can be input into the system. By performing further sweeps to additional potentials the degree of hysteresis can be investigated. The size of hysteresis loops are linked to repassivation of the metal. Zero hysteresis suggests no corrosion, while a small degree of hysteresis suggests that the surface has repassivated. A large hysteresis loop indicates that the surface has changed considerably from corrosion damage.

\subsubsection{Reference Electrodes}

The standard hydrogen electrode (SHE) is the gold standard RE for corrosion electrochemistry applications. Due to its large size and bulk however a smaller, more compact electrode was used. There are many types of reference electrode, but have a key requirement to maintain a steady potential with respect to time in the electrolyte of interest. A SCE was used in all experiments, and a diagram of its principle features is shown in a schematic in figure 2.5. The SCE uses elemental mercury $(\mathrm{Hg})$ which is in contact with calomel (mercury-chloride, $\mathrm{Hg}_{2} \mathrm{Cl}_{2}$ ). This is encased within glass, similar to a test tube, filled with saturated potassium chloride aqueous solution $(\mathrm{KCl})$. The SCE works works through the redox reaction between $\mathrm{Hg}$ and the mercury-chloride calomel, which is steady for within a range of elecrolytes. The potential of the SCE reference electrode is highly dependant on the chloride anion, but can be calculated using the Nerst equation. Saturated solutions of $\mathrm{KCl}$ result in a potential of $+244.4 \mathrm{mV}$ (wrt. $\mathrm{SHE}$ ) at room temperature, whereas for $3.5 \mathrm{~mol} \mathrm{dm}^{-3} \mathrm{KCl}$ the reference potential 


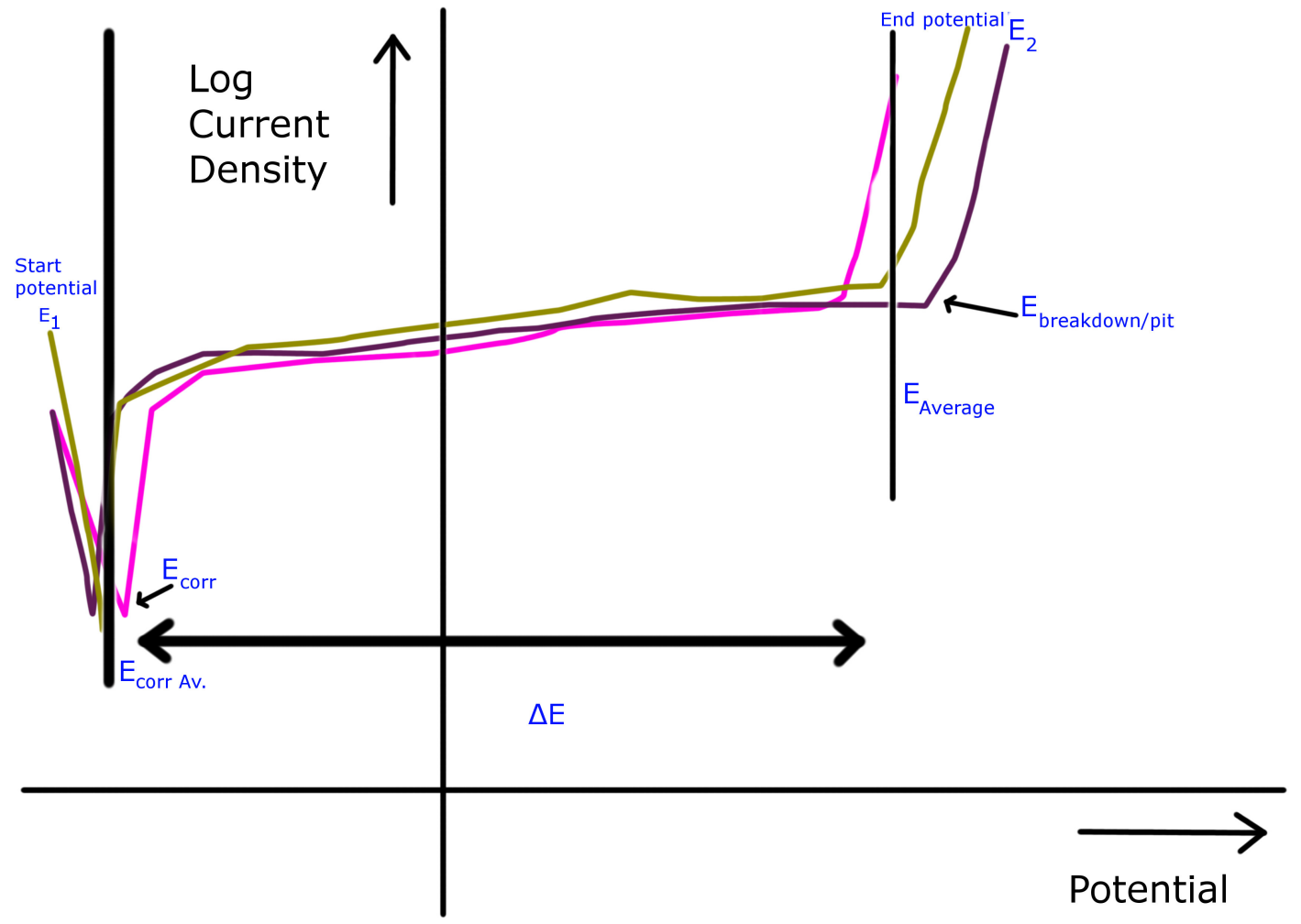

Figure 2.4: Example potentiodynamic curve (shown with a starting and final potential).

shifts to $+250 \mathrm{mV}$ (wrt. SHE). For all experiments saturated $\mathrm{KCl}$ solution was used.

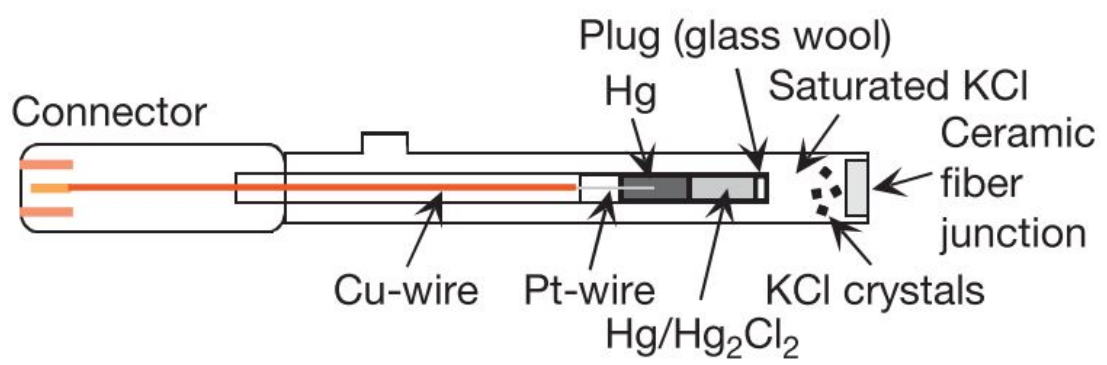

Figure 2.5: The saturated calomel electrode [58]

\subsection{SVET}

The Scanning Vibrating Reference Electrode Technique (SVRET) shares similarities with the traditional scanning reference electrode technique (SRET) [119].

The SVET has increased in spatial resolution and sensitivity when compared to 
SRET. The SVRET is commonly shortened as SVET, the instrument uses a vibrating probe. The SVET uses a Pt microdisc electrode which oscillates normal to the specimen surface, and is scanned in $\mathrm{x}$ and $\mathrm{y}$ planes. The SVET measures iso-potential gradient within the solution, as a function of space $(\mathrm{x}, \mathrm{y})$.

The SVET Pt microdisc electrode ('probe') is constructed of a short length of Pt wire $(125 \mu \mathrm{m}$ diameter), insulated in glass and is polished flat. This oscillates at a set amplitude and frequency. A diagram of the probe is shown in figure 2.6 courtesy of Williams [87]. A large area (c. $2.5 \times 2.5 \mathrm{~cm})$, low impedance $\mathrm{Ag} / \mathrm{AgCl}$ foil electrode was used as a pseudo-reference electrode and located c. $5 \mathrm{~cm}$ away from the vibrating probe. Prior to a corrosion experiment, the probe is immersed in $\mathrm{HCl}$ for a period of 5 minutes and rinsed with distilled water to remove any contamination. As the Pt probe oscillates, an alternating potential in solution is measured. When the SVET probe/electrode oscillates it intersects ('cuts') through lines of constant potential, and therefore measures the change in potential gradient within the z-plane. The measured potential difference in $\mathrm{nV}$ can be converted into current density by a simple calibration experiment. It is important to perform a calibration experiment each time the equipment is used. This is to ensure that the lock-in amplifier uses the correct phase and is synchronised with the signal from the SVET speaker setup. The correct phase not only ensures that the maximum signal can be attained, but has the correct sign (i.e. positive, negative). If the signal attained was out of phase by $180^{\circ}$, anodes would appear as cathodes and vice versa. The correct phase also ensures that the signal measured accurately reflects the intensity of corrosion events witnessed in an experiment. An additional reason for calibration of the SVET is that it allows the conductivity of the electrolyte to be estimated. The conductivity is assumed to be constant for the duration of the experiment. The SVET was calibrated using a two compartment tube cell, such as is shown in figure 2.7. In these calibrations a known current can be passed though a known cross sectional area.

A bespoke perspex tube cell is placed within a perspex tank of approximately 


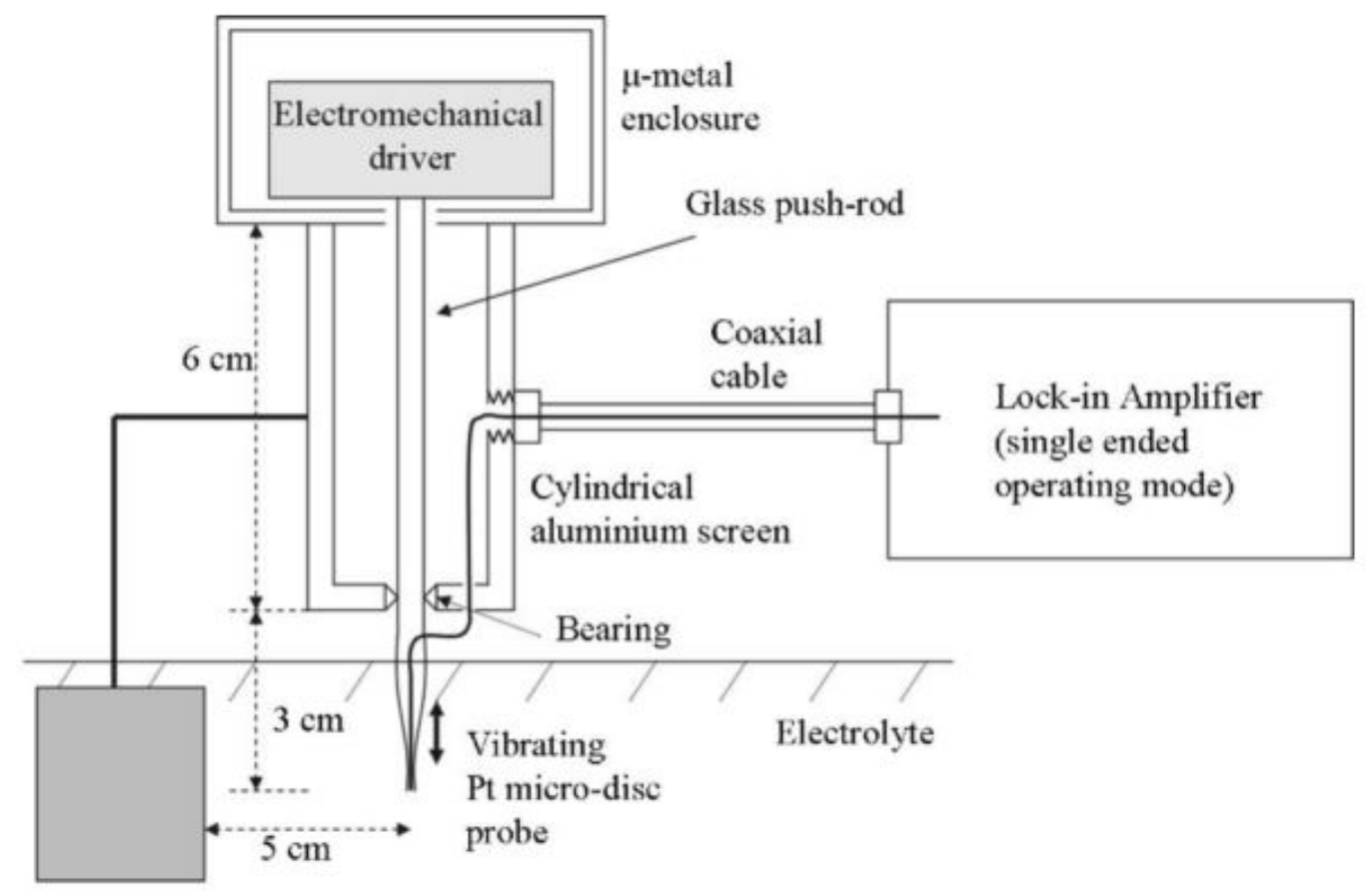

Large, low impedance

$\mathrm{AgCl}$ reference electrode

(instrument earth)

Figure 2.6: Diagram of the SVET probe and speaker set-up obtained from Williams [87] 


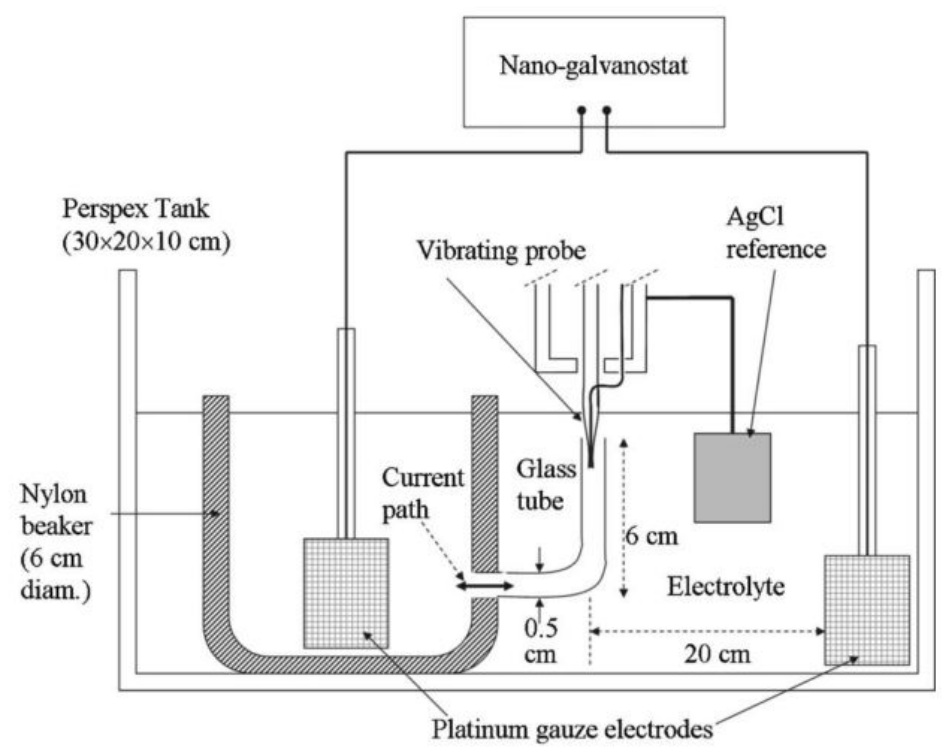

Figure 2.7: SVET tube cell used for calibration. Two Pt electrodes are seperated by a narrow vertical tube. This image was obtained from Williams [87]

5 litre capacity, and filled to the top of the tube cell with the desired electrolyte. The vibrating microtip is placed approximately $2 \mathrm{~cm}$ within a hole of known diameter, in which there is a flux of ionic current. The probe is moved in the $\mathrm{z}$ direction in $100 \mu \mathrm{m}$ steps in order to find the location of maximum signal. Once a stable signal is recorded, the lock-in amplifier is auto-phased; such that the speaker and lock-in are synchronised. The $\mathrm{Ag} / \mathrm{AgCl}$ electrode is placed within the perspex tank. A nano-galvanostat supplies a user selected current between two Pt electrodes; one of which is within a perspex beaker, the other is connected to the outside of this. As the two electrodes are separated by some distance of over $10 \mathrm{~cm}$, the only route for current flux is through the vertical tube. Furthermore, the majority of current will be in the $\mathrm{z}$ direction, parallel to the axis of vibration. Assuming that the current flux is constant across the diameter of the tube, and knowing its radius, a current density can be obtained. By plotting a graph of supplied current, and corresponding the SVET detection signal on a graph calibration slopes can be obtained. This can also be determined mathematically by Ohm's law, using the peak to peak SVET voltage $V_{p p}=j_{z}\left(a_{p p} / K\right)$, where $a_{p p}$ is the SVET probe vibration amplitude and $j_{z}$ the current flux density along the 
axis of vibration [87]. The units for the calibration factor (where $\mathrm{m}$ is the gradient) are $m=n V m^{2} A^{-1}$; assuming a plot of current density $\left(A m^{-2}\right)$ on the $\mathrm{x}$ axis, and SVET potential $(\mathrm{nV})$ on the y axis. A typical graph is shown in figure 2.8. The calibration factor is a function of the electrolyte conductivity. Once the value is known it can be used to convert SVET signal (nV) into current density values $\left(A m^{-2}\right)$, which is what is plotted using cartography software.

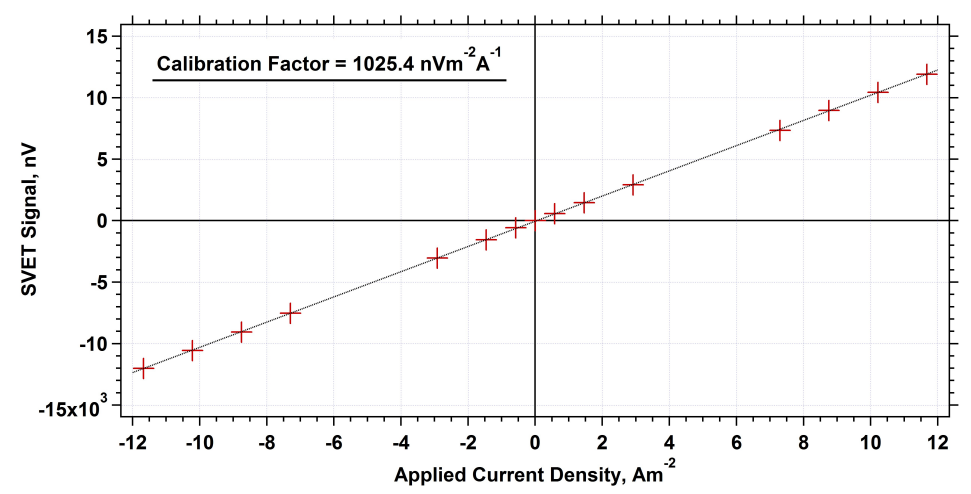

Figure 2.8: Typical SVET calibration plot of SVET detection signal against user selected current density. There exists a linear relationship over a range of current density values

\section{Height Profiling}

As discussed previously, in order to resolve local point current sources by a distance of $w h m=1.553 \cdot Z$ a user specified distance must be maintained throughout scanning. The scan height is not only critical to the ability to resolve localised anodes and cathodes but also fundamental to the technique itself. $F_{\max }$, the maximum field strength, is related to Z by means of an inverse square relationship, and thus it is vital that the scan height is maintained.

The theory suggests that specimens be flat, and free of features which would otherwise affect probe to specimen distance, therefore giving a constant scan height. Once a probe calibration has been completed within the desired electrolyte a specimen can be prepared for scanning. Manual levelling and planar interpolated scanning were used in the SVET experiments. The full list of height profiling to ensure probe to specimen distance is maintained are given below: 
- Manual levelling

- Planar interpolated scanning

- Three-dimensional SVET (3D-SVET)

Manual Levelling The most effective way of levelling, is to perform the task manually using a stage with height adjustment. This type of levelling is very effective for flat specimens as once the surface is levelled, it is orientated perpendicular to the scan, thereby making any movement in the z-axis unnecessary. As there is no need for the probe to travel toward, or away from the specimen, scan times are reduced.

Planar Interpolation This method relies on the mathematical method of interpolation. The probe takes height readings from four points on the specimen surface at each corner. Using these co-ordinates the SVET computes the difference in $\mathrm{z}$ that the probe must move to maintain a constant distance. This method is ineffective when there are irregularities on the surface due to the height being a function of only four data points. Aside from this; the method works well for flat, featureless surfaces, and a combination of rough manual levelling alongside planar interpolated scans can result in much reduced height scan times when compared to 3D levelling.

3D-SVET 3 dimensional SVET height levelling allows a wide variety of surfaces to be measured, including those that are highly non-planar. The technique works by taking a height measurement for each spatial data point on the surface without an electrolyte present. This set-up allows profiling of heights that differ by $2 \mu \mathrm{m}$ and works through measuring the change in inductance between the SVET probe speaker and the reference speaker, and once the program is initiated the process is automatic. The surface is 'detected' once the change in impedance crosses a user-defined threshold. Stepper motors controlling the z-direction move 
the probe toward the sample in small increments at a slow rate. Once the surface is detected the probe is retracted by a user-defined roughness value, $\mathrm{x}-\mathrm{y}$ stepper motors then move the probe to the next point in the raster pattern. The computer stores the number of steps within memory for this point. The process is repeated and the SVET program controls the SVET Z stepper motor at each data point, allowing the user specified distance to be maintained. As such, it is suitable for mapping weld locations and coins. Further information on the SVET 3D height profiling can be obtained in [120].

\subsection{Scanning Probe Microscopy}

\section{Atomic Force Microscopy}

Atomic force microscopy (AFM) is a surface probe (microscopy) technique and is similar to the scanning tunnelling microscope but uses atomic forces rather than quantum mechanical effects. A sharp tip connected to the end of a cantilever is raster scanned across the surface to map the sample topography. The upper surface of the cantilever is usually coated with a high reflectivity material such as silicon, or silicon nitride. In the non-contact mode experiments (topography, Kelvin probe force microscopy) the AFM tips used were made from single crystal silicon (Nanoworld FM50). For liquid cell experiments (contact mode) 3-lever n-type silicon tips were used (Mikromasch HQ:CSC37). As the tip interacts with the surface through forces between the sample and the cantilever, the cantilever undergoes a deflection. This deflection is measured by a laser which is reflected from the upper surface of the cantilever to a position sensitive detector which allows the AFM to have very high sensitivity measurement in the z-direction in the order of 0.01 angstroms. The AFM uses a feedback mechanism to enable tracking, control and position of the probe, as the instrument is very sensitive to surface conditions. 


\section{Scanning Kelvin Probe Force Microscopy}

Scanning Kelvin probe force microscopy (SKPFM) can be used to map the relative Volta potential difference of different phases within the microstructure of a conducting solid sample. This is conducted in non-contact mode using the same set-up as the AFM. The AFM is used to obtain a topography measurement on the trace, whilst the AFM feedback mechanism is used to maintain a set sample to probe distance on the retrace. SKPFM works in a similar fashion to the macroscale scanning Kelvin probe, as the sample and probe form a capacitor given that the distance is maintained throughout the measurement. The AFM tip and the sample will have different work functions (difference between the Fermi and vacuum levels) and thus when the two are connected externally the Fermi levels align. These align due to a a flow of current as the electrons flow between the two conducting metals until equilibrium is reached. Thus a charge and contact potential difference will arise. By using a external bias potential to null the contact potential difference such that the difference in work function between the sample and probe can be determined. Application of alternating and direct current voltages to the probe allows the measurement of the sample work function. This is explained in greater detail in [121] and [122].

\subsection{Overview of Experiments Undertaken in this Thesis}

A number of experiments were performed in this thesis and are displayed in table 2.1. Table 2.2 shows the conversion between different units of concentration used, in order to convert between $\mathrm{NaCl}$ molarity and parts per million $\left[\mathrm{Cl}^{-}\right]$. 


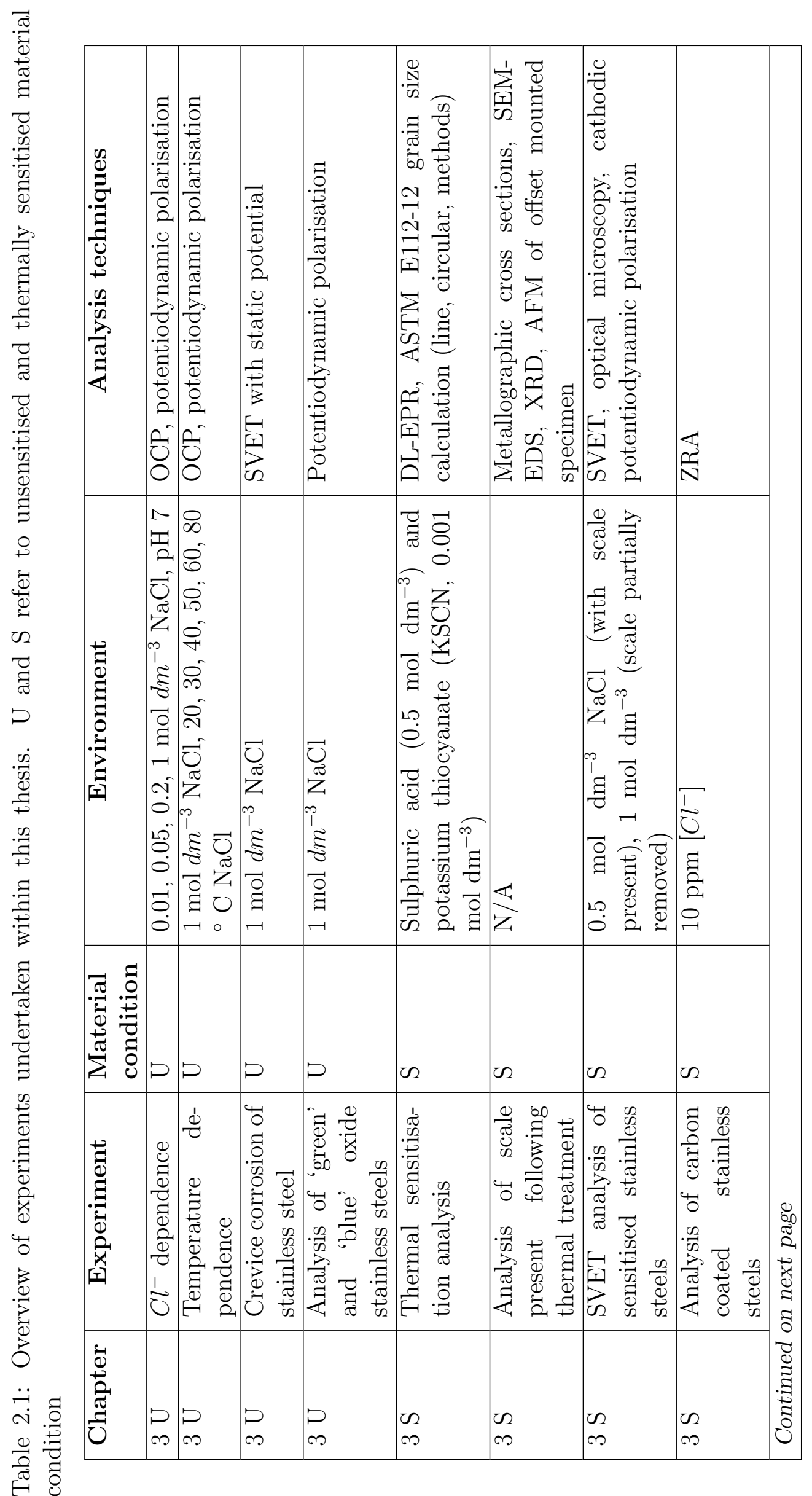




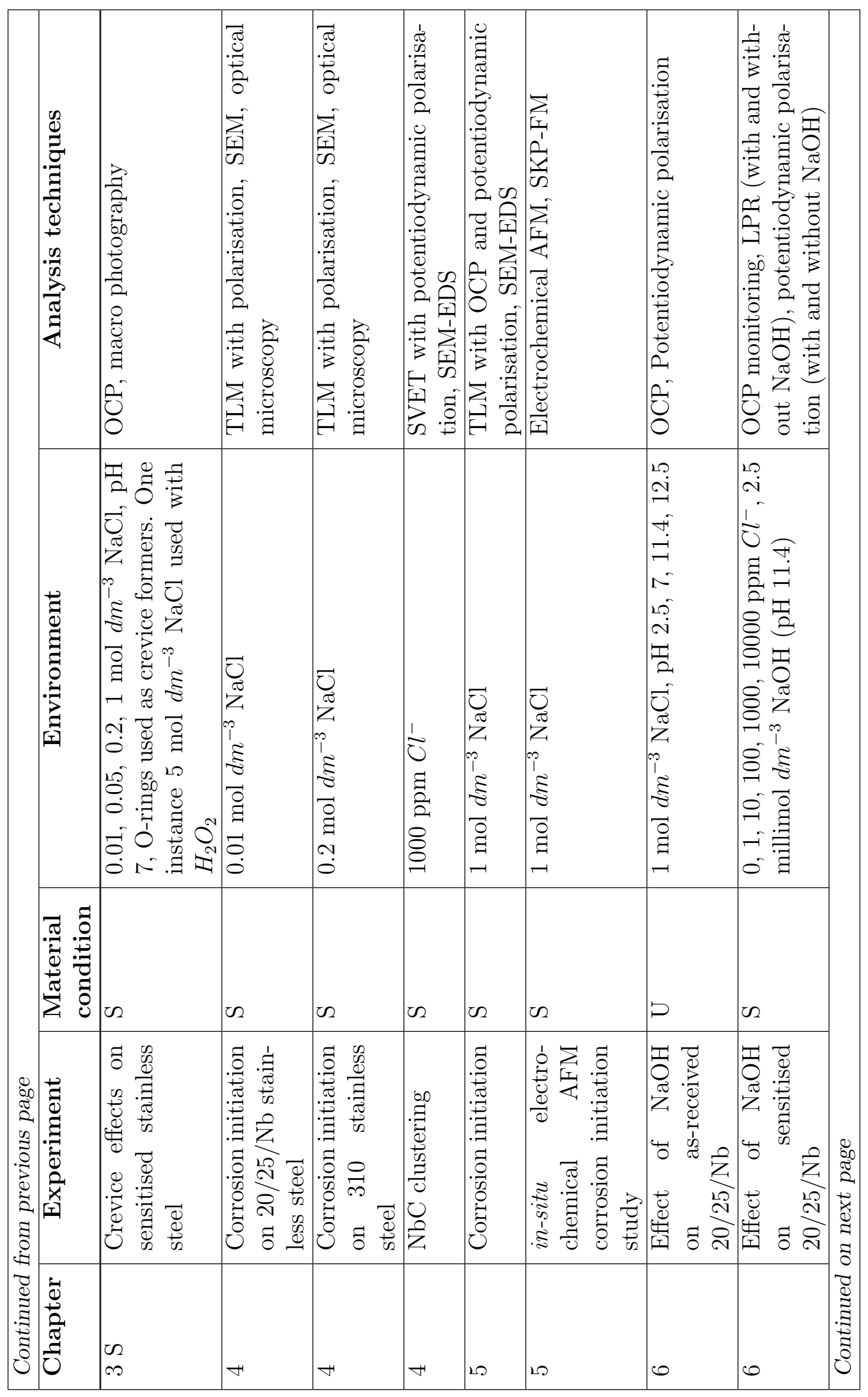




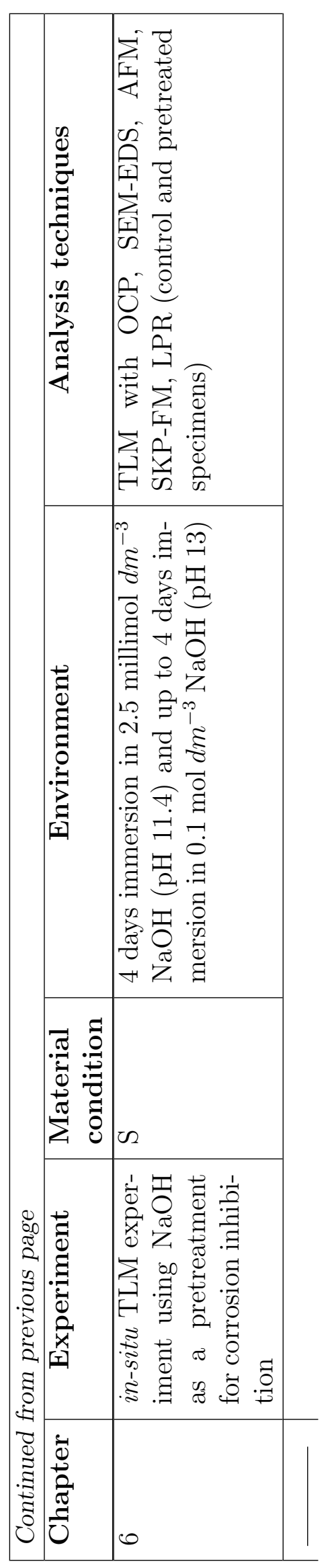




\begin{tabular}{|l|l|l|l|}
\hline $\mathrm{NaCl}$ molarity, mol & {$[\mathrm{NaCl}]$,} & {$\left[C l^{-}\right]$,} & {$\left[C l^{-}\right], \mathrm{ppm}$} \\
$d m^{-3} \mathrm{NaCl}$ & $g L^{-1}$ & $g L^{-1}$ & \\
\hline 1 & 58.44 & 35.45 & 35450 \\
0.2 & 11.69 & 7.09 & 7090 \\
0.05 & 2.92 & 1.77 & 1772.5 \\
0.01 & 0.58 & 0.35 & 354.5 \\
\hline
\end{tabular}

Table 2.2: Conversion between sodium chloride in units of molarity and as parts per million chloride 
Chapter 3

Corrosion of 20/25/Nb Stainless

Steel Studied using DC

Electrochemistry and SVET

Methods 


\subsection{Introduction}

20/25/Nb AGR fuel cladding has been investigated using a variety of aqueous electrolytes in order to study the corrosion mechanisms and kinetics. Such conditions are more aggressive than pond storage, which reflects the need to initiate corrosion in the orders of hours and days rather than decades. This chapter is split into two main sections according to the material condition: unsensitised and thermally sensitised.

Unsensitised Material Condition Within the first section a conventional assessment stainless steel was undertaken using standard DC electrochemistry, in order to properly characterise the material. It is known that the corrosion of SS is both $\left[\mathrm{Cl}^{-}\right]$and temperature dependent [12] [123]. Storage ponds are maintained to ensure that bulk water temperatures do not exceed specified limits. Whilst bulk pond water temperatures will be at the nominal target it is likely that local water temperatures adjacent to the cladding will be higher. As denser packing is scheduled to be used, these local temperatures are likely to rise in the future, compared to the present temperatures. It is expected that local temperatures will be over $50^{\circ} \mathrm{C}$ under normal operating conditions; and in a fault condition temperatures could rise even further [37]. Therefore, electrolyte temperatures will become increasingly relevant, as these reflect local conditions, even if the bulk temperature is low. Subsequently ferric Chloride $\left(\mathrm{FeCl}_{3}\right)$ was investigated for its use as a corrosive medium to produce localised corrosion in the unsensitised SS at, or near open circuit conditions in combination with the scanning vibrating electrode technique (SVET) to map local activity occurring on the metal surface in the aqueous electrolyte, in-situ. $\mathrm{FeCl}_{3}$ is an aggressive, low $\mathrm{pH}$, metal chloride containing solution, which shares similarities with pit and crevice solutions. $\mathrm{Fe}^{3+}$ is also highly oxidising and will promote $E_{\text {corr }}$. Within a pit or crevice, a concentration gradient of ions forms between the anodic and cathodic sites. Pit covers hinder mass transport; leading to a high concentration 
of metal ions, which in turn, causes electromigration. This induces a flux of $\mathrm{Cl}^{-}$ toward the pit mouth; and metal ions toward the free surface. Therefore, the pit solution is a highly concentrated $\mathrm{Cl}^{-}$environment. If the SS is immersed in acidic media an excess of protons $\left(H^{+}\right)$will migrate to the pit; leading to a highly acidic local pit solution. These protons are eventually reduced to form hydrogen. $\mathrm{FeCl}_{3}$ solutions are used for testing the critical pitting temperature of SS [124]. Though CPT tests were not used in this study, as $\mathrm{FeCl}_{3}$ is a very aggressive electrolyte, it was investigated for its ability to provide spontaneous corrosion initiation. Green and blue oxide specimens, those with specially prepared oxides, were analysed in this section to investigate the effects of thick deposition resistant cladding on corrosion performance.

Thermally Sensitised Condition In this section the stainless steel was thermally processed in order to attain a sensitised microstructure. The double-loop electropotentiokinetic reactivation (DL-EPR) was used to quantify the degree of sensitisation (DOS) in the material. Grain sizes were also measured to compare the as-received and sensitised microstructures. Following the sensitisation procedure the stainless steel was covered by a coating, which was analysed by X-ray diffraction and scanning electron microscopy (SEM). The SVET was then used to study thermally specimens fully and partially covered by this scale. Carbon deposition was then studied using the zero resistance ammeter technique to investigate if carbon forms a Galvanic couple when deposited on the stainless steel. Finally crevice corrosion was studied by the use of o-rings for crevice formers in $\mathrm{Cl}^{-}$containing electrolytes.

\subsection{Experimental}

Unsensitised Material Condition The SS was examined in various electrolytes $\left(0.01,0.05,0.2,1 \mathrm{~mol} \mathrm{dm}^{-3} \mathrm{NaCl}\right)$ in freely corroding conditions using a two-electrode cell. Specimens were immersed in electrolytes for 30 minutes for the 
free corrosion potential to stabilise. The concentrations were chosen to reflect the need to measure corrosion occurring over the relatively short timescales (hours, instead of tens of years). Following open circuit potential (OCP) experiments, specimens underwent potentiodynamic measurements to investigate the influence of $\left[\mathrm{Cl}^{-}\right]$and electrolyte temperature. As explained previously (chapter 2), all potentials were measured with respect to a saturated calomel electrode (SCE), with platinum (Pt) gauze used as a counter electrode. The start potential for potentiodynamic experiments was $-200 \mathrm{mV}$ polarised versus SCE with respect to $E_{\text {corr }}$, in order to capture detail on the cathodic oxygen reduction reaction slope. The electrolytes were deaerated by vigorous nitrogen bubbling for experiments where measuring the influence of $\left[\mathrm{Cl}^{-}\right]$on pitting potential was investigated.

A water bath was used to vary the experiment temperature. A number of different electrolyte temperatures were chosen $\left(20,30,40,50,60,80{ }^{\circ} \mathrm{C}\right)$; whilst

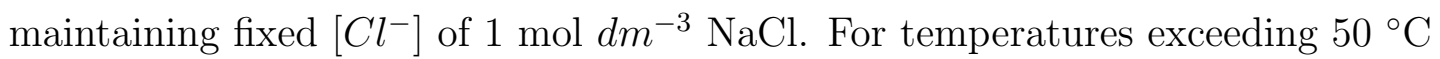
the time taken to reach the set point increased by a large margin. A thermometer was placed in the beaker to ensure the temperatures of electrolyte matched that of the bath. For the higher temperatures, a perspex cover was placed on top of the bath to re-condense vapour back into the bath. Ideally the SCE should be operated at lower temperatures as laws governing its relative potential vary with temperature. The SCE was chosen as it had been used in all other experiments. Corrections were made for the temperature dependence of the SCE reference electrode by the formula $\left(E(S C E)=+0.242-7.6^{-4} \cdot(T-298)\right)[125][126]$. This formula uses potentials in volts, and temperature in Kelvin. The slope for $\left[\mathrm{Cl}^{-}\right]$dependence was calculated using graph software (IGOR pro). A method of linear regression was used for the curve fitting, using weighting for error at each concentration. The error bars in the graph represent one standard deviation, calculated using Microsoft Excel, with any obvious outliers excluded.

Specially treated specimens, "green" and "blue" stainless steel specimens were studied using LPR experiment, with the colours relating to the thicknesses of the 
$\mathrm{Cr}_{2} \mathrm{O}_{3}$ oxide on the surface. In order to produce these different specimens, they were baked by NNL in a furnace containing different water vapour concentrations. The green oxide specimen had a target dew point of $-23{ }^{\circ} \mathrm{C}$ with a range of $-18{ }^{\circ} \mathrm{C}$ to $-28{ }^{\circ} \mathrm{C}$. The blue oxide specimen had a target dew point of $-41{ }^{\circ} \mathrm{C}$ with a range of $-36{ }^{\circ} \mathrm{C}$ to $-46{ }^{\circ} \mathrm{C}$. No information was given by $\mathrm{NNL}$ on time or temperature for this treatment other than the target and range of dew point. The starting potential for the experiment was more negative than $E_{c o r r}$, and was run to a suitable potential in which $E_{b}$ would be present. A reverse scan was then conducted back toward $E_{\text {corr }}$. This type of experiment was conducted to investigate the possibility for the specimens to undergo repassivation. Should the specimen show pitting (i.e. it has reached $E_{b}$ ); and the reverse scan cross the anodic sweep then repassivation has occurred. This is called the cross-over potential, $E_{r p}$ [127]. The smaller the hysteresis loop, the more successful the repassivation. The specimens were immersed in $1 \mathrm{~mol} d m^{-3} \mathrm{NaCl}$; with an open circuit measurement conducted beforehand to allow the potential to stabilise. All test specimens were subjected to anodic polarisation from $-300 \mathrm{mV}$ to $500 \mathrm{mV}$ (vs. SCE), with $500 \mathrm{mV}$ set as the initial vertex potential. All specimens showed transpassive behaviour before reaching the vertex potential.

Thermally Sensitised Condition Information on the DL-EPR test is given in section 2.2.1. Grain sizes were calculated using a Nikon MA200 inverted microscope its 100x objective lens. The study was conducted according to the ASTM E112 standard [128], using both linear and circular intercept procedures. 3 lines of $\approx 150 \mu \mathrm{m}$ were drawn and intercepts taken. This process was repeated at three different areas across the surface, and an average taken from these 9 points. The error was expressed as one standard deviation. In addition, circular intercepts were taken at each area, and averaged. The error in these measurements were expressed as the difference between the largest and smallest value.

The dull surface scale was analysed following sensitisation treatment to study thickness and also its composition. Specimens for thickness analysis were mounted 
in a cold set resin (Struers Durocit) allowing the cross section to be imaged. Specimens were ground, polished and subsequently imaged using SEM-EDS (Hitachi TM3000). Multiple areas of the cross section were imaged using SEM to gain a representative scale thickness. Further analysis was also undertaken using X-ray diffraction (XRD) in order to determine its crystal structure. The diffractometer scanned from $20-95^{\circ}$ in order to capture a large number of diffraction peaks for matching. In addition, to check the sensitised SS for texture, XRD was also performed on the SS in different orientations. In this experiment the SS specimen was placed in the diffractometer and fixed in place. A single scan of the same 20$95^{\circ}$ was undertaken before a $45^{\circ}$ rotation about the y plane. The specimen was then scanned through the same angles as before, this occurred multiple times until the specimen rotated a full $360^{\circ}$. The data from the texture experiment were processed using Bruker EVA software and displayed in the form of a waterfall plot (figure 3.23) for easy analysis. As a result, the y axis counts are relative.

A preliminary test in $\mathrm{NaCl}$ containing electrolyte $\left(0.5 \mathrm{~mol} d m^{-3} \mathrm{NaCl}\right)$ revealed the formation of red iron oxide rust on the surface of the sensitised SS after a duration of approx. 24 hours (see section 3.4.4). This indicated that a scanning probe technique could be used to assess the locations of anode formation. The SVET was used to study the unprepared Hematite coated specimen in $0.5 \mathrm{~mol} \mathrm{dm}^{-3} \mathrm{NaCl}$. Three tests were conducted using two specimens of similar area. The first experiment was undertaken using a $2 \mathrm{mV}$ SVET lock-in amplifier sensitivity, over a 24 hour period, using an 12x18 mm exposed area. The second experiment used the same concentration electrolyte, with an increased $(200 \mu \mathrm{V})$ sensitivity setting for a longer time period. This used a significantly smaller area using a 7x10 mm exposed area. A digital single lens reflex camera (Canon 600D) was used in this instance to image the surfaces post corrosion. In addition to analysing specimens with a full coating of the scale, the alloy was also prepared using polishing techniques to investigate the surface just below the scale where the SS is sensitised. The surface was ground at an angle to allow analysis of 
the sensitised area, as has been conducted previously (figure 3.15). $1 \mathrm{~mol} \mathrm{dm^{-3 }}$ $\mathrm{NaCl}$ was used and the SVET was set to $100 \mu \mathrm{V}$ sensitivity. Micrographs were taken using a Nikon MA200 inverted microscope, and stitched together using the Graphical Image Manipulation Program (GIMP).

The zero resistance ammeter (ZRA) technique was used to study the effects of carbon deposition on fuel cladding, with respect to corrosion in aqueous storage conditions.Sensitisation treatment for the SS tube was conducted at Swansea University according to the procedure described previously (Chapter 2). Once SS tubes were sensitised, carbon was deposited using a candle flame. Other methods (lighter, chefs blowtorch) were used but appeared to burn too cleanly for carbon deposition. A fine mist of oil (Frylight sunflower oil) was sprayed onto the tube before it was heat treated for 2 hours in a furnace $\left(250{ }^{\circ} \mathrm{C}\right)$ to help bind the carbon to the surface. Baked carbon is porous [129], but it is unlikely at the heating timescale that graphitisation, which has an even lower porosity had occurred. A length of uncoated SS tube was also cut such that the two tubes had the same area. The inside, and cut edges, of the two tubes were painted in a non-conductive laquer (Lacomit) in an attempt to order to only measure activity from the outer tube walls. The carbon coated and uncoated SS tubes were connected to the potentiostat, as CE and WE, respectively. The uncoated tube was immersed by $4 \mathrm{~cm}$. The carbon coated tube area was varied. A potential of zero volts was applied to the reference electrode (uncoated tube) in order to measure the current flux between the electrodes. The data had 50 point box smoothing applied by IGOR pro software.

As-received tube was immersed in $0.2 \mathrm{~mol} \mathrm{dm}^{-3} \mathrm{NaCl}$ (aq). For the sensitised tube experiments $10 \mathrm{ppm}\left[\mathrm{Cl}^{-}\right]$was used as an electrolyte to better simulate pond chemistry. The ZRA experiment measured the coupled current flux between the two species, and the sign of the current density gives details on which species is acting as the anode, or cathode. As such the magnitude of current density therefore defines the amount of activity between the two species. The sensitised 
SS was examined in an appropriate corrosive environment to investigate crevice corrosion. Preliminary tests had shown that the SS was responsive to crevice corrosion. Furthermore, crevices will tend to naturally form in environments where materials are in close proximity to each other (chapter 1). Crevice environments were formed by tight fitting rubber o-rings fastened around the SS tube structures. A separate SVET experiment studied the propagation of crevice corrosion for the SS when set in resin. The SS tube was thermally sensitised and subjected to varying levels of $\left[\mathrm{Cl}^{-}\right]\left(0.01,0.05,0.5,5 \mathrm{~mol} \mathrm{dm}^{-3} \mathrm{NaCl}\right)$. An investigation of the tubes corrosion behaviour in an even more extreme environment was also carried out. The sensitised tube was immersed in $5 \mathrm{~mol} d m^{-3} \mathrm{NaCl}$ and crevice formers used, but in addition 20 millimol $\mathrm{dm}^{-3}$ hydrogen peroxide $\left(\mathrm{H}_{2} \mathrm{O}_{2}\right)$ was also added to the electrolyte. The experiment represents a worst-case condition where a highly localised environment within a fuel pond a $\left[\mathrm{Cl}^{-}\right]$increased to close to saturation and radiolysis of the water has led to the formation of a higher than expected $\left[\mathrm{H}_{2} \mathrm{O}_{2}\right] . \mathrm{H}_{2} \mathrm{O}_{2}$ is an oxidiser which if present would act as a natural cathodic depolariser, thus raising the OCP. The specimens were immersed in electrolytes for a minimum of $160 \mathrm{Ks}$ (44.4 hours). Crevices were introduced by placing 4 tight fitting o-rings around the circumference of the tube within a 1.5 cm distance from each other (figure 3.1). Post corrosion images were taken with a digital single lens reflex camera (Canon 600D) and cropped according to relevant sizes for examination. Further images were taken using an infinity 1 camera and Meiji macroscope $\left(\mathrm{H}_{2} \mathrm{O}_{2}\right.$ experiment only).

\subsection{Unsensitised Stainless Steel}

\subsubsection{Chloride Dependence}

Figure 3.2 shows potentiodynamic curves for unsensitised 20/25/Nb stainless steel immersed in $0.01,0.05,0.2$ and $1 \mathrm{~mol} \mathrm{dm}^{-3} \mathrm{NaCl}$. Three repeats were taken for each concentration. The graph shows a plot of applied potential with 


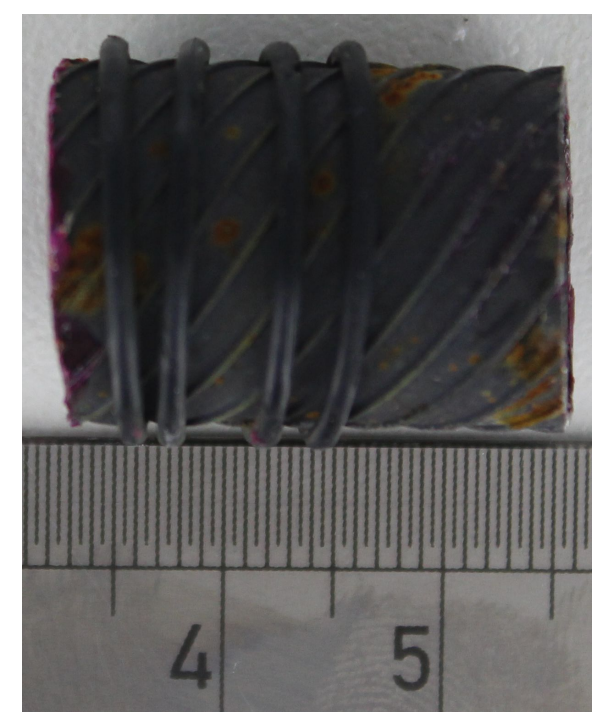

Figure 3.1: Tight fitting o-rings were used to introduce crevice corrosion. 4 o-rings were used within a maximum tube length of $1.5 \mathrm{~cm}$.

respect to the measured current density. The lowest concentration $(0.01 \mathrm{~mol}$ $\left.d m^{-3} \mathrm{NaCl}\right)$ showed the most anodic breakdown potential $\left(E_{b}\right)$, with an average change in potential $\left(\Delta E_{A v}\right.$.) of $744 \mathrm{mV}$ (vs. SCE). Specimens immersed in $0.05 \mathrm{~mol} \mathrm{dm}^{-3} \mathrm{NaCl}$ exhibited more current transients than in $0.01 \mathrm{~mol} \mathrm{dm}^{-3}$ $\mathrm{NaCl}$. This indicates that metastable pitting occurred, caused by ruptures in the passive $\mathrm{Cr}$ oxide film. In these cases the pit environment is not sufficient to allow continued pit growth, therefore repassivation occurs in the majority of events. $E_{b}$ was more cathodic, resulting in a smaller $\Delta E_{A v}$. of $680 \mathrm{mV}$ (vs. SCE). The effects of the higher conductivity are shown by the gradient of the slope at the $E_{b}$, which is greater for the $0.05 \mathrm{~mol} d m^{3}$ specimens. At $0.2 \mathrm{~mol}$ $d m^{-3} \mathrm{NaCl}$ transient events continue to manifest. The gradient of the curve at the breakdown potential is similar to that of the previous concentration, 0.05 mol $d m^{-3} \mathrm{NaCl}$. The value of $\Delta \mathrm{E}$ is comparable to that of $0.05 \mathrm{~mol} d m^{-3}$ $\mathrm{NaCl}$ (table 3.1). This is a result of the shift in free corrosion potential $\left(E_{\text {corr }}\right)$, even though $E_{b}$ is smaller value. The spread in results for both $E_{C o r r}$ and $E_{b}$ is more significant than that of the other data; with standard deviations of $17.4 \%$ and $11.4 \%$, respectively. As $\left[\mathrm{Cl}^{-}\right]$increases further, the expected decrease in $E_{b}$

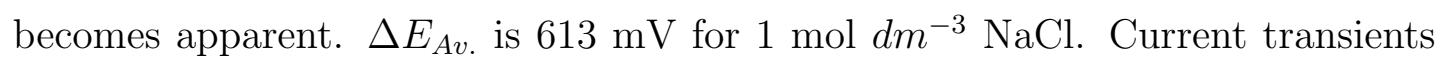


are still present in the curve suggesting that a number of pits had nucleated; but did not have the correct conditions for stable pit growth. A sharper transition from passive to active current was expected at $E_{b}$, however the transition was more in line with previous concentration results. The data from the separate experiments are tabulated in Table 3.1. This shows the average $E_{C o r r}, E_{b}$ and $\Delta E_{A v}$. values. Errors are quoted as one standard deviation from the mean.

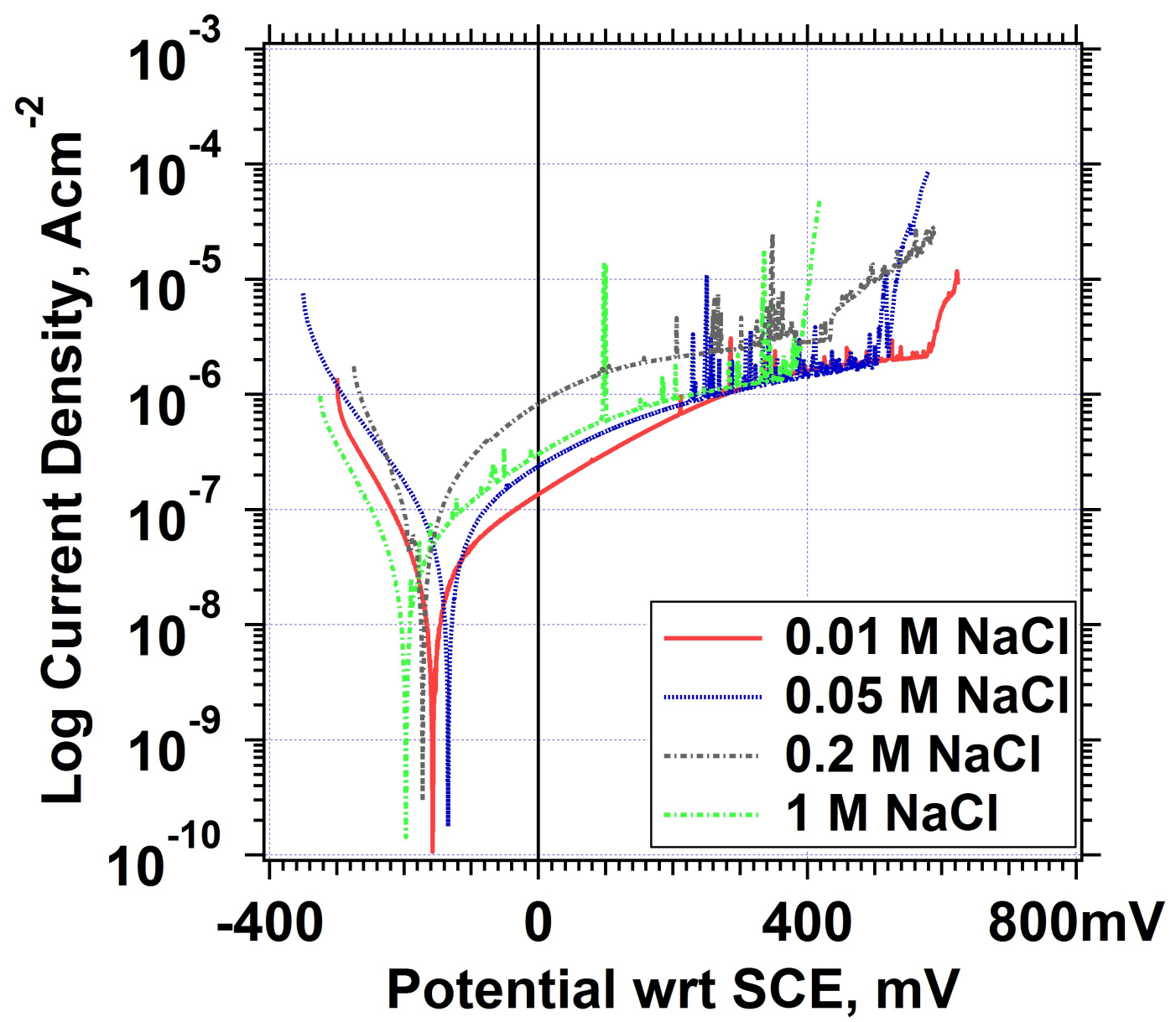

Figure 3.2: Comparison of typical potentiodynamic curves for unsensitised SS in a range of chloride ion concentrations.

The relationship between $C l^{-}$anion, $E_{C o r r}$, and $E_{b}$ as shown previously in figure 3.2 can be further investigated by the use of a log-linear graph. This is shown in figure 3.3. Using the logarithm of $\left[\mathrm{Cl}^{-}\right]$, results can be plotted with the same order of magnitude with respect to potential. As expected, the SS exhibits a negative $\mathrm{Cl}^{-}$dependence, i.e. as $\left[\mathrm{Cl}^{-}\right]$increases there is a resulting decrease in $E_{b}$. The $\left(E_{b}\right) \mathrm{Cl}^{-}$dependence is equal to the gradient, and for the unsensitised 


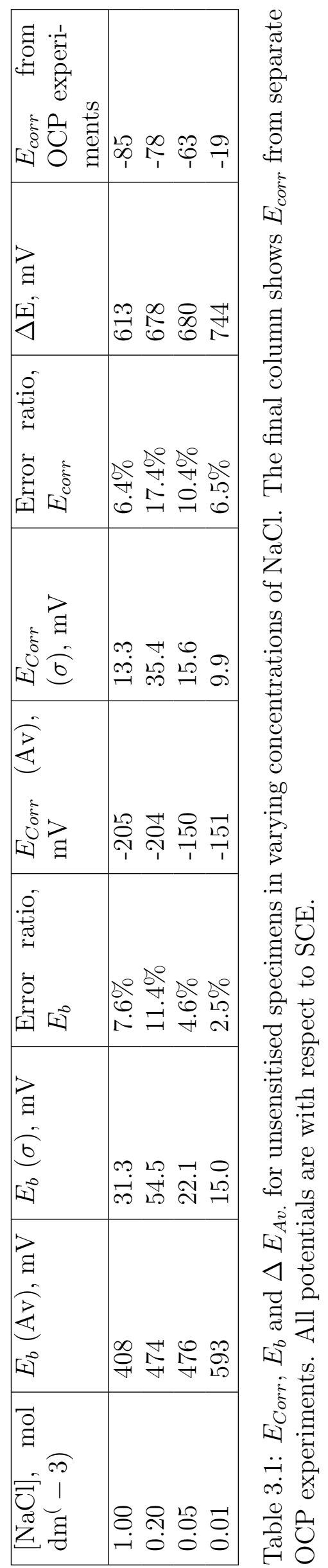


$20 / 25 / \mathrm{Nb}$ stainless steel is $-98 \mathrm{mVdecade^{-1 }}$.

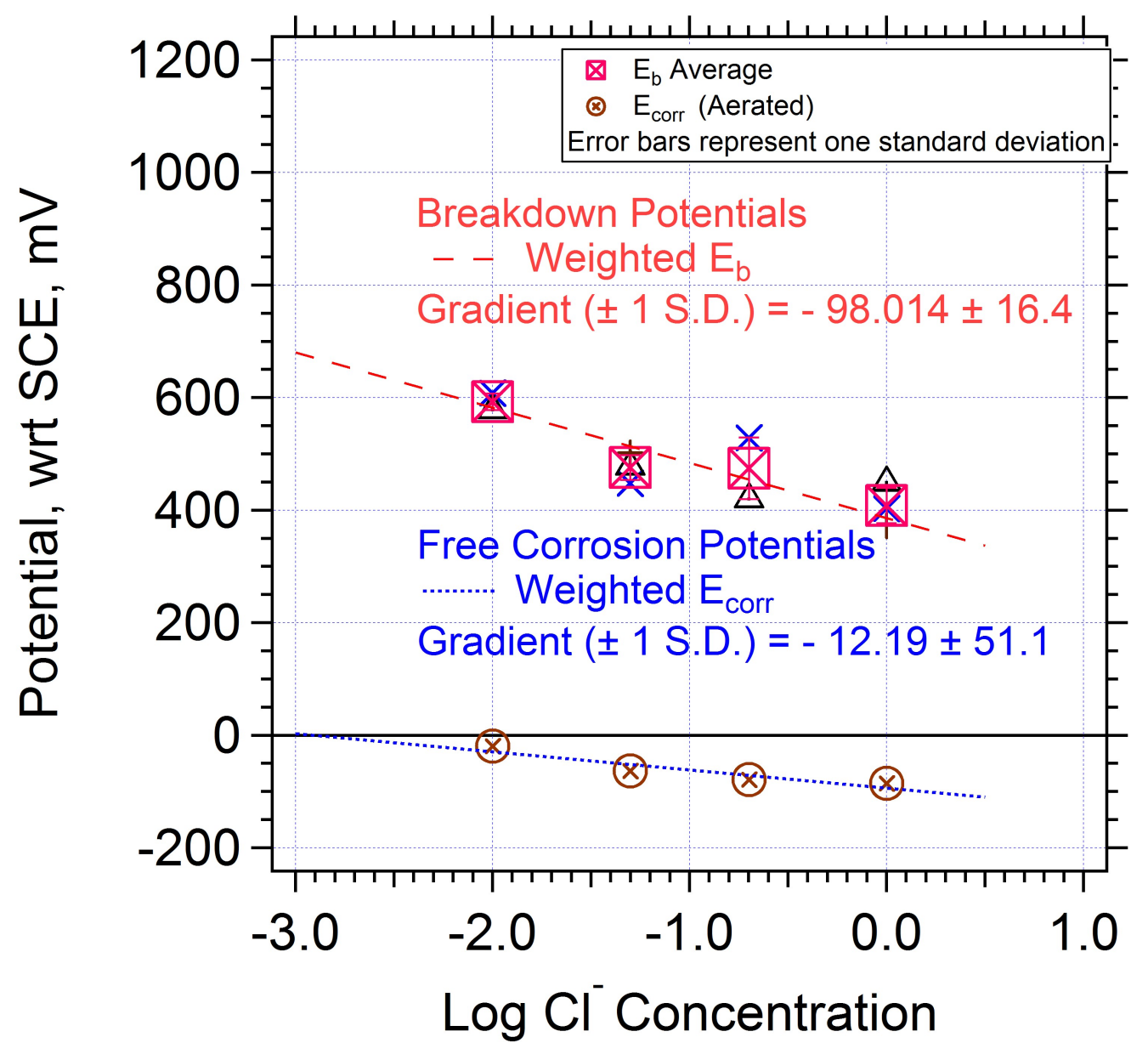

Figure 3.3: Graph showing the relationship between $\left[\mathrm{Cl}^{-}\right]$and potential. OCP values are from separate OCP experiments and are shown in table 3.1

A comparison may be made with the work by Abd El Meguid et al. [130] who studied type 904L and 316L stainless steels. 904L has a high Mo content of $4-5 \%$ (by mass), $\mathrm{Ni}$ and $\mathrm{Cr}$ contents of $23.5 \%$ and $20 \%$ respectively. 316L has Mo, Ni and $\mathrm{Cr}$ contents $2-3 \%, 9-8 \%$ and $17.5 \%$ respectfully. The authors quote a $\left[\mathrm{Cl}^{-}\right]$dependence of $-140 \mathrm{mVdecade} \mathrm{e}^{-1}$ for both materials, with the gradients for each running parallel although the $316 \mathrm{~L}$ is displaced by $-240 \mathrm{mV}$ relative to 904L. The results are shown in figure 3.4. These values are the same as those quoted by Williams et al. for turbine blade SS (-140 mVdecade $\left.{ }^{-1}\right)$ although these SS had Cr contents that were much lower (11.5 and 13.5 wt.\%) [12]. This figure is comparable to AISI $304 \mathrm{SS}(\mathrm{Fe}-18 \mathrm{Cr}-8 \mathrm{Ni})$ in $\mathrm{NaCl}$ at $-150 \mathrm{mVdecade}$ (between 20 and $80^{\circ} \mathrm{C}$ ) [63] [131]. For contrast, Fe metal $E_{b}\left[\mathrm{Cl}^{-}\right]$dependence is 
-59 mV decade ${ }^{-1}$. Laycock [132] analysed $302 \mathrm{SS}$ in $\mathrm{NaCl}$, similar in composition to 316 SS but without the $2.5 \%$ Mo addition which was found to increase $E_{b}$ by $+100 \mathrm{mV}$ relative to $302 \mathrm{SS}$ in all $\left[\mathrm{Cl}^{-}\right]$. The $302 \mathrm{SS}$ showed a $\left[\mathrm{Cl}^{-}\right]$dependence for $E_{b}$ of $-93 \mathrm{mVdecade}{ }^{-1}$ when prepared to a 120 grit surface finish. A higher quality surface finish (1200 grit) resulted in an increase to $-100 \mathrm{mVdecade}{ }^{-1}$. The gradient calculated here $\left(-98 m V\right.$ decade $\left.{ }^{-1}\right)$ is in line with results from Laycock [132] for 302 SS. Interestingly, the dependence results for low Cr SS studied by Williams [12] are similar to 304 SS [63] and 316 SS [130], whilst the 20/25/Nb SS has a similar dependence to 302 SS [132]. Future research may look to measure the protection potential $E_{\text {prot }}$. Abd El Meguid et al. emphasise that the pitting performance of a material is not solely dependent on $E_{\text {pit }}$, but also the $E_{\text {prot }}$, the point in which a reverse polarisation curve intersects a forward polarisation curve.

\subsubsection{Temperature Dependence}

As table 3.2 shows, $E_{b}$ is proportional to electrolyte temperature, as expected. In fact, the total change in potential arising from a $10{ }^{\circ} \mathrm{C}$ increase $\left(20-30{ }^{\circ} \mathrm{C}\right)$ is substantial enough to produce a $100 \mathrm{mV}$ decrease in $E_{b}$. Increasing this temperature to $40{ }^{\circ} \mathrm{C}$ yields a further $150 \mathrm{mV}$ change in potential.

\begin{tabular}{|l|l|l|l|l|l|l|l|}
\hline$T,{ }^{\circ} C$ & $\begin{array}{l}E_{b}(\mathrm{Av}), \\
\mathrm{mV}\end{array}$ & $\begin{array}{l}E_{b}(\sigma), \\
\mathrm{mV}\end{array}$ & $\begin{array}{l}\text { Error } \\
\text { ratio, } \\
E_{b}\end{array}$ & $\begin{array}{l}E_{\text {Corr }} \\
(\mathrm{Av}), \mathrm{mV}\end{array}$ & $\begin{array}{l}E_{\text {Corr }}(\sigma) \\
\mathrm{mV}\end{array}$ & $\begin{array}{l}\text { Error } \\
\text { ratio, } \\
E_{\text {corr }}\end{array}$ & $\begin{array}{l}\Delta \\
\mathrm{mV}\end{array}$ \\
\hline 20 & 412.5 & 37.2 & 9.0 & -204.4 & 10.9 & 5.3 & 616.9 \\
30 & 407.7 & 77.9 & 19.1 & -98.2 & 53.6 & 54.5 & 505.9 \\
40 & 245.1 & 13.3 & 5.4 & -118.7 & 17.8 & 14.9 & 363.8 \\
50 & 223.8 & 15.2 & 6.7 & -128.3 & 9 & 7.0 & 352.1 \\
60 & 178.9 & 9.2 & 5.1 & -131.7 & 20 & 15.1 & 310.6 \\
80 & 108.4 & 23 & 21.2 & -115.1 & 8.6 & 7.4 & 223.5 \\
\hline
\end{tabular}

Table 3.2: Table showing free corrosion and breakdown potentials for unsensitised specimens at a fixed $\left[\mathrm{Cl}^{-}\right]$, with varying $\mathrm{T}$. All potentials are with respect to SCE; and error values quoted represent one standard deviation from the mean.

The graph in figure 3.5 shows that $E_{b}$ decreases linearly with increasing temperature. Whilst this decrease is in accordance with previous research by Abd 


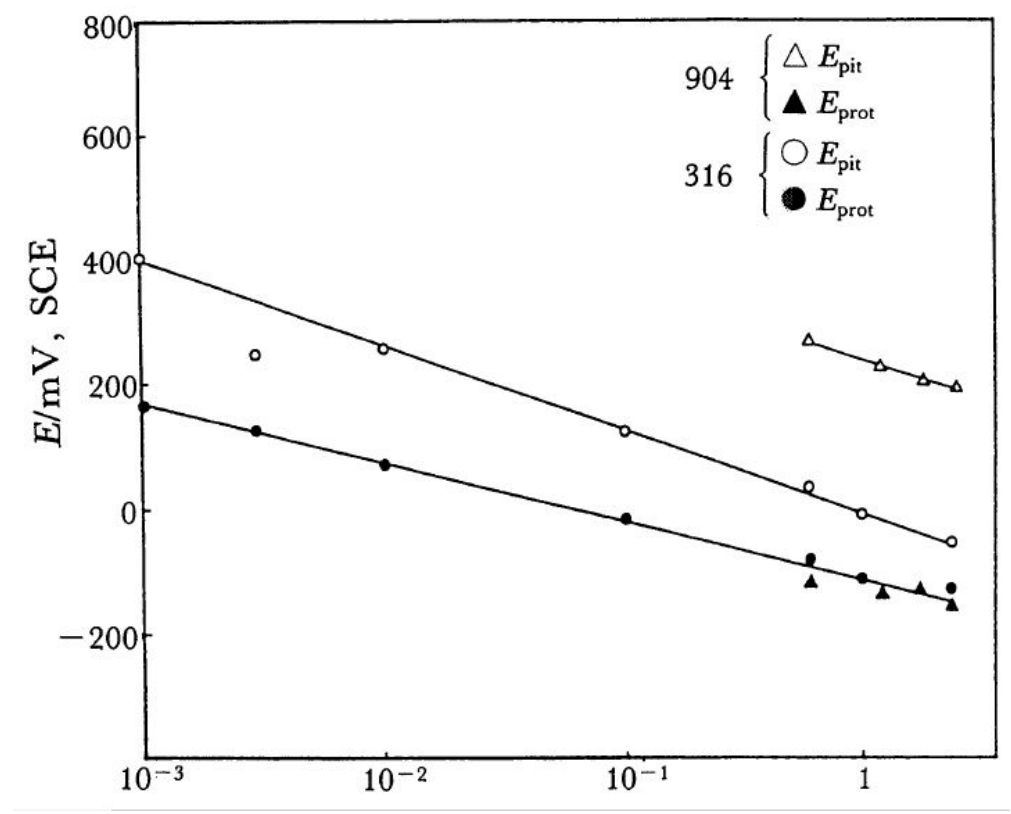

Figure 3.4: $\left[\mathrm{Cl}^{-}\right]$dependence at $60{ }^{\circ} \mathrm{C}$ at $\mathrm{pH} 5.8$ for $316 \mathrm{~L}$ and $904 \mathrm{~L}$ stainless steels [130]

El Meguid et al. [130] in the results shown here the dependence is linear, however for the results by Abd El Meguid et al. a parabolic dependence exists as shown in figure 3.6. These experiments are different however: type 904L and 316L stainless steels were used, alongside a lower $\left[\mathrm{Cl}^{-}\right]$concentration.

The temperature-potential graph shows that the SS exhibits a linear dependence on electrolyte temperature with a slope of $-7.3 \mathrm{mVK} \mathrm{K}^{-1}$. For example, a modest increase of $10{ }^{\circ} \mathrm{C}$ results in $E_{b}$ reducing by $70 \mathrm{mV}$, which could promote the SS to a the region of metastable pitting. A comparison of the potentiodynamic experiments is shown in figure 3.7. In this figure the curves have been shifted by the temperature correction (given in brackets) in the legend. Unexpectedly the $20{ }^{\circ} \mathrm{C}$ and $30{ }^{\circ} \mathrm{C}$ tests show more short lived current transients than at the higher temperatures. The sharp increase in current density, classic behaviour with more aggressive environments, is shown even in the increase from $20{ }^{\circ} \mathrm{C}$ and $30^{\circ} \mathrm{C}$. 


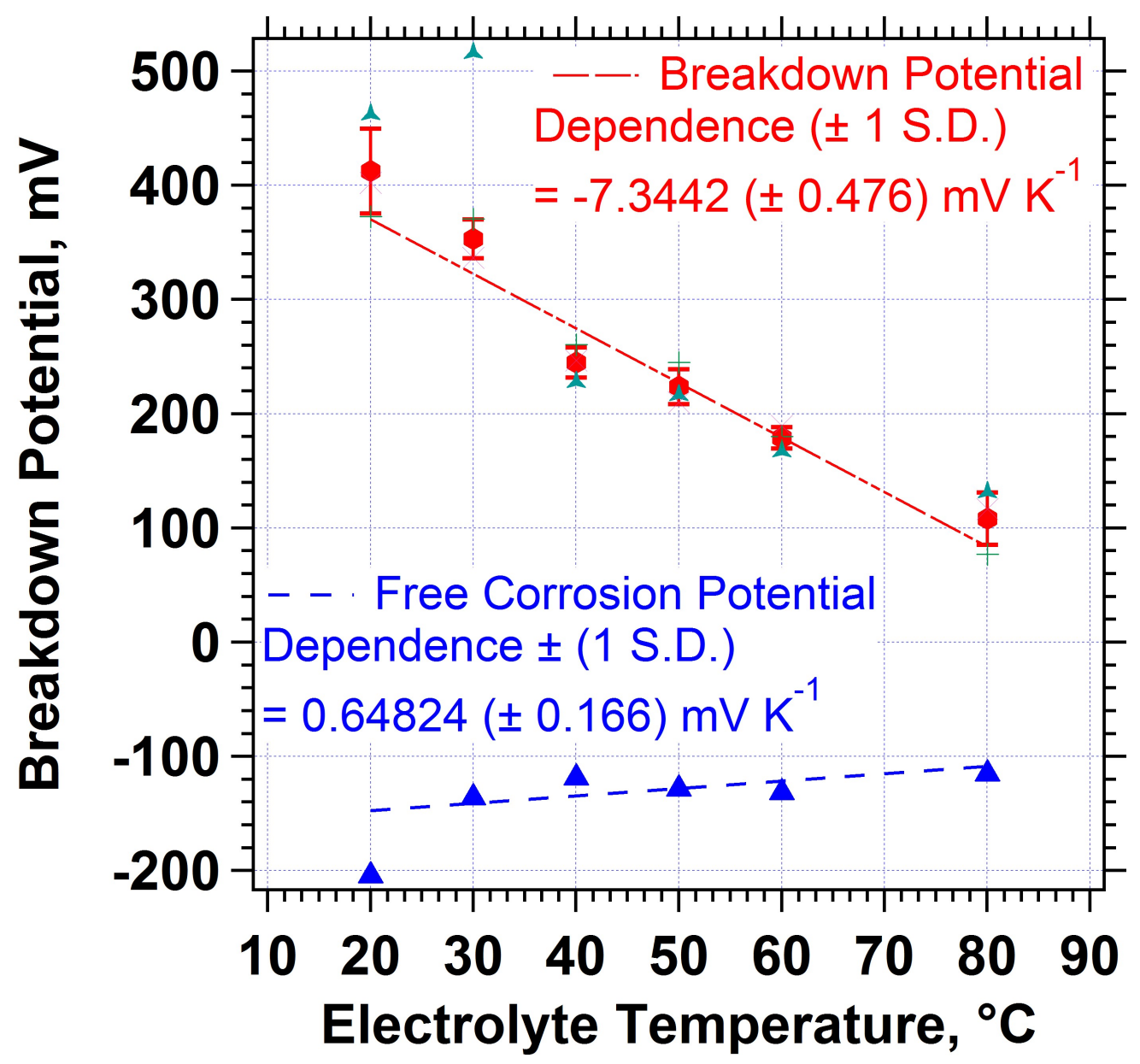

Figure 3.5: Temperature dependence of $E_{b}\left(-7.3 \mathrm{mVK} \mathrm{K}^{-1}\right)$ and $E_{\text {corr }}$ $\left(0.65 \mathrm{mVK}^{-1}\right)$ observed in fixed, $1 \mathrm{~mol} \mathrm{dm}^{-3} \mathrm{NaCl}$ electrolyte at a nominal $\mathrm{pH}$ of 7. 


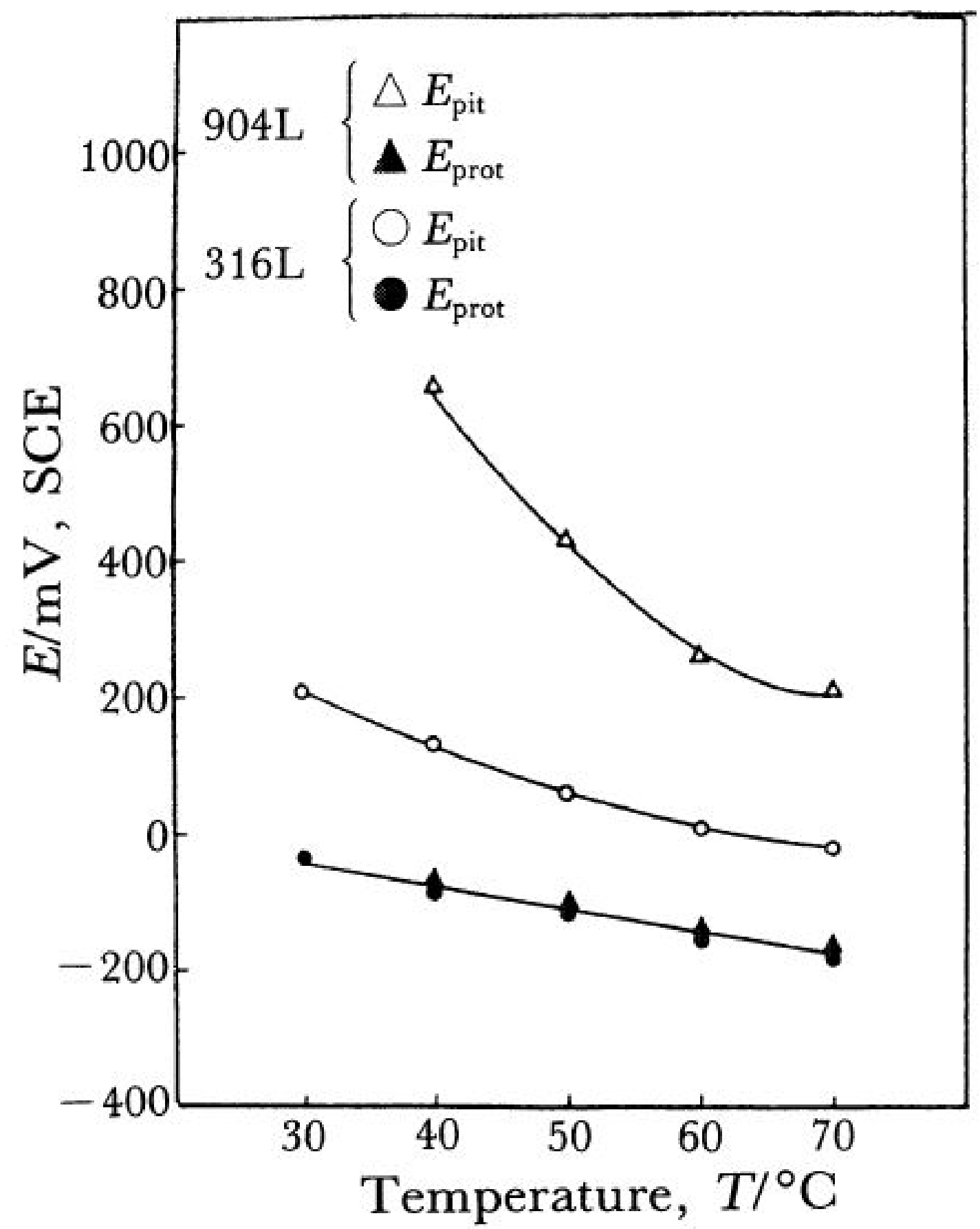

Figure 3.6: Temperature dependence of 316L and 904L stainless steels in $0.6 \mathrm{~mol}$ $d m^{-3} \mathrm{NaCl}[130]$ 


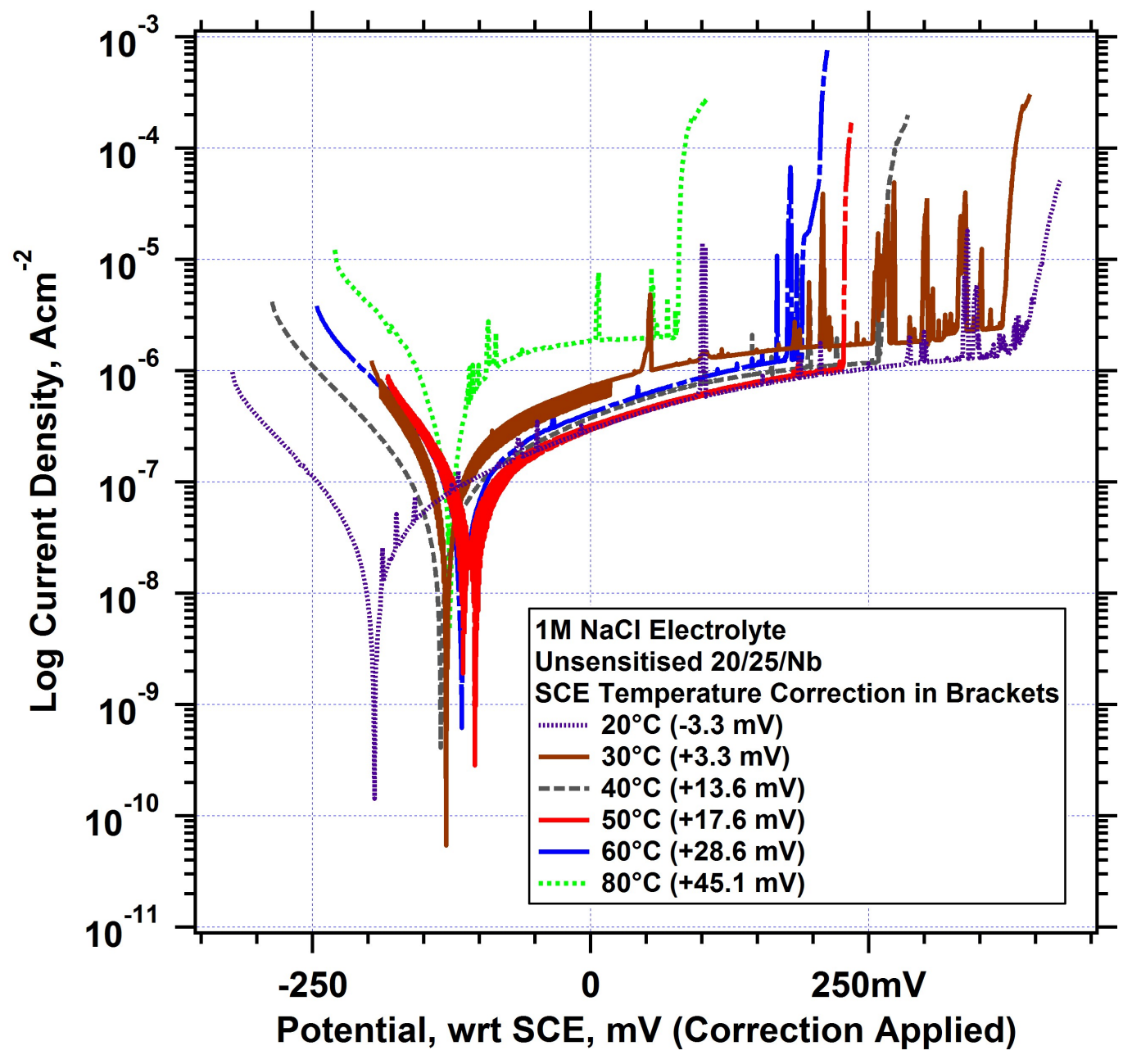

Figure 3.7: Comparison of typical potentiodynamic curves for unsensitised SS at a fixed chloride ion concentrations $\left(1 \mathrm{~mol} d m^{-3} \mathrm{NaCl}\right)$ and varying electrolyte temperature. 


\subsubsection{SVET Crevice Corrosion}

The unsensitised SS was subjected to immersion in $1 \mathrm{~mol} d m^{-3} \mathrm{NaCl}$ and polarised to $375 \mathrm{mV}$ (SCE). Previous experiments using SVET on the unsensitised 20/25/Nb SS showed little activity, even at high concentrations (1 mol $\mathrm{dm}^{-3}$ $\mathrm{NaCl}$ ). Therefore the earlier results using the three electrode cell (previously displayed in section 3.3.1) were used to assist in the study of localised corrosion using SVET. The specimen was immersed in the highest concentration electrolyte used previously and a potentiostatic potential of $375 \mathrm{mV}$ vs. SCE was applied (10s of $\mathrm{mV}$ away from its breakdown potential). The false colour maps show the anodic activity in red (positive current), little activity in white $(\approx 0)$, and cathodes as blue regions (negative currents). The intensity of the red and blue colours are directly proportional to the magnitude of current density. The lack of cathodic current density is caused as the net cathode is situated externaly (Pt gauze electrode) as a three electrode cell was used. As the graphics show in figure 3.8, the surface contour maps show a change in colour from largely white to red within less than 24 hours. The peak current density at the anodic front (upper anode in images) is sustained at $2000 \mathrm{Am}^{-2}$ between hours 1 to 9 . By hour 10 peak current density increases to $2500 \mathrm{Am}^{-2}$ and subsequently by hour 15 the peak is reduced to $1000 \mathrm{Am}^{-2}$. 

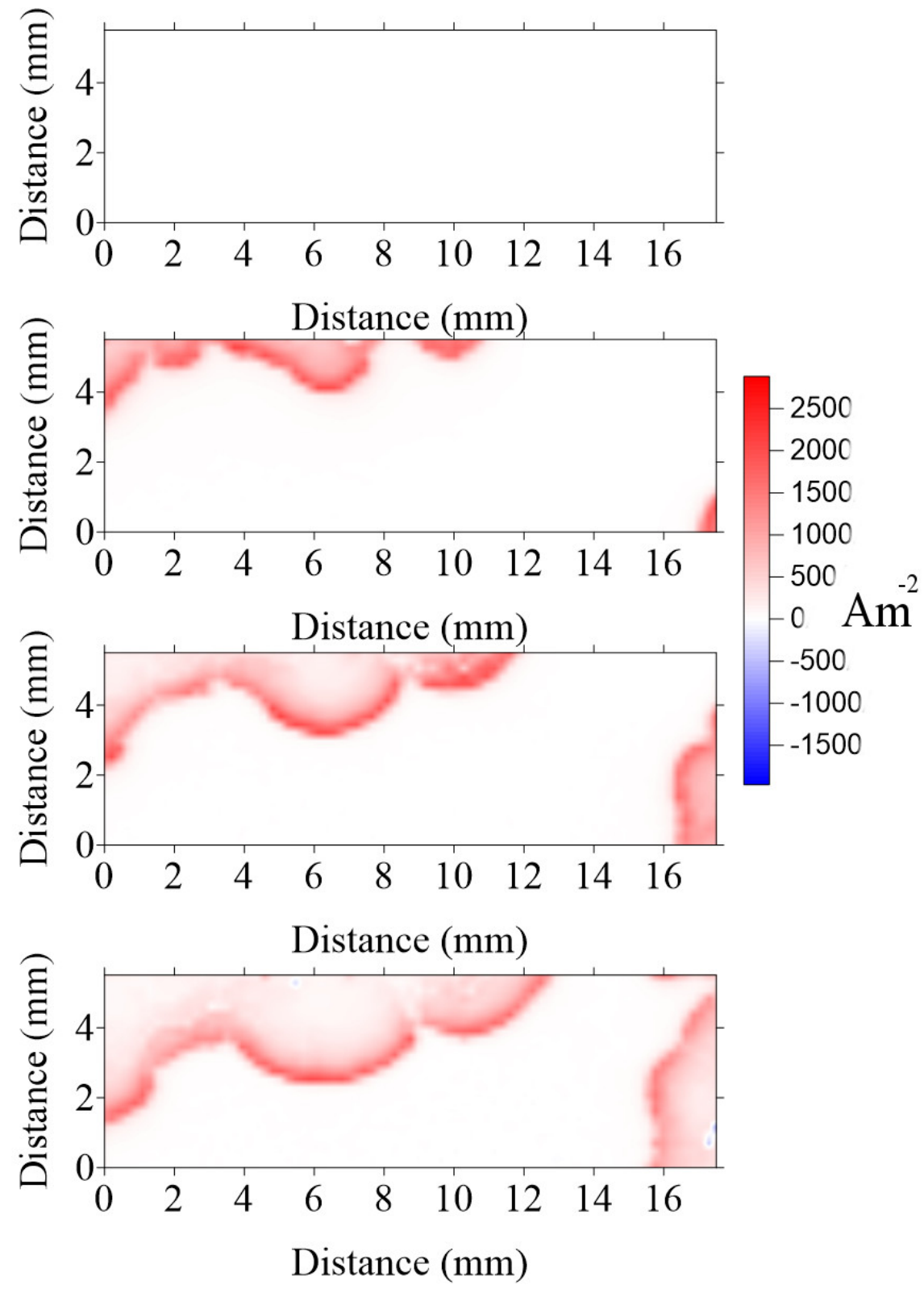

Figure 3.8: SVET scans obtained after 0, 3, 6, 9 hours in $1 \mathrm{~mol} d m^{-3} \mathrm{NaCl}$ for unsensitised SS, polarised to $375 \mathrm{mV}$ (Vs. SCE). 

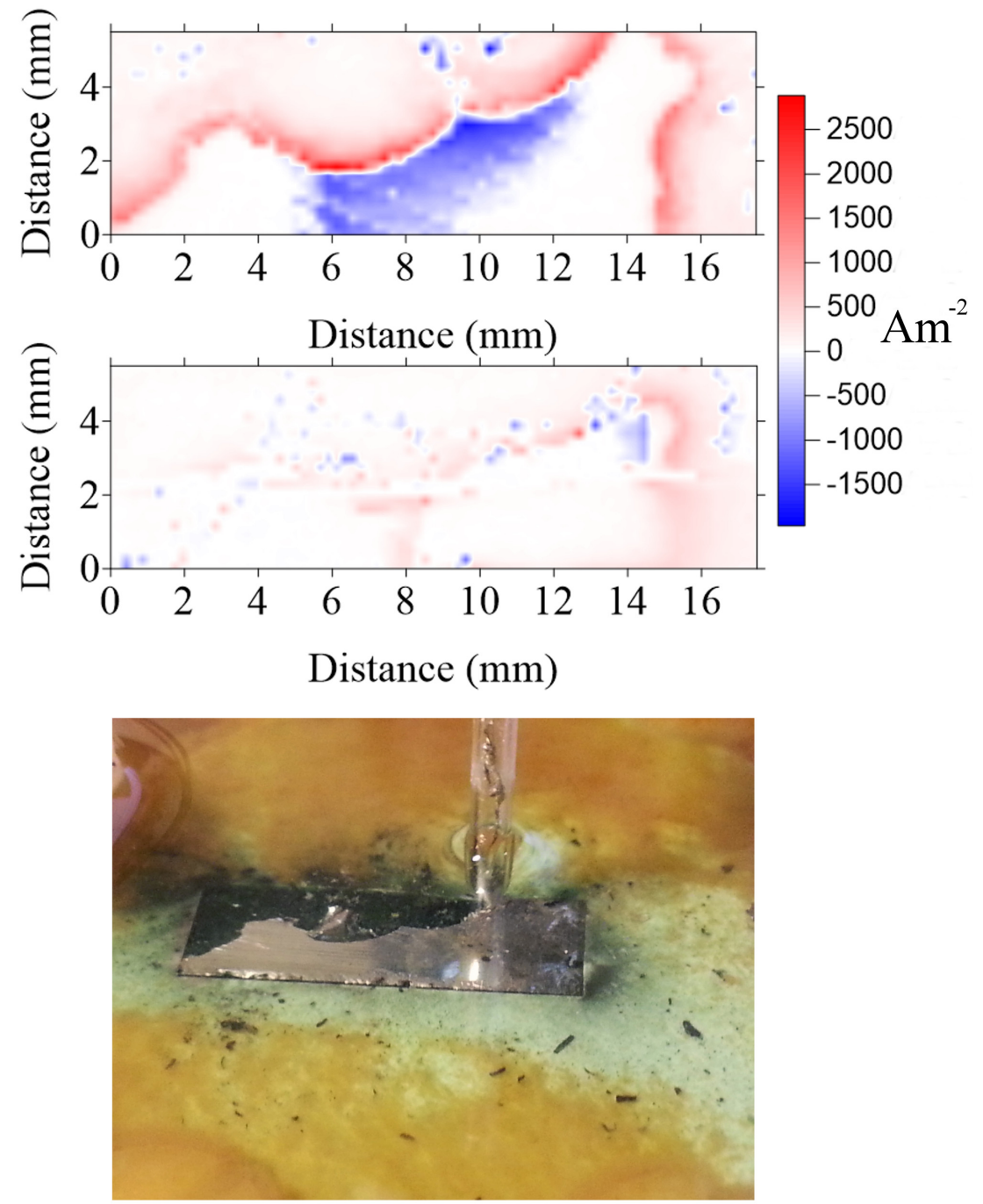

Figure 3.9: SVET scans at 11 and 14 hours in $1 \mathrm{~mol} d m^{-3} \mathrm{NaCl}$, polarised to 375 $\mathrm{mV}$. A photograph of the surface following 15 hours immersion is also included 
Materials that undergo pitting corrosion have large current density values focussed at the pit opening, with the larger free surface acting as a large cathode. In this experiment however a three electrode cell was used and so the net cathode is located at the counter electrode. The localised corrosion patterns under external polarisation do not resemble either crevice corrosion or pitting. For the former, the anode would be expected to remain stationary and active at the SSresin interface (i.e. around the scan area edges). The latter would give highly focal local anodes, which would either remain active or initiate and passivate, but would not necessarily traverse the surface. Anodes appear within 3 hours (top left and lower right), propagating along the surface at a rate of c. $30 \mu m h r^{-1}$ (100 $\mu \mathrm{m}$ between 3 hours, each division on graph is $1 \mathrm{~mm}$ ). The scans show that the corrosion initiated from a crevice, formed at the resin-specimen interface indicating the susceptibility of the material to crevice corrosion. However the system shown here shows an anodic front attacking a previously uncorroded region leaving behind a darkened surface, this is atypical behaviour and does not represent IGC, thus this study was discontinued. By the time of hour 15 the surface changed dramatically. The SVET current density map in figure 3.9 is a result of the specimen having undergone large amounts of corrosion, such that large amounts of the SS fragmented into solution. This impacts the SVET in two ways: SVET tip to specimen distance would have increased, causing anodecathode current loops to be less visible. Furthermore, the physical impact of fragments on the tip itself may have given false readings.

\subsubsection{Green and Blue Cladding}

In this section specially prepared custom specimens which represented a trial of deposition resistant fuel was assessed. The custom specimens (unsensitised SS) had an artificially thick chromium oxide which resulted in the colour change from transparent to green and blue due to different vapour concentrations in a furnace. The SS was prepared with these oxides to prevent the deposition of 
carbon explained at the start of the chapter (section 3.1), and the introduction of carbon acting as a cathode in the previous section 3.4.5. Temperatures of the target and actual range of dew points used to artificially grow these oxides can be found in the experimental section ( 3.2$)$. The specimens were immersed in $1 \mathrm{~mol} \mathrm{dm}{ }^{-3} \mathrm{NaCl}$ and subjected to potentiodynamic polarisation. The plot in figure 3.10 shows potentiodynamic curves for green oxide specimens, with an untreated specimen is also shown for reference. The first test specimen showed several pitting events in the transpassive region of the scan $(-170$ to $400 \mathrm{mV}$ vs. $\mathrm{SCE}$ ). The largest event lasted for a region of $40 \mathrm{mV}$; the equivalent of $40 \mathrm{~s}$, given the $1 \mathrm{mV} \mathrm{s}^{-1}$ scan rate. The difference in $E_{b}$ for test specimens 1 and 2 is c. $100 \mathrm{mV}$ vs. SCE. The two 'green' oxide specimens potentiodynamic curves sit between the control specimen. Specimen 1 shows a lower current density throughout the experiment and a larger change in potential $\left(E_{b}-E_{c o r r}\right)$ although large transients were present indicating pitting corrosion initiation. This is in contrast to specimen 2 which showed a higher current density throughout the experiment when compared to both specimen 1 and the control specimen, but with a lower number of transients. The current density exhibited by specimen 2 is almost an order of magnitude greater the standard oxide. As with specimen 1 the total change in potential for specimen 2 was larger than the control specimen. The plots indicate, from the more positive $E_{c o r r}$, that deactivation of the anodic dissolution occurred in the control specimen. This is in contrast to the two 'green' oxide specimens indicating that the surface changed due to pitting corrosion.

The 'blue' oxide specimens all showed notably higher current densities throughout the anodic sweep; which were in some instances three orders of magnitude greater than the control specimen. The first and second specimen exhibited transpassive behaviour; with no sharp increase in current. Whilst this might indicate that the specimens either did not undergo pitting corrosion, the current throughout the sweep was much greater. When compared to the green specimens the hysteresis loops are much smaller, but do not repassivate until the potential is 


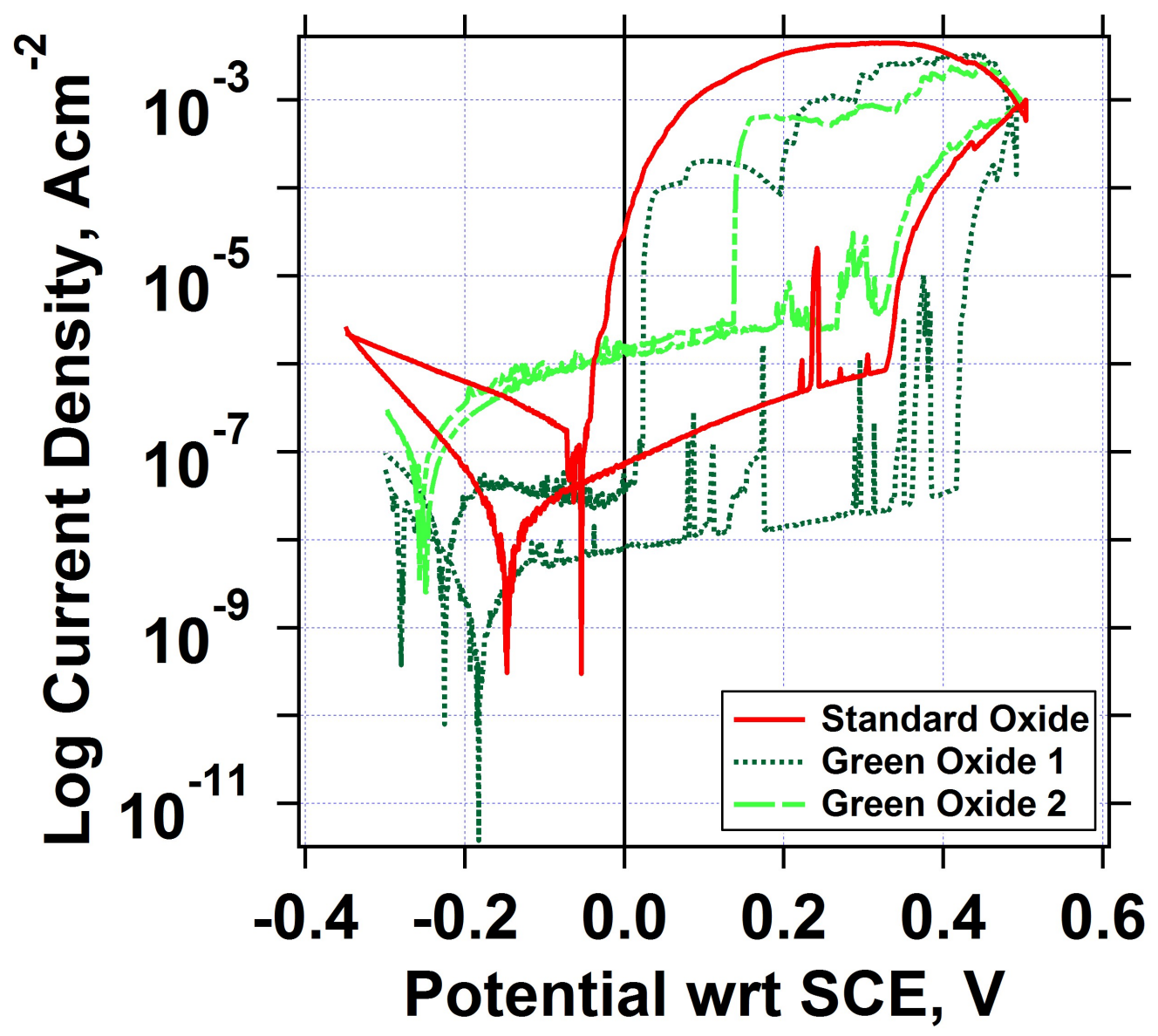

Figure 3.10: 'Green' oxide unsensitised SS compared to the unsensitised control specimen in $1 \mathrm{~mol} \mathrm{dm}^{-3} \mathrm{NaCl}$ 
close to $E_{\text {corr }}$ within $\approx 150 \mathrm{mV}$. Though sharp breakdown potentials were not observed, the passive current density is a greater for these specimens with a thinner oxide (blue) when compared to both the thicker (green), and control specimens, suggesting corrosion could be occurring throughout the anodic sweep.

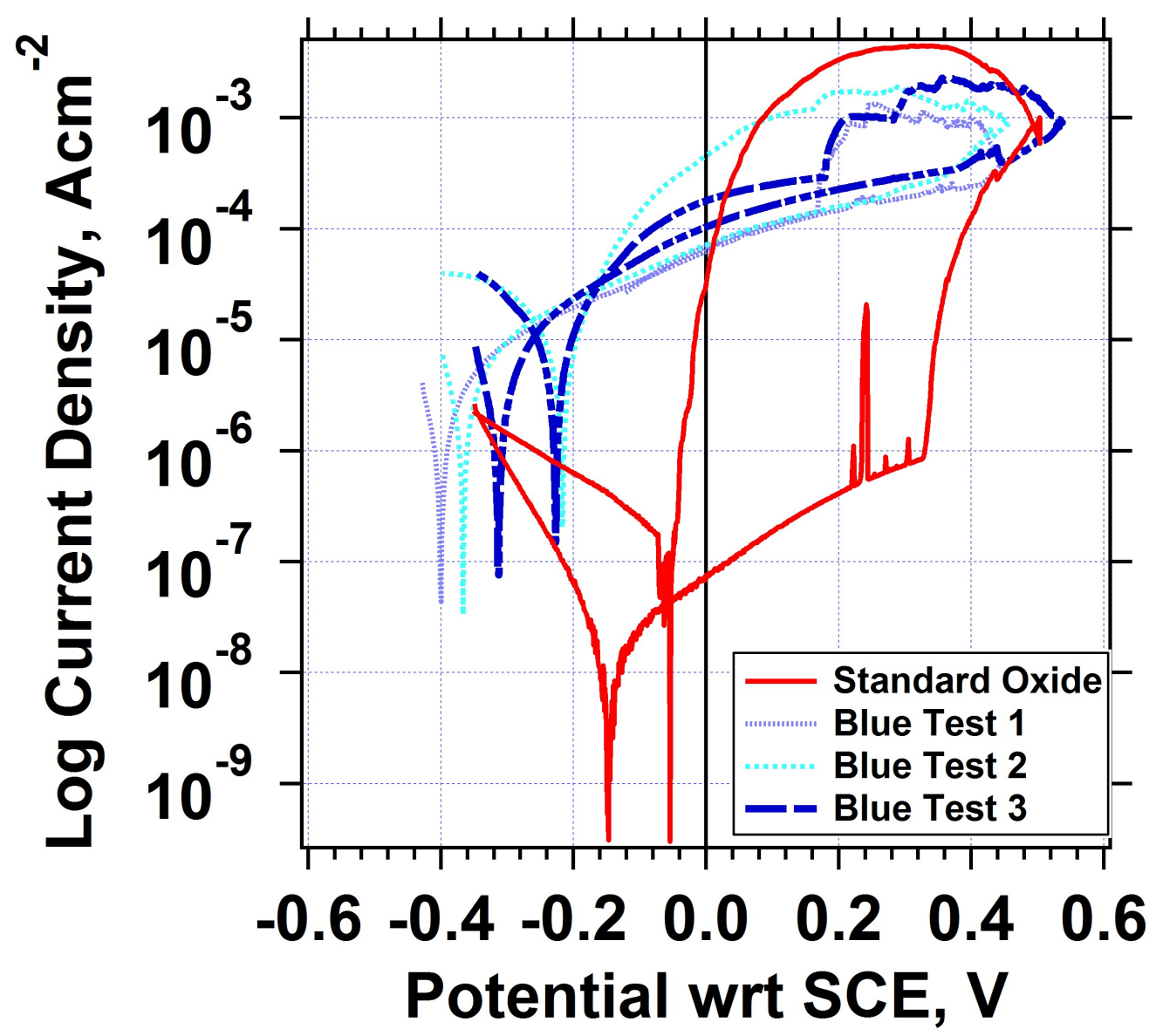

Figure 3.11: 'Blue' oxide unsensitised SS compared to the unsensitised control specimen in $1 \mathrm{~mol} \mathrm{dm}^{-3} \mathrm{NaCl}$

An assessment of two trial samples produced using a new process to decrease the effects of carbon deposition have been studied. The impact of these new production methods was investigated to measure the impact on their corrosion characteristics. Neither of the two specimens showed promising results when compared to the untreated standard oxide SS. Both types of treated specimens showed increased current densities; with the blue oxide specimens showing current densities up to three magnitudes greater. The green specimens showed a number of metastable events; whereas the blue oxide specimens exhibited transpassive 
behaviour. These treated specimens show the inverse of the expected, given that the oxides are thicker than the standard specimen - such that a colour change of the cladding is witnessed. A thicker film might act in a similar manner to a coating, providing a physical barrier to the underlying metal, however; the results suggest otherwise; with higher currents being measured throughout.

Another possible issue with the use of these new processes is that there is no current operational experience in-reactor. As such, the impact of RIS is not known for these materials. There is the possibility that the SS residing directly under the film becomes depleted of Cr during the treatment stage. This might lead to further challenges in the coming years if the underlying metal has a lower than normal $\mathrm{Cr}$ content where RIS may cause further depletion of $\mathrm{Cr}$ at areas adjacent to the GBs. This in effect has the ability therefore to render the SS in a more sensitised state than the untreated material, given that variables such as dose rate are constant.

\subsection{Thermally Sensitised Stainless Steel}

\subsubsection{Degree of Sensitisation}

Table 3.3 shows that current (and charge) are proportional to polish depth. The current and charge ratios are quantifiers used for degree of sensitisation (DOS). This suggests that sensitisation does not appear to be uniform throughout the specimen. This seems difficult to understand as at the temperatures used for the sensitisation treatment, heating should be homogeneous. At the high temperature used for the solution anneal $\left(1150{ }^{\circ} \mathrm{C}, 30 \mathrm{mins}\right)$ it would be expected that a large quantity of $\mathrm{NbC}$ inclusions would have dissolved, increasing free carbon within the matrix. Given that the ageing step is a two week period at $650{ }^{\circ} \mathrm{C}$ it would be expected that the entire specimen would be at temperature, leading to a uniform DOS. However, the results do not match the expectation. Reviewing the literature on the double-loop electropotentiokinetic reactivation (DL-EPR) 
experiments published by Chan on $20 / 25 / \mathrm{Nb}$ show that the as-received, stabilised and thermally sensitised microstructures had a Ir/Ip ratio of $0.022,0.48$ and 2.32 [45]. Chan's 20/25/Nb specimens were solution annealed at $1300{ }^{\circ} \mathrm{C}$ for 30 minutes and subsequently aged at $550{ }^{\circ} \mathrm{C}$ for 1000 hours to produce a sensitised microstructure. The stabilised microstructure used the same solution anneal time and temperature but the second step differed and was held at $930{ }^{\circ} \mathrm{C}$ for 30 minutes. The results by Chan indicate that the sensitised microstructure in this research is more representative of the stabilised microstructure although the heat treatment temperatures and duration differ.

\begin{tabular}{|l|l|l|l|l|l|l|}
\hline $\begin{array}{l}\text { Cumu- } \\
\text {-lative } \\
\text { Polish } \\
\begin{array}{l}\text { Depth } \\
(\mu \mathrm{m})\end{array}\end{array}$ & $\begin{array}{l}\text { Anodic } \\
\text { Charge, } \\
(\mathrm{C})\end{array}$ & $\begin{array}{l}\text { Rp* } \\
\text { Charge } \\
(\mathrm{C})\end{array}$ & $\begin{array}{l}\text { Max } \\
\text { Anodic } \\
\text { Current } \\
(\mathrm{mA})\end{array}$ & $\begin{array}{l}\text { Max } \\
\text { Rp.* } \\
\text { Current } \\
(\mathrm{mA})\end{array}$ & Qr/Qp & Ir/Ip \\
\hline $\begin{array}{l}\text { Unpolished } 179.2 \\
22\end{array}$ & 247.5 & 66.3 & 117 & 56 & 0.37 & 0.48 \\
69.2 & 298.4 & 54.48 & 141.1 & 55.2 & 0.2 & 0.39 \\
\hline
\end{tabular}

Table 3.3: Degree of Sensitisation - the DL-EPR test $\left({ }^{*} \mathrm{Rp}\right.$ - repassivation)

The surface was inspected after DL-EPR test as per the ISO specification. The surface appeared roughened (figure 3.12), this was confirmed by optical microscopy (figure 3.13 and 3.14). Therefore, it was decided that the grain area measurement would be omitted due to the difficulty in making measurements. The test solution and parameters were followed, as per the ISO specification.

As the DOS varied through the gauge of the material, a method of evaluating the corrosion behaviour of the sensitised material needed to be obtained. Cross sections of the sensitised SS were used to estimate the thickness of the dark scale (haematite) using SEM and was determined to have an approximate thickness of 6-9 $\mu \mathrm{m}$ (figure 3.21), as explained in section 3.4.3. Therefore, if the cut square specimen was mounted at a slight angle from the horizontal plane, different areas of the surface would have more material removed from the grinding stage. Figure 3.15 shows the process used to prepare the sensitised SS surfaces so that a range of DOS could be evaluated. Figure 3.16 shows an example of the offset 


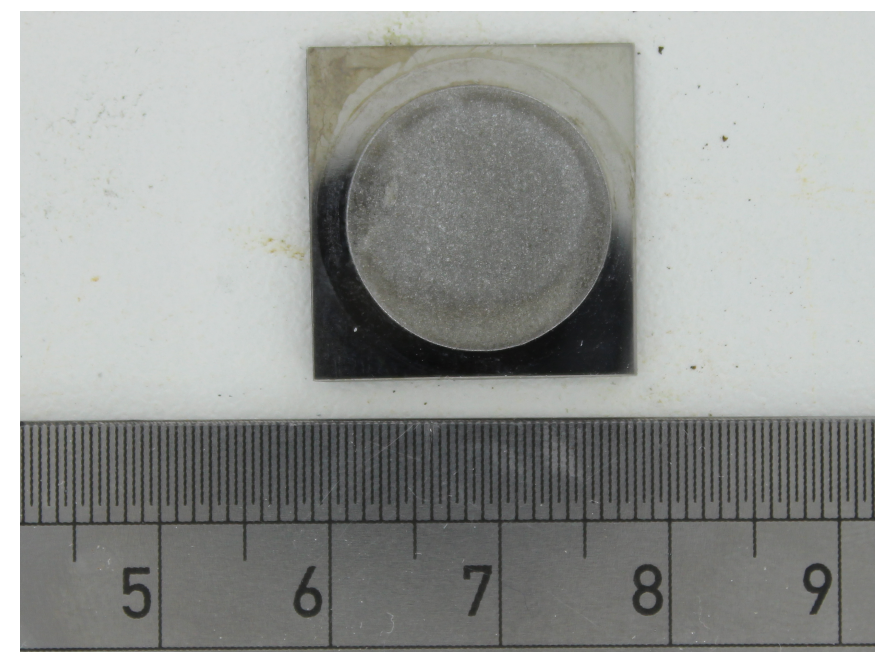

Figure 3.12: Surface of the sensitised 20/25/Nb specimen following the DL-EPR test

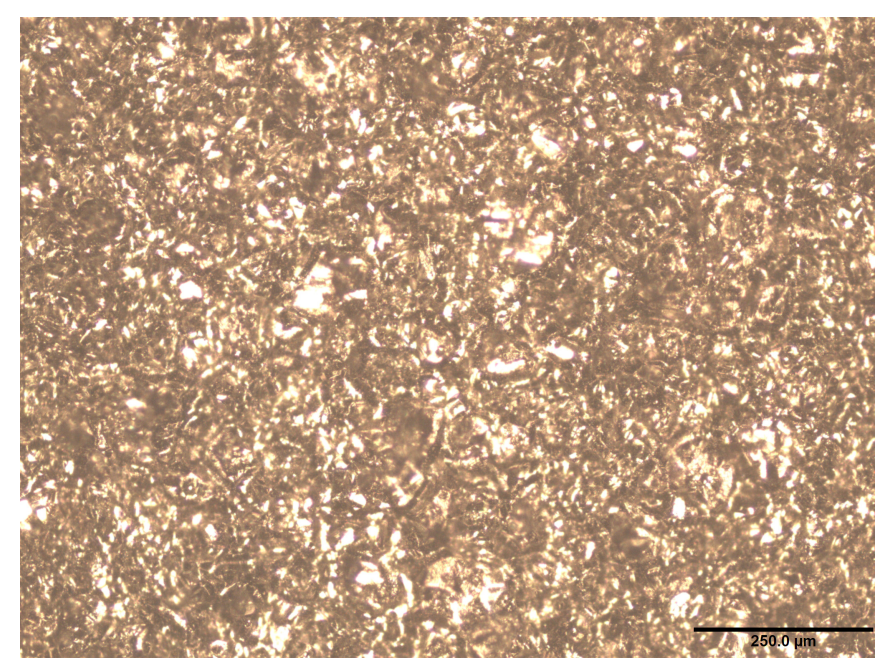

Figure 3.13: Micrograph of roughened surface following DL-EPR test, 10x

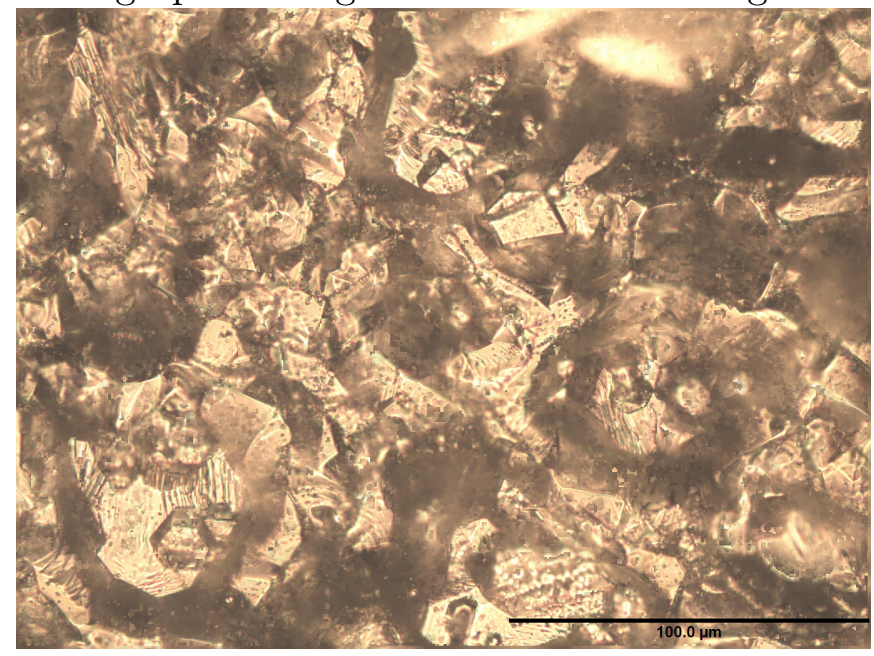

Figure 3.14: Micrograph of roughened surface following DL-EPR test, 50x 


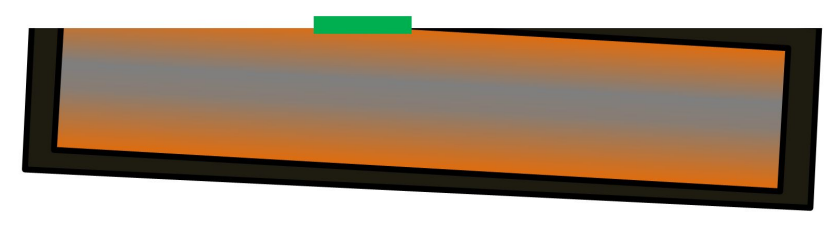

Figure 3.15: Offset mounted SS specimen schematic of the specimen cross section, where the dark box represents the oxide layer. The orange colouring represents highly sensitised grain boundaries relative to the grey area (less sensitised material). The green box is an area suitable for TLM or SVET investigation of a highly sensitised area adjacent to the oxide.

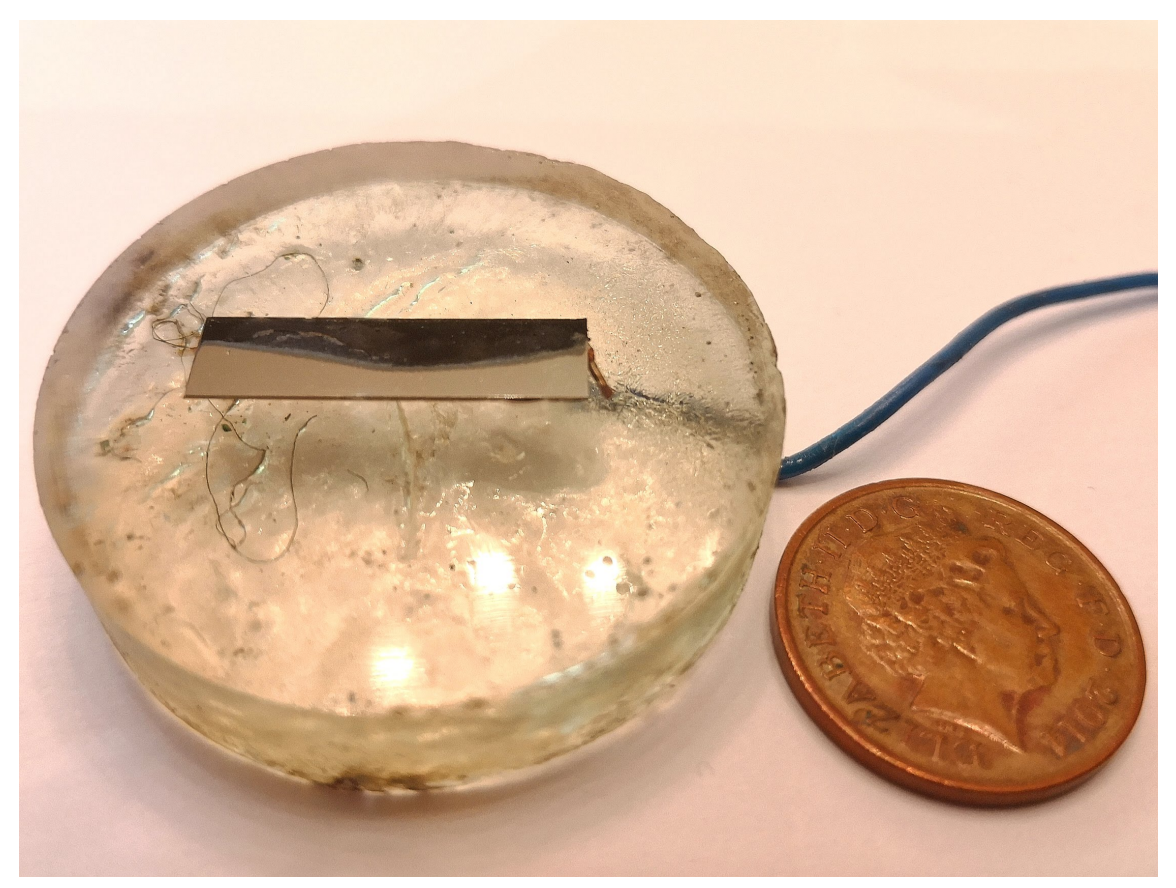

Figure 3.16: Offset mounted SS specimen in resin with the oxide layer clearly shown, the polished metal surface along with the interface between the two (the more sensitised material). 
preparation an actual specimen.

\subsubsection{Grain Size Calculation}

Table 3.4 displays the results of grain size calculations (ASTM E112-12 standard test methods for determining average grain size [128]). Both line and circle intercept procedures were used to calculate sizes at the recommended 100x magnification. Both methods work by counting the number of grain boundary intersections for a line of fixed length or a circle with a fixed diameter. The circular method has the benefit of automatically compensating for non-equiaxed grains within the microstructure. The grain sizes were calculated from polished specimens.

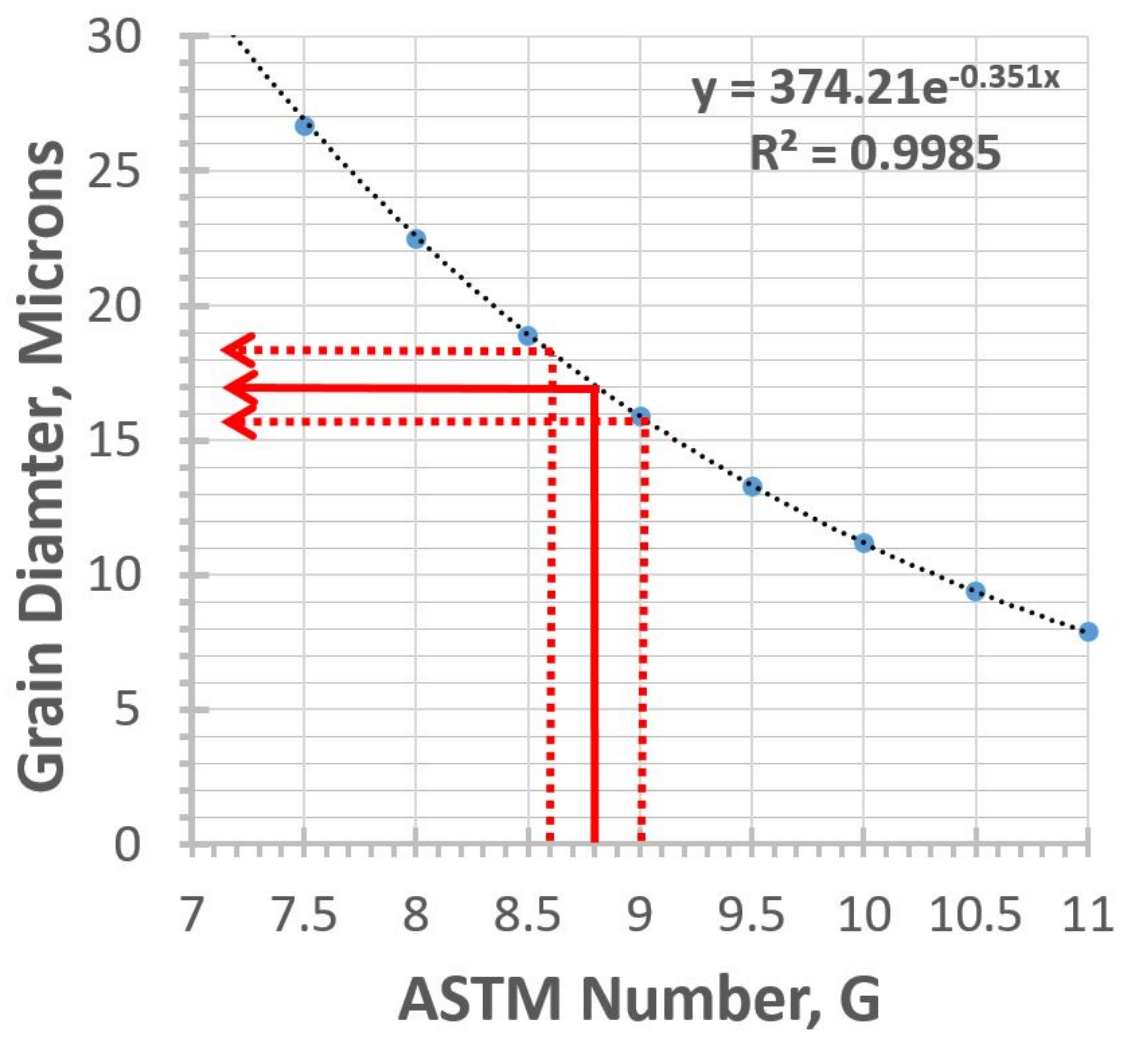

Figure 3.17: Grain size measurements for the as received microstructure - ASTM E112 data plotted [128]. Solid lines represent the average result, dotted lines indicate minimum and maximum sizes corresponding to one standard deviation.

The estimated grain sizes for the as received and sensitised microstructures are shown in figures 3.17 and 3.18 respectively. The ASTM grain size number, $\mathrm{G}$, is first obtained through either line or circular intercept procedures and then 


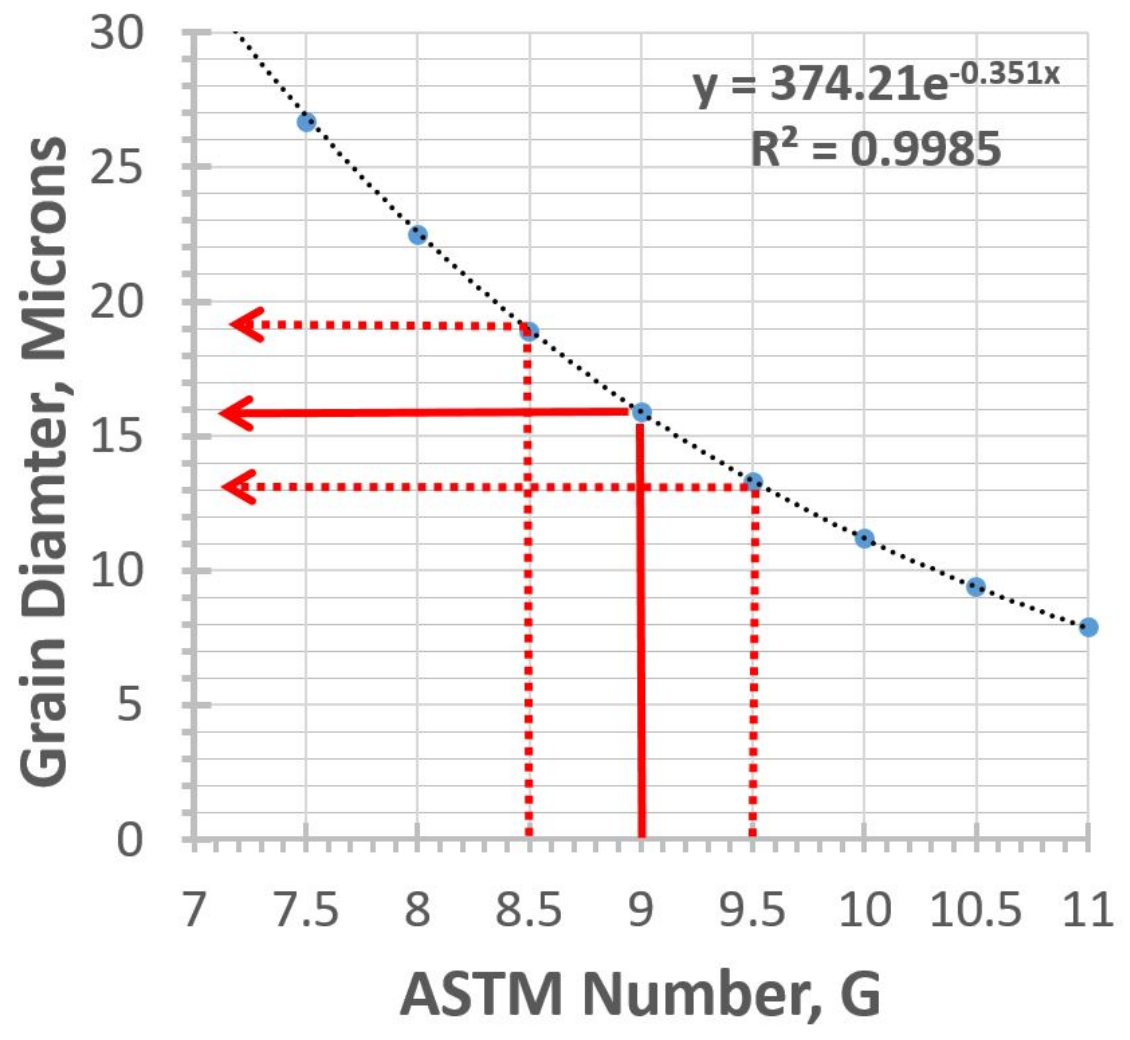

Figure 3.18: Grain size measurements for the sensitised microstructure. The solid lines represent the average result, dotted lines indicate minimum and maximum sizes corresponding to one standard deviation. 
cross referenced against grain diameters. The estimated average grain size is $17 \mu \mathrm{m}$ for the as-received microstructure, with minimum and maximum grain sizes of $16 \mu \mathrm{m}$ and $18.5 \mu \mathrm{m}$. The average values and one standard deviation for grain size measurement are displayed in table 3.5. The average grain size for the thermally sensitised microstructure is $16 \mu \mathrm{m}$, with a minimum and maximum of $13 \mu \mathrm{m}$ and $19 \mu \mathrm{m}$ respectively. The thermal sensitisation procedure does not appear to change the grain sizes; which wouldn't be expected at $600{ }^{\circ} \mathrm{C}$ unless the sample was cold worked. Phuah [51] reported larger grain sizes using electron microscopy for the as-received $20 / 25 / \mathrm{Nb}(25 \mu \mathrm{m})$ and also a smaller grain size with increasing ageing temperatures during thermal processing. Phuah measured an average grain size of $15 \mu \mathrm{m}$ for both $500{ }^{\circ} \mathrm{C}$ and $600{ }^{\circ} \mathrm{C}$, and $10 \mu \mathrm{m}$ at $800{ }^{\circ} \mathrm{C}$ (all 192 hours). Though both the methods of measuring grain size, and the duration of the heat treatment were different, the grain size in general was been shown to decrease with thermal processing.

\begin{tabular}{|l||l|l|l|l|}
\hline As received & Line & & & Circle \\
\hline Area & 1 & 2 & 3 & \\
\hline 1 & 8.6 & 7.7 & 9.9 & 8.9 \\
2 & 7.4 & 7.9 & 8.9 & 8.8 \\
3 & 9.1 & 9.4 & 9.3 & 9.3 \\
\hline Sensitised & Line & & & Circle \\
\hline Area & 1 & 2 & 3 & \\
\hline 1 & 8.4 & 10.5 & 7.6 & 9.6 \\
2 & 9.6 & 9.1 & 9.6 & 9.1 \\
3 & 8.8 & 9.4 & 8.8 & 8.3 \\
\hline Specimen & Average line & S.D. line & Average circle & S.D. circle \\
\hline As received & 8.78 & 0.77 & 9.00 & 0.22 \\
Sensitised & 9.09 & 0.78 & 9.00 & 0.54 \\
\hline
\end{tabular}

Table 3.4: Grain size measurement ASTM E112 for as received and sensitised SS specimens for line and circle intercept procedures. The numbers shown are ASTM grain size numbers 'G' and is unitless. 'S.D.' represents one standard deviation. 


\begin{tabular}{|l||l|l|l|}
\hline Specimen & $\begin{array}{l}\text { Average grain size } \\
\mu \mathrm{m}\end{array}$ & $\begin{array}{l}\text { Minimum } \\
\mu \mathrm{m}\end{array}$ & $\begin{array}{l}\text { Maximum } \\
\mu \mathrm{m}\end{array}$ \\
\hline Sensitised & 16 & 13 & 19 \\
As received & 17 & 16 & 18.5 \\
\hline
\end{tabular}

Table 3.5: Average grain sizes and respective minimum and maximums (one standard deviation) derived from grain size measurement using the circular intercept procedure. These were taken from the circular intersect results. The values are derived from ASTM E112 standard datasheet. 


\subsubsection{Analysis of Scale}

The appearance of the SS differs after the solution anneal has occurred, with the surface no longer having a metallic grey luster, but instead a dull dark, black appearance. This was analysed in order to investigate both the composition and thickness of the scale. Images of the surface before and after the anneal are shown in figures 3.19, 3.20.

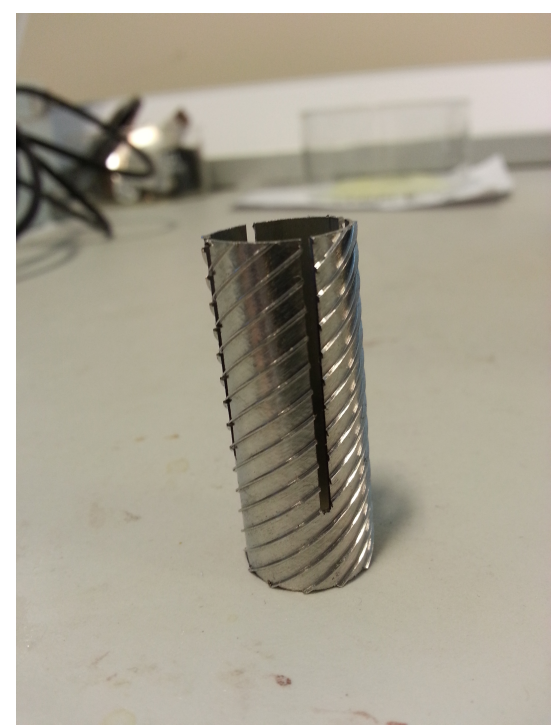

Figure 3.19: Tube before furnace treatment

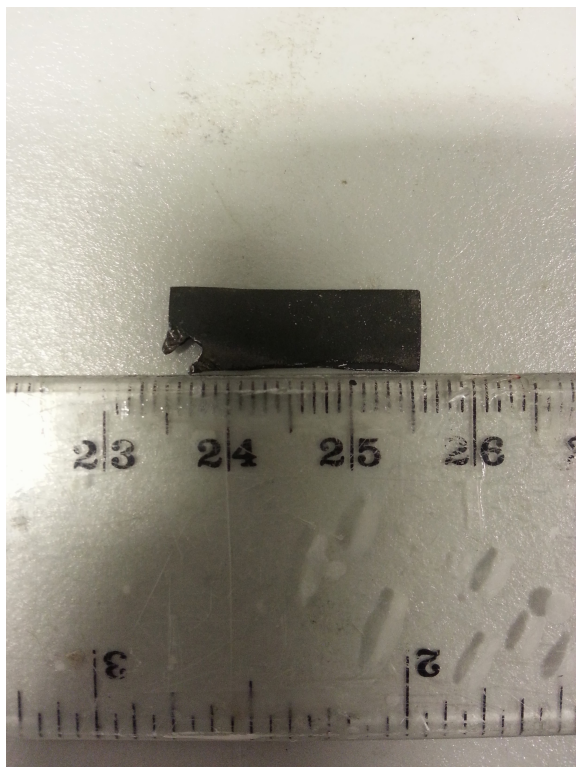

Figure 3.20: 20-25-Nb SS after solution anneal and ageing treatment

The thermally sensitised SS was cut into sections and mounted to form cross sections so that the thickness of the scale could be estimated by microscopy. 


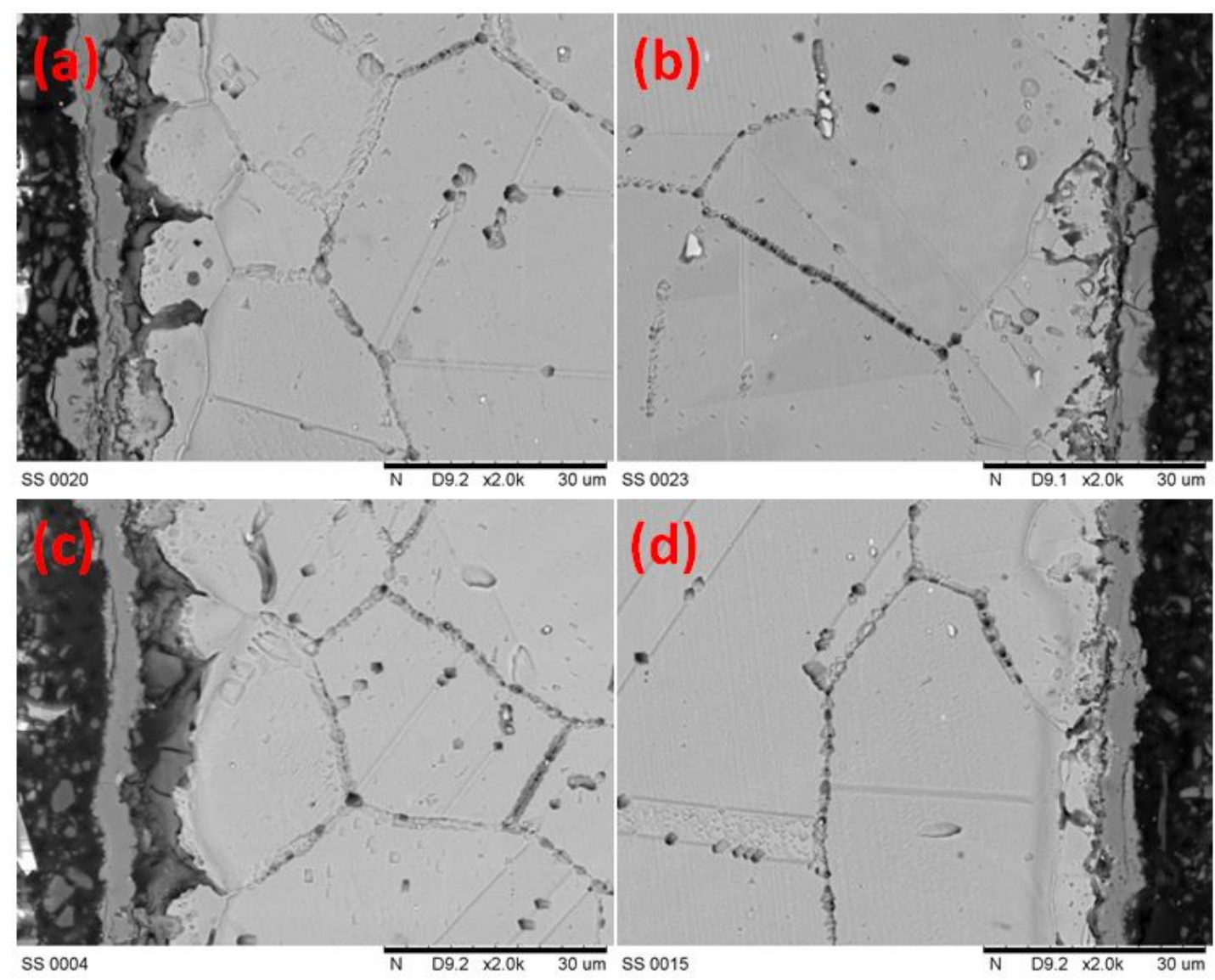

Figure 3.21: Thermally sensitised SS cross sections imaged using SEM. The micrographs show the thickness of the scale at different locations on the sample (a-d). 
The images from the cross section imaging experiment (figure 3.21) shows the thickness of the scale was approximately 6-9 $\mu \mathrm{m}$ for four locations on the sample (a-d). Therefore the scale is not a thin film, and is almost half the size of a grain (the grains were calculated to be $15-20 \mu \mathrm{m}$ ). The cross section shows from left to right the mounting resin, scale and grains of the stainless steel (figure 3.21a).

Analysis by X-ray diffraction the thermally sensitised SS showed peaks for iron $(\mathrm{Fe})$ and nickel $(\mathrm{Ni})$. Fe and $\mathrm{Ni}$ have the same face centred cubic (FCC) crystal structure, and their peaks are shown by the blue and red sticks on the diagram, which differ only in intensity. Interestingly, the data matches the peak listings for haematite, an iron oxide $\left(\mathrm{Fe}_{2} \mathrm{O}_{3}\right)$. The solution anneal was performed in an inert argon atmosphere, though after the initial treatment the surface no longer has a metallic luster. Most likely, oxidation, causing the formation of haematite, had occurred during the ageing treatment, which is performed in air where there would be a larger quantity of available oxygen.

The XRD spectra for the thermally sensitised SS (figure 3.23 and figure 3.24) shows that the peaks at $24^{\circ}, 33^{\circ}, 36^{\circ}, 41^{\circ}, 46^{\circ}, 51^{\circ}, 54^{\circ}, 63^{\circ}$ and $75^{\circ}$ all vary in size. The specimen shows that there is an apparent change in the diffraction of different crystal orientations with rotation. Crystallographic texture can also be seen when there is a large intensity single peak, where there are no reflections from other peaks. The sensitised SS has some form of texture, whether this may be from the work performed on the material during manufacture, or the consequence of cold rolling. The effect of texture may have been decreased by annealing during the manufacture, but also the sensitisation heat treatments that were undertaken. The cross sections for the thermally sensitised SS were analysed using EDS to identify the chemical composition of the base metal and scale, the results of which are shown in figure 3.26. The EDS false colour map shows clusters of niobium, which are the solid solution $\mathrm{NbC}$ inclusions. The corresponding SEM micrograph is shown annotated with regions of interest in figure 3.25. Away from these clusters an oxygen rich signal is apparent, which 

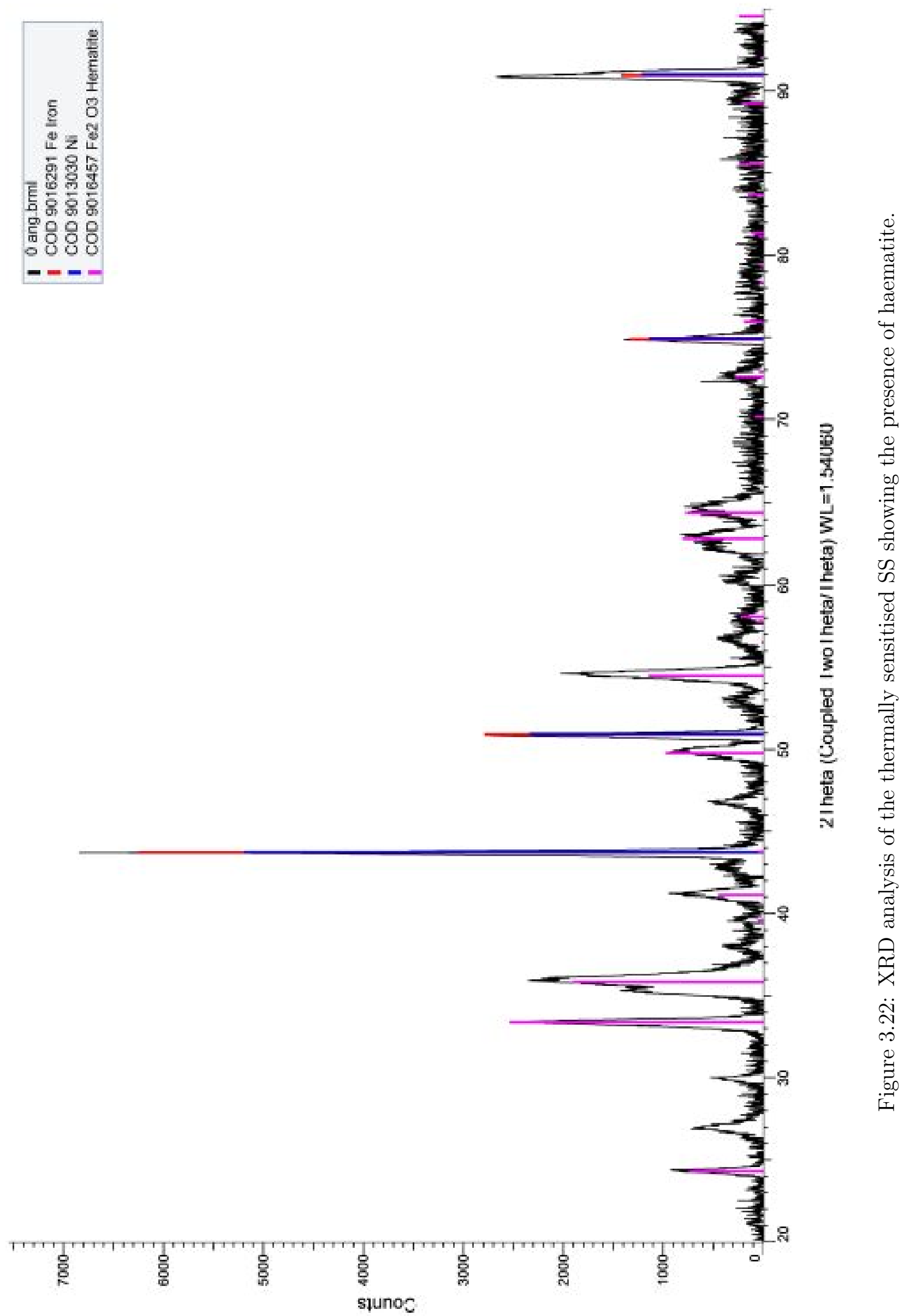

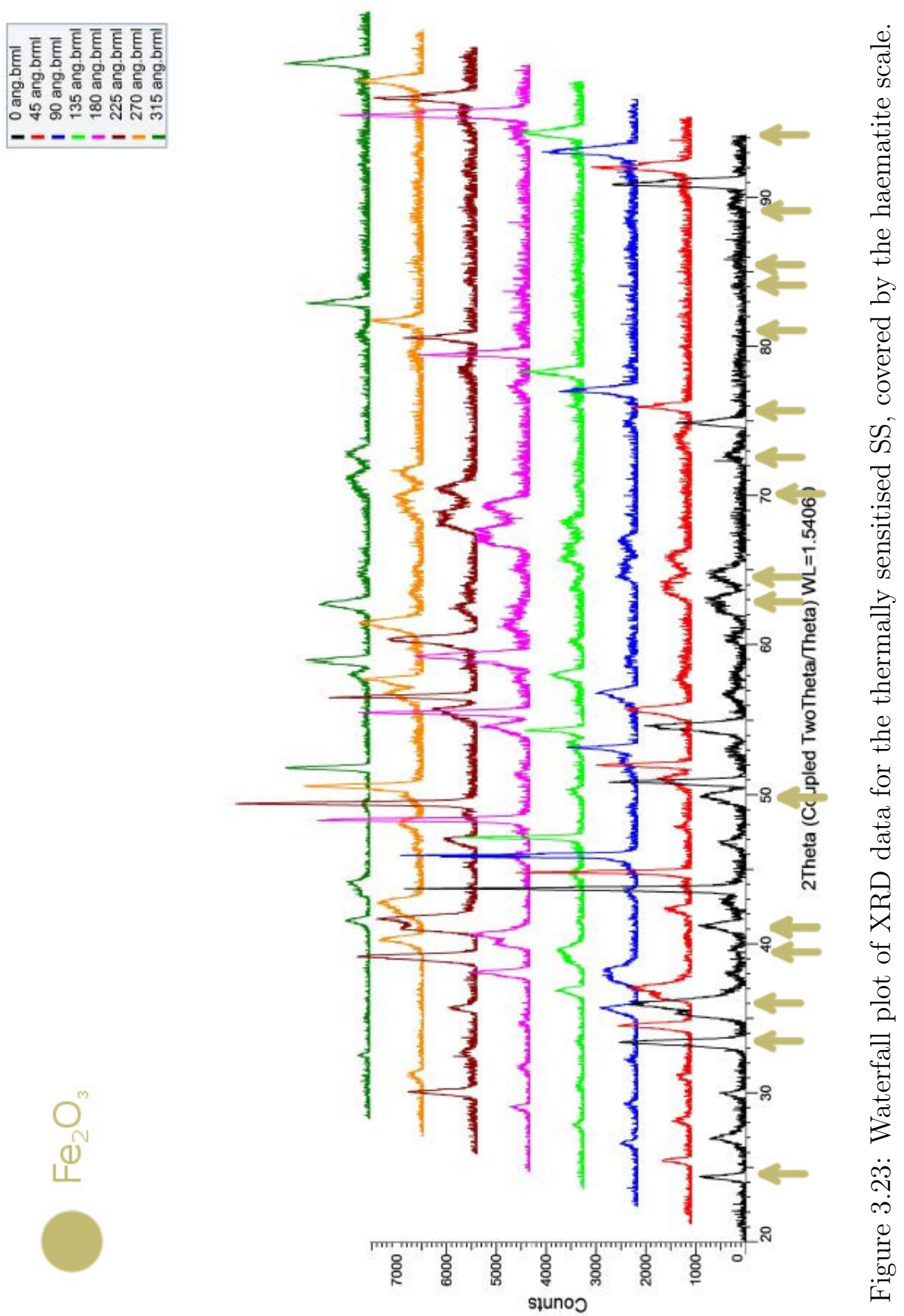

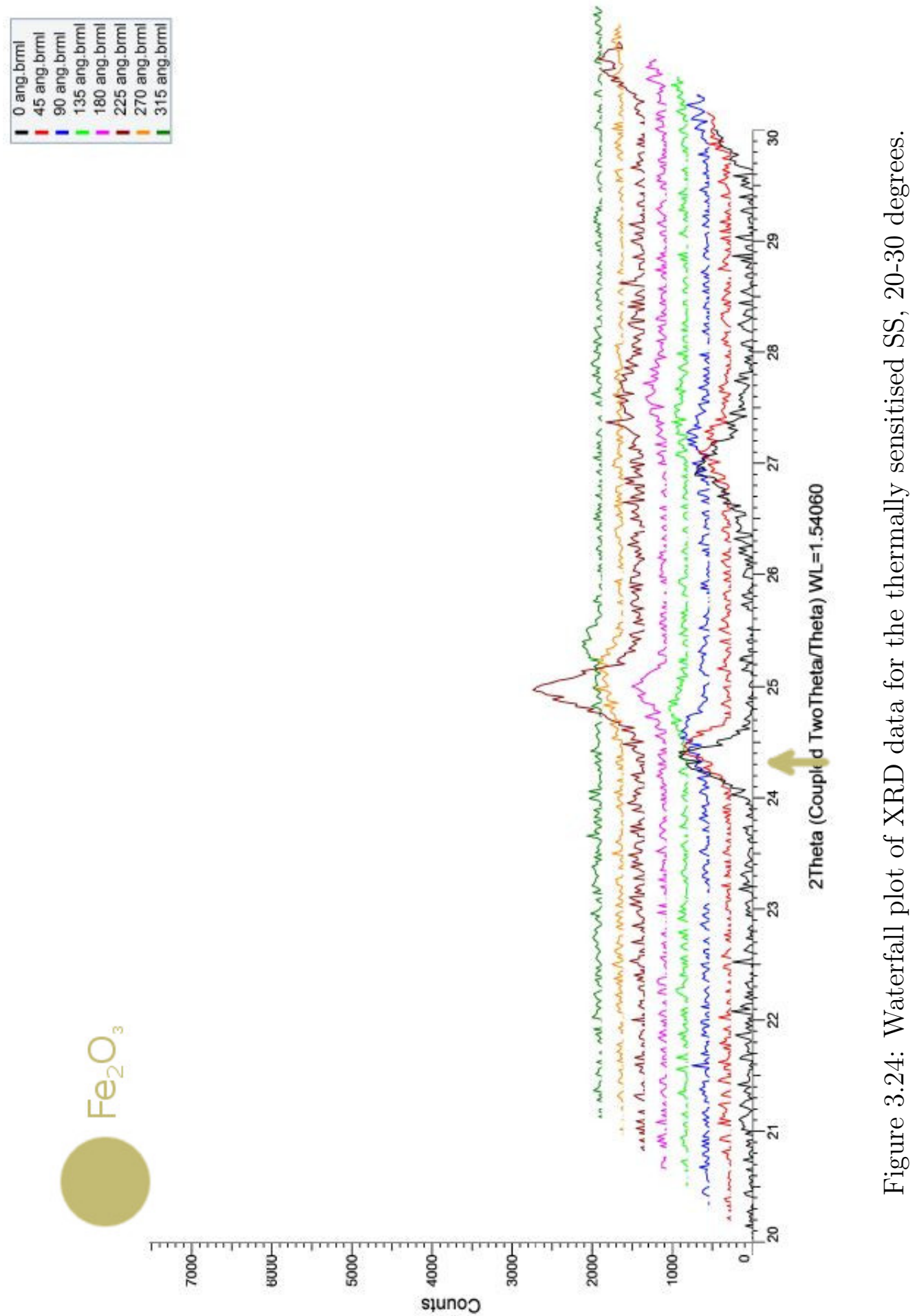
is in line with the previous results from XRD. The carbon signal is a result of X-rays from the cold mount resin.

An AFM image of the scale is shown in figure 3.27. The image shows the surface following surface preparation (grinding and polishing at an angle). The image clearly shows carbides which decorate the grain boundaries of the sensitised metal, without the need for an etchant. 


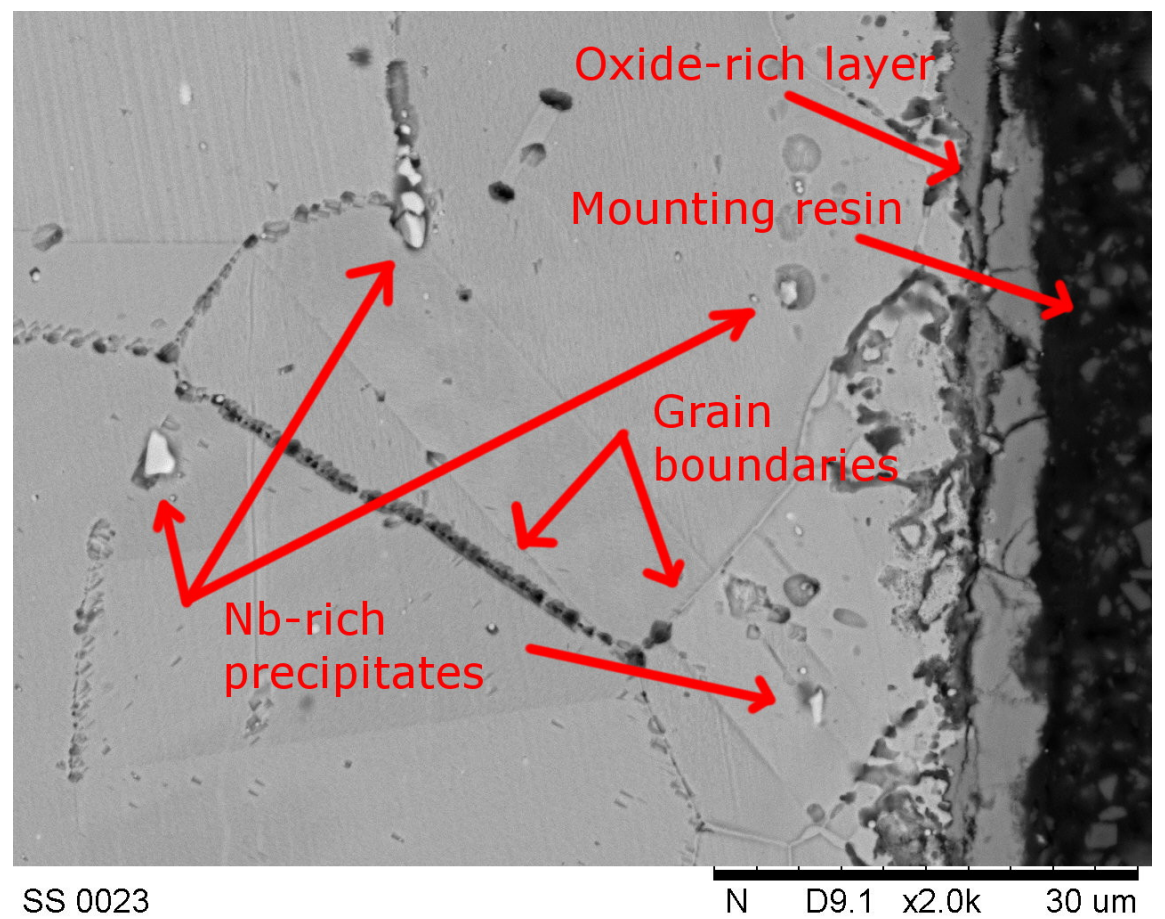

Sensitised

Figure 3.25: SEM image of thermally sensitised SS cross section showing areas of interest, corresponding to the EDS map in figure 3.21.

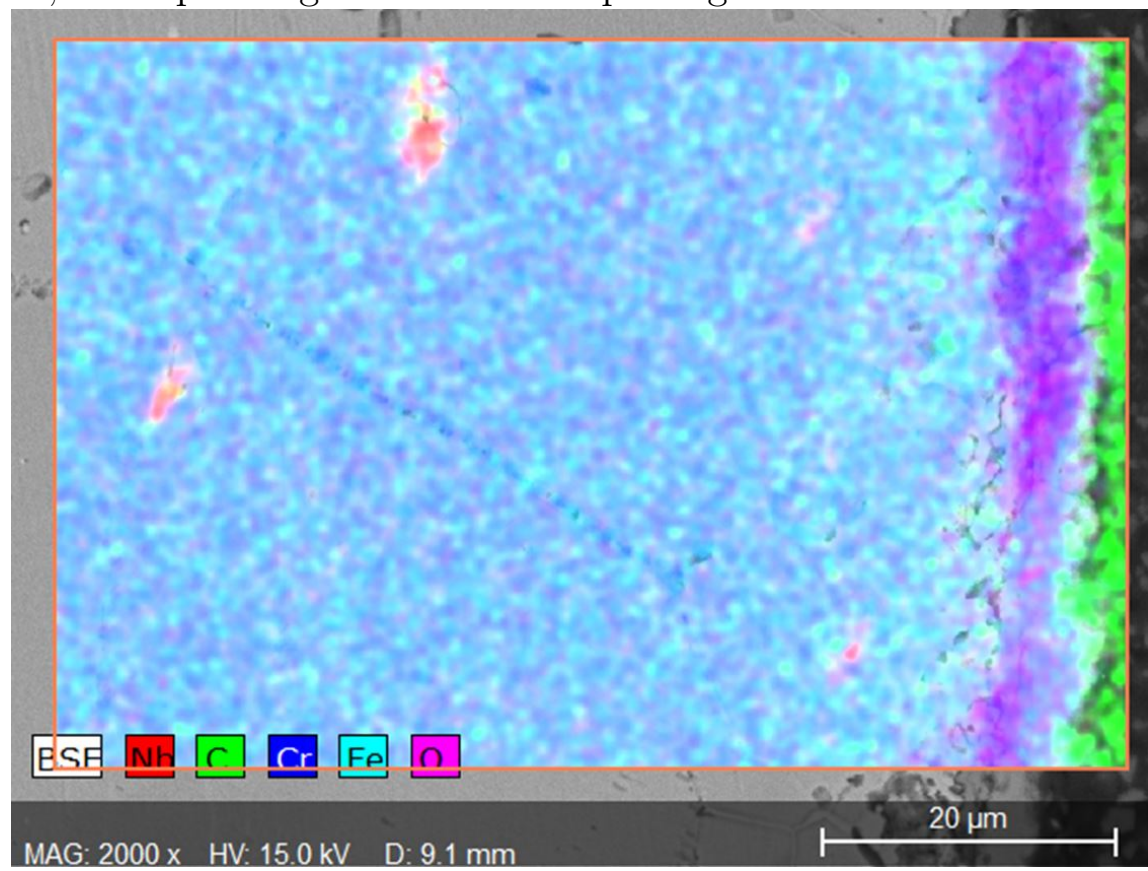

Figure 3.26: Cross section of the SEM micrograph in figure 3.25 analysed using EDS. The false colour map shows an oxide rich layer adjacent to the metal and the presence of niobium precipitates. 


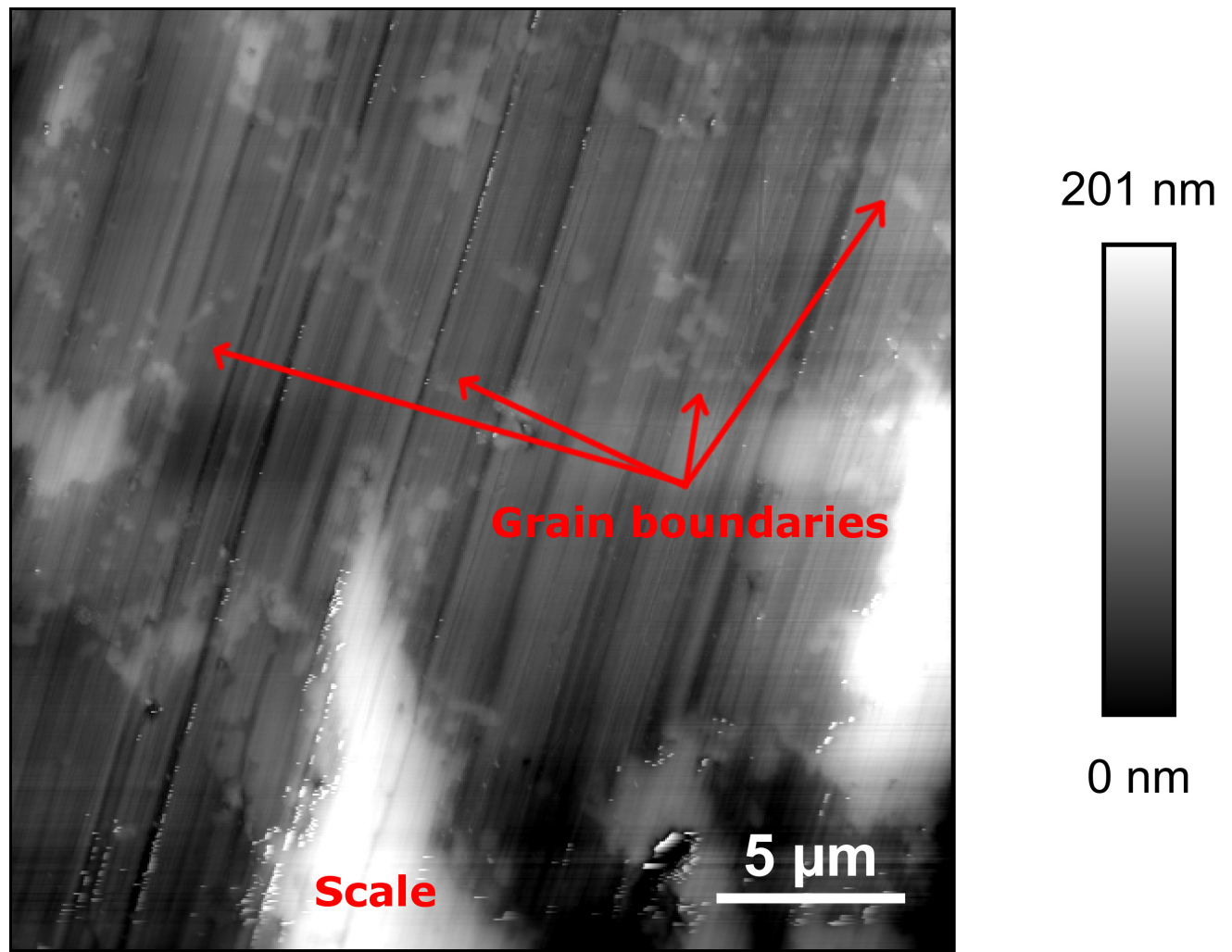

Figure 3.27: Annotated AFM topographical image of the thermally sensitised SS. The lower half of the image shows the scale (white), whereas the upper part of the image shows the metal surface and carbides which decorate the grain boundaries. The grain boundaries at these locations could also contain residual haematite. 


\subsubsection{SVET Analysis of Specimens Containing Scale}

\section{Full Scale Coating}

From the previous section it had been determined that SS specimens taken from thermal processing were not sensitised throughout the bulk of the material. Furthermore, the processing resulted in the nucleation and subsequent growth of a thick 6-9 $\mu \mathrm{m}$ coating of haematite, masking the entire surface. As a result, specimens were introduced to corrosive electrolyte to investigate localised corrosion behaviour of the thermally sensitised material prior to any grinding and polishing. Furthermore, by forgoing the grinding step, the vital highly sensitised material beneath the scale would not be either partially or fully removed.

The results of the first experiment are shown in figure 3.28. The SVET did not show the expected formation of anodes but instead the exposed surface was shown to be electrochemically inactive. The SVET was however able to resolve local anodes located in the top left region of the scans. A possibility is that many of the anodes could have initiated, but subsequently passivated during the time between the scans. The digital photographs clearly show that oxidation had occurred (inset in figure 3.28(vi)). Giving rise to numerous red-rust locations over the scale covered surface. To rule out instrument error, instrument sensitivity was increased such that other anodes (clear in the photograph) could be detected/resolved. The SVET surface current density maps recorded at the higher sensitivity are given in figure 3.29. The second experiment again did not show any activity related to the corrosion that was visually evident (figure 3.29(v)) .

The reasoning for the inability to detect this oxidation occurring on the surface of the SS is a fundamental drawback of this scanning electrode technique. The theory behind the SVET has been provided earlier in chapter 2. The SVET is able to monitor the change in potential gradient above the corroding surface which itself is proportional to current flux density in solution. However, for a potential gradient to be detected, the lines of ionic current flux which couple anodic and cathodic regions must pass through the plane of scan. One way of attaining better 

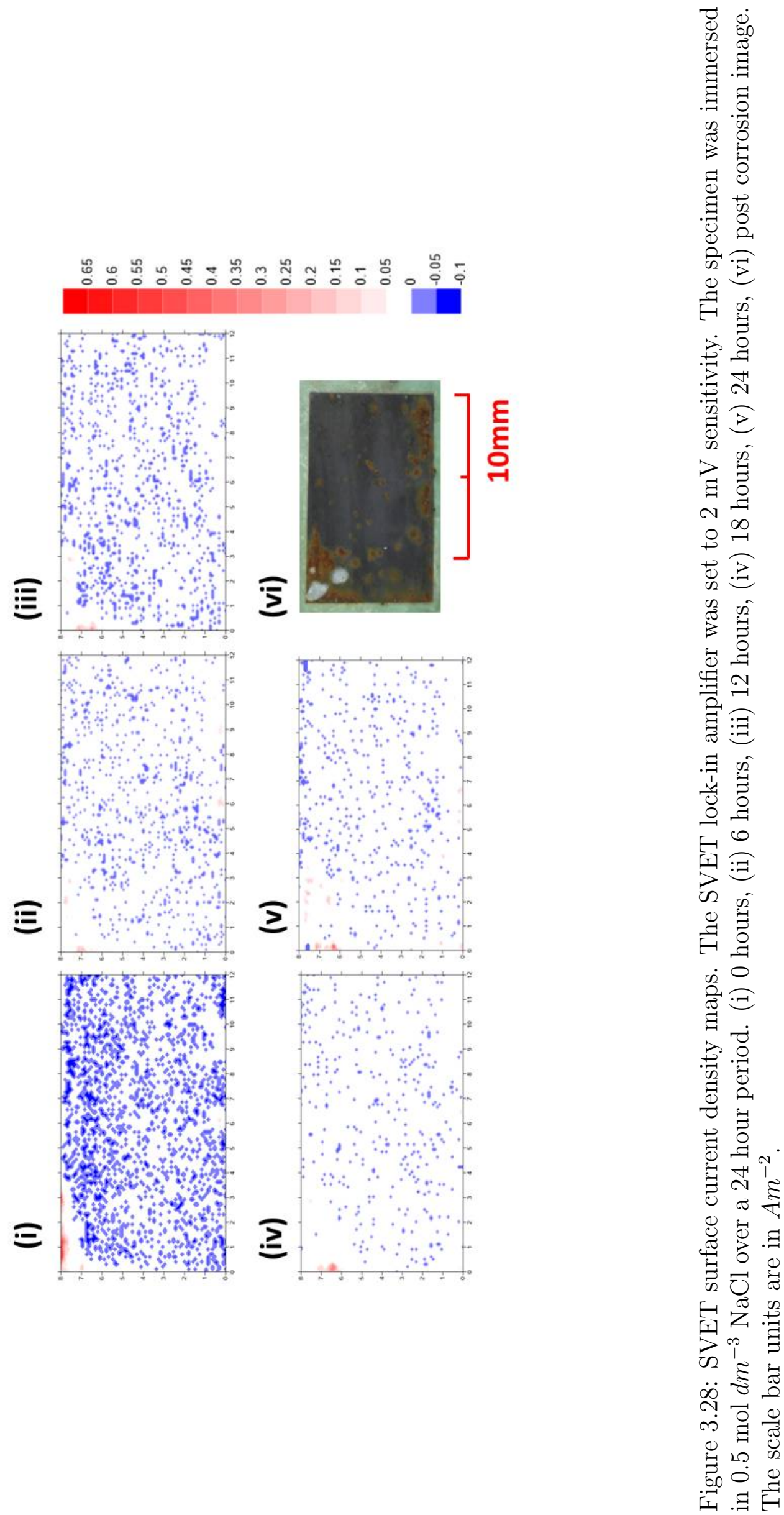


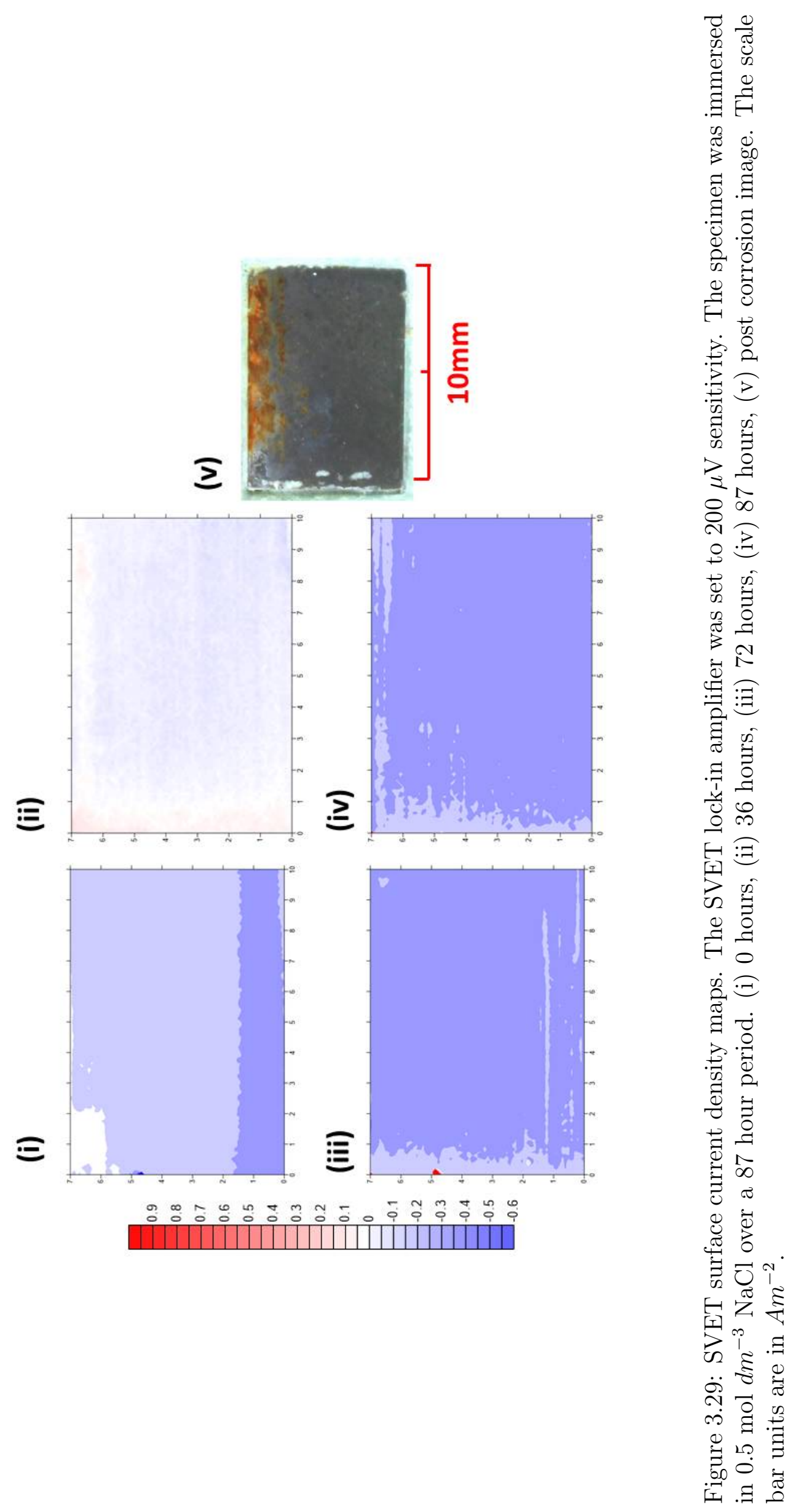


resolution is to decrease the probe to sample distance, which in itself requires a smaller diameter microelectrode, otherwise there will be blurring of point current sources. This comes to a cost, as electrical impedance is inversely proportional to diameter. The Swansea University SVET is at near optimal operation, c. both $100 \mu \mathrm{m}$ distance and microelectrode diameter. Thus, if current loops between anode and cathode are large enough, which for localised corrosion they usually are, then the SVET will detect the sources of ionic current. However, SVET will be unable to detect point current sources should the loops be very small the i.e. below the plane of the scan. Such an occurrence will occur where the corrosion cell is confined to small areas. Therefore it is evident that the discrepancy between visual appearance and SVET-derived current density maps arises because both anodic and cathodic events take place at the same locations and so cannot be detected. It is plausible therefore the scale acts to occlude the metallic surface, giving rise to highly localised crevices. 


\section{Polished Scale Section}

The image in figure 3.30 shows the surface before and after corrosion in $1 \mathrm{~mol}$ $d m^{-3} \mathrm{NaCl}$ electrolyte over the time period of 24 hours in freely corroding conditions. When highly sensitised grain boundaries are present at a site occluded of oxygen, the surface undergoes rapid corrosion initiation. The SVET maps show surface current density in 3 hourly intervals (figures $3.30(\mathrm{~d}-\mathrm{h})$ ) and the maps show that corrosion initiates almost immediately, within the first scan. Peak current density is the greatest in this time period, decreasing thereafter, with an unexpected increase in the final scan (figure 3.30(h)). It is possible that the decrease in current density in scans e, $\mathrm{g}, \mathrm{f}$, are a result of the local areas undergoing passivation due to a build up of corrosion product, blocking the principal anode and cathode sites. In figure 3.30(h) the perforated scale is removed and the extent of crevice IGC was fully detected. The optical micrographs in figure 3.31 show IGC at different magnifications. Figure 3.31(a) shows distinct different regions: the black scale on the top left, IGC within the crevice environment and the polished metal surface. In figure 3.31(c) highly localised IGC pitting can be seen, where discrete areas of the grain boundary have been attacked. These results were included in a special issue of Corrosion, Engineering, Science and Technology by this author [133]. The results from this experiment are in broad agreement with the previous experiment in section 3.4.4, whereby corrosion initiated at the haematite scale/bulk metal. IGC in this instance, did not manifest at pores on the surface of the scale, but formed underneath the scale where electrolyte had permeated through the apparently porous haematite layer. It should be noted that the concentrations used are different, and the higher concentration used here was undertaken in order to initiate corrosion on the metal surface in freely corroding conditions.

Following the previous experiments to understand the surface scale (EDS, $\mathrm{XRD}, \mathrm{SVET}$ ) there are two scenarios: (a) the haematite is a good cathode allowing a Galvanic cell to form, or (b) the haematite acts to occlude the surface and 

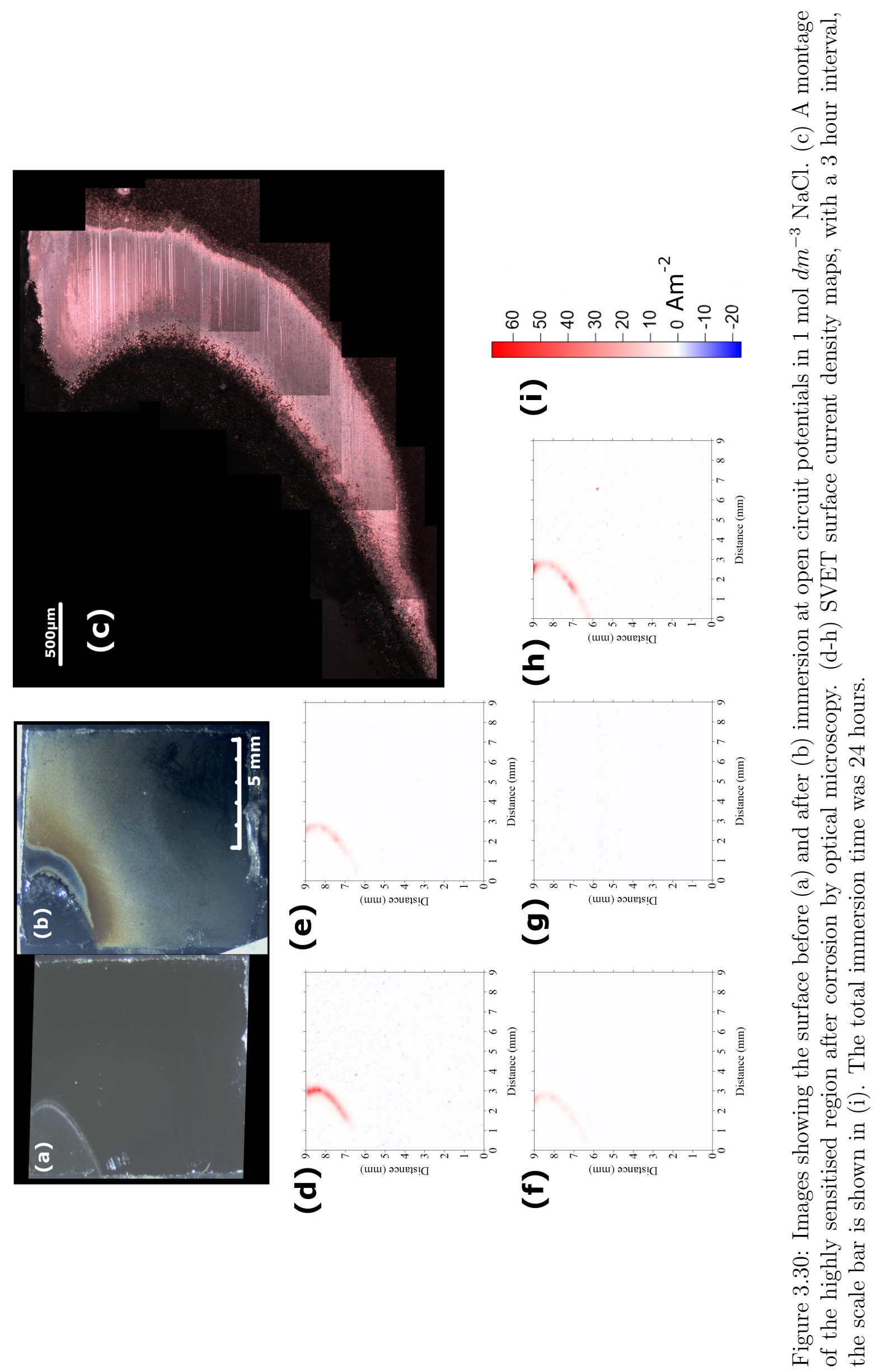

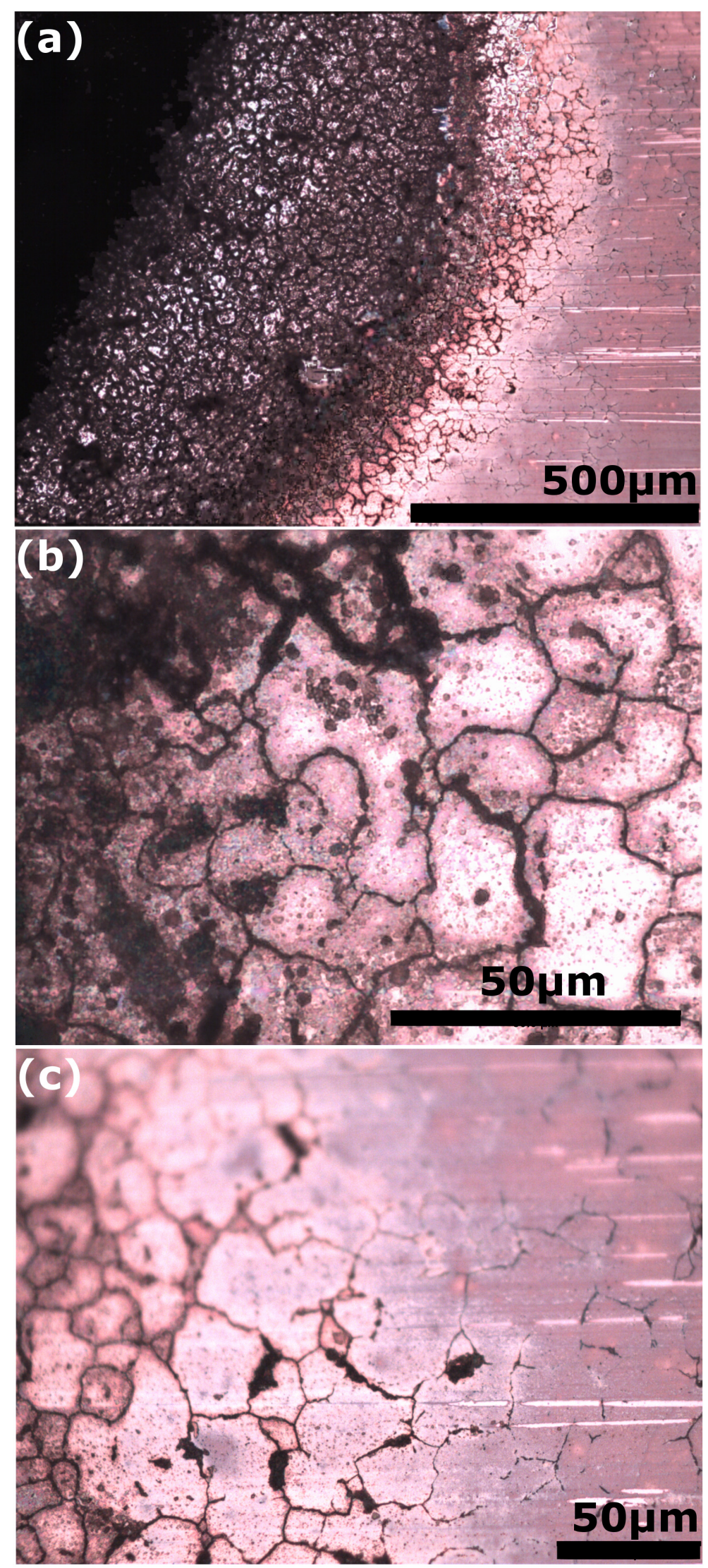

Figure 3.31: Optical micrographs of IGC in the region in figure 3.30(c) recorded at different magnifications. 
is relatively inert, but is porous. This experiment was conducted to understand which scenario was active. A specimen with the intact haematite surface was immersed in a $0.5 \mathrm{~mol} \mathrm{dm}^{-3} \mathrm{NaCl}$ containing electrolyte and polarised from cathodic potentials of -650 to $-250 \mathrm{mV}$ (vs. SCE). The same specimen was removed and polished, such that the Haematite scale was removed from the area of exposure. The specimen was reintroduced into the electrolyte and a polarisation curve (using the same start and end potentials) was taken. If polarised in the cathodic region of the curve, one of two reactions should occur: the oxygen reduction reaction (ORR), or the hydrogen evolution reaction. Both were described previously in the literature review (chapter 1 ). The most likely reaction to occur on this type of stainless steel in $\mathrm{NaCl}$ at a neutral $\mathrm{pH}$ is the ORR, whereby dissolved oxygen is reduced to form hydroxide ions $\left(\mathrm{OH}^{-}\right)$as shown in equation 3.1.

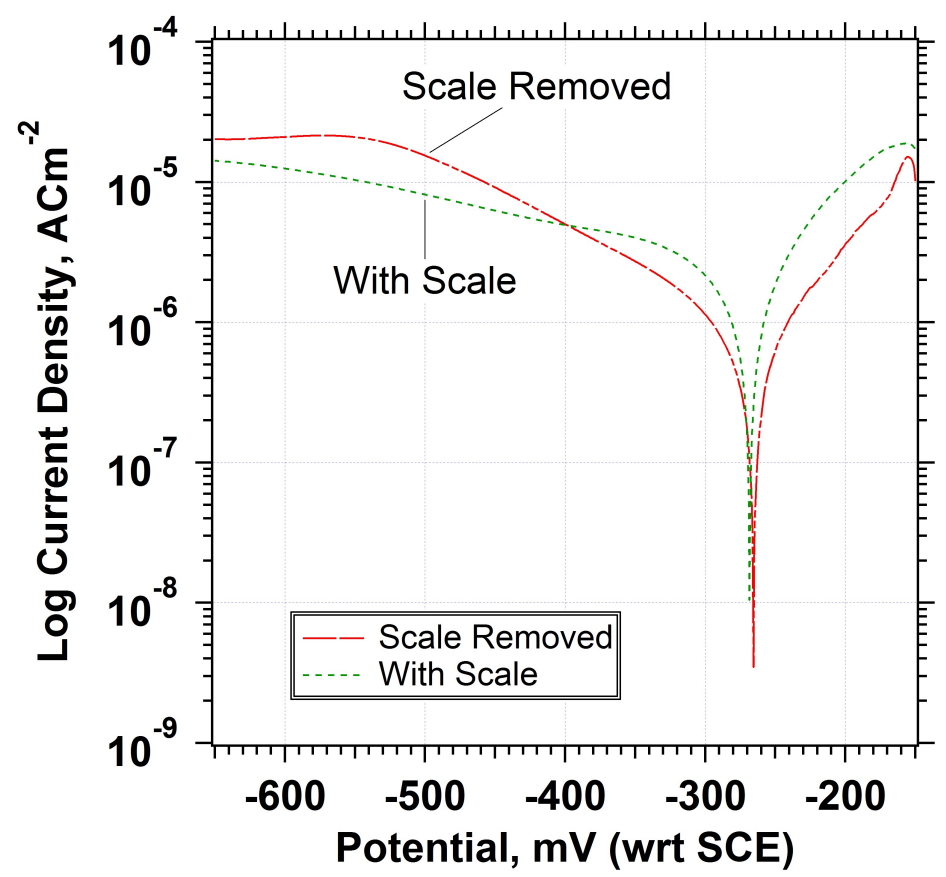

Figure 3.32: Cathodic branch scan of thermally sensitised SS with and without scale removed. The sample was polarised from $-650 \mathrm{mV}$ (vs. SCE) to $-150 \mathrm{mV}$ (vs. SCE).

$$
\mathrm{O}_{2}+2 \mathrm{H}_{2} \mathrm{O}+4 e^{-} \rightleftharpoons 4 O \mathrm{H}^{-}
$$

The plots in figure 3.32 show the results of the cathodic polarisation. The 
cathodic corrosion current density was greater once the scale was removed, contradictory to scenario (a) that the scale is a good cathode. The haematite scale does not promote the ORR as cathodic current density is smaller with the scale present. Therefore the results indicate that scenario (b) is the active mechanism for corrosion initiation, providing a microcrevice environment whereby corrosion cells can initiate. It is postulated that differential aeration corrosion cells form within the pores of the haematite. This is in agreement with the results of the SVET experiments in the earlier sections 3.4.4, where localised corrosion events were not detected though the specimen surfaces showed red-rust. This indicates that the current loops must be small which leads to the theory that the corrosion cells exist within the pores of the haematite. 


\subsubsection{Investigation of Carbon Coated SS}

In service SS cladding can become covered in carbon, a result of using carbon dioxide coolant, which lowers thermal conductivity decreasing plant efficiency. Carbon deposition is described in the introduction (section 3.1). The carbon is predicted to be a much better cathode than the SS itself and may cause acceleration of corrosion when stored in cooling ponds. In this section ZRA experiments were conducted to the evaluate this theory. The plot in figure 3.33 shows the impact of carbon deposition on the sensitised SS tubes in $10 \mathrm{ppm}\left[\mathrm{Cl}^{-}\right]$. This electrolyte had a low concentration to be representative of cladding stored in pond under a fault condition. The net current flux is positive, which indicates that the uncoated SS is coupling with the tube that has had carbon deposition treatment. This follows the expectation (Galvanic series) that carbon is acting as a cathode relative to the SS. The scale of currents is small, within the order of $1 \mu \mathrm{A}$. The current responds to a change in area of the carbon coated tube, again indicating that carbon is acting as a cathode, with greater areas yielding larger currents. As shown, the area of the cathode controls the rate of reaction (corrosion), and is well known by corrosion engineers not to design systems with small anodes and large cathodes [82] [134] [41] [135]. 


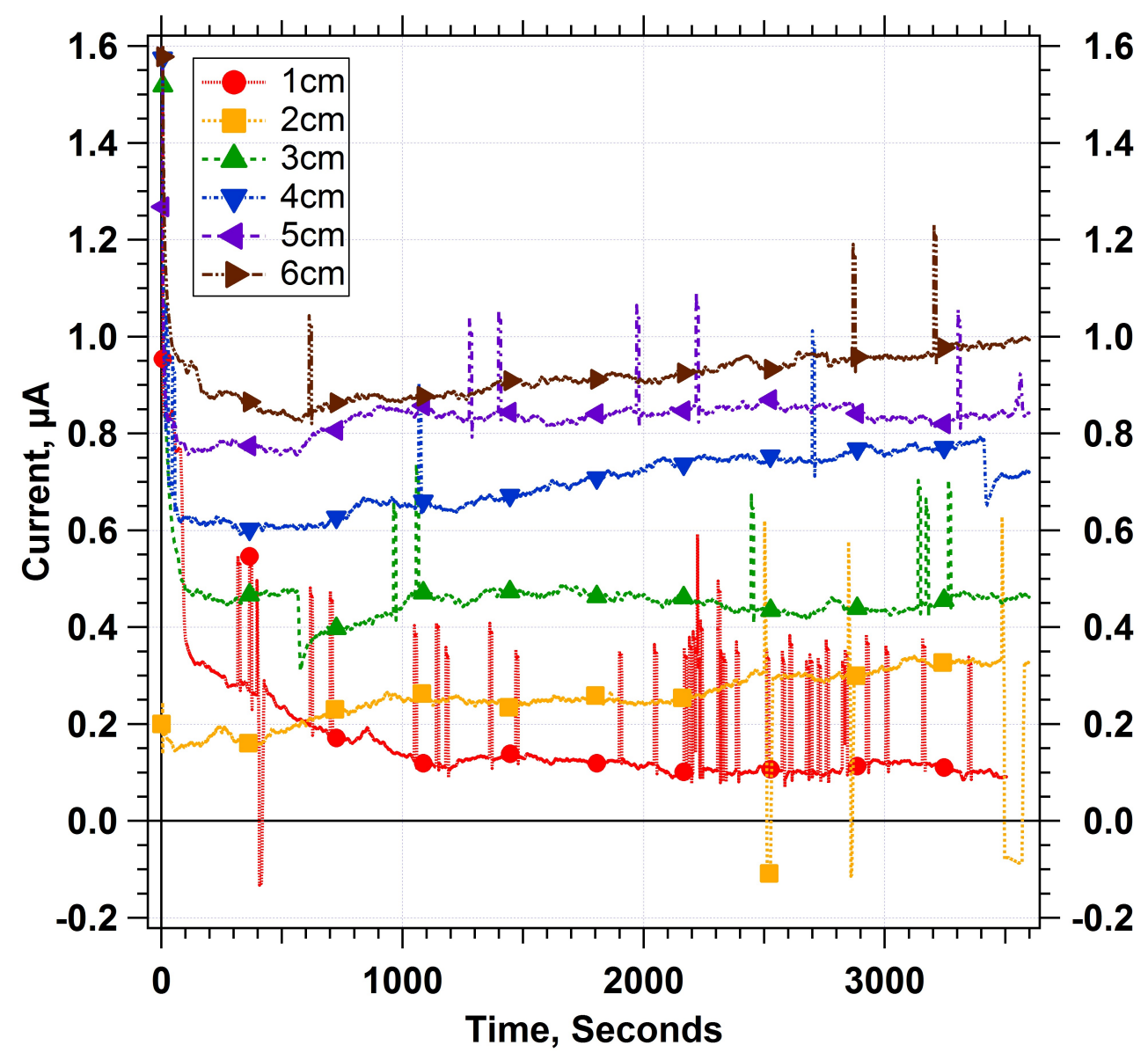

Figure 3.33: ZRA experiment sensitised SS. The uncoated sensitised tube was the working electrode, the counter electrode and reference was connected to the carbon coated sensitised SS tube. The SS electrodes were immersed in $10 \mathrm{ppm}$ $\left[\mathrm{Cl}^{-}\right]$. The lengths in the legend refer to the length of working electrode immersed within the electrolyte. The length of the non-carbon coated, sensitised counter electrode was $4 \mathrm{~cm}$ and kept constant. 


\subsubsection{Effects of Crevice Formers on Sensitised Tube}

These experiments were used to investigate the susceptibility to crevice corrosion, which was evident in the previous SVET experiments in section 3.3.3. This is applicable to the industry as multiple fuel pins are stored in slotted cans within cooling ponds, leading to a number of crevice environments. In contrast to the industry however the concentrations used in these experiments are much greater. The graphs in figure 3.35 show the effect of $\mathrm{Cl}^{-}$on the OCP of the sensitised tubes without any additional o-rings. In this type of experiment a significant drop in potential indicates the point in which crevice corrosion initiates. The lowest concentration has the most positive OCP (figure 3.35), which becomes successively more negative with an increasing $\left[\mathrm{Cl}^{-}\right]$. The same trend is witnessed with figure 3.36. The most positive potential is $0.01 \mathrm{~mol} \mathrm{dm}^{-3} \mathrm{NaCl}$, and the least positive being $5 \mathrm{~mol} \mathrm{dm}^{-3} \mathrm{NaCl}$. These have the tight fitting o-rings attached. It is expected that these would exhibit crevice corrosion due to the artificial differential aeration cell that was formed from multiple o-rings. As a comparison between the specimens with and without o-rings final rest potentials are very similar to one another, apart from the lowest concentration, $0.01 \mathrm{~mol} \mathrm{dm}^{-3} \mathrm{NaCl}$. Both specimens exhibit an exponential relationship between potential and time from the starting period. The non o-ring specimen starts c. -200 mV SCE, decaying to $-250 \mathrm{mV} \mathrm{SCE}$. Whereas the specimen that has o-rings displays an starting OCP of -240 mV SCE, rising exponentially to -200 mV SCE (with orings).

Images of the tubes after corrosion are shown in figure 3.34. Figures A, B, C, D ([Cl- $]$ low:high, left:right) show the specimens that had tightly fitting o-rings attached, figures E, F, G, H show the tubes that had no crevice treatment. It was expected that the specimens that had the o-rings would show more corrosion, however the images show that this is not always the case. The specimens that did not have o-rings showed more propagation of visible corrosion features, whereas those with the o-rings showed corrosion that was even more localised. Comparing 
specimens with the same $\left[\mathrm{Cl}^{-}\right]$, the most striking difference is at $0.5 \mathrm{~mol} \mathrm{dm}^{-3}$ $\mathrm{NaCl}$, where there is a brightly orange coloured oxide present on the specimen without o-rings (figure 3.34G). For the specimen with o-rings there appears to be less corrosion product present (figure 3.34C). At the lowest concentration, there is corrosion product evident when o-rings are used (figure A), but a fragmentation of the scale in figure $3.34 \mathrm{E}$.

The images show that the use of crevice formers does not change the amount of corrosion that can be seen optically using a camera. The images do show that the sensitised tube with an iron oxide scale does undergo spontaneous corrosion when immersed in at least $0.05 \mathrm{~mol} \mathrm{dm}^{-3}$ (without crevices), and at $0.01 \mathrm{~mol}$ $d m^{-3}$ (with crevice) $\mathrm{NaCl}$.

Electrochemical OCP data is displayed in figure 3.37 for specimens immersed in $5 \mathrm{~mol} \mathrm{dm}^{-3} \mathrm{NaCl}$ with and without $\mathrm{H}_{2} \mathrm{O}_{2}$. The addition of the oxidiser $(\approx 20$ $\mathrm{mM} \mathrm{H}_{2} \mathrm{O}_{2}$ ) at 1500 s (see inset figure 3.37) leads to a transient increase in potential from $-330 \mathrm{mV}$ SCE to $-220 \mathrm{mV}$ SCE. After reaching the peak of $-220 \mathrm{mV}$ SCE the potential decays and thus the presence of $\mathrm{H}_{2} \mathrm{O}_{2}$ only temporarily promotes the free corrosion potential to more positive values. The onset of increased anodic intensity means that the free corrosion potential drops to more negative values as predicted by mixed potential theory. This decrease in OCP is to offset the additional rise in anodic current. Uchida et al. [136] studied the effect of $\mathrm{H}_{2} \mathrm{O}_{2}$ on stainless steels in BWR (boiling water reactor) water on the free corrosion potential, and included an Evans diagram showing the effect of $\mathrm{H}_{2} \mathrm{O}_{2}$ addition (figure 3.38). Macrograph collages of the specimen after immersion are shown in figure 3.40. Images of the surface after immersion without the oxidant, $\mathrm{H}_{2} \mathrm{O}_{2}$, added are displayed in figure 3.39. Both specimens show corrosion spread across multiple sites. $\mathrm{H}_{2} \mathrm{O}_{2}$ appears to increase the amount of corrosion, and amount of loose corrosion product on the surface of the SS. 


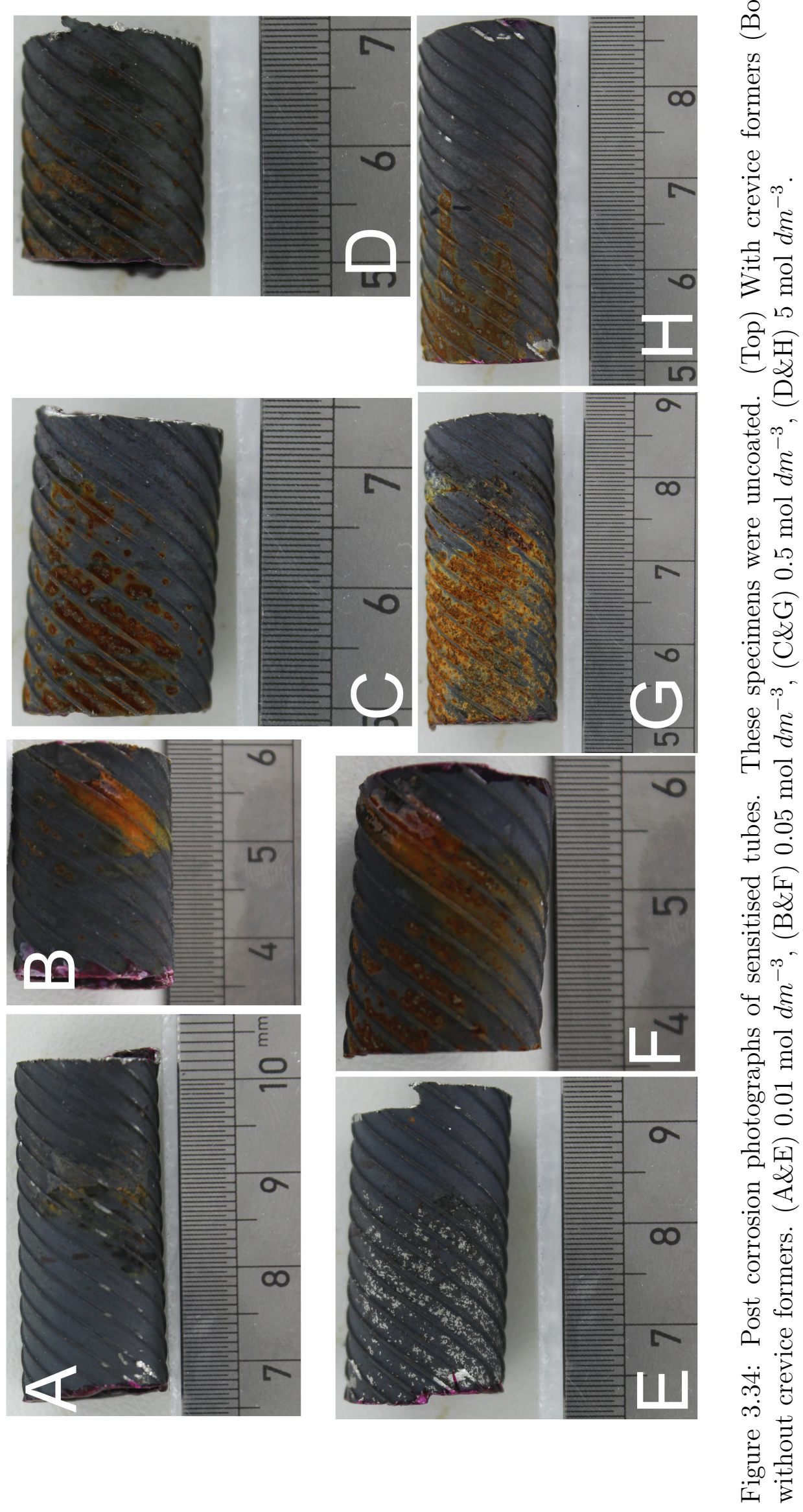




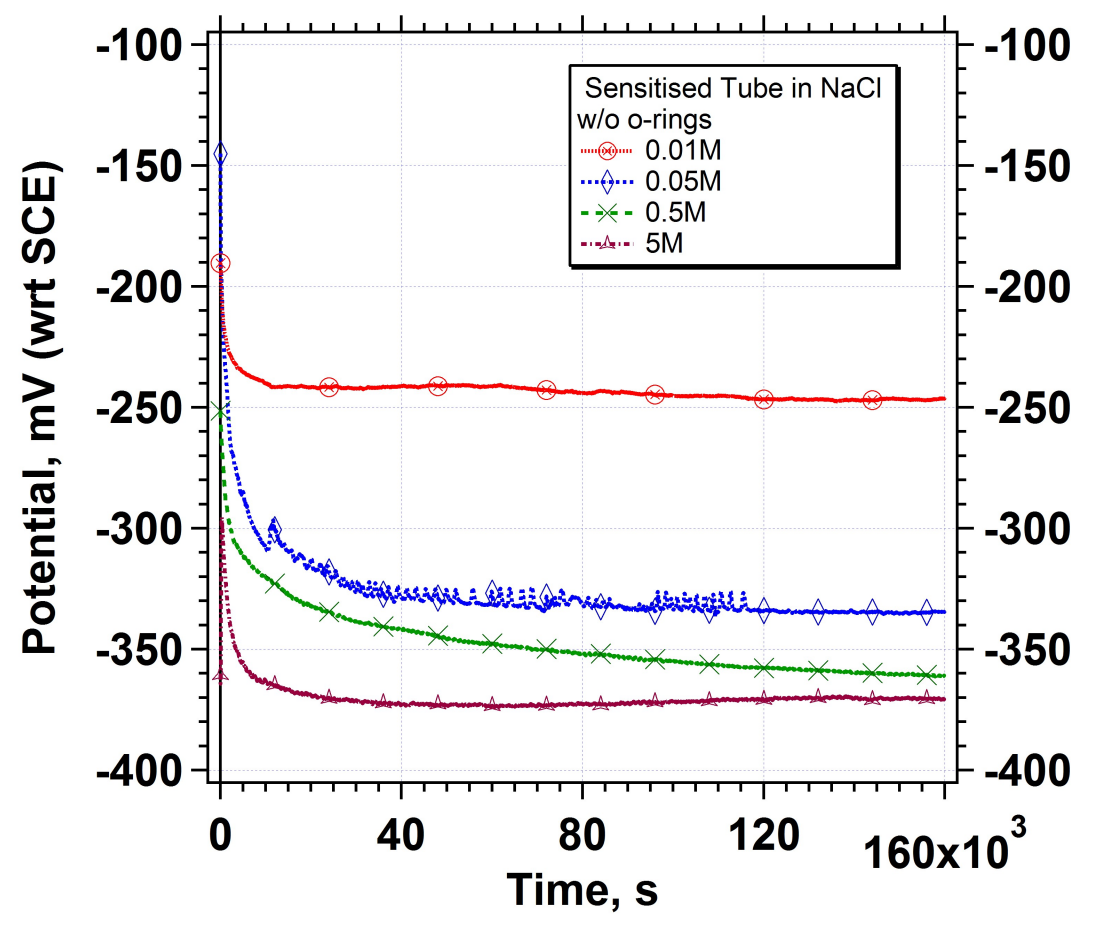

Figure 3.35: OCP of sensitised tube in varying $\left[\mathrm{Cl}^{-}\right]$.

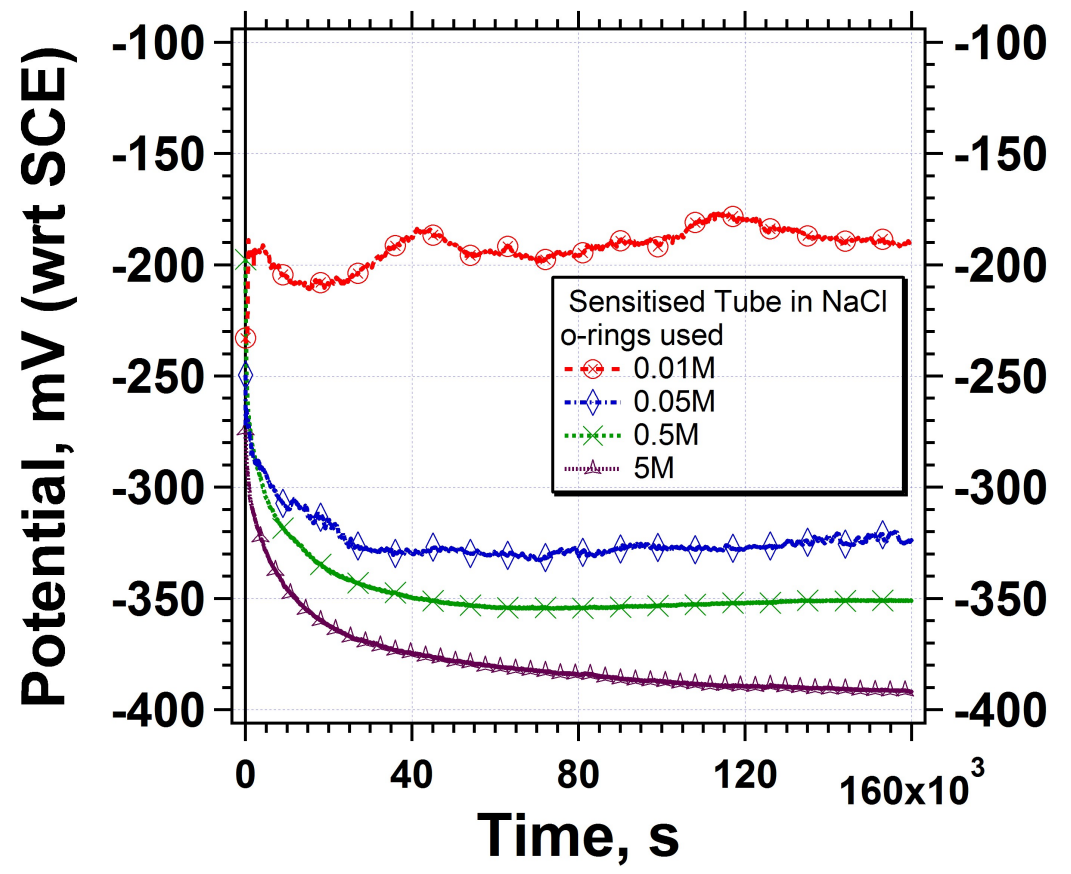

Figure 3.36: OCP of sensitised tube in varying $\left[\mathrm{Cl}^{-}\right]$. A crevice environment has been induced on the surface of the tubes. 


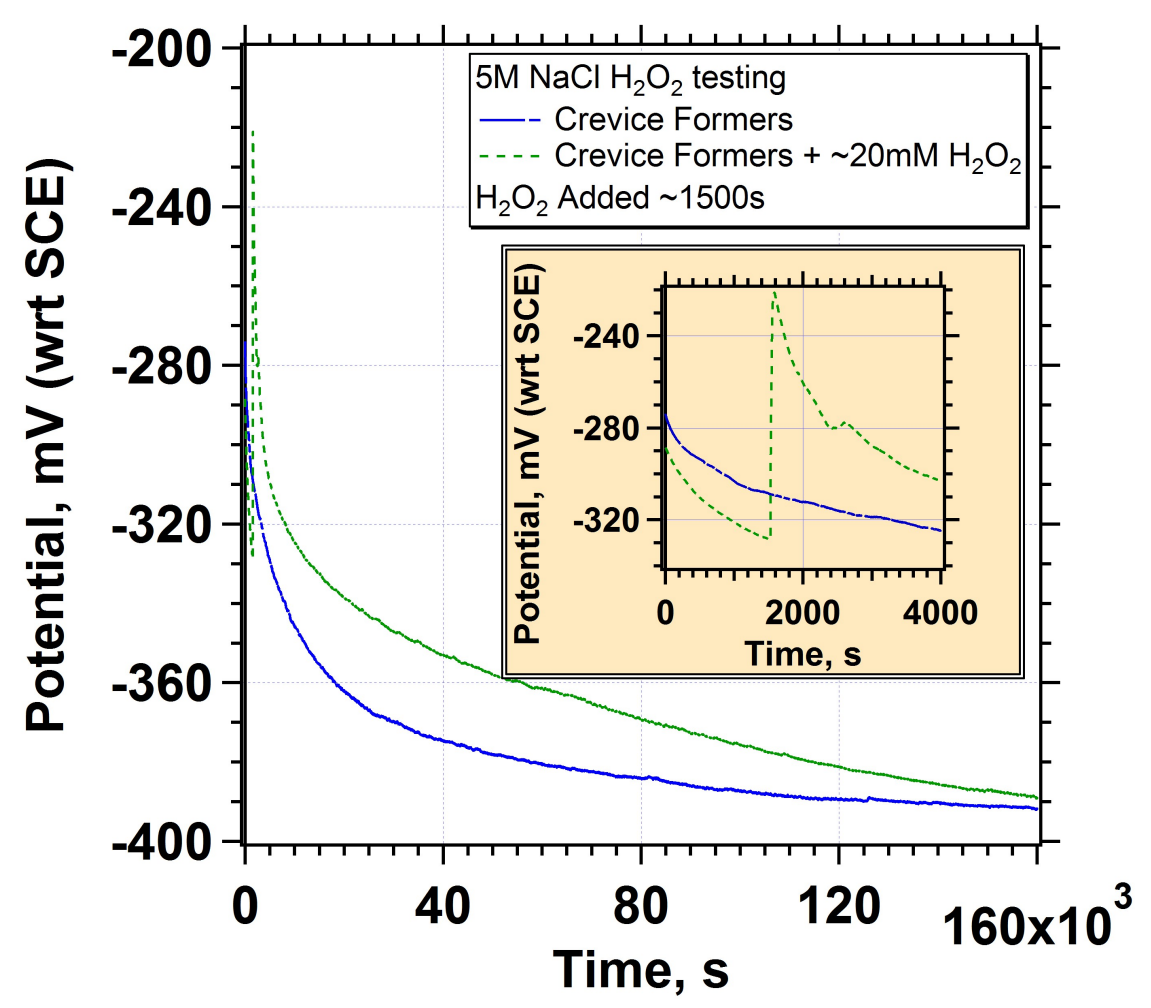

Figure 3.37: OCP of sensitised tubes. Crevice formers were used, and specimens immersed in $5 \mathrm{~mol} d m^{-3} \mathrm{NaCl}$. The green curve shows the specimen with additional $\mathrm{H}_{2} \mathrm{O}_{2}$ added.

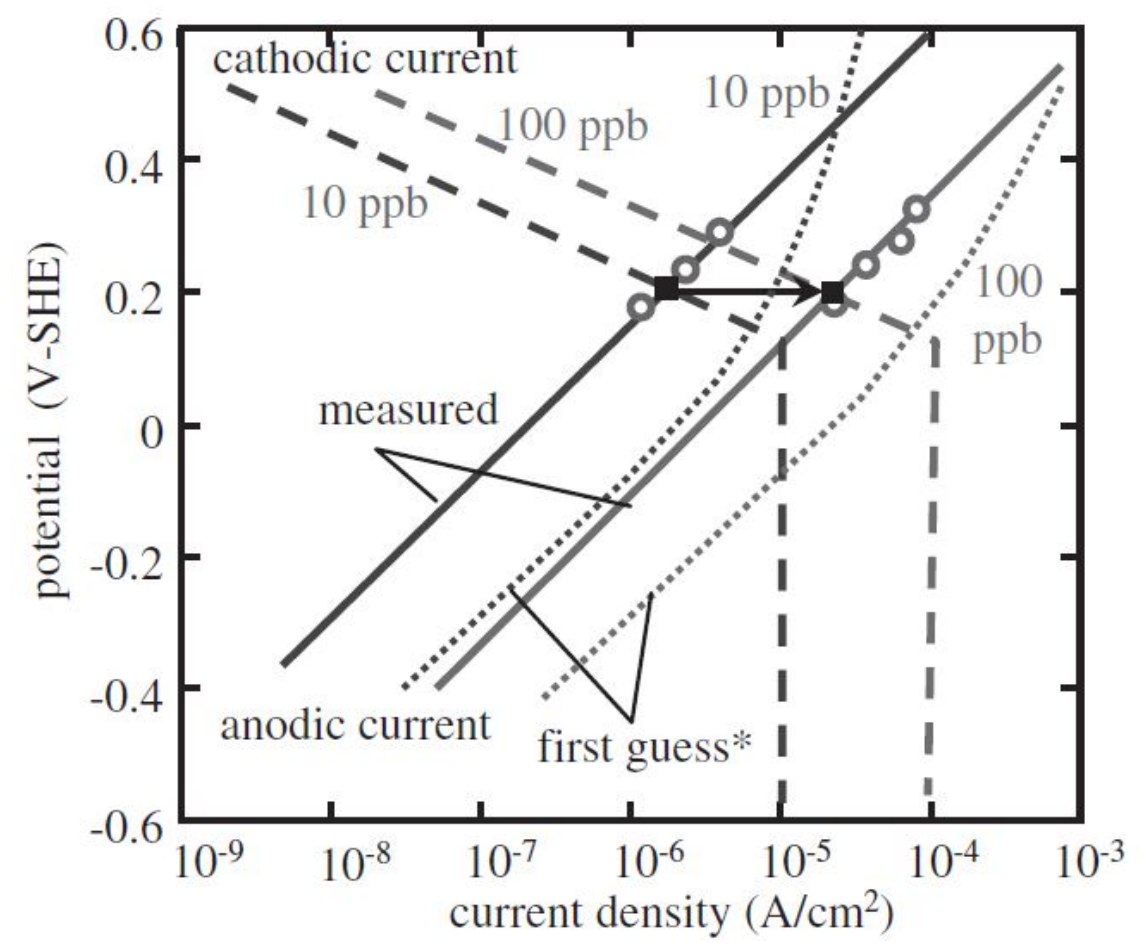

Figure 3.38: Evans diagram showing the effect of $\mathrm{H}_{2} \mathrm{O}_{2}$ addition on current density [136]. The two black squares show the increase in current density following a shift in concentration from $10 \mathrm{ppb}$ to $100 \mathrm{ppb}$ 


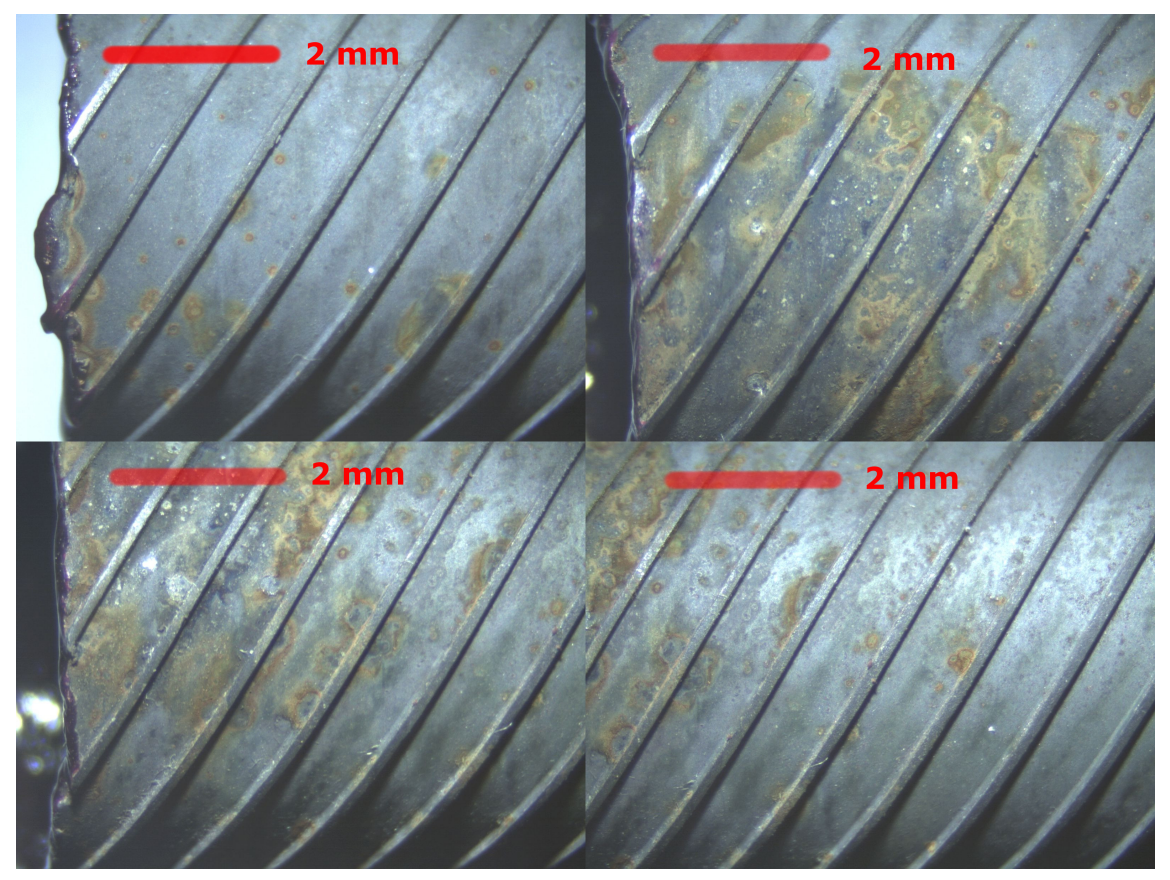

Figure 3.39: Macrographs of surface after immersion in $5 \mathrm{~mol} \mathrm{dm}^{-3} \mathrm{NaCl}$ with crevice formers

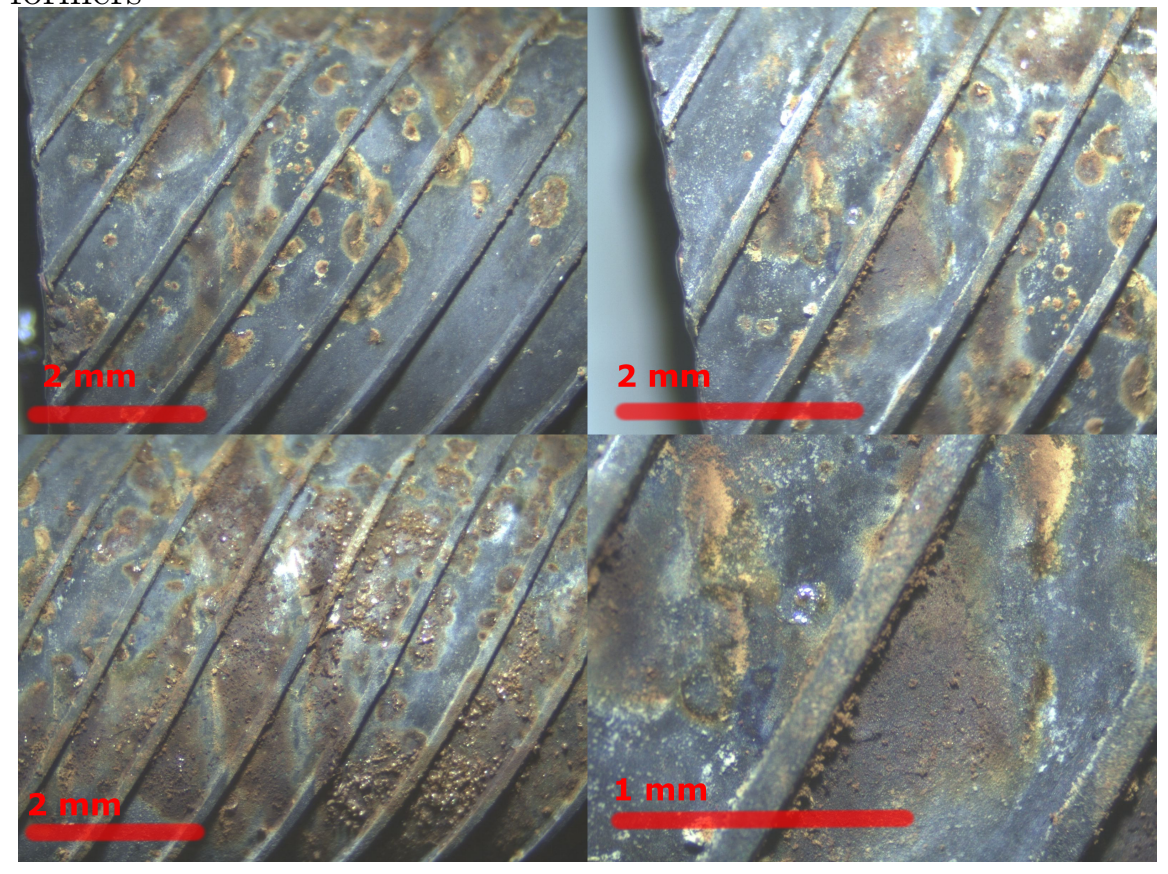

Figure 3.40: Macrographs of surface after immersion in $5 \mathrm{~mol} d m^{-3} \mathrm{NaCl}$ crevice formers and $20 \mathrm{mM} \mathrm{H}_{2} \mathrm{O}_{2}$ added 


\subsection{Conclusions}

Unsensitised Material Condition In this chapter, 20/25/Nb SS has been examined using different electrochemical techniques in various chloride containing electrolytes. The study investigated the dependence of the $\mathrm{Cl}^{-}$anion, and also temperature dependence of the material in an as-received condition. As expected, there is a dependence on both factors. The $\left[\mathrm{Cl}^{-}\right]$dependence for $\mathrm{E}_{b}$ was $-98 m V$ decade ${ }^{-1}$ and showed a temperature dependence of $-7.3 \mathrm{mVK}^{-1}$ at fixed $\left[\mathrm{Cl}^{-}\right]\left(1 \mathrm{~mol} \mathrm{dm}^{-3}\right)$.

Crevice corrosion was also studied using the SVET to demonstrate the ability to measure localised corrosion. As the SS does not undergo spontaneous corrosion initiation even in $1 \mathrm{~mol} \mathrm{dm}^{-3} \mathrm{NaCl}$, the specimen was polarised and crevices were induced around the edges of the specimen. The SVET surface current density maps showed it is possible to measure corrosion propagation. Anodic activity can be correlated to SVET-derived current density maps and verified by optical imaging.

Finally, the unsensitised SS was subjected to a special treatment to grow thicker surface Cr-oxides. The impact of this treatment, leading to 'blue' and 'green' oxide specimens was investigated using potentiodynamic experimentation.

The results of the "green" specimen experiments were mixed. One specimen showed lower current densities, and the other showed higher current densities when compared to the control specimen. The "blue" specimens both performed worse than the control specimen. Due to the size and number of specimens that were available for experiment, it is recommended that further testing be conducted, which would lead to a better understanding of oxide performance.

Thermally Sensitised Condition Following thermal processing of specimens, the amount of sensitisation was quantified using the DL-EPR test, following ISO procedures. The investigation revealed that the degree of sensitisation was not homogeneous throughout the bulk of the material. The sensitisation varied 
through the specimen, and as such a new preparation technique was theorised. This technique was used for many more experiments in this thesis. The grain size remained similar despite the thermal processing, decreasing in size by an average of $2 \mu \mathrm{m}$ from $17 \mu \mathrm{m}$ to $15 \mu \mathrm{m}$.

The scale resulting from the thermal processing was analysed using XRD and EDS techniques. XRD indicated the presence of haematite, an oxygen rich iron oxide $\left(\mathrm{Fe}_{2} \mathrm{O}_{3}\right)$. EDS was in agreement with the XRD, as a cross section revealed the oxide was enriched with Fe and O. Once the haematite was removed, the sensitised SS showed minimal crystallographic texture.

As spontaneous corrosion of the sensitised SS was apparent visually, SVET was used to map the surface in $0.5 \mathrm{~mol} \mathrm{dm}^{-3} \mathrm{NaCl}$ at two different sensitivity settings, without prior metal preparation (i.e. the specimen was not ground or polished). Post corrosion imaging of the specimens showed distinguishable corrosion product on the surface of the haematite, although SVET scans did not show the formation of anodes. Another specimen was polarised in the cathodic region of the polarisation curve, with, and without haematite to study if the coating had any impact on the oxygen reduction reaction (ORR). The findings of both the cathodic scanning, and the SVET indicate that the coating does not impact the cathodic ORR, leading to a small decrease when present. The crevice environment within the pores may support water hydrolysis whereby water reacts with metal cations to form metal hydroxide and protons within the crevice. This acidic environment causes an inward diffusion of hydroxides and anions (e.g. $\mathrm{Cl}^{-}$) into the crevice to neutralise the positive charge; thus an autocatalytic process ensues. In such an scenario the crevice is populated by a high density of metal chloride and high density of protons, hence it is possible that hydrogen evolution may occur within the crevice environment. The SVET study indicated that current loops between anode and cathode must be of sufficiently small size, so that the SVET cannot detect these events. The cells must be highly localised, most likely present within the pores of the haematite itself. 
The SS was investigated with and without a coating of carbon, in order to simulate the surface of material after it has been removed from operation. The sensitised SS showed an agreement with the theory that coated carbon acts as a cathode, creating a Galvanic cell.

In order to study the effect of crevice corrosion on the sensitised SS, tube structures were used with and without tightly fitting O-rings. The experiments were carried out at free corrosion potential, as a function of $\left[\mathrm{Cl}^{-}\right]$. The study revealed that although O-rings formed tight crevices, accelerating corrosion by forming differential aeration cells, they produced more localised corrosion. Without the crevice environment the corrosion products are more dispersed. Corrosion is evident in $0.01 \mathrm{~mol} d m^{-3} \mathrm{NaCl}$ when the crevices are present, and at $0.05 \mathrm{~mol} \mathrm{dm}^{-3} \mathrm{NaCl}$ without crevices. A short test using hydrogen peroxide $\left(\mathrm{H}_{2} \mathrm{O}_{2}\right)$ was also conducted on the sensitised tubes. The test used sensitised SS tubes immersed in the highest concentration tested $\left(5 \mathrm{~mol} d m^{-3} \mathrm{NaCl}\right)$ with the tightly fitting crevice formers attached. In order to promote further corrosion, 20 milimolar $\mathrm{H}_{2} \mathrm{O}_{2}$ was added. The aim of this test was to study the material in an extreme 'fault' scenario: sensitisation, high $\left[\mathrm{Cl}^{-}\right]$, crevices and an oxidant present. Corrosion was evident in both specimens, but when $\mathrm{H}_{2} \mathrm{O}_{2}$ was added there was a noticeable increase in corrosion product, when post corrosion imaging was conducted.

Summary A summary of the conclusions from this chapter are itemised below:

- The unsensitised SS displays a $\left[\mathrm{Cl}^{-}\right]$of $-98 \mathrm{mVdecade^{-1 }}$. In $1 \mathrm{~mol} \mathrm{dm}^{-3}$ $\mathrm{NaCl}$, the fuel cladding material is exhibits a temperature dependance of $-7.3 m V K^{-1}$.

- SVET can be used to study the effect of crevice corrosion propagation, if polarisation is used. The SVET technique can be cross referenced with optical imaging.

- It was found that spontaneous corrosion could occur on sensitised SS tubes 
immersed in $\mathrm{NaCl}$. Corrosion was initiated at $0.05 \mathrm{~mol} d m^{-3} \mathrm{NaCl}$. If a crevice environment was present the concentration needed for corrosion initiation reduced to $0.01 \mathrm{~mol} d m^{-3} \mathrm{NaCl}$.

- A mix of sensitisation, high $\left[\mathrm{Cl}^{-}\right], \mathrm{H}_{2} \mathrm{O}_{2}$ and crevices were used to create an extreme 'fault' scenario. Images of the SS were taken and analysed, along with electrochemical data (OCP).

- DL-EPR testing revealed that thermal sensitisation did not progress through to the bulk of the specimen. A thick scale formed after the thermal processing, XRD and EDS indicate that this is an iron-rich oxide known as haematite.

- SVET was used again to investigate spontaneous corrosion of the sensitised $\mathrm{SS}$ in $\mathrm{NaCl}$ with no prior surface preparation (haematite was not removed). Photographic evidence showed spontaneous corrosion which had occurred, but the SVET failed to detect localised anodes and cathodes. This indicates that current loops are small, and may exist within the pores of the oxide.

- Cathodic polarisation indicated that the haematite does not have an impact on the ORR. This is further evidence to support the claim that corrosion is promoted due the oxide structure being porous.

- It was found that carbon provides a cathode on sensitised SS tubes if connected as a Galvanic cell to non coated specimens. As carbon coated SS cathode area increased, the current increased when the anode (uncoated SS) area was fixed.

- Special thicker 'green' Cr-oxide specimens showed mixed performance compared to the control specimen. The 'blue' specimens showed worse performance compared to the control. 
Chapter 4

Where does IGC Initiate? A

Combined SVET and Time-Lapse

Microscopy Approach 


\subsection{Introduction}

Bulk 20/25/Nb stainless steel (SS) was studied using a variety of techniques in the previous chapter. This chapter aims to identify the areas in which corrosion initiates in the SS, which has been thermally processed to simulate sensitisation which may occur through the operating lifetime of the material. A time-lapse microscopy (TLM) technique has been used to study a small region of the SS (a circle of $\approx 500 \mu \mathrm{m}$ diameter was exposed to electrolyte). This is in contrast to the larger areas used in the previous chapter, as the areas exposed were $\geq 1 \mathrm{~cm}^{2}$. TLM was used to study the development of intergranular corrosion (IGC) in-situ. A further experiment used the scanning vibrating electrode technique (SVET) to provide electrochemical information on areas in which corrosion initiated. This was correlated with light and electron microscopy. When Chan [45] performed post corrosion SEM analysis of thermally sensitised 20/25/Nb following potentiodynamic experiments in $500 \mathrm{ppm}\left[\mathrm{Cl}^{-}\right]$and found that corrosion was 'often observed' within or at triple point grain boundaries and thus suggested that triple junction grain boundaries were more susceptible to corrosion. The suggestion was that in these areas the degree of sensitisation is greater in these areas in comparison to other grain boundaries. Double loop electrokinetic repassivation (DL-EPR) experiments showed that athough the grain boundaries showed severe corrosion, as expected, the areas around $\mathrm{NbC}$ inclusions were also attacked. Chan performed heat treatments by the following method to achieve a sensitised microstructure: $1300{ }^{\circ} \mathrm{C}$ solution anneal for 30 minutes and 1000 hours ageing at $550{ }^{\circ} \mathrm{C}$. Chan also performed experiments on a 'stabilised' microstructure. For this treatment the same solution anneal parameters were used, but with a $930{ }^{\circ} \mathrm{C}$ treatment for 30 minutes. Using the same parameters for the potentiodynamic experiment, pitting corrosion was found to initiate around $\mathrm{NbC}$ inclusions. Phuah [51], who also conducted research on the thermally sensitised 20/25/Nb stainless steel concludes that NbC secondary phases were found to be 'critical the pitting corrosion processes'. Phuah adds that the size of these 
$\mathrm{NbC}$ inclusions are important as this microstructures containing $0.1 \mu \mathrm{m}$ diameter precipitates differed in $\delta \mathrm{E}$, the difference in breakdown and free corrosion potentials, by $\approx 800 \mathrm{mV}$ compared to those containing larger 5 -12 $\mu \mathrm{m}$ diameter precipitates. In such examples pits formed around these inclusions of up to 2 by $5 \mu \mathrm{m}$ in size, confirmed by focussed ion beam scanning electron microscopy (FIB-SEM) methods. This allowed Phuah to mill a trench adjacent, or on, NbC particulates and examine the microstructure hidden by corrosion products and lacy covers. There was a plan for this research to secure time on a FIB-SEM to conduct similar analyses with an industrial partner (milling and subsequent imaging) due to reasons beyond the National Nuclear Laboratory and Swansea University's control this did not take place.

\subsection{Experimental}

TLM experiments were conducted on the as-received 20/25/Nb, thermally sensitised 20/25/Nb and thermally sensitised AISI 310 (UNS S31000). The thermally sensitised 20/25/Nb SS were prepared as described previously (chapter 3, figure 3.15) by mounting, then grinding and polishing at an angle to attain a graduation in the amount of sensitisation at the surface. The thermally sensitised 310 SS was prepared as standard and was used to compare IGC initiation for a different thermally sensitised material without $\mathrm{NbC}$ inclusions. The composition is given in table 4.1 and was thermally sensitised by NNL $\left(600{ }^{\circ} \mathrm{C}\right.$ for 50 hours).

The specimens had wires spot welded for an electrical connection, with the exposed wire encased within the resin. The specimens were immersed in $\mathrm{NaCl}$ electrolytes, and connected to a potentiostat (Palmsens 3) to allow either monitoring of OCP, or polarisation. The first experiment studied the thermally sensi-

\begin{tabular}{|l|l|l|l|l|l|l|l|l|}
\hline $\mathrm{C}$ & $\mathrm{Mn}$ & $\mathrm{Si}$ & $\mathrm{P}$ & $\mathrm{S}$ & $\mathrm{Cr}$ & $\mathrm{Mo}$ & $\mathrm{Ni}$ & $\mathrm{N}$ \\
\hline 0.25 & 2.00 & 1.50 & 0.045 & 0.030 & $24.0-$ & - & $19.0-$ & - \\
& & & & & 26.0 & & 22.0 & \\
\hline
\end{tabular}

Table 4.1: Composition of 310 stainless steel [137]. 
tised $20 / 25 / \mathrm{Nb}$ SS. The location exposed adjacent to the haematite scale (oxide surface scale classified in chapter 3). $0.01 \mathrm{~mol} \mathrm{dm}^{-3} \mathrm{NaCl}$ was used and the specimen was studied under the influence of external polarisation using an SCE and Pt gauze counter electrode both positioned as close as possible to the exposed area. A potentiodynamic experiment was conducted, polarising the specimen from free corrosion potential $\left(E_{C o r r},-130 \mathrm{mV} \mathrm{SCE}\right)$ to breakdown potential $\left(E_{b},+850 \mathrm{mV}\right.$ SCE). The TLM still images were cropped for ease of the viewer. The second experiment investigated the microstructure at the scale:metal interface in freely corroding conditions, using a more aggressive $\mathrm{NaCl}$ electrolyte $\left(3 \mathrm{~mol} \mathrm{dm^{-3 }}\right)$. The final experiment was a comparison to study the initiation and propagation of IGC for a different thermally sensitised SS, for this AISI 310 SS was used. This TLM experiment involved immersion in $0.2 \mathrm{~mol} \mathrm{dm}^{-3} \mathrm{NaCl}$ combined with a potentiodynamic sweep. Experiments using other $\mathrm{NaCl}$ concentrations were undertaken but due to issues with underfilm crevice corrosion (corrosion under the tape) these results were omitted. The specimen was polarised from $-300 \mathrm{mV}$ vs. $\mathrm{Ag} / \mathrm{AgCl}(\mathrm{OCP}-130 \mathrm{mV} \mathrm{Ag} / \mathrm{AgCl})$ to $1400 \mathrm{mV}$ vs. $\mathrm{Ag} / \mathrm{AgCl}\left(E_{b} 900 \mathrm{mV}\right.$ vs. $\mathrm{Ag} / \mathrm{AgCl}$ ). In the thermally sensitised 310 SS TLM experiment an $\mathrm{Ag} / \mathrm{AgCl}$ reference electrode was used. The relative potential of the $\mathrm{Ag} / \mathrm{AgCl}$ electrode relative to the SHE is $+210 \mathrm{mV}$ [58] (the SCE is $+240 \mathrm{mV}$ relative to SHE). As before, Meiji (MT7000) microscopes were used (x20 magnification) with the objective lens (x10 magnification) covered with a waterproof shroud to give x200 effective magnification. To perform the analysis of anode distance, first a zero line was chosen to indicate the scale-SS boundary (figure 4.1).

Following this, perpendicular lines were drawn to each of the anodes. The distances were converted from pixels to microns by using an image of a graticule taken at the same magnification. This was conducted to identify if there was a trend in the distance pits initiated relative to the haematite scale-metal interface. Further sensitised specimens were prepared for a study using SVET. The specimens were polished using 6 and $1 \mu \mathrm{m}$ diamond pastes, followed by $0.05 \mu \mathrm{m}$ 


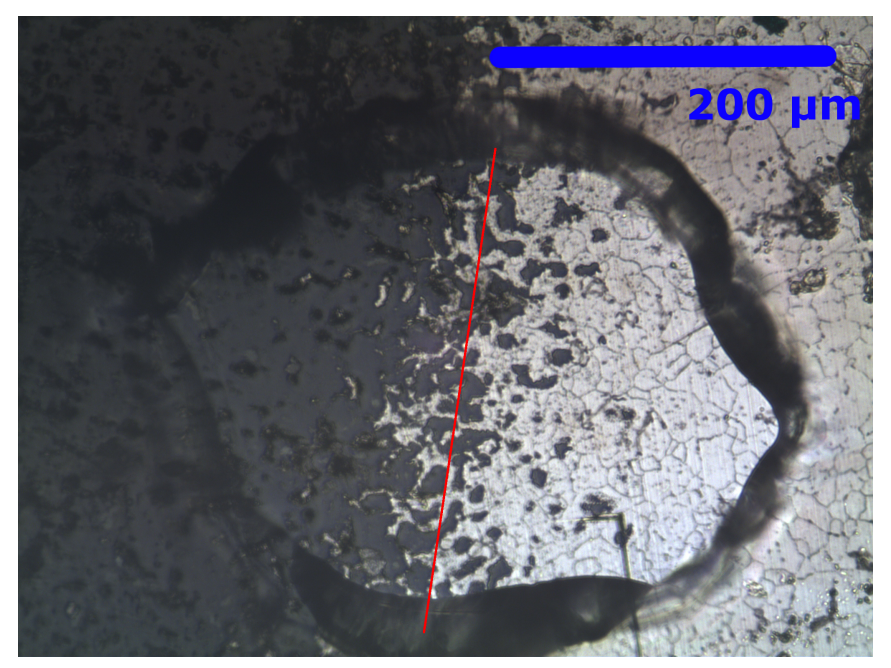

Figure 4.1: Annotated micrograph of the exposed area showing the location of the zero line for measurement of distance of anode to scale. The scale bar is $\approx 200 \mu \mathrm{m}$.

alumina paste to prepare the surface.

For the next experiment, a pre corrosion map was taken using a Zeiss Axial Observer microscope. Over 400 images were taken and stitched together to form a high resolution montage. The exposed surface was larger $(3.5 \times 3.5 \mathrm{~mm})$ so the montage was created optically. The specimen was also immersed in 1000 ppm $\left[\mathrm{Cl}^{-}\right]$and polarised to $E_{b}$. Tests using single static potentials (close to $E_{b}$ ) were already found to be inappropriate as $E_{b}$ varied depending on the amount of material ground away in the preparation process (chapter 3). Thus the potential was stepped at $50 \mathrm{mV}$ intervals from $0 \mathrm{mV}$ (SCE). Once the scan area was set using SVET, a scan was undertaken and timed. From this, a step time was calculated, at a rate of 4 SVET scans/step. 4 scans were chosen in order to provide more steady conditions (hence why a potentiodynamic sweep was not used). Prior to polarisation, 4 SVET scans were conducted at $E_{\text {corr }}$ to attain surface current density maps under freely corroding conditions. Following the corrosion experiment using SVET, a SEM (JEOL 6010-PLUS) was used to provide post corrosion microscopy and chemical analysis through EDS. 


\subsection{Results and Discussion}

\subsection{1 molar $\mathrm{NaCl}-20 / 25 / \mathrm{Nb}$}

The results of polarisation of the thermally sensitised SS in $0.01 \mathrm{~mol} \mathrm{dm}^{-3} \mathrm{NaCl}$ are shown in figure 4.2 .

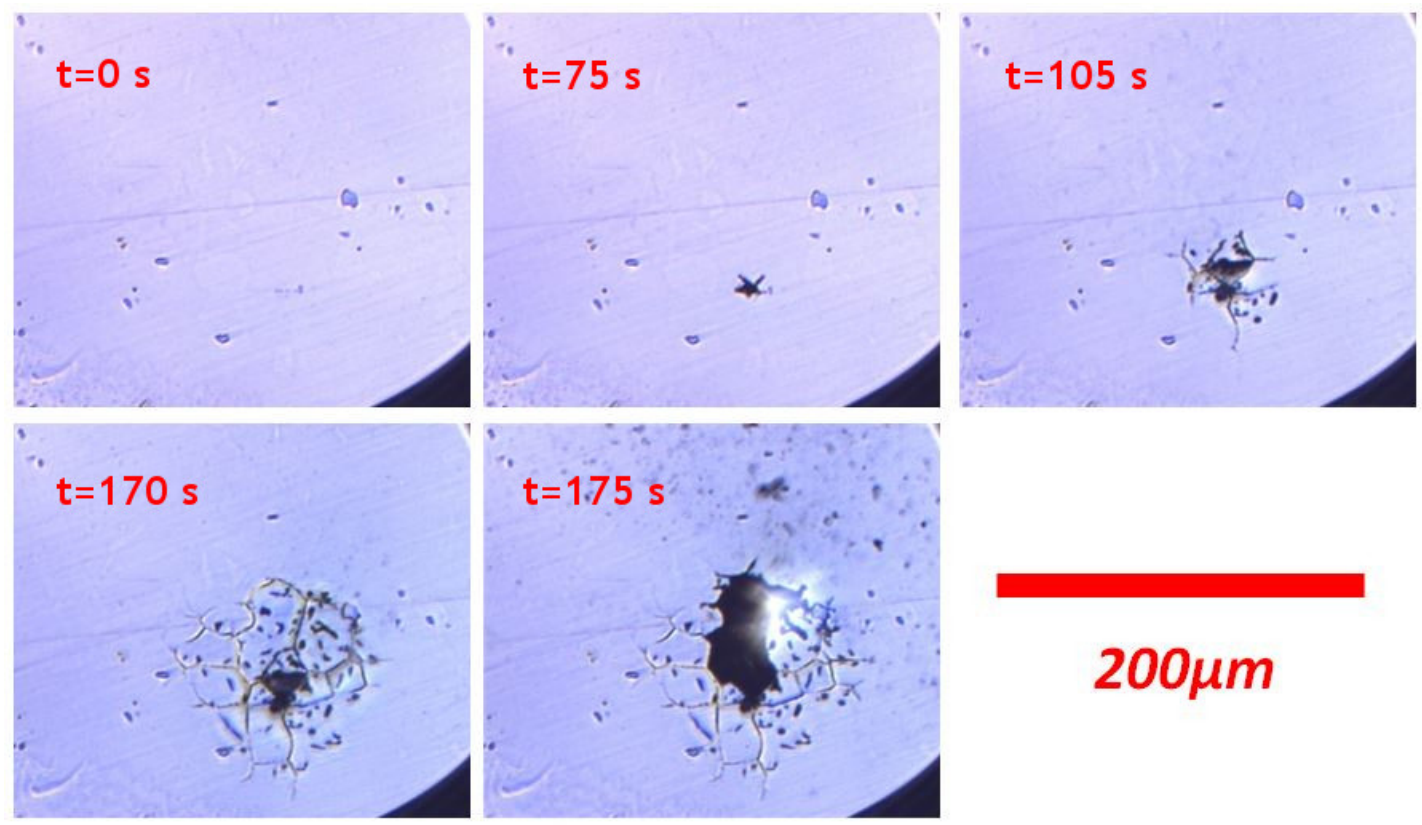

Figure 4.2: TLM stills from $0.01 \mathrm{~mol} \mathrm{dm}^{-3} \mathrm{NaCl}$ time lapse polarisation study. The study was published as an extended abstract at NACE Corrosion 2016 by Clark [114]

The specimen was polarised to $E_{b}\left(850 \mathrm{mV}\right.$ vs. SCE) from $E_{\text {Corr }}(-130 \mathrm{mV}$ vs. SCE). This indicates that the SS specimen is not highly sensitised, as in previous results using as-received materials showed a significantly smaller $\Delta \mathrm{E}$ (see chapter 3, $E_{\text {Corr }}$ (c.-150 mV vs. SCE), $E_{b}$ (c.600 mV vs. SCE)). The SS specimen was from a different part of the bar, but the same material which had received the same treatment as other thermally sensitised specimens. This could of course be an artefact to the used of a small exposed area, $0.2 \times 10^{-3} \mathrm{~cm}^{2}$, compared to $1 \mathrm{~cm}^{2}$ for conventional DC electrochemistry prepared specimens. This has an adverse effect on both the reference-working electrode distance, and more importantly, a decrease in the number of initiation sites. If a smaller area is exposed, the occurrence of suitable sites is smaller, leading to a lower initiation 
probability. If a site that might have been susceptible was not present then higher potentials would be required to cause corrosion to initiate.

The TLM stills show the initiation of IGC at a grain boundary intersection which is in line with the expectation that the grain boundary in a sensitised material is a less corrosion-resistant location, due to the nucleation and growth of $\mathrm{Cr}$ carbides causing the surrounding area to be depleted of $\mathrm{Cr}$, thus leaving areas sensitised and susceptible to corrosion. The grain boundaries in sensitised 20/25/Nb may also comprise of other phases, as described by Powell [113], such as nickel-niobium-silicide 'G-phase' and sigma phase, but are only found after 500 and 100 hours ageing at $600{ }^{\circ} \mathrm{C}$, so are unlikely to be present in the material described here. The initiation of IGC at a grain boundary intersection is in agreement with Chan et al. [45] who conducted potentiodynamic experiments $(-200$ to +1000 vs. OCP $)$ on the sensitised $20 / 25 / \mathrm{Nb}$ microstructure in $500 \mathrm{ppm}$ $\left[\mathrm{Cl}^{-}\right]$. The presence of an $\mathrm{NbC}$ inclusion (top right corner of figure 4.3) did not lead to initiation of IGC, at or in close vicinity. If the $\mathrm{NbC}$ inclusions are tracked through the still images, the inclusions themselves are largely unaffected (figure 4.3).

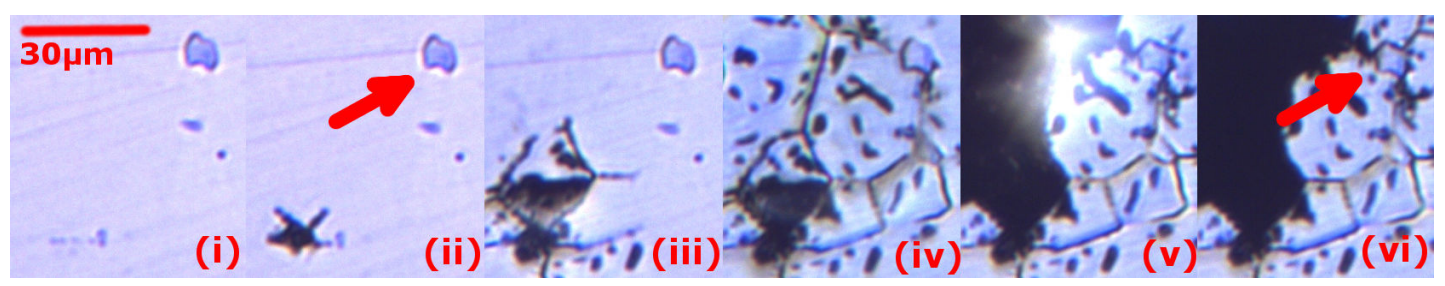

Figure 4.3: Crops of figure 4.2. Annotations show the NbC inclusion which is not affected by the IGC.

IGC propagates along the grain boundaries (Cr depleted areas) until it meets an NbC inclusion, whereby the corrosion traces around the impurity. The metal surrounding Cr carbides at grain boundaries is removed by corrosion, as it is sensitised, thereby undercutting the Cr-carbides and causing precipitates to become detached from the microstructure. Regarding the NbCs Phuah has previously suggested that the $\mathrm{NbC}$ act as a cathodic impurity, creating a micro-Galvanic 
cell whereby the surrounding metal undergoes anodic attack [51]. Post corrosion analysis has shown that the process of dissolution around the NbC follows two possible scenarios, (a) the $\mathrm{NbC}$ is removed from the microstructure and becomes aggregated within the electrolyte (i.e. it has not dissolved), (b) the $\mathrm{NbC}$ fall by gravity to the bottom of the pit with the surrounding matrix being dissolved. It is thought in case (b) that $\mathrm{NbC}$ become detached from the microstructure where the SS matrix is no longer attached to the inclusion. A schematic of this is provided for the viewer in figure 4.4. Evidence of this has been shown for other specimens using SEM (figure 4.6) corrosion pits have been observed at the matrix around NbC inclusions with some examples of where case (b) has occurred. An alternative for stage 2 in this figure is given in 4.5 . 
(i)

(ii)

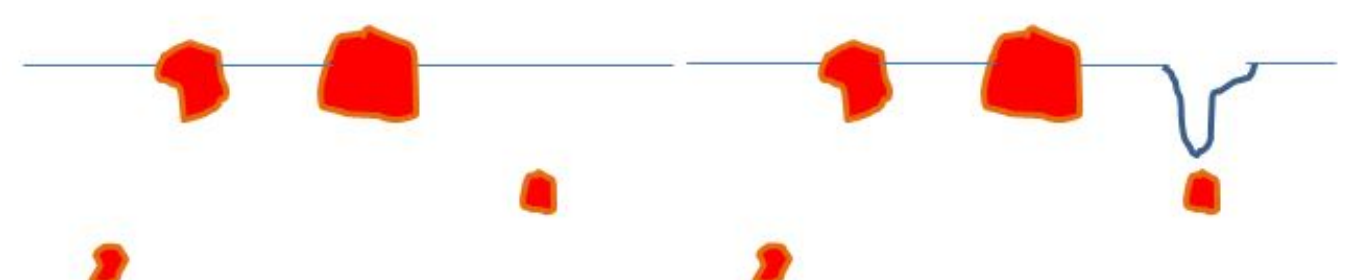

(iii)

(iv)

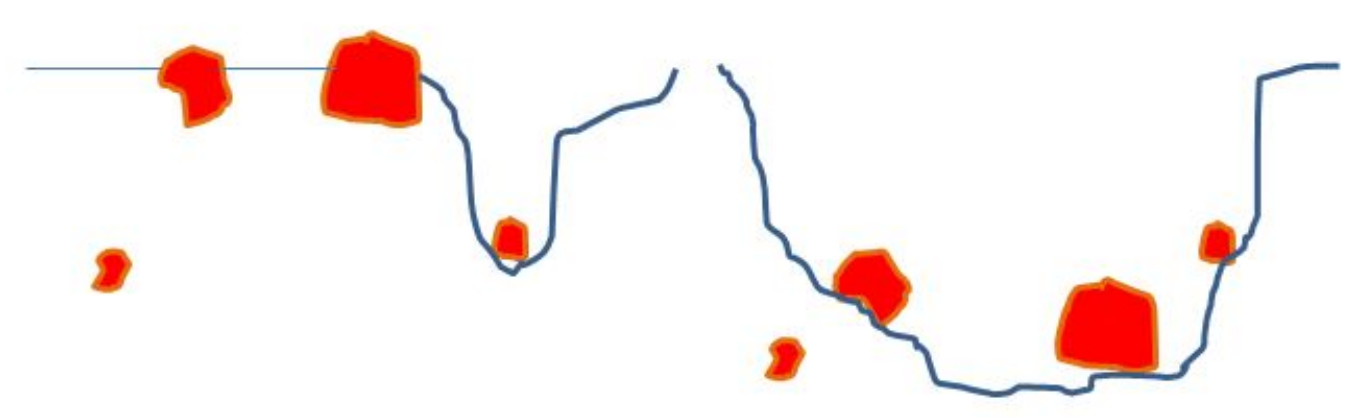

Figure 4.4: Schematic of how NbC inclusions are affected by the corrosion of the surrounding alloy. The red particulates are NbC inclusions. At (i) no corrosion has yet occurred, at (ii) a pit initiates, (iii) the pit propagates, but does not affect the inclusion, (iv) the corrosion causes other $\mathrm{NbC}$ to fall by gravity to the bottom of the pit. An alternative schematic for stage (ii) is provided in figure 4.5 
i)

ii)
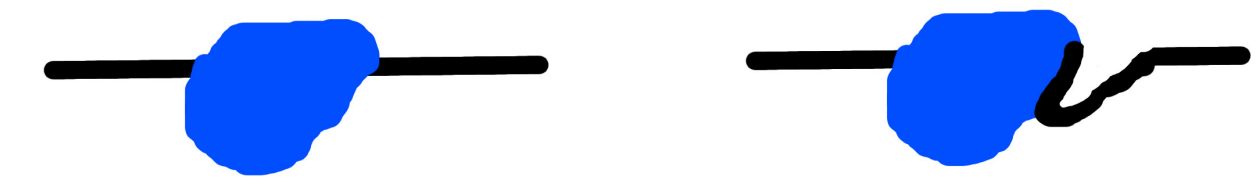

iii)

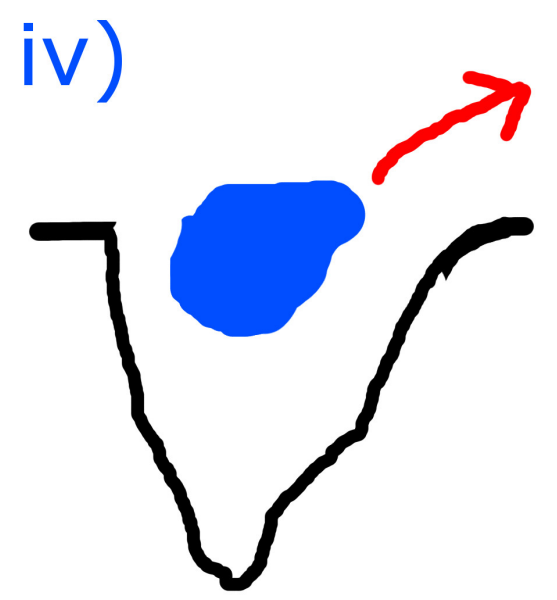

Figure 4.5: An alternative schematic for the case provided in figure 4.4(ii) 

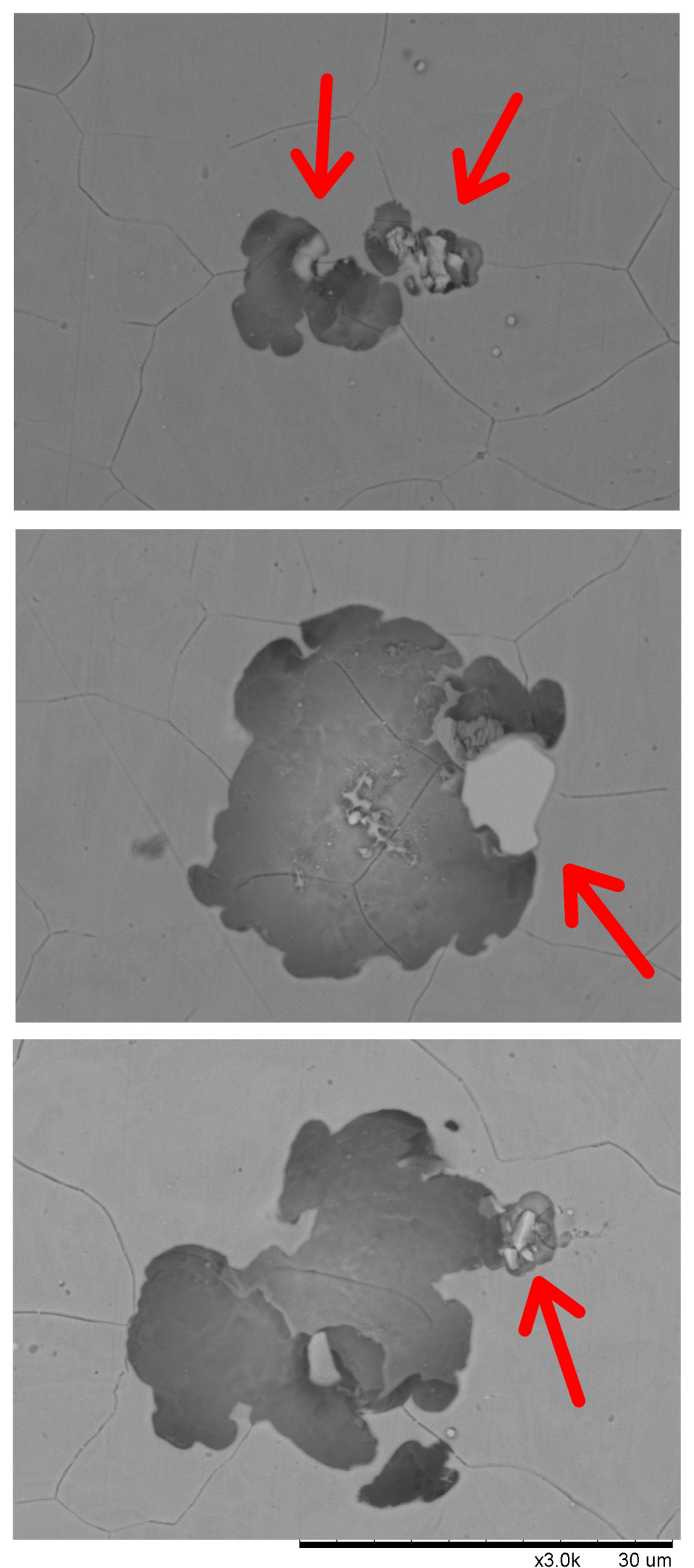

Figure 4.6: Post corrosion SEM micrographs of (unsensitised) SS in $0.01 \mathrm{~mol}$ $d m^{-3} \mathrm{NaCl}$ with pits witnessed at locations around $\mathrm{NbC}$. The example supports case (b), where $\mathrm{NbC}$ fall by gravity into pits, whilst the surrounding matrix is dissolved 


\subsubsection{3 molar $\mathrm{NaCl}-20 / 25 / \mathrm{Nb}$}

This experiment differs from the previous experiment as a much higher $[\mathrm{NaCl}]$ was used. IGC was found to occur spontaneously in the absence of external polarisation. A series of TLM micrographs showing the initiation of anodes within the sensitised SS are indicated in figure 4.7. Within $3 \mathrm{~mol} d m^{-3} \mathrm{NaCl}$ at $E_{\text {corr }}$ anode initiation occurred at a rapid rate, at the area previously described as being highly sensitised with the first initiating almost instantly and the last initiating at 4.5 hours. All anodes that initiated within this timeframe passivated by the next image (5 minute intervals were used), showing no further development. This infers that the corrosion current became concentrated in another area (next IG pit initiation point) that was more susceptible. The IG-pitting initiated at highly sensitised grain boundary intersections, but as post corrosion micrographs show, crevice corrosion was more dominant (figure 4.8). The post corrosion micrographs show that crevice corrosion propagated underneath the masked area. The locations of anodes were distributed within the exposed area. The sites were studied to understand if there was a location dependence, the results are shown in figure 4.13. The maximum distance away from the haematite scale-metal interface was $\approx 200 \mu \mathrm{m}$. This would indicate either that there is no dependence whatsoever, or that the distance was not great enough to get a relevant number of results. Previous tests showed corrosion preferentially propagates at the scale, as had occurred in this case, through the mechanism of crevice corrosion.

Anodes that initiated rapidly were analysed using post corrosion optical microscopy and SEM techniques. Optical microscopy images of the surface before and after corrosion are given in figure 4.9. The image has been annotated to show the locations in which corrosion initiated from the post corrosion overlay. Therefore, it is easy to see the same location before and after corrosion. The surface was imaged using an SEM in two different modes 5 and $20 \mathrm{KeV}$ shown in figures 4.11 and 4.12 , respectively. At the higher acceleration voltage the electron beam has a greater interaction volume and thus is more penetrating to the 
sample allowing images obscuring surface detail (such as corrosion product). 

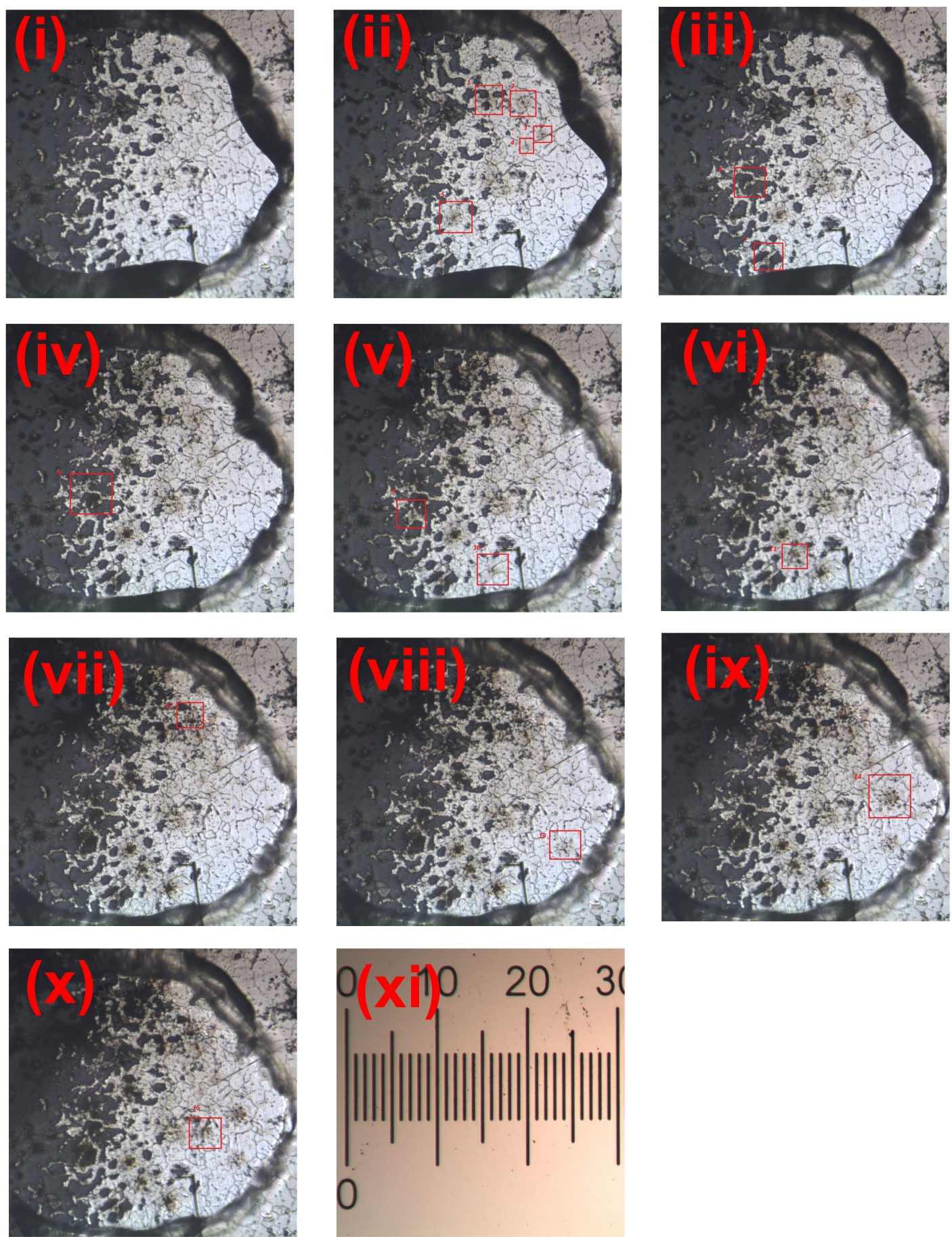

Figure 4.7: (i-x) Crops of SS in $3 \mathrm{~mol} \mathrm{dm}^{-3} \mathrm{NaCl}$ under freely corroding conditions. The crops have been selected to show the locations in which anodes initiated, from the TLM images at 20x. These have been marked in red boxes for ease of viewing. (xi) graticule scale bar, with the same crop factor applied. Each division represents $10 \mu \mathrm{m}$. 


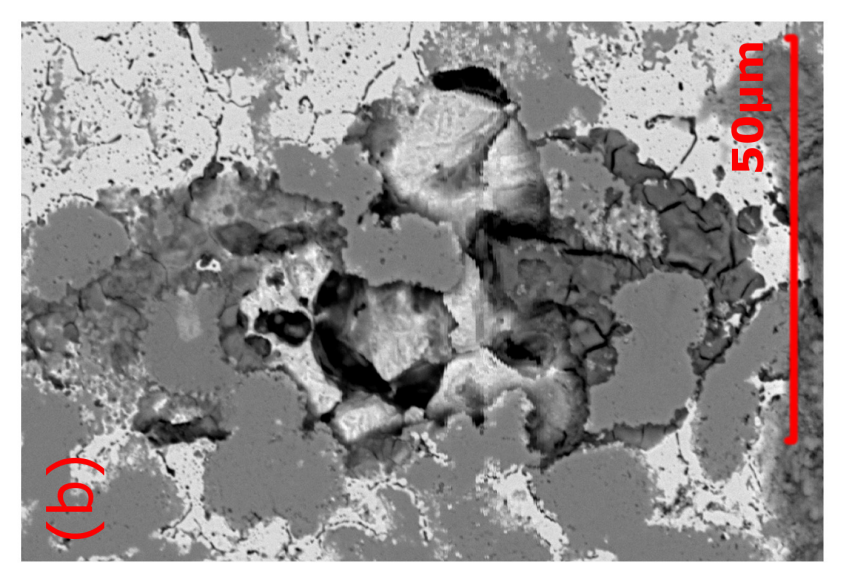

0
0
0
0
0
0
3
$\frac{0}{0}$
07
3
0
0
.7
0
0
0
0
0
0
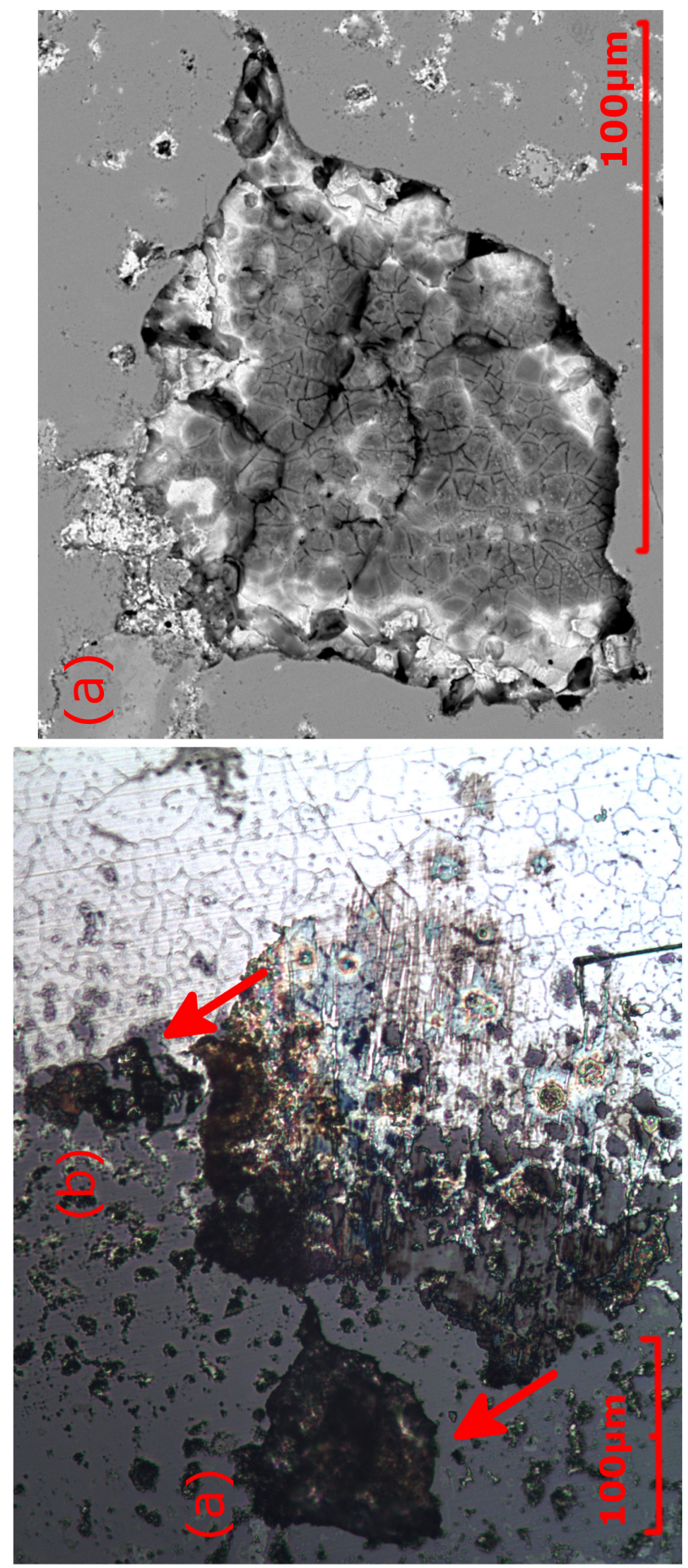

章

क

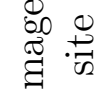

$\exists$

हैं

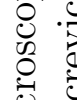

.

苞焉

क्ष

离

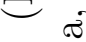

ن.

$\Xi$

毛.

की

壳

虫

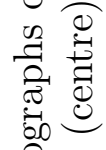

.

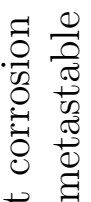

范

$\ddot{\infty}$

車

$\underset{:}{0}$. 


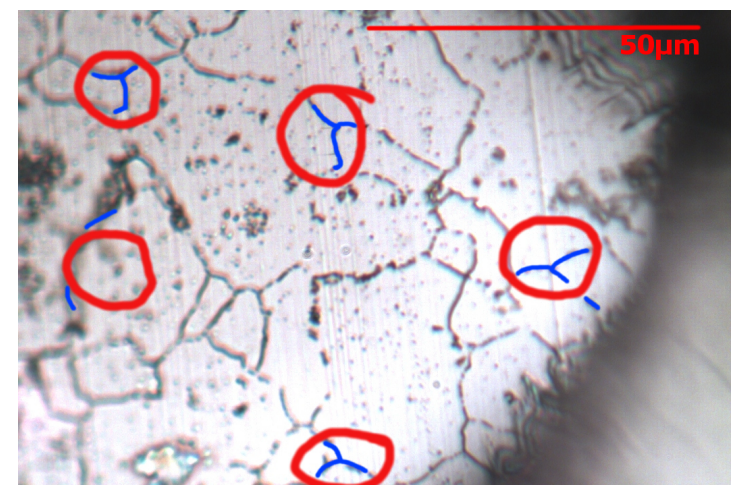

Figure 4.9: The exposed surface before corrosion had occurred.

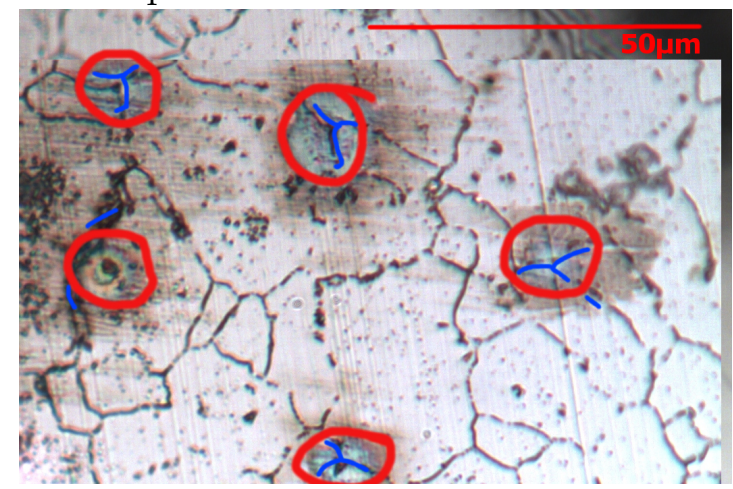

Figure 4.10: The exposed surface following corrosion.

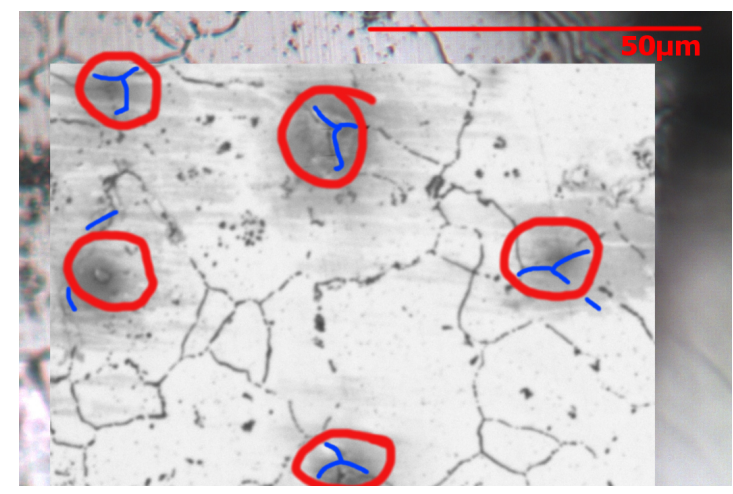

Figure 4.11: SEM micrograph of the surface $(5 \mathrm{KeV})$, annotated to show the location of anodes from the post corrosion optical image.

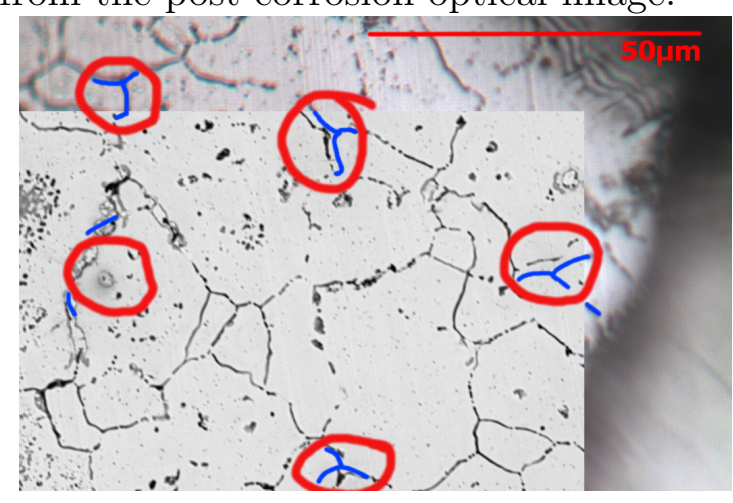

Figure 4.12: SEM micrograph of the surface (20 KeV), annotated showing the location of anodes from the post corrosion optical image. 


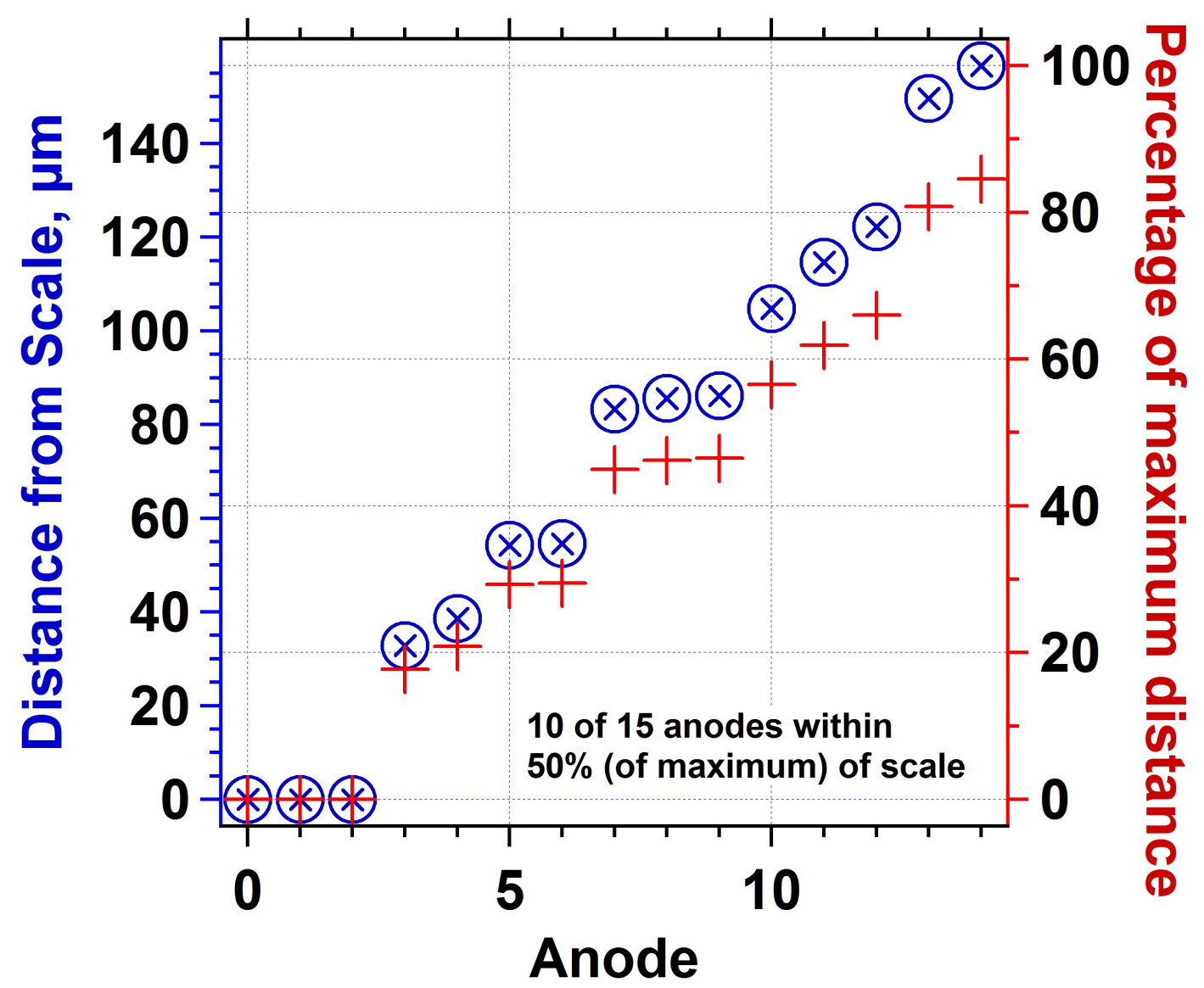

Figure 4.13: Distances in microns and percent of anodes from haematite scale. $2 / 3$ of anodes initiated within $50 \%$ of the scale. 


\subsubsection{Sensitised 310 Stainless Steel in 0.2 molar $\mathrm{NaCl}$}

The results of the comparison experiment using thermally sensitised 310 SS are shown in figure 4.14. In this experiment the SS was immersed in $0.2 \mathrm{~mol} \mathrm{dm}^{-3}$ and polarised using a three electrode cell set up. The duration of the experiment was 184 minutes and the specimen was polarised from $-300 \mathrm{mV}$ vs. $\mathrm{Ag} / \mathrm{AgCl}$ (OCP -130 mV Ag/AgCl) to $1400 \mathrm{mV}$ vs. $\mathrm{Ag} / \mathrm{AgCl}\left(E_{b} 900 \mathrm{mV}\right.$ vs. $\left.\mathrm{Ag} / \mathrm{AgCl}\right)$. Image 4.14(ii) shows transient events which had all initiated by 120 minutes but did not subsequently propagate. Stable IGC, where corrosion is no longer transient or random, occurred much later, at 167 minutes, shown in figure 4.14(iii) relating to $E_{b}$. Following these images IGC propagates rapidly, as the SS passed $E_{b}$, shown in successive images (figure 4.14 iv-vi).

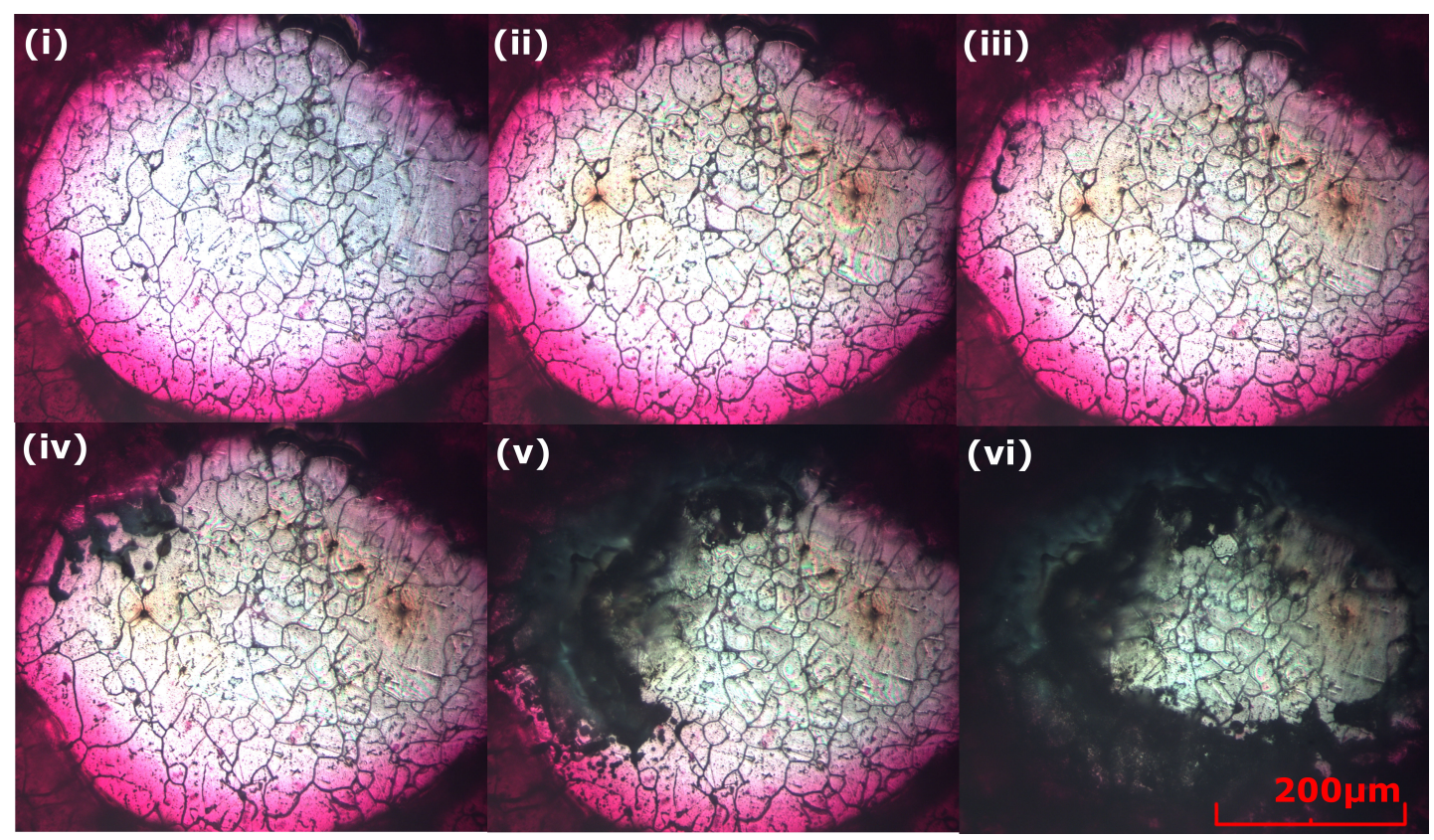

Figure 4.14: Thermally sensitised $310 \mathrm{SS}$ polarised to past $E_{b}(900 \mathrm{mV}$ vs $\mathrm{Ag} / \mathrm{AgCl}$ ) (image iii). Images (iv - vi) show rapid IGC propagation caused by polarisation past $E_{b}$

Crops of the metastable events are shown in figure 4.15. The image shows the sites before and after corrosion had occurred. Corrosion sites A, B, C, D, F, G and $\mathrm{H}$ all occur at grain boundary intersections. E, and I occur at locations along the grain boundary. The data shows that IGC initiation is most commonly confined to intersections or triple points, in line with the previous experiments in this 
chapter using the $20 / 25 / \mathrm{Nb}$ austenitic SS. The reasoning for this increased susceptibility to corrosion is not clearly understood, but two reasons are suggested. Possibility (A): grain boundaries are locations where voids and defects are located, and intersections are areas where crystals (grains) meet. Consequently at these points increased localised stress may be present. Such a possibility would indicate that the added stress gives rise to corrosion initiation, similar to SCC, but without the crack propagation. Possibility (B): intersections between two or more grain boundaries (for example a triple point) show increased Cr-depletion. Clark et al. presented this theory at the NACE corrosion conference [114].

Stable IGC occurs once $E_{b}$ is reached, and initiates at two locations, in the centre of a grain boundary and at an intersection (figure 4.16(ii)). IGC propagates between the two initiation locations (figure 4.16(iii)). Interestingly, IGC does not necessarily follow a straight path, instead re initiating at locations close to the anode, possibly at areas where Cr-carbides are located, as these locations will be more highly depleted of Cr (figure 4.16(iv)). 

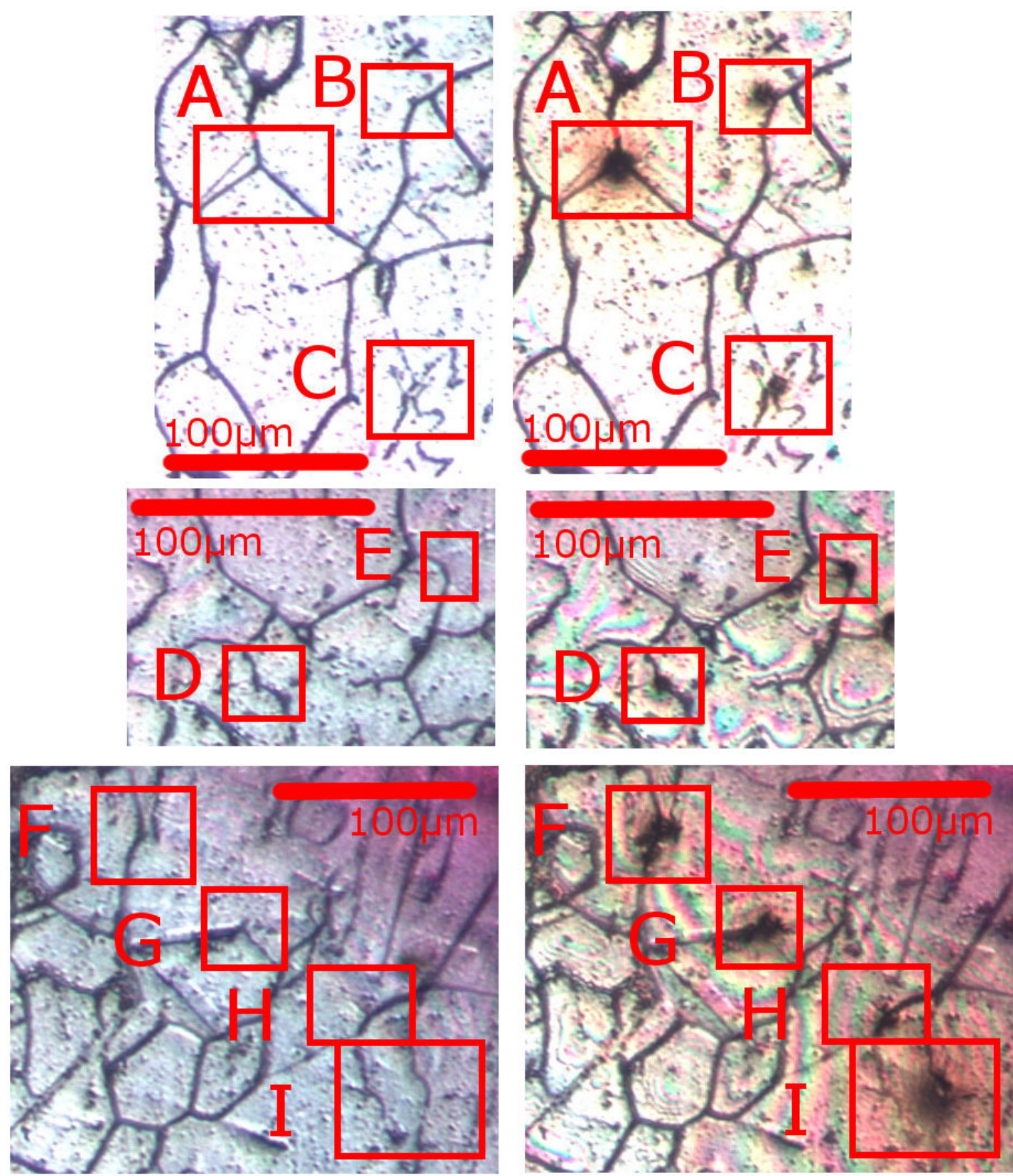

Figure 4.15: Crops of metastable events. Each of these sites passivated.

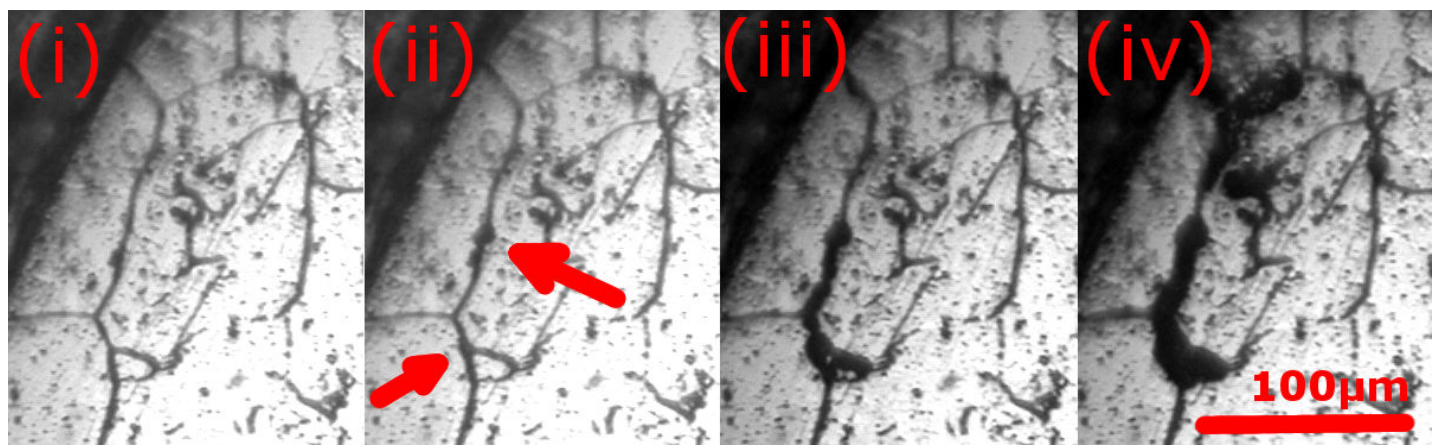

Figure 4.16: Cropped images of stable IGC initiation on the sensitised 310 SS in $0.2 \mathrm{~mol} \mathrm{dm}^{-3}$. Image (ii) has been annotated to show the initiation location. (iv) shows the propagation of IGC to areas more heavily depleted of Cr. 


\subsubsection{SVET Analysis - 1000 ppm Chloride - Sensitised 20/25/Nb Stainless Steel}

The surface of the thermally sensitised 20/25/Nb SS was mapped optically as shown in figure 4.17(i) and a comparison after the corrosion experiment is shown in figure 4.17 (viii). The rest potential $\left(E_{\text {corr }}\right)$ was $-480 \mathrm{mV}$ (vs. SCE) after 600 $\mathrm{s}$ immersion in $1000 \mathrm{ppm}\left[\mathrm{Cl}^{-}\right]$. This concentration equates to approximately 0.03 moldm $^{-3} \mathrm{NaCl}$ (see table 2.2). This was used as lower concentrations had resulted in difficulties using SVET given the low conductivity of the electrolyte. As the potential was changed in $50 \mathrm{mV}$ steps (every $1000 \mathrm{~s}$ from $0 \mathrm{mV}$ (vs. SCE)) it was calculated that $E_{b}$ had to have occurred at $750 \mathrm{mV}$ (vs. SCE), a total change of potential of over 1 volt. As suggested in the previous sections such a figure is large for a material which should have much reduced corrosion resistance, suggesting that the grinding stage removed much of the most sensitised area.

The surface current density maps are shown in figure 4.17. Corrosion initiation is shown by the formation of anodes, which initiate between image 4.17 (ii) and image 4.17 (iii). The image shows 6 anodes, the centre of the frame shows two anodes. The anodes do not appear to be well resolved, this is limited by the SVET sensitivity that was selected and the large step in over-potential. There is also a small contribution from the spatial resolution of the instrument itself. Higher sensitivities allow for small changes in current density to be measured, i.e. initiation, but may cause an overload on the instrument. The current densities have become 'topped out' and therefore only an estimate can be provided, which represents a minimum value. Nevertheless, the electrochemical (SVET) data shows correlation between the spatially resolved SVET current density map and microscope images (see image 4.17 (viii)).

It is expected that the anode at the top left of image 4.17 (iii) was first to initiate as it shows the largest current density (labelled as site B in figure 4.17 (iv)). Between the images (iii, 4 hours 11 minutes) and (iv, 4 hours 16 minutes) anode G initiates. An optical macro image of the exposed surface is shown in figure 4.19, 
and an annotated SEM micrograph of the same location is given in figure 4.20. 

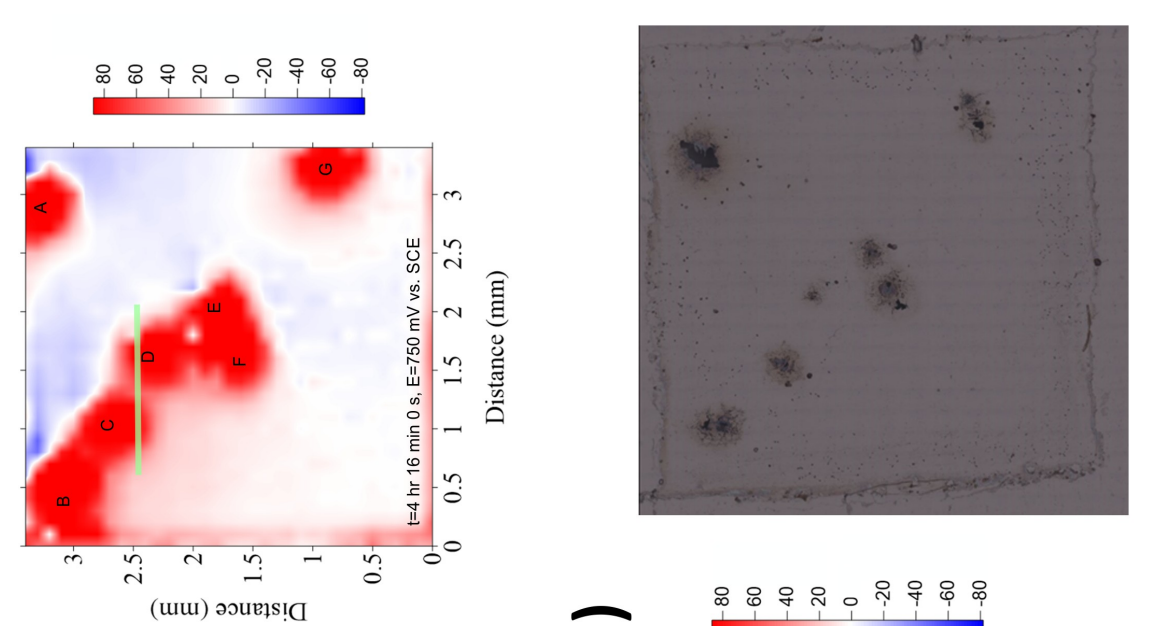

๘)

品

党言

:ᄏ

$\infty_{0} \infty_{0} \infty$

घี

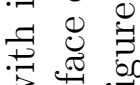

胥

हैं क ज

.

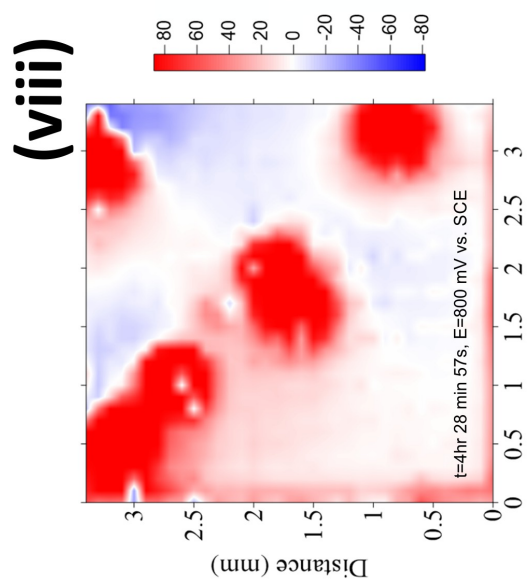

Чै山

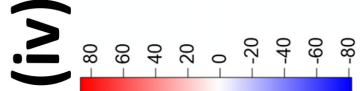

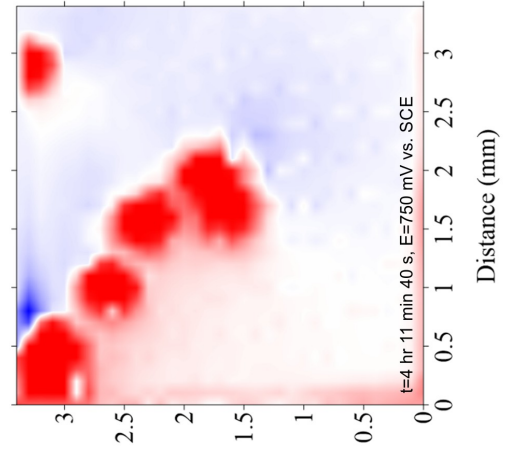

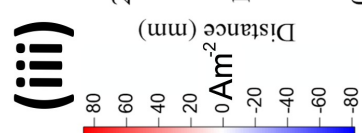

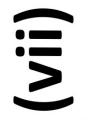

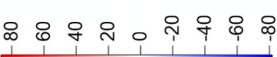

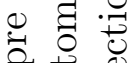

(1)

○.

苛息

必艺

Чับ

8 ह $\frac{\pi}{\pi}$

疍.

.

$\approx 0 \mathrm{H}$

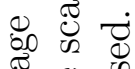

胥
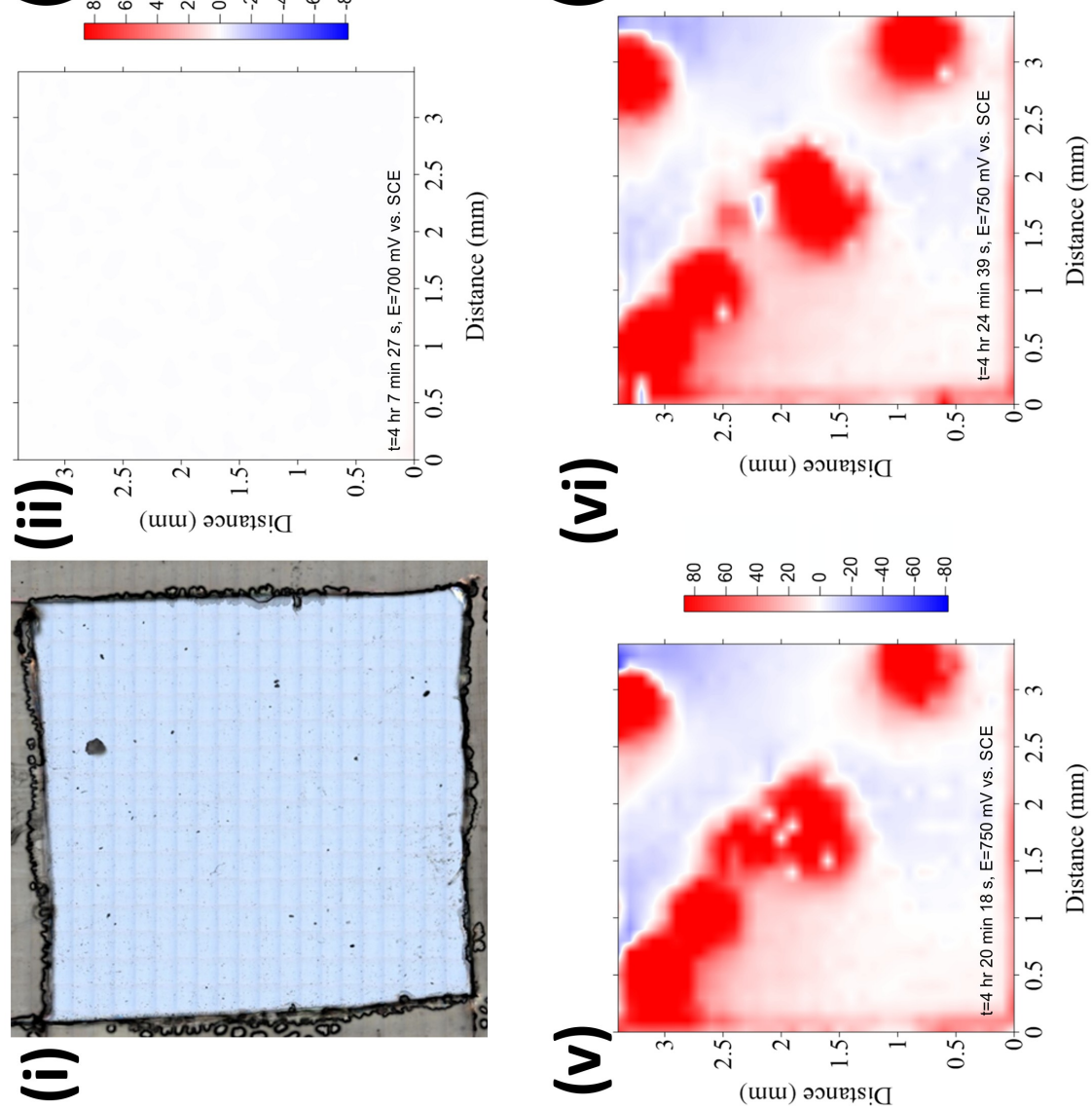

$\because \nsubseteq$

:F

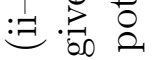

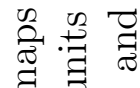

卷苛

ठै

氙

夏

040

晃 00

क ष

륨

成 क

क ज

등

मे 0.

$\stackrel{\pi}{3}$

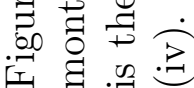




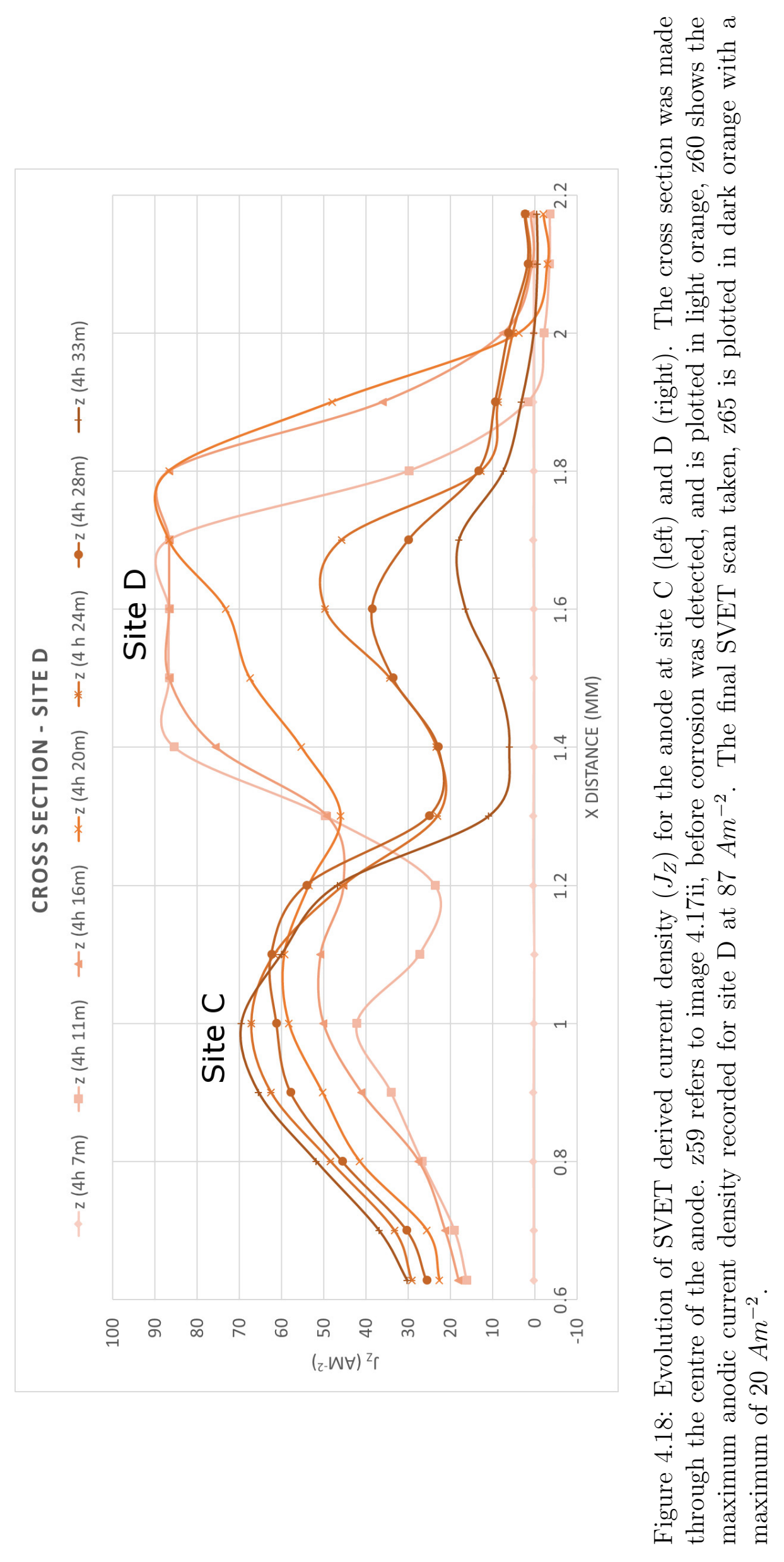




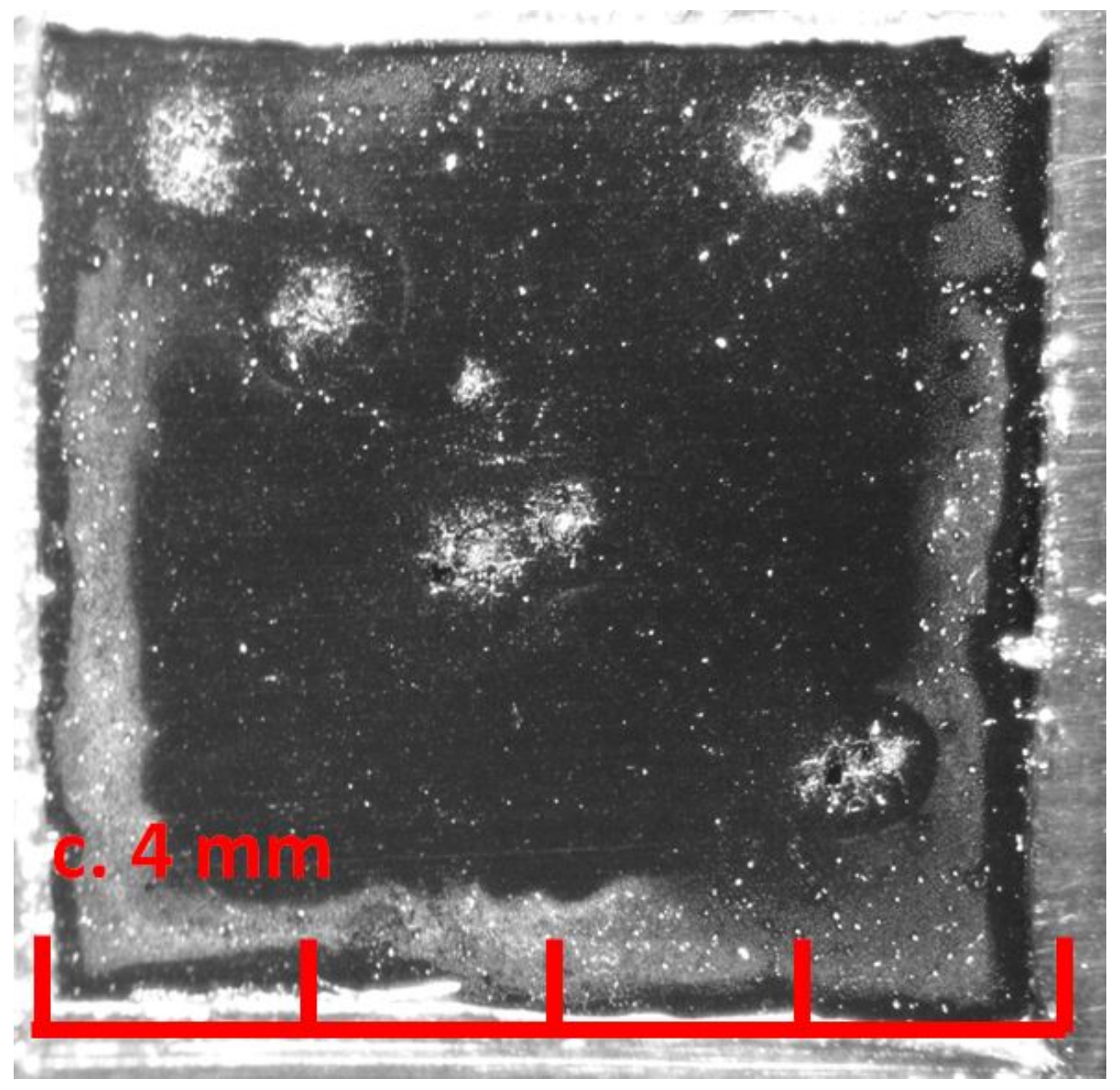

Figure 4.19: Macroscope image of the surface post corrosion. Each division is equivalent to $1 \mathrm{~mm}$, the entire bar is $4 \mathrm{~mm}$.

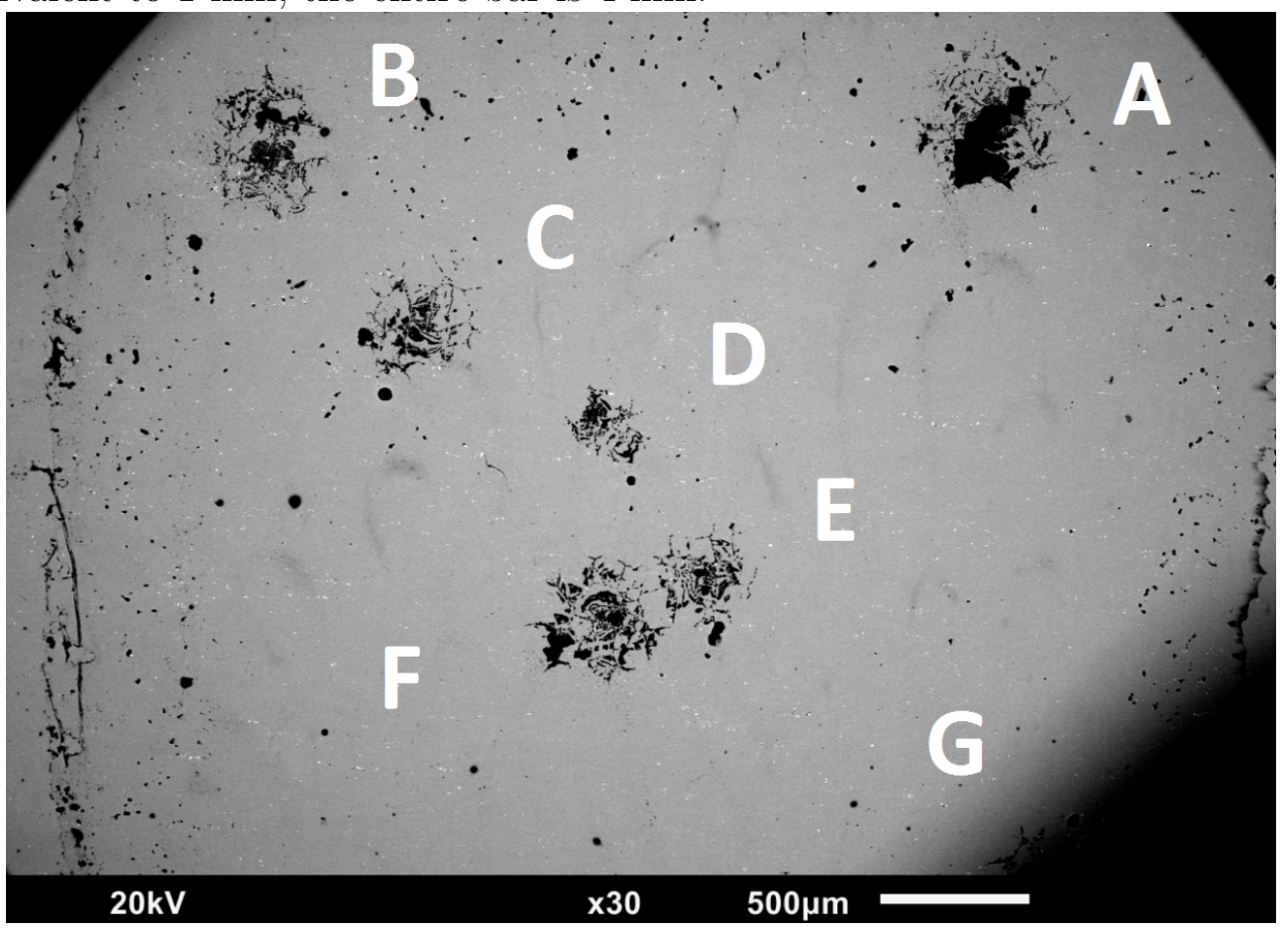

Figure 4.20: SEM map of the surface post immersion 
Anode D, which initiated in image (iii, 4 hours 11 minutes) shows passivation by image (vi, 4 hours 24 minutes), evidence that the site struggled to compete against nearby sites of high current density. A cross section of the surface current density map was taken to analyse this further. The plot in figure 4.18 shows the SVET derived current density $\left(J_{Z}\right)$ as a function of distance across site D the location of the different sites are given in figure 4.20). The plot in figure 4.18 shows two peaks, the left peak arises from site $\mathrm{C}$ whilst the second peak represents the cross section for site D. The first SVET scan shown (4 hours 7 minutes) shows no net current density change across the site. By the time of the next scan (4 hours 11 minutes) the SVET reaches signal of $87 \mathrm{Am}^{-2}$ and in this occurrence the signal has reached a maximum. The profile of current density around the pit is reflective of what is happening to the current distribution around the pit. At the pit edges the current flux is constrained at the pit edges, when compared to the centre of the pit, which is reflected in the current density cross sections in 4.18. The maximum current density is sustained for the following 3 scans, although by 4 hours 20 minutes the pit appears to start repassivation as the signal does not appear topped out. The scans thereafter show the decrease of current density over time from peak current density of $87 \mathrm{Am}^{-2}$ (4 hours 11 minutes) to $20 \mathrm{Am}^{-2}$ (4 hours 28 minutes). This decrease in peak current density at site D is typical of pitting corrosion whereby repassivation at this site has occurred, thus allowing the adjacent pit (site C) to increase in peak current density over time. An SEM image of the pit at site $\mathrm{D}$ is shown in figure 4.21 , which compares to the pits at $\mathrm{E}$ and F (current density map in figure 4.22). The number concentration of Nb-rich particulates (showing as white in the backscatter electron (BSE) micrographs) is greater at sites $\mathrm{E}$ and $\mathrm{F}$, compared to $\mathrm{D}$. The passivation of the anode $\mathrm{D}$ might also be attributed to the formation of a new anode at site $\mathrm{G}$ in image (iv), as this becomes larger, anode D decays. The surface current density map indicates some regions of the surface were acting as a cathode (shown as blue). This is not a true representation of the current, as the specimen surface is under potential 
control externally.

Post corrosion EDS chemical analysis shows an $\mathrm{O}, \mathrm{Cl}$ and $\mathrm{Nb}$ enrichment at the pit site. EDS reveals that $\mathrm{Cl}$ and $\mathrm{O}$ are present together at the same locations in figure 4.23. Interestingly, a greater concentration of $\mathrm{Nb}$ appears to be associated with the IG-pitting corrosion at site A. A more detailed view of the pit is shown in figure 4.24. In order to check $\mathrm{Nb}$ was more strongly associated with pit sites, background EDS scans were taken away from the pit and this finding was confirmed. Figure 4.24(ii) shows a smaller Nb signal, when compared to (ii), where there are a greater number of Nb-rich inclusions present. This is in agreement with Chan [45] who indicated that the 'Nb-rich precipitates play an important role in the corrosion process' as through post corrosion analysis by SEM showed that pits formed around Nb precipitates in stabilised microstructure of 20/25/Nb. Phuah [51] indicated that NbC inclusions in 20/25/Nb may form a Galvanic couple with the matrix, causing cathodic protection of the inclusion. 


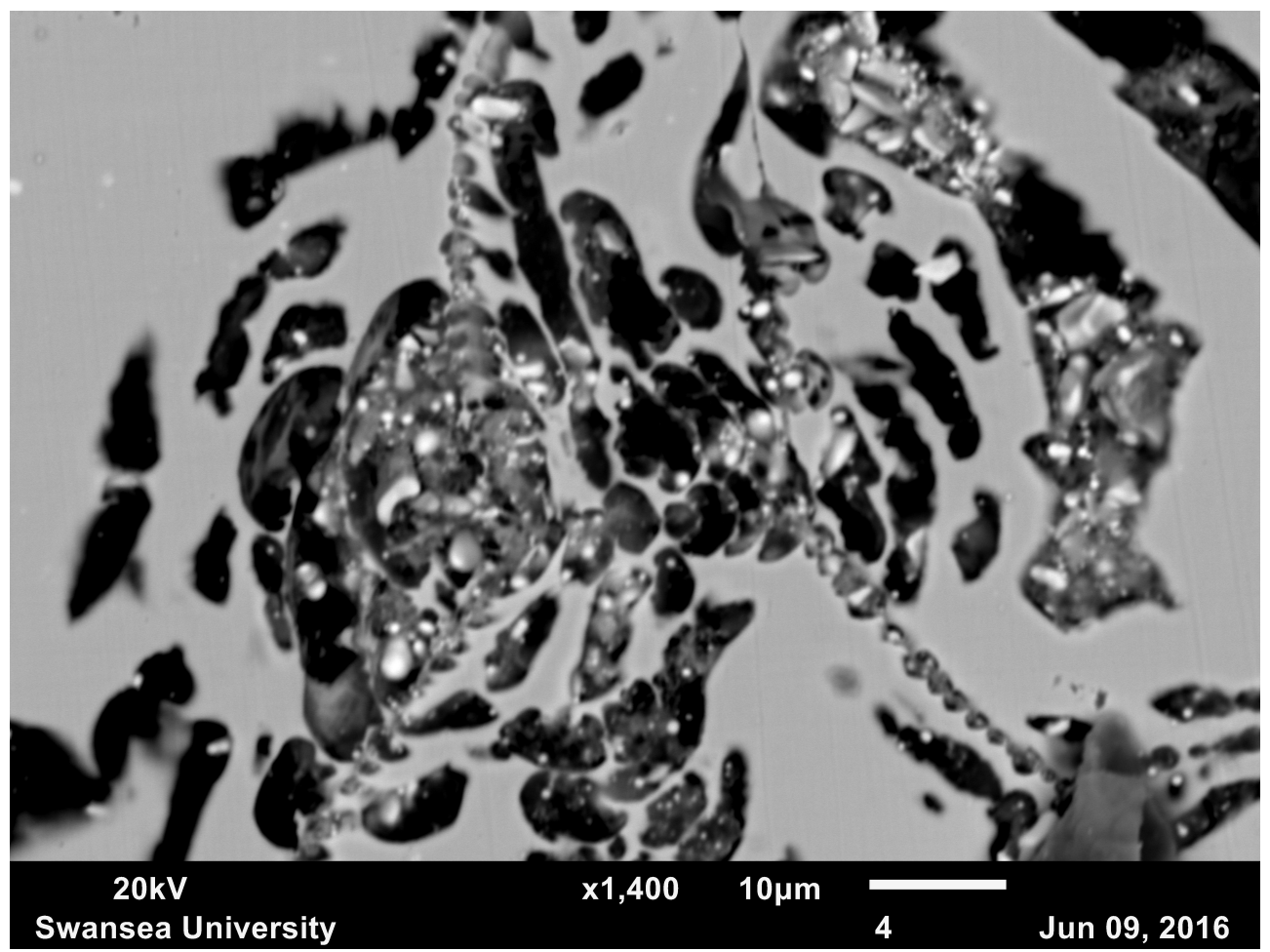

Figure 4.21: Site D (1400x). Nb-rich inclusions are present within the pit itself, shown as white in the BSE micrograph.

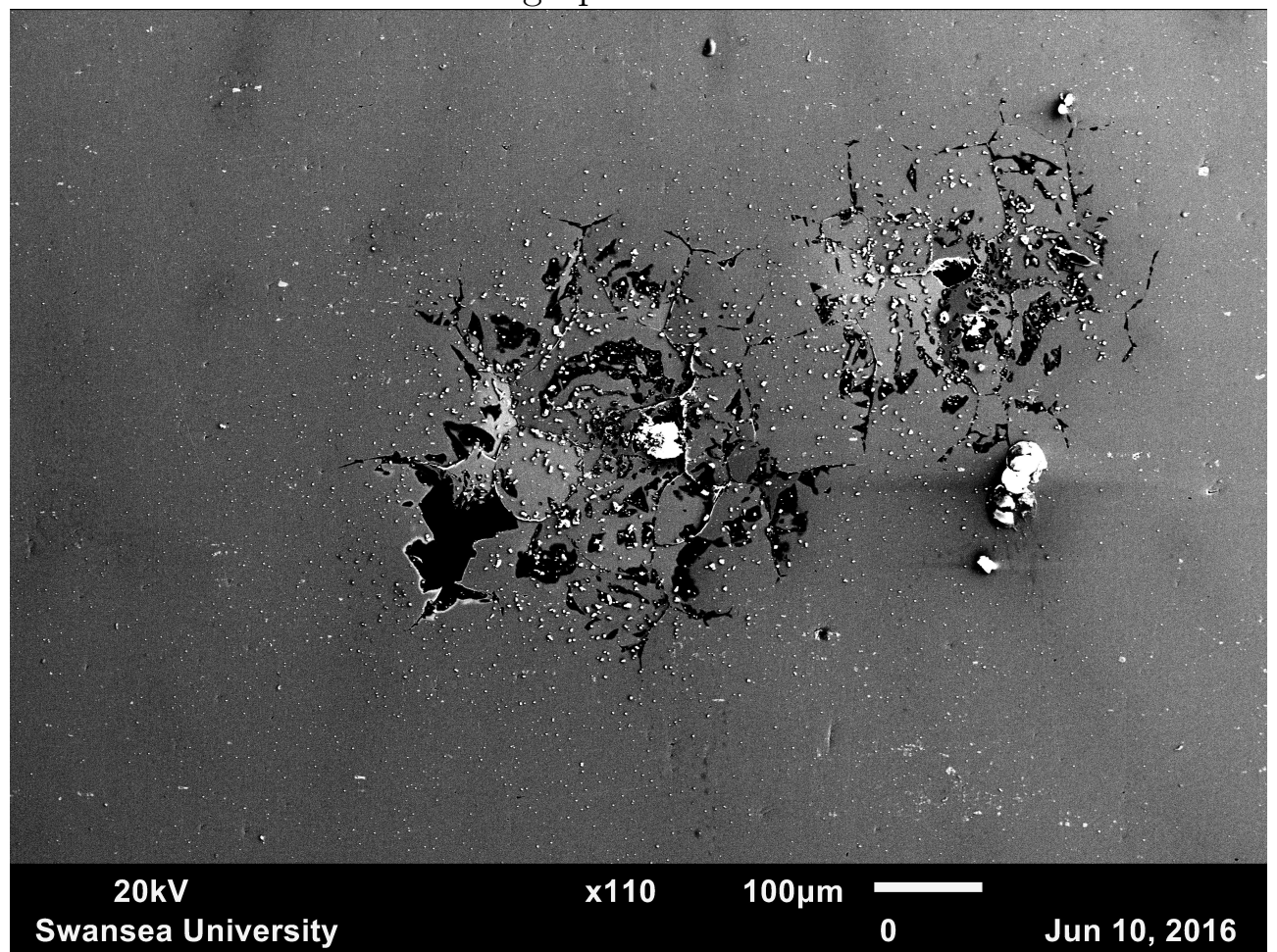

Figure 4.22: SEM of intergranular pitting corrosion at site F (left) and E (right). Nb-rich inclusions are shown as white. The large $\approx 50 \mu \mathrm{m}$ particulates are a result of contaminants and not $\mathrm{NbC}$. 

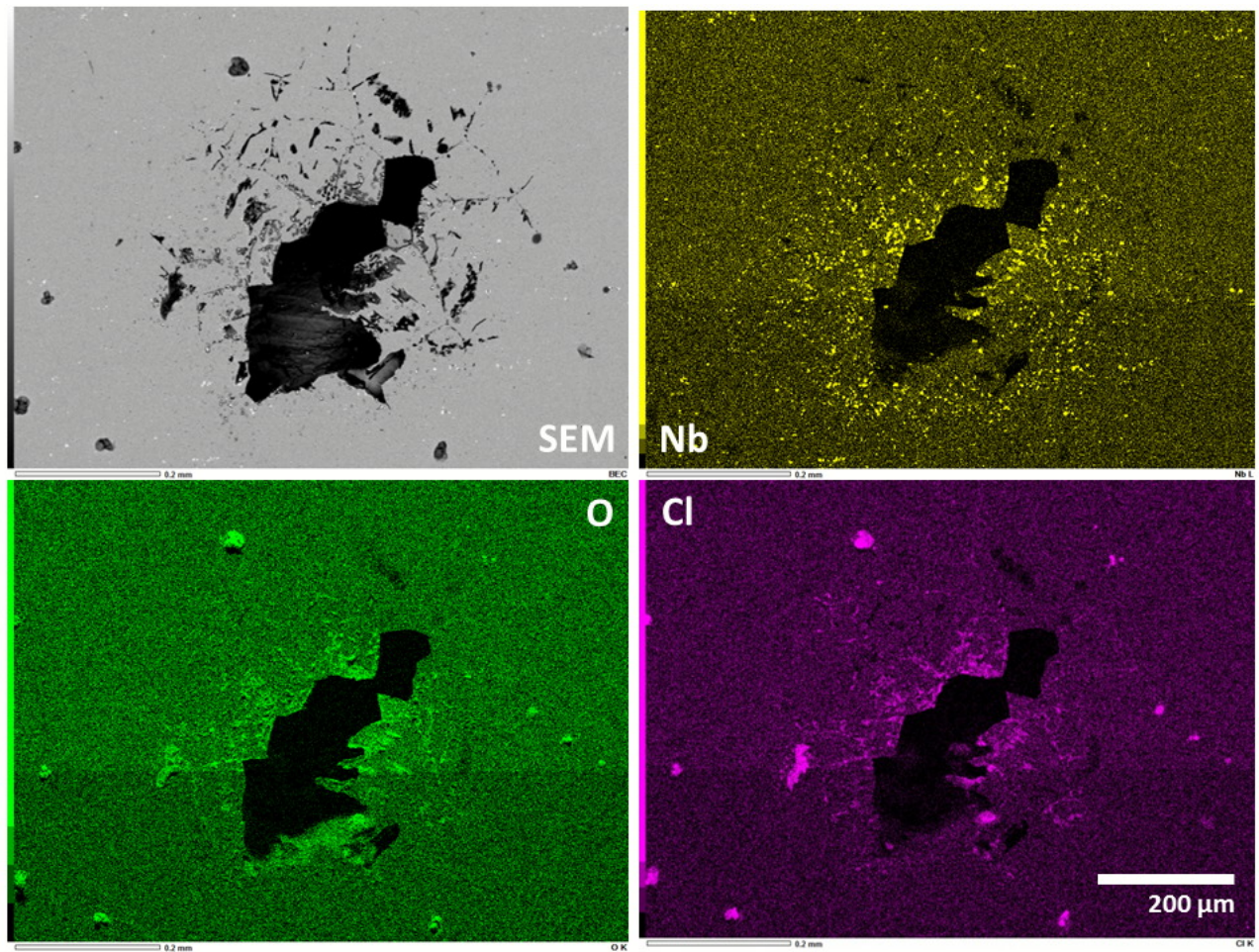

Figure 4.23: SEM and respective EDS measurement for site A (150x).
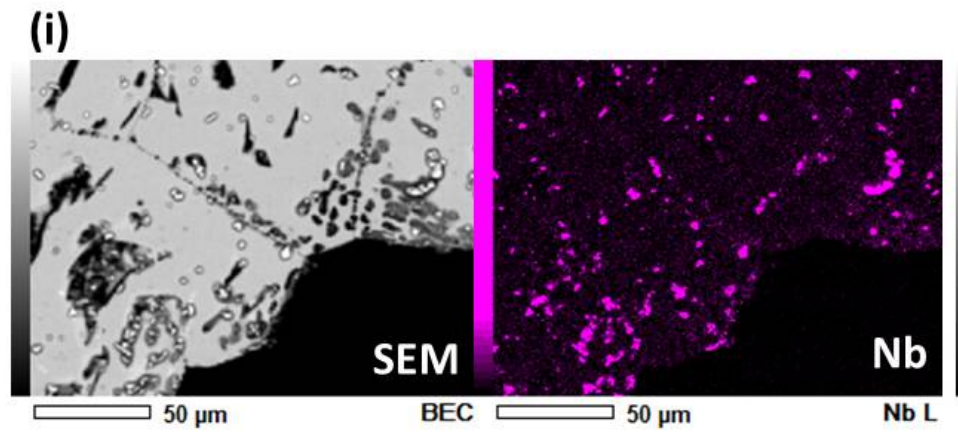

\section{(ii)}
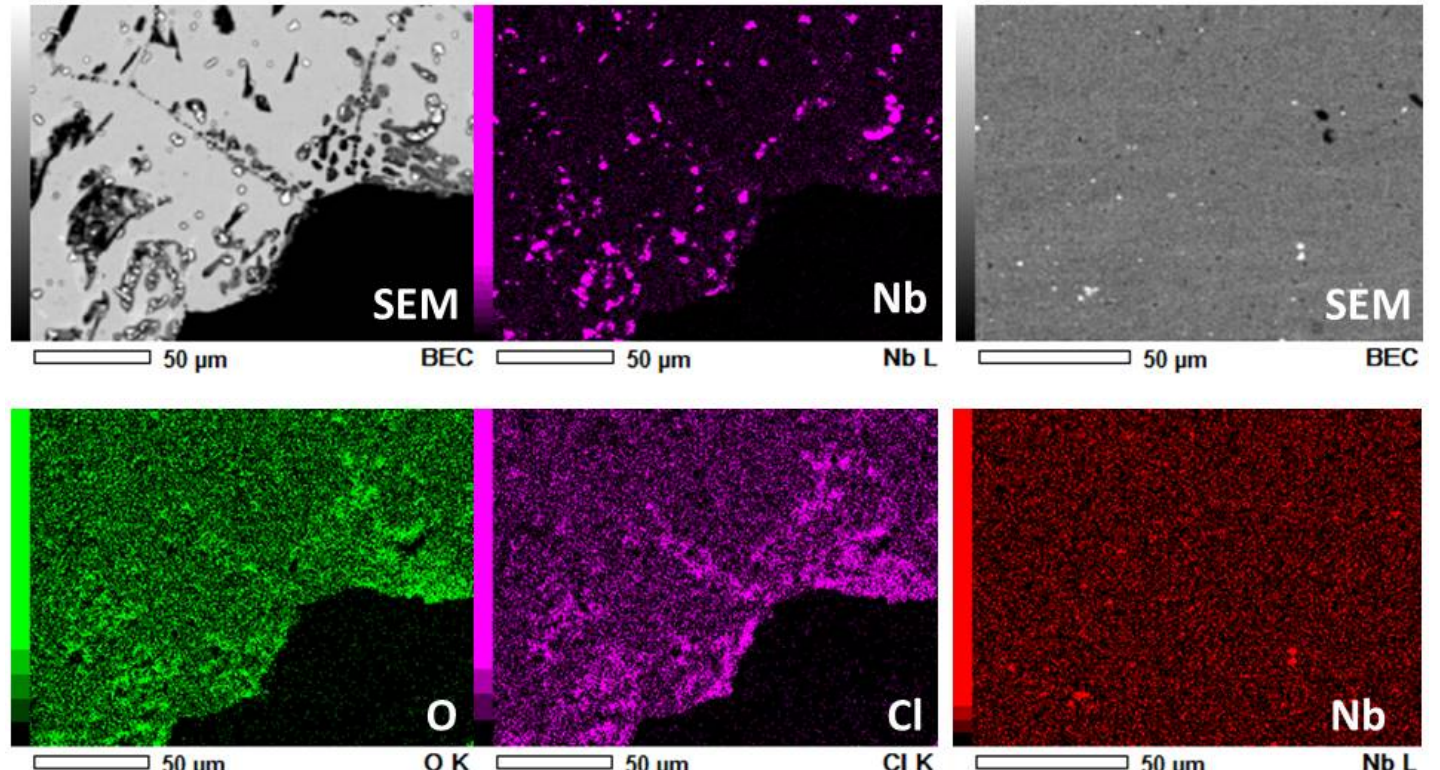

Figure 4.24: SEM and respective EDS measurement at (i) pit site A. Note in these images the $\mathrm{Nb}$ and $\mathrm{Cl}$ signals are both shown in pink. (ii) away from the pit site. In this image the $\mathrm{Nb}$ signal is shown in red. 
The pit at site B (figure 4.25) shows a strong Nb signal at the lacy cover. Again, $\mathrm{O}$ and $\mathrm{Cl}$ appear to be associated together, a consequence of corrosion in $\mathrm{NaCl}$, leading to the formation of iron oxides. This suggests that $\mathrm{Cl}$ has an affinity for areas around where $\mathrm{Nb}$ is located. $\mathrm{O}$ is enriched due to the oxidation reaction.

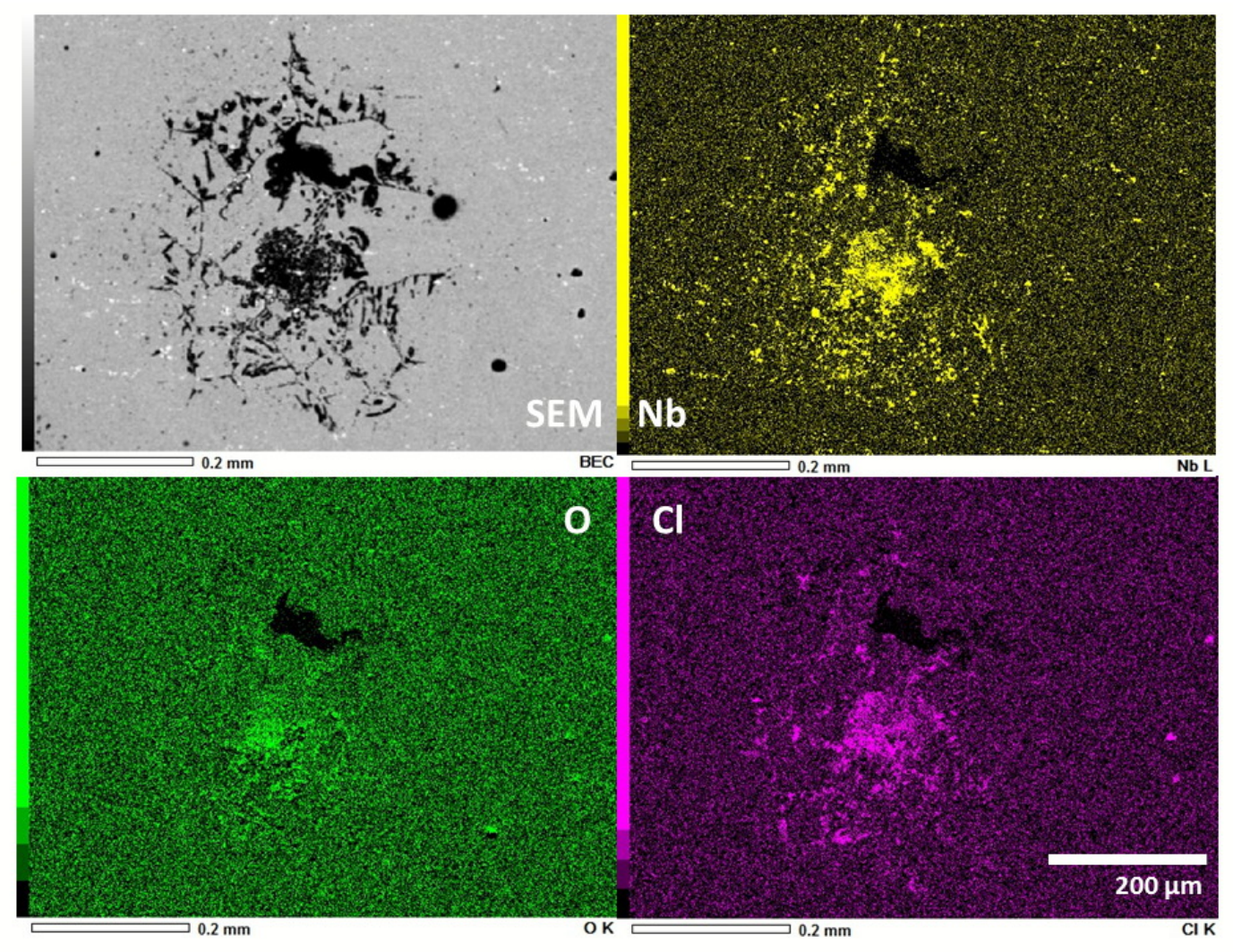

Figure 4.25: SEM and respective EDS measurement for site B (170x). There is distinct $\mathrm{Nb}$ enrichment at the lower location of the image close to the pit. $\mathrm{O}$ and $\mathrm{Cl}$ signals are also associated with the lower part of the image.

The SEM and EDS images (Nb signal overlaid) at this magnification are shown in figure 4.26. At the low magnification it is difficult to understand where exactly and why the $\mathrm{Nb}$ is associated with pit sites, so both 400x and 800x maps were conducted at the site, for more detailed analysis. Again, the SEM and EDS images were overlaid for ease of viewing. The 400x image (figure 4.27) shows that $\mathrm{Nb}$ is present as inclusions, with the largest cluster at the pit site itself. A closer look at the microstructure $(800 \mathrm{x})$ in figure 4.28 reveals that the $\mathrm{Nb}$ is in fact dispersed within the pit itself. $\mathrm{Nb}$ rich inclusions are present outside of the pit, but the $\mathrm{Nb}$ inside the pit itself appears more finely divided. 


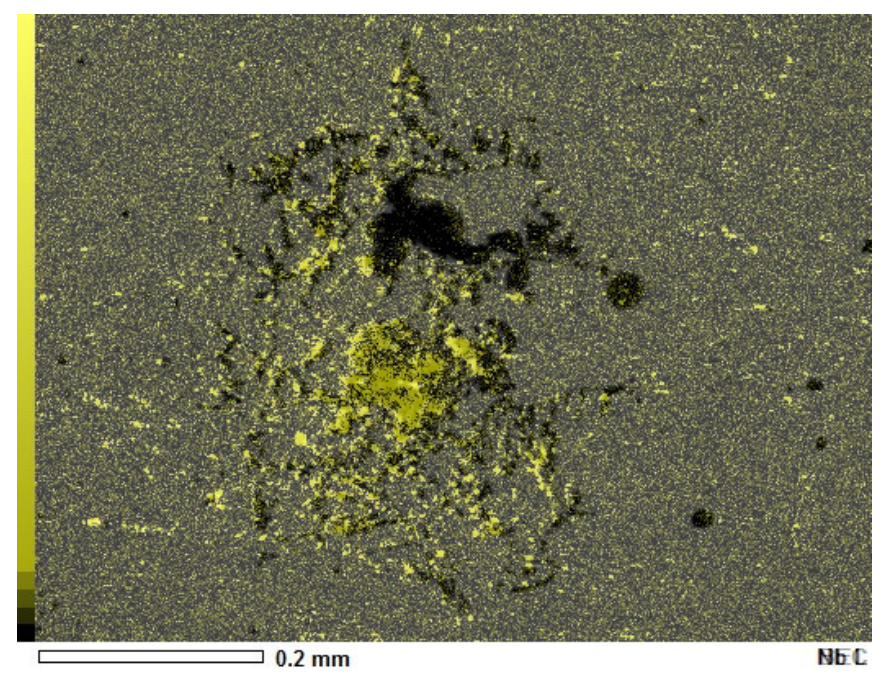

Figure 4.26: EDS map for site B (170x) with the SEM image overlaid. Yellow represents $\mathrm{Nb}$. The scale bar is $200 \mu \mathrm{m}$.

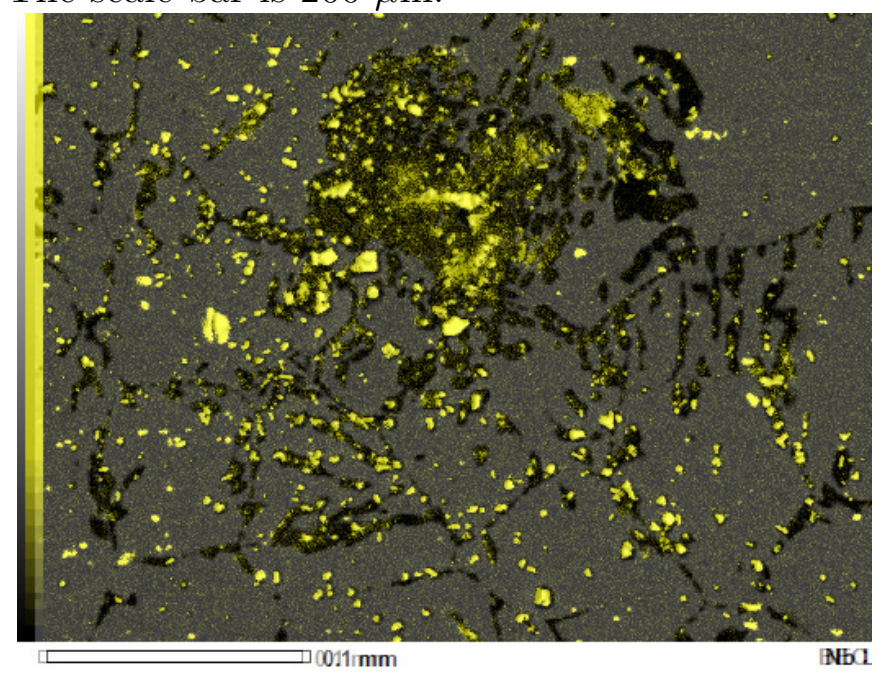

Figure 4.27: As above, site B, at 400x magnification. The scale bar is $100 \mu \mathrm{m}$

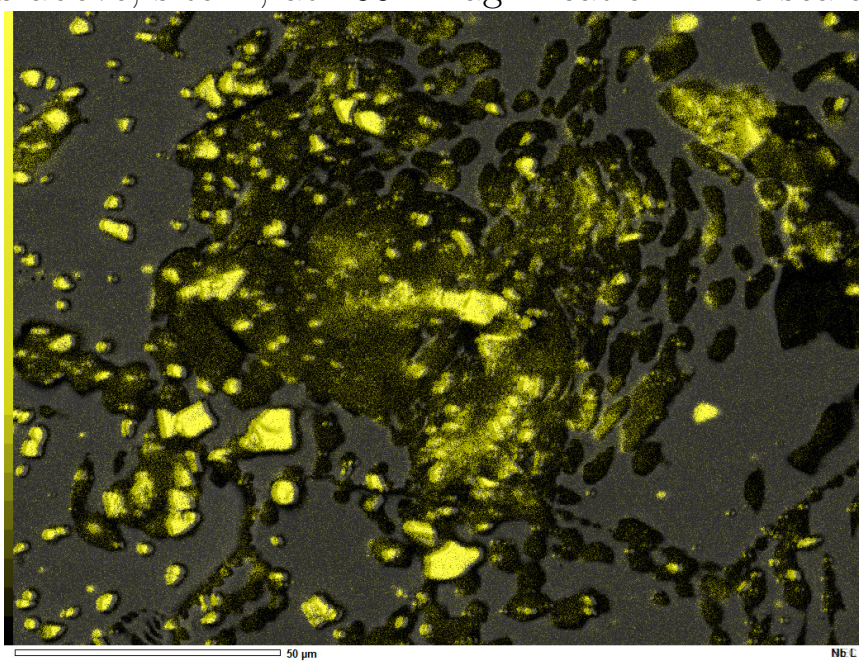

Figure 4.28: As above, site B, at 800x magnification. The scale bar is $50 \mu \mathrm{m}$ 
As EDS is not truly a surface science technique (its interaction volume is measured in microns, not nanometers) the dispersion could be caused by quantities of $\mathrm{Nb}$, present subsurface, rather $\mathrm{Nb}$ dispersed as fine particulates at the surface. This adds to the speculation that $\mathrm{NbC}$ inclusions are a precursor for corrosion initiation. The SVET has shown the locations in which corrosion has initiated, and SEM/EDS has indicated this is in areas where Nb is clustered. The higher magnification images indicate that corrosion propagates through to areas where $\mathrm{Nb}$ is even more concentrated, at the subsurface. Further evidence of the enrichment of $\mathrm{Nb}$ at the mouth of the pit is shown in the SEM image at site $\mathrm{D}$ (figure 4.21 and figure 4.29). This $\mathrm{Nb}$ enrichment continues with other pit sites, C (figure 4.30), F (figure 4.31), G (figure 4.32).
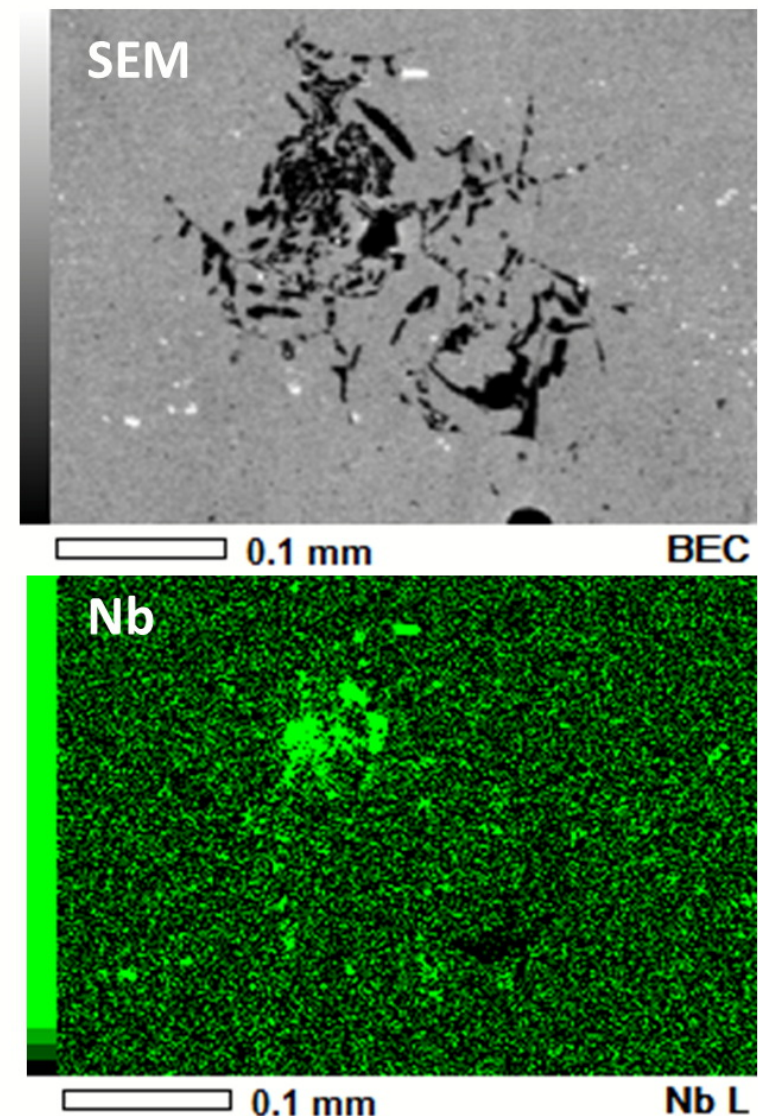

Figure 4.29: SEM and respective EDS measurement for site D (figure 4.21) at a lower magnification, 200x. The map confirms the increased concentration of $\mathrm{Nb}$ rich particulates closer to the pit. 


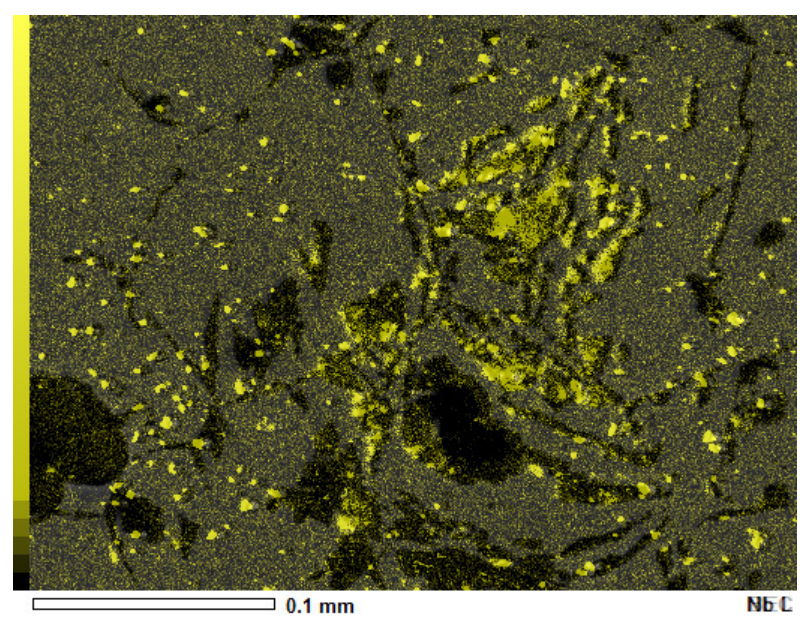

Figure 4.30: SEM and respective EDS measurement for site C (400x). The scale bar is $100 \mu \mathrm{m}$.

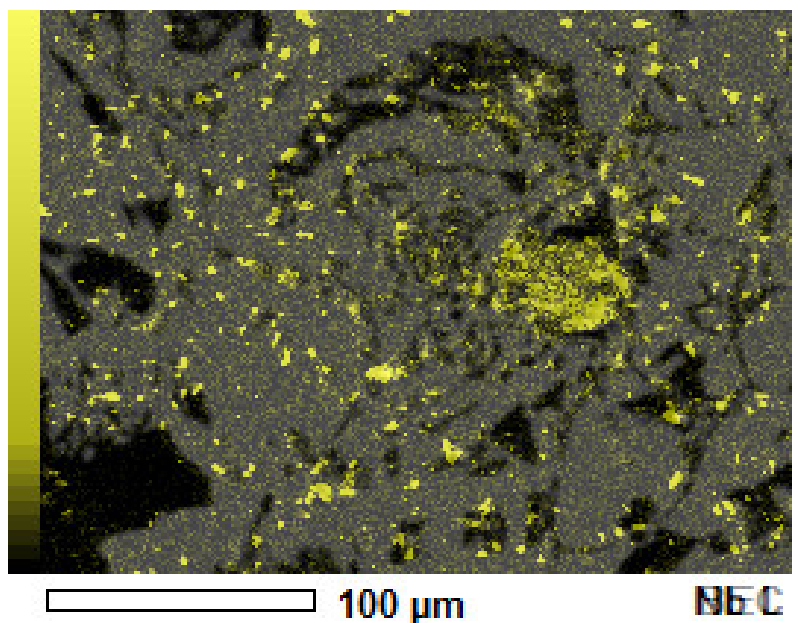

Figure 4.31: EDS measurement for site F (450x) (figure 4.22). The image is overlaid above the SEM image for easier viewing.

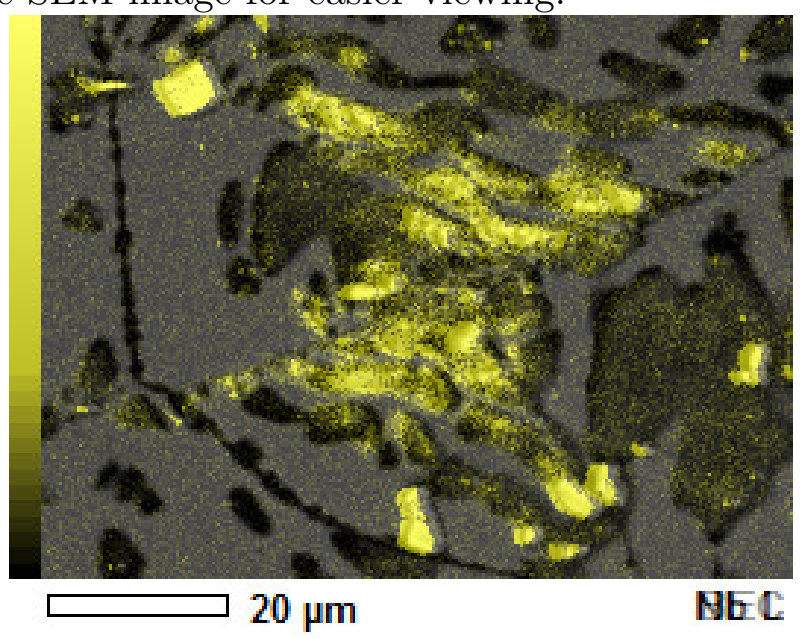

Figure 4.32: Site G lacy cover from figure 4.37. The SEM and EDS maps have been overlaid at 1500x. The scale bar is $20 \mu \mathrm{m}$. 
Again Nb-rich inclusions are present within the pit at site G (figures 4.36, 4.38 and 4.37). The amount of $\mathrm{Nb}$ can easily be seen from the micrograph at site E also (figure 4.22) as Nb-rich inclusions appear white, scattering due to the different atomic number. Figures 4.33 and 4.34 show the location of one of two pits at site G . An EDS map was taken of the pit cavity in figure 4.35 but failed to detect elements, it is thought that this was due to the inclusions present within the cavity being too far from the focus point of the detector. It is easy to see that there are a number of Nb-rich inclusions within the cavity, within a small area (the scale bar is $5 \mu \mathrm{m}$.) 


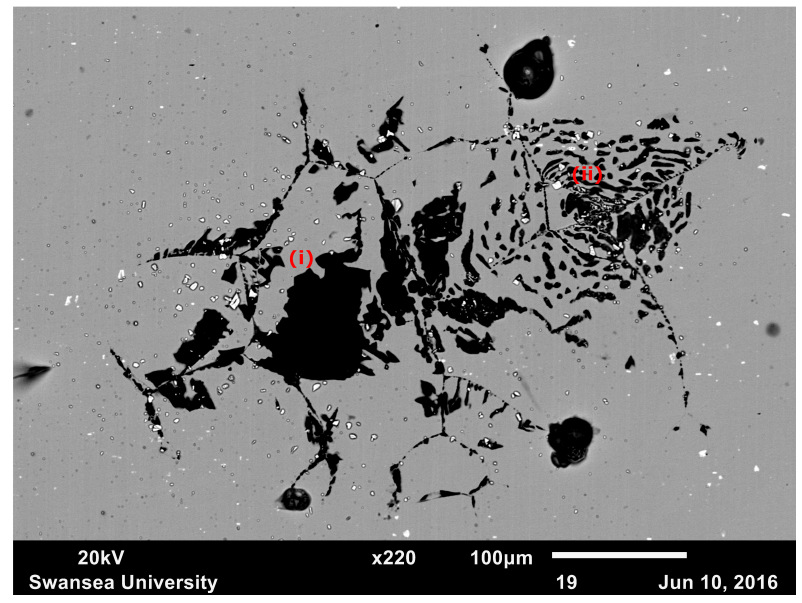

Figure 4.33: Site G (i) pit cavity, (ii) lacy cover.

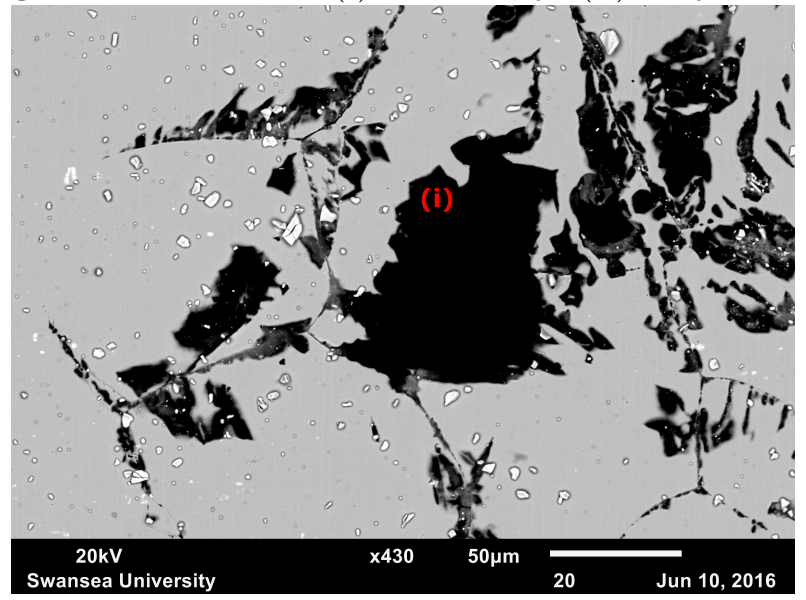

Figure 4.34: Higher magnification image of the pit site (i) in area G.

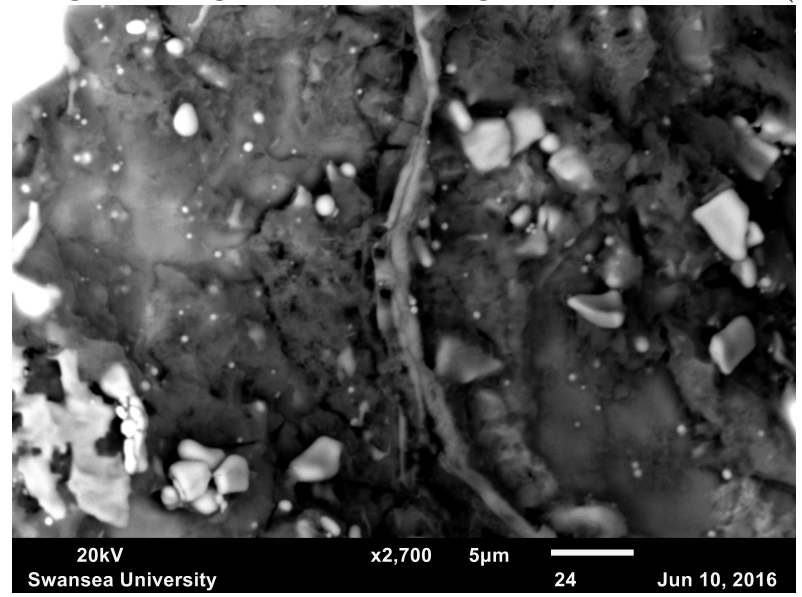

Figure 4.35: An image from location (i) in images 4.33 4.34, inside the pit cavity at site $\mathrm{G}$. Note the number of Nb-rich inclusions present, and the scale bar, showing many inclusions within a small area. 


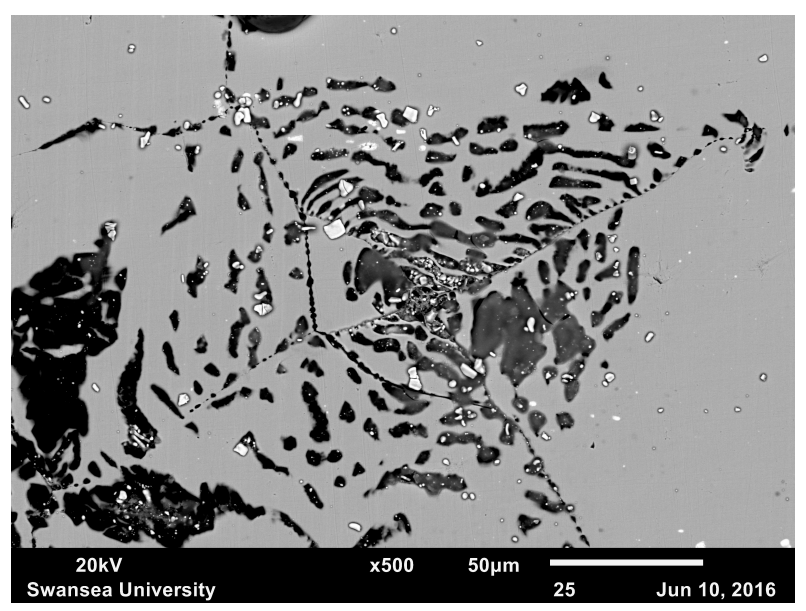

Figure 4.36: Site G lacy cover (figure 4.33(ii)) (500x).

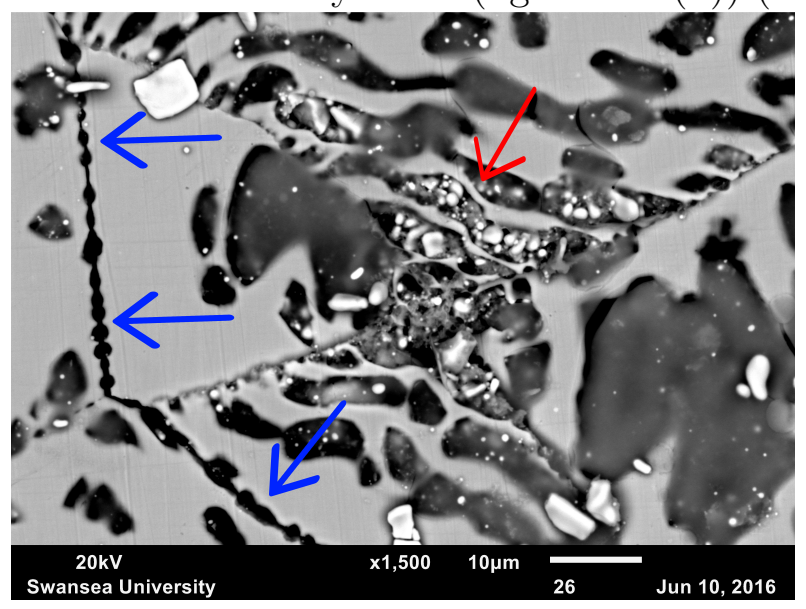

Figure 4.37: Site G lacy cover (1500x). The red arrow shows the high density of Nb-rich inclusions present in the pit, and the blue arrow shows the locations where IGC has caused the removal of $\mathrm{Cr}$ carbides through undercutting of the adjacent metal.

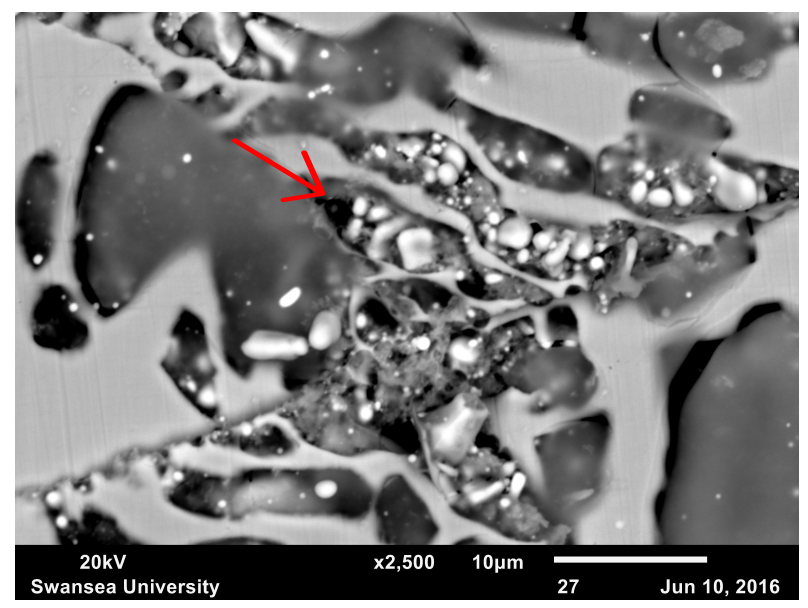

Figure 4.38: Site G lacy cover (2500x). The arrow shows the abundance of Nb-rich inclusions present within the pit (under the lacy cover). 


\subsection{Conclusions}

A light optical microscope has been used to identify locations in which corrosion has manifest in $20 / 25 / \mathrm{Nb}$ SS in two different concentration electrolytes. The TLM technique has been used to assess corrosion in a highly sensitised area (50:50, scale:metal) in $3 \mathrm{~mol} \mathrm{NaCl} d^{-3}$ at free corrosion potential (section 4.3.2). With this highly sensitised TLM example multiple pits initiated in rapid succession, following passivation, and subsequent crevice corrosion at a pore within the haematite oxide scale. The pits occurred mainly at triple point grain boundaries, and one instance of dissolution occurred adjacent to a Nb-rich inclusion. The technique was also used at a location expected to be less sensitised away from the scale in $0.01 \mathrm{~mol} \mathrm{NaCl} d m^{-3}$ under the influence of polarisation. In this less sensitised area, corrosion initiated again at a triple point grain boundary. IGC propagated along grain boundaries and into the metal, but avoided the inclusions present, instead migrating around them. This is in agreement with previous studies by Phuah [51] and Chan [45]. A study using a different austenitic SS, (sensitised AISI 310), showed similar results. Metastable events were confined to grain boundary intersections, then passivated, leading to stable IGC initiation at another site. Once the potential had reached a given potential, $E_{b}$, the transient metastable events are no longer witnessed, in favour of corrosion which can continue from the event initiation to the propagation phase, thus more stable. Stable IGC initiated in two locations at the grain boundary, within its centre, and at another intersection (in the form of a triple point). A grain boundary intersection is the location where grain boundaries meet, which can include any number of boundaries.

In the second phase of the study the SVET was used to map the surface elec-

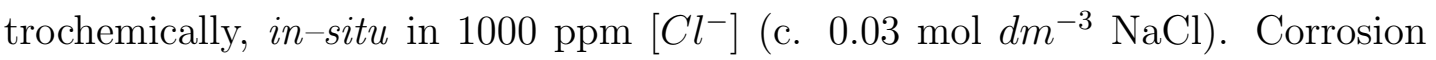
was initiated by stepping the overpotential. Pits initiated rapidly, due to the step in polarisation. Post corrosion electron microscopy revealed that pits were located where there were a high number density of Nb-rich inclusions. Further 
chemical analysis showed pits had propagated at areas where Nb-rich inclusions are present within the subsurface. Indicating that Nb-rich clusters are also a key initiation point in the SS.

The data shows that the triple point grain boundaries are also areas where corrosion initiates preferentially, and it is expected that these triple points (and grain boundary intersections) are locally more depleted of $\mathrm{Cr}$ than others. It is speculated that highly sensitised SS IGC will initiates at these locations. For specimens that are less sensitised, i.e. with the SVET experiment, the surface was prepared by grinding and polishing, leaving the surface less sensitised, the initiation mechanism changes. Should the less sensitised microstructure contain quantities of clustered Nb-rich inclusions, IGC seeks to initiate at that location. It is thought that the grain boundaries may not be sensitised enough for stable IGC $\left([\mathrm{Cr}]>\left[\mathrm{Cr}_{\text {threshold } 12 w t \%}\right]\right)$, and instead undergoes an intergranular pitting mechanism. In such a scenario corrosion initiates at a grain boundary intersection close to where Nb-rich precipitates are present and a pit starts to form. As the sub-surface volume is removed, areas adjacent to Cr-carbides (present at grain boundaries) are also removed which is more characteristic of IGC.

An ideal material, based on the results displayed here, will have Nb-rich inclusions dispersed within the material in a homogeneous fashion, rather than clustering together. Furthermore, the SVET experiment, combined with post exposure SEM-EDS, has shown that should a large number density of Nb-rich inclusions be present subsurface, IGC will seek to continue at these locations. In the TLM specimens the probability of $\mathrm{Nb}$ clusters is decreased, because of the smaller exposed area. This is possibly why corrosion has only been found to initiate at intersections using TLM specimens. If a porous material is present (such as haematite), a differential aeration cell is formed, and corrosion will seek to initiate at oxygen occluded regions, and should the SS be residing at its free corrosion potential, pitting sites may passivate. If the metal is undergoing polarisation, pitting sites can continue to propagate, due to the constant supply of current 
provided by the potentiostat system as in the case of the SVET experiment (section 4.3.4) where unless nearby pits are competing, corrosion continues to propagate. 
Chapter 5

A Combined in-situ

Investigation into Intergranular

Corrosion on Stainless Steel 


\subsection{Introduction}

This chapter focuses on characterising localised intergranular corrosion (IGC) behaviour of the cladding alloy post thermal sensitisation, over different length scales, in an in-situ environment. Optical time-lapse microscopy (TLM) was first used to attain information on the corrosion for hundreds of grains. A higher resolution method, time-lapse electrochemical atomic force microscopy (EC-AFM), was used to gather information on tens of grains. EC-AFM shows the mechanism for attack appears different to what is apparent over the larger length scale; and is more akin to intergranular-pitting than IGC. The in-situ investigation was further complimented by scanning Kelvin probe force microscopy (SKPFM) to identify areas susceptible to attack. This study uses two complimentary techniques, both the conventional optical time-lapse method, capable of mapping hundreds of grains, with the highly resolved AFM technique providing mapping on the individual grain scale. EC-AFM is suitable for spatially resolving inclusions and grain boundaries, and monitoring in-situ. The technique has been successfully used in the past with $\mathrm{Cu}$ alloys under potential control [138], Al alloys [139], and also sensitised AISI 304 SS [140]. In addition a new development in atomic force microscopy was demonstrated by Laferrere et al. at the Eurocorr conference in 2015 [141]. The conference paper was adapted by Clark (2017) as part of a special issue in Corrosion, Engineering, Science and Technology [133]. This conference paper showcased the high-speed AFM technique which authors state has a resolution comparable to TEM and demonstrated its uses for corrosion science, and showed the removal of Cr carbides on sensitised 20/25/Nb when subjected to polarisation in-situ. Figure 5.1 shows a mosaic of the surface obtained by HS-AFM. An image of the carbide dissolution from the paper is shown in figure 5.2. This chapter aimed to develop the use of the AFM for corrosion purposes where the sample is immersed under an electrolyte droplet. 


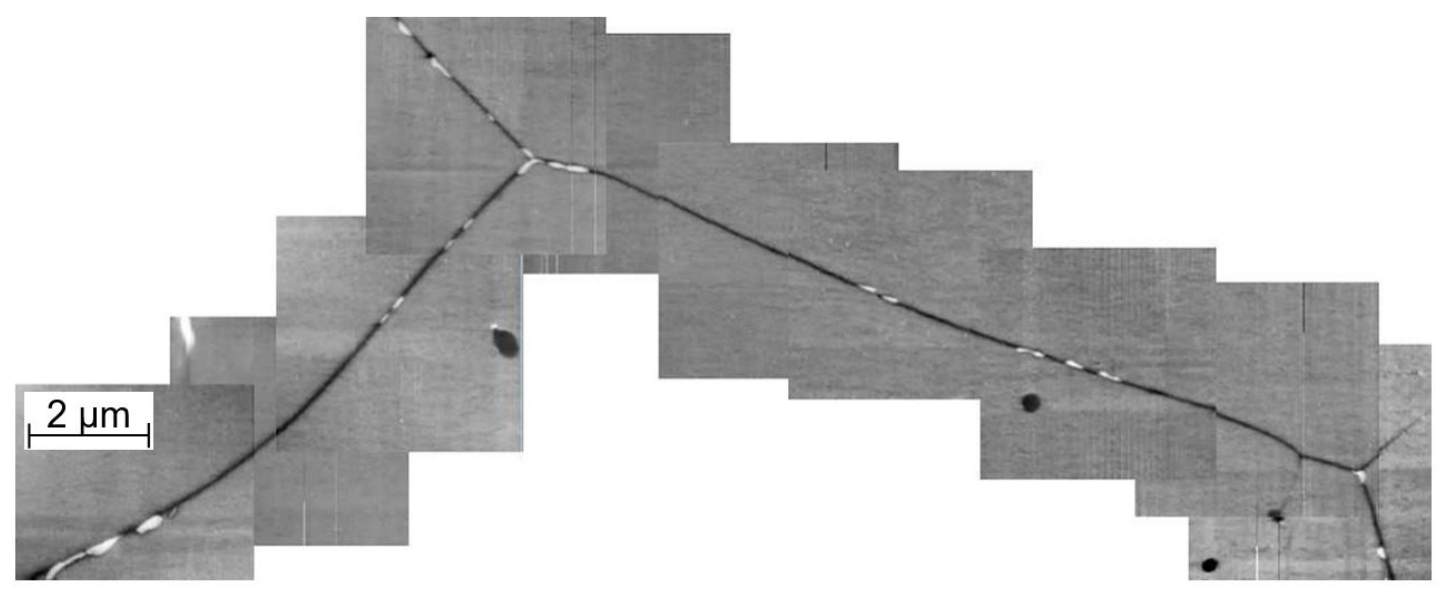

Figure 5.1: Carbides decorate the grain boundaries of thermally sensitised 20/25/Nb stainless steel. The image was obtained using a HS-AFM.

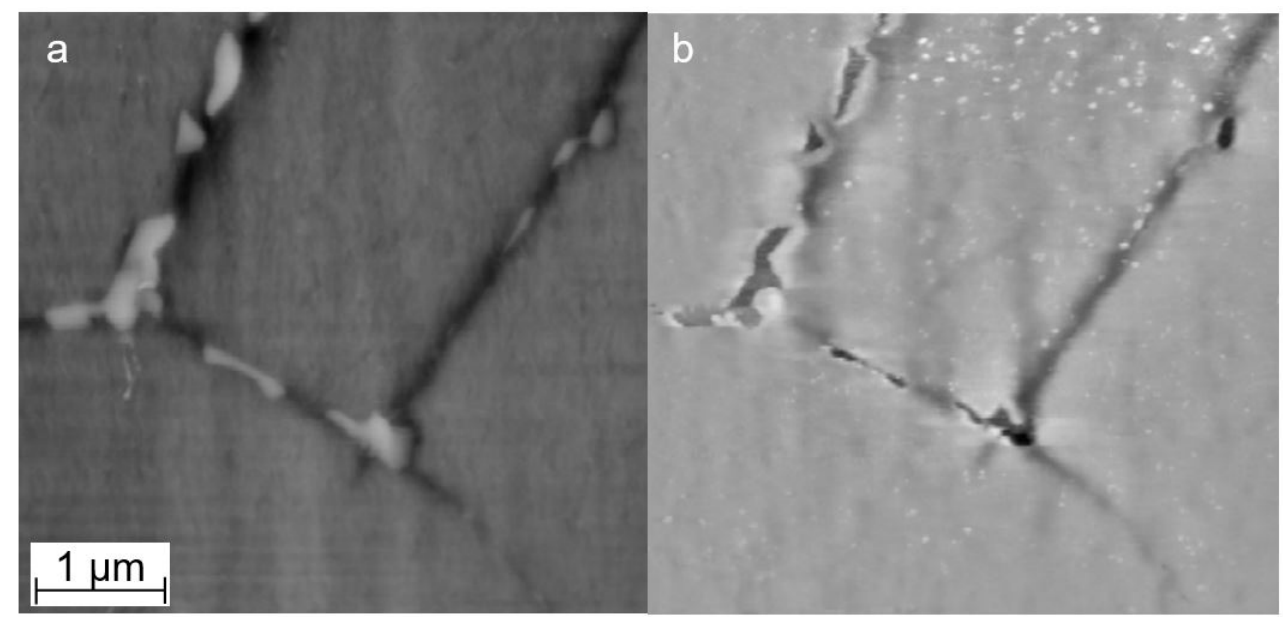

Figure 5.2: Carbides present along grain boundaries of thermally sensitised $20 / 25 / \mathrm{Nb}$ stainless steel (a). (b) shows the surface following polarisation (500 $\mathrm{mV}$ vs. Pt) in $5 \mathrm{mgkg}^{-1}\left[\mathrm{Cl}^{-}\right]$. 


\subsection{Experimental}

\subsubsection{Specimen and Solution Preparation}

Following thermal processing $\left(1150^{\circ} \mathrm{C}, 30\right.$ minutes under $\mathrm{Ar}, 600{ }^{\circ} \mathrm{C}, 336$ hours) specimens were cut, then ground and polished, as explained in the main experimental chapter (Chapter 2). A previous chapter (section 3.4.1) showed that sensitisation appeared depth-limited, as such preparation technique shown in figure 3.15 was used.

For metallographic observation of unsensitised specimens electrolytic etching was used to expose grain boundaries. The specimen and Pt gauze counter electrode were connected to a DC power supply and immersed 10 wt.\% Oxalic acid. A DC potential of $6 \mathrm{v}$ was applied for a period of 10 seconds in order to reveal grain boundaries. The specimen was rinsed in distilled water and finally cleaned with ethanol. The electro-etch procedure has been used in the past for $316 \mathrm{~L}$ SS [142] and is recommended by ASTM E407-07 16 [116] and was used by both Chan [45] and Phuah [51] on 20/25/Nb. Heat treated specimens did not require an etch, as grain boundaries within the highly sensitised area adjacent to the darkened surface, once polished, were visible.

Solutions were prepared with sodium chloride $(\mathrm{NaCl})$ (Sigma Aldrich, ACS reagent, $>99.0 \%$ ) and distilled water. Tests were conducted at neutral $\mathrm{pH}$. At low concentrations (ppm levels), such as those found in storage ponds, there is a high probability that the electrolyte would evaporate prior to any corrosion initiation during the in-situ EC-AFM experiment, given the low quantity of liquid as the specimen was not polarised. As such $1 \mathrm{~mol} d m^{3} \mathrm{NaCl}$ was used for both optical TLM and Electrochemical AFM. This $\left[\mathrm{Cl}^{-}\right]$was chosen for the AFM studies as the corrosion rate was compatible with the scan time achievable; such that corrosion progress could be followed effectively during the course of a few hours. The chosen concentration provided an insight to what may happen if $\left[\mathrm{Cl}^{-}\right]$underwent a large increase such as in a very extreme fault scenario. 


\subsubsection{In-situ Time-Lapse Microscopy}

Images were taken using a Meiji MT8000 metallurgical microscope with 0.7x Cmount and Infinity 1-3C camera. In order to increase the probability of corrosion initiation in the viewing area, and not elsewhere on the specimen a tape mask was used (PTFE, 3M, HD5490). Once a suitable area of the specimen had been identified, the PTFE tape was perforated using a $0.35 \mathrm{~mm}$ diameter biopsy pen and placed onto the surface, reducing corrosion susceptibility elsewhere on the specimen.

Prior to subjecting the specimen to experiment, multiple images of the exposed surface were mapped using a 50x optic enclosed in a protective waterproof shroud; in order to form a high-resolution montage, created using Microsoft image composition editor (ICE). Pre-corrosion mapping was also repeated using a Hitachi TM3000 SEM with energy-dispersive x-ray spectroscopy (EDS) capability. Once pre-mapping was conducted; the specimen was fixed to a large petri dish and immersed in c.300 ml electrolyte. Images were taken every 60 s over a 24 hour period, with the exception for the specimen under potential control, where images were taken every $30 \mathrm{~s}$, in order to more accurately follow the corrosion. In conjunction with TLM; a Palmsens 3 potentiostat was used to measure the open circuit potential, with respect to an SCE. In addition; a three-electrode cell (Pt gauze, SCE) was used in order to investigate the effects of linear polarisation.

\subsubsection{Atomic Force Microscopy}

A JPK Instruments Nanowizard III AFM was used for taking measurements. AFM collects topographical information by using a small, sharp tip attached to the end of a cantilever. This allows the very high resolution in the z-direction within the order of picometers. Further information on the AFM technique can be found elsewhere [143] [144]. For in-situ electrochemical AFM experiments a special o-ring was used which allowed imaging in a liquid environment. The surface area occupied by the o-ring was $\approx 1.8 \mathrm{~cm}^{2}$. The AFM images were captured 
in a freely corroding environment; with no external polarisation applied. Prior to the introduction of the electrolyte, an in-air topographic image was recorded using contact mode, the electrolyte was then added slowly, to avoid damage of the AFM tip. Once fully immersed, the instrument was re-configured for measurement under electrolyte (including compensation for change in refractive index) during reconfiguration approximately 15 minutes had elapsed. Individual scans were performed over a $50 \mu \mathrm{m}^{2}$ area, using 512 x 512 pixel resolution with a 0.3 $\mathrm{Hz}$ scan speed. This resulted in a $\approx 30$ minute duration per scan.

Low stiffness Nanoworld FM50 AFM cantilevers were used for SKP mapping (single crystal silicon). For the electrochemical AFM experiments 3-lever n-type silicon tips were used (Mikromasch HQ:CSC37) in contact mode.

\subsubsection{Scanning Kelvin Probe Force Microscopy}

In order to collect a Kelvin probe measurement, an electrical connection was required, which was achieved by attaching copper foil to the specimen outside of the scan area. The scanning Kelvin probe force microscope (SKPFM) determines the Volta potential difference $\Delta \Phi$ between the specimen and probe reference, and has the ability to resolve Volta potential differences for inclusions. Previous examples of such measurements include Al alloys [145] [146], high-entropy alloys [147], filaform corrosion [148] and Cobalt-Chromium-molybdenum alloys undergoing hot isostatic pressing (HIP) [14]. The SKP-FM probe performs a raster scan collecting topographical information and Kelvin potential information line by line. Using feedback from the topography; the probe hovers the surface at a user defined height $(50 \mathrm{~nm})$ when conducting the SKP-FM mapping, and as such the recorded potentials are independent of feature height.

Specimen topography was firstly measured for the desired area in AC mode; and once surface tracking was optimised, SKP-FM mapping was undertaken. SKPFM methodology has been compared the conventional SKP previously by Rohwerder [122]. 


\subsubsection{AFM Post Processing}

AFM data post-processing was undertaken by JPK instruments data processing software version spm5.1.4. First order levelling was applied to all data; with the lower $5 \%$ and upper $90 \%$ being excluded from the baseline fit. Line cross sections were completed by choosing the same horizontal line on each scan, in order to avoid line-to-line effects.

\subsection{Results and Discussion}

\subsubsection{In-situ TLM}

\section{Freely Corroding Conditions}

Figure 5.3(a) shows a montage of the surface before immersion in $1 \mathrm{~mol} \mathrm{dm}^{-3}$ $\mathrm{NaCl}$. The lower part of the image shows part of the haematite scale, above this is the highly sensitised area that appears as though it has been etched. Figure 5.3b shows the same area using SEM. Differences in optical and electron microscopy can been seen in these different images; as NbC show bright white on the BSE electron microscopy image. Fig. 5.4 shows the presence of niobium whilst carbon appears more homogeneous. $\mathrm{Nb}$ is is highlighted in red in the EDS map whilst $\mathrm{C}$ is highlighted in green, which is difficult to detect using EDS due to its low atomic number.

From the TLM stills, in a freely corroding environment, there is the lack of visible IGC on the exposed surface of the SS indicating that the SS has passivated since no gross attack seems to have occurred. After 6 hours of immersion a quantity of corrosion product was witnessed on the exposed surface in figure 5.5 (b). These deposits increased over time and gas appeared to nucleate after 18 hours at the top of figure 5.5. This may be due to trapped gas simply diffusing toward the surface; as gas evolution should not be active at neutral $\mathrm{pH}$ without external polarisation. The primary cathodic reaction is the oxygen reduction reaction; 
forming hydroxide ions.

Figure 5.3(c) shows the surface, post corrosion, after 24 hours immersion in electrolyte. Again, with previous evidence (sections 3.4.4, 4.3.2) it is expected that IGC manifests within pores of the haematite; creating one or more differential aeration cells, similar to a crevice. As corrosion product increases as a function of time the two corrosion half reactions must be occurring somewhere, with the corrosion occurring under the tape. Electrochemical data gathered insitu from the same specimen is given in figure 5.6. A fluctuation in potential occurred within $5000 \mathrm{~s}$; suggesting there is some competition between the two half reactions. However, for the course of the first 10,000 s (3 hours) there is a net drift in electrochemical potential to more positive anodic values, from $-350 \mathrm{mV}$ (vs. SCE) to $-200 \mathrm{mV}$ (vs. SCE), indicating an increase in free corrosion current density and cathodic depolarisation.

Over the course of the next $5000 \mathrm{~s}$ there is a plateau, suggesting passivation of the surface. However; potential decreases sharply from c. 13,000 s, which is evidence that the specimen has reached breakdown potential, and corrosion had initiated. Electrolyte may have migrated through the pores within the haematite, leading to the formation of a differential aeration cell, as corresponding TLM images do not show the effect of this sharp change in potential. This indicates that the anode may be present in the form of a crevice, helping explain the relative low breakdown potential. As the potential shifts toward more positive values corrosion is occurring. By the time of the next arrow (6 hours, Figure $5.5 \mathrm{~b}$ ) the effects of the sharp increase in current can be seen as an increase in corrosion product deposits on the surface. At c.65,000 s (18 hours, Figure 5.5 c) the potential shows a shift of $\Delta \mathrm{E} 50 \mathrm{mV}$ (vs. SCE) to more anodic values, which lasts $\approx 4000 \mathrm{~s}$. Following this we see again that the surface becomes covered in corrosion product Figure 5.5(d). 

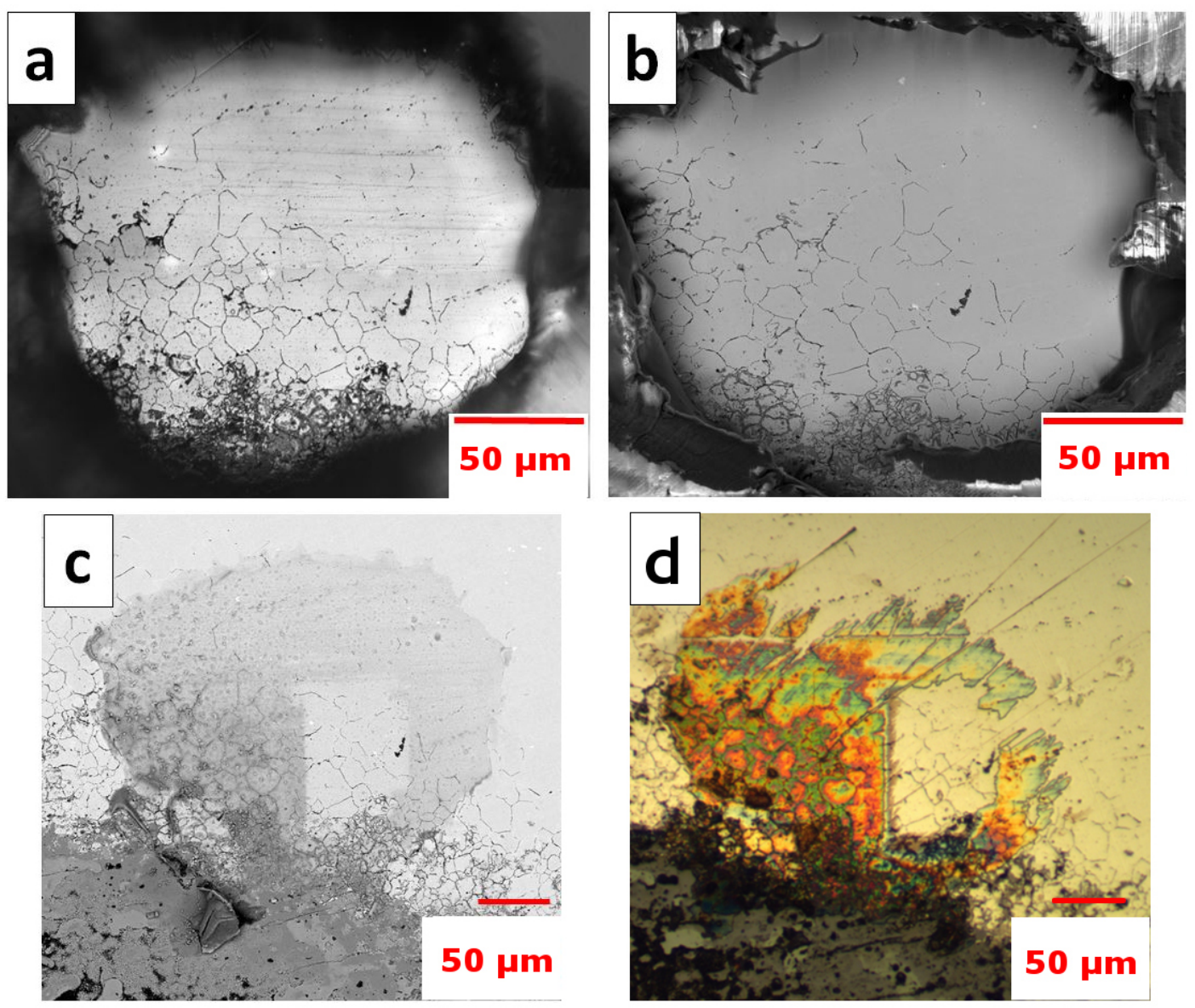

Figure 5.3: (a) 50x optical montage (22 composited images) of the surface before immersion, (b) SEM micrograph of surface before immersion, (c) SEM micro-

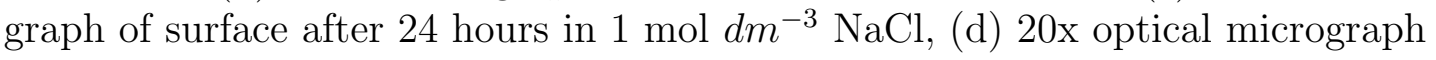
post corrosion 


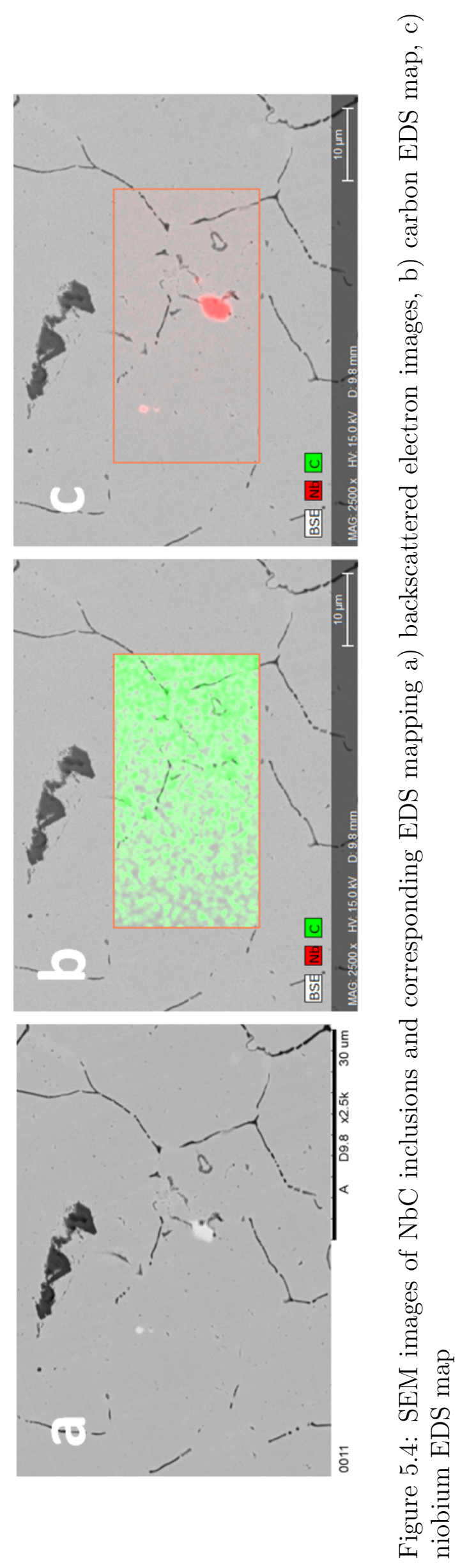




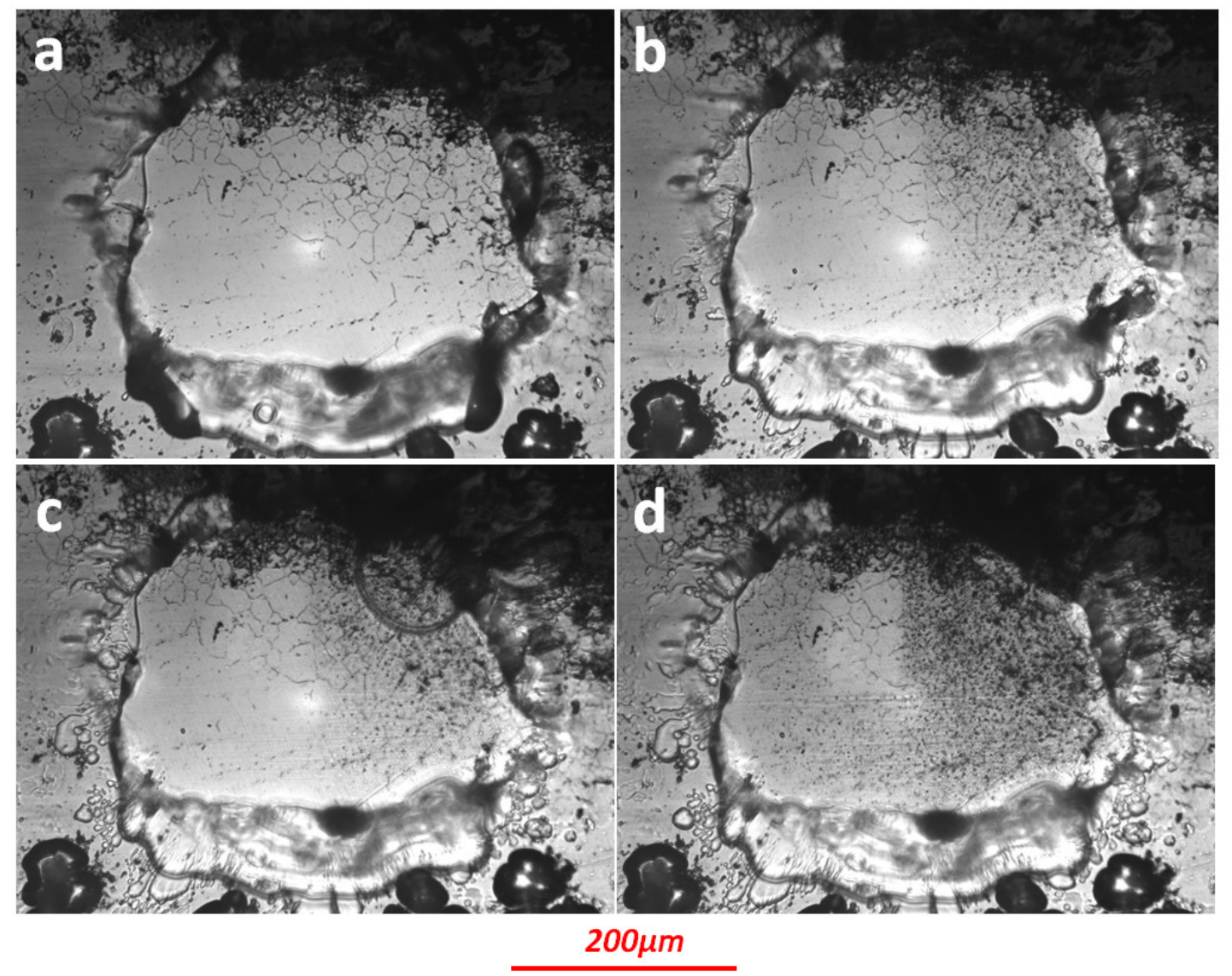

Figure 5.5: Optical TLM images of the sensitised SS 20-25-Nb immersed in 1 mol $d m^{-3} \mathrm{NaCl}$ showing the progression of corrosion product depositing on the exposed surface. a) 0, b) 6, c) 18, d) 24 hours 


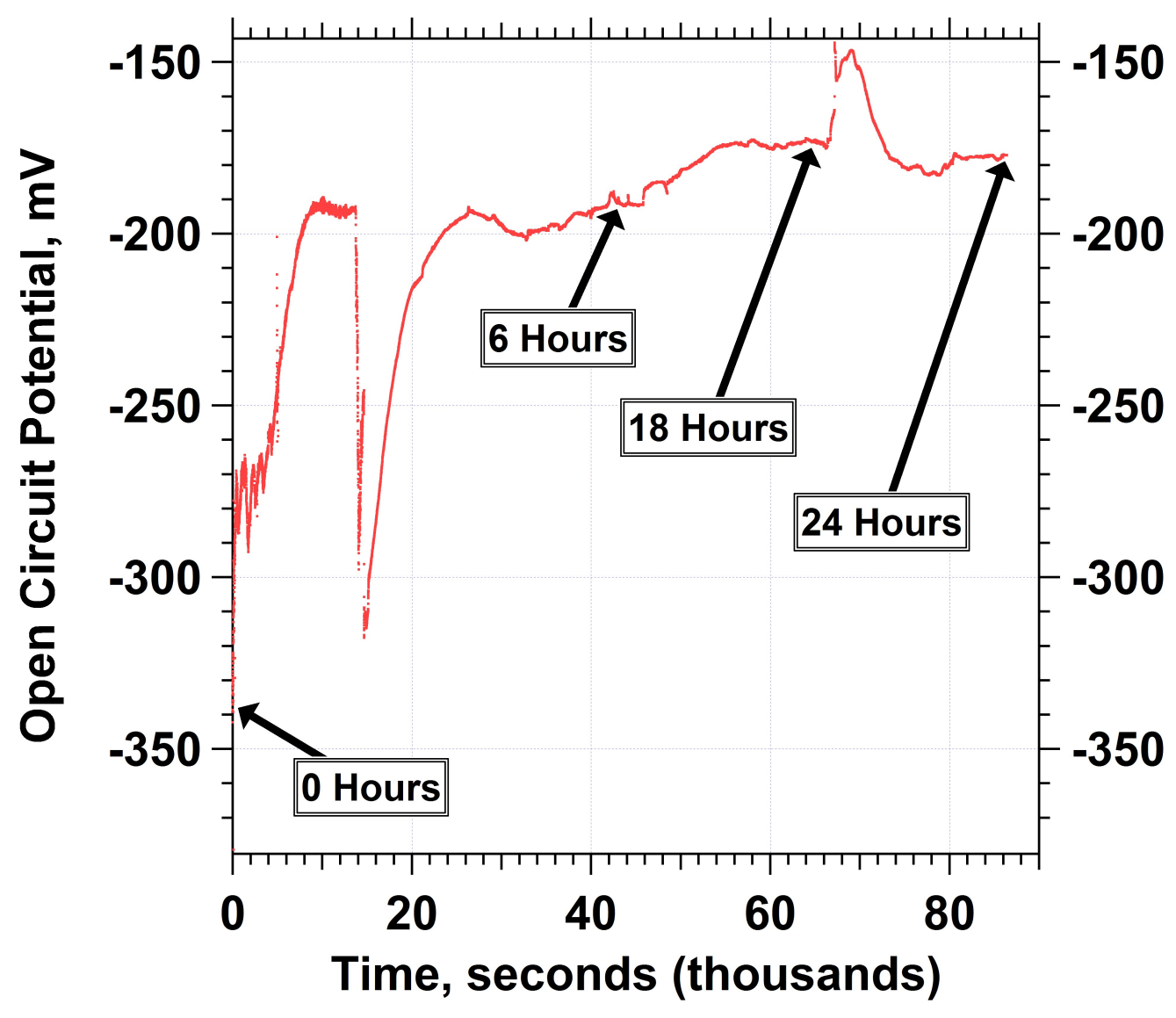

Figure 5.6: Open circuit potential as a function of time (vs. SCE) for the TLM specimen immersed in $1 \mathrm{~mol} \mathrm{dm}^{-3} \mathrm{NaCl}$. Arrows at c.0, 6, 18, 24 hours have been added to assist the reader. The peak at c. 65,000 s (18 hour) corresponds to the trapped gas movement, or gas evolution seen in figure 5.5 


\section{Potentiodynamic Experimentation}

Potentiodynamic experiments were used to assess the difference between corrosion mechanisms for the sensitised SS within a freely corroding environment and under potential control. The specimen was allowed to rest for 10 minutes to allow for the open circuit potential to equilibrate the starting potential was $\approx-100 \mathrm{mV}(-350$ $\mathrm{mV}$ vs. SCE) below the recorded OCP. Figure 5.7 shows the development of IGC over a short period of time (the experiment lasted $1000 \mathrm{~s}$ ). A graph of potential against time is also shown in figure 5.8. Given the time interval for the images $(30 \mathrm{~s})$, the number of images from where IGC is visible, and linear sweep rate $(0.5$ $\left.m V s^{-1}\right)$, the potential in which breakdown occurred was back calculated. It is estimated that breakdown $\left(E_{b}\right)$ occurred at $-150 \mathrm{mV}$ vs. SCE, suggesting a $\Delta \mathrm{E}$ of $\approx 80 \mathrm{mV}$ vs. SCE. This corresponds with a shift in corrosion current. From the images (figure 5.7) IGC is more visible when polarised, and there is less corrosion product deposition. Multiple anodes appear to be present; which nucleate as points upon the GB, suggesting that highly localised corrosion occurred at specific points on the grain boundary, possibly at the most Cr-depleted regions. IGC then appears to trace the GB, but over time, IGC becomes less localised and the matrix is also attacked, with grains themselves eventually dissolving into solution. This is an effect of potential increasing (and current increasing exponentially) as a function of time. Figure 5.9 shows the surface post-polarisation. IGC continued past the boundary where the PTFE tape was present confirming that the IGC is not only related to the surface, but also tracks in 3D. The perforated lacy cover of the haematite scale suggests crevice corrosion within the pores had occurred. This has been recorded previously in chapter 3, section 3.4.4. 

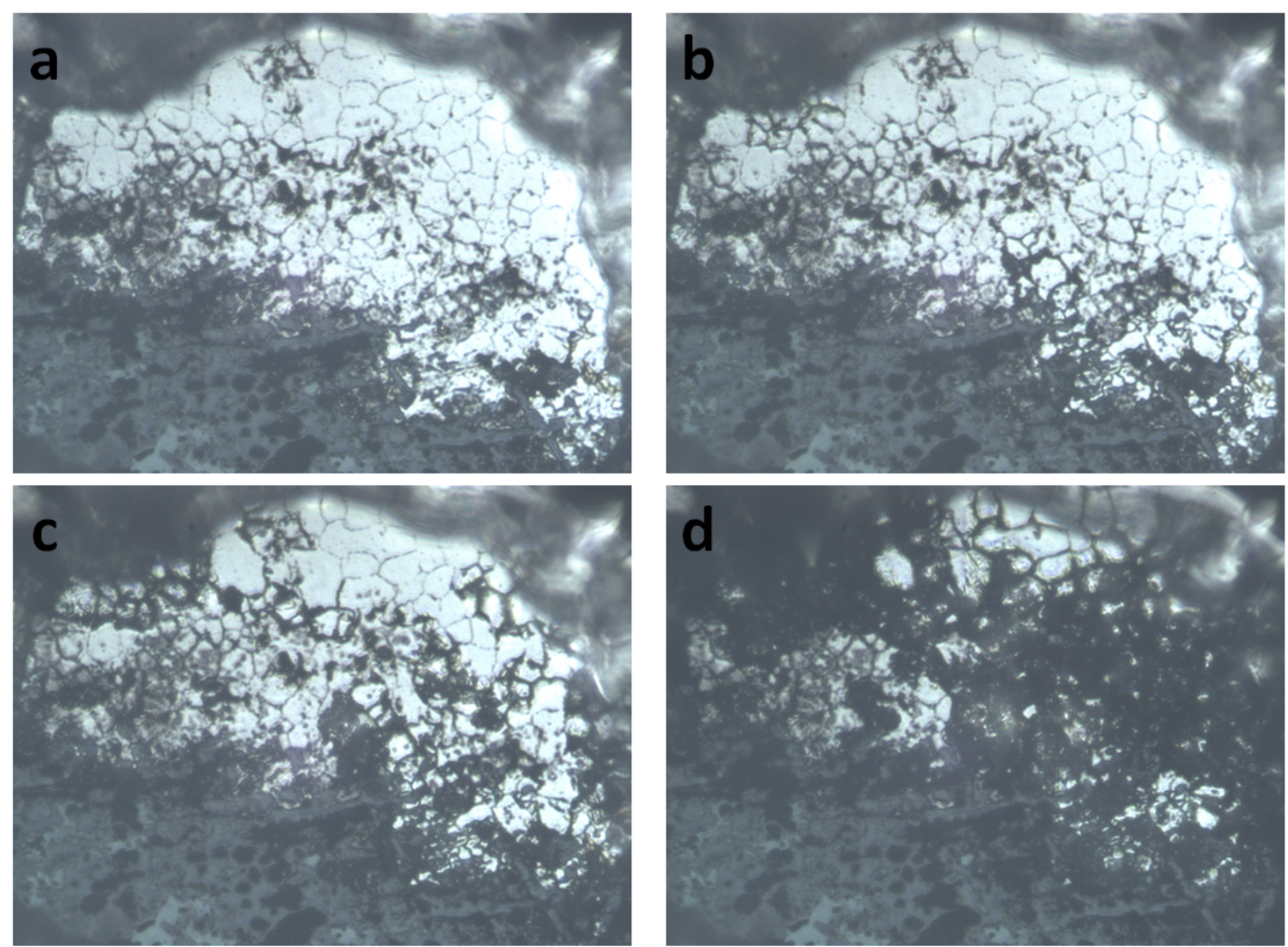

$200 \mu$

Figure 5.7: Cropped optical TLM images of the sensitised SS alloy in $1 \mathrm{~mol} d \mathrm{~m}^{-3}$ $\mathrm{NaCl}$ showing the development of IGC over time under the influence of potential control. (a-d) Show the progression of several anodes within a period of $270 \mathrm{~s}$ where the current density increased exponentially from $\approx-150 \mathrm{mV}$. 


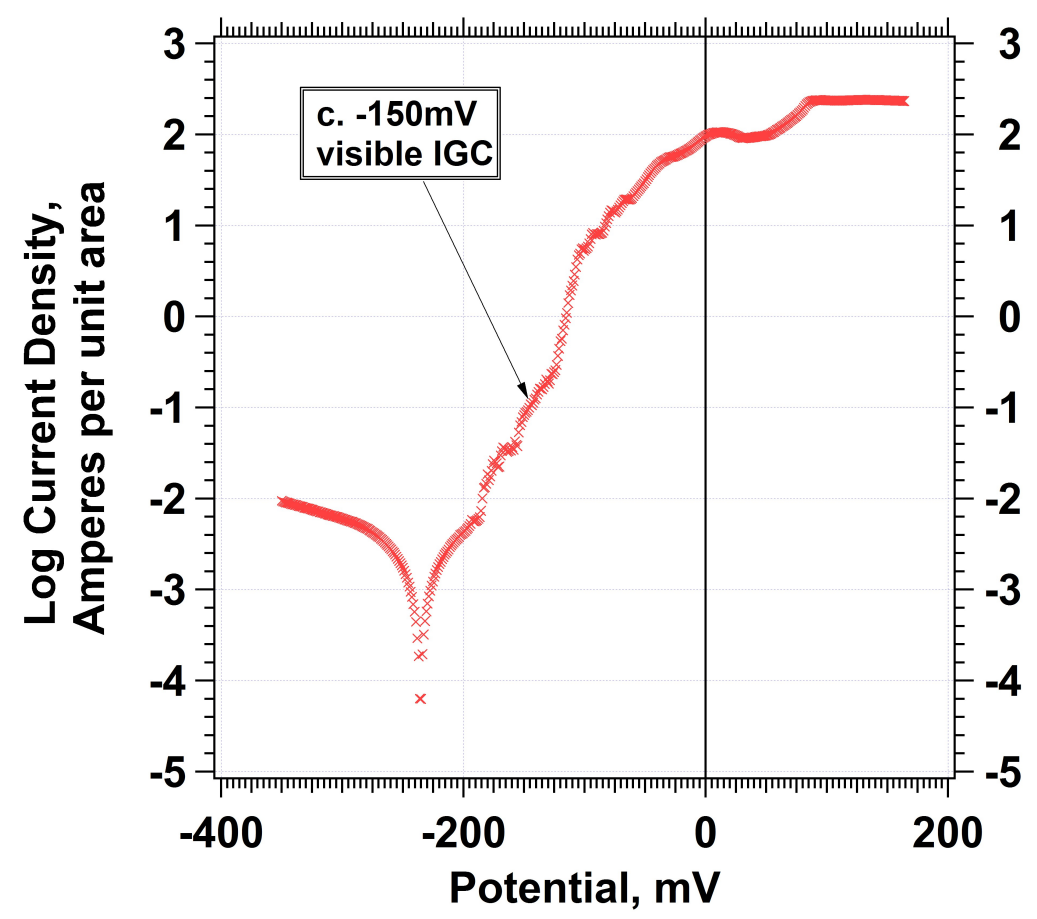

Figure 5.8: Potentiodynamic curve for the above TLM specimen immersed in 1 mol $d m^{-3} \mathrm{NaCl}$ undergoing polarisation. Current density is shown as a function of applied potential (vs. SCE). The experiment started at $-350 \mathrm{mV}$ vs. SCE. We have annotated the graph to show the specimen breakdown potential at c. -150 $\mathrm{mV}$ vs. SCE. The graph appears to plateau at $86 \mathrm{mV}$ vs. SCE.

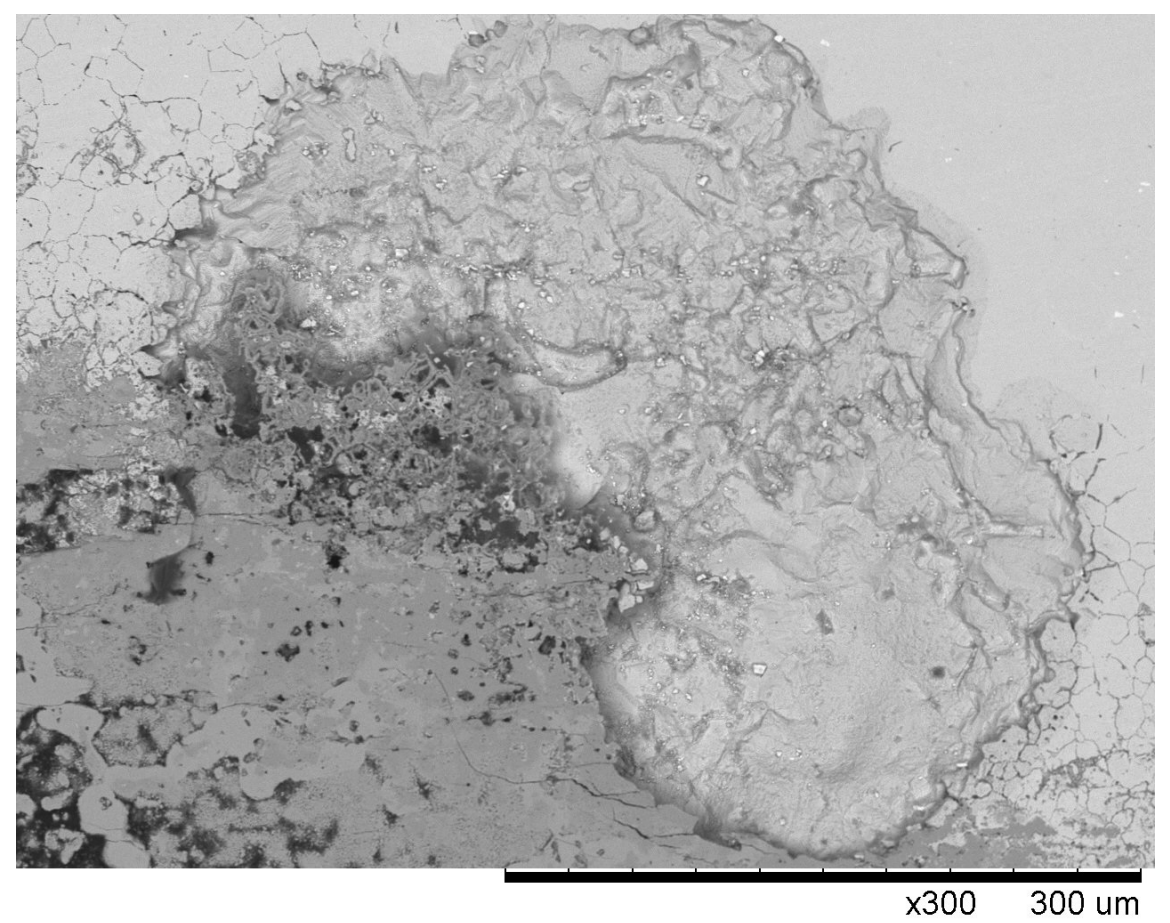

Figure 5.9: SEM montage of surface after immersion in $1 \mathrm{~mol} d m^{-3} \mathrm{NaCl}$. The specimen has been subjected to polarisation. The perforated cover at the scale is visible in this image, as is the large amount of $\mathrm{NbC}$ present within the excavation. 


\subsubsection{In-situ Electrochemical Atomic Force Microscopy}

Figure 5.10 shows the series of AFM measurements on the sensitised SS immersed in $1 \mathrm{~mol} \mathrm{dm}^{-3} \mathrm{NaCl}$ over the period of 4.5 hours, in-situ. A total of nine images were collected. Shown are scans (a) 30 minutes, (b) 60 minutes, (c) 120 minutes, (d) 240 minutes.
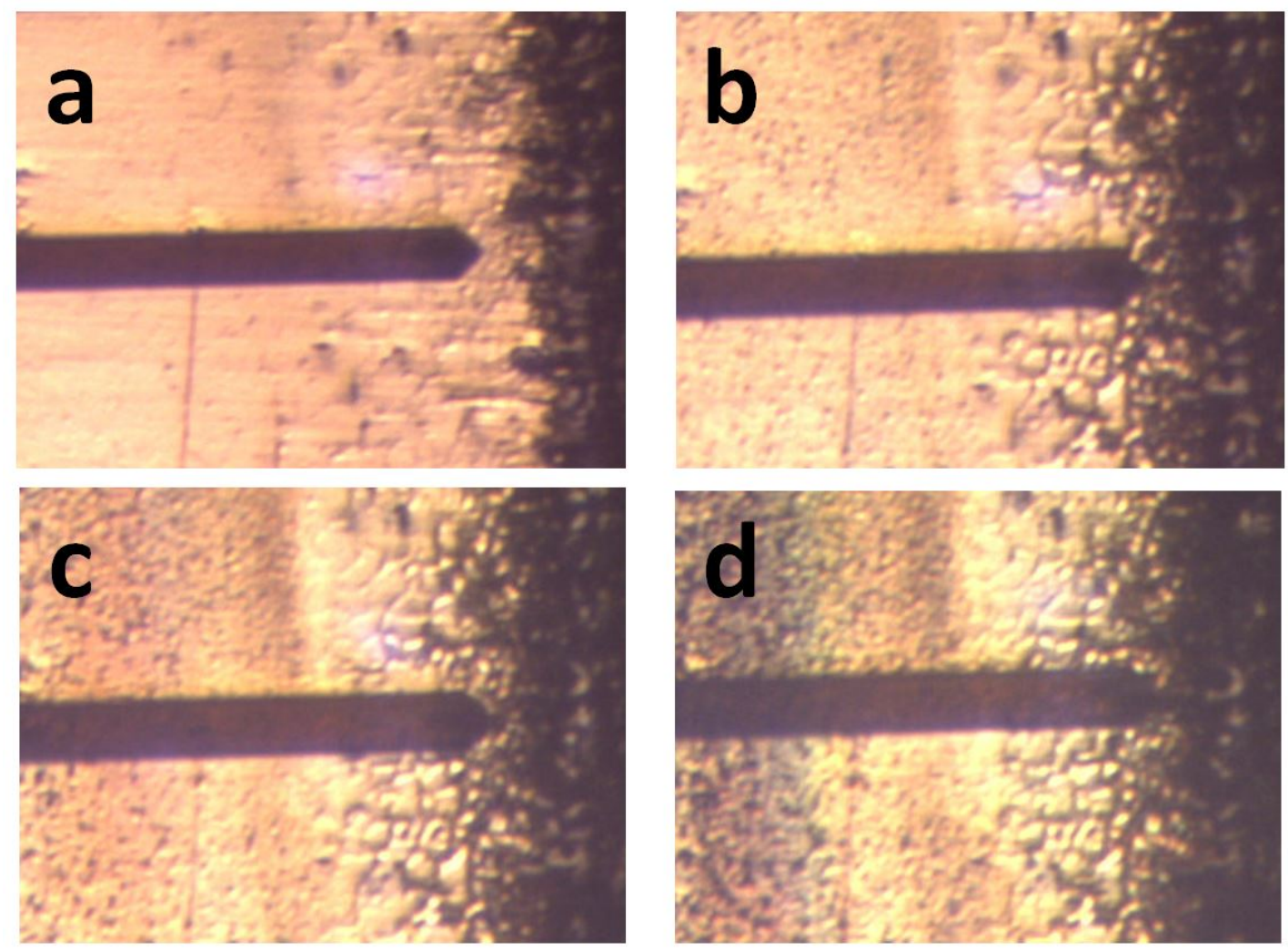

Figure 5.10: In-situ optical TLM images of the development of IGC and increase

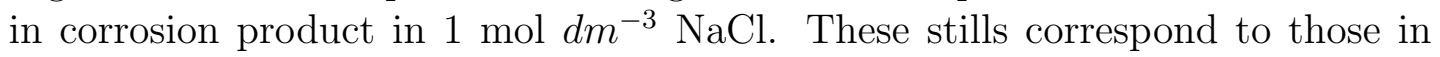
figure 5.11; (a) 30 minutes, (b) 60 minutes, (c) 120 minutes, (d) 240 minutes.

Localised corrosion events initiated quickly on the surface, with the first occurring adjacent to an inclusion near the centre of the frame in the first image (figure 5.11(a)). The bulk of the attack occurs approximately 1 hour after immersion, as by figure 5.11(b) a pit cover forms around the inclusion (shown by the arrow in figure 5.11(a)), which was ruptured by the next image. There is evidence that the surface was modified even after the first image (30 minutes). Figure 5.10 a shows swelling of the GB, and this is even more prominent in future images. It is believed that this may be a visual artefact caused by the relative 
height scale used. By showing different length scales (optical, AFM); the mechanisms for attack can be better understood. Optical TLM in figure 5.11 shows
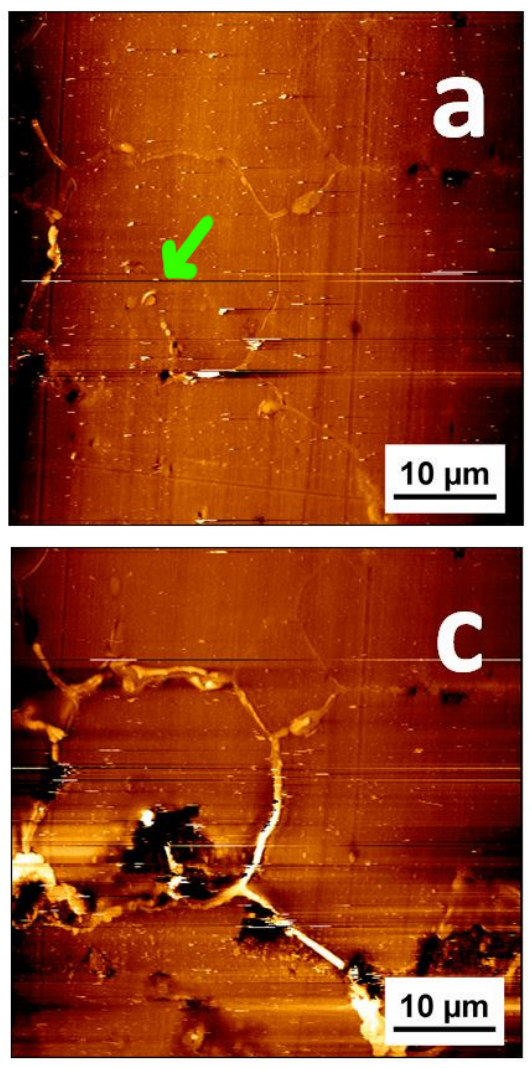
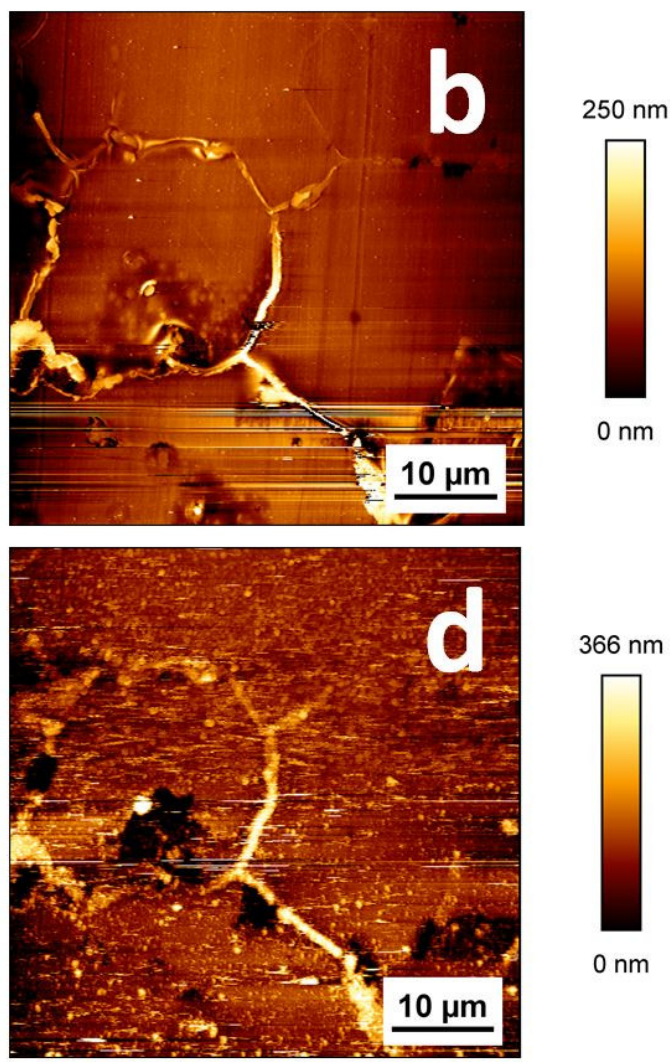

$306 \mathrm{~nm}$

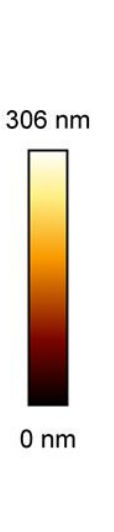

Figure 5.11: In-situ EC-AFM images of the sensitised SS 20-25-Nb immersed

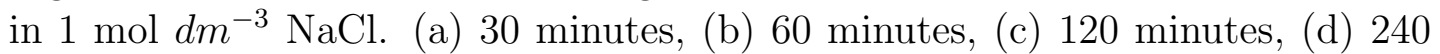
minutes. The images depict the development of a grain boundary swelling effect, pit covers, intergrannular pitting and consequential corrosion product covering the exposed surface. The arrow in image (a) shows what is a possible NbC inclusion. The swelling effect may be an artefact of the relative height scaling used

IGC, tracing GB along the highly sensitised region adjacent to the scale. The corrosion behaviour displayed is similar to when polarisation was employed on the optical TLM specimen previously (section 5.3.1). From the images in figure 5.10, corrosion products on the surface appear to deposit in a radial fashion, suggesting the scan was conducted adjacent to an anode; where a large amount of corrosion had occurred. At the grain level (EC-AFM), corrosion does not appear to trace the GB; as expected from the optical images in the previous figure, local events appear to take the form of intergranuar pits. Of the pits visible in the scan area $4 / 5$ nucleated adjacent to the GB. The pits form a perforated lacy 
cover which is known widely in literature to offer pit protection [16] [13]. Within local pit environments acidity and $\left[\mathrm{Cl}^{-}\right]$increase, due to the charge imbalance caused by increased metal ions, with the lacy cover decreasing mass transport, keeping the environment aggressive enough for pit propagation. In the second image (figure 5.11(b)) lacy covers form; but by the next scan the cover is removed, possibly by the AFM tip. Zhang et al. using AFM, witnessed the removal of a lacy cover while performing similar experiments on sensitised 304SS in 3.5Wt.\% $\mathrm{NaCl}[140]$. Once the pit cap was removed, the pit ceased to propagate.

Whilst the SS would normally undergo passivation in an aqueous environment; in order to retain passivity the surface must form an invisible Cr-oxide film, (with minor concentrations of Fe close to the metal-film interface [57]). However; localised corrosion can occur once the local concentration of $\mathrm{Cr}$ drops below $12 \mathrm{Wt} . \%$, as stated previously. At these locations highly localised attack can occur; such as pitting. As has been seen using electrochemical scanning techniques, many pits appear to initiate in a rapid fashion, as is shown in figure 5.11; but over time anodic current density at the most competent pit site increases, and thus this single pit continues to grow. Most other pits repassivate; and the number of these active pit sites decrease as a function of time. This was shown by Issacs with the scanning reference electrode technique (SRET) on type 304 stainless steels [82] and previously in an SVET experiment (section 4.3.4) which showed a decrease in SVET derived current density one pit, but an increase at an adjacent one. The large free surface on a stainless steel becomes a net cathode; as compensation for the highly localised anodic current sources. Previous studies by Williams et al. show a similar effect in the pitting of magnesium; when using the more spatially-resolved scanning vibrating electrode technique (SVET) [87]. Once the pit ceases to propagate, pitting either continues at another location, or repassivation occurs. It would appear that stainless steel has undergone repassivation; as there is no continued IG-pitting, and no single large pit growing. It is postulated that IGC continues outside the scan area, at the pores within the 
scale, as corrosion product continues to deposit radially, increasing with time. This is in agreement with earlier work in this thesis. This intergranular pitting phenomenon is caused by variances in $\mathrm{Cr}$ depletion even along the grain. From an optical standpoint (thousands to hundreds of grains) this IGC may appear to trace along the GB, but from the small area viewed under AFM; intergranuar pitting ensues in open circuit conditions. From figure 5.11c (120 minutes) the AFM image becomes more noisy, up until figure $5.11 \mathrm{~d}$ (240 minutes) when it becomes more difficult to make out features. In order to assess the noise; a smaller working area, $20 \mu \mathrm{m}^{2}$, was selected (centre of frame) with a higher setpoint in figure 5.12 (310 minutes). This surface is representative of what occurs at 290 minutes. False lines can appear on AFMs when the set point and gains are not optimised; causing the trace and retrace to not follow each other. In this experiment, both lines showed agreement. Though the traces matched, features from one line did not necessarily follow on the next line, indicating removal of features has occurred. It is expected that this is caused by a weakly adhering iron (II, III) oxide corrosion products being moved by the AFM tip.

\section{SKPFM}

SKPFM maps provided in figure 5.13 show the spatial variation of Kelvin potential on sensitised, and the unsensitised SS. Figure 5.13 (a, b) show the variance in potential between matrix and GB, along with the relevant topography. The data shows that whilst areas of the GB appear to be less noble; the variation is not significant. The image shows a discrete area of the GB with an increased Kelvin potential to that of the matrix, and other grain boundaries. Figure $5.13(\mathrm{c}, \mathrm{d})$ is a magnified view of the GB in figure 5.13 (a, b). Again, as per the previous figure, the GB appears to have a slightly higher potential with respect to the matrix. Interestingly, this one region of the GB appears to be more susceptible to attack. Consequently; this suggests that there may be three possibilities: a) a different phase is present at this location, or more likely, b) there is a greater extent of 
Cr depletion at this site thereby creating a corrosion 'hot-spot'. Figure 5.13 (e, f) show the surface and SKP-FM maps of the electro-etched, as-received, polished SS. The AFM image shows NbC inclusions which appear to be more noble (electrochemically) and more proud, with the shape of these being different to Cr carbides located on heat treated specimens, such as those described above.
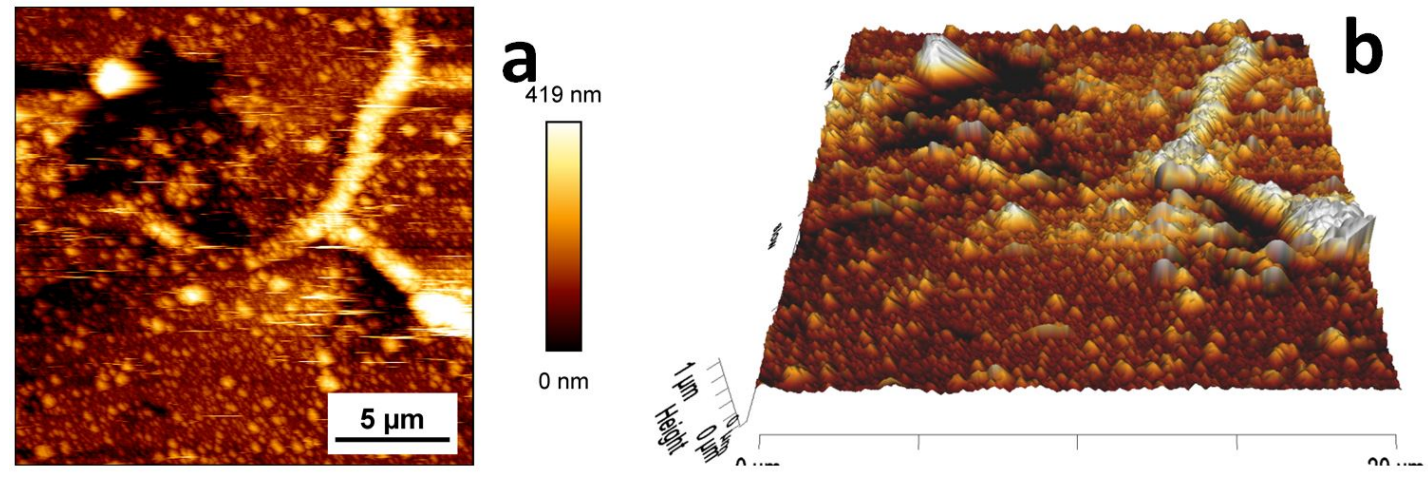

Figure 5.12: Centre frame AFM image at 310 minutes (a) 2D map (b) 3D map

\subsection{Conclusions}

A method for assessing the corrosion of an austenitic stainless steel in an aqueous environment over different length scales has been presented. It has been shown that, in a freely corroding environment the actively corroding surface can be mapped using both optical and AFM in-situ techniques. As a comparison, the sensitised SS has also been investigated using polarisation, accelerating the process. The SS appears to show IGC when polarised; whilst using the highly resolved AFM the corrosion appears to be more in line with intergranular pitting. EC-AFM showed intergranular pit initiation around an inclusion. The AFM images show that these intergranular pits cease to propagate once the lacy cover is removed; therefore attack is not occurring into the surface. It is believed that IGC continued at pores within the haematite. 

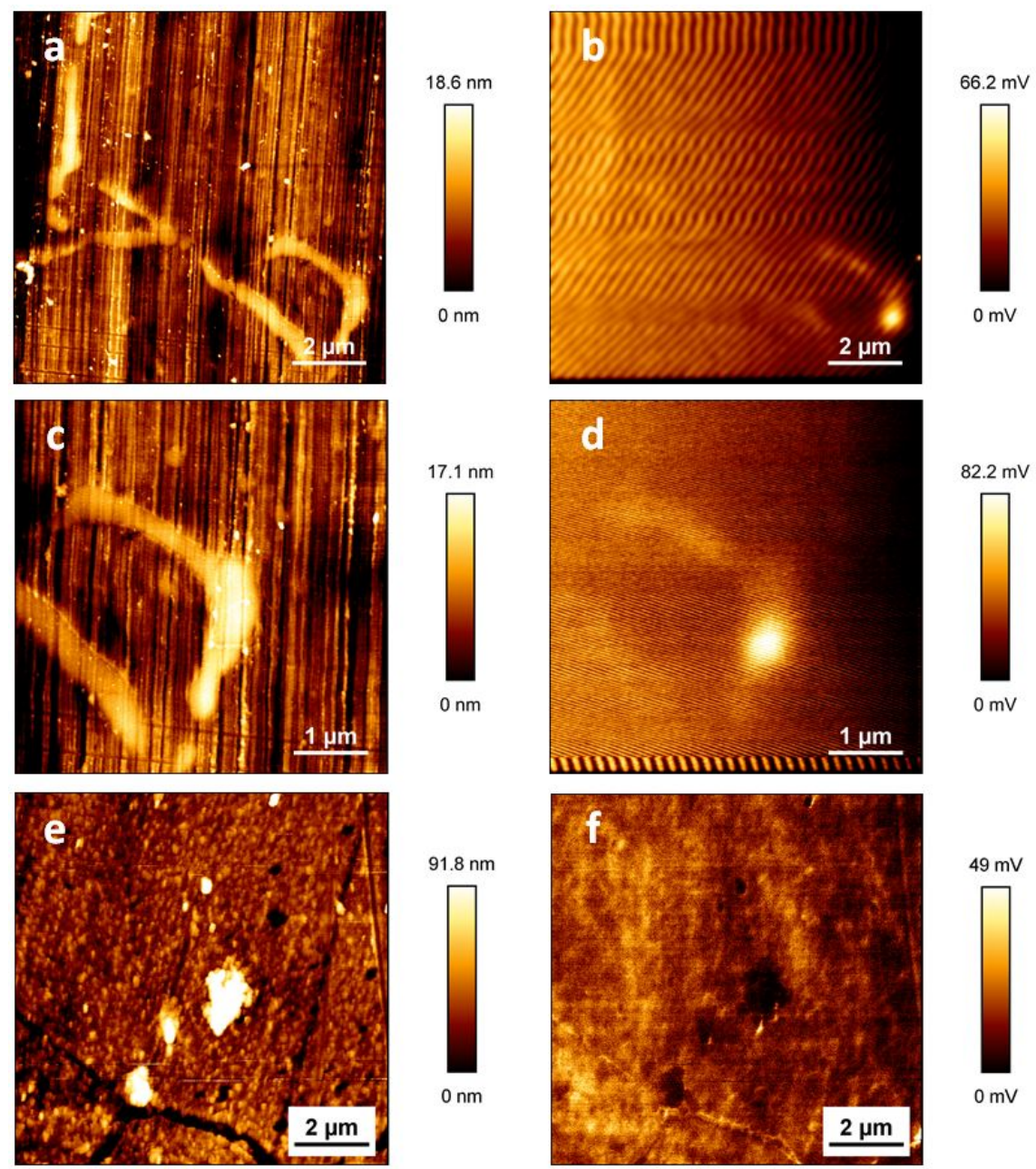

Figure 5.13: AFM topography and KPM maps. (a) Sensitised SS Metal:scale interface, (b) Corresponding SKP-FM map, (c) Cr carbide present on sensitised SS, (d) Corresponding SKP-FM map, (e) Unsensitised, etched SS NbC, (f) Corresponding SKP-FM map 
Chapter 6

\section{Inhibition of IGC: The Caustic \\ Dosing Approach}




\subsection{Introduction}

The effect of using a basic inhibitor has been investigated by both electrochemical polarisation, and high-resolution imaging techniques. $2.5 \mathrm{mmol} \mathrm{dm}^{-3}(\mathrm{pH}$ 11.4 ) and $0.1 \mathrm{~mol} d m^{-3}$ (pH 13) sodium hydroxide ( $\mathrm{NaOH}$, caustic soda) have been used to condition the surface, as a pretreatment stage. The pretreatment leads to lower corrosion rates and, through microscopy analysis, appears to affect niobium carbide $(\mathrm{NbC})$ inclusions. It is thought that the dissolution of $\mathrm{NbC}$ precipitates, or possibly an additional lamellar phase within the inclusion, leads to a reduction in corrosion initiation sites, which may have the effect of thereby lowering corrosion rates. 


\subsubsection{Experimental Procedure}

AGR SS fuel cladding, of composition $20 \mathrm{wt} \% \mathrm{Cr}, 25 \mathrm{wt} \% \mathrm{Ni}, 0.05 \mathrm{wt} \% \mathrm{Nb}$ was studied, the full composition is given in figure 6.1. A sheet (0.3 mm thickness) of $20 / 25 / \mathrm{Nb}$ stainless steel underwent a thermal processing treatment in order to develop sensitisation, which followed the same processes as in earlier chapters. Both thermally sensitised and as-received material was used in this Chapter. The as-received stainless steel was studied in the first instance to understand the impact of $\mathrm{pH}$ on the material before studying in a thermally sensitised condition, where the stainless steel was immersed in electrolytes with and without $\mathrm{NaOH}$. Following the treatments the flat sheet was cut, ground, and polished into $1.5 \mathrm{~cm}^{2}$ pieces. Sensitisation was depth dependant for these specimens, as shown from previous DL-EPR experiments. The Haematite scale was ground and polished. This was to ensure a surface free of the iron oxide, such that corrosion did not preferentially initiate within the pores of the oxide.

\begin{tabular}{|l|l|l|l|l|l|l|}
\hline $\mathrm{Cr}$ & $\mathrm{Ni}$ & $\mathrm{Nb}$ & $\mathrm{Mn}$ & $\mathrm{Si}$ & $\mathrm{C}$ & $\mathrm{N}$ \\
\hline 20.12 & 24.75 & 0.70 & 0.62 & 0.57 & 0.049 & 0.016 \\
\hline $\mathrm{S}$ & $\mathrm{P}$ & $\mathrm{Co}$ & $\mathrm{Ta}$ & $\mathrm{Al}, \mathrm{Co}, \mathrm{Zr}, \mathrm{Ti}$ & $\mathrm{Fe}$ \\
\hline 0.0004 & 0.006 & 0.0025 & $<0.001$ & $<0.05$ & Balance \\
\hline
\end{tabular}

Figure 6.1: AGR Fuel cladding, 20/25/Nb stainless steel fuel cladding composition [7]

DL-EPR experiments were not conducted on samples due to the destructive nature of the experiments. Whilst grinding depth was considered, the assessment was on the impact of the surface treatment, not the depth of sensitisation. The microstructure was not etched for microscopy analysis, which allowed the material to be studied without prior dissolution of phases taking place. The main focus of this study was to investigate the role of $\mathrm{NaOH}$ on the material and how it might be used to inhibit corrosion. This investigation comprised of the following work packages:

1. Investigating the effects of different $\mathrm{pH}$ in an as-received condition 
2. Investigation of effect of $\mathrm{NaOH}$ on sensitised $\mathrm{SS}$ in ppm $\left[\mathrm{Cl}^{-}\right]$

3. An in-situ time-lapse microscopy experiment 


\subsection{Study 1: The Effect of $\mathrm{NaOH}$ on Unsensi- tised SS}

\subsubsection{Experimental Procedure}

All experiments were conducted in $400 \mathrm{ml}$ electrolyte prepared from a large batch of distilled water with electroyte $\mathrm{pH}$ ranging from 2.5 to 12.5. Basic electrolytes were created by adding in droplets of $1 \mathrm{~mol} \mathrm{dm}^{-3} \mathrm{NaOH}$. An acidic environment was produced by adding in droplets of concentrated $\mathrm{HCl}$. For these experiments, no prior furnace treatment was needed. These experiments represent the large quantity of unsensitised AGR SNF that the industry have in storage conditions. Potentiodynamic experiments were repeated 3 times for each $\mathrm{pH}$, resulting in an average. Error bars quoted represent one standard deviation.

\subsubsection{Results and Discussion}

Immersed in $1 \mathrm{~mol} d m^{-3} \mathrm{NaCl}$ at neutral $\mathrm{pH}$, the $\mathrm{SS}$ is separated by many hundreds of millivolts from its breakdown potential (figure 6.2). The total change in potential is approximately $600 \mathrm{mV}$. The $\mathrm{SS}$ is passive even in $1 \mathrm{~mol} \mathrm{dm^{-3 }}$ $\mathrm{NaCl}$ at neutral $\mathrm{pH}$, with current densities remaining below $10^{-5} \mathrm{Acm}^{-1}$ for the majority of the potential range. $\mathrm{NaOH}$ appears to both increase not only the total change in potential $\left(E_{b}-E_{c}\right)$, but also the current at open circuit, and passive current density. Higher concentrations of $\mathrm{NaOH}$ result in shifting $E_{c}$ to less noble values, but also increasing $E_{b}$ to more noble potentials. This leads to a greater change in potential ( $\Delta \mathrm{E}$ ) such that the average $\Delta \mathrm{E}$ is increased by 267 $\mathrm{mV}$ from $\mathrm{pH} 7$ to $\mathrm{pH}$ 12.5. The results of experimentation, including averages and error, are given in table 6.1. 


\begin{tabular}{|l||l|l|l|l|}
\hline $\mathrm{pH}$ & 2.5 & 7 & 11.4 & 12.5 \\
\hline$E_{b}(\mathrm{Av}), \mathrm{mV}$ & 342.4 & 412.5 & 368.4 & 560.9 \\
$E_{b}(\sigma), \mathrm{mV}$ & 44.8 & 18.6 & 27.0 & 1.3 \\
$E_{\text {Corr }}$ & -259.4 & -204.4 & -271.4 & -323.3 \\
$E_{\text {Corr }}(\sigma), \mathrm{mV}$ & 6.9 & 10.9 & 45.9 & 32.3 \\
\hline$\Delta \mathrm{E}$ & 601.8 & 616.8 & 639.8 & 884.2 \\
\hline
\end{tabular}

Table 6.1: Average potentials of unsensitised SS, and respective uncertainty (one standard deviation).

In electrolyte without $\mathrm{pH}$ adjustment (figure 6.6) more transient pitting events are present, some of which are up to an order of magnitude in size. These occur at potentials well below the breakdown potential, where stable pitting occurs. Only when the SS is immersed in neutral pH electrolyte do large transient events occur, in both an acidic (hydrochloric) and basic (sodium hydroxide) such events do not appear. The results from acidic and neutral environments are presented in figures 6.5 and 6.6. Both $\mathrm{NaOH}$ graphs show large fluctuations in current density around $E_{c}$, which is believed to be caused by experimental error, caused by the instrument filtering working incorrectly. The experiment for both $\mathrm{pH} 11.4$ and $\mathrm{pH} 12.5(\mathrm{NaOH})$ was re-run with the same parameters, but the same trend around $E_{c}$ occurred (figures 6.3, 6.4).

A plot of $E_{c}$ and $E_{b}$ as a function of electrolyte $\mathrm{pH}$ is given in figure 6.7. The graph shows both the results from the individual experiments, averages, error bars (1 S.D.) and lines of best fit. The results are in line with what was to be expected, with acidic electrolytes leading to a reduction in $E_{b}$. 


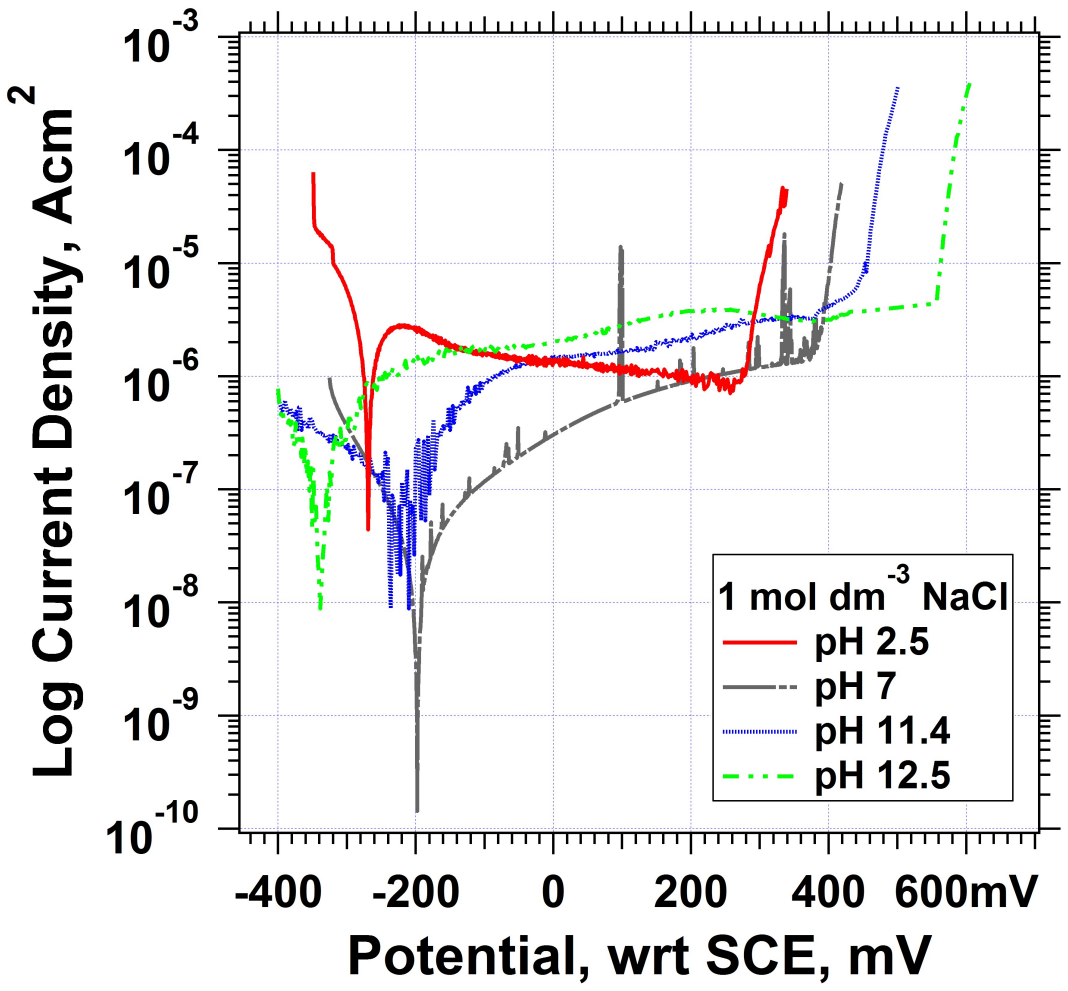

Figure 6.2: Unsensitised SS in $1 \mathrm{~mol} d^{-3} \mathrm{NaCl}$, varying additions of $\mathrm{HCl}$ and $\mathrm{NaOH}$. 


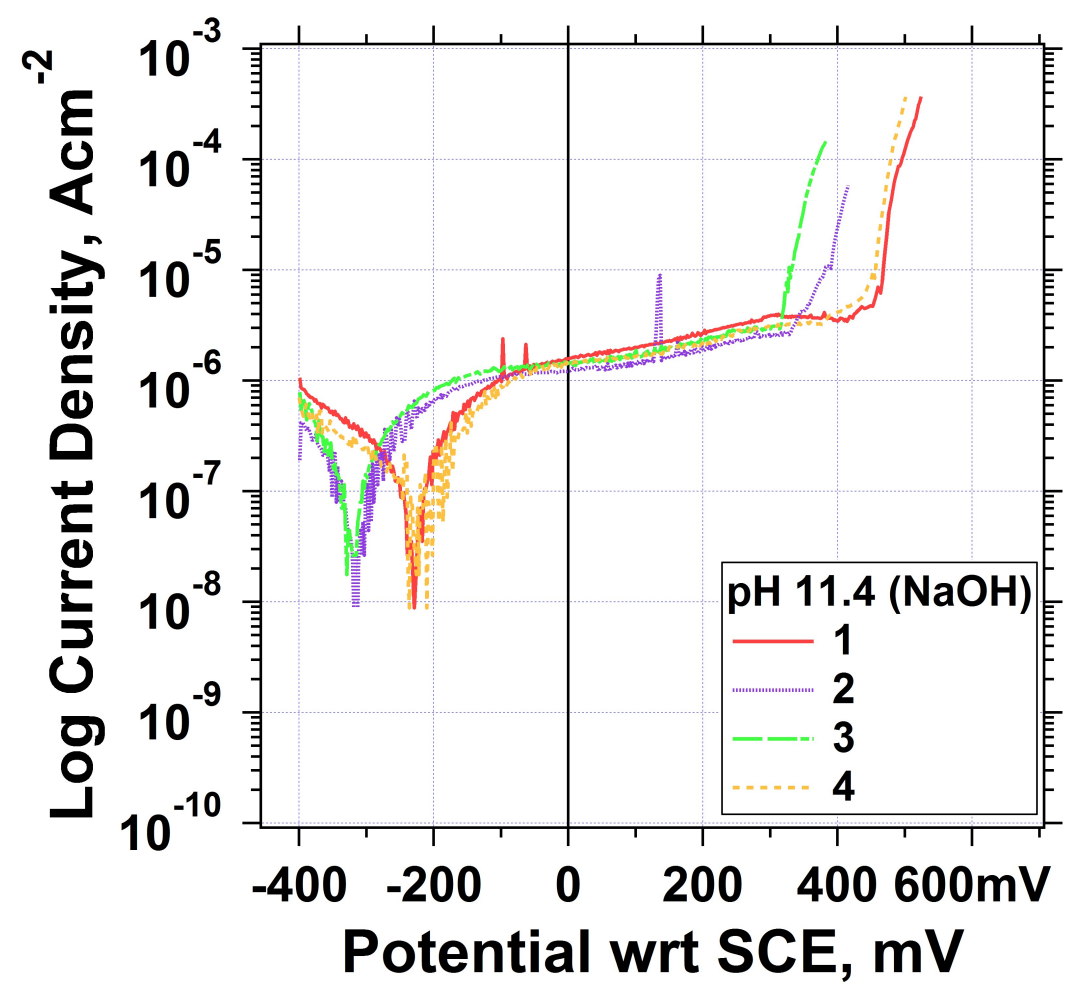

Figure 6.3: Potentiodynamic experiment on unsensitised specimen in $1 \mathrm{~mol} \mathrm{dm}^{-3}$ $\mathrm{NaCl}$ at $\mathrm{pH} 11.4(\mathrm{NaOH})$.

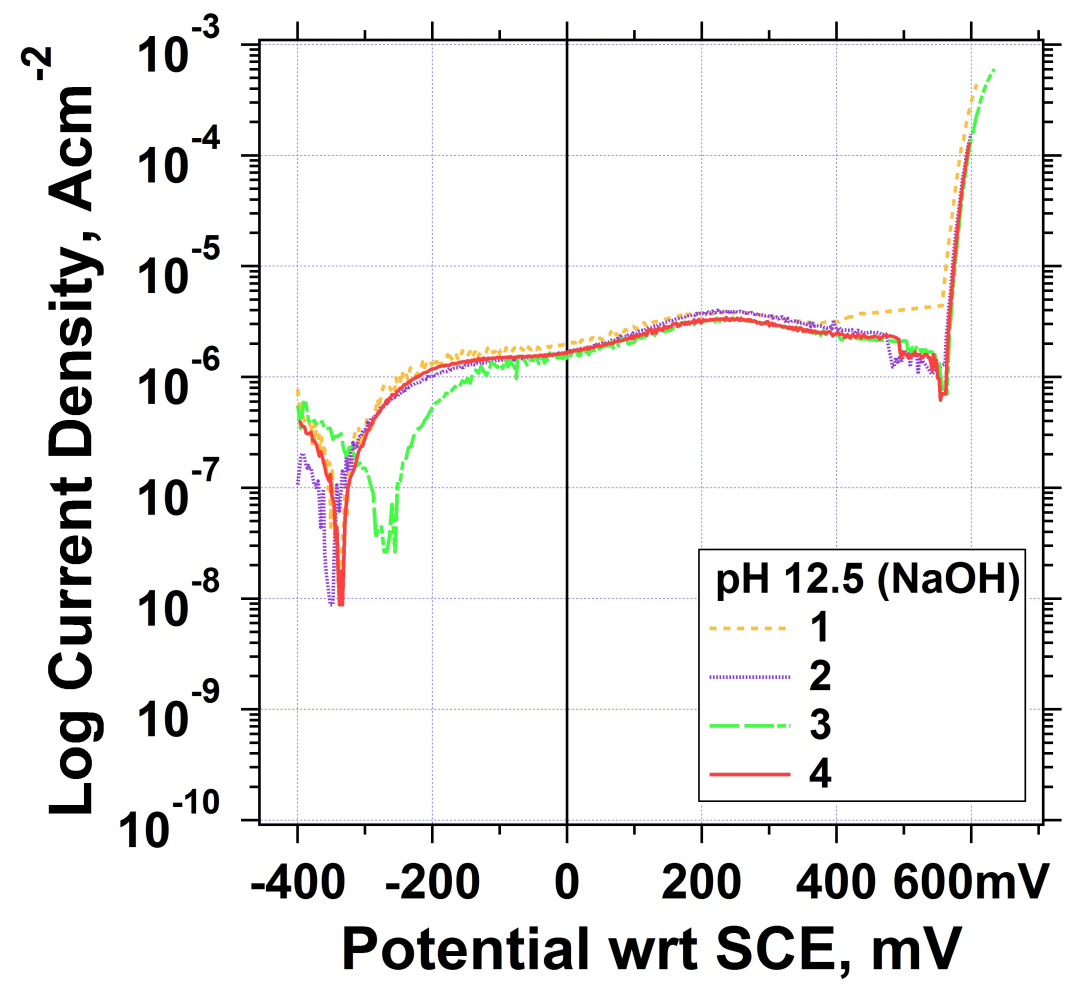

Figure 6.4: Potentiodynamic experiment on unsensitised specimen in $1 \mathrm{~mol} \mathrm{dm^{-3 }}$ $\mathrm{NaCl}$ at $\mathrm{pH} 12.5(\mathrm{NaOH})$. 


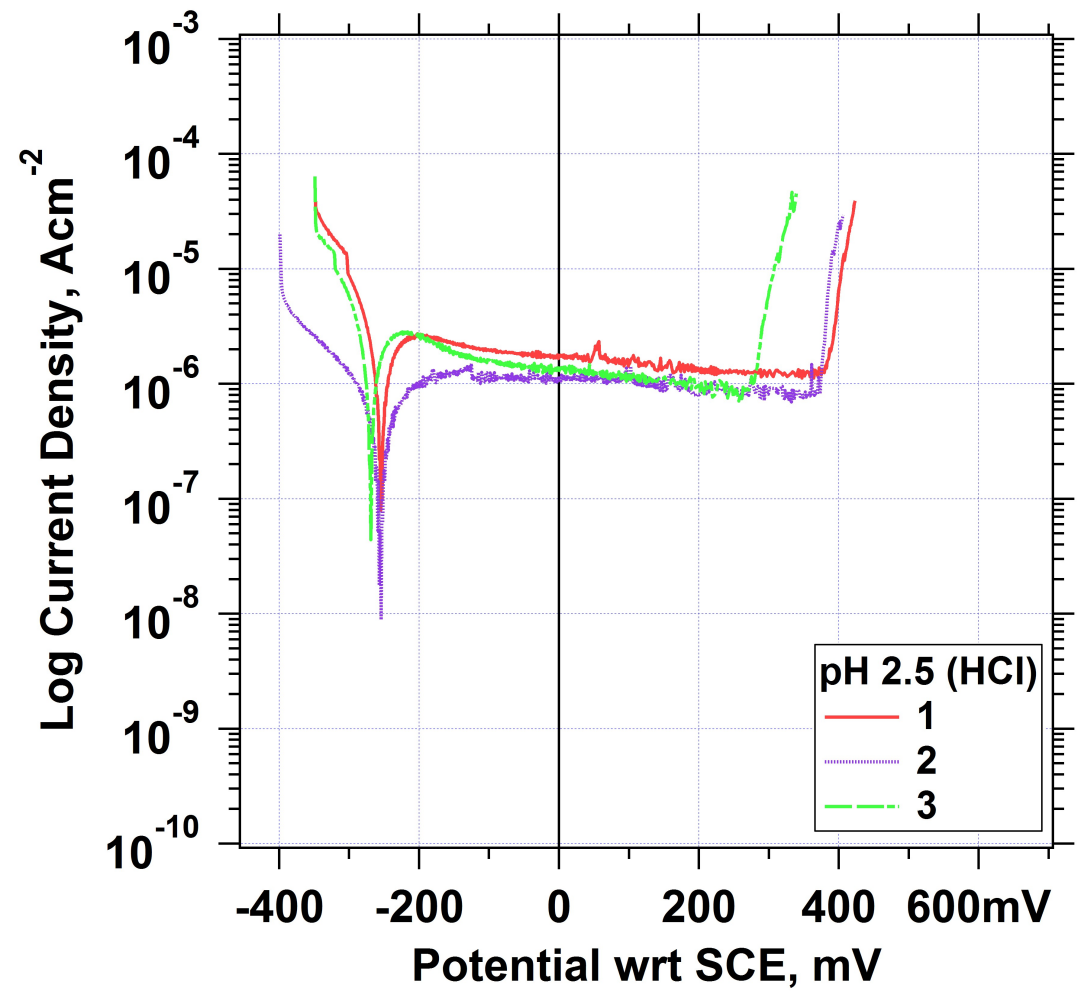

Figure 6.5: Potentiodynamic experiment on unsensitised specimen in $1 \mathrm{~mol} \mathrm{dm}$ $\mathrm{NaCl}$ at $\mathrm{pH} 2.5(\mathrm{HCl})$.

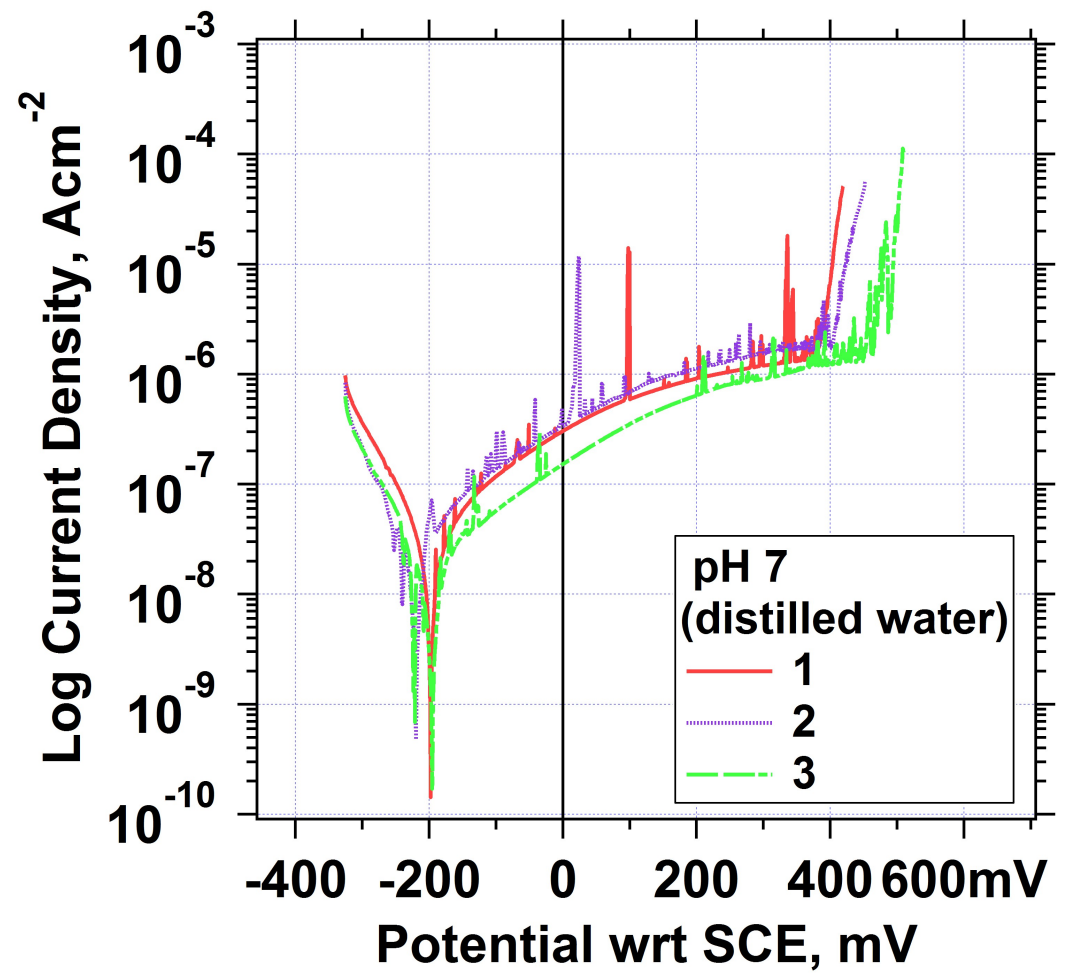

Figure 6.6: Potentiodynamic experiment on unsensitised specimen in $1 \mathrm{~mol} \mathrm{dm^{-3 }}$ $\mathrm{NaCl}$. 


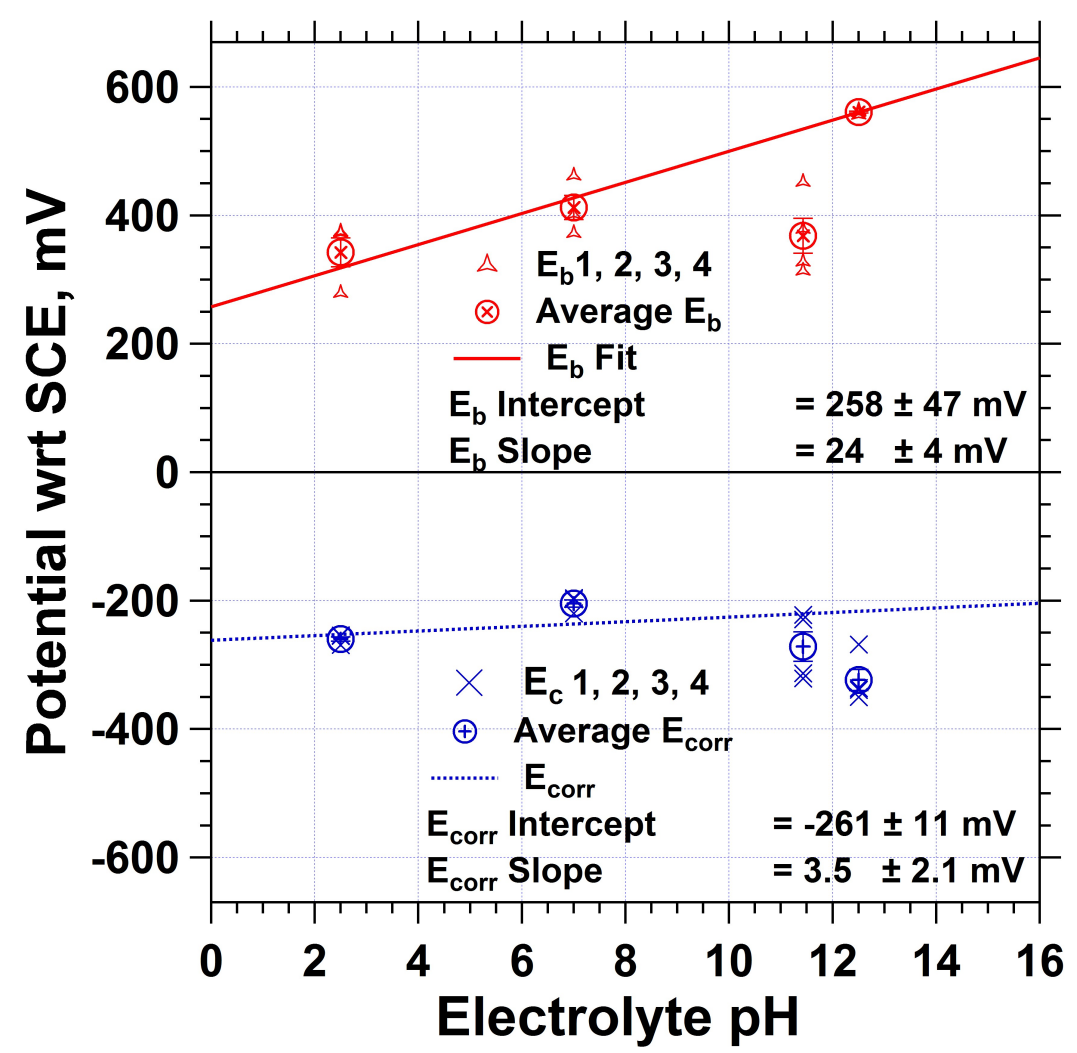

Figure 6.7: Breakdown and free corrosion potentials as a function of electrolyte $\mathrm{pH}$ for unsensitised SS. 


\subsection{Study 2: The Effect of $\mathrm{NaOH}$ on Sensitised SS}

\subsubsection{Introduction}

Following the results from the previous study, $\mathrm{NaOH}$ does appear to delay the onset of corrosion on unsensitised SS. $\mathrm{NaOH}$ also leads to a decrease in the large metastable events that were present when immersed in neutral pH. As some cooling ponds are dosed with $\mathrm{NaOH}$, this investigation was conducted as a function of $\left[\mathrm{Cl}^{-}\right]$and $\mathrm{NaOH}$, rather than $\mathrm{pH}$ at fixed $\left[\mathrm{Cl}^{-}\right]$. In this study sensitised SS specimens were analysed as a function of chloride with, and without $\mathrm{NaOH}$ additions of $2.5 \mathrm{mmol} \mathrm{dm}^{-3}$ ( $\mathrm{pH}$ 11.4). Industry cooling ponds are tightly controlled for contaminants, and thus have many orders of magnitude less $\left[\mathrm{Cl}^{-}\right]$ than were used in the first study $\left(1 \mathrm{~mol} \mathrm{dm}^{-3} \mathrm{NaCl}\right)$. Open-air cooling ponds have a low $\left[\mathrm{Cl}^{-}\right]$, at parts per million (ppm) levels, nominally these are below trace levels at 1-2 ppm $\left[\mathrm{Cl}^{-}\right]$at $\mathrm{pH} 11.4(\mathrm{NaOH})$. To reflect this, units of ppm are quoted.

\subsubsection{Experimental Procedure}

Parts per million (ppm) solutions prepared for experiments were prepared in the same way described in section 6.1.1. Ultra high purity $18 \mathrm{M} \Omega \mathrm{cm}^{-1}$ distilled water (Purite Select Fusion) was used for the 0 ppm solution. For $\mathrm{NaOH}$ (Aldrich, $\geq 98 \%, \leq 1.0 \%$ sodium carbonate) electrolytes, a large quantity of the ultra pure distilled water was dosed to $\mathrm{pH} 11.4$ using a $\mathrm{pH}$ meter (Thermo Scientific Orion star A) and then diluted to the desired ppm concentrations. A quantity of the original batch was kept separate to form the $0 \mathrm{ppm}$ baseline. The $\mathrm{NaOH}$ electrolytes were prepared in this way, rather than adding in droplets of concentrated $\mathrm{NaOH}$ to reduce the amount of dilution. Efforts were made to reduce errors as much as possible in this preparation stage as the concentrations 
of $\mathrm{Cl}^{-}$were at ppm levels. Specimens were sensitised by thermal treatment as shown in section $2.2\left(1150{ }^{\circ} \mathrm{C}, 650{ }^{\circ} \mathrm{C}\right)$. The specimens were prepared by grinding flat specimens using successive grit papers (rough:fine). The surfaces were polished using 6 and $1 \mu \mathrm{m}$ diamond suspension, then rinsed using ethanol to assist in the removal of contaminants before experimentation took place. Open circuit potential (OCP) experiments were run for $3600 \mathrm{~s}$ to allow for both equilibration,

and comparison of the rest potential for different concentrations of $\mathrm{NaCl}$, and with and without $\mathrm{NaOH}$. Linear polarisation resistance (LPR) experiments were conducted with a three electrode cell set-up, using Pt gauze as the counter electrode and an SCE reference. The experiment was set to scan $-10 \mathrm{mV}$ to $+10 \mathrm{mV}$ away from the steady state OCP.

\subsubsection{Results and Discussion}

Results of immersion in a series of $\mathrm{Cl}^{-}$electrolytes are presented in figure 6.8 and 6.9. Generally, apart from the $1000 \mathrm{ppm}\left[\mathrm{Cl}^{-}\right]$specimen at neutral $\mathrm{pH}$, which showed a drop in OCP at $2700 \mathrm{~s}$ by $20 \mathrm{mV}$, all potentials appeared steady, after a period of $1800 \mathrm{~s}$ (30 minutes).

$\mathrm{NaOH}$ appears to push the rest potential to more negative values by at least $100 \mathrm{mV}$. For concentrations $10 \mathrm{ppm}$ and above (neutral) rest potentials are more varied $(\approx 100 \mathrm{mV})$, as opposed to those in $\mathrm{NaOH}$, where the potentials are within $20 \mathrm{mV}$ of one another. $\mathrm{NaOH}$ may be suppressing the effect of $\left[\mathrm{Cl}^{-}\right]$ on the system at higher concentrations, as there is far less potential shift. At lower concentrations, the potentials shift more, but the total decrease in potential when using $\mathrm{NaOH}$ is larger. There is a maximum change of $\approx 300 \mathrm{mV}$ (1 ppm $\left.\left[\mathrm{Cl}^{-}\right]\right)$. Polarisation resistance experiment data is shown in figure 6.10. The data shown is the polarisation resistance determined from the slope of $\mathrm{dE} / \mathrm{di}$. Polarisation resistance is a component the corrosion rate calculation, and thus a larger polarisation resistance is appropriate for greater resistance to corrosion.

For specimens in neutral $\mathrm{pH}$ an increase in $\mathrm{Cl}^{-}$leads to a decrease in polar- 


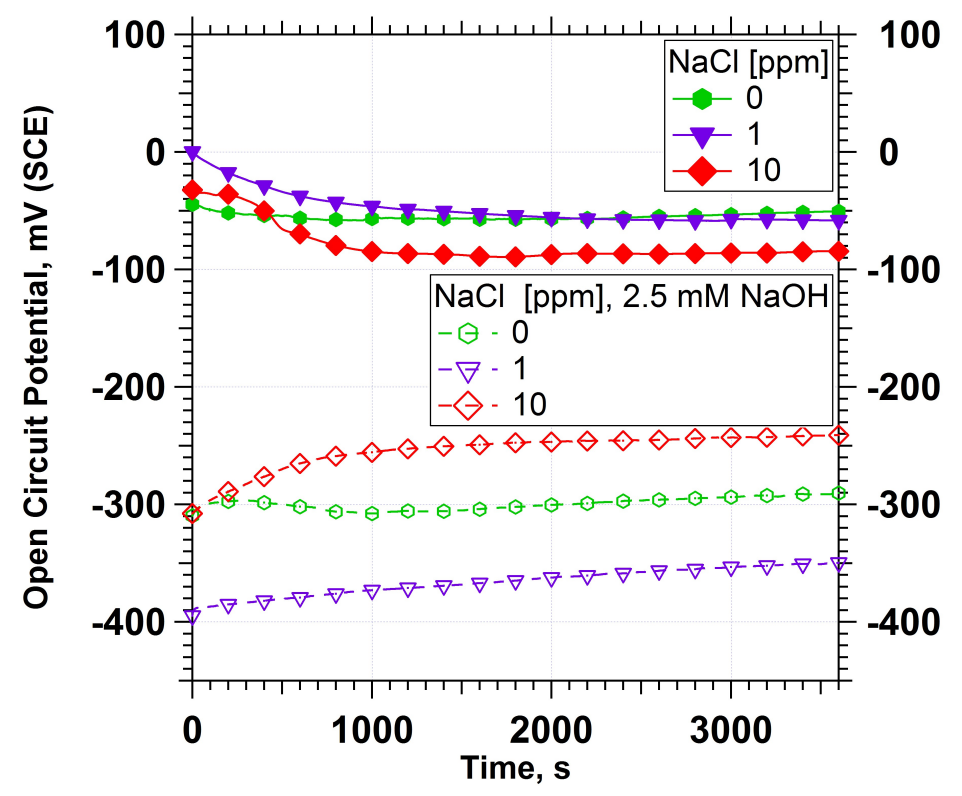

Figure 6.8: Sensitised SS with and without $\mathrm{NaOH}$ inhibitor $\left(2.5 \mathrm{mM} d m^{-3}\right)$, as a function of $\left[\mathrm{Cl}^{-}\right](0,1$ and $10 \mathrm{ppm})$

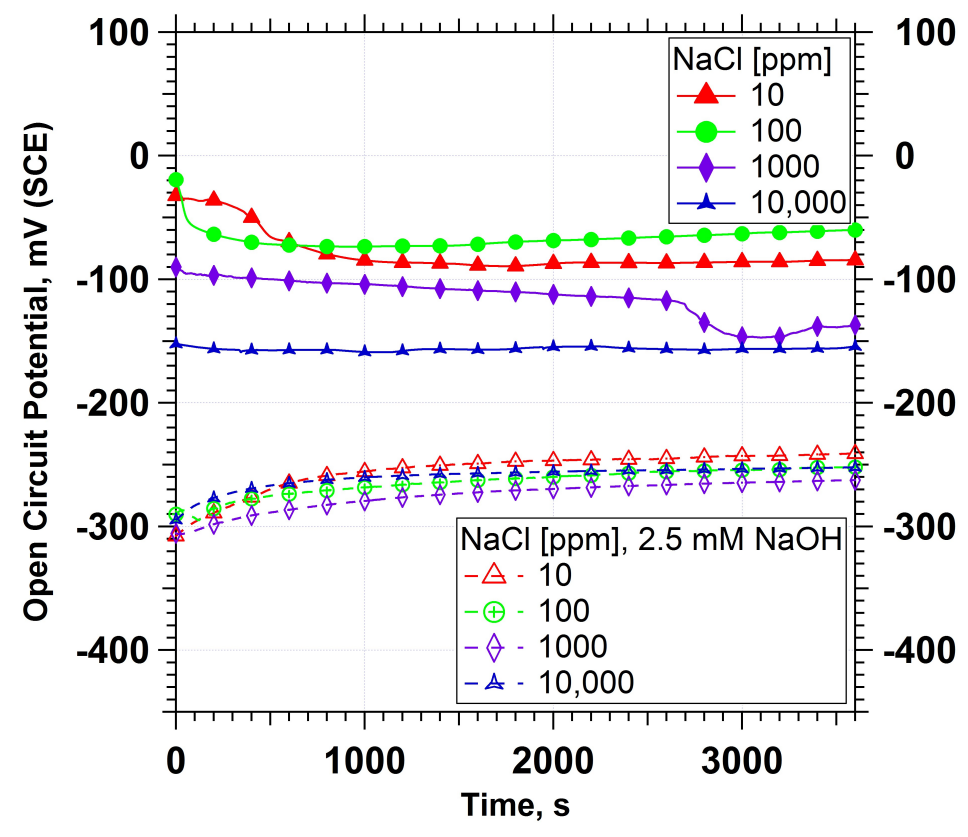

Figure 6.9: Sensitised SS with and without $\mathrm{NaOH}$ inhibitor $\left(2.5 \mathrm{mM} d m^{-3}\right)$, as a function of $\left[\mathrm{Cl}^{-}\right](10,100,1000,10000 \mathrm{ppm})$ 
isation resistance. There is a decrease in resistance of over $80 \%$ when moving from 0 to 10,000 ppm $\left[\mathrm{Cl}^{-}\right]$. The results for specimens immersed in $\mathrm{NaOH}$ show no trend. Polarisation resistance values are lower or equal when compared to those in neutral pH in the majority of experiment electrolytes. For higher values of $\left[\mathrm{Cl}^{-}\right](1000,10,000 \mathrm{ppm})$ polarisation resistance is greater. Oddly, the experiment results for typical pond water chemistry (1 ppm $\left.\left[\mathrm{Cl}^{-}\right]\right)$show a reduction in polarisation resistance when using $\mathrm{NaOH}$. In $1 \mathrm{ppm}\left[\mathrm{Cl}^{-}\right]$the polarisation resistance is over $100 \mathrm{~K} \Omega$, but decreases by $27 \mathrm{~K} \Omega$ when the inhibitor is used ( $\mathrm{NaOH}$ at $\mathrm{pH} 11.4$ ). This occurs again with the $10 \mathrm{ppm}$ experiment, polarisation resistance decreases by over $2 / 3$ when $\mathrm{NaOH}$ is used $(67.8 \mathrm{~K} \Omega$ to $20.2 \mathrm{~K} \Omega$ ). 


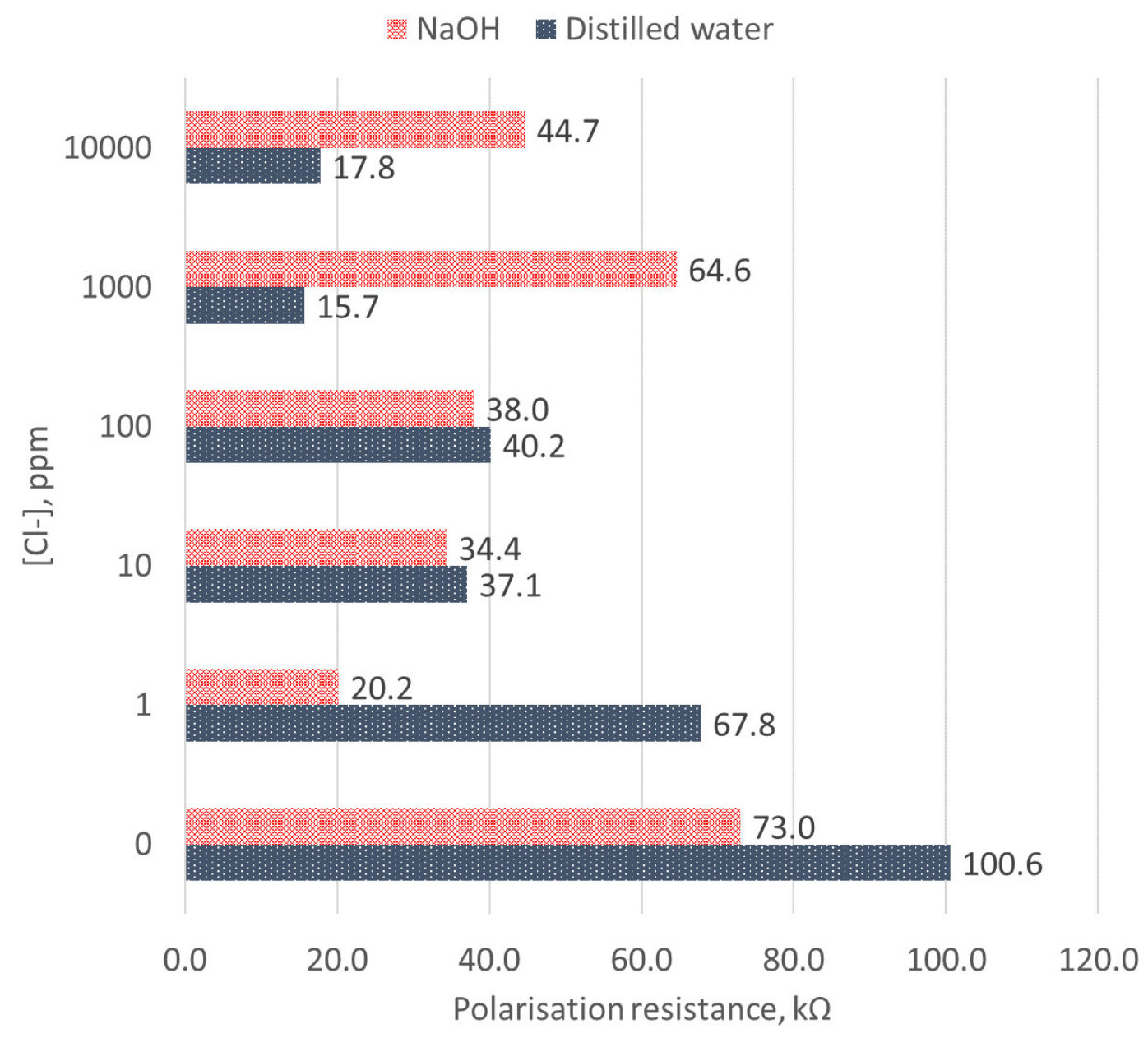

Figure 6.10: Polarisation resistance experiments as a function of $\left[\mathrm{Cl}^{-}\right]$in distilled water and $2.5 \mathrm{mM} d m^{-3} \mathrm{NaOH}(\mathrm{pH} 11.4)$ 


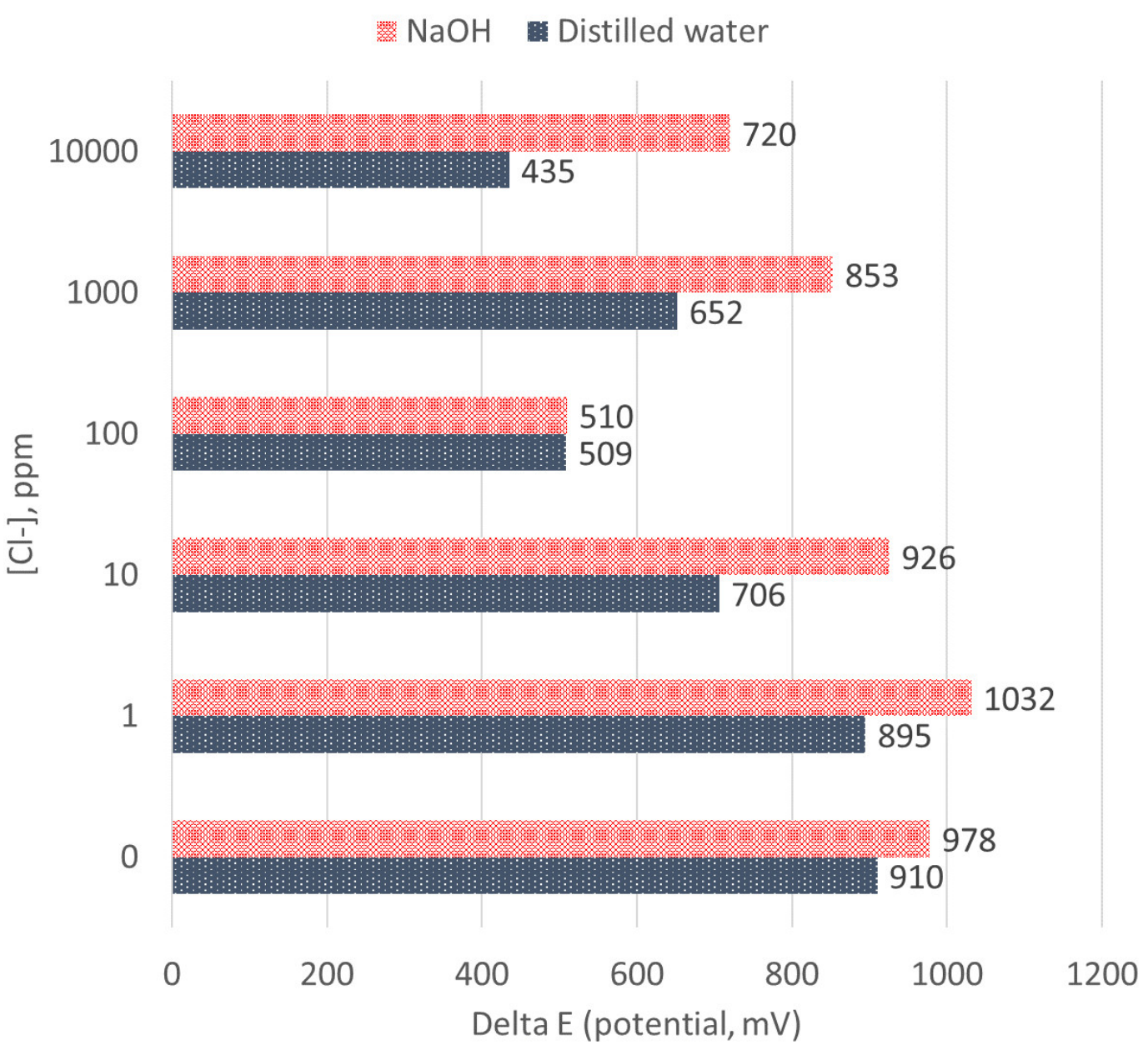

Figure 6.11: Change in potential (Eb-Ecorr) for sensitised SS specimens immersed in neutral $\mathrm{pH}$ and $\mathrm{NaOH}$ as a function of $\left[\mathrm{Cl}^{-}\right]$ 
Figure 6.11 shows results of potentiodynamic experiments on specimens with and without an $\mathrm{NaOH}$ addition. The bar chart shows the total change in potential from $\Delta E=E_{b}-E_{\text {corr }}$. Larger values suggest a delay in stable corrosion propagation, as the specimen rest potential is $E_{\text {corr }}$, and stable IGC occurs where there is an exponential increase in current density, at $E_{b}$. As $\left[\mathrm{Cl}^{-}\right]$increases, $\Delta \mathrm{E}$ decreases, as susceptibility to undergo stable corrosion at either an earlier $E_{b}$, or a shift in $E_{\text {corr }}$ to more positive values, or both occur. The 100 ppm result for both neutral and $\mathrm{NaOH}$ electrolytes is not in line with the trend. For all experiments (excluding 0 and $100 \mathrm{ppm}\left[\mathrm{Cl}^{-}\right]$), $\mathrm{NaOH}$ increases $\Delta \mathrm{E}$ by a sizeable margin, for most by at least $200 \mathrm{mV}$. The change in potential is a useful quantifier, however more detail could be attained from the traces themselves, rather than the difference between two points on a line. These are displayed in figure 6.12 and figure 6.13.

Without $\mathrm{NaOH}$, current density over the passive region rises with $\left[\mathrm{Cl}^{-}\right]$(figure 6.12). The increase in current density at $E_{b}$ is not substantial until $\left[\mathrm{Cl}^{-}\right]$ rises above $100 \mathrm{ppm}$. This is a consequence of the ability to pass current due to the limited conductivity of the electrolyte itself. In contrast, the use of $\mathrm{NaOH}$ (figure 6.13) gives increased current densities over the passive region. Current density reaches a plateau at $\approx 200 \mathrm{mV}$.

Potentiodynamic traces in low $\left[\mathrm{Cl}^{-}\right](0,1,10 \mathrm{ppm})$ show that $E_{\text {corr }}$ is stable at $\approx-100 \mathrm{mV}$ vs. SCE for the sensitised SS in neutral conditions (figure 6.12). $E_{\text {corr }}$ for the specimens in low $\left[\mathrm{Cl}^{-}\right](0,1,10 \mathrm{ppm}$, in $\mathrm{NaOH})$ is more cathodic, at -300 $\mathrm{mV}$ (figure 6.13 ). $E_{b}$ for 0 and $1 \mathrm{ppm}\left[\mathrm{Cl}^{-}\right]$occurs at lower potentials when in the basic electrolyte, but $E_{\text {corr }}$ is still greater. The current density through the entire trace (including the active and passive regimes) is greater when using $\mathrm{NaOH}$. Given the very small concentration of $\mathrm{Cl}^{-}$ions, both seem to show very similar traces, having similar $E_{\text {corr }}, E_{b}$ and current density values. The $10 \mathrm{ppm}$ trace shows an example where $E_{\text {corr }}$ has shifted to more noble potential, such that it is more positive than in neutral $\mathrm{pH}$. These experiments are of particular importance, 


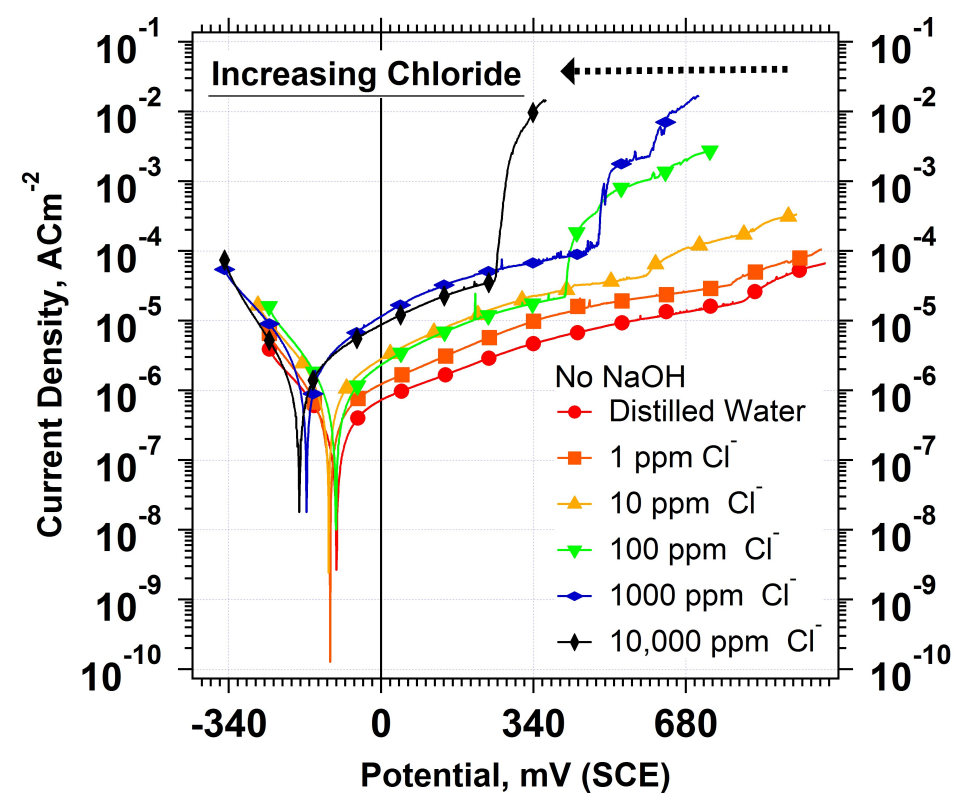

Figure 6.12: ppm $\left[\mathrm{Cl}^{-}\right]$potentiodynamic experiments without $\mathrm{NaOH}$

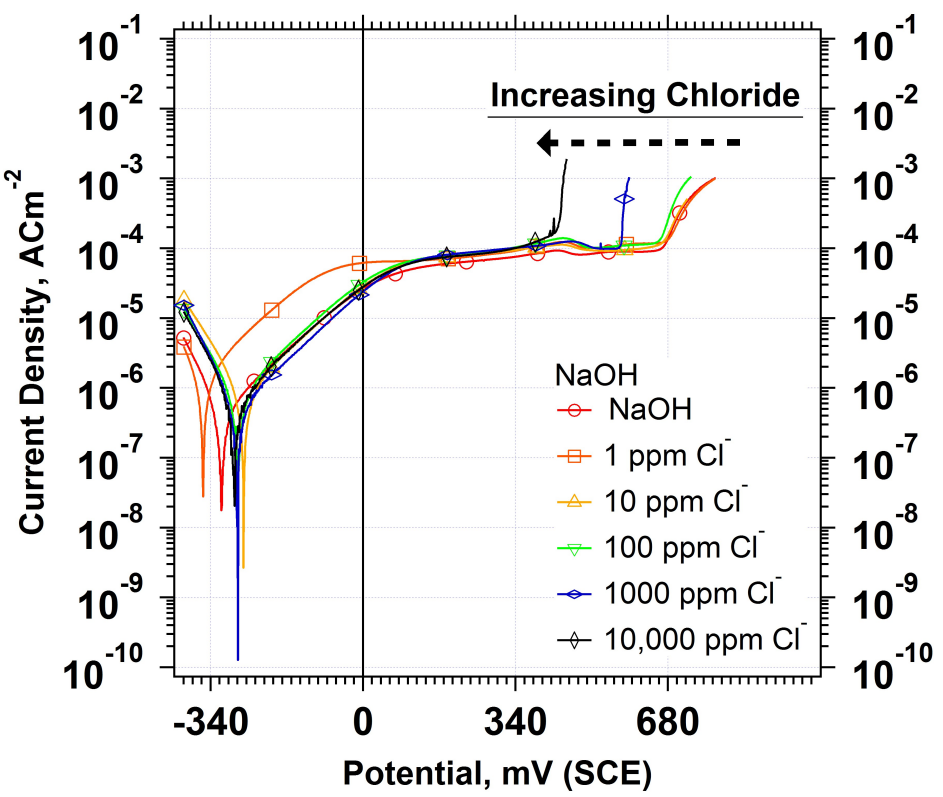

Figure 6.13: ppm $\left[\mathrm{Cl}^{-}\right]$potentiodynamic experiments with $\mathrm{pH} 11.4 \mathrm{NaOH}$ 
as less than $1 \mathrm{ppm}\left[\mathrm{Cl}^{-}\right]$would be expected in the industrial context. From the reverse $\mathrm{NaOH}$ traces, repassivation is delayed, occurring at more negative potentials in $10 \mathrm{ppm}\left[\mathrm{Cl}^{-}\right]$when compared to both distilled water and $1 \mathrm{ppm}$ $\left[\mathrm{Cl}^{-}\right]$. The effects of having a higher $\left[\mathrm{Cl}^{-}\right](100,1000,10,000 \mathrm{ppm})$ are easily seen in figures $6.17,6.18,6.19$, compared with $6.14,6.15,6.16$. The passive current density in $\mathrm{NaOH}$ remains similar to that of the lower concentrations, although for specimens without $\mathrm{NaOH}$, the current density during the reverse scan almost reaches $0.1 \mathrm{~A}$ for specimens immersed in 100,000 ppm. In addition, hysteresis loops are much larger, even for specimens which have been dosed with $\mathrm{NaOH}$. 


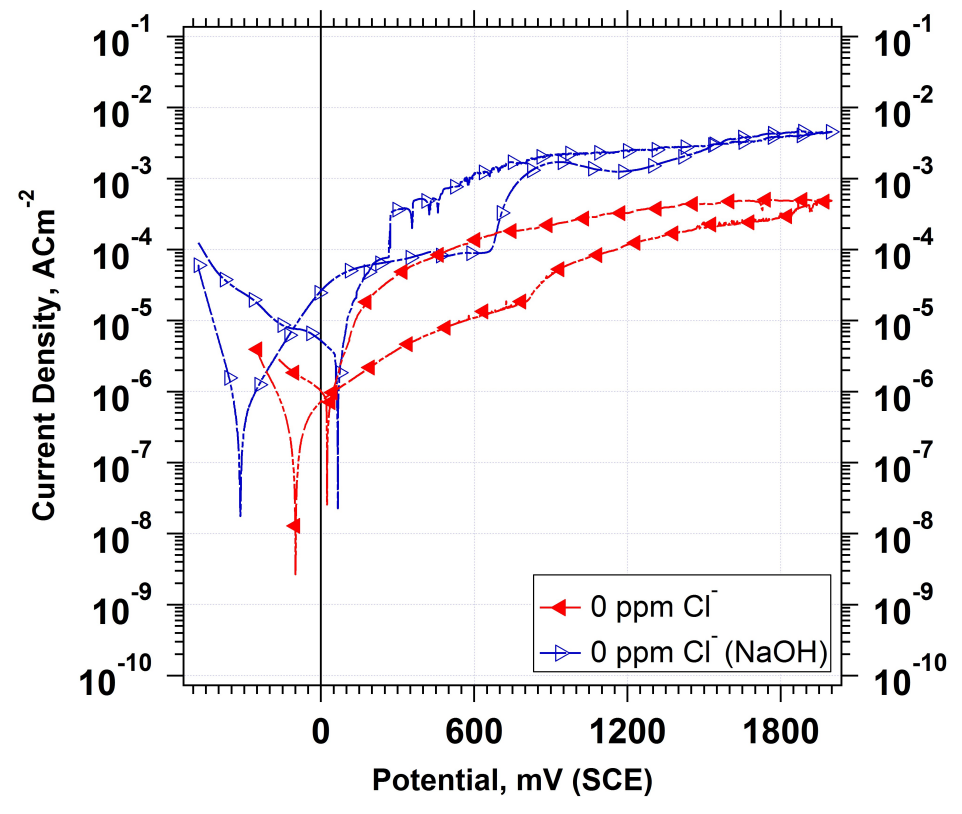

Figure 6.14: $0 \mathrm{ppm}\left[\mathrm{Cl}^{-}\right]$potentiodynamic experiments in neutral $\mathrm{pH}$ and 11.4 $(\mathrm{NaOH})$.

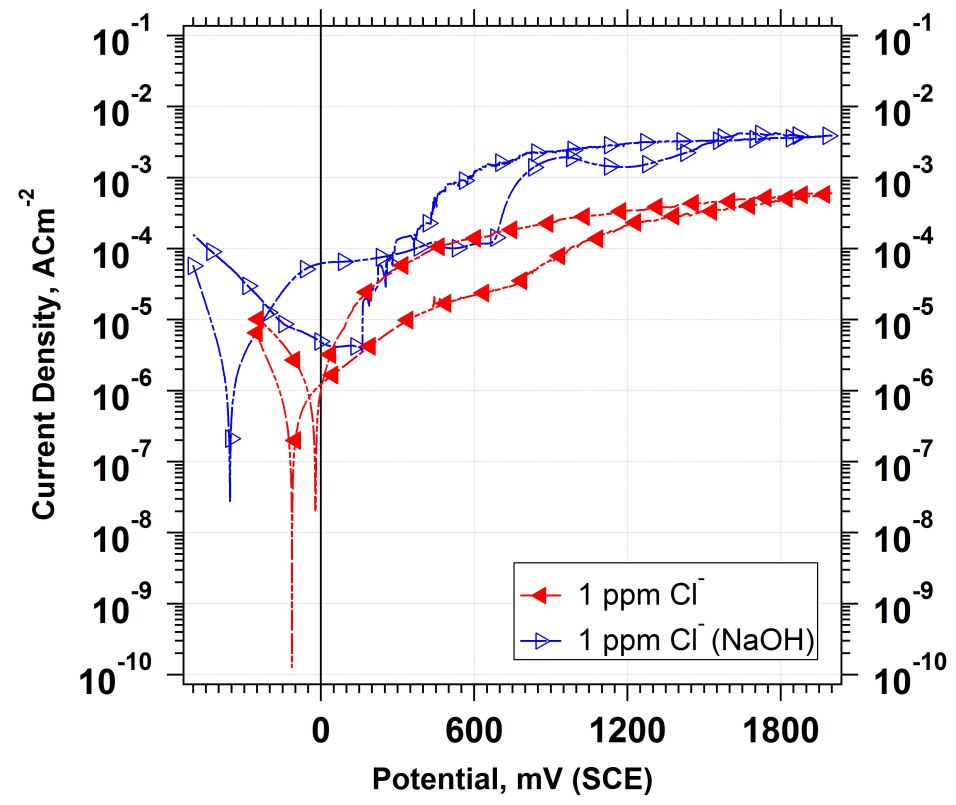

Figure 6.15: $1 \mathrm{ppm}\left[\mathrm{Cl}^{-}\right]$potentiodynamic experiments in neutral $\mathrm{pH}$ and 11.4 $(\mathrm{NaOH})$. 


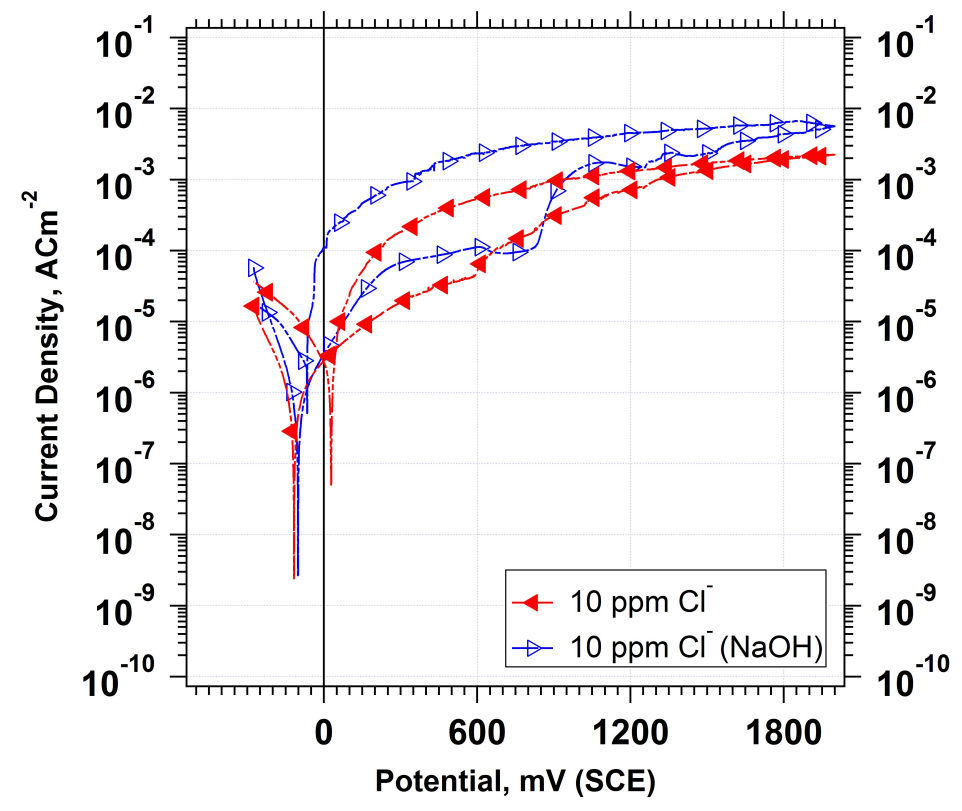

Figure 6.16: $10 \mathrm{ppm}\left[\mathrm{Cl}^{-}\right]$potentiodynamic experiments in neutral $\mathrm{pH}$ and 11.4 $(\mathrm{NaOH})$.

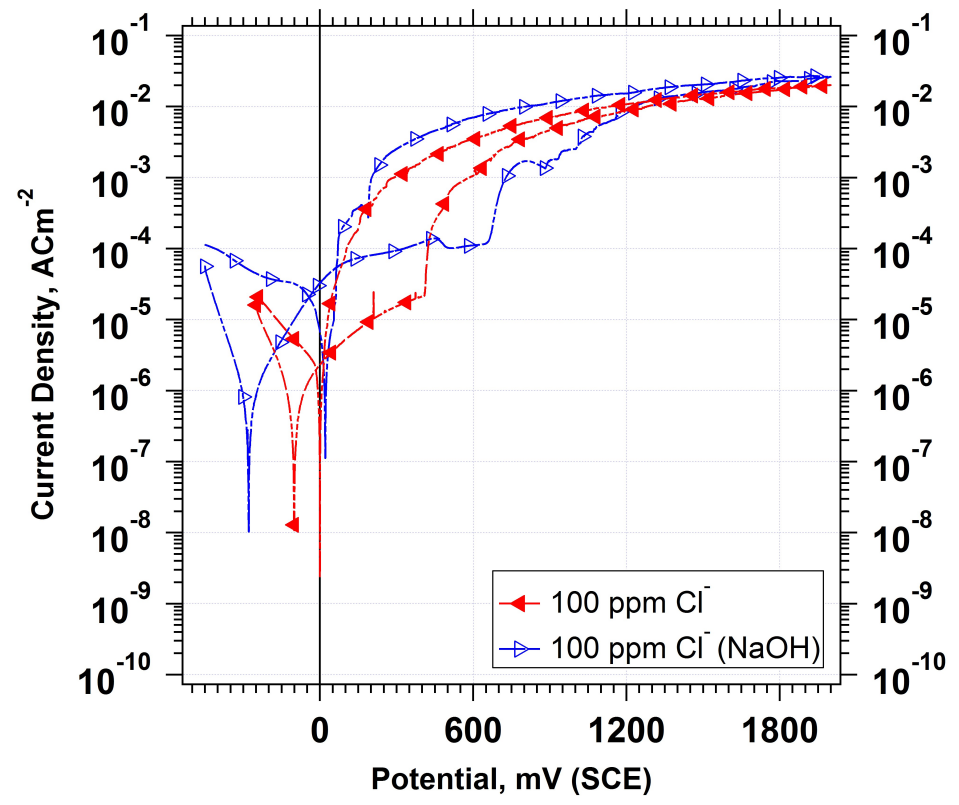

Figure 6.17: $100 \mathrm{ppm}\left[\mathrm{Cl}^{-}\right]$potentiodynamic experiments in neutral $\mathrm{pH}$ and 11.4 $(\mathrm{NaOH})$. 


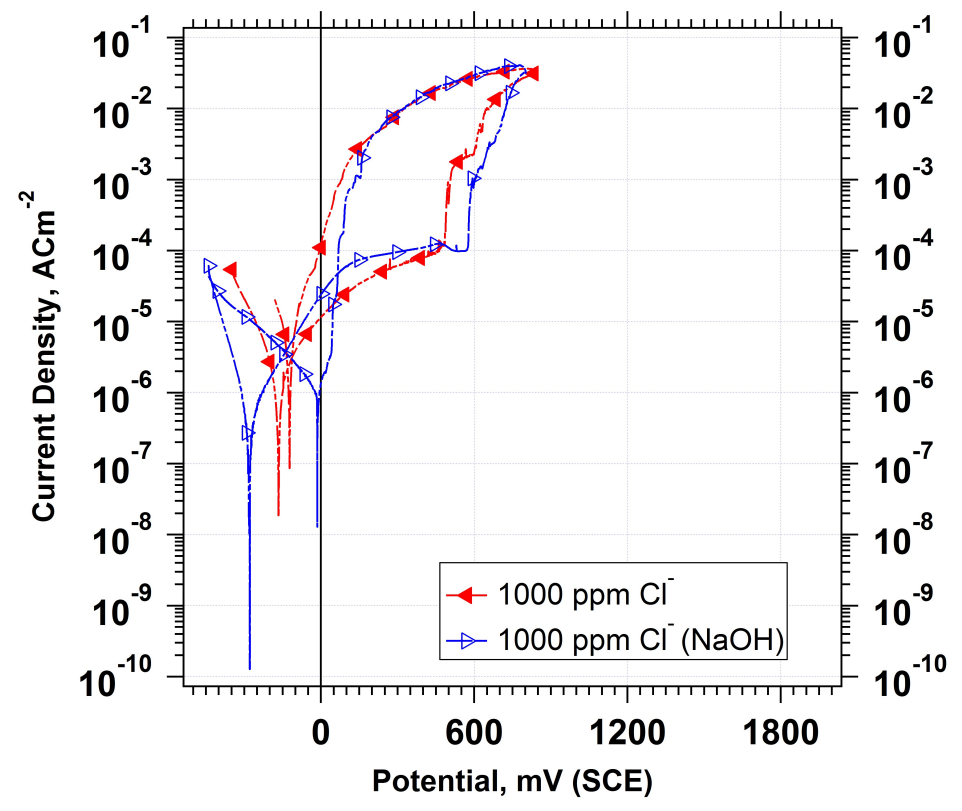

Figure 6.18: $1000 \mathrm{ppm}\left[\mathrm{Cl}^{-}\right]$potentiodynamic experiments in neutral $\mathrm{pH}$ and $11.4(\mathrm{NaOH})$.

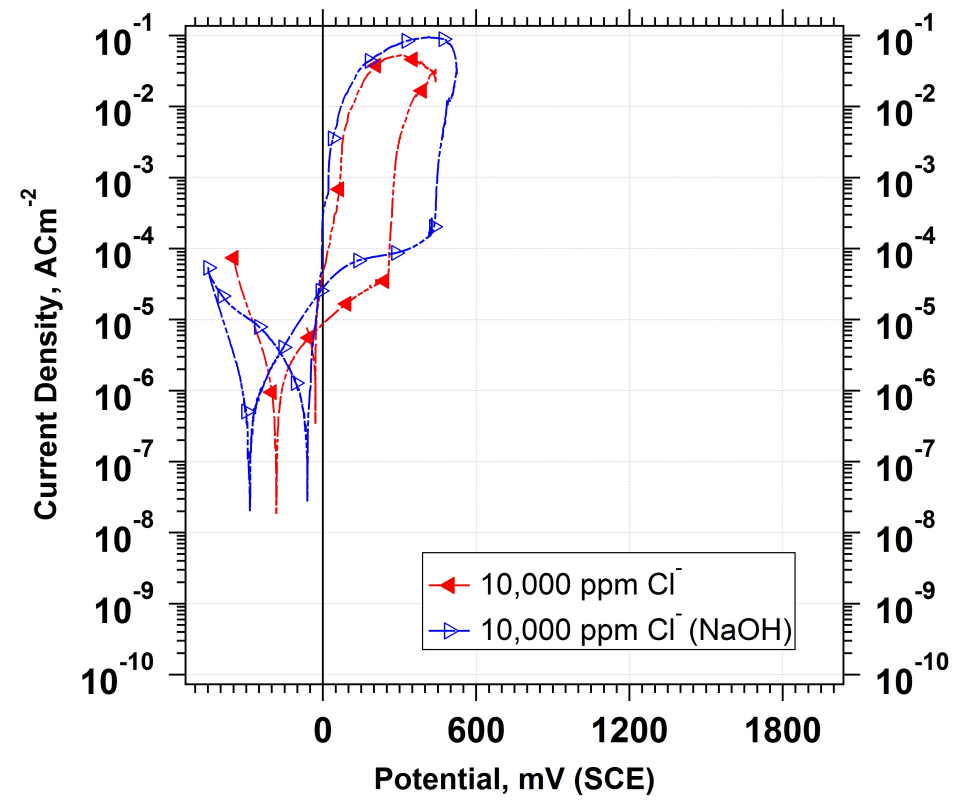

Figure 6.19: $10,000 \mathrm{ppm}\left[\mathrm{Cl}^{-}\right]$potentiodynamic experiments in neutral $\mathrm{pH}$ and $11.4(\mathrm{NaOH})$. 


\subsection{Study 3: An In-situ Experiment Using $\mathrm{NaOH}$ as a Pretreatment for Corrosion Inhibition}

\subsubsection{Introduction}

As $\mathrm{NaOH}$ has delayed the onset of pitting and IGC for the SS in its unsensitised and thermally sensitised condition, a trial was conducted to investigate its use as a pretreatment. A variety of in-situ techniques used throughout this thesis to analyse the effect of $\mathrm{NaOH}$. Using a combination of time-lapse microscopy (with polarisation), EDS chemical mapping, electron microscopy, atomic and scanning Kelvin probe force microscopy the effect of $\mathrm{NaOH}$ on the surface could be analysed. The $\mathrm{NaOH}$ pretreatment was conducted using $\mathrm{NaOH}$ at a higher $\mathrm{pH}$ than 11.4 to see if a short term pretreatment (relative to industry timescale) could facilitate added corrosion resistance. Of particular importance was to be able to image the $\mathrm{NbC}$ inclusions to see how these changed with respect to time in a strong base. $\mathrm{pH} 13 \mathrm{NaOH}\left(0.1 \mathrm{~mol} \mathrm{dm}^{-3}\right)$ was chosen as one of the pretreatments as it is already used in the industry for magnox fuel containment as an inhibitor [42].

\subsubsection{Experimental Procedure}

The specimen was sensitised and prepared using the same furnace treatment as has been described previously. In addition, a wire was spot welded to the specimen for OCP monitoring. The weld and specimen were encased in a cold set resin, and the surface was ground using successive grit papers to remove Haematite and then polished to a $1 \mu m$ finish. The $\mathrm{NaOH}$ pretreatment process was as follows:

1. Immersion in $\mathrm{pH} 11.4\left(2.5 \mathrm{mmol} \mathrm{dm}{ }^{-3} \mathrm{NaOH}\right)$ for 4 days

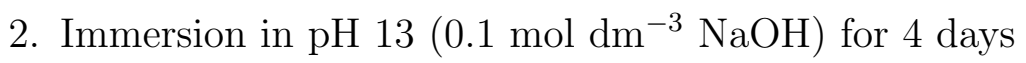


Ultra pure distilled water was used for the experiment. When stage 1 of the pretreatment was complete the electrolyte was removed and dosed to $\mathrm{pH} 13$ (0.1 mol $\left.\mathrm{dm}^{-3} \mathrm{NaOH}\right)$. This was measured using a $\mathrm{pH}$ meter, once the correct $\mathrm{pH}$ had been reached the electrolyte was re-used. Time-lapse Microscopy (TLM) was conducted using a Meiji MT8000 metallurgical microscope, fitted with an Infinity 2-5C camera. The technique has been well described in previous studies [149] [88] and in the experimental chapter. Once an $\mathrm{NbC}$ inclusion of suitable size was located (a set of three inclusions in a $\approx 20 \mu m^{2}$ area was selected), the specimen was connected to a Palmsens 3 potentiostat. A saturated calomel electrode (SCE) was used as a reference, with datapoints collected every 10 seconds. A 20x optical lens, protected by a waterproof shroud, was used for imaging the inclusion as a function of time. Due to the relative long experiment time, images were captured every 600 seconds. Following pretreatment in $\mathrm{NaOH}$, the specimen was analysed using a JEOL JSM-6010 PLUS/LA scanning electron microscope (SEM) with energy dispersive X-ray spectroscopy (EDS) capability. The set of inclusions from the same location were found and imaged using secondary electron imaging (SEI) and EDS maps taken. In addition, AFM and SKP-FM (JPK Nanowizard 3) provided high resolution maps of the inclusions. The set up for the SKP-FM is the same as described in the main experimental chapter. Finally a longer term pretreatment was conducted skipping stage 1 and implimenting stage 2 directly (immersion in $0.1 \mathrm{~mol} d m^{3} \mathrm{NaOH}$ ) for four weeks. Specimens were immersed in $\mathrm{NaOH}$ in $100 \mathrm{ml}$ beakers covered with parafilm to limit the effects of acidification from the environment and SEM-EDS analysis undertaken following immersion.

\subsubsection{Results and Discussion}

The specimen used for the experiment was immersed in $2.5 \mathrm{mM} d m^{3} \mathrm{NaOH}(\mathrm{pH}$ 11.4) for a period of 4 days. A set of three $\mathrm{NbC}$ inclusions were imaged. However there was no development of corrosion visually within this timeframe. Figure 6.20 (inset) shows the open circuit potential as a function of time, which stabilises at 
$-200 \mathrm{mV}$ vs. SCE after $\approx 200 \mathrm{Ks}$. Subsequent immersion in $0.1 \mathrm{~mol} d m^{-3} \mathrm{NaOH}$ causes an increase in the mixed potential to $\approx-110 \mathrm{mV}$ vs. SCE after $75 \mathrm{Ks}$. By working through the TLM images an approximate time could be calculated in which the inclusions underwent dissolution.

Following the immersion in a higher concentration of $\mathrm{NaOH}$, the three inclusions darken, after $28 \mathrm{Ks}$, as shown in figure 6.21. Suggesting NbC modification had occurred in the strong base. After a period of 1 day the experiment was stopped, and specimen removed. OCP data does not show any variation in potential relating to this apparent dissolution. This could be related to the fact the area imaged was not masked. As the instrument is collecting data on the whole sample $\left(1.5 \mathrm{~cm}^{2}\right)$ many inclusions could be undergoing dissolution at different rates; given the area covered by the inclusions imaged $\left(0.0025 \mathrm{~cm}^{2}\right)$. Therefore the general increase in potential could be a result of widespread NbC modification at differing rates. The electrochemical data does show an increase in potential of c. $+100 \mathrm{mV}$ from $2.5 \mathrm{mmol}$ to $0.1 \mathrm{~mol} \mathrm{dm}^{-3} \mathrm{NaOH}$ (see inset figure 6.20). The elevated potentials suggest resistance to corrosion, as SS are a passivating system.

The area in which the three inclusions occupied were located and then imaged using SEM. Interestingly it appeared that $\mathrm{NbC}$ were still present using SEM, in contrast to the in-situ TLM experiment where the inclusions appeared to have undergone modification 6.22.

It is expected that a light scattering effect caused the darkening. Roughening of the inclusions may have caused incident light to scatter at different angles, rather than reflecting toward the camera sensor. It is postulated that the inclusion was partially dissolved by the $\mathrm{NaOH}$, and the SEM micrograph shows lines across the surface of all three inclusions (figure 6.22). It is expected that the dissolution of $\mathrm{NbC}$ occurred along the polishing direction. The very hard diamond particulates used in the preparation stage may be the precursor to dissolution along surface scratches. Chemical mapping shows the presence of $\mathrm{Nb}$ and $\mathrm{C}$ in each of the inclusions, post pretreatment, figure 6.23. Suggesting that if Nb has 


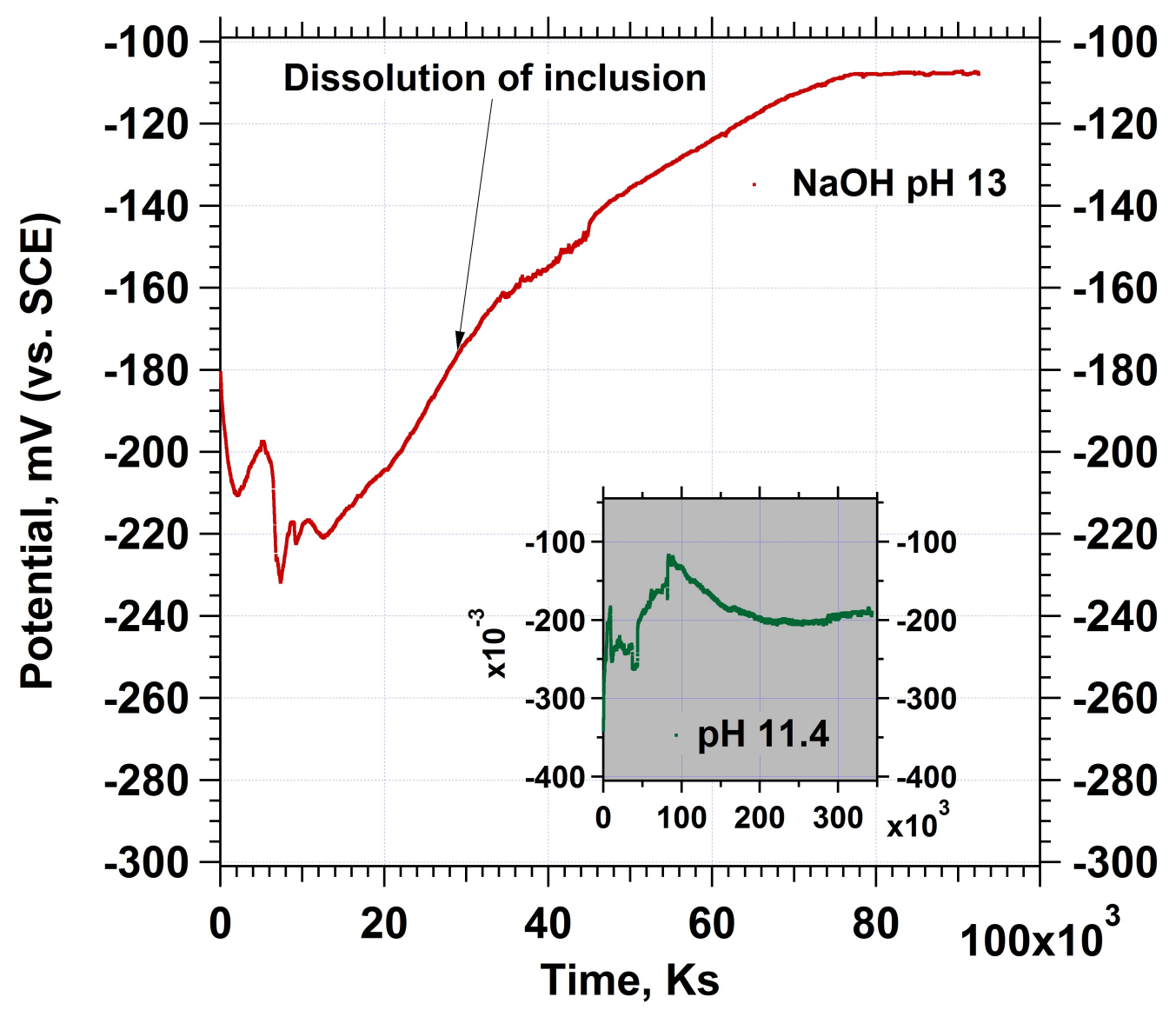

Figure 6.20: Specimen used for the $\mathrm{pH} 13 \mathrm{NaOH}$ experiment following 1 day of immersion. The inclusion dissolution occurred at 28,800 s. OCP data at 2.5 mmol $d m^{-3}$ for 4 days shown in inset
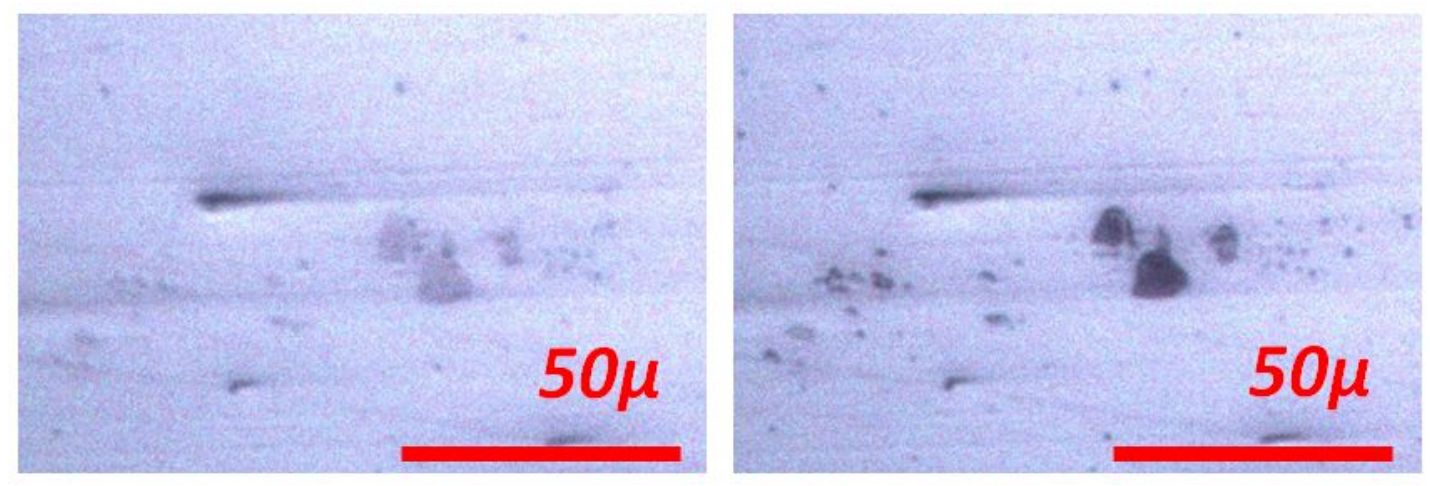

Figure 6.21: Images of inclusions in $0.1 \mathrm{~mol} d^{-3} \mathrm{NaOH}$ (20x crop). Colour change witnessed after $\approx 28 \mathrm{Ks}$ 
dissolved, it is still present in a large enough quantity to be detected.

The specimen underwent further analysis using an AFM/SKPFM for acquisition of topography and Volta potential maps. The same set of inclusions were mapped using AFM and SKPFM techniques. AFM topography shows the inclusions are still present, with the inclusions appearing rough, showing a structure which appears lamellar. This is in broad agreement with the SEM imaging. Cross sections for typical $\mathrm{NbC}$ inclusions after immersion in $2.5 \mathrm{mmol} d \mathrm{~m}^{-3}$ $\mathrm{NaOH}$ show the roughening effect in figures $6.24,6.24$. The cross section of the inclusion shows the majority of troughs are $\approx 60 \mathrm{~nm}$ in depth. A trace of the background is included for comparison. It could be argued that the inclusion cross section trace (shown in red within figure 6.24) suggests a swelling of the inclusion between 3 and $7 \mu \mathrm{m}$. The reason for this is due to the gradient of the cross section trace at the edges of the inclusion. A suggested cross sectional area for the inclusion above the surface, prior to dissolution, is given in figure 6.26.

The ability to detect Volta potential differences of two different materials is shown, even when an inclusion is present beneath the surface (figure 6.29). SKPFM reveals that NbC inclusions are active with respect to the matrix. This is seen in all three of the inclusions. Thus the inclusions themselves appear susceptible to dissolution, which corresponds with the AFM topography images. For the material without pretreatment, $\mathrm{NbC}$ inclusions are noble to the matrix as shown in previous chapters. $\mathrm{NbC}$ is a hard material (Mohs hardness of 8 ), and is
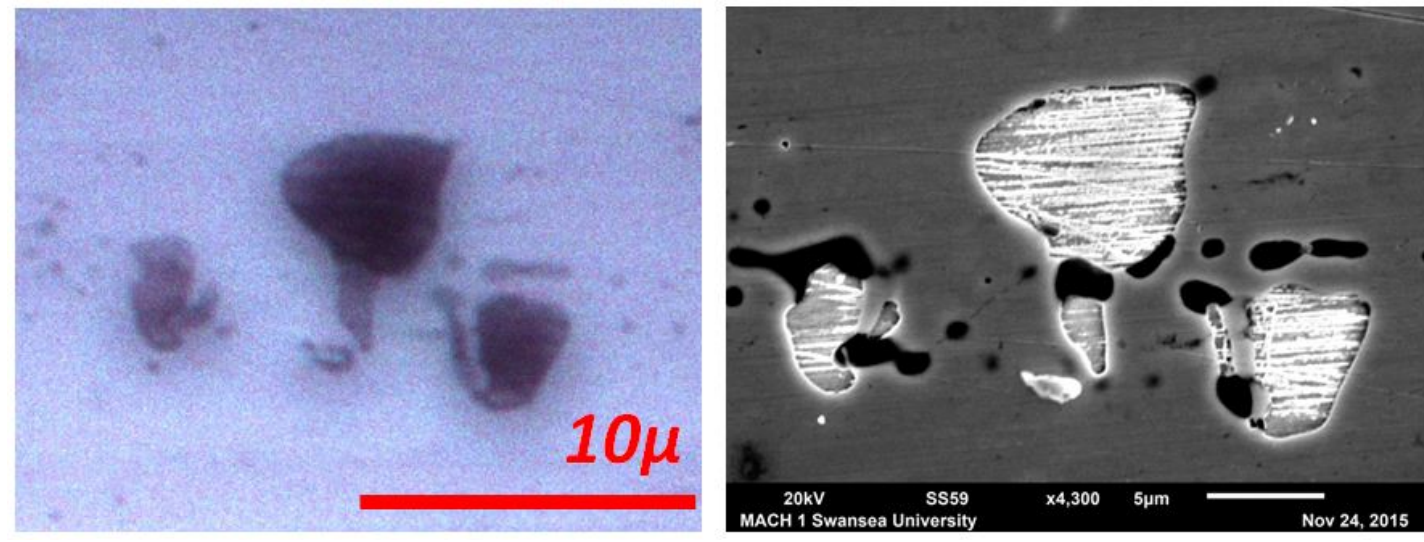

Figure 6.22: Inclusions captured using optical (50x crop) and SEM techniques 

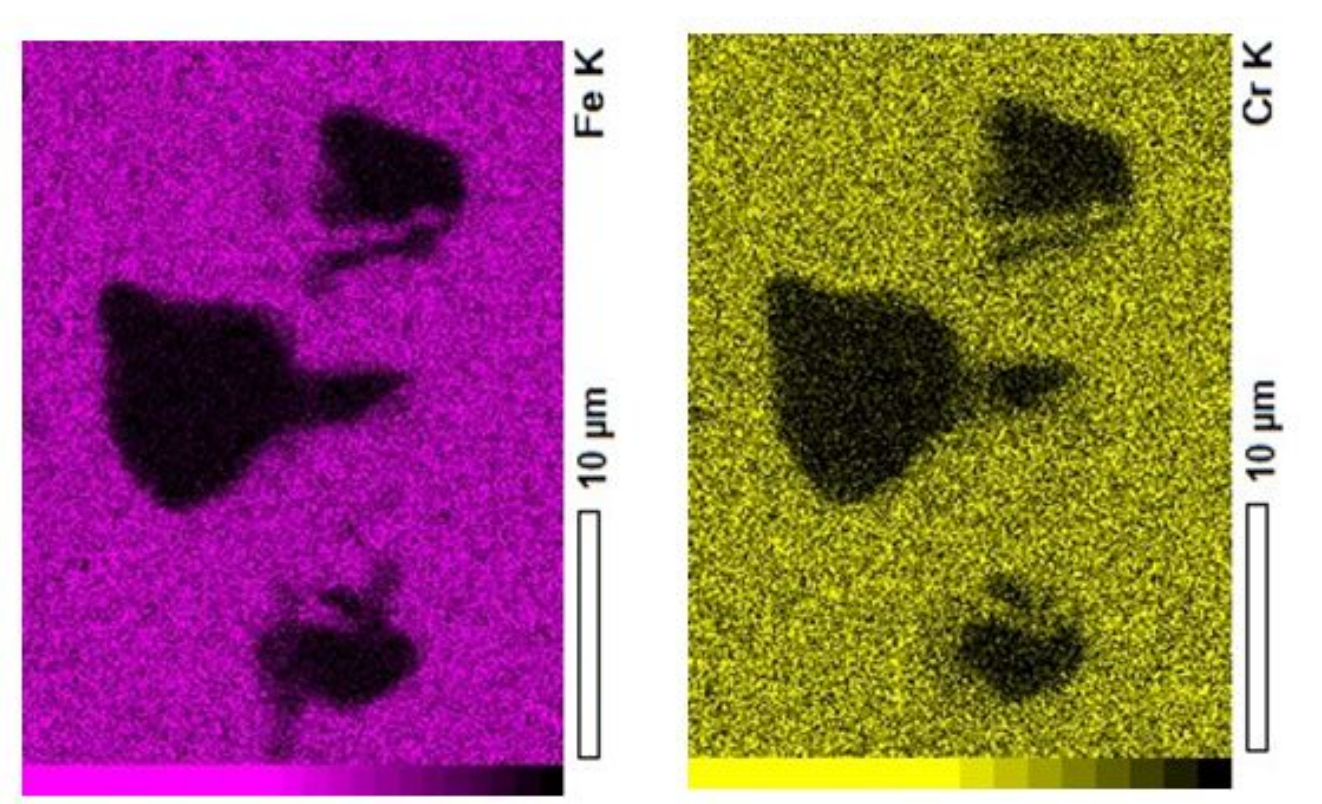

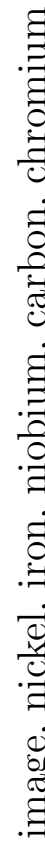
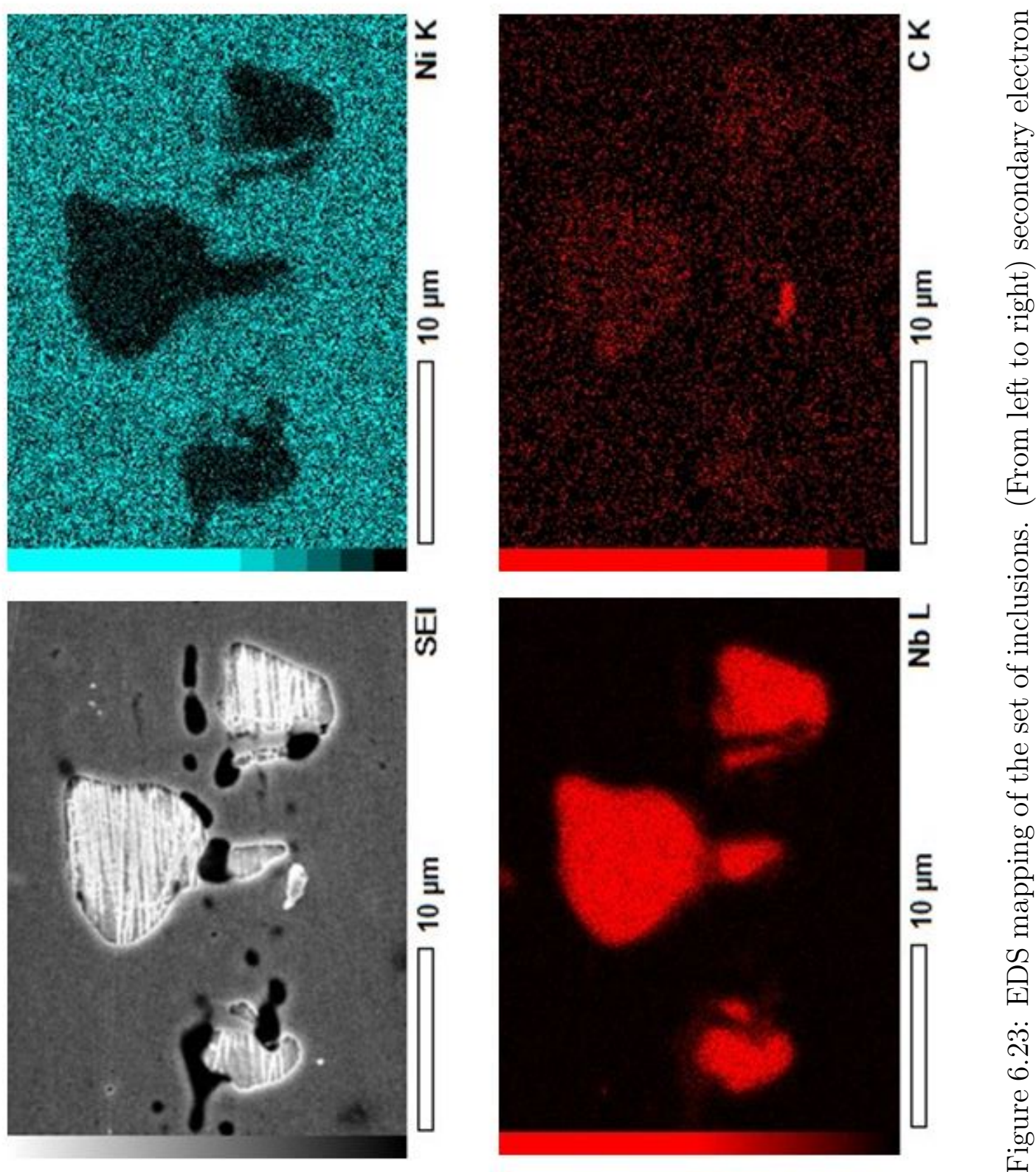


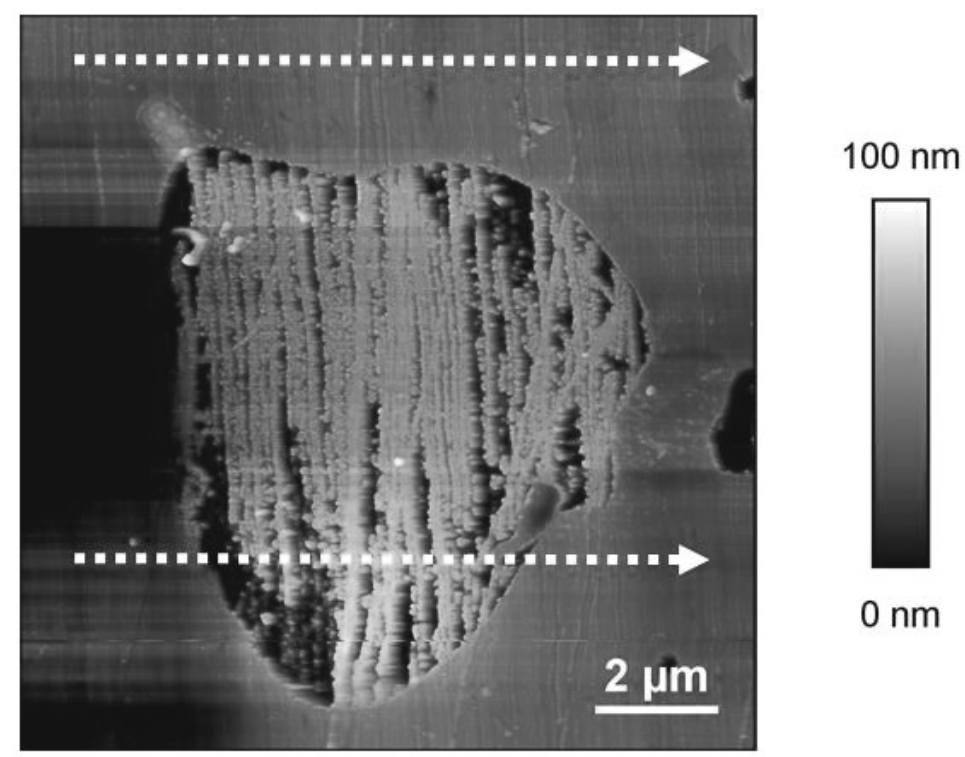

Figure 6.24: Annotated AFM image to show location of background and inclusion cross sections. This inclusion was present in the in-situ TLM experiment.

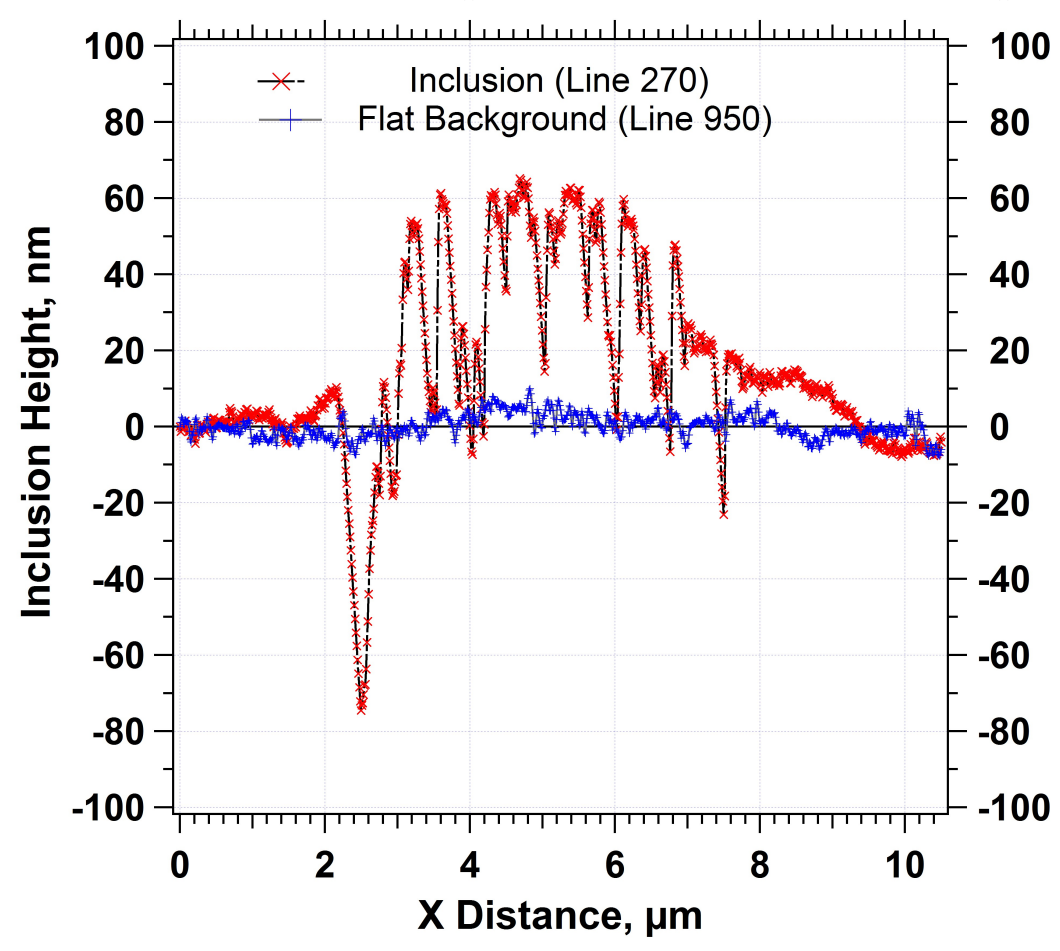

Figure 6.25: Cross section of background and inclusion. 


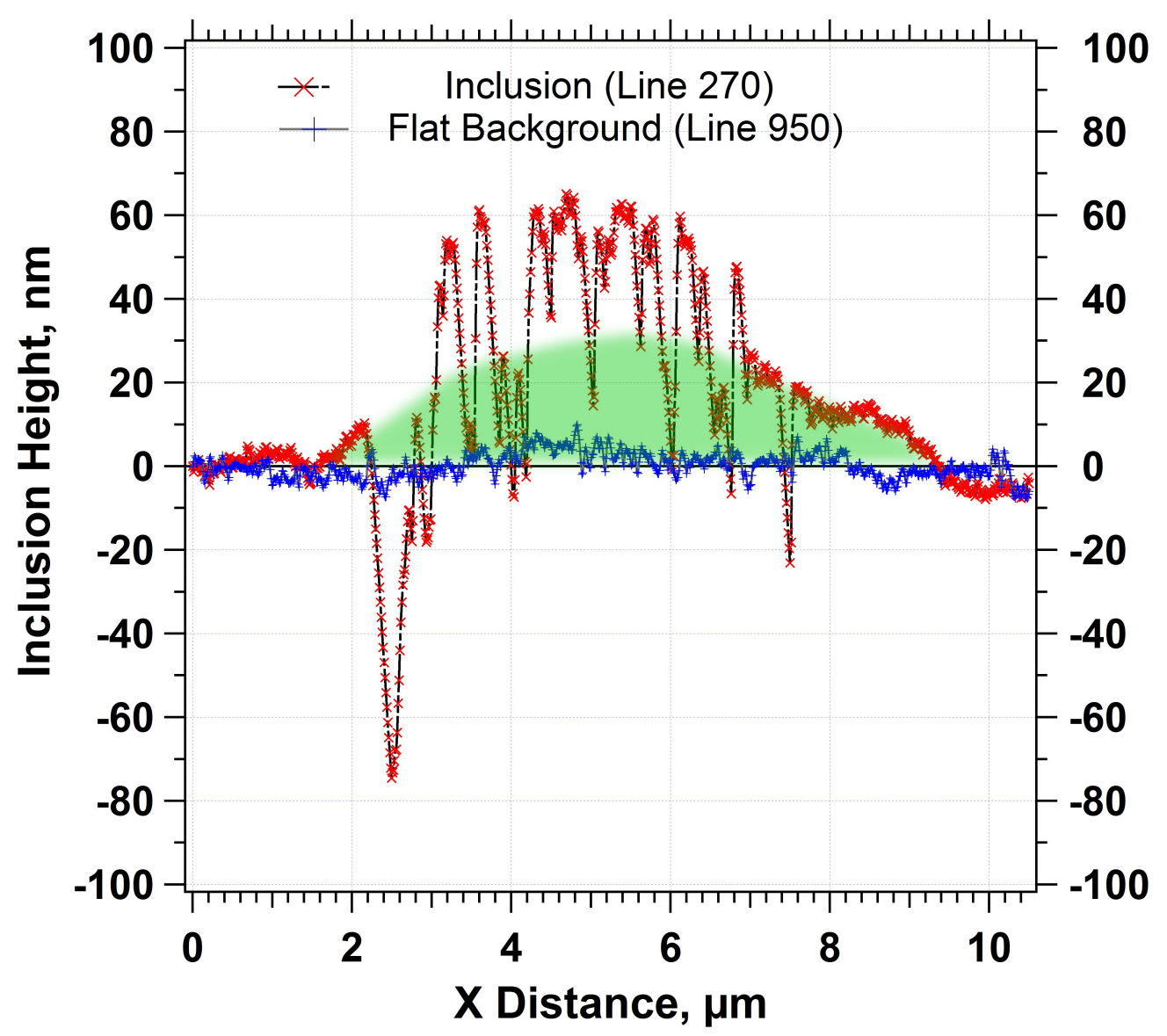

Figure 6.26: Suggested cross sectional area for the inclusion above the surface (shown in green). 


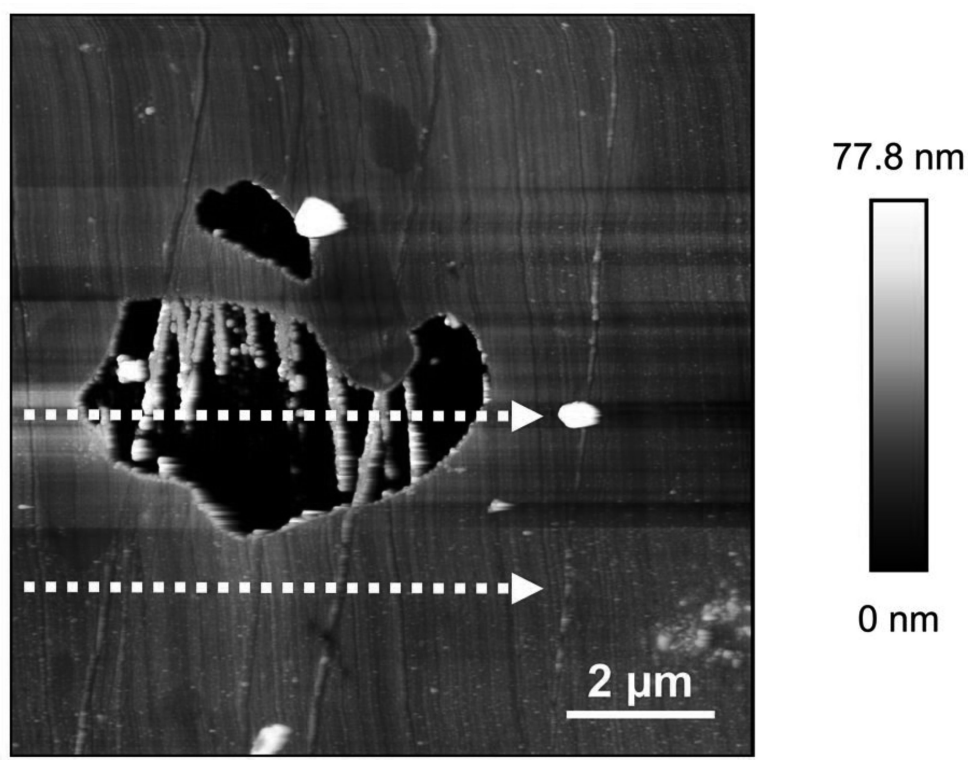

Figure 6.27: Annotated AFM image to show location of background and inclusion cross sections. This inclusion was present in the in-situ TLM experiment.

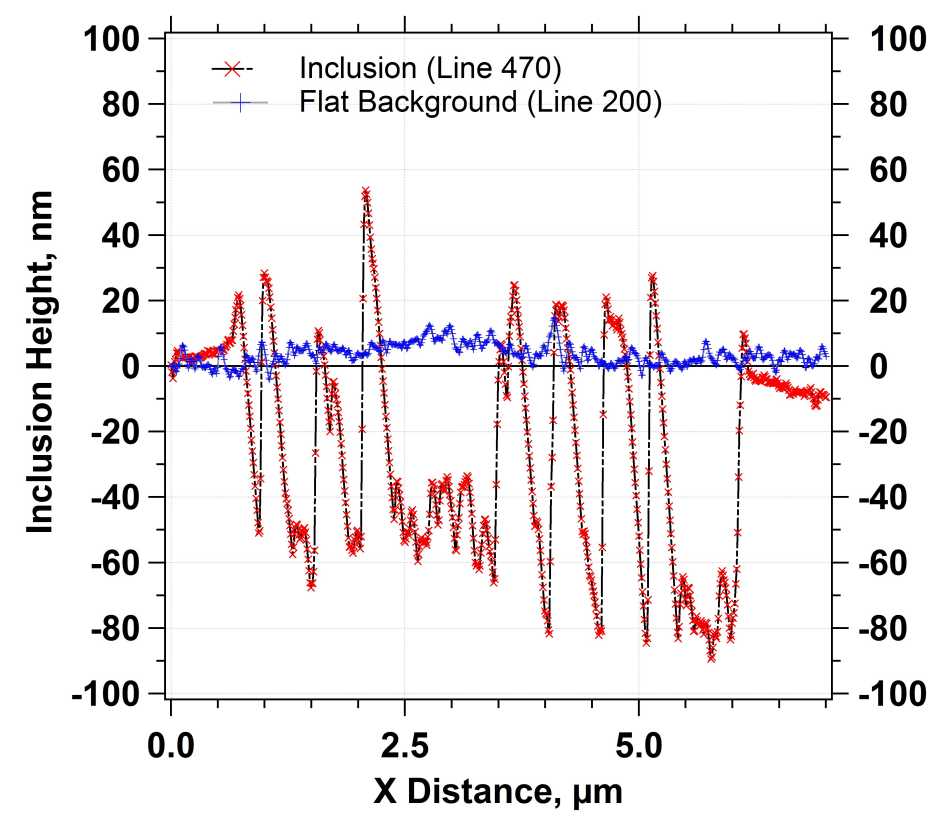

Figure 6.28: Cross section of background and inclusion. 
used as a coating for cutting tools. Still, it is expected that both silicon carbide abrasive papers (9-9.5 Mohs hardness) and diamond (10 Mohs) used within the preparation process caused the small surface scratches on the inclusions. It is expected that this led to the initiation of dissolution at the inclusion, along the scratches. It is also possible that the grinding process itself is what had caused the modification, rather than simple dissolution itself. As with SEM, AFM confirms that dissolution has propagated along the polishing direction; as can be seen in figure 6.29.
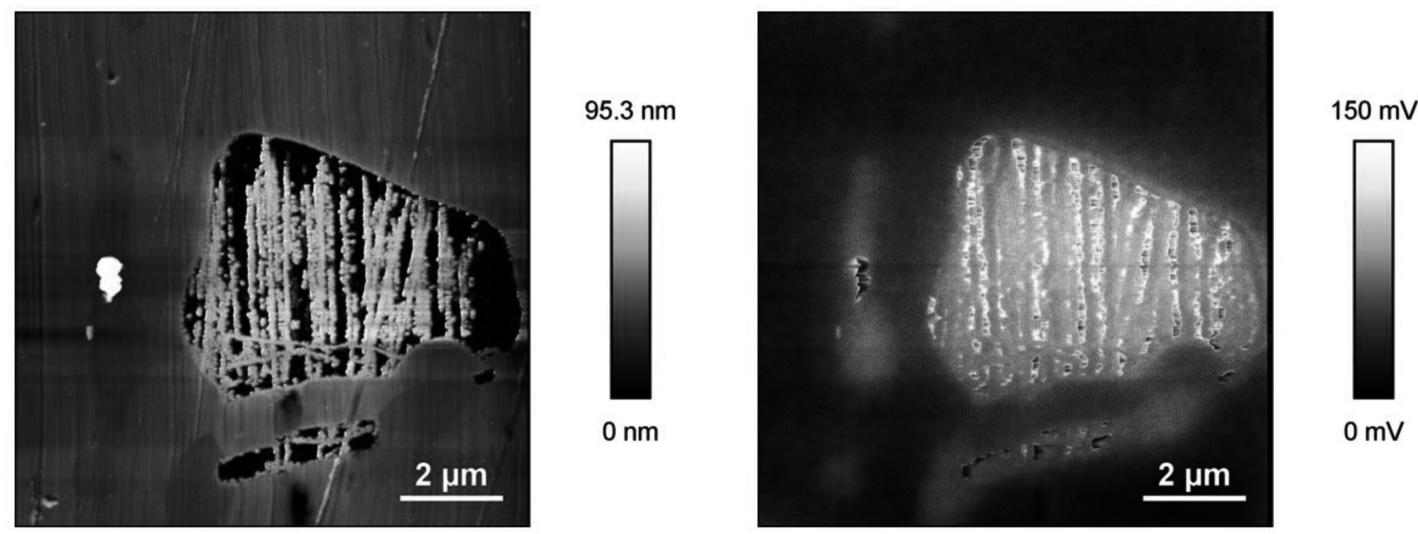

Figure 6.29: NbC inclusion; AFM topography (left), SKPFM Volta potential map (right)

In addition to the in-situ TLM experiment, additional specimens were immersed for the full 4 days in both $\mathrm{NaOH}$ concentrations. LPR experiment results are displayed in the table 6.2 . 
Table 6.2: Sodium Hydroxide LPR results (neutral pH included for comparison at $10 \mathrm{ppm}\left[\mathrm{Cl}^{-}\right]$.

\begin{tabular}{lll}
\hline Sample & Cl- Concentration in $\mathrm{NaOH}(\mathrm{pH} 11.4)$ & Polarisation Resistance \\
& $(\mathrm{ppm})$ & $(\mathrm{M} \Omega)$ \\
\hline Pretreated & 0 & 11.66 \\
& 10 & 11.35 \\
& $10^{*}$ & 8.54 \\
& 100 & 11.67 \\
& 1000 & 15.65 \\
& 10,000 & 12.28 \\
\hline 0 & 10 & 8.65 \\
$10 *$ & 7.97 \\
100 & 0.51 \\
1000 & 6.82 \\
\hline 10,000 & 5.67 \\
\hline
\end{tabular}

${ }^{*}$ Neutral $\mathrm{pH}$

The LPR graphs (figure 6.30) show plots for both the NaOH treated specimen, and the control specimen that underwent no treatment. The $\mathrm{NaOH}$ treated specimen showed a greater resistance to corrosion at all $\mathrm{Cl}^{-}$(higher polarisation resistance). The control specimen on the other hand displayed decreasing polarisation resistance with $\mathrm{Cl}^{-}$. This may be due to a decrease in initiation sites. Previously, LPR experiments did not show a clear trend, so reservations should be kept with the data, until more repeat experiments are conducted (see next chapter).

AFM analysis is presented below. Two inclusion sites are shown in figure 6.31. From the 3D topography in figures $6.31 \mathrm{a}, 6.31 \mathrm{~b}$ it appears that a larger amount of dissolution may have occurred compared to when immersed for less than one 


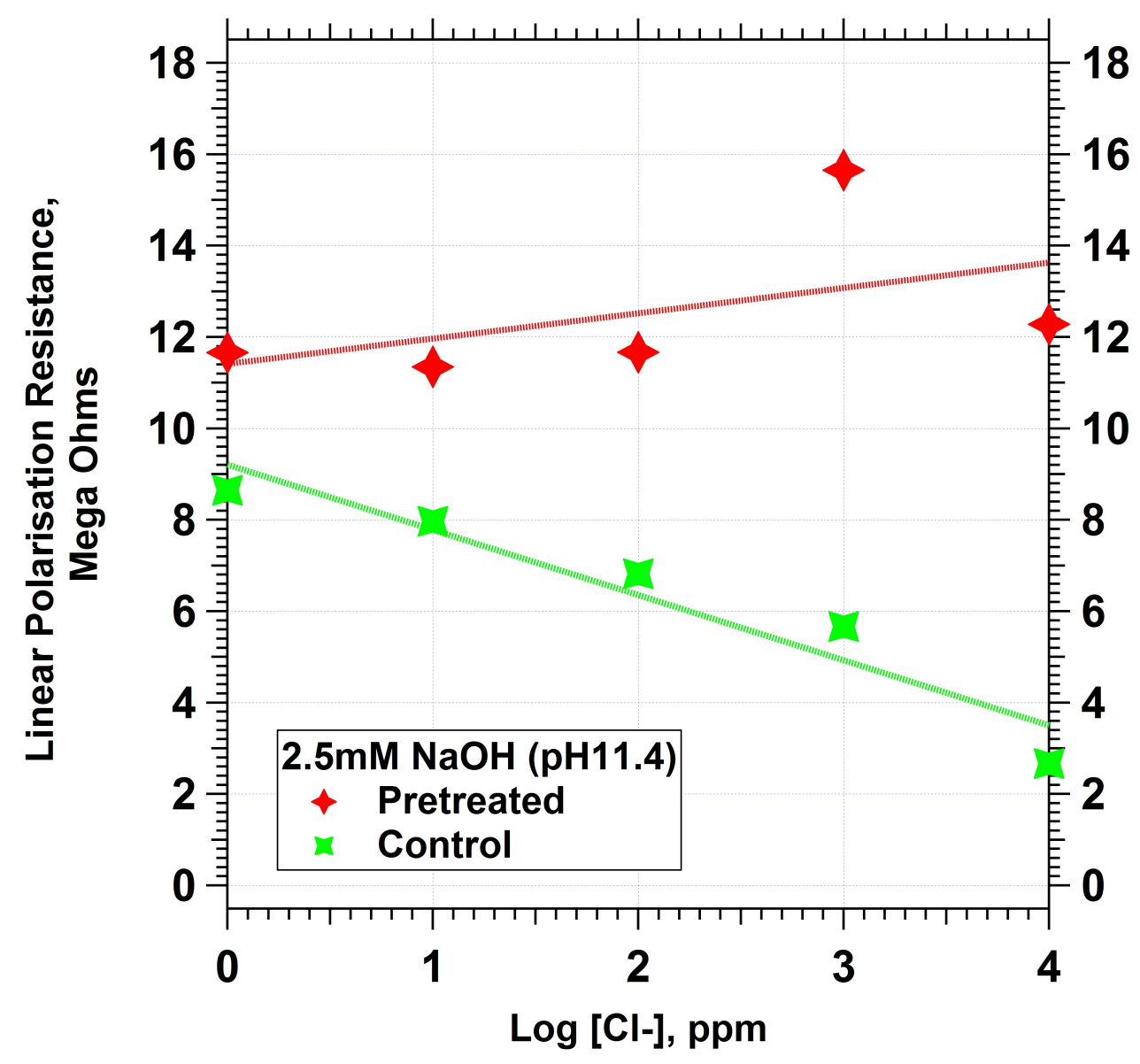

Figure 6.30: LPR dependency as a function of $\left[\mathrm{Cl}^{-}\right]$ 

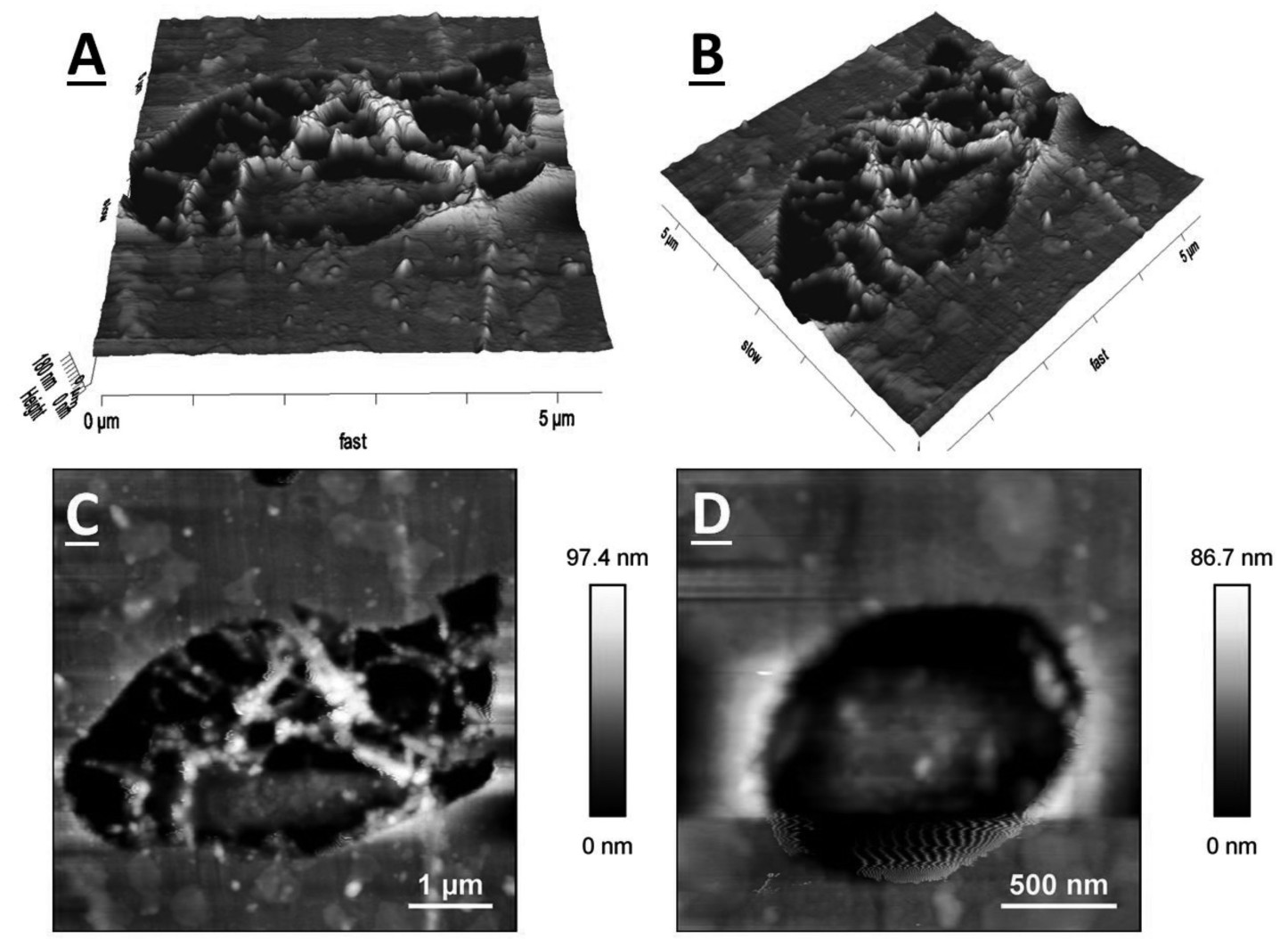

Figure 6.31: Images of inclusions after 4 days immersion in $\mathrm{pH} 13 \mathrm{NaOH}$. (A, B) 3D topography of image in (C). Image (D) shows an inclusion which has large excavation around edges. The lower half of the image shows an artefact, relating to the AFM instrumentation 
day. Chan et al. showed a similar phenomenon of NbC dissolution on sensitised 20/25/Nb when polarised in $500 \mathrm{ppm}\left[\mathrm{Cl}^{-}\right]$dosed with $\mathrm{NaOH}(\mathrm{pH} 11.5)$ [45]. The authors showed that $\mathrm{NbC}(5-7 \mu \mathrm{m})$ appeared to fragment under the influence of external polarisation, although not to the extreme that has been seen in the examples shown here. This represents evidence that the dissolution is a real effect, and is limited by the exposure time in the strong base. The NbC structure shown here appears less like strands, and more lamellar-like. Either NbC has not been fully dissolved away, but would do given additional time, or it is expected that perhaps two different phases of NbC must be present. Initially, dissolution must occur via any scratches in the carbide, then a $\mathrm{NbC}$ phase less resistant to $\mathrm{NaOH}$ corrosion is first dissolved, leaving behind a lamellar bridge network. Figure 6.31c shows the same site, but in 2D. Figure 6.31d shows an inclusion that has shown a larger amount of dissolution.

Cross sections of $\mathrm{NbC}$ sites are shown in figure 6.32 and figure 6.33. The AFM image itself, follows what has been shown in the earlier images, with a lamellar-like network present. The cross section reveals that a up to $100 \mathrm{~nm}$ has been dissolved away. Figure 6.32 is an interesting example that shows a large inclusion c. $10 \mu m^{2}$ which has undergone comprehensive dissolution. The majority of the inclusion has been dissolved, leaving behind a shallow dish region, with a large void in the centre. The cross section depth is on par with the previous example, with depths reaching c. $90 \mathrm{~nm}$.

Inclusion sites following the full 4 day treatment analysis using undertaken again using SEM and AFM. SEM images showed the inclusions were no longer strand-like, or whole, but instead appeared separated in separate pieces, appearing fractured. Example SEM micrographs are shown in figure 6.34, showing the fracturing effect which has taken place. SEM-EDS figures are shown in figures $6.35,6.36,6.37$ confirming that the inclusions contain $\mathrm{Nb}$. $\mathrm{NbC}$ have only been observed in this way when using an $\mathrm{NaOH}$ pretreatment.

Chemical analysis reveal that although the inclusions have undergone further 


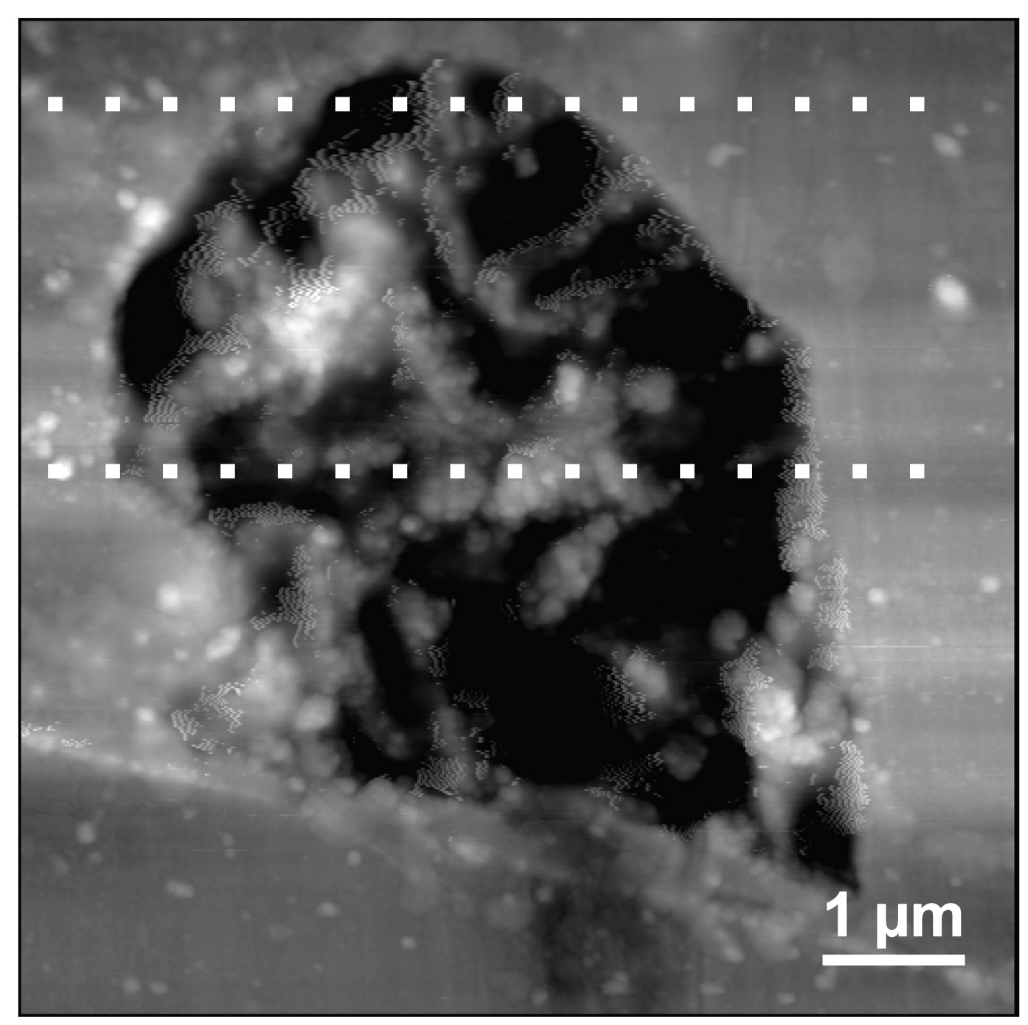

$142 \mathrm{~nm}$

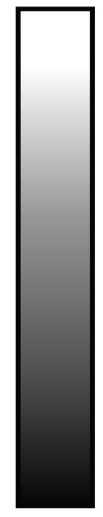

$0 \mathrm{~nm}$

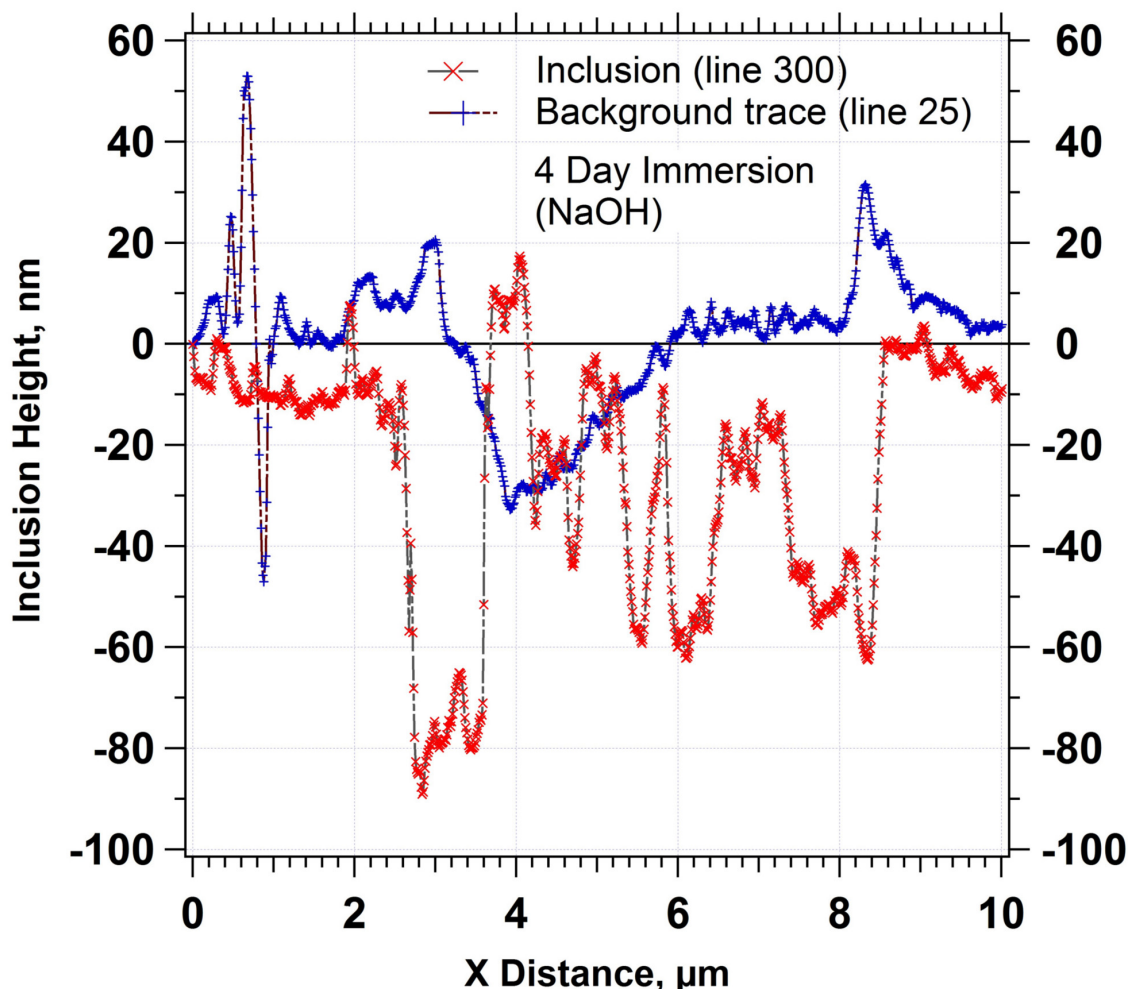

Figure 6.32: Cross section of inclusion site following 4 days immersion in 0.1 moldm $^{-3} \mathrm{NaOH}$ and corresponding AFM image 

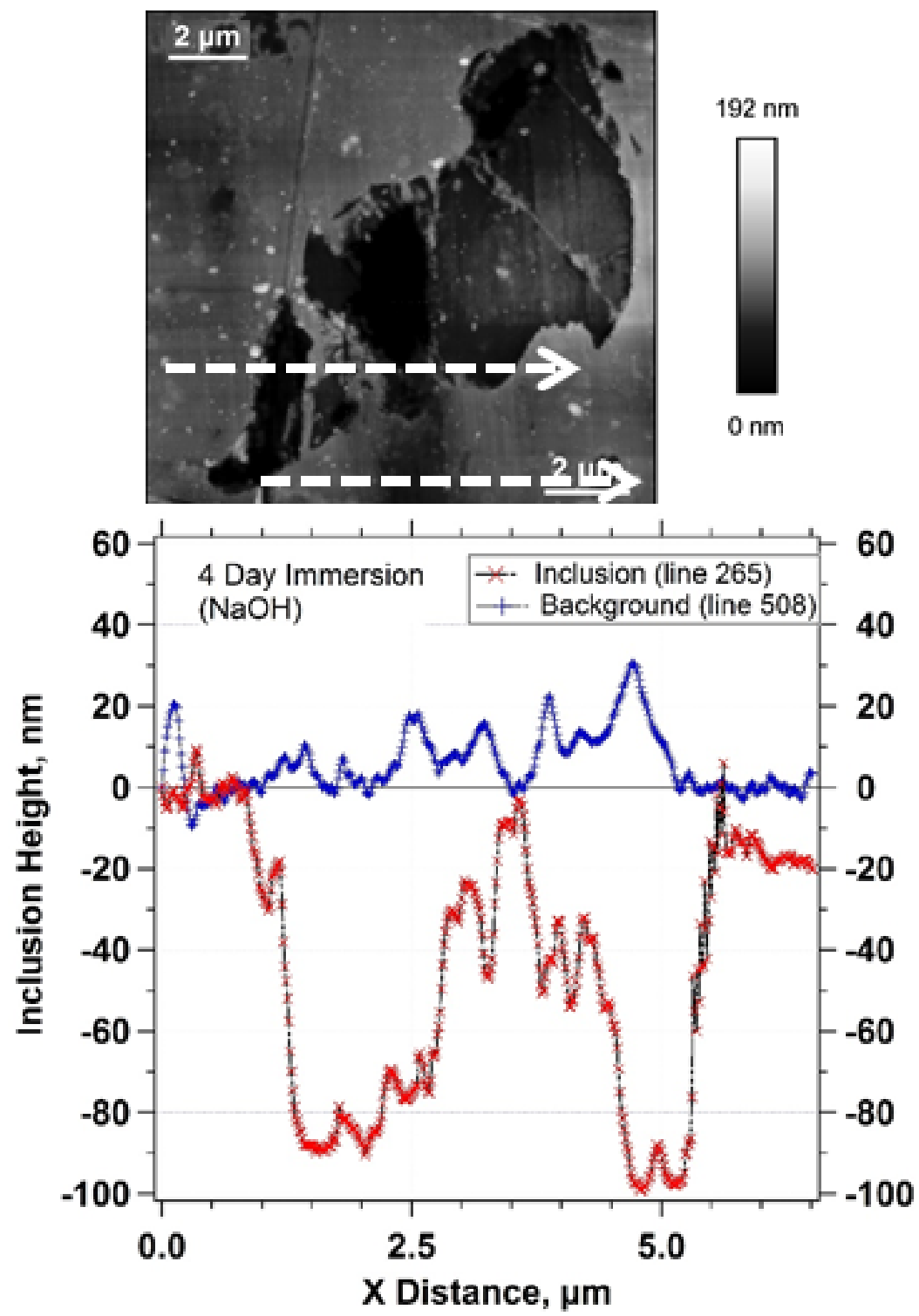

Figure 6.33: Cross section of inclusion site following 4 days immersion in 0.1 moldm $^{-3} \mathrm{NaOH}$ and corresponding AFM image. 


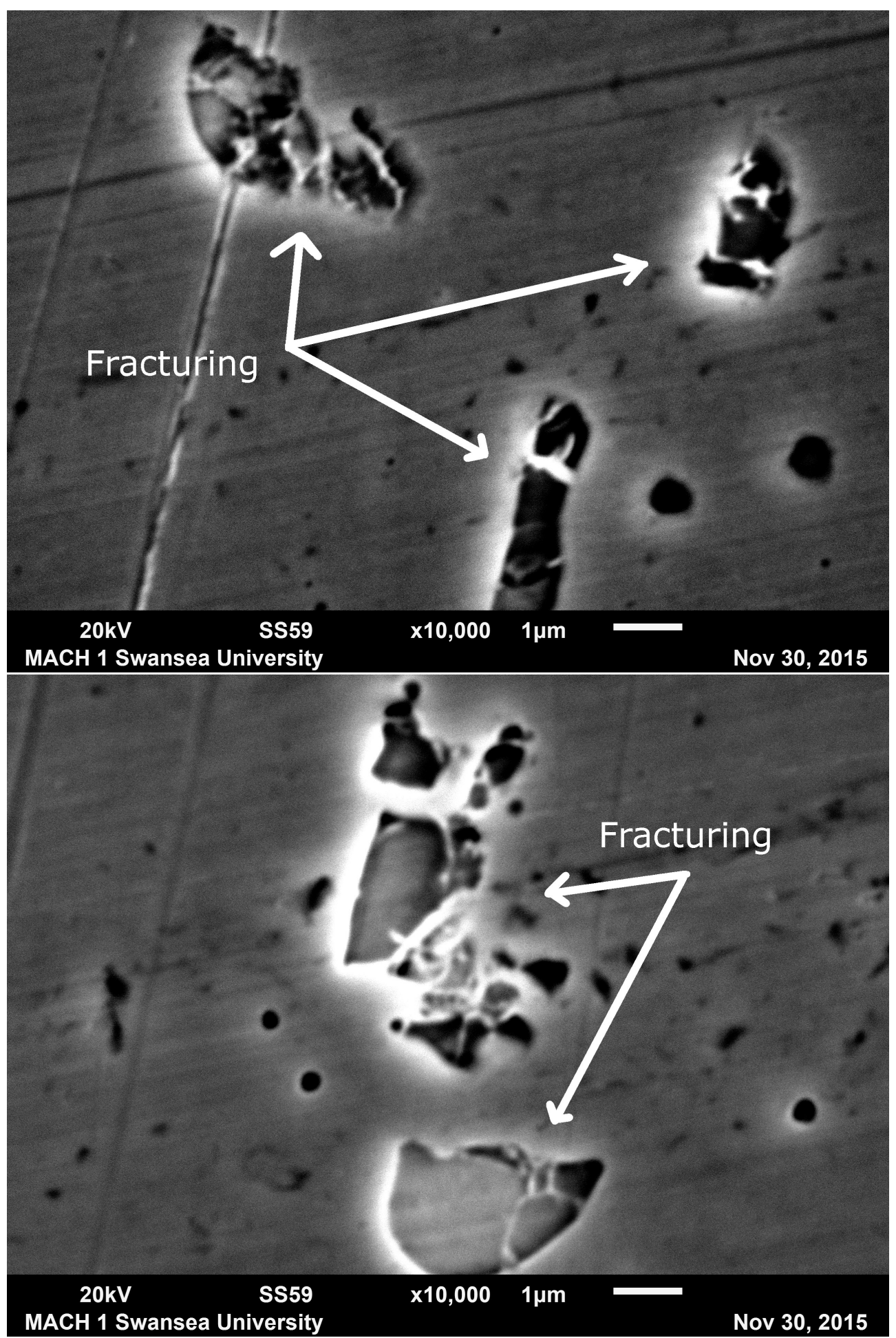

Figure 6.34: SEM micrographs of the stainless steel following 4 days immersion in 0.1 moldm $^{-3} \mathrm{NaOH}(\mathrm{pH} 13)$. The arrows show the fracturing effect which appears to have modified the inclusions. 

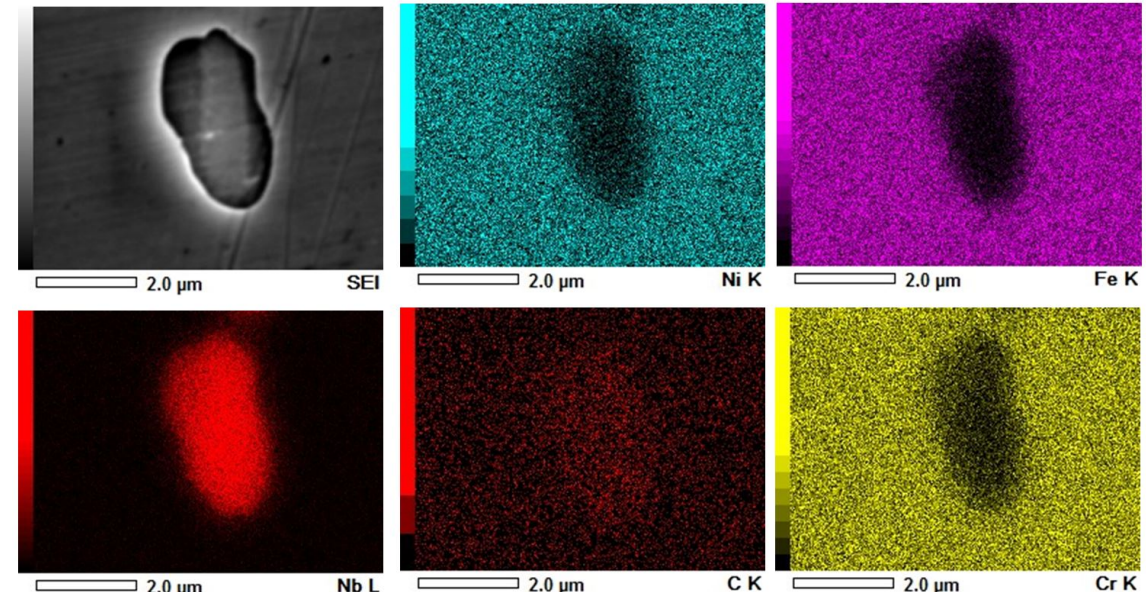

Figure 6.35: 4 day immersion in $\mathrm{NaOH}$ SEM-EDS showing a single NbC inclusion.
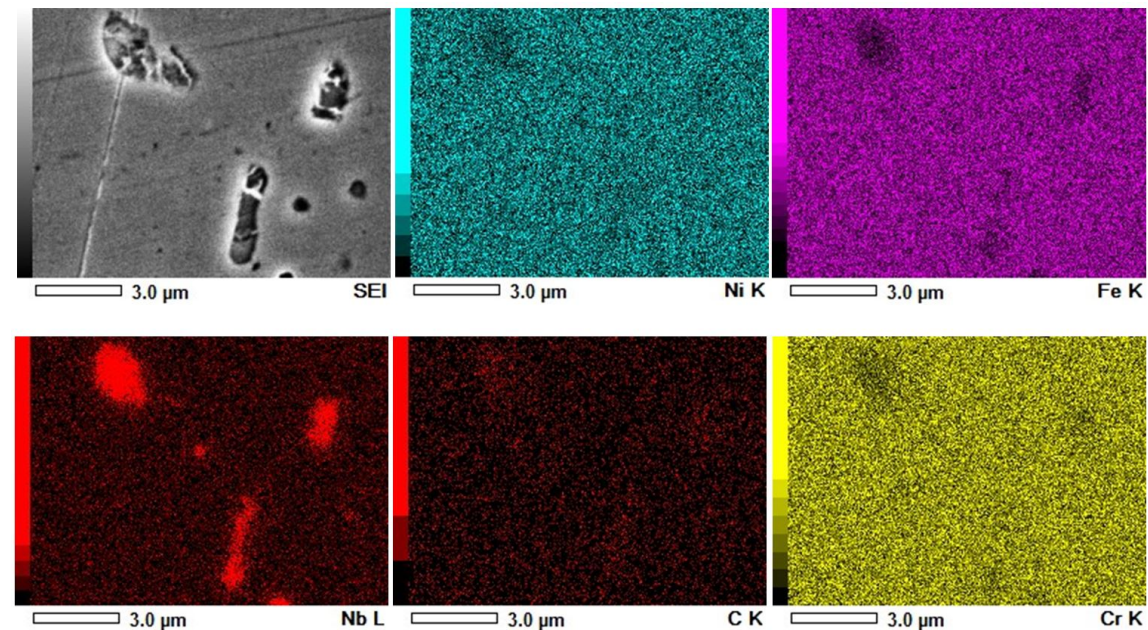

Figure 6.36: 4 day immersion in $\mathrm{NaOH}$ SEM-EDS showing a group of $\mathrm{NbC}$ inclusions. The fractured effect in the SEM image is either visual, or the EDS detector cannot resolve these features.
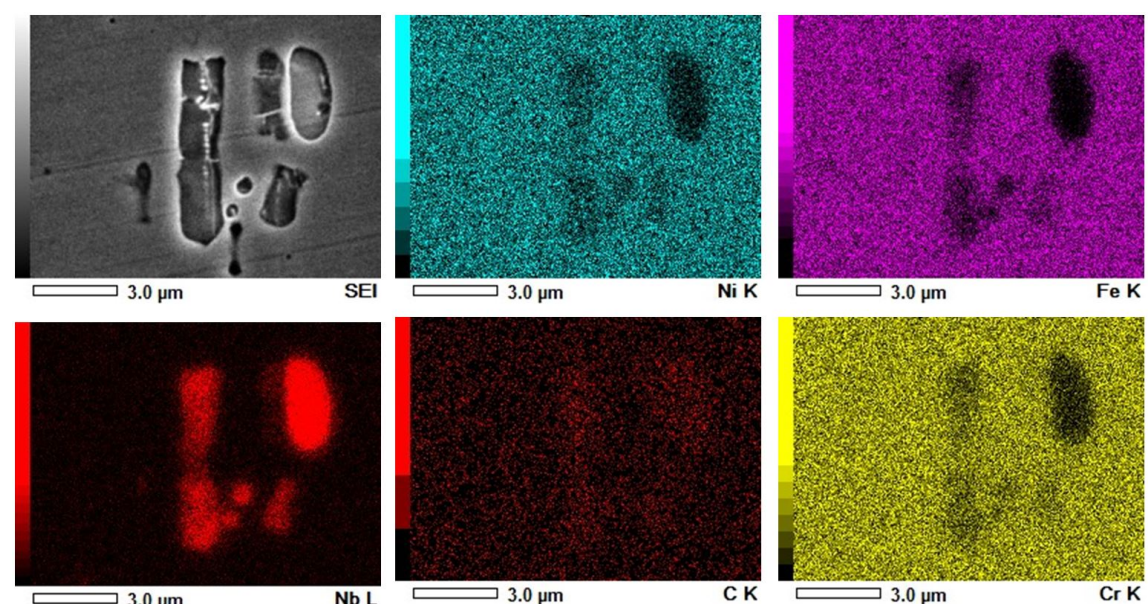

Figure 6.37: 4 day immersion in NaOH SEM-EDS. The fracture witnessed with a second set of $\mathrm{NbC}$ inclusions. The EDS maps show no change in Nb enrichment at the crack sites. 
dissolution (see AFM images), $\mathrm{Nb}$ is still present. Even for the inclusions in figure 6.36 which which appear fragmented there is still an Nb signal. Figure 6.37 shows how the EDS is not able to resolve the possible brighter area within the inclusion. This appears to be undissolved NbC. The limits of the EDS detector that is used on the SEM may be at its limits in regard to spatial resolution. SEM-EDS by design interacts with a hemisphere beneath the surface, giving an area averaged result. For further accuracy, different instrumentation would need to be used such as TEM. This would use a specially prepared lamella cut from the specimen (thickness less than $100 \mathrm{~nm}$ ) which reduce scattering, leading to a more accurate measurement using EDS.

In the 4 week long term pretreatment in $0.1 \mathrm{~mol} d \mathrm{~m}^{3}$ SEM-EDS revealed inclusions that showed the same type of dissolution as before, with inclusions appearing 'fractured', much like the study by Chan et al. [45]. Figure 6.39 shows that $\mathrm{Nb}$ is still present in some of the inclusions imaged. Image 6.38 indicates that a portion of $\mathrm{Nb}$ may have been removed, as only half the inclusion is showing a signal for $\mathrm{Nb}$. Point based detection, allowing quantification, shows $\mathrm{Nb}$ (figure 6.40). The $\mathrm{Nb}$ was not detected in the matrix, but was detected at levels of $16 \%$ at the inclusion site. Therefore $\mathrm{Nb}$ is still present and detectable using SEM/EDS even after 4 weeks in pH $13 \mathrm{NaOH}$. 

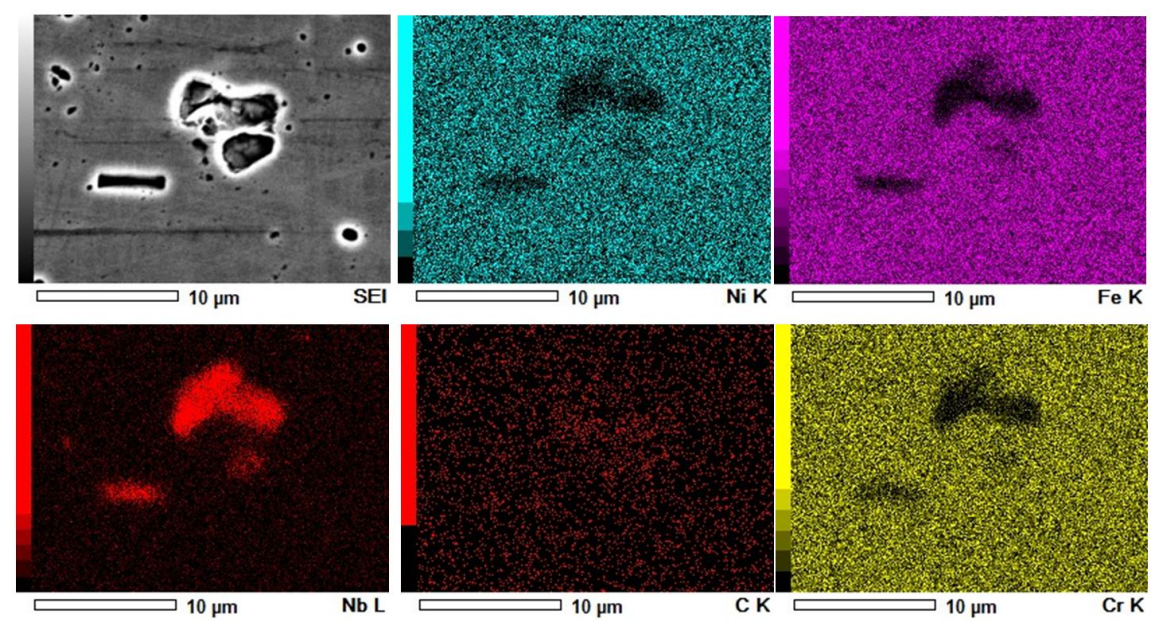

Figure 6.38: NbC site following $\mathrm{pH} 13 \mathrm{NaOH}$ treatment for 4 weeks
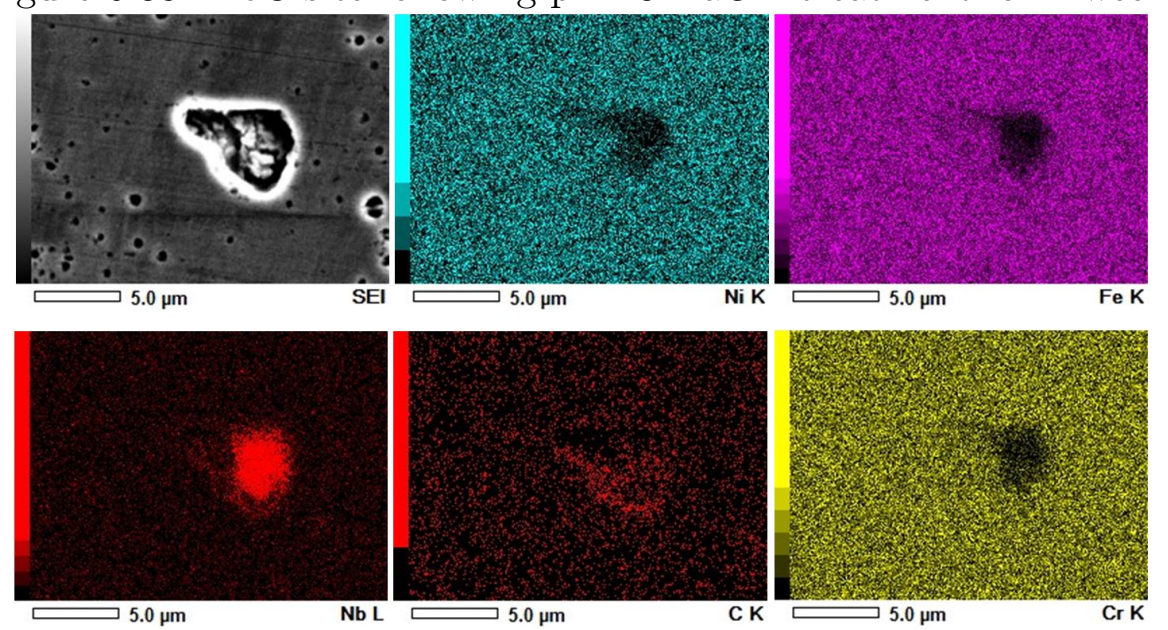

Figure 6.39: NbC site following $\mathrm{pH} 13 \mathrm{NaOH}$ treatment for 4 weeks 


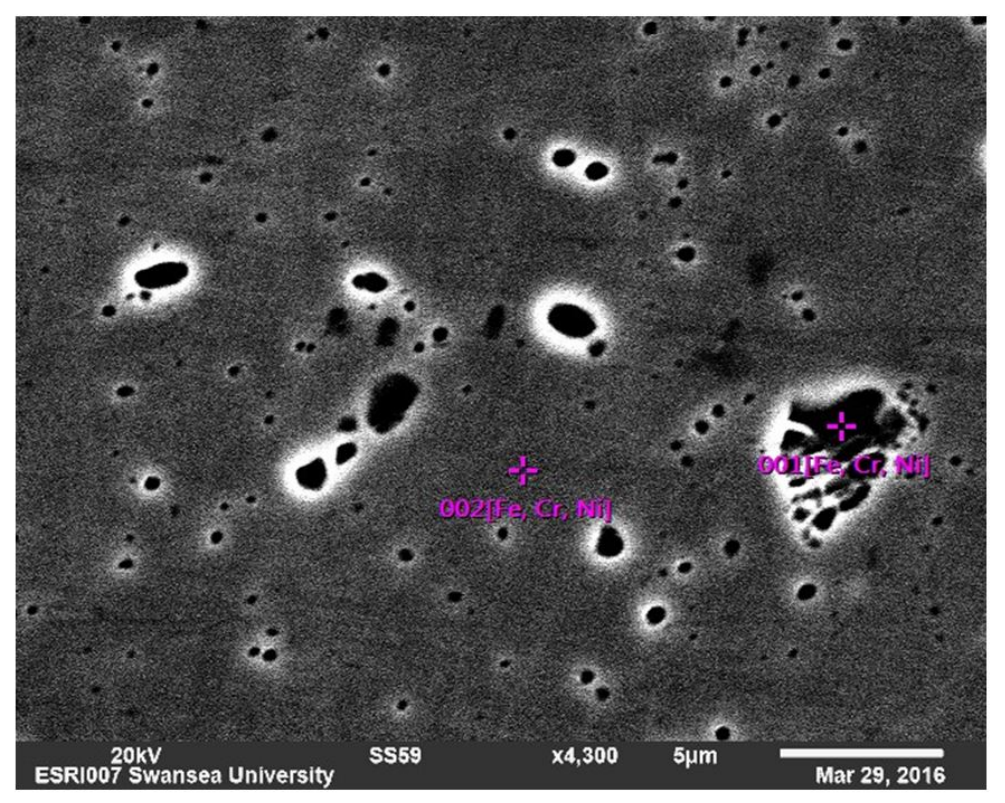

\begin{tabular}{|l|l|l|l|l|l|l|l|l|l|l|l|l|}
\hline Element & $\mathrm{C}$ & $\mathrm{Si}$ & $\mathrm{Cr}$ & $\mathrm{Fe}$ & $\mathrm{Ni}$ & $\mathrm{O}$ & $\mathrm{Nb}$ & $\mathrm{Ti}$ & $\mathrm{Mg}$ & $\mathrm{Al}$ & $\mathrm{Ca}$ & $\mathrm{Na}$ \\
\hline $\begin{array}{l}\text { Matrix } \\
\text { (Mass \%) }\end{array}$ & 5.13 & 0.55 & 18.80 & 51.50 & 24.02 & - & - & - & - & - & - & - \\
\hline $\begin{array}{l}\text { Inclusion } \\
\text { (Mass\%) }\end{array}$ & 8.13 & 1.01 & 12.44 & 30.69 & 13.76 & 14.00 & 16.08 & 0.66 & 0.54 & 0.33 & 0.46 & 1.90 \\
\hline
\end{tabular}

Figure 6.40: Point based acquisition of NbC inclusion following pH $13 \mathrm{NaOH}$ treatment

\subsection{Conclusions}

Experiments have been conducted on $20 / 25 / \mathrm{Nb}$ SS to investigate the effect of $\mathrm{NaOH}$, initially in an as-received condition, as a function of $\mathrm{pH}$. The data showed that $\mathrm{NaOH}$ delayed the onset of pitting corrosion, as the electrolyte became more basic. $\mathrm{NaOH}$ increased the average change in potential over the range $\mathrm{pH} 7$ 12.5 by c. $43 \%$, interestingly the change from $\mathrm{pH} 7$ to 11.4 showed only a c. $4 \%$ increase. Shifting the $\mathrm{pH}$ to more basic values also decreased the number of metastable events occurring, which are needed for stable pitting to manifest. This study determined that $\mathrm{NaOH}$ was successful in delaying corrosion initiation, and led to the next investigation into its use with the SS in a sensitised form. $\left[\mathrm{Cl}^{-}\right]$was reduced to ppm from molar, in order to provide a closer look into levels of $\mathrm{Cl}^{-}$that may be present in an industrial context. LPR experiments did not show the expected results, with polarisation resistance not showing any clear trend with $[\mathrm{NaOH}]$. Over a 1 hour period $(\mathrm{OCP})$ rest potentials showed a 
clear shift to cathodic potentials when $\mathrm{NaOH}$ dosing was used, a good indication of nobility if the $E_{b}$ is maintained. $\mathrm{NaOH}$ increased $E_{\text {corr }}$ by up to $300 \mathrm{mV}$ vs. SCE.

It was shown that corrosion could be somewhat mitigated by using $\mathrm{NaOH}$ as an inhibitor. The next investigation studied the effects of using $\mathrm{NaOH}$ as a pretreatment method at two different basic pHs. This was conducted on the sensitised SS. An in-situ study was used to analyse a set of three inclusions present within the SS. No optical change was shown over 4 days in $2.5 \mathrm{mmol} d m^{-3}$ (pH 11.4). However upon immersion in $0.1 \mathrm{~mol} \mathrm{dm}^{-3}(\mathrm{pH} \mathrm{13)}$, the inclusions darkened within 8 hours of immersion, a similar effect to what is seen when corrosion is witnessed. This was attributed to a light scattering effect and was later discovered using high resolution microscopy techniques that the $\mathrm{NbC}$ inclusions underwent dissolution along the polishing direction. AFM topography images demonstrated a lamellar bridge-like structure present after the $\mathrm{NaOH}$ pretreatment. Finally immersion experiments on the sensitised SS following a 4 week pretreatment in $0.1 \mathrm{~mol} d m^{-3}$ (pH 13) NaOH were conducted. SEM-EDS still showed the presence of $\mathrm{Nb}$, although some examples showed what appeared to be a decrease in $\mathrm{Nb}$ at some inclusion sites. In conclusion, $\mathrm{NaOH}$ is effective in delaying the onset of corrosion, both in unsensitised, and sensitised SS. If $0.1 \mathrm{~mol} d m^{3}(\mathrm{pH} \mathrm{13)}$ is used as a pretreatment $\mathrm{NbC}$ inclusions appear to undergo modification, which may have a beneficial impact on corrosion. 
Chapter 7

\title{
Conclusions and Discussion of
}

\author{
Future Work
}




\section{Corrosion of Nuclear Alloys using DC Electrochemistry and SVET}

A conventional assessment of the AGR fuel cladding alloy was made in the first chapter. Pitting corrosion was dependent on $\left[\mathrm{Cl}^{-}\right]$. Corrosion onset followed a similar trend as a function of electrolyte temperature. Breakdown of the protective oxide film occurred at lower breakdown potentials with increasing $\left[\mathrm{Cl}^{-}\right]$ and electrolyte temperatures. The $\left[\mathrm{Cl}^{-}\right]$dependence for this stainless steel is $98 m V$ decade ${ }^{-1}$, in line with Laycock between -93 and $100 m$ Vdecade ${ }^{-1}$ [132]. Interestingly the stainless steel had a linear temperature dependence $\left(-7.3 \mathrm{mVK}^{-1}\right)$ whilst other stainless steels such as 904L and 316L show a parabolic dependence [130]. Using the SVET method it was possible to observe crevice corrosion propagation in-situ. The alloy in an unsensitised condition is prone to crevice corrosion, in environments containing $\mathrm{Cl}^{-}$ions where corrosion seeks to initiate occluded sites preferentially. When the alloy was sensitised, corrosion was found to initiate at highly sensitised areas close to an oxide scale, identified as haematite by XRD. An investigation using the sensitised tube to study crevice corrosion showed that corrosion initiated at lower $\left[\mathrm{Cl}^{-}\right]$when a crevice former was present, compared to without. Corrosion also appeared more localised at the crevice site. Interestingly, the SVET method was unable to detect corrosion occurring in $0.05 \mathrm{~mol} \mathrm{dm}^{-3}$ if the haematite scale covered the specimen. In these cases red rusting was visible by eye. As it is known that the alloy is prone to crevice corrosion, it was postulated that the haematite scale acts as a porous scale which allows the formation of differential aeration cells causing microcrevice corrosion.

\section{Where does IGC Initiate? SVET and Time-Lapse Microscopy}

The second chapter sought identify IGC initiation sites in sensitised alloys. The AGR fuel cladding, 20/25/Nb, and AISI 310 were both thermally sensitised and analysed using an in-situ time lapse microscopy (TLM) technique. The 20/25/Nb showed that when haematite was not present, IG pitting corrosion occurred at a grain boundary intersection. The corrosion propagated along grain 
boundaries, appearing to form a lacy cover remaining pit-like, rather than traversing along the surface grain boundaries. Inclusions visible on the surface appeared to be unaffected, with corrosion tracing around them. When the haematite scale was present IG pits formed at grain boundary triple points, although crevice corrosion eventually initiated at occluded sites, causing the metastable IG pits to passivate. This is in line with the above hypothesis that the haematite scale acts as a porous layer. The sensitised AISI 310 stainless steel specimen showed similar results, with metastable IG pitting occurring at triple points. Stable IGC initiated in the centre of a grain boundary and at a triple point. Using in-situ SVET, in conjunction with post corrosion SEM/EDS techniques on the sensitised 20/25/Nb specimens immersed in $1000 \mathrm{ppm}\left[\mathrm{Cl}^{-}\right]$and subjected to external polarisation, it was shown that IGC was present at locations where Nbrich inclusions were clustered. Each of the IG pit sites showed a large presence of $\mathrm{Nb}$. Some pits showed evidence that propagation of IGC might continue to large Nb-rich inclusions subsurface.

\section{A Combined in-situ Investigation into Intergranular Corrosion on Stain- less Steel}

To provide a closer insight into the processes occurring on the surface of the SS, within the highly sensitised region, a time-lapse atomic force microscopy technique was used. This allowed visualisation of the process of IGC on the length scale of tens of grains, providing a highly resolved topographical map of the surface undergoing aqueous corrosion in-situ. Metastable IGC pitting occurred at areas along the grain boundary. Using scanning Kelvin probe force microscopy select grain boundaries showed Volta potentials that were higher than others (i.e. more anodic). This could be caused by different grain boundaries having increased $\mathrm{Cr}$ depletion (i.e. more sensitised), which would explain why metastable IGC initiated at certain grain boundaries. The study was able to visualise IGC initiation at an inclusion site, before the alloy passivated. It was speculated that corrosion had not completely ceased, but instead re-initiated at 
another location, most likely within the pores of the haematite layer.

\section{Inhibition of IGC: The Caustic Dosing Approach}

$2.5 \mathrm{mmol} d m^{-3} \mathrm{NaOH}(\mathrm{pH} 11.4$ ) was used to delay the onset of pitting and IGC (unsensitised, sensitised) in the $20 / 25 / \mathrm{Nb}$ alloy. By immersing the alloy in a higher concentration of $\mathrm{NaOH}\left(0.1 \mathrm{~mol} \mathrm{dm}^{-3}, \mathrm{pH} 13\right)$ for periods of up to one month, a change in the physical appearance of $\mathrm{NbC}$ inclusions was witnessed. Immersion in high concentration $\mathrm{NaOH}$ showed modification of the $\mathrm{NbC}$ inclusions using the AFM technique. The inclusions appeared to partially dissolve in $\mathrm{NaOH}$, becoming more columar in nature.

\section{Future Work}

At the outset of this thesis, the question was asked: where does corrosion initiate? The stainless steel, when sensitised using a heat treatment process, is vulnerable to intergranular corrosion (IGC) which has been shown to propagate at grain boundaries. The initiation process was studied using in-situ microscopy methods and showed initiation at grain boundary intersections (and triple points) as small intergranular pits when there was a low concentration of $\mathrm{NbC}$ inclusions present. Using SVET in combination with SEM-EDS over a larger area than TLM, it was attributed that clusters of high number density Nb-rich inclusions were prone to intergranular pit initiation. This is in agreement with the works of Phuah [51], who stated that NbC inclusions were critical to corrosion processes in this $20 / 25 / \mathrm{Nb}$ stainless steel, and that of Chan [45] who showed that corrosion initiated around $\mathrm{NbC}$ precipitates for a stabilised 20/25/Nb microstructure, and at or within the vicinity of triple point grain boundaries. The suggestion by Chan was that corrosion initiated in these locations because the triple point grain boundary intersections had a higher degree of sensitisation (DOS) than others. Following the work of Chan [45] it was found that the stainless steel heat treatment used in this work is comparable to the 'stabilised' and sensitised microstructures (similar DOS) used by Chan although the time and temperature 
of both the solution anneal and ageing were different. Using a high resolution imaging technique (EC-AFM), IGC developed around an inclusion, forming a lacy cover. The most prominent form of corrosion witnessed was if a porous coating happened to cover the stainless steel, as a network of differential aeration cells form, leading to crevice corrosion. The formation of a lacy cover around $\mathrm{NbC}$ precipiates is in accordance to Phuah [51], who found that corrosion pits formed around $\mathrm{NbC}$, leading to lacy cover formation. Below are a list of suggestions for future study in the field of sensitised $20 / 25 / \mathrm{Nb}$ stainless steel corrosion.

Immersion of the alloy in basic solutions $\left(0.1 \mathrm{~mol} d m^{-3} \mathrm{NaOH}, \mathrm{pH} 13\right)$ showed that it may be possible to mitigate corrosion initiation. Immersion in sodium hydroxide leads to a modification of the Nb-rich inclusions, partially dissolving them. Should a large quantity of $\mathrm{Nb}$ inclusions be removed through this dissolution stage, initiation at these NbC inclusion sites (shown with SVET and EC-AFM) would not occur. This leads to the question: if a pretreatment step was used, but did not remove all NbC inclusions, would this lead to increased corrosion? This is unlikely; if a micro Galvanic couple does exist (SKPFM experiments provide an insight this being a possibility) the current loops between the $\mathrm{NbC}$ inclusion and the adjacent $20 / 25 / \mathrm{Nb}$ matrix will be very locallised. If the number of $\mathrm{NbC}$ inclusions were reduced, then the current density will not be constrained to the remaining $\mathrm{NbC}$ sites. It is suggested that further lab-based study be undertaken using $\mathrm{NaOH}$, at higher concentrations, and at elevated potentials. For further work it is advised following the $\mathrm{NaOH}$ treatment, cross sections (using FIB) of the inclusions be imaged, using electron microscopy and EDS chemical analysis. This would provide further understanding on the processes that cause the modification to the $\mathrm{NbC}$. $\mathrm{NaOH}$ pretreament could be compared to control specimens through development of spatial x-ray photoelectron spectroscopy (XPS) techniques, which would help quantify the effect of $\mathrm{NaOH}$. Otherwise low magnification, high resolution EDS may be another avenue. This would allow $\mathrm{Nb}$ rich sites to be identified. 
The development of EC-AFM for high resolution imaging of IGC initiation and propagation, in-situ. The technique has been shown to work, but further developments could allow the study of the effect and rate of IGC propagation on the microscale at different $\left[\mathrm{Cl}^{-}\right]$. This work could include collaboration with the University of Bristol where the high-speed AFM is being developed, which was used by Laferrere on thermally sensitised 20/25/Nb stainless steel [141][133].

Further work would be beneficial using the potential-controlled SVET for specimens that do not undergo spontaneous corrosion, as in the current work an optimal sensitivity was chosen for the lock-in amplifier. In further work the polarisation could perhaps be controlled by a feedback loop. An example could be that the specimen is polarised to the region around its breakdown potential, and once a critical current is reached the SVET experiment stops to ensure post corrosion analysis of the surface features could be conducted. As was determined in section 4.3.4, the outputs of the SVET technique (SVET maps) are best when combined with other techniques. Complimentary techniques such as OCP, photography, microscopy and SEM-EDS chemical analysis are suitable. If the full suite of techniques is used, SVET gives information on local current densities (potentially initiation points), OCP gives the bulk electrochemical potential, whilst ex-situ microscopy and EDS gives detailed information on the point at which IGC has initiated.

Stress corrosion cracking is a widely researched mechanism of corrosion, but there has been relatively little work conducted on the characterisation of local anodic activity with an applied stress. Although the $20 / 25 / \mathrm{Nb}$ stainless steel is annealed during manufacturing, locations such as the anti-stacking grooves are a possible location for SCC initiation as they are produced by stressing the tube. An adapted SVET set-up could incorporate a tensile test machine to produce a static tensile stress whilst the specimen is immersed. In such an experiment the advanced scanning probe technique could provide information on corrosion 
initation, at locations where the chromium oxide film ruptures thereby allowing diffusion of aggressive chloride species to promote corrosion initiation. Using the 3D SVET capability to provide height mapping even contoured surfaces such as the tube material itself might be analysed, and this could include tubes with anti-stacking grooves present. The aim of these experiments on the tube material would be to investigate if corrosion initiates at the locations of higher stress and if a 'safe' limit for ionic contaminants such as chloride could be set. SKP could also be used to provide complimentary information on the Volta potential of the previously exposed specimen. Areas in which corrosion had not occurred are likely to correspond to areas of nobility (areas where the passive film has not ruptured). Further, more highly resolved, information could be obtained using SKPFM to identify areas of relative nobility on a much smaller length scale (e.g. inclusions, grain boundaries, different phases). 
Chapter 8

\section{Bibliography}




\section{Bibliography}

[1] S. Taylor, Privatisation and Financial Collapse in the Nuclear Industry. Oxon: Routledge, 1st ed., 2007.

[2] E. Nonbel, "Description of the Advanced Gas Cooled Type of Reactor (AGR)," Tech. Rep. November 1996, Riso national laboratory, Roskilde, 1996.

[3] EDF Energy, "Our Energy - Power Stations https://www.edfenergy.com/energy," Accessed 06/09/2016.

[4] H. J. Christie, Molecular Dynamics Simulations in Graphite and Carbon Materials. PhD thesis, University of Salford, 2014.

[5] G. B. Neighbour and P. J. Hacker, "The variation of compressive strength of AGR moderator graphite with increasing thermal weight loss," Materials Letters, vol. 51, no. 4, pp. 307-314, 2001.

[6] T. Korkut, "A molecular dynamics study about graphite and boron coated graphite at reactor temperatures," Annals of Nuclear Energy, vol. 63, pp. 100-106, 2014.

[7] M. S. Adam, "The Characteristics of Failed AGR Fuel," tech. rep., NNL Internal Report, Issue 8, (10) 10931, 2012.

[8] ASM, "Introduction to Stainless Steels," in Alloy Digest Sourcebook: Stainless Steels, ch. 1, pp. 1-7, Materials Park, Ohio: ASM International, 2000. 
[9] A. U. Malik, N. A. Siddiqi, S. Ahmad, and I. N. Andijani, "The effect of dominant alloy additions on the corrosion behavior of some conventional and high alloy stainless steels in seawater," Corrosion Science, vol. 37, no. 10, pp. 1521-1535, 1995.

[10] K. R. Trethewey and J. Chamberlain, Corrosion: For Science and Engineering. Longman Sci. \& Tech., 2nd ed., 1996.

[11] J. Horvath and H. Uhlig, "Critical Potentials for Pitting Corrosion of Ni, CrNi, CrFe, and Related Stainless Steels," Journal of the Electrochemical society, vol. 115, no. 8, pp. 791-795, 1968.

[12] G. Williams and H. N. McMurray, "Pitting corrosion of steam turbine blading steels: The influence of chromium content, temperature, and chloride ion concentration," Corrosion, vol. 62, no. 3, pp. 231-242, 2006.

[13] M. Ryan, D. E. Williams, and R. Chater, "Why stainless steel corrodes," Nature, vol. 415, no. February 2002, pp. 770-774, 2002.

[14] E. Bettini, C. Leygraf, C. Lin, P. Liu, and J. Pan, "Influence of Grain Boundaries on Dissolution Behavior of a Biomedical CoCrMo Alloy: InSitu Electrochemical-Optical, AFM and SEM/TEM Studies," Journal of the Electrochemical Society, vol. 159, pp. C422-C427, aug 2012.

[15] B. Stellwag, "The mechanism of oxide film formation on austenitic stainless steels in high temperature water," Corrosion Science, vol. 40, no. 2/3, pp. 337-370, 1998.

[16] S. Lyon, "Overview of Corrosion Science," in Nuclear Corrosion Science and Engineering (D. Féron, ed.), ch. 1, pp. 1-24, Woodhead Publishing Series in Energy, illustrate ed., 2012.

[17] J. M. Bastidas, C. L. Torres, E. Cano, and J. L. Polo, "Influence of molybdenum on passivation of polarised stainless steels in a chloride environment," Corrosion Science, vol. 44, no. 3, pp. 625-633, 2002. 
[18] R. C. Newman, "Understanding the corrosion of stainless steel," Corrosion, vol. 57, no. 12, pp. 1030-1041, 2001.

[19] Avesta Sheffield, Corrosion Handbook. Sheffield: Sandvikens Tryckeri, 1994.

[20] A. S. Hamdy, E. El-Shenawy, and T. El-Bitar, "The corrosion behavior of niobium bearing cold deformed austenitic stainless steels in $3.5 \% \mathrm{NaCl}$ solution," Materials Letters, vol. 61, no. 13, pp. 2827-2832, 2007.

[21] H. Yanliang, B. Kinsella, and T. Becker, "Sensitisation identification of stainless steel to intergranular stress corrosion cracking by atomic force microscopy," Materials Letters, vol. 62, pp. 1863-1866, apr 2008.

[22] G. Bai, S. Lu, D. Li, and Y. Li, "Influences of niobium and solution treatment temperature on pitting corrosion behaviour of stabilised austenitic stainless steels," Corrosion Science, vol. 108, pp. 111-124, 2016.

[23] Praxair, "Argon Oxygen Decarburization http://www.praxair.com/industries/metal-production/argon-oxygendecarburization-aod," Accessed 07/09/2016.

[24] J. Nutting, E. Wondris, and E. Wente, "Argon-Oxygen Decarburization - https://www.britannica.com/technology/steel/Ladlemetallurgy\#ref622946," Accessed 07/09/2016.

[25] C. Schade, R. Causton, and T. Cimino-Corey, "Improved Stainless Steel Processing Routes - http://www.gkn.com/hoeganaes/media/Tech Library/135. Improved Stainless Steel Processing Routes.pdf," Accessed $27 / 08 / 2017$

[26] J. Olsson and M. Snis, "Duplex - A new generation of stainless steels for desalination plants," Desalination, vol. 205, no. 1-3, pp. 104-113, 2007. 
[27] ASM International, "Austenitic Stainless Steels," in Stainless Steels for Design Engineers, ch. Chapter 6, pp. 69-78, ASM International, 2008.

[28] W. S. Walters, T. Atkins, R. M. Boothby, C. J. Donohoe, S. Dumbill, and H. E. Sims, "Replication of reactor-induced sensitsiation of AGR fuel cladding," tech. rep., National Nuclear Laboraory, 2012.

[29] Westinghouse, "Oxide Fuels Complex Brouchure http://www.westinghousenuclear.com/springfields/Products," Accessed $27 / 08 / 2017$.

[30] International Atomic Energy Agency, "Spent Fuel Suveillance at BNFL, Sellafield Nuclear Reprocessing Plant," Tech. Rep. IAEA-TECDOC-461, IAEA, Vienna, 1987.

[31] International Atomic Energy Agency and E. Agency, "Further analysis of extended storage of spent fuel," IAEA-TECDOC-944, pp. 1991-1996, 1996.

[32] I. Dickson, P. Dulson, D. Edens, and S. Fisher, "Fuel management and handling for AGR high burn-up fuel cycles," in Fuel Management and Handling: Proceedings of the International Conference (T. Telford, ed.), (Edinburgh), pp. 81-86, British Nuclear Energy Society, 1995.

[33] C. Taylor, "The formation of sensitised microstructures during the irradiation of AGR fuel cladding," in Symposium on Radiation-Induced Sensitisation of Stainless Steels, (Sellafield), pp. 60-73, Berkley Nuclear Laboratories, 1986.

[34] ASTM International, "Standard practices in detecting susceptibility to intergranular corrosion in austenitic stainless steels A-262-10," tech. rep., ASTM International, West Conshohocken, 2010.

[35] British Energy, "How an AGR power station works - $\quad$ https://archive.uea.ac.uk/ e680/energy/energy _links/nuclear/How_an_AGR_power_station_works.pdf," 27/08/2017. 
[36] Nuclear Decomissioning Authority, "Oxide Fuels," Tech. Rep. June, NDA, 2012.

[37] J. Kyffin, "The Technical Case for Interim Storage of AGR Fuel," in Sustainable Nuclear Energy Conference 2014, 2014.

[38] Sellafield, "Sellafield Plan Issue 1 (2011) - https://www.cumbria.gov.uk/elibrary/ Content/Internet/538/755/1929/6478/41333114920.pdf," Date accessed $27 / 08 / 2017$.

[39] J. Hunt, A. Ferrari, A. Lita, M. Crosswhite, B. Ashley, and A. E. Stiegman, "Microwave-Specic Enhancement of the Carbon-Carbon Dioxide (Boudouard) Reaction," The Journal of Physical Chemistry C, vol. 117, pp. 26871-26880, 2013.

[40] K. Verrall, "AGR Coolant Chemistry and Carbon Deposition Overview 2012 - http://www.rsc.org/images/Karen_Verrall_tcm18-219419.pdf," Accessed $27 / 08 / 2017$.

[41] ASTM International, "Standard Guide for Development and Use of a Galvanic Series for Predicting Galvanic Corrosion Performance - G82-98," tech. rep., ASTM International, West Conshohocken, 2014.

[42] IAEA, "Spent fuel performance Assessment and research - IAEATECDOC-1680," tech. rep., IAEA, Vienna, 2012.

[43] C. Addison and R. Shaw, "Irradiated Nuclear Fuel Storage at the Sellafield (UK) Reprocessing Plant - IAEA-TECDOC-418," Tech. Rep. Long Term Wet Spent Nuclear Fuel Storage - IAEA-TECDOC-418, IAEA, Vienna, 1987.

[44] A. Iversen, "Aqueous Corrosion of Stainless Steels," in Shreir's Corrosion, ch. 3.04, Elsevier B.V., 2010. 
[45] C. M. Chan, D. L. Engelberg, and W. S. Walters, "Performance Characterisation of AGR Fuel Cladding Relevant to Long-term in-Pond Storage in pH-moderated Aqueous Environment," in Top Fuel, 2015.

[46] Outokumpu, "Handbook of Stainless Steel http://www.outokumpu.com/sitecollectiondocuments/outokumpustainless-steel-handbook.pdf," Accessed 27/08/2017.

[47] INCO, "Corrosion Resistance of the Austenitic Chromium-Nickel Stainless Steels in Chemical Environments," tech. rep., The International Nickel Company Incorporated, New York, 1963.

[48] J. A. Richardson, "Corrosion in alkalis," in Shreir's Corrosion, pp. 11911206, Elsevier B.V., 2010.

[49] A. Bhattacharya and P. M. Singh, "Corrosion of Duplex Stainless Steels in High pH Caustic Solution," NACE Corrosion 2008, no. 08194, pp. 1-14, 2008 .

[50] T. L. S. L. Wijesinghe and D. J. Blackwood, "Real time pit initiation studies on stainless steels: The effect of sulphide inclusions," Corrosion Science, vol. 49, no. 4, pp. 1755-1764, 2007.

[51] C. H. Phuah, Corrosion of Thermally-Aged Advanced Gas Reactor Fuel Cladding. Thesis, Imperial College London, 2012.

[52] S. Lyon, "Corrosion of Tantalum and Niobium and their Alloys," in Shreir's Corrosion, ch. 3.15, pp. 2135-2150, Elsevier B. V., 2010.

[53] A. Robin, "Corrosion behaviour of niobium in sodium hydroxide solutions," Journal of Applied Electrochemistry, vol. 34, no. 6, pp. 623-629, 2004.

[54] G. S. Frankel and R. A. Cottis, "Principles of corrosion in liquids," in Shreir's Corrosion, ch. 2.01, pp. 725-730, Elsevier B.V., 2010. 
[55] G. Hinds and J. Thomas, "The Electrochemistry of Corrosion," Journal of The Electrochemical Society, vol. 74, no. 1, pp. 495-517, 1938.

[56] R. Akid, "Corrosion of Engineering Materials," in Handbook of Advanced Materials (J. Wessel, ed.), ch. Corrosion, pp. 487-542, Sheffield: John Wiley and Sons, 2004.

[57] G. T. Burstein, "Passivity and localized corrosion," in Shreir's Corrosion, vol. 1, ch. 2.02, pp. 731-752, Elsevier B.V., 2010.

[58] C. Lefrou, R. P. Nogueira, F. Huet, and H. Takenouti, "Electrochemistry," in Shreir's Corrosion, ch. 1.02, pp. 13-51, Elsevier B.V., 2010.

[59] T. P. Hoar and W. R. Jacob, "Breakdown of Passivity of Stainless Steel by Halide Ions," Nature, vol. 216, pp. 1299-1301, dec 1967.

[60] G. S. Frankel, "Pitting Corrosion of Metals," Journal of The Electrochemical Society, vol. 145, no. 6, p. 2186, 1998.

[61] P. Schmutz, "Surfaces, Interfaces, and their Applications II," in Surfaces, Interfaces, and their Applications II, ch. 6 Pitting, pp. 1-25, Dübendorf: EMPA Dubendorf, mar 2013.

[62] H. Bohni and H. Uhlig, "Effect of alloyed Re on the critical pitting potentials of $18 \% \mathrm{Cr} / 10 \% \mathrm{Ni}$ stainless steels," Corrosion Science, vol. 9, no. 5, pp. 353-355, 1968.

[63] J. R. Galvele, "Transport Processes and the Mechanism of Pitting of Metals," Journal of The Electrochemical Society, vol. 123, no. April, p. 464, 1976.

[64] A. I. Almashad and S. A. Hossain, "Environmental effects and local chemistry development," in Prevention of metal corrosion : new research (M. Nunez, ed.), ch. Phenomenol, p. 310, Nova Science Publishers, 2007. 
[65] M. Alvarez and J. Galvele, "Pitting Corrosion," in Shreir's Corrosion, ch. 2.04, pp. 772-800, Elsevier B.V., 2010.

[66] W. Schwenk, "Theory Of Stainless Steel Pitting," Corrosion, vol. 20, pp. 129t-137t, apr 1964.

[67] M. P. Ryan, N. J. Laycock, H. S. Isaacs, and R. C. Newman, "Corrosion Pits in Thin Films of Stainless Steel," Journal of The Electrochemical Society, vol. 146, no. 1, p. 91, 1999.

[68] N. J. Laycock, S. P. White, J. S. Noh, P. T. Wilson, and R. C. Newman, "Perforated Covers for Propagating Pits," Journal of The Electrochemical Society, vol. 145, p. 1101, apr 1998.

[69] P. Ernst, N. Laycock, M. Moayed, and R. Newman, "The mechanism of lacy cover formation in pitting," Corrosion Science, vol. 39, no. 6, pp. 1133$1136,1997$.

[70] I. Rosenfeld and I. Danilov, "Electrochemical aspects of pitting corrosion," Corrosion Science, vol. 7, no. 3, pp. 129-142, 1967.

[71] S. M. Ghahari, A. J. Davenport, T. Rayment, T. Suter, J.-P. Tinnes, C. Padovani, J. A. Hammons, M. Stampanoni, F. Marone, and R. Mokso, "In situ synchrotron X-ray micro-tomography study of pitting corrosion in stainless steel," Corrosion Science, vol. 53, pp. 2684-2687, sep 2011.

[72] S. M. Ghahari, In Situ Synchrotron X-Ray Characterisation and Modelling of Pitting Corrosion of Stainless Steel. Thesis, University of Birmingham, 2012.

[73] J. Soltis, "Passivity breakdown, pit initiation and propagation of pits in metallic materials Review," Corrosion Science, vol. 90, pp. 5-22, jan 2015.

[74] G. S. Frankel, L. Stockert, F. Hunkeler, and H. Boehni, "Metastable Pitting of Stainless Steel.," Corrosion, vol. 43, no. 7, pp. 429-436, 1987. 
[75] H. Boehni, "Breakdown of passivity and localized corrosion processes," Langmuir, vol. 3, pp. 924-930, nov 1987.

[76] T. Hoar, D. Mears, and G. Rothwell, "The relationships between anodic passivity, brightening and pitting," Corrosion Science, vol. 5, no. 4, pp. 279-289, 1965.

[77] G. Burstein, P. Pistorius, and S. Mattin, "The nucleation and growth of corrosion pits on stainless steel," Corrosion Science, vol. 35, pp. 57-62, jan 1993.

[78] A. Riley, D. Wells, and D. Williams, "Initiation events for pitting corrosion of stainless steel?," Corrosion Science, vol. 32, pp. 1307-1313, jan 1991.

[79] T. Suter, T. Peter, and H. Böhni, "Microelectrochemical Investigations of MnS Inclusions," Materials Science Forum, vol. 192-194, pp. 25-40, 1995.

[80] G. T. Burstein and R. M. Souto, "Observations of Localised Instability of Passive Titanium in Chloride Solution," Electrochimica Acta, vol. 40, no. 12 , pp. 1881-1888, 1995.

[81] Y. Zhu and D. E. Williams, "Scanning Electrochemical Microscopic Observation of a Precursor State to Pitting Corrosion of Stainless Steel," Journal of The Electrochemical Society, vol. 144, no. 3, p. L43, 1997.

[82] H. S. Isaacs and B. Vyas, "Scanning Reference Electrode Techniques in Localised Corrosion," Tech. Rep. BNL-26094, Brookhaven National Laboratory, Upton, New York, 11973, 1979.

[83] R. Ke and R. Alkire, "Surface-Analysis of Corrosion Pits Initiated at MnS Inclusions in 304 Stainless-Steel," Journal of the Electrochemical Society, vol. 139, no. 6, pp. 1573-1580, 1992. 
[84] R. Ke and R. Alkire, "Initiation of corrosion pits at inclusions on 304 stainless steel," Journal of the Electrochemical Society, vol. 142, no. 12, pp. 4056-4062, 1995.

[85] N. Alonso-Falleiros and S. Wolynec, "Correlation between Corrosion Potential and Pitting Potential for AISI 304L Austenitic Stainless Steel in 3.5\% NaCl Aqueous Solution," Materials Research, vol. 5, no. 1, pp. 77-84, 2002.

[86] C. Honess and A. Harrison, "Importance of surface finish in the design of stainless steel - http://www.bssa.org.uk/publications.php?id=97," Accessed 27/08/2017.

[87] G. Williams and H. N. Mcmurray, "Localized Corrosion of Magnesium in Chloride-Containing Electrolyte Studied by a Scanning Vibrating Electrode Technique," Journal of the Electrochemical Society, vol. 155, no. 7, pp. C340-C349, 2008.

[88] J. Sullivan, N. Cooze, C. Gallagher, T. Lewis, T. Prosek, and D. Thierry, "In-situ monitoring of corrosion mechanisms and phosphate inhibitor surface deposition during corrosion of Zinc Magnesium Aluminium (ZMA) alloys using novel time-lapse microscopy.," Faraday Discuss., pp. 1-19, 2015.

[89] U.S Atomic Energy Commission, "Control of the use of Sensitised Stainless Steel," Tech. Rep. Regulatory guide 1.44, U.S Atomic Energy Commission, 1973.

[90] A. S. El-Amoush, "Intergranular corrosion behavior of the 7075-T6 aluminum alloy under different annealing conditions," Materials Chemistry and Physics, vol. 126, pp. 607-613, apr 2011.

[91] S. M. Bruemmer and G. S. Was, "Microstructural and microchemical mechanisms controlling intergranular stress corrosion cracking in light-water- 
reactor systems," Journal of Nuclear Materials, vol. 216, pp. 348-363, oct 1994.

[92] D. L. Engelberg, "Intergranular Corrosion," in Shreir's Corrosion, ch. 2.06, pp. 810-827, Elsevier B.V., 2010.

[93] ASM International, "Basic Understanding of Weld Corrosion," Tech. Rep. Ref 1, ASM International, 2006.

[94] S. Jain, N. D. Budiansky, J. L. Hudson, and J. R. Scully, "Surface spreading of intergranular corrosion on stainless steels," Corrosion Science, vol. 52 , pp. 873-885, mar 2010.

[95] D. Norris, C. Baker, C. Taylor, and J. Titchmarsh, "Radiation-induced segregation in $20 \mathrm{Cr} / 25 \mathrm{Ni} / \mathrm{Nb}$ Stainless Steel," in 15th Symposium on Effects of Radiation on Materials, (Berkeley), pp. 1-28, Nuclear Electric plc, 1990.

[96] E. Simonen and S. Bruemmer, "Mechanistic issues for modeling radiationinduced segregation," in Corrosion 1993, (Lousiana), Pacific Northwest Laboratory, NACE corrosion series, 1993.

[97] J. M. Perks and S. M. Murphy, "Modelling the Major Element Radiationinduced Segregation in Concentrated Fe-Cr-Ni Alloys," in Materials for nuclear core applications, BNES, pp. 165-169, UKAEA Atomic Energy Research Establishment, 1987.

[98] T. Allen and G. Was, "Modeling radiation-induced segregation in austenitic FeCrNi alloys," Acta Materialia, vol. 46, no. 10, pp. 3679-3691, 1998.

[99] C. A. English, S. M. Murphy, and J. M. Perks, "Radiation-induced Segregation in Metals," J. Chem Soc., Faraday Trans, vol. 86, no. 8, pp. 1263-1271, 1990. 
[100] A. D. Marwick, "Segregation in irradiated alloys: The inverse Kirkendall effect and the effect of constitution on void swelling," Journal of Physics F: Metal Physics, vol. 8, pp. 1849-1861, sep 1978.

[101] S. M. Bruemmer, E. P. Simonen, P. M. Scott, P. L. Andresen, G. S. Was, and J. L. Nelson, "Radiation-induced material changes and susceptibility to intergranular failure of light-water-reactor core internals," Journal of Nuclear Materials, vol. 274, no. 3, pp. 299-314, 1999.

[102] F. V. Nolfi, Phase transformations during irradiation. Applied Science Publishers, 1983.

[103] K. Nakata and I. Masaoka, "Solute segregation along non-migrated and migrated grain boundaries during electron irradiation in austenitic stainless steels," Journal of Nuclear Materials, vol. 150, no. 2, pp. 186-193, 1987.

[104] T. Allen and G. S. Was, "Radiation-enhanced Diffusion (RED) and Radiation-induced Segregation (RIS)," 2004.

[105] J. M. Perks, C. A. English, and A. D. Marwick, "Fundamental Aspects of Radiation-induced Segregation in Fe-Cr-Ni alloys," in Symposium on radiation-induced sensitisation of stainless steels, (CEGB), 1987.

[106] J. M. Perks, A. D. Marwick, and C. A. English, "A computer code to calculate radiation-induced segregation in concentrated ternary alloys.," tech. rep., UKAEA, AERE-R-12121, 1986.

[107] D. I. R. Norris, C. Baker, and J. M. Titchmarsh, "Compositional Profiles at Grain Boundaries in 20\% Cr/ 25\%Ni/ Nb Stainless Steel," in Symposium on Radiation-Induced Sensitisation of Stainless Steels, p. 86, 1986.

[108] D. I. R. Norris, C. Baker, and J. M. Titchmarsh, "A Study of Radiationinduced Sensitisation in 20/25/Nb Steel by Compositional Profile Measurements at Grain Boundaries," in Materials for nuclear core applications, BNES, pp. 277-283, 1987. 
[109] C. Barcellini, S. Pimblott, S. Dumbill, and M. G. Burke, "Grain boundary damage mechanisms in strained AGR claddings under irradiation," in DISTINCTIVE meeting: Theme 1 - AGR, Magnox and Exotic Spent Fuels, (Bristol), 2015.

[110] C. Moss and J. Sykes, "Thermal Sensitisation of 20Cr/25Ni/Nb Stainless Steel," in Symposium on radiation-induced sensitisation of stainless steels (D. I. R. Norris, ed.), (Berkeley), pp. 43-59, Department of metallurgy and science of materials, university of oxford, parks road, oxford, OX1 3PH, CEGB, 1987.

[111] G. Yi, A. T. Derrick, Y. Zhu, and M. L. Free, "A Collector Plate Mechanism-Based Classical Intergranular Precipitation Model for Al Alloys Sensitized at Different Temperatures," Metallurgical and Materials Transactions A, vol. 46, pp. 5393-5406, nov 2015.

[112] R. C. Ecob, R. C. Lobb, and V. L. Kohler, "The formation of G-phase in 20/25 Nb stainless steel AGR fuel cladding alloy and its effect on creep properties," Journal of Materials Science, vol. 22, no. 8, pp. 2867-2880, 1987.

[113] D. J. Powell, R. Pilkington, and D. A. Miller, "The Precipitation Characteristics of $20 \%$ Cr/25\% Ni-Nb Stabilised Stainless Steel," Acta Meturgica, vol. 36, no. 3, pp. 713-724, 1988.

[114] R. N. Clark, W. S. Walters, and G. Williams, "A Scanning Probe Investigation of Inter-Granular Corrosion in Sensitised Stainless Steel Nuclear Fuel Cladding," in NACE Corrosion 2016, (Vancouver), pp. 293-298, 2016.

[115] T. L. Burnett, S. A. McDonald, A. Gholinia, R. Geurts, M. Janus, T. Slater, S. J. Haigh, C. Ornek, F. Almuaili, D. L. Engelberg, G. E. Thompson, and P. J. Withers, "Correlative Tomography," Scientific Reports, vol. 4, p. 4711, 2014. 
[116] ASTM International, "Standard Practice for Microetching Metals and Alloys," tech. rep., ASTM International, West Conshohocken, 2012.

[117] British Standard (BS ISO 12732:2006), "Corrosion of metals and alloys Electrochemical potentiokinetic reactivation measurement using the double loop method (based on Cihal's method)," tech. rep., British Standards, 2006.

[118] A. P. Majidi and M. A. Streicher, "Potentiodynamic Reactivation Method for Detecting Sensitization in AISI 304 and 304L Stainless Steels," Corrosion, vol. 40, no. 8, pp. 393-408, 1984.

[119] H. N. McMurray and D. A. Worsley, "Research in Chemical Kinetics," in Research in Chemical Kinetics Volume 4 (R. Compton and G. Haancock, eds.), Oxford, UK: Blackwell Science, 1997.

[120] B. P. Wilson, Scanning Electrochemical Techniques for the Monitoring of Localised Corrosion on Automotive Steels. PhD thesis, Swansea University, 2003.

[121] W. Melitz, J. Shen, A. C. Kummel, and S. Lee, "Kelvin probe force microscopy and its application," Surface Science Reports, vol. 66, no. 1, pp. 1$27,2011$.

[122] M. Rohwerder and F. Turcu, "High-resolution Kelvin probe microscopy in corrosion science: Scanning Kelvin probe force microscopy (SKPFM) versus classical scanning Kelvin probe (SKP)," Electrochimica Acta, vol. 53, no. 2, pp. 290-299, 2007.

[123] J. W. Fielder, B. A. Hobson, and M. L. Pickett, "The stress corrosion cracking behaviour of stainless steel at temperatures below 50 degrees C," tech. rep., British Steel plc, London, 1990.

[124] ASTM International, "Standard Test Methods for Pitting and Crevice Corrosion Resistance of Stainless Steels and Related Alloys by use of Ferric 
Chloride Solution - G48-11," tech. rep., ASTM International, West Conshohocken, 2003.

[125] Research Solutions and Resources, "The Potential of Calomel Reference Electrodes - http://www.consultrsr.net/resources/ref/calomelpotl.htm," Accessed 19/08/2016.

[126] E. L Meites, Handbook of Analytical Chemistry. McGraw-Hill Inc.,US, 1st ed., 1963.

[127] M. Iannuzzi, "About pitting and repassivation potentials http://www.aboutcorrosion.com/2014/04/03/about-pitting-andrepassivation-potentials/," Accessed 19/08/2016.

[128] ASTM, "Standard Test Methods for Determining Average Grain Size," tech. rep., ASTM International, E112-12, 2014.

[129] S. Lyon, "Degradation of carbon and graphite," in Shreir's Corrosion, ch. 3.24, pp. 2271-2281, Elsevier B.V., 2010.

[130] E. A. Abd El Meguid, V. K. Gouda, and N. A. Mahmoud, "Pitting Corrosion Behaviour of Type SUS904L and SUS316L Stainless Steels in Chloride Solutions," Materials Transactions, vol. 35, no. 10, pp. 699 - 702, 1994.

[131] H. P. Leckie and H. H. Uhlig, "Environmental Factors Affecting the Critical Potential for Pitting in 188 Stainless Steel," Journal of The Electrochemical Society, vol. 113, no. 12, p. 1262, 1966.

[132] N. J. Laycock and R. C. Newman, "Localised dissolution kinetics, salt films and pitting potentials," Corrosion Science, vol. 39, no. 10-11, pp. 17711790, 1997.

[133] A. Laferrere, R. Burrows, R. N. Clark, C. Glover, G. Williams, O. Payton, and L. Picco, "In Situ Imaging of Corrosion Processes in Nuclear Fuel 
Cladding," Corrosion, Engineering, Science and Technology, vol. 52, no. 8, pp. 596-604, 2017.

[134] International Atomic Energy Agency, "Durability of spent nuclear fuels and facility components in wet storage," tech. rep., Nuclear fuel cycle and materials section, IAEA, Wagramerstrasse 5, P.O. Box 100, A-1400 Vienna, Austria, 1998.

[135] S. B. Lyon, "Corrosion of tin and its alloys," in Shreir's Corrosion, vol. 1, ch. 3.12, pp. 2068-2077, Elsevier, 2010.

[136] S. Uchida, Y. Morishima, T. Hirose, T. Miyazawa, T. Satoh, Y. Satoh, and W. Wada, "Effects of Hydrogen Peroxide on Corrosion of Stainless Steel (VI)," Journal of Nuclear Science and Technology, vol. 44, pp. 758-766, 2007.

[137] AZO Materials, "Stainless Steel - Grade 310 (UNS S31000) - $\quad$ http://www.azom.com/article.aspx?ArticleID=966," Accessed $18 / 08 / 2017$.

[138] J. Li and D. Lampner, "In-situ AFM study of pitting corrosion of Cu thin films," Colloids and Surfaces A: Physicochemical and Engineering Aspects, vol. 154, no. 1-2, pp. 227-237, 1999.

[139] V. Guillaumin, P. Schmutz, and G. S. Frankel, "Characterization of Corrosion Interfaces by the Scanning Kelvin Probe Force Microscopy Technique," Journal of The Electrochemical Society, vol. 148, no. 5, p. B163, 2001.

[140] Q. Zhang, R. Wang, M. Kato, and K. Nakasa, "Observation by atomic force microscope of corrosion product during pitting corrosion on SUS304 stainless steel," Scripta Materialia, vol. 52, pp. 227-230, feb 2005.

[141] A. Laferrere, R. Burrows, R. N. Clark, C. Glover, G. Williams, O. Payton, and L. Picco, "In situ imaging of corrosion processes in nuclear fuel cladding," in Eurocorr, 2015. 
[142] M. Matula, L. Hyspecka, M. Svoboda, V. Vodarek, C. Dagbert, J. Galland, Z. Stonawska, and L. Tuma, "Intergranular corrosion of AISI 316L steel," Materials Characterization, vol. 46, pp. 203-210, feb 2001.

[143] P. Marcus and V. Maurice, "Scanning Probe Microscopies," Shreir's Corrosion, no. UMR 7045, pp. 1430-1442, 2010.

[144] P. Eaton and P. West, Atomic Force Microscopy. OUP Oxford, 2010.

[145] L. Lacroix, L. Ressier, C. Blanc, and G. Mankowski, "Combination of AFM, SKPFM, and SIMS to Study the Corrosion Behavior of S-phase particles in AA2024-T351," Journal of The Electrochemical Society, vol. 155, no. 4, p. C131, 2008.

[146] P. Schmutz and G. S. Frankel, "Corrosion Study of AA2024-T3 by Scanning Kelvin Probe Force Microscopy and In Situ Atomic Force Microscopy Scratching," The Electrochemical Society, vol. 145, no. 7, pp. 2295-2305, 1998.

[147] S. Riva, K. V. Yusenkoa, C. M. Fung, J. R. Searle, R. N. Clark, N. P. Lavery, and S. G. Brown, "Formation and disruption of W-phase in High-Entropy Alloys," Metals, vol. 106, no. 6, 2016.

[148] P. P. Leblanc and G. S. Frankel, "Investigation of Filiform Corrosion of Epoxy-Coated 1045 Carbon Steel by Scanning Kelvin Probe Force Microscopy," Journal of The Electrochemical Society, vol. 151, no. 3, p. B105, 2004 .

[149] J. Sullivan, S. Mehraban, and J. Elvins, "In situ monitoring of the microstructural corrosion mechanisms of zinc magnesium aluminium alloys using time lapse microscopy," Corrosion Science, vol. 53, pp. 2208-2215, jun 2011. 